\title{
A]l100 988884
}

\section{Hinc Parameters and Computed Speatra}

For Water Vanor Bands at $2.7 \mu$

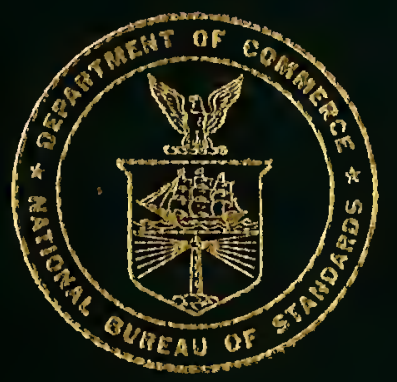






\section{Line Parameters and Computed Spectra for Water Vapor Bands at $2.7 \mu$}

David M. Gates, Robert F. Calfee, David W. Hansen, and W. S. Benedict

This work was supported by the Advanced Research Projects Agency under contract number 250-61: code number 7400-DO.
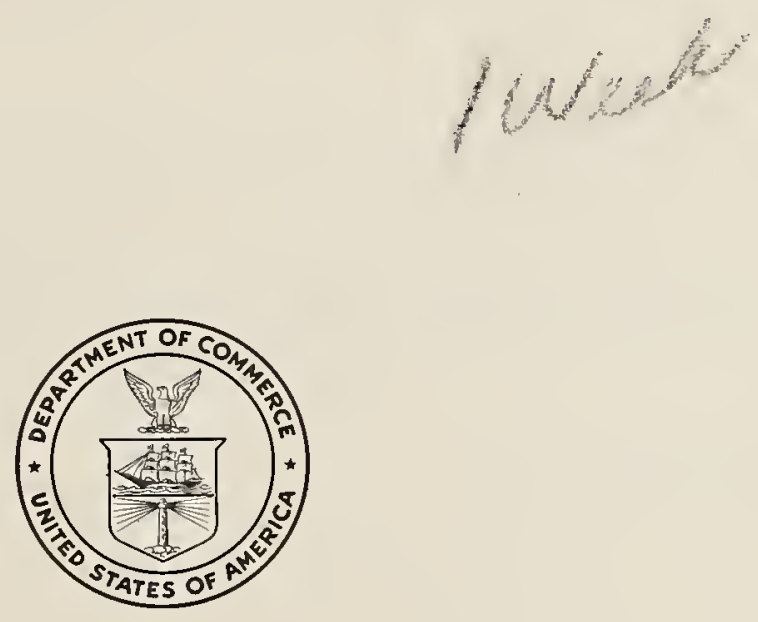

National Bureau of Standards Monograph 71

Issued August 3, 1964

For sale by the Superintendent of Documents, U.S. Government Printing Office

Washington, D.C., 20402 - Price $\$ 2.00$ 


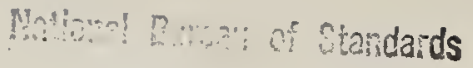

JAN - 1935

122,859

QC100
.4556

cop. 2

Library of Congress Catalog Card Number: $63-62353$ 


\section{Foreword}

Knowledge concerning the infrared transmittance properties of the atmosphere is important to the study of the radiative balance of the atmosphere, astronomical observation, missile detection and tracking, laser communication, satellite weather reconnaissance, and optical communication. This Monograph is one of a series intended to provide fundamental information required for the estimation and interpretation of atmospheric transmittance at infrared frequencies.

Part I of this Monograph presents the fundamental properties of the $2.7 \mu$ water vapor band in terms of line absorptions and the integrated transmittance at any frequency for infinite resolution.

Part II presents examples of the "degraded" transmittance for finite resolution, as a function of the spectral slit width, concentration, and pressure with which direct observations may be compared.

A. V. Astin, Director. 


\section{Contents}

Part I Page

1. Introduction.

2. Line positions

3. Line strengths and half-widths

4. Discussion .

5. References..... VIII

\section{Part II}

1. Introduction

2. Transmission

3. Computational procedure

4. Degraded spectra_._. 87

5. References_... 88 


\title{
Line Parameters and Computed Spectra for Water Vapor Bands at $2.7 \mu$
}

\section{Part I. Line Positions, Strengths, and Half-Widths for Water Vapor Bands $\nu_{1}, 2 \nu_{2}$, and $\nu_{3}$ in the Interval 2857 to $4444 \mathrm{~cm}^{-1}$}

\author{
David M. Gates, Robert F. Calfee, David W. Hansen, and W. S. Benedict \\ The spectral line positions, strengths and half-widths of the $\nu_{1}, 2 \nu_{2}$, and $\nu_{3}$ bands con- \\ stituting the $2.7 \mu$ region of water vapor are presented for the range $2857-4444 \mathrm{~cm}^{-1}$. Lines \\ attributed to the $\mathrm{H}_{2} \mathrm{O}^{17}$ and $\mathrm{H}_{2} \mathrm{O}^{1:}$ molecules in this region have also been calculated and \\ included along with the "hot lines" produced by 030-010, 011-010, and 110-010 transitions. \\ The computations were carried out for values of $J$ through 15 .
}

\section{Introduction}

It has been evident for many years that the only fundamental approach to atmospheric transmission problems at infrared frequencies would be through an understanding of the complete detailed spectrum, band for band and line for line. However, the enormous complexity of this procedure has deterred many investigators from this approach. The problem is further complicated by the fact that the strength (intensity) and half-width of each line is dependent upon temperature and pressure as well as being dependent upon the concentration of the absorbing gas. Faced with the need to intercompare various spectroscopic observations of atmospheric transmission at infrared frequencies, both at low resolution and at high resolution, it became evident that one had to tackle the problem of computing the positions, strengths, and halfwidths of all of the spectral lines within any region of the spectrum. It would be possible, at least in principle, to compute the integrated effect of all these lines on the transmission at any given position in the spectrum.

Two absorbing constituents, which are of greatest importance for atmospheric transmission problems in the infrared region of the spectrum, are water vapor and carbon dioxide. It was decided to work with water vapor first and to analyze the broad absorption region around $2.7 \mu$, which involves the bands $\nu_{3}, \nu_{1}$, and $2 \nu_{2}$. The many excellent experimental observations and analyses which have now been made of the water vapor spectrum, coupled with computations of line width and intensity, make it possible to systematically undertake such a program. Furthermore, the advent of the large computer makes the task of computing the transmission for given atmospheric conditions a manageable problem.

The water vapor molecule possesses a very complicated spectrum with irregularly spaced lines. It is triatomic, has three normal modes of vibration, and possesses three very different moments of inertia, hence falling into the category known as asymmetric top rotators. The general theory concerning such a molecule is to be found fully discussed by Herzberg [1954]. The analytical formulation of the rotational structure of $\mathrm{H}_{2} \mathrm{O}$ is well known [Mecke, 1933; Randall, Dennison, Ginsburg, and Weber, 1937; and Benedict and Plyler, 1951], and will not be repeated here.

The strong, broad absorption region around $2.7 \mu$ is caused by the following vibrations: $\nu_{3}$, a strong stretching fundamental with the change of dipole moment during the vibration along the least axis of inertia, giving rise to a type $\mathrm{A}$ band with the origin at $3755.92 \mathrm{~cm}^{-1} ; \nu_{1}$, a weaker stretching fundamental with the change of dipole moment along the intermediate axis of inertia (type $\mathrm{B}$ band), with the origin at $3657.08 \mathrm{~cm}^{-1}$; and $2 \nu_{2}$, the first overtone of the bending fundamental (type $B$ band) with the band origin at $3151.60 \mathrm{~cm}^{-1}$. The relative intensities of these 
bands are approximately $\nu_{3}: \nu_{1}: 2 \nu_{2}=120: 12: 1$. In addition there are lines due to bands of isotopic configurations of $\mathrm{H}_{2} \mathrm{O}$ whose normal abundances are $\mathrm{H}_{2} \mathrm{O}^{18}, 0.20$ percent; $\mathrm{H}_{2} \mathrm{O}^{17}, 0.037$ percent; and $\mathrm{HDO}, 0.027$ percent. Lines are also due to bands in which the lower level is vibrationally excited. At atmospheric temperatures, $<320^{\circ} \mathrm{K}$, the only such level which must be considered is the lowest, $\nu_{2}$, and its abundance is very small, 0.033 percent at $287^{\circ} \mathrm{K}$.

In this note we will present a tabulation of the relevant parameters; frequency, strength, width and lower-level energy, for all water-vapor lines which can make significant contributions to atmos- pheric absorption between 2857-4444 $\mathrm{cm}^{-1}$ (3.5$2.25 \mu$ ). In the "windows" beyond these limits there is a small contribution from very weak lines belonging to the three principal bands, however the main contribution to the weak water vapor absorption in the "windows" is due to other bands. At the lower frequency side the $\nu_{1}$ band of HDO predominates $\left(\nu_{0}=2723.66 \mathrm{~cm}^{-1}\right)$, while at the high frequency side the weak absorption is due to $3 \nu_{2}\left(\nu_{0}=4666.72 \mathrm{~cm}^{-1}\right)$. Because of their extreme weakness, the information concerning these bands is not as extensive as those treated in this paper.

\section{Line Positions}

Table I contains a listing in order of frequency of approximately 4500 lines giving their position $\left(\mathrm{cm}^{-1}\right)$, intensity $\left(\mathrm{cm}^{-1} / \mathrm{g} \mathrm{cm}^{-2}\right)$, half-width $\left(\mathrm{cm}^{-1} / \mathrm{atm}\right)$, rotational identification for upper and lower states, energy of the lower state $\left(\mathrm{cm}^{-1}\right)$, rotational transition strength, and band designation. The positions of the lines as tabulated here are computed from the energy levels. The energy levels in turn are derived from observed line positions. All lines observed either in the solar spectrum [Benedict, 1955 and 1957], or in laboratory spectra [Plyler and Tidwell, 1957 and Gloersen, 1956], with the exception of a few very weak lines, are accounted for. It is possible to predict weaker lines that have not been observed due to insufficient resolving power under the proper conditions. These weak lines fall into several categories. Some of them are transitions of low inherent probability between levels whose energies have been established from several observed lines of higher probability. For these the line position can be predicted to within \pm 0.05 $\mathrm{cm}^{-1}$, since the ground state energy levels are known to within this limit. We have, however, also included lines whose positions are much less certain, involving transitions between higher energy states, observed weakly or inferred by extrapolation of one or two lines in observed series. Most of the lines attributed to $\mathrm{H}_{2} \mathrm{O}^{18}$ and $\mathrm{H}_{2} \mathrm{O}^{17}$ also have not been observed, but their positions should be calculable to within $\pm 2 \mathrm{~cm}^{-1}$, using for $\mathrm{H}_{2} \mathrm{O}^{18}$ the rotational energy shifts tabulated [Benedict, 1957], and the following vibrational shifts:

$\nu_{3}=-15.3 \mathrm{~cm}^{-1} ; \nu_{1}=-7.4 \mathrm{~cm}^{-1} ; 2 \nu_{2}=-12.5 \mathrm{~cm}^{-1}$.

The values of $\mathrm{H}_{2} \mathrm{O}^{16}$ energy levels used in constructing table I do not differ greatly from those previously published [Benedict 1957], but are believed to be more self-consistent, the ground-state levels being based on more recent precise observations [Rao et al., 1962; Delbouille et al., 1963]. 'They also include higher levels than previously published, the intention being to include an entry for all levels, even if dubious, that would lead to transitions whose strength at $287^{\circ} \mathrm{K}$ is greater than $10^{-2} \mathrm{~cm}^{-1} / \mathrm{g} \mathrm{cm}^{-2}$; in the most completely analyzed band, $\nu_{3}$, the strengths extend to $10^{-3} \mathrm{~cm}^{-1} / \mathrm{g} \mathrm{cm} \mathrm{cm}^{-2}$. The upper state levels may be found from the table by adding the value of the line position in the first column to the lower energy state in the $E^{\prime \prime}$ column.

The notation used to designate the rotational states of the asymmetric rotator [King et al., 1943] is $J K_{a} K_{c}$, where $K_{a}$ and $K_{c}$ run from 0 to $J$. $J$ is the rotational quantum number of total angular momentuin; $K_{a}$ and $K_{c}$ are indices that in the limiting case of the symmetric rotator would become the quantum numbers associated with the angular momentum of the molecule about $A$ and $C$ axes, respectively. The other notation in common use is $J_{\tau}$, where $\tau=K_{a}-K_{c}$. The selection rules for the asymmetric top molecule are given as follows:

$$
\Delta J=0, \pm 1
$$

Axis of Dipole Moment

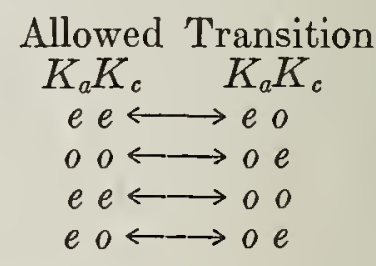

$$
\begin{aligned}
& A \text { (least) } \\
& B \text { (intermediate) } \\
& e=\text { even, } o=\text { odd, }
\end{aligned}
$$

Hence, it is seen from the selection rules for type $A$ bands $\Delta K_{a}-\Delta K_{c}$ is odd and for type $B$ bands $\Delta K_{a}-\Delta K_{c}$ is even.

'The energy levels for the four vibrational states for each rotational level $J K_{a} K_{c}$ were punched on cards. A computer program was devised to select the correct transitions in terms of the selection rules just given. The line positions were calculated for each of the three bands by subtracting the energy value of the ground state from that of each of the upper states. These are the line positions in $\mathrm{cm}^{-1}$ listed in the first column of table $\mathrm{I}$.

The quantum number identifications of the upper and lower states are given in columns 4 and 5. Each column contains the $J, K_{a}$, and $K_{c}$ values in that order. The sixth column lists the ground state energy value in $\mathrm{cm}^{-1}$. 


\section{Line Strengths and Half-Widths}

The intensity of every line in a rotationvibration band, assuming no interaction effects between vibration and rotation, may be written as the product of a vibrational factor, $S_{v}$, common to all lines in the band and a rotational factor $S_{r}$, for the particular rotational transition involved. It is customary to define $S_{v}$ as the sum of all the transitions in the band so that

$$
S=S_{o} S_{r}\left(\nu / \nu_{0}\right) / Q_{r}
$$

where $Q_{r}$ is the rotational partition function given by

$$
Q_{r}=\sum_{J}(2 J+1) g \exp \left(-E^{\prime \prime} / k T\right)
$$

where $g$ is a statistical weighting factor depending upon the symmetry of the level, and $E^{\prime \prime}$ is the ground level energy. The values of $g$ are:

$$
\begin{aligned}
& g=1 \text { for even } \tau^{\prime \prime} \\
& g=3 \text { for odd } \tau^{\prime \prime}
\end{aligned}
$$

where $\tau=K_{a}-K_{c}$. The value of $Q_{r}$ is evaluated to be 167.7 at a temperature of $287^{\circ} \mathrm{K}$ and varies with temperature approximately with $T^{3 / 2}$.

The rotational line strength, $S_{r}$ is:

$$
S_{r}=L \cdot g \cdot \exp \left(-E^{\prime \prime} / k T\right)
$$

where $L$ is called the rotational transition strength, defined to be the sum of the squares of the magnitude of the direction cosine matrix elements evaluated for the asymmetric rotator.

A full discussion of how $L$ is evaluated for the rigid asymmetric rotator has been given by Cross, Hainer, and King [1944]. Very useful tables of $L$ as a function of the asymmetry parameter have been computed by Schwendeman and Laurie [1958] for all transitions up to $J \leq 12$ at intervals of 0.1 in $\kappa$, and by Wacker and Pratto [1963] for higher $J$ at a slightly coarser interval in $\kappa$. For the ground state of $\mathrm{H}_{2} \mathrm{O}, \quad \kappa=\frac{2 B-(A+C)}{A-C}=$ -0.4377 . It is slightly different from that value in the three excited vibrational states considered here. We have calculated $L$ by interpolation to $\kappa=-0.4377$. The resulting values appear in the seventh column of table $I$. It is recognized that this results only in a first approximation to the true line strength; in addition to the neglect of the changed $\kappa$ in the upper state, other interaction effects may be expected to enter. Their importance will be discussed shortly.

The values used for $S_{v}$ in the present computation were as follows:

$$
\begin{aligned}
\nu_{3}, S_{v} & =2.63 \times 10^{5} \mathrm{~cm}^{-1} / \mathrm{g} \mathrm{cm}^{-2} \\
\nu_{1}, S_{v} & =2.7 \times 10^{4} \mathrm{~cm}^{-1} / \mathrm{g} \mathrm{cm}^{-2} \\
2 \nu_{2}, S_{v} & =2.21 \times 10^{3} \mathrm{~cm}^{-1} / \mathrm{g} \mathrm{cm}^{-2} .
\end{aligned}
$$

The value for $\nu_{3}$ is believed to be the most reliable; it is based on a recent determination, Jaffe and
Benedict [1963], of the strength of a well resolved line near the band center by the dispersion method and agrees well with other measurements of the total band absorption. The values for $\nu_{1}$ and $2 \nu_{2}$ are based on intercomparisons of the relative strengths of lines of the three bands, principally in the region $3250-3500 \mathrm{~cm}^{-1}$. Some confirmation of the estimate for $2 \nu_{2}$ may be obtained from the low resolution studies of Burch and Williams [1960] for the low-frequency portion of the band which is free from overlapping by $\nu_{1}$.

The value for $\nu_{1}$ is the least certain, particularly as this relatively weak fundamental vibration appears to show interaction effects similar to those encountered in $\nu_{2}$, with lines in the high frequency branches showing decreases in strength, and those in the low frequency branches showing increases. The general theory for these effects suggests that a number of processes may be acting in combination so that it is difficult to make a calculation. The dominant processes in $\nu_{1}$ would appear to be interaction with the pure rotation (permanent dipole moment), increasing principally with rotation about $A$ axis, and Coriolis coupling of the $\nu_{1}$ and $\nu_{3}$ vibrations. In the present computation, no attempt was made to correct for these general effects. This may result in an overestimate of many of the high $J$ lines in the high frequency branches by factors of 200 to 300 percent, and in underestimates of the highest $J$ lines in the low frequency branches by 50 to 100 percent.

The interaction effects do not appear to be large in $2 \nu_{2}$, except possibly in some of the weaker branches, such as $P_{3 \overline{1}}$.

In $\nu_{3}$, present indications are that interaction effects do not have a noticeable influence on the high $L$ lines of the $R_{0 \overline{1}}$ and $P_{01}$ branches, although experimental confirmation would be desirable. However, some of the weaker branches appear to show interaction effects [Benedict, 1955]; in particular the $Q_{2 \overline{1}}$, and $Q_{3 \overline{2}}$, and $Q_{4 \overline{3}}$ branches are increasingly weaker than calculated. In addition to the general interaction effects just mentioned, there are instances of accidental perturbation when lines from a common lower state to upper state levels of the same $J$ in different bands lie close together. In these cases the weaker line, usually in $\nu_{1}$, "borrows" strength from the stronger, $\nu_{3}$ line. For these cases the values of $L$ have been adjusted so that the total strength remains constant, but shared in a desirable proportion. In the table such enhanced lines are designated "f" at the right margin. The "lending" line is designated "- "

Although the rigid line strengths may be in some cases uncertain for the reasons outlined, it is hoped that their publication in this form will be useful. Comparison of the composite transmission spectra derived from table $I$ in various spectral regions with observed transmission spectra at various pressures and temperatures, and further measurements 
of the absolute or relative strength of well-resolved lines, should reveal whether systematic deviations exist. If so, these may be accounted for by variations in $S_{v}$, or introduction of empirical or theoretical factors with the correct rotational dependence on the several bands.

Values of the half width of the lines are listed in column 3, table I of this paper. The halfwidths for the $\nu_{1}$ and $2 \nu_{2}$ lines were taken directly from the body of the tables published by Benedict and Kaplan [1959]; the half-widths for the $\nu_{3}$ lines were calculated from the average half width, $\bar{\gamma}$, as given in the last column of these tables. Estimates for higher $J$ lines not appearing in the tables by Benedict and Kaplan [1963] were made by extrapolations of these tables. It should be noted [Benedict and Kaplan, 1964] that the widths apply to water vapor in air rather than $\mathrm{N}_{2}$ as implied by Benedict and Kaplan [1959]. The accuracy of the widths is not known, but recent measurements [Saiedy, 1961] confirm that they should be satisfactory for absorption computations.

The band identification is given in the last column of table I. As mentioned, the symbols "+" and "-" denote lines in which the band is mixed by perturbation. An asterisk indicates a line in the corresponding band of the isotopic molecule $\mathrm{H}_{2} \mathrm{O}^{18}$, a double asterisk $\mathrm{H}_{2} \mathrm{O}^{17}$, and three asterisks a "hot" line.

\section{Discussion}

Line strengths were calculated at $287.7^{\circ} \mathrm{K}$ for all possible transitions in the three principal bands up to $J_{\max }=15$. The strongest line (404503 in $\nu_{3}$, at $3838 \mathrm{~cm}^{-1}$ ) has $S=9.23 \times 10^{3} \mathrm{~cm}^{-1} / \mathrm{gm}$ $\mathrm{cm}^{-2}$. Since the purpose of the table is to list all lines of possible significance in atmospheric transmission problems, the lower limit of $S$ was set at $10^{-3} \mathrm{~cm}^{-1} / \mathrm{g} \mathrm{cm} \mathrm{cm}^{-2}$ in the band wings and at $10^{-2}$ in the central region $3503-3950 \mathrm{~cm}^{-1}$, which includes all lines with $S>2 \times 10^{2}$. In addition weak lines were excluded if within 0.25 $\mathrm{cm}^{-1}$ of a strong line whose strength exceeded the strength of the weak line by $10^{3}$ or if within 0.5 $\mathrm{cm}^{-1}$ of a strong line whose strength exceeded the strength of the weak line by $4 \times 10^{3}$. This limitation excludes from the table a large proportion of the lines in the weak branches of the original bands and in the weak satellite bands due to $\mathrm{H}_{2} \mathrm{O}^{18}, \mathrm{H}_{2} \mathrm{O}$ ", and the "hot" transitions
011-010, 110-010, 030-010. No attempt was made to list lines in the $\nu_{3}$ band of HDO $\left(\nu_{0}=\right.$ $3704.25 \mathrm{~cm}^{-1}$ ) as it was determined that its strongest lines, with few exceptions, would be excluded by the above criteria. The very weak combination bands of HDO, $\nu_{1}+\nu_{2}\left(\nu_{0}=4100.05\right.$ $\left.\mathrm{cm}^{-1}\right)$, and $3 \nu_{2},\left(\nu_{0}=4145.59 \mathrm{~cm}^{-1}\right)$, also are not considered.

It must be emphasized that the exclusion of extremely weak lines from table I was for purposes of saving space in printing and time in transmission computations based on line-by-line integrations. The actual distribution in strength of the lines in the spectrum includes additional weak lines in the categories mentioned and other weaker lines of vibrational and rotational states, which have not been included here. These higher-state lines are important when considering the absorptivity or emissivity of water vapor at elevated temperatures.

\section{References}

Benedict, W. S. (1955), Comments on a table of solar spectrum wavelengths $11984 \mathrm{~A}$ to $25578 \mathrm{~A}$, O. C. Mohler, Univ. of Mich. Press.

Benedict, W. S. (1957), Comments on the spectra of telluric $\mathrm{H}_{2} \mathrm{O}$ and $\mathrm{CO}_{2}$ as observed in the solar spectrum, 2.8-23.7 microns, Mém. Soc. Roy. Sci. Liège, Special Vol. 2, 18-30.

Benedict, W. S. (1957), Identification of $\mathrm{H}_{2} \mathrm{O}^{18}$ and $\mathrm{H}_{2} \mathrm{O}^{17}$ telluric lines in the infrared solar spectrum, Mém. Soc. Roy. Sci. Liège, 4th Series, 18, 557-571.

Benedict, W. S., and L. D. Kaplan (1959), Calculation of line widths in $\mathrm{H}_{2} \mathrm{O}-\mathrm{N}_{2}$ collisions, J. Chem. Phys. 30, 338-399.

Ben sdict, W. S., and L. D. Kaplan (1963), Calculation of water-vapor line widths, self-broadened and $\mathrm{O}_{2}$-broadened, J. Quant. Spect. Rad. Trans. 3 (in press).

Benedict, W. S., and Earle K. Plyler (1951), Absorption spectrum of water vapor and carbon dioxide in the region of 2.7 microns, J. Res. NBS 46, 246-265.

Burch, Darrell E., and Dudley Williams (1960), Infrared absorption by minor atmospheric constituents, Scientific Renort 1 (AFCRL TN-60-674).

Cross, P. C., R. M. Hainer, and G. W. King (1944), The asymmetric rotor, II. Calculation of dipole intensities and line classification, J. Chem. Phys. 12, 210-243.

Delbouille, L., G. Roland, M. Migeotte, and J. W. Swensson (1963), A table of solar spectrum wavelengths, 7500-12000 $\AA$ (private communication).

Gloersen, Per (1956), High resolution spectroscopy in the near infrared, Dissertation, The Johns Hopkins University $(80 \mathrm{pp})$.
Herzberg, G. (1954), Infrared and Raman spectra of polyatomic molecules (632 pp). (Van Nostrand Co., Inc., New York).

Jaffe, J. H., and W. S. Benedict (1963), Strength of $\nu_{3}$ in $\mathrm{H}_{2} \mathrm{O}$. J. Quant. Spect. Rad. Trans. 3, 87-88.

King, G. W., R. M. Hainer, and P. C. Cross (1943), The asymmetric rotor, I. Calculation and symmetry classification of energy levels, J. Chem. Phys. 11, 27-42.

Mecke, R. (1933), Das Rotationschwingungsspektrum des Wasserdampfes. I, Z. Physik, 81, 313-335.

Plyler, Earle K., and E. D. Tidwell (1957), The precise measurement of the infrared spectrum of molecules of the atmosphere, Mém. Soc. Roy. Sci. Liège, 4th Series 18, 426-449.

Randall, H. M., D. M. Dennison, N. Ginsburg, and L. R. Weber (1937), The far infrared spectrum of water vapor, Phys. Rev. 52, 160-174.'

Rao, K. Nahahari, W. W. Brim, V. L. Sinnett, and Ralph H. Wilson (1962), Wavelength calibrations in the infrared IV. Use of a 1000-lines-per-inch Bausch and Lomb plane replica grating, J. Opt. Soc. 52, No. 8, 862-865.

Saiedy, F. (1961), Atmospheric observations of line intensity and half-width in the rotational and $\nu_{2}$ rotationvibration bands of water vapor, Quart. Roy. Met. Soc. 13, 578-587.

Schwendeman, R. H., and V. W. Laurie (1958), Table of line strengths for rotational transitions of asymmetric rotor molecules (Pergamon Press, Ltd., New York).

Wacker, Paul F., and Marlene R. Pratto (1963), Microwave spectral tables, Vol. II. Line strengths of asymmetric rotors, NBS Mono. 70. 
TABLE 1

PARAMETERS OF THE $2.7 \mu$ BAND OF WATER VAPOR

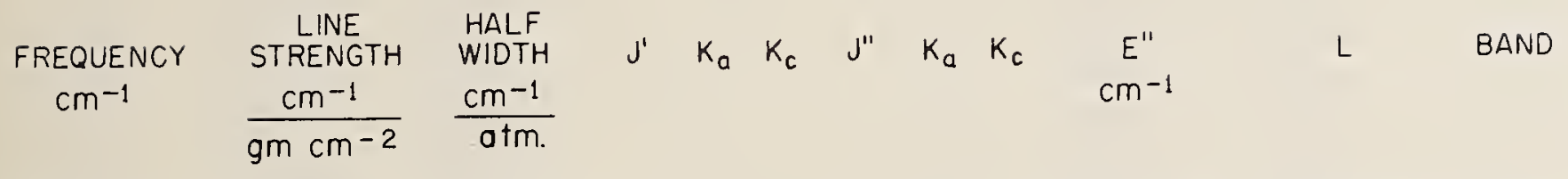

\begin{tabular}{|c|c|c|c|c|c|c|c|c|c|c|c|}
\hline $2864 \cdot 34$ & 0.0623 & 0.0769 & 8 & 3 & 6 & 9 & 4 & 5 & 1360.28 & 1.797448 & 2 NU2 2 \\
\hline 2867.99 & 0.0043 & 0.032 & 13 & 1 & 13 & 14 & 0 & 14 & 2073.66 & $12 \cdot 99955$ & $2 \mathrm{NU} 2$ \\
\hline 2867.99 & 0.0122 & 0.032 & 13 & 0 & 13 & 14 & 1 & 14 & 2073.66 & 12.99957 & $2 N U 2$ \\
\hline 2871.28 & 0.0804 & 0.0874 & 4 & 0 & 4 & 5 & 3 & 3 & 504.00 & 0.095779 & $2 N \cup 2$ \\
\hline $2872 \cdot 38$ & 0.0018 & 0.0743 & 5 & 1 & 5 & 5 & 4 & 2 & 610.12 & 0.003573 & $2 \mathrm{NU} 2$ \\
\hline 2874.17 & 0.0019 & 0.0701 & 10 & 4 & 6 & 11 & 5 & 7 & 1985.83 & 3.652101 & $2 \mathrm{NU} 2$ \\
\hline $2876 \cdot 28$ & 0.0062 & 0.0677 & 9 & 4 & 6 & 10 & 5 & 5 & 1724.72 & 3.272354 & 2 NU 2 \\
\hline 2879.73 & 0.1306 & 0.0868 & 5 & 1 & 5 & 6 & 2 & 4 & 602.77 & 0.254241 & 2 NU2 \\
\hline $2881 \cdot 42$ & 0.0011 & 0.0388 & 9 & 0 & 9 & 10 & 1 & 10 & 2705.18 & 9.013676 & 2 NU $2 * * *$ \\
\hline 2884.83 & 0.0014 & 0.0703 & 5 & 2 & 3 & 5 & 5 & 0 & $742 \cdot 10$ & 0.001850 & $2 N \cup 2$ \\
\hline 2884.88 & 0.0029 & 0.0571 & 10 & 5 & 6 & 11 & 6 & 5 & $2144 \cdot 12$ & 4.582064 & $2 \mathrm{NU} 2$ \\
\hline 2888.11 & 0.0023 & 0.0771 & 5 & 3 & 2 & 6 & 4 & 3 & 2398.39 & 3.153879 & $2 N \cup 2 * * *$ \\
\hline 2889.31 & 0.0010 & 0.0603 & 10 & 5 & 5 & 11 & 6 & 6 & 2142.69 & 4.667626 & $2 \mathrm{NU} 2$ \\
\hline $2890 \cdot 24$ & 0.0454 & 0.0324 & 12 & 1 & 12 & 13 & 0 & 13 & $1806 \cdot 78$ & 11.99672 & $2 \mathrm{NU} 2$ \\
\hline 2890.27 & 0.0151 & 0.0325 & 12 & 0 & 12 & 13 & 1 & 13 & 1806.78 & 11.99676 & $2 \mathrm{NU} 2$ \\
\hline 2890.82 & 0.0047 & 0.0871 & 4 & 2 & 3 & 5 & 3 & 2 & 2130.50 & 1.841317 & $2 \mathrm{~N} \cup 2 * * * *$ \\
\hline 2892.29 & 0.003 .1 & 0.0852 & 5 & 2 & 3 & 6 & 3 & 4 & 2271.70 & 2.452523 & $2 \mathrm{NU} 2 * * *$ \\
\hline 2893.09 & 0.0222 & 0.0711 & 9 & 4 & 5 & 10 & 5 & 6 & 1718.77 & 3.697402 & 2 NU 2 \\
\hline 2893.63 & 0.0034 & 0.0762 & 4 & 1 & 4 & 4 & 4 & 1 & 488.10 & 0.001222 & 2 NU2 \\
\hline 2893.77 & 0.4658 & 0.0839 & 6 & 2 & 5 & 7 & 3 & 4 & 842.38 & 0.996364 & $2 \mathrm{NU} 2$ \\
\hline 2896.00 & 0.0013 & 0.0729 & 6 & 2 & 4 & 6 & 5 & 1 & 888.63 & 0.010677 & $2 \mathrm{NU} 2$ \\
\hline 2896.21 & 0.0156 & 0.0741 & 7 & 0 & 7 & 7 & 3 & 4 & $842 \cdot 38$ & 0.033256 & 2 NU2 2 \\
\hline 2897.84 & 0.0014 & 0.0948 & 3 & 1 & 3 & 4 & 2 & 2 & 1922.92 & 0.610552 & $2 \mathrm{NU} 2 * * *$ \\
\hline $2899 \cdot 32$ & 0.0024 & 0.0327 & 13 & 0 & 13 & 13 & 1 & 12 & $2042 \cdot 33$ & 1.90653 & $2 \mathrm{NU} 2$ \\
\hline 2901.75 & 0.0026 & 0.0443 & 8 & 1 & 8 & 9 & 0 & 9 & $2512 \cdot 36$ & 8.007087 & $2 \mathrm{NU} 2 * * *$ \\
\hline 2901.82 & 0.0855 & 0.0782 & 7 & 3 & 5 & 8 & 4 & 4 & 1131.76 & 2.323327 & 2 NU2 2 \\
\hline 902.83 & 0.0041 & 0.0665 & 9 & 1 & 8 & 9 & 4 & 5 & 1360.28 & 0.116860 & $2 N \cup 2$ \\
\hline 904.46 & 0.0723 & 0.0676 & 8 & 4 & 5 & 9 & 5 & 4 & 1477.31 & 3.682999 & $2 N \cup 2$ \\
\hline 905.98 & 0.0043 & 0.0684 & 10 & 3 & 7 & 11 & 4 & 8 & 1843.02 & 4.032477 & $2 \mathrm{NU} 2$ \\
\hline 906.02 & 0.0031 & 0.0616 & 11 & 3 & 8 & 12 & 4 & 9 & 2124.98 & 4.836759 & $2 N \cup 2$ \\
\hline 906.71 & 0.2389 & 0.0890 & 3 & 0 & 3 & 4 & 3 & 2 & 382.52 & 0.051049 & $2 \mathrm{NU} 2$ \\
\hline 2907.23 & 0.0020 & 0.0865 & 4 & 2 & 2 & 5 & 3 & 3 & 2126.41 & 2.324407 & $2 \mathrm{NU} 2 * * *$ \\
\hline 2907.65 & 0.0060 & 0.0734 & 7 & 2 & 5 & 7 & 5 & 2 & 1059.85 & 0.038149 & 2NU2 \\
\hline 2908.10 & 0.0035 & 0.033 & 13 & 1 & 12 & 14 & 2 & 13 & $2327 \cdot 90$ & 11.01049 & $2 N U 2$ \\
\hline 2908.42 & 0.0012 & 0.033 & 13 & 2 & 12 & 14 & 1 & 13 & 2327.88 & 11.00954 & $2 N \cup 2$ \\
\hline 2910.47 & 0.0041 & 0.0569 & 9 & 5 & 5 & 10 & 6 & .4 & 1875.53 & 4.830967 & 2 NU 2 \\
\hline 2910.75 & 0.0050 & 0.0733 & 4 & 3 & 2 & 5 & 4 & 1 & $2251 \cdot 86$ & 3.250024 & $2 N \cup 2 * \ldots$ \\
\hline $2911 \cdot 34$ & 0.0217 & 0.0708 & 8 & 4 & 4 & 9 & 5 & 5 & 1474.99 & 3.26 & 2NU2- \\
\hline 2911.40 & 0.0016 & 0.0608 & 5 & 4 & 1 & 6 & 5 & 2 & 2552.85 & 4.266963 & $2 \mathrm{NU} 2 * *$ \\
\hline 2911.57 & 0.0017 & 0.0734 & 4 & 3 & 1 & 5 & 4 & 2 & 2251.71 & 3.270203 & $2 \mathrm{~N} \cup 2 * * *$ \\
\hline 2911.90 & 0.1434 & 0.0335 & 11 & 0 & 11 & 12 & 1 & 12 & 1557.91 & 11.021891 & 2NU2 \\
\hline 2911.95 & 0.0478 & 0.0331 & 11 & 1 & 11 & 12 & 0 & 12 & 1557.91 & 11.021861 & $2 N \cup 2$ \\
\hline 2912.00 & 0.0123 & 0.0583 & 9 & 5 & 4 & 10 & 6 & 5 & 1875.00 & 4.857731 & 2 NU 2 \\
\hline 2912.26 & 0.0077 & 0.0547 & 8 & 0 & 8 & 9 & 5 & 5 & 1474.99 & 0.0557 & NUI+ \\
\hline
\end{tabular}



FREQUENCY
$\mathrm{cm}^{-1}$
LINE
STRENGTH

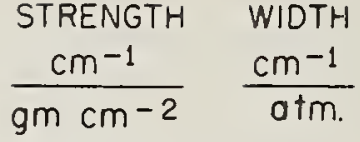
$\begin{array}{llllll}J^{\prime} & K_{a} & K_{c} & J^{\prime \prime} & K_{a} & K_{c}\end{array}$
$E^{\prime \prime}$
$\mathrm{cm}^{-1}$

$\begin{array}{ll}2913.06 & 0.0019 \\ 2913.26 & 0.0407 \\ 2914.48 & 0.0019 \\ 2917.36 & 0.0019 \\ 2919.00 & 0.0055 \\ 2919.58 & 0.0410 \\ 2920.90 & 0.0035 \\ 2921.33 & 0.0018 \\ 2921.39 & 0.0013 \\ 2922.00 & 0.0036 \\ 2922.33 & 0.0083 \\ 2922.45 & 0.0028 \\ 2923.10 & 0.0027 \\ 2923.16 & 0.0017 \\ 2924.62 & 0.0400 \\ 2925.25 & 0.0128 \\ 2925.38 & 0.0011 \\ 2926.46 & 0.0112 \\ 2928.70 & 0.0065 \\ 2930.16 & 0.3523 \\ 2930.54 & 0.0787 \\ 2931.10 & 0.0054 \\ 2933.14 & 0.1399 \\ 2933.18 & 0.2406 \\ 2933.26 & 0.4196 \\ 2933.69 & 0.0464 \\ 2933.73 & 0.8408 \\ 2934.73 & 0.0155 \\ 2935.23 & 1.3260 \\ 2935.65 & 0.0032 \\ 2935.77 & 0.0097 \\ 2936.00 & 0.0035 \\ 2936.01 & 0.0012 \\ 2936.18 & 0.0058 \\ 2936.74 & 0.0343 \\ 2936.82 & 0.0035 \\ 2936.95 & 0.5512 \\ 2937.48 & 0.0033 \\ 2937.60 & 0.0019 \\ 2938.50 & 0.0203 \\ 2939.65 & 0.0106 \\ 2939.66 & 0.0407 \\ 2940.92 & 0.0105 \\ 2941.00 & 0.0136 \\ 2942.48 & 0.0022 \\ 2943.45 & 0.0111 \\ 2944.02 & 0.0025 \\ 2944.73 & 0.0097 \\ 2944.94 & 0.0291 \\ 2945.75 & 0.0024 \\ 2945.95 & 0.3158 \\ 2945.98 & 0.0013 \\ 2946.57 & 0.0134 \\ 2946.96 & 0.0018\end{array}$

0.048

0.0737

0.0653

0.0712

0.0534

0.0775

0.0858

0.0542

0.0764

0.0688

0.0337

0.0337

0.0920

0.0776

0.0338

0.0349

0.0864

0.0851

0.0842

0.0796

0.0665

0.0709

0.0354

0.0686

0.0348

0.0557

0.0776

0.0565

0.0920

0.0680

0.0683

0.0545

0.0546

0.0475

0.0895

0.0647

0.0864

0.0901

0.0473

0.0793

0.0950

0.0361

0.0671

0.0379

0.0388

0.0465

0.0470

0.0358

0.0359

0.0559

0.0790

0.0643

0.0544

0.0656 $\begin{array}{rrrrrrr}10 & 6 & 5 & 11 & 7 & 4 & 2321.94 \\ 9 & 3 & 6 & 10 & 4 & 7 & 1581.34 \\ 7 & 1 & 6 & 8 & 2 & 7 & 2495.18 \\ 8 & 2 & 6 & 8 & 5 & 3 & 1255.92 \\ 7 & 0 & 7 & 8 & 1 & 8 & 2337.70 \\ 8 & 3 & 5 & 9 & 4 & 6 & 1340.89 \\ 3 & 2 & 2 & 4 & 3 & 1 & 2005.92 \\ 7 & 1 & 7 & 8 & 0 & 8 & 2337.53 \\ 6 & 1 & 5 & 7 & 2 & 6 & 2318.53 \\ 9 & 2 & 7 & 9 & 5 & 4 & 1477.31\end{array}$

$\begin{array}{lllllll}12 & 1 & 12 & 12 & 2 & 11 & 1774.74\end{array}$

$\begin{array}{llllll}12 & 0 & 12 & 12 & 1 & 11\end{array}$

$\begin{array}{lll}4 & 1 & 4\end{array}$

$\begin{array}{lll}6 & 3 & 4\end{array}$

$\begin{array}{lll}12 & 1 & 11\end{array}$

$\begin{array}{lll}12 & 2 & 11\end{array}$

$\begin{array}{lll}5 & 2 & 4\end{array}$

$\begin{array}{lll}5 & 1 & 4\end{array}$

$\begin{array}{lll}7 & 3 & 4\end{array}$

$\begin{array}{lll}7 & 4 & 4\end{array}$

$\begin{array}{lll}8 & 1 & 7\end{array}$

$10 \quad 0 \quad 10$

$\begin{array}{lll}7 & 4 & 3\end{array}$

10110

$\begin{array}{lll}8 & 5 & 4\end{array}$

634

$$
\begin{array}{lll}
8 & 5 & 3 \\
4 & 1 & 4
\end{array}
$$$$
4
$$$$
3
$$

4

$\begin{array}{lll}4 & 4 & 1\end{array}$

440

963

$\begin{array}{lll}2 & 0 & 2\end{array}$

606

524

413

964

606

$\begin{array}{lll}2 & 1 & 2\end{array}$

11110

$\begin{array}{rrr}6 & 1 & 6 \\ 11 & 2 & 10\end{array}$

$\begin{array}{lll}11 & 2 & 9\end{array}$

12310

11111

$\begin{array}{lll}11 & 0 & 11\end{array}$

845

633

643

$\begin{array}{lll}10 & 2 & 8\end{array}$
1774.60

446.50

931.22

2042.38

2042.33

661.56

2004.81

2161.30

1122.72

1255.92

1131.76

1327.14

1255.16

1327.14

1631.41

931.22

1631.27

446.50

2129.65

2129.63

2406.15

$2406 \cdot 15$

2054.40

285.23

2181.12

661.56

2024.17

20.54 .40

661.56

1819.34

1774.74

2180.65

1774.60

1114.56

1962.55

2246.98

1525.13

1524.87

1810.63

927.77

1059.85

1695.03

$2309 \cdot 74$ $\begin{array}{lll}3 & 2 & 1\end{array}$

$\begin{array}{rrr}11 & 2 & 10 \\ 9 & 0 & 9\end{array}$
L.

BAND
$5.877228 \quad 2 \mathrm{NU} 2$

$3.465920 \quad 2 \mathrm{NU} 2$

4. 996649 2NU2****

$0.0980242 \mathrm{NU} 2$

7.0002912 NU2***

3.141455 2NU2

2. 164905 2NU2***

6.998008 2NU2***

4.008107 2NU2***

0.180415 2NU2

1.854861 2NU2

1.854934 2NU2

0.386397 2NU2*

2.767638 2NU2*

8.71329 NU1

9.99731 2NU2

1.4111712 NU2*

2.320352 2NU2***

3. 129447 2NU2***

3.022183 2NU2

3.953657 2NU2

0.146118 2NU2

10.018239 2NU2

4.002077 2NU2

$10.0181532 \mathrm{NU} 2$

$5.006530 \quad 2 \mathrm{NU} 2$

2.767638 2NU2

5.013867 2NU2

0.386397 2NU2

3.390390 2NU2***

3.393148 2NU2****

4. 396748 2NU2****

$4.3970102 \mathrm{NU} 2 * * *$

6.019433 2NU2

0.013376 2NU2

5.990506 2NU2****

1.411171 2NU2

2.444582 2NU2***

6.018378 2NU2

0.051901 2NU2

$0.925282 \quad 2 N \cup 2 * * *$

9.078065 2NU2

5. 983681 2NU2***

9.076485 2NU2

9.013676 2NU2*

7.003088 2NU2

7.82232 2NU2

$1.851690 \quad 2 \mathrm{NU} 2$

1.851910 2NU2

0.0668 NU1+

3.050978 2NU2

4. 131192 2NU2*

5.954956 2NU2

1.838288 2NU2**** 


\begin{tabular}{|c|c|c|c|c|c|c|c|c|c|c|c|}
\hline $\begin{array}{c}\text { FREQUENCY } \\
\mathrm{cm}^{-1}\end{array}$ & $\begin{array}{l}\text { LINE } \\
\text { STRENGTH } \\
\frac{\mathrm{cm}^{-1}}{\mathrm{gm} \mathrm{cm}^{-2}}\end{array}$ & $\begin{array}{l}\text { HALF } \\
\text { WIDTH } \\
\frac{\mathrm{cm}^{-1}}{\text { otm. }}\end{array}$ & $J^{\prime}$ & $\mathrm{K}_{\mathrm{a}}$ & $\mathrm{K}_{\mathrm{c}}$ & J" & $\mathrm{K}_{\mathrm{a}}$ & $K_{c}$ & $\begin{array}{c}E^{\prime \prime} \\
\mathrm{cm}^{-1}\end{array}$ & L & BAND \\
\hline $\begin{array}{l}2947.61 \\
2948.08\end{array}$ & $\begin{array}{l}0.0188 \\
0.0415\end{array}$ & $\begin{array}{l}0.0841 \\
0.0748\end{array}$ & $\begin{array}{l}2 \\
7\end{array}$ & $\begin{array}{l}2 \\
1\end{array}$ & $\begin{array}{l}1 \\
6\end{array}$ & $\begin{array}{l}3 \\
7\end{array}$ & $\begin{array}{l}3 \\
4\end{array}$ & $\begin{array}{l}0 \\
3\end{array}$ & $\begin{array}{r}1907.63 \\
931.22\end{array}$ & $\begin{array}{l}2.366160 \\
0.135960\end{array}$ & $\begin{array}{l}2 \mathrm{NU} 2 * * * \\
2 \mathrm{NU} 2\end{array}$ \\
\hline 2948.65 & 0.0063 & 0.0830 & 2 & 2 & 0 & 3 & 3 & 1 & 1907.47 & 2.394805 & $2 \mathrm{NU} 2 \%$ \\
\hline 2948.89 & 0.0145 & 0.0930 & 3 & 1 & 2 & 4 & 2 & 3 & 1907.99 & 1.970843 & $2 \mathrm{NU} 2 * * *$ \\
\hline 2950.44 & 0.0056 & 0.0777 & 4 & 1 & 3 & 4 & 4 & 0 & 488.13 & 0.005990 & 2NU2 \\
\hline $2951 \cdot 26$ & 0.0014 & 0.0795 & 7 & 2 & 5 & 8 & 3 & 6 & $1006 \cdot 12$ & 3.262769 & $2 \mathrm{NU} 2 *$ \\
\hline 2951.38 & 0.0015 & 0.0769 & 5 & 3 & 3 & 6 & 4 & 2 & 757.78 & 3.066224 & $2 \mathrm{NU} 2 *$ \\
\hline 2953.15 & 0.1143 & 0.0637 & 9 & 2 & 7 & 10 & 3 & 8 & $1446 \cdot 16$ & 4.932263 & $2 \mathrm{NU} 2$ \\
\hline 2953.47 & 0.0176 & 0.0771 & 5 & 0 & 5 & 6 & 1 & 6 & 2042.77 & 4.979001 & $2 \mathrm{NU} 2^{*} * *$ \\
\hline 2954.17 & 1.1029 & 0.0388 & 9 & 0 & 9 & 10 & 1 & 10 & 1114.56 & 9.013676 & $2 \mathrm{NU} 2$ \\
\hline 2954.43 & 0.3677 & 0.0382 & 9 & 1 & 9 & 10 & 0 & 10 & 1114.55 & 9.013420 & $2 \mathrm{NU} 2$ \\
\hline 2955.09 & 0.0486 & 0.0787 & 5 & 1 & 4 & 5 & 4 & 1 & 610.35 & 0.031887 & 2 NU2 \\
\hline $2955 \cdot 28$ & 0.0206 & 0.0785 & 6 & 1 & 5 & 6 & 4 & 2 & 757.78 & 0.084732 & $2 \mathrm{NU} 2$ \\
\hline 2955.57 & 0.0424 & 0.0401 & 10 & 1 & 9 & 11 & 2 & 10 & 1525.13 & 8.063213 & $2 \mathrm{NU} 2$ \\
\hline 2955.61 & 0.6655 & 0.0643 & 6 & 4 & 3 & 7 & 5 & 2 & 1059.85 & 4.131192 & 2 NU 2 \\
\hline 2956.02 & 0.0046 & 0.0771 & 5 & 3 & 2 & 6 & 4 & 3 & 756.75 & 3.153879 & $2 \mathrm{NU} 2 *$ \\
\hline $2956 \cdot 35$ & 0.2225 & 0.0656 & 6 & 4 & 2 & 7 & 5 & 3 & 1059.65 & $4 \cdot 143594$ & 2NU2 \\
\hline 2956.84 & 0.0475 & 0.0537 & 7 & 5 & 3 & 8 & 6 & 2 & 1411.68 & 5.142711 & $2 \mathrm{NU} 2$ \\
\hline 2956.97 & 0.1426 & 0.0539 & 7 & 5 & 2 & 8 & 6 & 3 & 1411.65 & 5.144364 & $2 \mathrm{NU} 2$ \\
\hline 2957.01 & 0.0981 & 0.0716 & 8 & 2 & 6 & 9 & 3 & 7 & $1216 \cdot 27$ & 4.004942 & $2 \mathrm{NU} 2$ \\
\hline 2958.41 & 0.1273 & 0.0442 & 10 & 2 & 9 & 11 & 1 & 10 & 1524.87 & 8.058917 & $2 \mathrm{NU} 2$ \\
\hline 2959.78 & 0.0045 & 0.0557 & 11 & 3 & 9 & 12 & 2 & 10 & 1960.22 & 6.964699 & 2NU2 \\
\hline 2960.27 & 0.0037 & 0.0743 & 6 & 1 & 6 & 6 & 2 & 5 & $2161 \cdot 30$ & 1.783035 & $2 \mathrm{NU} 2 * *$ \\
\hline 2960.78 & 0.0059 & 0.0806 & 5 & 1 & 5 & 6 & 0 & 6 & 2041.76 & $4.9585 \div 9$ & $2 \mathrm{~N} \cup 2 * * *$ \\
\hline 2961.38 & 0.6888 & 0.0795 & 7 & 2 & 5 & 8 & 3 & 6 & $1006 \cdot 12$ & 3.262769 & 2 NU2 2 \\
\hline 2961.69 & 0.7464 & 0.0769 & 5 & 3 & 3 & 6 & 4 & 2 & 757.78 & 3.066224 & $2 \mathrm{NU} 2$ \\
\hline 2962.11 & 0.0094 & 0.0871 & 4 & 2 & 3 & 5 & 3 & 2 & 508.81 & 1.841317 & $2 \mathrm{NU} 2^{*}$ \\
\hline 2962.86 & 0.0017 & 0.0447 & 8 & 0 & 8 & 9 & 1 & 9 & 920.21 & 8.007851 & $2 \mathrm{~N} \cup 2 *$ \\
\hline 2963.32 & 0.0052 & 0.0443 & 8 & 1 & 8 & 9 & 0 & 9 & 920.18 & 8.007087 & $2 \mathrm{~N} \cup 2 *$ \\
\hline 2963.42 & 0.0066 & 0.0959 & 2 & 1 & 1 & 3 & 2 & 2 & 1813.81 & 1.666667 & 2 NU 2 长米关 \\
\hline 2963.75 & 0.0214 & 0.0454 & 8 & 6 & 3 & 9 & 7 & 2 & 1810.63 & 5.53 & NU2- \\
\hline 2964.75 & 0.0055 & 0.0454 & 8 & 6 & 2 & 9 & 7 & 3 & 1810.63 & $4 \cdot 30$ & NU2- \\
\hline 2966.00 & 2.3187 & 0.0771 & 5 & 3 & 2 & 6 & 4 & 3 & 756.75 & 3.153879 & 2 Nu2 2 \\
\hline 2966.64 & 0.0037 & 0.0848 & 6 & 2 & 5 & 7 & 1 & 6 & 2309.74 & 3.799063 & 2 NU $2 * * *$ \\
\hline 2966.74 & 0.0926 & 0.0393 & 10 & 1 & 10 & 10 & 2 & 9 & 1293.66 & 1.847651 & 2NU2 \\
\hline 2967.24 & 0.0311 & 0.0397 & 10 & 0 & 10 & 10 & 1 & 9 & 1293.04 & 1.848326 & 2 NU2 \\
\hline 2967.65 & 0.0062 & 0.0852 & 5 & 2 & 3 & 6 & 3 & 4 & 648.97 & 2.452523 & $2 \mathrm{NU} 2 *$ \\
\hline 2967.68 & 0.0017 & 0.0871 & 4 & 2 & 3 & 5 & 3 & 2 & 508.81 & 1.841317 & $2 N \cup 2 * *$ \\
\hline 2967.91 & 0.4999 & 0.0835 & 6 & 2 & 4 & 7 & 3 & 5 & 816.72 & 2.751216 & 2NU2 \\
\hline 2968.85 & 0.0086 & 0.0880 & 4 & 0 & 4 & 5 & 1 & 5 & 1922.86 & 3.972803 & $2 \mathrm{NU} 2 * 2^{*} *$ \\
\hline $2969 \cdot 45$ & 0.3534 & 0.0463 & 9 & 1 & 8 & 10 & 2 & 9 & 1293.66 & 7.043661 & $2 \mathrm{NU} 2$ \\
\hline 2970.17 & 0.1589 & 0.0843 & 5 & 0 & 5 & 5 & 3 & 2 & 508.81 & 0.062476 & 2 NU2 \\
\hline 2970.62 & 0.0011 & 0.0604 & 5 & 4 & 2 & 6 & 5 & 1 & 888.63 & 4.264570 & $2 N \cup 2 *$ \\
\hline 2970.73 & 0.0033 & 0.0608 & 5 & 4 & 1 & 6 & 5 & 2 & 888.60 & 4.266963 & $2 \mathrm{NU} 2 *$ \\
\hline 2971.66 & 0.0014 & 0.0777 & 6 & 0 & 6 & 6 & 1 & 5 & $2146 \cdot 28$ & 1.852055 & $2 \mathrm{~N} \cup 2 * x^{2}$ \\
\hline $2972 \cdot 34$ & 0.0027 & 0.0948 & 3 & 1 & 3 & 4 & 2 & $2^{\prime}$ & 315.79 & 0.610552 & $2 \mathrm{NU} 2 *$ \\
\hline 2972.80 & 0.0011 & 0.0852 & 5 & 2 & 3 & 6 & 3 & 4 & 648.97 & 2.452523 & $2 \operatorname{NU} 2 * *$ \\
\hline 2973.25 & 4.6875 & 0.0871 & 4 & 2 & 3 & 5 & 3 & 2 & 508.81 & 1.841317 & $2 \mathrm{NU} 2$ \\
\hline 2974.00 & 0.0070 & 0.0947 & 1 & 1 & 1 & 2 & 2 & 0 & 1743.47 & 1.244747 & $2 \mathrm{NU} 2{ }^{*} *$ * \\
\hline 2974.63 & 0.8690 & 0.0447 & 8 & 0 & 8 & 9 & 1 & 9 & 920.21 & 8.007851 & $2 \mathrm{NU} 2$ \\
\hline 2975.10 & 2.6070 & 0.0443 & 8 & 1 & 8 & 9 & 0 & 9 & 920.18 & 8.007087 & $2 \mathrm{NU} 2$ \\
\hline 2975.13 & 0.1186 & 0.0534 & 9 & 2 & 8 & 10 & 1 & 9 & 1293.04 & 7.032041 & $2 \mathrm{NU} 2$ \\
\hline 2977.28 & 0.0100 & 0.0733 & 4 & 3 & 2 & 5 & 4 & 1 & 610.35 & 3.250024 & $2 \mathrm{NU} 2 *$ \\
\hline 2977.30 & 0.0399 & 0.0655 & 10 & 3 & 8 & 11 & 2 & 9 & 1690.70 & 5.859437 & $2 N_{2} 2$ \\
\hline
\end{tabular}




\begin{tabular}{|c|c|c|c|c|c|c|c|c|c|c|c|}
\hline $\begin{array}{c}\text { FREQUENCY } \\
\mathrm{cm}^{-1}\end{array}$ & $\begin{array}{l}\text { LINE } \\
\text { STRENGTH } \\
\frac{\mathrm{cm}^{-1}}{g m \mathrm{~cm}^{-2}}\end{array}$ & $\begin{array}{l}\text { HALF } \\
\text { WIDTH } \\
\frac{\mathrm{cm}^{-1}}{\text { atm. }}\end{array}$ & $J^{\prime}$ & $K_{a}$ & $\mathrm{~K}_{\mathrm{c}}$ & J" & $K_{a}$ & $K_{c}$ & $\begin{array}{c}E^{\prime \prime} \\
\mathrm{cm}^{-1}\end{array}$ & L & BAND \\
\hline $\begin{array}{l}2977.96 \\
2978.37\end{array}$ & $\begin{array}{l}3.1032 \\
0.0024\end{array}$ & $\begin{array}{l}0.0852 \\
0.0839\end{array}$ & $\begin{array}{l}5 \\
5\end{array}$ & $\begin{array}{l}2 \\
1\end{array}$ & $\begin{array}{l}3 \\
5\end{array}$ & $\begin{array}{l}6 \\
5\end{array}$ & $\begin{array}{l}3 \\
2\end{array}$ & $\begin{array}{l}4 \\
4\end{array}$ & $\begin{array}{r}648 \cdot 97 \\
2024 \cdot 17\end{array}$ & $\begin{array}{l}2.452523 \\
1.711792\end{array}$ & $\begin{array}{l}2 \mathrm{~N} \cup 2 \\
2 \mathrm{~N} \cup 2 * *\end{array}$ \\
\hline 2978.45 & 0.0034 & 0.0734 & 4 & 3 & 1 & 5 & 4 & 2 & $610 \cdot 12$ & 3.270203 & $2 \mathrm{~N} \cup 2 *$ \\
\hline 2980.25 & 0.5431 & 0.0604 & 5 & 4 & 2 & 6 & 5 & 1 & 888.63 & 4.264570 & $2 \mathrm{~N} \cup 2$ \\
\hline 2980.35 & 1.6302 & 0.0608 & 5 & 4 & 1 & 6 & 5 & 2 & 888.60 & 4.266963 & $2 \mathrm{NU} 2$ \\
\hline 2980.75 & 0.0018 & 0.0646 & 8 & 2 & 7 & 9 & 1 & 8 & 1079.07 & 5.987889 & $2 \mathrm{NU} 2 *$ \\
\hline $2981 \cdot 13$ & 0.3918 & 0.0498 & 6 & 5 & 2 & 7 & 6 & 1 & $1216 \cdot 20$ & 5.265222 & 2 NU2 \\
\hline $2981 \cdot 21$ & 0.0255 & 0.0993 & 1 & 1 & 0 & 2 & 2 & 1 & $1742 \cdot 28$ & 1.500000 & $2 \mathrm{NU} 2 * * *$ \\
\hline $2981 \cdot 24$ & 0.1306 & 0.0499 & 6 & 5 & 1 & 7 & 6 & 2 & $1216 \cdot 20$ & 5.265494 & $2 \mathrm{NU} 2$ \\
\hline $2981 \cdot 36$ & 0.0256 & 0.0914 & 4 & 1 & 4 & 5 & 0 & 5 & 1920.76 & 3.911752 & $2 \mathrm{NU} 2 * * *$ \\
\hline $2981 \cdot 43$ & 0.0041 & 0.0865 & 4 & 2 & 2 & 5 & 3 & 3 & .00 & 2.324407 & $2 \mathrm{NU} 2 *$ \\
\hline 2982 & 0.0039 & 0.0653 & 7 & 1 & 6 & 8 & 2 & 7 & 885.62 & 4.996649 & $2 \mathrm{NU} 2^{*}$ \\
\hline 2982 & 0.0019 & 0.0733 & 4 & 3 & 2 & 5 & 4 & 1 & $610 \cdot 35$ & 3.250024 & $2 \mathrm{~N} \cup 2 * *$ \\
\hline $2982 \cdot 48$ & 0.2942 & 0.0552 & 8 & 1 & 7 & 9 & 2 & $\varepsilon$ & 0.38 & 6.019178 & $2 \mathrm{NU} 2$ \\
\hline 2982.61 & 0.0111 & 0.0534 & 7 & 0 & 7 & 8 & 1 & $\varepsilon$ & $744 \cdot 16$ & 7.000291 & $2 \mathrm{NU} 2 *$ \\
\hline 2983. & 0.0326 & 0.0952 & 3 & 0 & 3 & 4 & 1 & 4 & 1821.61 & 3.004990 & $2 \mathrm{NU} 2 * * *$ \\
\hline 2983 & 0.0037 & 0.0542 & 7 & 1 & 7 & 8 & 0 & $\varepsilon$ & .09 & 6.998008 & $2 \mathrm{NU} 2 *$ \\
\hline 2983. & 0.0010 & 0.0438 & 12 & 2 & 11 & 13 & 3 & 10 & .73 & 0.27474 & NU1 \\
\hline $2984 \cdot 20$ & 1.3649 & 0.0948 & 3 & 1 & 3 & 4 & 2 & , & 315.79 & 0.610552 & $2 \mathrm{NU} 2$ \\
\hline 2987 & 5.0028 & 0.0733 & 4 & 3 & 2 & 5 & 4 & 1 & .35 & 3.250024 & 2 NU2 \\
\hline 2987. & 0.0238 & 0.0422 & 7 & 6 & 2 & 8 & 7 & 1 & .74 & 6.263414 & $2 \mathrm{NU} 2$ \\
\hline 2987. & 0.0714 & 0.0422 & 7 & 6 & 1 & 8 & 7 & 2 & 0.74 & 6.263444 & $2 \mathrm{NU} 2$ \\
\hline 2988 & 0.0020 & 0.0534 & 7 & 0 & 7 & 8 & 1 & 8 & $744 \cdot 16$ & 7.000291 & $2 \mathrm{~N} \cup 2 * *$ \\
\hline 2988 & 1.6807 & 0.0734 & 4 & 3 & 1 & 5 & 4 & 2 & $\cdot 12$ & 3.270203 & $2 \mathrm{NU} 2$ \\
\hline 2988. & 0.0902 & 0.0451 & 9 & 1 & 9 & 9 & 2 & 8 & 108 & 1.842036 & $2 \mathrm{NU} 2$ \\
\hline 2989 & 0.0012 & 0.0470 & 13 & 1 & 12 & 13 & 2 & 11 & 2246.98 & 3.76133 & $2 N \cup 2$ \\
\hline 2989 & 0.2728 & 0.0459 & 9 & 0 & 9 & 9 & 1 & $\varepsilon$ & 107 & 1.844131 & $2 \mathrm{NU} 2$ \\
\hline 2991 & 0.0760 & 0.0870 & 4 & 0 & 4 & 4 & 3 & 1 & .85 & 0.047652 & $2 \mathrm{NU} 2$ \\
\hline 2991 & 0.0010 & 0.0687 & 6 & 1 & 6 & 5 & 4 & 1 & .35 & 0.000636 & $2 N U 2$ \\
\hline 2991 & 2.0331 & 0.0865 & 4 & 2 & 2 & 5 & 3 & 3 & 504.00 & 2.324407 & $2 \mathrm{NU} 2$ \\
\hline 2992 & 0.0025 & 0.0764 & 6 & 1 & 5 & 7 & 2 & 6 & .60 & .008107 & $2 \mathrm{NU} 2 *$ \\
\hline 2992 & 0.8868 & 0.0646 & 8 & 2 & 7 & 9 & 1 & 8 & 107 & 5.987889 & $2 N \cup 2$ \\
\hline 2992 & 0.0069 & 0.0858 & 3 & 2 & 2 & 4 & 3 & 1 & 85 & 2.164905 & $2 \mathrm{NU} 2 *$ \\
\hline 2993 & 1.9468 & 0.0653 & 7 & 1 & 6 & 8 & 2 & 7 & .62 & 4.996649 & 2NU2 \\
\hline 2994 & 0.0117 & 0.0907 & 4 & 1 & 4 & 4 & 2 & 3 & 1907.99 & 1.567300 & $2 \mathrm{~N} \cup 2 * * * *$ \\
\hline 2994 & 5.5317 & 0.0534 & 7 & 0 & 7 & 8 & 1 & 8 & $\cdot 16$ & 7.000291 & 2NU2 \\
\hline 2995 & 0.0070 & 0.0545 & 4 & 4 & 1 & 5 & 5 & 0 & .10 & 4.396748 & $2 \mathrm{NU} 2^{*}$ \\
\hline 2995 & 0.0023 & 0.0546 & 4 & 4 & 0 & 5 & 5 & 1 & 10 & 4.397010 & $2 \mathrm{NU} 2 *$ \\
\hline 2995 & 0.0087 & 0.0871 & 5 & 0 & 5 & 5 & 1 & 4 & $\cdot 90$ & 1.912338 & $2 \mathrm{NU} 2 * * *$ \\
\hline $2995 \cdot 38$ & 1.8446 & 0.0542 & 7 & 1 & 7 & 8 & 0 & 8 & +.09 & 6.998008 & 2 NU2 \\
\hline $2996 \cdot 24$ & 0.0019 & 0.0447 & 5 & 5 & 0 & 6 & 6 & 1 & .07 & 5.399718 & $2 N \cup 2 *$ \\
\hline 2996 & 0.0016 & 0.0379 & 11 & 0 & 11 & 12 & 3 & 10 & .55 & 0.089303 & NU1 \\
\hline $2997 \cdot 64$ & 0.0119 & 0.1006 & 2 & 0 & 2 & 3 & 1 & 3 & - 51 & 2.156792 & $2 \mathrm{NU} 2 * * *$ \\
\hline 2997.70 & 0.0014 & 0.0530 & 8 & 1 & 8 & 8 & 2 & 7 & .62 & 1.833129 & $2 \mathrm{NU} 2 *$ \\
\hline $2998 \cdot 30$ & 0.0385 & 0.0747 & 9 & 3 & 7 & 10 & 2 & 8 & 8.00 & 4.706625 & 2NU2 \\
\hline 2998.37 & 0.0013 & 0.0858 & 3 & 2 & 2 & 4 & 3 & 1 & & 2.164905 & $2 \mathrm{NU} 2 * *$ \\
\hline 2998.63 & 0.0032 & 0.0378 & 8 & 7 & 1 & 9 & 8 & 2 & .87 & 7.261219 & 2 NU2 2 \\
\hline 2998.63 & 0.0095 & 0.0378 & 8 & 7 & 2 & 9 & 8 & 1 & 200 & 7.261215 & 2NU2 \\
\hline 2999.08 & 0.0013 & 0.0759 & 7 & 2 & 6 & 8 & 1 & 7 & 882.93 & 4.913532 & $2 \mathrm{NU} 2 *$ \\
\hline 2999.56 & 0.0225 & 0.0851 & 3 & 2 & 1 & 4 & 3 & 2 & $382 \cdot 52$ & 2.320352 & $2 N \cup 2 *$ \\
\hline 2999.63 & 0.0080 & 0.0531 & 6 & 4 & 3 & 7 & 7 & 0 & 1394.86 & 0.026 & $\mathrm{NUI+}$ \\
\hline 2999.82 & 0.0013 & 0.0545 & 4 & 4 & 1 & 5 & 5 & 0 & $742 \cdot 10$ & 4.396748 & 2 NU $2 * *$ \\
\hline $3001 \cdot 34$ & 0.0129 & 0.0842 & 5 & 1 & 4 & 6 & 2 & 5 & 552.92 & 3.129447 & $2 \mathrm{NU} 2 *$ \\
\hline 3001.70 & 0.0070 & 0.0647 & 6 & 0 & 6 & 7 & 1 & 7 & $5,86.48$ & 5.990506 & 2NU2* \\
\hline
\end{tabular}




\begin{tabular}{|c|c|c|c|c|c|c|c|c|c|c|c|}
\hline $\begin{array}{c}\text { FREQUENCY } \\
\mathrm{cm}^{-1}\end{array}$ & $\begin{array}{l}\text { LINE } \\
\text { STRENGTH } \\
\frac{\mathrm{cm}^{-1}}{\mathrm{gm} \mathrm{cm}^{-2}}\end{array}$ & $\begin{array}{l}\text { HALF } \\
\text { WIDTH } \\
\frac{\mathrm{cm}^{-1}}{\text { atm. }}\end{array}$ & $J^{\prime}$ & $K_{a}$ & $K_{c}$ & $J^{\prime \prime}$ & $K_{a}$ & $K_{c}$ & $\begin{array}{c}E^{\prime \prime} \\
\mathrm{cm}^{-1}\end{array}$ & $\mathrm{~L}$ & BAND \\
\hline $3002 \cdot 14$ & 0.0065 & 0.0680 & 3 & 3 & 1 & 4 & 4 & 0 & $488 \cdot 13$ & 3.390390 & $2 \mathrm{NU} 2 \ddot{x}$ \\
\hline & 0.0194 & 0.0683 & 3 & 3 & 0 & 4 & 4 & 1 & $488 \cdot 10$ & 3.393148 & $2 \mathrm{NU} 2 *$ \\
\hline .26 & 0.0104 & 0.0974 & 3 & 1 & 3 & 4 & 0 & 4 & 1817.50 & 2.831638 & $2 N \cup 2 * * *$ \\
\hline 3003.46 & 1.2588 & 0.0764 & 6 & 1 & 5 & 7 & 2 & 6 & 709.60 & 4.008107 & $2 \mathrm{NU} 2$ \\
\hline 3003 & 0.0210 & 0.0671 & 6 & 1 & 6 & 7 & 0 & 7 & $586 \cdot 26$ & 5.983681 & $2 N \cup 2 *$ \\
\hline 3003 & 0.1313 & 0.0874 & 3 & 0 & 3 & 3 & 3 & 0 & 285.43 & 0.016715 & 2 NU2 2 \\
\hline 3003.83 & 3.4667 & 0.0858 & 3 & 2 & 2 & 4 & 3 & 1 & 383.85 & 2.164905 & $2 \mathrm{Nu} 2$ \\
\hline 3004.65 & 3.5222 & 0.0545 & 4 & 4 & 1 & 5 & 5 & 0 & $742 \cdot 10$ & 4.396748 & 2 NU2 \\
\hline 3004.66 & 1.1741 & 0.0546 & 4 & 4 & 0 & 5 & 5 & 1 & 742.10 & 4.397010 & $2 \mathrm{NU} 2$ \\
\hline 3004.68 & 0.0039 & 0.0783 & 6 & 2 & 5 & 6 & 3 & 4 & 2271.70 & 2.963539 & $2 \mathrm{~N} \cup 2 * * *$ \\
\hline 3004.89 & 0.0042 & 0.0851 & 3 & 2 & 1 & 4 & 3 & 2 & 382.52 & $2 \cdot 32$ & $2 \mathrm{NU} 2 * *$ \\
\hline 3005 & 0.0060 & 0.0381 & 12 & 2 & 11 & 12 & 3 & 10 & 1962.55 & 3.64 & $2 \mathrm{NU} 2$ \\
\hline 3005 & 0.3172 & 0.0447 & 5 & 5 & 1 & 6 & 6 & 0 & 5.07 & 5.3 & 2 NU2 \\
\hline 3005.41 & 0.9517 & 0.0447 & 5 & 5 & 0 & 6 & 6 & 1 & .07 & 5.39 & 2 NU2 \\
\hline 3006.78 & 0.0024 & 0.0391 & 12 & 1 & 11 & 12 & 2 & 10 & .22 & 3.65 & $2 \mathrm{NU} 2$ \\
\hline 3006.93 & 0.0024 & 0.0842 & 5 & $i$ & 4 & 6 & 2 & 5 & .92 & 3.129447 & $2 \mathrm{~N} \cup 2 * *$ \\
\hline 3006.95 & 0.0052 & 0.0944 & 3 & 1 & 3 & 3 & 2 & 2 & 3.81 & 1.297651 & 2 NU $2 * * *$ \\
\hline 3007.07 & 0.0014 & 0.0547 & 6 & 4 & 2 & 7 & 7 & 1 & 1394.86 & 0.013 & NUI+ \\
\hline 3007.24 & 0.0012 & 0.0680 & 3 & 3 & 1 & 4 & 4 & 0 & 488.13 & 3.3 & $2 \mathrm{NU} 2 * *$ \\
\hline $3007 \cdot 42$ & 0.0036 & 0.0683 & 3 & 3 & 0 & 4 & 4 & 1 & $488 \cdot 10$ & $3 \cdot 3$ & $2 \mathrm{NU} 2 * *$ \\
\hline 3007.64 & 0.0013 & 0.0647 & 6 & 0 & 6 & 7 & 1 & 7 & 5.48 & 5.9 & $2 \operatorname{NU} 2 * *$ \\
\hline 3009.57 & 0.0039 & 0.0671 & 6 & 1 & 6 & 7 & 0 & 7 & $5 \cdot 26$ & $5 \cdot 9$ & $2 \mathrm{NU} 2 * *$ \\
\hline 3009.66 & 0.7180 & 0.0530 & 8 & 1 & 8 & 8 & 2 & 7 & 885.62 & 1.83 & $2 N \cup 2$ \\
\hline 3010.23 & 11.2452 & 0.0851 & 3 & 2 & 1 & 4 & 3 & 2 & 382.52 & 2.32 & 2 NU 2 \\
\hline $3011 \cdot 18$ & 0.0067 & 0.0901 & 4 & 1 & 3 & 5 & 2 & 4 & $416 \cdot 22$ & 2.444582 & 2 NU2* \\
\hline 3011.22 & 0.6505 & 0.0759 & 7 & 2 & 6 & 8 & 1 & 7 & 882.93 & 4.913532 & 2 NU2 \\
\hline 3011 & 0.0023 & 0.0833 & 5 & 2 & 4 & 5 & 3 & 3 & $2126 \cdot 41$ & 2.45 & $2 \mathrm{NU} 2 * * * *$ \\
\hline 301 & 0.2436 & 0.0548 & 8 & 0 & 8 & 8 & 1 & 7 & 882.93 & 1.839778 & $2 N \cup 2$ \\
\hline 3012 & 0.1679 & 0.0388 & 6 & 6 & 1 & 7 & 7 & 0 & 1394.86 & 5.44 & NU2 - \\
\hline 3012 & 3.2325 & 0.0680 & 3 & 3 & 1 & 4 & 4 & 0 & $488 \cdot 13$ & $3 \cdot 3$ & 2NU2 \\
\hline 3012 & 0.0659 & 0.0388 & 6 & 6 & 0 & 7 & 7 & 1 & 4.86 & 6.40 & 2 NU2 \\
\hline 3012 & 6.4740 & 0.0842 & 5 & 1 & 4 & 6 & 2 & 5 & .92 & 3.1 & $2 \mathrm{NU} 2$ \\
\hline . 52 & 9.7070 & 0.0683 & 3 & 3 & 0 & 4 & 4 & 1 & .10 & 3.39 & 2 NU2 \\
\hline .19 & 0.0359 & 0.0621 & 6 & 3 & 3 & 7 & 7 & 0 & +.86 & 0.01 & NU3+ \\
\hline 3013 & 0.0340 & 0.1037 & 1 & 0 & 1 & 2 & 1 & 2 & .08 & 1.5 & $2 \mathrm{NU} 2 * * * *$ \\
\hline .58 & 3.4942 & 0.0647 & 6 & 0 & 6 & 7 & 1 & 7 & .48 & 5.9 & 2 NU2 \\
\hline $3014 \cdot 14$ & 0.0211 & 0.0950 & 2 & 1 & 2 & 3 & 2 & 1 & .15 & 0.92 & $2 \mathrm{NU} 2 *$ \\
\hline .56 & 10.4892 & 0.0671 & 6 & 1 & 6 & 7 & 0 & 7 & .26 & 681 & 2 NU2 \\
\hline 3016.22 & 0.0058 & 0.0942 & 4 & 0 & 4 & 4 & 1 & 3 & 5.49 & 2.04 & $2 \mathrm{~N} \cup 2 * k$ \\
\hline 3016.51 & 0.0092 & 0.0845 & 4 & 2 & 3 & 4 & 3 & 2 & +.81 & 1.84 & $2 \mathrm{~N} \cup 2 * * *$ \\
\hline 3016.71 & 0.0144 & 0.0955 & 2 & 1 & 2 & 2 & 2 & 1 & 2.28 & 0.83 & $2 \mathrm{~N} \cup 2 * * *$ \\
\hline 3016.76 & 0.0012 & 0.0901 & 4 & 1 & 3 & 5 & 2 & 4 & $416 \cdot 22$ & 2.444582 & $2 \mathrm{~N} \cup 2 * *$ \\
\hline 3017.08 & 0.0027 & 0.0802 & 7 & 1 & 6 & 7 & 2 & 5 & $2392 \cdot 58$ & 3.98 & $2 N \cup 2 * * *$ \\
\hline 3017.91 & 0.0012 & 0.0634 & 7 & 1 & 7 & 7 & 2 & 6 & 9.60 & 1.8 & $2 \mathrm{~N} \cup 2 *$ \\
\hline 3019.35 & 0.0028 & 0.0833 & 3 & 2 & 2 & 3 & 3 & 1 & 1907.47 & 1.035682 & $2 N \cup 2 * * *$ \\
\hline 3019.49 & 0.0074 & 0.0848 & 6 & 2 & 5 & 7 & 1 & 6 & 704.22 & 3.799063 & $2 \mathrm{NU} 2 *$ \\
\hline 3019.84 & 0.0352 & 0.0771 & 5 & 0 & 5 & 6 & 1 & 6 & $447 \cdot 24$ & 4.979001 & $2 N \cup 2 *$ \\
\hline 3019.89 & 0.0375 & 0.0841 & 2 & 2 & 1 & 3 & 3 & 0 & 5.43 & 2.36 & $2 N \cup 2 *$ \\
\hline 3019.96 & 0.0039 & 0.0950 & 2 & 1 & 2 & 3 & 2 & 1 & 212.15 & 0.9 & $2 \mathrm{NU} 2 * \ddot{*}$ \\
\hline 3020.42 & 0.0075 & 0.0947 & 4 & 2 & 3 & 5 & 1 & 4 & $2000 \cdot 90$ & 1.6 & $2 N \cup 2 * * *$ \\
\hline 3020 . & 0.0084 & 0.0425 & 11 & 2 & 10 & 11 & 3 & 9 & 1695.03 & 3.6 & $2 \mathrm{NU} 2$ \\
\hline 3021.18 & 0.0127 & 0.0830 & 2 & 2 & 0 & 3 & 3 & 1 & 285.23 & 2.39 & $2 \mathrm{~N} \cup 2 *$ \\
\hline 3021.94 & 0.0036 & 0.0656 & 7 & 0 & 7 & 7 & 1 & 6 & $4 \cdot 22$ & 1.83 & $2 N \cup 2 *$ \\
\hline 3022.35 & 3.3500 & 0.0901 & 4 & 1 & 3 & 5 & 2 & 4 & $416 \cdot 22$ & 2.444582 & $2 \mathrm{NU} 2$ \\
\hline
\end{tabular}




\begin{tabular}{|c|c|c|c|c|c|c|c|c|c|c|c|}
\hline $\begin{array}{c}\text { FREQUENCY } \\
\mathrm{cm}^{-1}\end{array}$ & $\begin{array}{l}\text { LINE } \\
\text { STRENGTH } \\
\mathrm{cm}^{-1} \\
\end{array}$ & $\begin{array}{l}\text { HALF } \\
\text { WIDTH } \\
\mathrm{cm}^{-1} \\
\end{array}$ & $J^{\prime}$ & $\mathrm{K}_{0}$ & $K_{c}$ & $J^{\prime \prime}$ & $\mathrm{K}_{\mathrm{a}}$ & $K_{c}$ & $\begin{array}{c}E^{\prime \prime} \\
\mathrm{cm}^{-1}\end{array}$ & L & BAND \\
\hline & $\mathrm{gm} \mathrm{cm-2}$ & $\overline{a t m .}$ & & & & & & & & & \\
\hline $\begin{array}{l}3022.67 \\
3023.03 \\
3023.64\end{array}$ & $\begin{array}{l}0.2861 \\
0.0290 \\
0.0089\end{array}$ & $\begin{array}{l}0.0834 \\
0.0930 \\
0.0835\end{array}$ & $\begin{array}{l}8 \\
3 \\
3\end{array}$ & $\begin{array}{l}3 \\
1 \\
2\end{array}$ & $\begin{array}{l}6 \\
2 \\
1\end{array}$ & $\begin{array}{l}9 \\
4 \\
3\end{array}$ & $\begin{array}{l}2 \\
2 \\
3\end{array}$ & $\begin{array}{l}7 \\
3 \\
0\end{array}$ & $\begin{array}{r}1201 \cdot 95 \\
300 \cdot 35 \\
1907.63\end{array}$ & $\begin{array}{l}3.529968 \\
1.970843\end{array}$ & $\begin{array}{l}2 \mathrm{NU} 2 \\
2 \mathrm{NU} 2 * \\
2 \mathrm{NU} 2^{*} * *\end{array}$ \\
\hline 3023.70 & 0.0252 & 0.0445 & 11 & 1 & 10 & 11 & 2 & 9 & 1690.70 & 3.646421 & $2 \mathrm{~N} \cup 2$ \\
\hline 3023.71 & 0.0117 & 0.0806 & 5 & 1 & 5 & 6 & 0 & 6 & 446.71 & 4.958549 & $2 N \cup 2 *$ \\
\hline 3025.30 & 0.0069 & 0.0841 & 2 & 2 & 1 & 3 & 3 & 0 & 285.43 & 2.366160 & $2 \mathrm{NU} 2 *$ ** \\
\hline 3025.78 & 10.5645 & 0.0950 & 2 & 1 & 2 & 3 & 2 & 1 & $212 \cdot 15$ & 0.925282 & 2 NU2 \\
\hline 3026.57 & 0.0023 & 0.0830 & 2 & 2 & 0 & 3 & 3 & 1 & 285.23 & 2.394805 & $2 N \cup 2 * *$ \\
\hline 3026.77 & 0.0018 & 0.0653 & 8 & 1 & 8 & 9 & 4 & 5 & $1360 \cdot 28$ & 0.004551 & NUI. \\
\hline 3027.10 & 0.0297 & 0.0994 & 2 & 1 & 2 & 3 & 0 & 3 & 1731.89 & 1.741385 & $2 N \cup 2 * * *$ \\
\hline 3027.14 & 0.0126 & 0.0811 & 10 & 4 & 7 & 11 & 3 & 8 & 1813.26 & 3.178818 & $2 N \cup 2$ \\
\hline 3027.72 & 0.0036 & 0.0862 & 4 & 2 & 2 & 4 & 3 & 1 & 2005.92 & 2.167225 & $2 \mathrm{~N} \cup 2 * * *$ \\
\hline 3028.55 & 0.0019 & 0.0447 & 11 & 1 & 10 & 12 & 4 & 9 & 2124.98 & 0.265959 & NUI \\
\hline 3028.66 & 0.0054 & 0.0930 & 3 & 1 & 2 & 4 & 2 & 3 & $300 \cdot 35$ & 1.970843 & $2 \mathrm{NU} 2 * *$ \\
\hline 3028.71 & 0.0023 & 0.0877 & 6 & 1 & 5 & 6 & 2 & 4 & 2211.21 & 4.225862 & $2 \mathrm{~N} \cup 2 * * *$ \\
\hline 3029.75 & 0.0022 & 0.0806 & 5 & 1 & 5 & 6 & 0 & 6 & 446.71 & 4.958549 & $2 \mathrm{NU} 2 * *$ \\
\hline 3029.87 & 0.5756 & 0.0634 & 7 & 1 & 7 & 7 & 2 & 6 & 709.60 & 1.816721 & 2 NU2 \\
\hline 3030.72 & 18.7586 & 0.0841 & 2 & 2 & 1 & 3 & 3 & 0 & 285.43 & 2.366160 & $2 \mathrm{NU} 2$ \\
\hline 3031.27 & 0.0022 & 0.0739 & 6 & 3 & 4 & 6 & 4 & 3 & 2398.39 & 2.878858 & $2 N \cup 2 * * *$ \\
\hline 3031.74 & 17.5826 & 0.0771 & 5 & 0 & 5 & 6 & 1 & 6 & $447 \cdot 24$ & 4.979001 & $2 N \cup 2$ \\
\hline 3031.75 & 0.0113 & 0.0996 & 0 & 0 & 0 & 1 & 1 & 1 & 1634.97 & 1.000000 & $2 N \cup 2 * * *$ \\
\hline 3031.93 & 3.7126 & 0.0848 & 6 & 2 & 5 & 7 & 1 & 6 & 704.22 & 3.799063 & 2NU2 \\
\hline 3031.96 & 6.3375 & 0.0830 & 2 & 2 & 0 & 3 & 3 & 1 & 285.23 & 2.394805 & 2 NU 2 \\
\hline 3032.42 & 0.0312 & 0.0975 & 3 & 0 & 3 & 3 & 1 & 2 & 1772.41 & 2.185861 & $2 N \cup 2 * * *$ \\
\hline 3032.88 & 0.0011 & 0.0737 & 5 & 3 & 3 & 5 & 4 & 2 & 2251.71 & 2.024051 & $2 N \cup 2^{* * *} *$ \\
\hline 3032.98 & 0.0032 & 0.0699 & 4 & 3 & 2 & 4 & 4 & 1 & 2129.63 & 1.082946 & $2 N \cup 2 * * *$ \\
\hline 3033.49 & 0.0092 & 0.0884 & 5 & 2 & 3 & 5 & 3 & 2 & $2130 \cdot 50$ & 3.442901 & $2 \mathrm{NU} 2 * * *$ \\
\hline 3033.63 & 0.0011 & 0.0705 & 4 & 3 & 1 & 4 & 4 & 0 & 2129.65 & 1.088294 & $2 N \cup 2 * * *$ \\
\hline 3033.76 & 0.0072 & 0.0957 & 2 & 1 & 1 & 2 & 2 & 0 & 1743.47 & 1.258755 & $2 \mathrm{~N} \cup 2 * * *$ \\
\hline 3034.29 & 14.5185 & 0.0930 & 3 & 1 & 2 & 4 & 2 & 3 & 300.35 & 1.970843 & 2NU2 \\
\hline 3034.37 & 1.7979 & 0.0656 & 7 & 0 & 7 & 7 & 1 & 6 & $704 \cdot 22$ & 1.838288 & 2 NU2 \\
\hline 3034.64 & 0.0033 & 0.0759 & 5 & 3 & 2 & 5 & 4 & 1 & 2251.86 & 2.066632 & $2 N \cup 2 * * * *$ \\
\hline $3035 \cdot 14$ & 0.0028 & 0.0340 & 8 & 8 & 1 & 9 & 9 & 0 & $2225 \cdot 56$ & 8.403719 & 2NU2 \\
\hline 3035.79 & 5.8599 & 0.0806 & 5 & 1 & 5 & 6 & 0 & 6 & 446.71 & 4.958549 & 2NU2 \\
\hline 3036.02 & 0.0154 & 0.0912 & 5 & 1 & 4 & 5 & 2 & 3 & 2053.98 & 4.214107 & $2 N \cup 2 * * *$ \\
\hline 3036.71 & 0.0172 & 0.0880 & 4 & 0 & 4 & 5 & 1 & 5 & 326.64 & 3.972803 & $2 N \cup 2 *$ \\
\hline 3036.94 & 0.0075 & 0.0743 & 6 & 1 & 6 & 6 & 2 & 5 & 552.92 & 1.783035 & $2 N \cup 2 *$ \\
\hline 3037.08 & 0.0010 & 0.0927 & 6 & 3 & 4 & 7 & 2 & 5 & 2392.58 & 1.512999 & $2 N \cup 2 * * *$ \\
\hline 3037.12 & 0.0859 & 0.0486 & 10 & 2 & 9 & 10 & 3 & 8 & 6.16 & 3.605841 & 2 NU2 \\
\hline 3037.54 & 0.0299 & 0.0937 & 3 & 1 & 2 & 3 & 2 & 1 & 1819.34 & 2.538253 & $2 N \cup 2 * * *$ \\
\hline 3037.63 & 0.0132 & 0.0959 & 2 & 1 & 1 & 3 & 2 & 2 & 5.30 & 1.666667 & $2 N \cup 2 *$ \\
\hline 3038.73 & 0.0085 & 0.0934 & 4 & 1 & 3 & 4 & 2 & 2 & 2.92 & 3.642913 & $2 N \cup 2 * * *$ \\
\hline 3039.07 & 0.0020 & 0.0893 & 6 & 2 & 4 & 6 & 3 & 3 & 2.59 & 4.829435 & $2 N \cup 2 * * *$ \\
\hline 3041.25 & 0.0014 & 0.0832 & 7 & 3 & 4 & 7 & 4 & 3 & 2572.13 & 4.268667 & $2 N \cup 2 * * *$ \\
\hline 3041.30 & 0.0015 & 0.0532 & 11 & 2 & 9 & 12 & 5 & 8 & 2275.44 & 0.426841 & NUI \\
\hline 3042.33 & 0.0030 & 0.0886 & 7 & 2 & 5 & 7 & 3 & 4 & 2462.87 & 5.918411 & $2 \mathrm{~N} \cup 2 * * *$ \\
\hline 3042.67 & 0.0032 & 0.0880 & 4 & 0 & 4 & 5 & 1 & 5 & 326.64 & 3.972803 & $2 \mathrm{~N} \cup 2 * *$ \\
\hline 3042.70 & 0.0302 & 0.0520 & 10 & 1 & 9 & 10 & 2 & 8 & 1438.00 & 3.646815 & 2NU2 \\
\hline 3042.75 & 0.0039 & 0.0920 & 5 & 2 & 4 & 6 & 1 & 5 & 542.91 & 2.671788 & $2 \mathrm{~N} \cup 2 *$ \\
\hline 3042.92 & 0.0014 & 0.0743 & 6 & 1 & 6 & 6 & 2 & 5 & 552.92 & 1.783035 & $2 N \cup 2 * *$ \\
\hline 3043.20 & 0.0020 & 0.0678 & 9 & 3 & 6 & 10 & 6 & 5 & 1875.00 & 0.071662 & NUI \\
\hline 3043.31 & 0.0024 & 0.0959 & 2 & 1 & 1 & 3 & 2 & 2 & $206 \cdot 30$ & 1.666667 & $2 N \cup 2 * *$ \\
\hline 3043.50 & 0.0146 & 0.1021 & 2 & 0 & 2 & 2 & 1 & 1 & 1693.65 & 2.074578 & $2 N \cup 2 * * *$ \\
\hline $3044 \cdot 14$ & 0.0512 & 0.0914 & 4 & 1 & 4 & 5 & 0 & 5 & $325 \cdot 35$ & 3.911752 & $2 N \cup 2 *$ \\
\hline
\end{tabular}




\begin{tabular}{|c|c|c|c|c|c|c|c|c|c|c|c|}
\hline $\begin{array}{c}\text { FREQUENCY } \\
\mathrm{cm}^{-1}\end{array}$ & 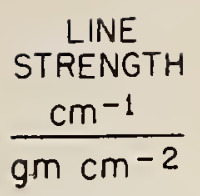 & $\begin{array}{l}\text { HALF } \\
\text { WIDTH } \\
\frac{\mathrm{cm}^{-1}}{\text { otm. }}\end{array}$ & $J^{\prime}$ & $K_{a}$ & $K_{c}$ & J" & $\mathrm{K}_{\mathrm{a}}$ & $K_{c}$ & $\begin{array}{c}E^{\prime \prime} \\
\mathrm{cm}^{-1}\end{array}$ & $L$ & BAND \\
\hline 3044.53 & 0.0027 & 0.0777 & 6 & 0 & 6 & 6 & 1 & 5 & 542.91 & 1.852055 & $2 N \cup 2 *$ \\
\hline 3046.20 & 0.0011 & 0.0623 & 7 & 3 & 5 & 8 & 6 & 2 & 1411.68 & 0.010619 & NU1 \\
\hline 3047.80 & 0.0010 & 0.0488 & 11 & 2 & 10 & 12 & 3 & 9 & 2105.90 & 0.278800 & NU 1 \\
\hline 3048.41 & 0.0140 & 0.0947 & 1 & 1 & 1 & 2 & 2 & 0 & $136 \cdot 17$ & 1.244747 & $2 N \cup 2 *$ \\
\hline 3048.64 & 8.5945 & 0.0880 & 4 & 0 & 4 & 5 & 1 & 5 & $326 \cdot 64$ & 3.972803 & 2 NU2 \\
\hline 3048.90 & 3.7332 & 0.0743 & 6 & 1 & 6 & 6 & 2 & 5 & 552.92 & 1.783035 & 2NU2 \\
\hline 3048.99 & 6.5817 & 0.0959 & 2 & 1 & 1 & 3 & 2 & 2 & $206 \cdot 30$ & 1.666667 & $2 N \cup 2$ \\
\hline 3050.04 & 0.0413 & 0.1075 & 1 & 0 & 1 & 1 & 1 & 0 & 1640.51 & 1.500000 & $2 N \cup 2 * * *$ \\
\hline 3050.26 & 0.0095 & 0.0914 & 4 & 1 & 4 & 5 & 0 & 5 & 325.35 & 3.911752 & $2 \mathrm{NU} 2 * *$ \\
\hline 3050.67 & 0.1971 & 0.0898 & 7 & 3 & 5 & 8 & 2 & 6 & 982.91 & 2.422109 & 2NU2 \\
\hline $3051 \cdot 33$ & 0.0024 & 0.0951 & 3 & 2 & 2 & 4 & 1 & 3 & 1875.49 & 0.835095 & $2 \mathrm{NU} 2 * * *$ \\
\hline 3051.90 & 0.0899. & 0.0563 & 9 & 2 & 8 & 9 & 3 & 7 & 1216.27 & 3.554957 & 2NU2 \\
\hline $3052 \cdot 47$ & 0.0652 & 0.0952 & 3 & 0 & 3 & 4 & 1 & 4 & 224.83 & 3.004990 & $2 \mathrm{NU} 2 *$ \\
\hline 3052.50 & 0.0069 & 0.1044 & 1 & 1 & 1 & 2 & 0 & 2 & 1664.97 & 0.755253 & $2 \mathrm{~N} \cup 2 * * *$ \\
\hline 3053.80 & 0.0015 & 0.0646 & 8 & 2 & 7 & 8 & 3 & 6 & $1006 \cdot 12$ & 3.456295 & 2NU2* \\
\hline 3054.17 & 0.0026 & 0.0947 & 1 & 1 & 1 & 2 & 2 & 0 & 136.17 & 1.244747 & $2 \mathrm{NU} 2 * *$ \\
\hline 3054.43 & 0.0048 & 0.0839 & 5 & 1 & 5 & 5 & 2 & 4 & $416 \cdot 22$ & 1.711792 & 2NU2* \\
\hline 3055.34 & 0.0024 & 0.0421 & 10 & 0 & 10 & 11 & 3 & 9 & 1695.03 & 0.097311 & NU1 \\
\hline 3055.57 & 0.0511 & 0.0993 & 1 & 1 & 0 & 2 & 2 & 1 & 134.91 & 1.500000 & $2 \mathrm{NU} 2 *$ \\
\hline 3055.60 & 1.9646 & 0.0920 & 5 & 2 & 4 & 6 & 1 & 5 & 542.91 & 2.671788 & 2 NU2 \\
\hline $3056 \cdot 38$ & 25.6158 & 0.0914 & 4 & 1 & 4 & 5 & 0 & 5 & $325 \cdot 35$ & 3.911752 & $2 N \cup 2$ \\
\hline 3057.15 & 1.3625 & 0.0777 & 6 & 0 & 6 & 6 & 1 & 5 & 542.91 & 1.852055 & $2 \mathrm{NU} 2$ \\
\hline 3058.01 & 0.0011 & 0.0694 & 8 & 3 & 5 & 9 & 6 & 4 & $1631 \cdot 27$ & 0.032042 & NU1 \\
\hline 3058 & 0.0121 & 0.0952 & 3 & 0 & 3 & 4 & 1 & 4 & 224.83 & 3.004990 & $2 \mathrm{NU} 2 * *$ \\
\hline 3058 & 0.0012 & 0.0622 & 10 & 1 & 10 & 9 & 2 & 7 & 1201.95 & 0.015027 & $2 \mathrm{NU} 2$ \\
\hline 3059 & 0.0019 & 0.0459 & 13 & 2 & 11 & 13 & 3 & 10 & $2414 \cdot 73$ & 5.73353 & $2 N U 2$ \\
\hline 3055 & 0.0074 & 0.0440 & 10 & 1 & 10 & 11 & 2 & 9 & .70 & 0.097873 & NU1 \\
\hline 3059.93 & 7.0055 & 0.0947 & 1 & 1 & 1 & 2 & 2 & 0 & 136.17 & 1.244747 & $2 N U 2$ \\
\hline $3061 \cdot 16$ & 0.3016 & 0.0613 & 9 & 1 & 8 & 9 & 2 & 7 & $1201 \cdot 95$ & 3.674255 & 2NU2 \\
\hline 3061 & 0.0095 & 0.0993 & 1 & 1 & 0 & 2 & 2 & 1 & 134.91 & 1.500000 & $2 \mathrm{NU} 2^{* *} *$ \\
\hline 3062.61 & 0.0035 & 0.0562 & 9 & 0 & 9 & 8 & 3 & 6 & $1006 \cdot 12$ & 0.015 & 2NU2 \\
\hline 3062.77 & 0.0111 & 0.0863 & 9 & 4 & 6 & 10 & 3 & 7 & .23 & 2.171641 & $2 N \cup 2$ \\
\hline 3064.40 & 32.6126 & 0.0952 & 3 & 0 & 3 & 4 & 1 & 4 & $224 \cdot 83$ & 3.004990 & 2NU2 \\
\hline 3065 & 0.0209 & 0.0974 & 3 & 1 & 3 & 4 & 0 & 4 & 222.06 & 2.831638 & $2 N \cup 2 *$ \\
\hline 3065.59 & 0.7553 & 0.0646 & 8 & 2 & 7 & 8 & 3 & 6 & $1006 \cdot 12$ & 3.456295 & 2 NU2 2 \\
\hline 3065.85 & 0.0012 & 0.0722 & 7 & 2 & 6 & 7 & 3 & 5 & $6 \cdot 72$ & 3.273499 & $2 \mathrm{NU} 2^{*}$ \\
\hline 3066.02 & 0.0036 & 0.0467 & 12 & 3 & 10 & 12 & 4 & 9 & 2124.98 & 5.350112 & 2NU2 \\
\hline 3066.28 & 2.3799 & 0.0839 & 5 & 1 & 5 & 5 & 2 & 4 & $416 \cdot 22$ & 1.711792 & $2 N \cup 2$ \\
\hline 3067.01 & 25.5426 & 0.0993 & 1 & 1 & 0 & 2 & 2 & 1 & 134.91 & 1.500000 & 2 NU 2 \\
\hline 3067.72 & 0.0237 & 0.1006 & 2 & 0 & 2 & 3 & 1 & 3 & $142 \cdot 28$ & 2.156792 & $2 N \cup 2 *$ \\
\hline 3068.43 & 0.0020 & 0.0927 & 6 & 3 & 4 & 7 & 2 & 5 & $782 \cdot 40$ & 1.512999 & $2 N \cup 2 *$ \\
\hline 3069.44 & 0.0149 & 0.0947 & 4 & 2 & 3 & 5 & 1 & 4 & 399.46 & 1.638916 & 2NU2* \\
\hline 3069.62 & 0.0234 & 0.0907 & 4 & 1 & 4 & 4 & 2 & 3 & $300 \cdot 35$ & 1.567300 & 2 NU2* \\
\hline 3071.73 & 0.0039 & 0.0974 & 3 & 1 & 3 & 4 & 0 & 4 & 222.06 & 2.831638 & $2 N \cup 2 * *$ \\
\hline 3072.20 & 0.0107 & 0.0356 & 7 & 7 & 1 & 8 & 8 & 0 & $1789 \cdot 10$ & 7.402783 & 2NU2 \\
\hline 3072.20 & 0.0322 & 0.0356 & 7 & 7 & 0 & 8 & 8 & 1 . & 1789.10 & 7.402783 & $2 \mathrm{NU} 2$ \\
\hline 3073.18 & 0.0032 & 0.0871 & 5 & 0 & 5 & 5 & 1 & 4 & 399.46 & 1.912338 & $2 N \cup 2 * *$ \\
\hline 3073 & 0.0042 & 0.0691 & 7 & 3 & 4 & 8 & 6 & 3 & 1.65 & 0.013422 & NU1 \\
\hline 3073.70 & 0.0044 & 0.1006 & 2 & 0 & 2 & 3 & 1 & 3 & 142.28 & 2.156792 & $2 N \cup 2 * *$ \\
\hline 3075.50 & 0.0043 & 0.0907 & 4 & 1 & 4 & 4 & 2 & 3 & $300 \cdot 35$ & 1.567300 & $2 \mathrm{~N} \cup 2 * *$ \\
\hline 3075.84 & 0.0078 & 0.0783 & 6 & 2 & 5 & 6 & 3 & 4 & 648.97 & 2.963539 & $2 N \cup 2 *$ \\
\hline 3076.02 & 0.0028 & 0.0947 & 4 & 2 & 3 & 5 & 1 & 4 & 399.46 & 1.638916 & $2 \operatorname{NU} 2 * *$ \\
\hline 3076.10 & 0.0018 & 0.0519 & 12 & 2 & 10 & 12 & 3 & 9 & 2105.90 & 5.509361 & 2NU2 \\
\hline 3076.70 & 0.0038 & 0.0617 & 6 & 3 & 4 & 7 & 6 & 1 & $1216 \cdot 20$ & 0.004557 & NU1 \\
\hline
\end{tabular}




\begin{tabular}{|c|c|c|c|c|c|c|c|c|c|c|c|}
\hline $\begin{array}{c}\text { FREQUENCY } \\
\mathrm{cm}^{-1}\end{array}$ & 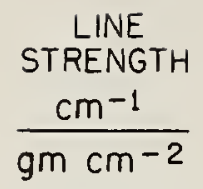 & $\begin{array}{l}\text { HALF } \\
\text { WIDTH } \\
\frac{\mathrm{cm}^{-1}}{\operatorname{atm} .}\end{array}$ & $J^{\prime}$ & $\mathrm{K}_{\mathrm{a}}$ & $K_{c}$ & Ј" & $K_{a}$ & $K_{c}$ & $\begin{array}{c}E^{\prime \prime} \\
\mathrm{cm}^{-1}\end{array}$ & $L$ & BAND \\
\hline 3076.98 & 0.0059 & 0.0528 & 11 & 3 & 9 & 11 & 4 & 8 & 1843.02 & 5.243402 & 2 NU2 \\
\hline 3077.34 & 0.0058 & 0.0753 & 5 & 1 & 4 & 4 & 4 & 1 & $488 \cdot 10$ & 0.001994 & $2 \mathrm{NU} 2$ \\
\hline 3077.43 & 0.6168 & 0.0722 & 7 & 2 & 6 & 7 & 3 & 5 & 816.72 & 3.273499 & $2 \mathrm{NU} 2$ \\
\hline 3077.93 & 10.4331 & 0.0974 & 3 & 1 & 3 & 4 & 0 & 4 & 222.06 & 2.831638 & $2 \mathrm{NU} 2$ \\
\hline 3078.90 & 0.0014 & 0.0711 & 7 & 2 & 5 & 6 & 5 & 2 & 888.60 & 0.003511 & $2 \mathrm{NU} 2$ \\
\hline 3079.52 & 8.7109 & 0.0871 & 5 & 0 & 5 & 5 & 1 & 4 & 399.46 & 1.912338 & $2 \mathrm{NU} 2$ \\
\hline 3079.69 & 11.8510 & 0.1006 & 2 & 0 & 2 & 3 & 1 & 3 & $142 \cdot 28$ & 2.156792 & $2 \mathrm{NU}_{2}$ \\
\hline 3079.95 & 0.3099 & 0.0706 & 8 & 1 & 7 & 8 & 2 & 6 & 982.91 & 3.773034 & $2 \mathrm{NU} 2$ \\
\hline $3081 \cdot 38$ & 11.7249 & 0.0907 & 4 & 1 & 4 & 4 & 2 & 3 & $300 \cdot 35$ & 1.567300 & 2 NU 2 \\
\hline 3082.03 & 0.0104 & 0.0944 & 3 & 1 & 3 & 3 & 2 & 2 & $206 \cdot 30$ & 1.297651 & $2 \mathrm{NU} 2 *$ \\
\hline 3082.55 & 1.0167 & 0.0927 & 6 & 3 & 4 & 7 & 2 & 5 & 782.40 & 1.512999 & 2 NU2 2 \\
\hline 3082.60 & 7.4729 & 0.0947 & 4 & 2 & 3 & 5 & 1 & 4 & 399.46 & 1.638916 & $2 \mathrm{~N} \cup 2$ \\
\hline 3083.17 & 0.0022 & 0.0595 & 10 & 2 & 8 & 11 & 5 & 7 & 1985.83 & 0.365425 & NUI \\
\hline 3083.33 & 0.0045 & 0.0833 & 5 & 2 & 4 & 5 & 3 & 3 & 504.00 & 2.492169 & $2 N \cup 2 *$ \\
\hline 3083.88 & 0.0054 & 0.0802 & 7 & 1 & 6 & 7 & 2 & 5 & 782.40 & 3.983004 & $2 N \cup 2 *$ \\
\hline 3083.90 & 0.0680 & 0.1037 & 1 & 0 & 1 & 2 & 1 & 2 & 79.48 & 1.500000 & $2 \operatorname{NU} 2 *$ \\
\hline 3085.32 & 0.0020 & 0.0445 & 9 & 6 & 4 & 10 & 8 & 3 & 2254.34 & 0.051950 & NU3 \\
\hline 3086.07 & 0.0015 & 0.0710 & 9 & 1 & 9 & 8 & 2 & 6 & 982.91 & 0.018354 & 2 NU2 \\
\hline 3086.30 & 0.0029 & 0.0438 & 12 & 1 & 11 & 13 & 3 & 10 & 2414.73 & 0.074330 & NU3 \\
\hline 3086.66 & 0.0629 & 0.0590 & 10 & 3 & 8 & 10 & 4 & 7 & $1581 \cdot 34$ & 5.051335 & 2NU2 \\
\hline 3087.15 & 0.0116 & 0.0942 & 4 & 0 & 4 & 4 & 1 & 3 & 275.52 & 2.047924 & $2 N \cup 2 *$ \\
\hline 3087.18 & 3.8873 & 0.0783 & 6 & 2 & 5 & 6 & 3 & 4 & 648.97 & 2.963539 & $2 \mathrm{NU} 2$ \\
\hline 3087.86 & 0.0019 & 0.0944 & 3 & 1 & 3 & 3 & 2 & 2 & $206 \cdot 30$ & 1.297651 & $2 N \cup 2 * *$ \\
\hline 3088.51 & 0.0184 & 0.0845 & 4 & 2 & 3 & 4 & 3 & 2 & 382.52 & 1.848533 & $2 \mathrm{NU} 2 *$ \\
\hline 3088.57 & 0.0594 & 0.0994 & 2 & 1 & 2 & 3 & 0 & 3 & 136.77 & 1.741385 & $2 \mathrm{NU} 2 *$ \\
\hline 3089.04 & 0.0011 & 0.0794 & 8 & 5 & 4 & 9 & 4 & 5 & 2998.78 & $7 \cdot 39$ & NUI*** \\
\hline 3089.62 & 0.0236 & 0.0684 & 7 & 0 & 7 & 6 & 3 & 4 & 648.97 & 0.017960 & $2 \mathrm{NU} 2$ \\
\hline 3089.92 & 0.0126 & 0.1037 & 1 & 0 & 1 & 2 & 1 & 2 & 79.48 & 1.500000 & $2 N \cup 2 * *$ \\
\hline 3090.05 & 0.0112 & 0.0845 & 4 & 0 & 4 & 3 & 3 & 1 & $285 \cdot 23$ & 0.004153 & $2 \mathrm{NU} 2$ \\
\hline 3091.03 & 0.0011 & 0.0705 & 8 & 3 & 6 & 8 & 4 & 5 & 1122.72 & 4.264036 & $2 \operatorname{NU} 2 *$ \\
\hline 3091.43 & 0.0287 & 0.0955 & 2 & 1 & 2 & 2 & 2 & 1 & 134.91 & 0.833333 & $2 \mathrm{NU} 2 *$ \\
\hline 3091.51 & 0.0056 & 0.0833 & 3 & 2 & 2 & 3 & 3 & 1 & $285 \cdot 23$ & 1.035682 & $2 N \cup 2 *$ \\
\hline 3091.98 & 0.0014 & 0.0663 & 6 & 3 & 3 & 7 & 6 & 2 & $1216 \cdot 20$ & 0.004975 & NU 1 \\
\hline 3092.30 & 0.0014 & 0.0597 & 9 & 1 & 8 & 10 & 5 & 5 & 1724.72 & 0.005774 & NU3 \\
\hline 3092.74 & 0.0228 & 0.0614 & 11 & 2 & 9 & 11 & 3 & 8 & 1813.26 & 5.642326 & $2 N \cup 2$ \\
\hline 3093.45 & 0.0022 & 0.0942 & 4 & 0 & 4 & 4 & 1 & 3 & 275.52 & 2.047924 & $2 \operatorname{NU} 2 * *$ \\
\hline 3093.69 & 5.1996 & 0.0944 & 3 & 1 & 3 & 3 & 2 & 2 & 206.30 & 1.297651 & $2 \mathrm{NU} 2$ \\
\hline 3094.02 & 0.0034 & 0.0845 & 4 & 2 & 3 & 4 & 3 & 2 & 382.52 & 1.848533 & $2 \operatorname{NU} 2 * *$ \\
\hline 3094.51 & 2.2546 & 0.0833 & 5 & 2 & 4 & 5 & 3 & 3 & 504.00 & 2.492169 & $2 \mathrm{NU} 2$ \\
\hline 3094.86 & 0.0110 & 0.0994 & 2 & 1 & 2 & 3 & 0 & 3 & 136.77 & 1.741385 & $2 \mathrm{NU} 2 * *$ \\
\hline 3095.41 & 0.0655 & 0.0651 & 9 & 3 & 7 & 9 & 4 & 6 & 1340.89 & 4.734521 & $2 \mathrm{NU} 2$ \\
\hline 3095.94 & 34.0199 & 0.1037 & 1 & 0 & 1 & 2 & 1 & 2 & 79.48 & 1.500000 & $2 \mathrm{NU} 2$ \\
\hline 3096.46 & 0.0551 & 0.0816 & 5 & 0 & 5 & 4 & 3 & 2 & $382 \cdot 52$ & 0.011053 & 2 NU2 \\
\hline 3096.54 & 0.0178 & 0.0835 & 3 & 2 & 1 & 3 & 3 & 0 & 285.43 & 1.092505 & $2 N \cup 2 *$ \\
\hline 3096.90 & 2.6891 & 0.0802 & 7 & 1 & 6 & 7 & 2 & 5 & 782.40 & 3.983004 & 2 NU2 \\
\hline 3096.95 & 0.0041 & 0.0656 & 7 & 2 & 6 & 8 & 5 & 3 & 1255.92 & 0.018297 & NUI \\
\hline 3097.22 & 0.0053 & 0.0955 & 2 & 1 & 2 & 2 & 2 & 1 & 134.91 & 0.833333 & $2 \mathrm{NU} 2 * *$ \\
\hline 3097.65 & 0.0047 & 0.0877 & 6 & 1 & 5 & 6 & 2 & 4 & 602.77 & 4.225862 & $2 N \cup 2 *$ \\
\hline 3097.76 & 0.0044 & 0.0739 & 6 & 3 & 4 & 6 & 4 & 3 & 756.75 & 2.878858 & $2 \mathrm{NU} 2 *$ \\
\hline 3098.81 & 0.0048 & 0.0951 & 3 & 2 & 2 & 4 & 1 & 3 & 275.52 & 0.835095 & $2 \mathrm{~N} \cup 2 *$ \\
\hline 3098.85 & 0.0785 & 0.0887 & 8 & 4 & 5 & 9 & 3 & 6 & 1282.92 & 1.416234 & $2 \mathrm{NU} 2$ \\
\hline 3099.01 & 0.0022 & 0.0737 & 5 & 3 & 3 & 5 & 4 & 2 & 610.12 & 2.024051 & $2 \mathrm{NU} 2 *$ \\
\hline $3099 \cdot 54$ & 9.2244 & 0.0845 & 4 & 2 & 3 & 4 & 3 & 2 & 382.52 & 1.848533 & $2 \mathrm{NU} 2$ \\
\hline 3099.76 & 5.8166 & 0.0942 & 4 & 0 & 4 & 4 & 1 & 3 & 275.52 & 2.047924 & 2 NU2 \\
\hline
\end{tabular}




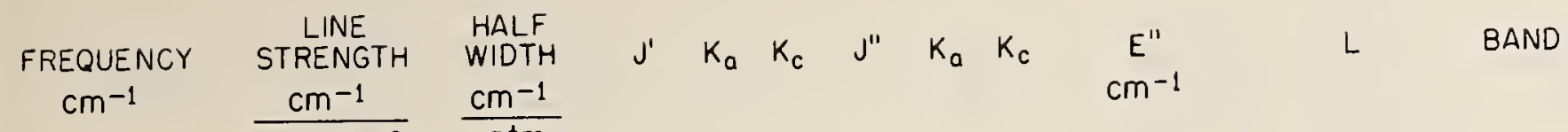

\begin{tabular}{|c|c|c|c|c|c|c|c|c|c|c|c|}
\hline $\begin{array}{l}3100.33 \\
3100.64\end{array}$ & $\begin{array}{l}0.0021 \\
0.0029\end{array}$ & $\begin{array}{l}0.0705 \\
0.0694\end{array}$ & $\begin{array}{l}4 \\
7\end{array}$ & $\begin{array}{l}3 \\
1\end{array}$ & $\begin{array}{l}1 \\
7\end{array}$ & $\begin{array}{l}4 \\
8\end{array}$ & $\begin{array}{l}4 \\
4\end{array}$ & $\begin{array}{l}0 \\
4\end{array}$ & $\begin{array}{r}488.13 \\
1131.76\end{array}$ & $\begin{array}{l}1.088294 \\
0.006860\end{array}$ & $\begin{array}{l}2 \text { NU2* } \\
\text { NUI }\end{array}$ \\
\hline 3101.16 & 29.7089 & 0.0994 & 2 & 1 & 2 & 3 & 0 & 3 & 136.77 & 1.741385 & $2 N \cup 2$ \\
\hline 3101.81 & 0.0020 & 0.0389 & 11 & 0 & 11 & 12 & 2 & 10 & 1960.22 & 0.025215 & NU3 \\
\hline 3101.90 & 0.5262 & 0.0705 & 8 & 3 & 6 & 8 & 4 & 5 & 1122.72 & 4.264036 & 2 NU 2 \\
\hline 3101.93 & 0.0033 & 0.0835 & 3 & 2 & 1 & 3 & 3 & 0 & 285.43 & 1.092505 & $2 N \cup 2 * *$ \\
\hline $3102 \cdot 18$ & 0.0073 & 0.1032 & 2 & 0 & 2 & 1 & 1 & 1 & 1634.97 & 0.755253 & $2 \mathrm{NU} 2 * * *$ \\
\hline $3102 \cdot 30$ & 0.0066 & 0.0759 & 5 & 3 & 2 & 5 & 4 & 1 & 610.35 & 2.066632 & $2 N \cup 2 *$ \\
\hline 3102.36 & 0.0226 & 0.0996 & 0 & 0 & 0 & 1 & 1 & 1 & 37.13 & 1.000000 & $2 N \cup 2 *$ \\
\hline 3102.45 & 2.8045 & 0.0833 & 3 & 2 & 2 & 3 & 3 & 1 & 285.23 & 1.035682 & 2 NU2 2 \\
\hline 3103.02 & 14.3570 & 0.0955 & 2 & 1 & 2 & 2 & 2 & 1 & 134.91 & 0.833333 & 2 NU2 \\
\hline 3103.37 & 0.0307 & 0.0701 & 10 & 2 & 8 & 10 & 3 & 7 & $1538 \cdot 23$ & 5.910419 & $2 N \cup 2$ \\
\hline 3103.44 & 0.0624 & 0.0975 & 3 & 0 & 3 & 3 & 1 & 2 & 173.36 & 2.185861 & 2 NU $2 *$ \\
\hline 3104.58 & 0.0012 & 0.0699 & 4 & 3 & 2 & 4 & 4 & 1 & $488 \cdot 10$ & 1.082946 & $2 \mathrm{NU} 2 * *$ \\
\hline $3104 \cdot 94$ & 0.0461 & 0.1111 & 1 & 1 & 0 & 1 & 0 & 1 & $1618 \cdot 55$ & 1.500000 & $2 \mathrm{~N} \cup 2 * * *$ \\
\hline 3105.81 & 0.3969 & 0.0732 & 7 & 3 & 5 & 7 & 4 & 4 & 927.77 & 3.637027 & $2 \mathrm{NU} 2$ \\
\hline 3106.02 & 0.0016 & 0.0801 & 6 & 3 & 3 & 6 & 4 & 2 & 757.78 & 3.075987 & $2 \mathrm{NU} 2 *$ \\
\hline 3106.72 & 0.0013 & 0.0862 & 4 & 2 & 2 & 4 & 3 & 1 & 383.85 & 2.167225 & $2 \mathrm{~N} \cup 2 * *$ \\
\hline 3106.73 & 0.0307 & 0.0912 & 5 & 1 & 4 & 5 & 2 & 3 & 446.50 & 4.214107 & $2 \mathrm{NU} 2 *$ \\
\hline 3107.32 & 8.8801 & 0.0835 & 3 & 2 & 1 & 3 & 3 & 0 & 285.43 & 1.092505 & $2 \mathrm{NU} 2$ \\
\hline 3107.35 & 0.0184 & 0.0884 & 5 & 2 & 3 & 5 & 3 & 2 & 508.81 & 3.442901 & $2 \mathrm{NU} 2 *$ \\
\hline 3107.60 & 0.0144 & 0.0957 & 2 & 1 & 1 & 2 & 2 & 0 & 136.17 & 1.258755 & $2 \mathrm{NU} 2 *$ \\
\hline $3108 \cdot 20$ & 2.2180 & 0.0739 & 6 & 3 & 4 & 6 & 4 & 3 & 756.75 & 2.878858 & 2 NU2 \\
\hline 3108.42 & 0.0042 & 0.0996 & 0 & 3 & 0 & 1 & 1 & 1 & 37.13 & 1.000000 & $2 \mathrm{NU}_{2 * *} *$ \\
\hline 3108.63 & 0.0016 & 0.0909 & 4 & 3 & 2 & 5 & 2 & 3 & 2053.98 & 0.436045 & $2 \mathrm{NU} 2^{* * * *}$ \\
\hline 3108.89 & 0.0025 & 0.0627 & 5 & 3 & 2 & 6 & 6 & 1 & 1045.07 & 0.001286 & NU1 \\
\hline 3109.35 & 1.0823 & 0.0737 & 5 & 3 & 3 & 5 & 4 & 2 & $610 \cdot 12$ & 2.024051 & $2 N \cup 2$ \\
\hline 3109.65 & 0.0115 & 0.0975 & 3 & 0 & 3 & 3 & 1 & 2 & $173 \cdot 36$ & 2.185861 & $2 \mathrm{~N} \cup 2 * *$ \\
\hline 3109.74 & 3.1980 & 0.0699 & 4 & 3 & 2 & 4 & 4 & 1 & 488.10 & 1.082946 & 2 NU2 \\
\hline 3110.29 & 2.3448 & 0.0877 & 6 & 1 & 5 & 6 & 2 & 4 & 602.77 & 4.225862 & $2 \mathrm{NU} 2$ \\
\hline 3110.57 & 1.0714 & 0.0705 & 4 & 3 & 1 & 4 & 4 & 0 & $488 \cdot 13$ & 1.088294 & $2 \mathrm{NU} 2$ \\
\hline 3110.87 & 0.0598 & 0.0937 & 3 & 1 & 2 & 3 & 2 & 1 & 212.15 & 2.538253 & $2 \mathrm{NU} 2 *$ \\
\hline 3110.93 & 0.0170 & 0.0934 & 4 & 1 & 3 & 4 & 2 & 2 & 315.79 & 3.642913 & $2 \mathrm{NU} 2 *$ \\
\hline 3111.99 & 0.0040 & 0.0893 & 6 & 2 & 4 & 6 & 3 & 3 & 661.56 & 4.829435 & $2 \operatorname{NU} 2 *$ \\
\hline 3112.08 & 3.5955 & 0.0862 & 4 & 2 & 2 & 4 & 3 & 1 & 383.85 & 2.167225 & $2 \mathrm{NU} 2$ \\
\hline 3112.16 & 2.3813 & 0.0951 & 3 & 2 & 2 & 4 & 1 & 3 & 275.52 & 0.835095 & $2 \mathrm{NU} 2$ \\
\hline 3112.26 & 0.0194 & 0.1044 & 2 & 1 & 1 & 2 & 0 & 2 & 1664.97 & 2.074578 & $2 \mathrm{NU} 2 * * *$ \\
\hline 3112.40 & 3.3142 & 0.0759 & 5 & 3 & 2 & 5 & 4 & 1 & 610.35 & 2.066632 & $2 \mathrm{NU} 2$ \\
\hline 3112.67 & 0.0050 & 0.0425 & 8 & 6 & 2 & 9 & 8 & 1 & 2009.87 & 0.032025 & NU3 \\
\hline 3112.73 & 0.0034 & 0.0884 & 5 & 2 & 3 & 5 & 3 & 2 & 508.81 & 3.442901 & $2 \operatorname{NU} 2 * * *$ \\
\hline 3112.83 & 0.0057 & 0.0912 & 5 & 1 & 4 & 5 & 2 & 3 & $446 \cdot 50$ & 4.214107 & $2 \mathrm{NU} 2 * *$ \\
\hline 3112.88 & 0.0165 & 0.0801 & 8 & 1 & 8 & 7 & 2 & 5 & 782.40 & 0.024347 & 2 NU2 \\
\hline 3113 & 0.0014 & 0.0504 & 10 & 5 & 6 & 11 & 7 & 5 & 2321.89 & 0.107152 & NU3 \\
\hline 12 & 0.0139 & 0.0567 & 10 & 2 & 9 & 11 & 3 & 8 & 1813.26 & 0.316624 & NU I \\
\hline 29 & 0.0139 & 0.1044 & 1 & 1 & 1 & 2 & 0 & 2 & 70.08 & 0.755253 & $2 \mathrm{NU} 2 *$ \\
\hline 3113.36 & 0.0027 & 0.0957 & 2 & 1 & 1 & 2 & 2 & 0 . & $136 \cdot 17$ & 1.258755 & $2 \mathrm{NU} 2 * *$ \\
\hline 3113.49 & 0.0060 & 0.0886 & 7 & 2 & 5 & 7 & 3 & 4 & $842 \cdot 38$ & 5.918411 & $2 N \cup 2 *$ \\
\hline 3113.57 & 0.0280 & 0.0482 & 9 & C & 9 & 10 & 3 & 8 & $1446 \cdot 16$ & 0.106449 & NU 1 \\
\hline 3113.69 & 0.0011 & 0.0825 & 4 & 2 & 3 & 3 & 3 & 0 & 1907.63 & 0.131472 & $2 \mathrm{NU} 2 * * * *$ \\
\hline 3114.47 & 11.3161 & 0.0996 & 0 & 0 & 0 & 1 & 1 & 1 & 37.13 & 1.000000 & 2NU2 \\
\hline 3114.53 & 0.0293 & 0.1021 & 2 & 0 & 2 & 2 & 1 & 1 & 95.17 & 2.074578 & $2 \mathrm{~N} \cup 2 *$ \\
\hline 3115.25 & 0.0018 & 0.0613 & 11 & 3 & 9 & 12 & 4 & 8 & 2205.65 & 0.720922 & NU1 \\
\hline 3115.87 & 31.2022 & 0.0975 & 3 & 0 & 3 & 3 & 1 & 2 & 173.36 & 2.185861 & $2 \mathrm{NU} 2$ \\
\hline 3115.94 & 0.7877 & 0.0801 & 6 & 3 & 3 & 6 & 4 & 2 & 757.78 & 3.075987 & $2 \mathrm{NU} 2$ \\
\hline
\end{tabular}




\begin{tabular}{|c|c|c|c|c|c|c|c|c|c|c|c|}
\hline $\begin{array}{l}\text { FREQUENCY } \\
\mathrm{cm}^{-1}\end{array}$ & $\begin{array}{l}\text { LINE } \\
\text { STRENGTH } \\
\frac{\mathrm{cm}^{-1}}{\mathrm{am} \mathrm{cm}^{-2}}\end{array}$ & $\begin{array}{l}\text { HALF } \\
\text { WIDTH } \\
\frac{\mathrm{cm}^{-1}}{\mathrm{a}+\mathrm{m}}\end{array}$ & $J^{\prime}$ & $\mathrm{K}_{\mathrm{a}}$ & $K_{c}$ & $J^{\prime \prime}$ & $K_{a}$ & $\mathrm{~K}_{\mathrm{c}}$ & $\begin{array}{c}E^{\prime \prime} \\
\mathrm{cm}^{-1}\end{array}$ & L & BAND \\
\hline 3116.39 & 0.3480 & 0.0783 & 9 & 2 & 7 & 9 & 3 & 6 & 1282.92 & 6.247031 & $2 \mathrm{NU} 2$ \\
\hline 3116.68 & 0.0111 & 0.0937 & 3 & 1 & 2 & 3 & 2 & 1 & $212 \cdot 15$ & 2.538253 & $2 \mathrm{NU} 2 * * *$ \\
\hline 3116.70 & 0.4801 & 0.0925 & 5 & 3 & 3 & 6 & 2 & 4 & 602.77 & 0.863547 & 2 NU2 2 \\
\hline 3116.85 & 0.0032 & 0.0934 & 4 & 1 & 3 & 4 & 2 & 2 & 315.79 & 3.642913 & $2 \operatorname{NU} 2 * *$ \\
\hline 3117.10 & 0.0018 & 0.0578 & 5 & 4 & 1 & 5 & 5 & 0 & $742 \cdot 10$ & 1.101100 & $2 \mathrm{NU} 2 *$ \\
\hline 3117.12 & 0.0017 & 0.0620 & 6 & 4 & 3 & 6 & 5 & 2 & $888 \cdot 60$ & 2.085226 & $2 \operatorname{NU} 2 *$ \\
\hline 3118.12 & 9.1917 & 0.0884 & 5 & 2 & 3 & 5 & 3 & 2 & $508 \cdot 81$ & 3.442901 & $2 N \cup 2$ \\
\hline 3118.93 & 0.0186 & 0.0659 & 9 & 2 & 7 & 10 & 5 & 6 & 1718.77 & 0.266747 & NU 1 \\
\hline $3118 \cdot 94$ & 15.3668 & 0.0912 & 5 & 1 & 4 & 5 & 2 & 3 & 446.50 & 4.214107 & 2NU2 \\
\hline $3119 \cdot 12$ & 7.2213 & 0.0957 & 2 & 1 & 1 & 2 & 2 & 0 & $136 \cdot 17$ & 1.258755 & 2NU2 \\
\hline 3119. & 0.0026 & 0.1044 & 1 & 1 & 1 & 2 & 0 & 2 & 70.08 & 0.755253 & 2 NU $2 * *$ \\
\hline 3120. & 0.0043 & 0.0569 & 10 & 5 & 5 & 11 & 7 & 4 & 2321.94 & 0.108914 & NU3 \\
\hline 3120.66 & 0.0054 & 0.1021 & 2 & 0 & 2 & 2 & 1 & 1 & 95.17 & 2.074578 & $2 \mathrm{~N} U 2 * *$ \\
\hline 3120.86 & 0.0826 & 0.1075 & 1 & 0 & 1 & 1 & 1 & 0 & $42 \cdot 37$ & 1.500000 & 2 NU $2 *$ \\
\hline 3121.63 & 0.0354 & 0.0613 & 10 & 4 & 7 & 10 & 5 & 6 & 1718.77 & 5.482102 & $2 N \cup 2$ \\
\hline $3121 \cdot 66$ & 1.3799 & 0.0832 & 7 & 3 & 4 & 7 & 4 & 3 & 931.22 & 4.268667 & $2 \mathrm{NU} 2$ \\
\hline 3121.76 & 0.0099 & 0.0511 & 9 & 1 & 9 & 10 & 2 & 8 & 1438.00 & 0.108277 & NU1 \\
\hline 3122.49 & 29.9070 & 0.0937 & 3 & 1 & 2 & 3 & 2 & 1 & $212 \cdot 25$ & 2.538253 & $2 \mathrm{NU} 2$ \\
\hline $3122 \cdot 78$ & 8.5222 & 0.0934 & 4 & 1 & 3 & 4 & 2 & 2 & $315 \cdot 79$ & 3.642913 & $2 N U 2$ \\
\hline 3123.07 & 2.0057 & 0.0893 & 6 & 2 & 4 & 6 & 3 & 3 & 661.56 & 4.829435 & $2 N U 2$ \\
\hline 3123.13 & 0.3787 & 0.0840 & 8 & 2 & 6 & 8 & 3 & 5 & $1050 \cdot 15$ & 6.368783 & 2NU2 \\
\hline 3124.99 & 0.0385 & 0.0994 & 3 & 1 & 2 & 3 & 0 & 3 & 1731.89 & 2.185861 & $2 \mathrm{NU} 2 * * *$ \\
\hline 3125.12 & 2.9878 & 0.0886 & 7 & 2 & 5 & 7 & 3 & 4 & $842 \cdot 38$ & 5.918411 & 2NU2 \\
\hline 3126.01 & 0.0339 & 0.0639 & 9 & 4 & 6 & 9 & 5 & 5 & 1474.99 & 4.736397 & $2 \mathrm{NU} 2$ \\
\hline 3126.02 & 6.9402 & 0.1044 & 1 & 1 & 1 & 2 & 0 & 2 & 70.08 & 0.755253 & $2 \mathrm{NU} 2$ \\
\hline 3126.61 & 0.2500 & 0.0650 & 8 & 4 & 5 & 8 & 5 & 4 & $1255 \cdot 16$ & 3.901175 & $2 N \cup 2$ \\
\hline 3126.78 & 0.3058 & 0.0574 & 5 & 4 & 2 & 5 & 5 & 1 & $742 \cdot 10$ & $1 \cdot 10$ & $2 \mathrm{NU} 2$ \\
\hline 3126.80 & 14.6442 & 0.1021 & 2 & 0 & 2 & 2 & 1 & 1 & 95.17 & 2.074578 & 2NU2 \\
\hline 3126.81 & 0.1711 & 0.0645 & 7 & 4 & 4 & 7 & 5 & 3 & 1059.65 & 3.012848 & $2 N \cup 2$ \\
\hline 3126.85 & 0.9180 & 0.0578 & 5 & 4 & 1 & 5 & 5 & 0 & $742 \cdot 10$ & 1.101 & $2 \mathrm{NU} 2$ \\
\hline 3126.86 & 0.8358 & 0.0620 & 6 & 4 & 3 & 6 & 5 & 2 & $888 \cdot 60$ & 2.085226 & 2NU2 \\
\hline 3126.95 & 0.0153 & 0.1075 & 1 & 0 & 1 & 1 & 1 & 0 & .37 & 1.500000 & $2 \mathrm{NU} 2 * *$ \\
\hline $3127 \cdot 37$ & 0.2793 & 0.0637 & 6 & 4 & 2 & 6 & 5 & 1 & .63 & 2.089996 & 2NU2 \\
\hline 3127.75 & 0.0409 & 0.0994 & 3 & 0 & 3 & 2 & 1 & 2 & 1677 & 1.741385 & $2 N \cup 2 * * * *$ \\
\hline 3127.89 & 0.0054 & 0.0654 & 8 & J & 8 & 9 & 4 & 5 & $\cdot 28$ & 0.001245 & NU3 \\
\hline 3128. & 0.5182 & 0.0680 & 7 & 4 & 3 & 7 & 5 & 2 & 1059 & 3.039271 & $2 \mathrm{NU} 2$ \\
\hline 3128. & 0.2265 & 0.0844 & 8 & 3 & 5 & 8 & 4 & 4 & 1131 & 5.709388 & $2 \mathrm{~N} \cup 2$ \\
\hline 312 & 0.0087 & 0.0949 & 2 & 2 & 1 & 3 & 1 & 2 & $173 \cdot 36$ & 0.3005 & $2 \mathrm{NU} 2 *$ \\
\hline 3129. & 0.0066 & 0.0546 & 11 & 4 & 8 & 12 & 6 & 7 & .85 & 0.16 & NU3 \\
\hline 3129. & 0.0037 & 0.0812 & 10 & 5 & 6 & 11 & 4 & 7 & .06 & 1.370216 & $2 N \cup 2$ \\
\hline 3130. & 0.0747 & 0.0715 & 8 & 4 & 4 & 8 & 5 & 3 & .92 & 3.41 & NU2- \\
\hline 3130.41 & 0.0061 & 0.0446 & 11 & 2 & 10 & 12 & 4 & 9 & 2124.98 & 0.077267 & NU3 \\
\hline 3131 . & 0.1309 & 0.0554 & 8 & 0 & 8 & 8 & 5 & 3 & .92 & 0.580 & NU1+ \\
\hline $3131 \cdot 94$ & 0.0232 & 0.0755 & 11 & 3 & 8 & 11 & 4 & 7 & .06 & 8.484859 & $2 \mathrm{NU} 2$ \\
\hline 3132.49 & 0.0289 & 0.0806 & 10 & 3 & 7 & 10 & 4 & 6 & $1616 \cdot 51$ & 8.179783 & 2 NU2 \\
\hline 3133.05 & $41 \cdot 3221$ & 0.1075 & 1 & 0 & 1 & 1 & 1 & 0 & $42 \cdot 37$ & 1.500000 & $2 \mathrm{NU} 2$ \\
\hline $3134 \cdot 32$ & 0.2717 & 0.0835 & 9 & 3 & 6 & 9 & 4 & 5 & $1360 \cdot 28$ & 7.165263 & 2 NU2 \\
\hline 3134.54 & 0.0017 & 0.0681 & 6 & 2 & 4 & 7 & 6 & 1 & $1216 \cdot 20$ & 0.000189 & NU3 \\
\hline 3134.55 & 0.1083 & 0.0740 & 9 & 4 & 5 & 9 & 5 & 4 & $1477 \cdot 31$ & 5.113359 & $2 N \cup 2$ \\
\hline 3134.69 & 0.0388 & 0.0567 & 9 & 1 & 8 & 10 & 4 & 7 & $1581 \cdot 34$ & 0.284777 & NU 1 \\
\hline $3135 \cdot 09$ & 0.0041 & 0.0659 & 8 & 1 & 7 & 7 & 4 & 4 & 927.77 & 0.037317 & $2 \mathrm{NU} 2$ \\
\hline 3135.28 & 0.0133 & 0.0755 & 10 & 4 & 6 & 10 & 5 & 5 & 1724.72 & 6.455380 & $2 \mathrm{NU} 2$ \\
\hline 3136.07 & 0.0016 & 0.0949 & 2 & 2 & 1 & 3 & 1 & 2 & $173 \cdot 36$ & 0.300506 & $2 \mathrm{NU} 2 * *$ \\
\hline 3136.31 & 0.0537 & 0.0894 & 7 & 4 & 4 & 8 & 3 & 5 & $1050 \cdot 15$ & 0.898681 & $2 \mathrm{NU} 2$ \\
\hline
\end{tabular}




\begin{tabular}{|c|c|c|c|c|c|c|c|c|c|c|c|}
\hline $\begin{array}{c}\text { FREQUENCY } \\
\mathrm{cm}^{-1}\end{array}$ & $\begin{array}{l}\text { LINE } \\
\text { STRENGTH } \\
\frac{\mathrm{cm}^{-1}}{\mathrm{gm} \mathrm{cm}^{-2}}\end{array}$ & $\begin{array}{l}\text { HALF } \\
\text { WIDTH } \\
\frac{\mathrm{cm}^{-1}}{\mathrm{~atm} .}\end{array}$ & $J^{\prime}$ & $\mathrm{K}_{\mathrm{a}}$ & $\mathrm{K}_{\mathrm{c}}$ & $J^{\prime \prime}$ & $K_{a}$ & $K_{c}$ & $\begin{array}{c}E^{\prime \prime} \\
\mathrm{cm}^{-1}\end{array}$ & L & BAND \\
\hline 3136.70 & 0.0200 & 0.0861 & 7 & 1 & 7 & 6 & 2 & 4 & 602.77 & 0.035782 & 2NU 2 \\
\hline 3137.00 & 0.0032 & 0.0909 & 4 & 3 & 2 & 5 & 2 & 3 & $446 \cdot 50$ & 0.436045 & $2 \mathrm{NU} 2$ * \\
\hline 3139.10 & 0.0092 & 0.0524 & 9 & 5 & 5 & 10 & 7 & 4 & $2054 \cdot 40$ & 0.078635 & NU3 \\
\hline 3139.53 & 0.0218 & 0.0687 & 6 & 2 & 5 & 7 & 5 & 2 & 1059.85 & 0.011802 & NUI \\
\hline 3139.95 & 0.0025 & 0.0398 & 7 & 6 & 1 & 8 & 8 & 0 & $1789 \cdot 10$ & 0.014826 & NU3 \\
\hline 3139.95 & 0.0076 & 0.0398 & 7 & 6 & 2 & 8 & 8 & 1 & 1789.10 & 0.014826 & NU 3 \\
\hline 3140.39 & 0.0057 & 0.0601 & 9 & 1 & 8 & 8 & 4 & 5 & $1122 \cdot 72$ & 0.045574 & 2 NU2 \\
\hline 3140.44 & 0.0467 & 0.1054 & 2 & 1 & 2 & 1 & 0 & 1 & 1618.55 & 1.500000 & $2 \mathrm{~N} \cup 2 * * *$ \\
\hline 3142.21 & 0.0031 & 0.0548 & 9 & 5 & 4 & 10 & 7 & 3 & 2054.40 & 0.079027 & NU3 \\
\hline 3142.79 & 4.3267 & 0.0949 & 2 & 2 & 1 & 3 & 1 & 2 & $173 \cdot 36$ & 0.300506 & $2 \mathrm{NU} 2$ \\
\hline 3144.15 & 0.0020 & 0.0662 & 9 & 2 & 7 & 8 & 5 & 4 & $1255 \cdot 16$ & 0.030877 & 2NU2 \\
\hline $3144 \cdot 15$ & 0.0079 & 0.0974 & 4 & 1 & 3 & 4 & 0 & 4 & 1817.50 & 2.047924 & $2 \mathrm{~N} \cup 2 * * *$ \\
\hline 3145.83 & 0.0056 & 0.0513 & 11 & 3 & 9 & 12 & 5 & 8 & 2275.44 & 0.143644 & NU3 \\
\hline 3146.97 & 0.0137 & 0.0634 & 8 & 1 & 7 & 9 & 5 & 4 & 1477.31 & 0.005631 & NU 3 \\
\hline 3147.84 & 0.0035 & 0.0947 & 4 & 1 & 3 & 3 & 2 & 2 & 1813.81 & 0.835095 & $2 \mathrm{~N} \cup 2 * * *$ \\
\hline 3147.92 & 0.0122 & 0.0704 & 8 & 2 & 6 & 9 & 5 & 5 & 1474.99 & 0.157672 & NUI \\
\hline 3149.35 & 0.0031 & 0.0489 & 11 & 1 & 10 & 12 & 3 & 9 & 2105.90 & 0.080345 & NU3 \\
\hline $3151 \cdot 34$ & 1.6066 & 0.0909 & 4 & 3 & 2 & 5 & 2 & 3 & $446 \cdot 50$ & 0.436045 & 2NU2 \\
\hline 3152.00 & 0.0012 & 0.0960 & 3 & 1 & 3 & 2 & 2 & 0 & 136.17 & 0.101693 & $2 \mathrm{NU} 2^{*}$ \\
\hline $3152 \cdot 20$ & 0.0163 & 0.0961 & 4 & 0 & 4 & 3 & 1 & 3 & 1739.51 & 2.831638 & $2 \mathrm{NU} 2 * * *$ \\
\hline 3152.26 & 0.2055 & 0.0477 & 6 & 5 & 2 & 6 & 6 & 1 & 1045.07 & 1.111607 & 2 NU2 \\
\hline $3152 \cdot 32$ & 0.0556 & 0.0518 & 7 & 5 & 3 & 7 & 6 & 2 & $1216 \cdot 20$ & 2.119434 & $2 \mathrm{NU} 2$ \\
\hline 3152.37 & 0.0685 & 0.0478 & 6 & 5 & 1 & 6 & 6 & 0 & 1045.07 & 1.111654 & 2 NU2 \\
\hline 3152.42 & 0.1668 & 0.0521 & 7 & 5 & 2 & 7 & 6 & 1 & $1216 \cdot 20$ & 2.119964 & $2 \mathrm{NU} 2$ \\
\hline $3153 \cdot 45$ & 0.0909 & 0.0545 & 8 & 5 & 4 & 8 & 6 & 3 & 1411.65 & 3.073714 & $2 N \cup 2$ \\
\hline 3154.00 & 0.0135 & 0.0554 & 10 & 5 & 6 & 10 & 6 & 5 & 1875.00 & 4.913223 & $2 \mathrm{NU} 2$ \\
\hline $3154 \cdot 32$ & 0.0303 & 0.0556 & 8 & 5 & 3 & 8 & 6 & 2 & 1411.68 & 3.077042 & $2 \mathrm{NU} 2$ \\
\hline 3154.73 & 0.0133 & 0.0558 & 9 & 5 & 5 & 9 & 6 & 4 & 1631.27 & 4.001691 & $2 \mathrm{NU}_{2}$ \\
\hline 3155.04 & 0.0028 & 0.0914 & 3 & 2 & 1 & 4 & 1 & 4 & 224.83 & 0.123609 & $2 \mathrm{NU} 2 *$ \\
\hline 3155.32 & 0.2107 & 0.0902 & 6 & 1 & 6 & 5 & 2 & 3 & 446.50 & 0.057105 & $2 \mathrm{NU} 2$ \\
\hline 3155.59 & 0.0401 & 0.0583 & 9 & 5 & 4 & 9 & 6 & 3 & 1631.41 & 4.017231 & $2 \mathrm{NU} 2$ \\
\hline 3155.79 & 0.0205 & 0.1026 & 3 & 1 & 3 & 2 & 0 & 2 & 1664.97 & 2.156792 & $2 \mathrm{NU} 2 * * *$ \\
\hline $3156 \cdot 47$ & 0.0046 & 0.0608 & 10 & 5 & 5 & 10 & 6 & 4 & 1875.53 & 4.974368 & $2 \mathrm{NU} 2$ \\
\hline 3156.63 & 0.0014 & 0.0814 & 5 & 3 & 2 & 6 & 2 & 5 & $552 \cdot 92$ & 0.312103 & $2 \mathrm{NU} 2$ 关 \\
\hline 3157.41 & 0.0010 & 0.0829 & 3 & 3 & 0 & 4 & 2 & 3 & 1907.99 & 0.131472 & $2 \mathrm{NU} 2 *{ }^{\circ} *$ \\
\hline $3157 \cdot 46$ & 0.0030 & 0.0948 & 4 & 1 & 4 & 3 & 2 & 1 & $212 \cdot 15$ & 0.123609 & $2 \mathrm{NU} 2^{*} *$ \\
\hline 3157.71 & 0.0076 & 0.0421 & 10 & 1 & 10 & 11 & 3 & 9 & 1695.03 & 0.027489 & NU3 \\
\hline 3158.15 & 0.0107 & 0.0951 & 4 & 2 & 2 & 4 & 1 & 3 & 1875.49 & 3.642913 & $2 \operatorname{NU} 2 * * * *$ \\
\hline 3158.86 & 0.0374 & 0.0949 & 3 & 2 & 1 & 3 & 1 & 2 & 1772.41 & 2.538253 & $2 \mathrm{NU} 2 * * * *$ \\
\hline 3159.18 & 0.0023 & 0.0886 & 5 & 2 & 3 & 4 & 3 & 2 & 2004.81 & 0.436045 & $2 \mathrm{NU} 2 * * * *$ \\
\hline 3161.73 & 0.0012 & 0.0941 & 2 & 2 & 0 & 3 & 1 & 3 & $142 \cdot 28$ & 0.101693 & $2 \mathrm{NU} 2 *$ \\
\hline 3161.98 & 0.0038 & 0.0574 & 10 & 4 & 7 & 11 & 6 & 6 & 2142.69 & 0.142262 & NU3 \\
\hline 3162.04 & 0.0229 & 0.0440 & 10 & 0 & 10 & 11 & 2 & 9 & 1690.70 & 0.027627 & NU3 \\
\hline 3162.47 & 0.0092 & 0.0979 & 2 & 2 & 0 & 2 & 1 & 1 & 1693.65 & 1.258755 & $2 \mathrm{NU} 2^{* * * *}$ \\
\hline 3163.09 & 0.0201 & 0.0947 & 5 & 2 & 3 & 5 & 1 & 4 & $2000 \cdot 90$ & 4.214107 & $2 \mathrm{NU} 2 *$ 头* \\
\hline 3163.82 & 0.5918 & 0.0960 & 3 & 1 & 3 & 2 & 2 & 0 & 136.17 & 0.101693 & $2 \mathrm{NU} 2$ \\
\hline 3163.97 & 0.0052 & 0.0569 & 11 & $j$ & 7 & 12 & 6 & 6 & 2437.56 & 4.215931 & NUI \\
\hline $3164 \cdot 12$ & 0.0897 & 0.0780 & 6 & 3 & 3 & 7 & 2 & 6 & 709.60 & 0.271048 & 2 NU2 \\
\hline 3164.61 & 0.0292 & 0.0736 & 6 & 1 & 6 & 7 & 4 & -3 & $931 \cdot 22$ & 0.008274 & NUI \\
\hline 3164.80 & 0.0026 & 0.0653 & 11 & 4 & 8 & 12 & 5 & 7 & $2300 \cdot 80$ & 2.124861 & NU 1 \\
\hline 3165.32 & 0.0923 & 0.1111 & 1 & 1 & 0 & 1 & 0 & 1 & 23.79 & 1.500000 & $2 \mathrm{NU} 2 *$ \\
\hline 3165.38 & 0.0085 & 0.0512 & 8 & 5 & 4 & 9 & 7 & 3 & 1810.63 & 0.053898 & NU3 \\
\hline 3165.70 & 0.0102 & 0.0689 & 9 & 4 & 5 & 10 & 3 & 8 & $1446 \cdot 16$ & 0.409296 & $2 \mathrm{NU} 2$ \\
\hline $3166 \cdot 41$ & 0.0257 & 0.0523 & 8 & 5 & 3 & 9 & 7 & 2 & 1810.63 & 0.053971 & NU3 \\
\hline
\end{tabular}




\begin{tabular}{|c|c|c|c|c|c|c|c|c|c|c|c|}
\hline $\begin{array}{c}\text { FREQUENCY } \\
\mathrm{cm}^{-1}\end{array}$ & 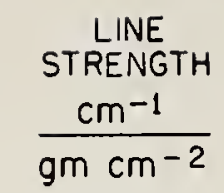 & $\begin{array}{l}\text { HALF } \\
\text { WIDTH } \\
\frac{\mathrm{cm}^{-1}}{a+m .}\end{array}$ & $J^{\prime}$ & $K_{0}$ & $\mathrm{~K}_{\mathrm{c}}$ & $J^{\prime \prime}$ & $\mathrm{K}_{\mathrm{a}}$ & $K_{c}$ & $\begin{array}{c}\mathrm{E}^{\prime \prime} \\
\mathrm{cm}^{-1}\end{array}$ & L & BAND \\
\hline 3166.71 & 0.2166 & 0.0929 & 5 & 1 & 5 & 4 & 2 & 2 & 315.79 & 0.091324 & $2 \mathrm{NU} 2$ \\
\hline 3167.26 & 0.0795 & 0.0737 & 7 & 3 & 4 & 8 & 2 & 7 & 885.62 & 0.192937 & $2 \mathrm{NU} 2$ \\
\hline 3167.92 & 1.3868 & 0.0914 & 3 & 2 & 1 & 4 & 1 & 4 & 224.83 & 0.123609 & $2 \mathrm{NU} 2$ \\
\hline 3169.24 & 0.0133 & 0.0914 & 5 & 1 & 4 & 5 & 0 & 5 & 1920.76 & 1.912338 & $2 \operatorname{NU} 2 * * *$ \\
\hline 3169.29 & 0.2054 & 0.0872 & 4 & 2 & 2 & 5 & 1 & 5 & 326.64 & 0.091324 & 2 NU2 2 \\
\hline 3169.49 & 0.0034 & 0.0813 & 9 & 5 & 5 & 10 & 4 & 6 & 1616.51 & 0.959644 & $2 N \cup 2$ \\
\hline 3169.58 & 1.4784 & 0.0948 & 4 & 1 & 4 & 3 & 2 & 1 & $212 \cdot 15$ & 0.123609 & $2 \mathrm{NU} 2$ \\
\hline 3169.83 & 0.6794 & 0.0814 & 5 & 3 & 2 & 6 & 2 & 5 & 552.92 & 0.312103 & 2NU2 \\
\hline 3170.06 & 0.0162 & 0.0720 & 8 & 4 & 4 & 9 & 3 & 7 & $1216 \cdot 27$ & 0.616 & NU2+ \\
\hline 3170.23 & 0.0536 & 0.0976 & 4 & 1 & 4 & 3 & 0 & 3 & 1731.89 & 3.004990 & $2 \mathrm{NU} 2 * * *$ \\
\hline 3170.92 & 0.0520 & 0.0740 & 7 & 2 & 5 & 8 & 5 & 4 & 1255.16 & 0.074366 & NU1 \\
\hline 3170.98 & 0.0283 & 0.0559 & 8 & 0 & 8 & 9 & 3 & 7 & $1216 \cdot 27$ & 0.10 & NU I- \\
\hline 3171.72 & 0.0171 & 0.1111 & 1 & 1 & 0 & 1 & 0 & 1 & 23.79 & 1.500000 & $2 \mathrm{NU} 2 * *$ \\
\hline 3172.48 & 0.0388 & 0.1044 & 2 & 1 & 1 & 2 & 0 & 2 & 70.08 & 2.074578 & $2 \operatorname{Nu} 2 *$ \\
\hline 3172.57 & 0.0145 & 0.1032 & 2 & 0 & 2 & 1 & 1 & 1 & 37.13 & 0.755253 & $2 N \cup 2 *$ \\
\hline 3173.08 & 0.2821 & 0.0876 & 6 & 4 & 3 & 7 & 3 & 4 & 842.38 & 0.550367 & 2 NU2 \\
\hline 3174.63 & 0.0451 & 0.0880 & 5 & 0 & 5 & 4 & 1 & 4 & 1821.61 & 3.911752 & $2 \mathrm{NU} 2 * * *$ \\
\hline 3174.91 & 0.5760 & 0.0941 & 2 & 2 & 0 & 3 & 1 & 3 & $142 \cdot 28$ & 0.101693 & $2 N \cup 2$ \\
\hline 3175.05 & 0.0219 & 0.0481 & 10 & 6 & 5 & 11 & 7 & 4 & $2321 \cdot 94$ & 5.877228 & NUl \\
\hline $3175 \cdot 24$ & 0.0073 & 0.0470 & 10 & 6 & 4 & 11 & 7 & 5 & 2321.89 & 5.881509 & NU I \\
\hline 3175.38 & 0.0033 & 0.0920 & 6 & 2 & 4 & 6 & 1 & 5 & $2146 \cdot 28$ & 4.225862 & $2 N \cup 2 * * *$ \\
\hline 3175.56 & 0.0089 & 0.0403 & 9 & 7 & 3 & 10 & 8 & 2 & $2254 \cdot 34$ & 7.138077 & NUI \\
\hline 3175.56 & 0.0266 & 0.0403 & 9 & 7 & 2 & 10 & 8 & 3 & 2254.34 & 7.138103 & NUI \\
\hline 3176.22 & 0.0082 & 0.0700 & 5 & 2 & 4 & 6 & 5 & 1 & 888.63 & 0.005627 & NUI \\
\hline 3177.08 & 0.0285 & 0.0675 & 10 & 3 & 8 & 11 & 4 & 7 & 1899.06 & 0.954846 & NUI \\
\hline 3178.13 & 46.1336 & 0.1111 & 1 & 1 & 0 & 1 & 0 & 1 & 23.79 & 1.500000 & 2 NU2 2 \\
\hline 3178.70 & 0.0027 & 0.1032 & 2 & 0 & 2 & 1 & 1 & 1 & 37.13 & 0.755253 & $2 \mathrm{NU} 2 * *$ \\
\hline 3178.84 & 0.0218 & 0.0641 & 9 & 2 & 8 & 10 & 3 & 7 & $1538 \cdot 23$ & 0.380414 & NU 1 \\
\hline 3178.84 & 0.0072 & 0.1044 & 2 & 1 & 1 & 2 & 0 & 2 & 70.08 & 2.074578 & $2 \operatorname{NU} 2 * *$ \\
\hline 3179.69 & 0.2115 & 0.0812 & 5 & 2 & 3 & 6 & 1 & 6 & $447 \cdot 24$ & 0.057105 & 2 NU 2 \\
\hline 3179.95 & 0.0130 & 0.0467 & 9 & 6 & 3 & 9 & 7 & 2 & 1810.63 & 3.116419 & 2 NU 2 \\
\hline 3180.09 & 0.0066 & 0.0687 & 8 & 3 & 5 & 9 & 2 & 8 & $1080 \cdot 38$ & 0.126905 & $2 \mathrm{NU} 2$ \\
\hline 3180.60 & 0.0042 & 0.0475 & 10 & 6 & 5 & 10 & 7 & 4 & 2054.40 & 4.058996 & $2 \mathrm{NU} 2$ \\
\hline 3180.70 & 0.0014 & 0.0484 & 10 & 6 & 4 & 10 & 7 & 3 & $2054 \cdot 40$ & 4.061130 & $2 \mathrm{NU} 2$ \\
\hline 3181.37 & 0.0043 & 0.0463 & 9 & 6 & 4 & 9 & 7 & 3 & 1810.63 & 3.116005 & $2 N \cup 2$ \\
\hline 3182.01 & 0.0130 & 0.0918 & 5 & 1 & 4 & 4 & 2 & 3 & 1907.99 & 1.638916 & $2 \mathrm{NU} 2 * * *$ \\
\hline 3182.22 & 0.1178 & 0.0741 & 7 & 4 & 3 & 8 & 3 & 6 & $1006 \cdot 12$ & 0.519243 & 2 NU2 \\
\hline 3182.48 & 0.3777 & 0.0829 & 4 & 3 & 1 & 5 & 2 & 4 & $416 \cdot 22$ & 0.261722 & 2 NU 2 \\
\hline 3182.96 & 0.0413 & 0.0640 & 8 & 1 & 7 & 9 & 4 & 6 & 1340.89 & 0.269351 & NUI \\
\hline 3183.21 & 0.0232 & 0.0996 & 1 & 1 & 1 & 0 & 0 & 0 & 0.00 & 1.000000 & $2 \mathrm{NU} 2 *$ \\
\hline 3183.30 & 0.0105 & 0.0340 & 8 & 8 & 0 & 9 & 9 & 1 & 2225.56 & 8.403719 & NUI \\
\hline 3183.30 & 0.0314 & 0.0340 & 8 & 8 & 1 & 9 & 9 & 0 & 2225.56 & 8.403719 & NUI \\
\hline 3183.64 & 0.0122 & 0.0409 & 7 & 6 & 2 & 7 & 7 & 1 & 1394.86 & 1.119792 & 2 NU2 \\
\hline 3183.64 & 0.0247 & 0.0440 & 8 & 6 & 3 & 8 & 7 & 2 & 1590.74 & 2.03 & NU2- \\
\hline 3183.64 & 0.0365 & 0.0409 & 7 & 6 & 1 & 7 & 7 & 0 & 1394.86 & 1.119797 & $2 \mathrm{NU} 2$ \\
\hline 3184.51 & 0.0111 & 0.0499 & 10 & 2 & 9 & 11 & 4 & 8 & 1843.02 & 0.082847 & NU3 \\
\hline 3184.64 & 0.0078 & 0.0441 & 8 & 6 & 2 & 8 & 7 & 1 & 1590.74 & 1.93 & NU2- \\
\hline 3184.69 & 0.3855 & 0.0884 & 3 & 3 & 1 & 4 & 2 & 2 & 315.79 & 0.161593 & 2NU 2 \\
\hline 3184.84 & 7.2584 & 0.1032 & 2 & 0 & 2 & 1 & 1 & 1 & 37.13 & 0.755253 & 2 NU2 2 \\
\hline 3185.10 & 0.1125 & 0.0601 & 8 & 1 & 8 & 9 & 2 & 7 & 1201.95 & 0.122288 & NU I \\
\hline 3185.21 & 19.4248 & 0.1044 & 2 & 1 & 1 & 2 & 0 & 2 & 70.08 & 2.074578 & 2NU 2 \\
\hline 3185.30 & 0.0769 & 0.0994 & 3 & 1 & 2 & 3 & 0 & 3 & 136.77 & 2.185861 & $2 \operatorname{NU} 2 *$ \\
\hline 3185.49 & 0.0022 & 0.0825 & 4 & 2 & 3 & 3 & 3 & 0 & 285.43 & 0.131472 & 2 NU2* \\
\hline 3186.41 & 0.0020 & 0.0829 & 3 & 3 & 0 & 4 & 2 & 3 & $300 \cdot 35$ & 0.131472 & 2NU2* \\
\hline
\end{tabular}




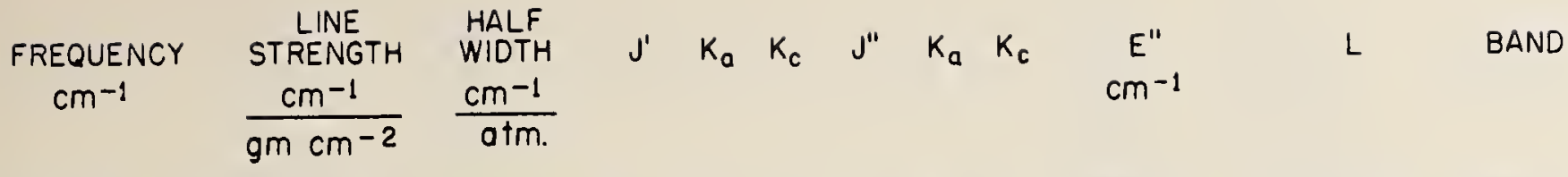

\begin{tabular}{|c|c|c|c|c|c|c|c|c|c|c|c|}
\hline $\begin{array}{l}3187 \cdot 31 \\
3187 \cdot 54\end{array}$ & $\begin{array}{l}0.0075 \\
0.0167\end{array}$ & $\begin{array}{l}0.0939 \\
0.0613\end{array}$ & $\begin{array}{r}3 \\
11\end{array}$ & $\begin{array}{l}2 \\
5\end{array}$ & $\begin{array}{l}2 \\
6\end{array}$ & $\begin{array}{r}3 \\
12\end{array}$ & $\begin{array}{l}1 \\
6\end{array}$ & $\begin{array}{l}3 \\
7\end{array}$ & $\begin{array}{l}1739.51 \\
2433.85\end{array}$ & $\begin{array}{l}1.297551 \\
4.458582\end{array}$ & $\begin{array}{l}2 \mathrm{~N} \cup 2 * * * \\
N \cup 1\end{array}$ \\
\hline 3188.16 & 0.0107 & 0.0951 & 3 & 1 & 2 & 2 & 2 & 1 & 134.91 & 0.300506 & $2 \mathrm{NU} 2 *$ \\
\hline $3188 \cdot 30$ & 0.0031 & 0.0685 & 5 & 1 & 5 & 6 & 5 & 2 & 888.60 & 0.000066 & NU3 \\
\hline 188.98 & 0.0009 & 0.0633 & 10 & 5 & 5 & 11 & 4 & 8 & 1843.02 & 0.753106 & $2 \mathrm{NU} 2$ \\
\hline 189.66 & 0.0043 & 0.0996 & 1 & 1 & 1 & 0 & 0 & 0 & 0.00 & 1.000000 & $2 \mathrm{NU} 2 * *$ \\
\hline 189.90 & 0.0179 & 0.0748 & 6 & 2 & 4 & 7 & 5 & 3 & 1059.65 & 0.028700 & NUI \\
\hline 190.09 & 0.0093 & 0.0566 & 10 & 3 & 8 & 11 & 5 & 7 & 1985.83 & 0.140157 & NU3 \\
\hline 190.86 & 0.0225 & 0.0571 & 10 & 5 & 6 & 11 & 6 & 5 & 2144.12 & 3.00 & NU1- \\
\hline 191.58 & 0.0142 & 0.0994 & 3 & 1 & 2 & 3 & 0 & 3 & 136.77 & 2.185861 & $2 N \cup 2 * *$ \\
\hline $191 \cdot 93$ & 0.0458 & 0.0489 & 7 & 5 & 3 & 8 & 7 & 2 & 1590.74 & 0.032804 & NU3 \\
\hline 192.06 & 0.0359 & 0.0589 & 9 & 4 & 6 & 10 & 6 & 5 & 1875.00 & 0.112325 & NU3 \\
\hline 192.17 & 0.0153 & 0.0493 & 7 & 5 & 2 & 8 & 7 & 1 & 1590.74 & 0.032814 & NU3 \\
\hline 3193.04 & 0.0101 & 0.0676 & 7 & 1 & 6 & 8 & 5 & 3 & 1255.92 & 0.004077 & NU3 \\
\hline 3193.94 & 0.0020 & 0.0951 & 3 & 1 & 2 & 2 & 2 & 1 & 134.91 & 0.300506 & $2 \mathrm{NU} 2 * *$ \\
\hline $3194 \cdot 2.4$ & 0.0036 & 0.0678 & 11 & 4 & 7 & 12 & 6 & 6 & $2437 \cdot 56$ & 0.265013 & NU3 \\
\hline 3195.08 & 0.0115 & 0.0760 & 6 & 0 & 6 & 5 & 1 & 5 & 1922.86 & 4.958549 & $2 \mathrm{NU} 2 * * *$ \\
\hline 3195.46 & 0.0042 & 0.0848 & 7 & 2 & 5 & 7 & 1 & 6 & $2309 \cdot 74$ & 3.983004 & $2 N \cup 2 * * *$ \\
\hline 3196.10 & 11.6127 & 0.0996 & 1 & 1 & 1 & 0 & 0 & 0 & 0.00 & 1.000000 & $2 \mathrm{NU} 2$ \\
\hline 3196.63 & 1.0993 & 0.0825 & 4 & 2 & 3 & 3 & 3 & 0 & $285 \cdot 43$ & 0.131472 & 2 NU2 2 \\
\hline $3197 \cdot 34$ & 0.0819 & 0.0994 & 3 & 0 & 3 & 2 & 1 & 2 & 79.48 & 1.741385 & $2 \mathrm{~N} \cup 2 *$ \\
\hline 3197.87 & 38.4548 & 0.0994 & 3 & 1 & 2 & 3 & 0 & 3 & 136.77 & 2.185861 & 2NU2 \\
\hline 3198.15 & 0.0221 & 0.0747 & 6 & 2 & 4 & 7 & 1 & 7 & $586 \cdot 48$ & 0.035782 & $2 \mathrm{NU} 2$ \\
\hline 3199.28 & 0.0844 & 0.0743 & 6 & 4 & 2 & 7 & 3 & 5 & $816 \cdot 72$ & 0.431025 & $2 \mathrm{NU} 2$ \\
\hline 3199.71 & 0.0181 & 0.0896 & 4 & 2 & 3 & 4 & 1 & 4 & 1821.61 & 1.567300 & $2 \mathrm{~N} \cup 2 * * *$ \\
\hline 3199.73 & 5.3386 & 0.0951 & 3 & 1 & 2 & 2 & 2 & 1 & 134.91 & 0.300506 & $2 \mathrm{NU} 2$ \\
\hline 3200.27 & 1.0215 & 0.0829 & 3 & 3 & 0 & 4 & 2 & 3 & $300 \cdot 35$ & 0.131472 & $2 \mathrm{~N} \cup 2$ \\
\hline $3200 \cdot 36$ & 0.0049 & 0.0765 & 10 & 2 & 9 & 9 & 3 & 6 & $1282 \cdot 92$ & 0.084934 & 2 NU2 2 \\
\hline 3200.81 & 0.0350 & 0.0779 & 6 & 1 & 6 & 5 & 0 & 5 & $1920 \cdot 76$ & 4.979001 & $2 \mathrm{~N} \cup 2 * * *$ \\
\hline 3200.94 & 0.0046 & 0.0633 & 9 & 3 & 6 & 10 & 2 & 9 & 1293.66 & 0.084934 & 2NU2 \\
\hline 3201.03 & 0.0088 & 0.0696 & 7 & 0 & 7 & 8 & 4 & 4 & 1131.76 & 0.001880 & NU3 \\
\hline 3201.18 & 0.0933 & 0.1054 & 2 & 1 & 2 & 1 & 0 & 1 & 23.79 & 1.500000 & $2 \mathrm{NU} 2 *$ \\
\hline 3201.64 & 0.0018 & 0.0638 & 8 & 3 & 5 & 8 & 7 & 2 & 1590.74 & 0.001180 & NU3+ \\
\hline 3201.84 & 0.0226 & 0.0473 & 9 & 6 & 4 & 10 & 7 & 3 & 2054.40 & 6.018378 & NU 1 \\
\hline 3201.96 & 0.0679 & 0.0475 & 9 & 6 & 3 & 10 & 7 & 4 & 2054.40 & 6.019433 & NUI \\
\hline 3203.17 & 0.0364 & 0.0378 & 8 & 7 & 1 & 9 & 8 & 2 & 2009.87 & 7.261219 & NUI \\
\hline 3203.17 & 0.1092 & 0.0378 & 8 & 7 & 2 & 9 & 8 & 1 & 2009.87 & 7.261215 & NUl \\
\hline $3203 \cdot 54$ & 0.0152 & 0.0994 & 3 & 0 & 3 & 2 & 1 & 2 & 79.48 & 1.741385 & $2 \mathrm{NU} 2 * *$ \\
\hline 3204.12 & 0.0158 & 0.0974 & 4 & 1 & 3 & 4 & 0 & 4 & 222.06 & 2.047924 & $2 \mathrm{NU} 2 *$ \\
\hline $3204 \cdot 82$ & 0.0260 & 0.0794 & 8 & 5 & 4 & 9 & 4 & 5 & $1360 \cdot 28$ & 0.669979 & $2 \mathrm{NU} 2$ \\
\hline 3205.66 & 0.0093 & 0.0641 & 9 & 5 & 4 & 10 & 4 & 7 & $1581 \cdot 34$ & 0.720783 & $2 N \cup 2$ \\
\hline 3206.01 & 0.0749 & 0.0949 & 3 & 2 & 1 & 3 & 1 & 2 & $173 \cdot 36$ & 2.538253 & $2 \mathrm{NU} 2 *$ \\
\hline 3207.20 & 0.0402 & 0.0733 & 5 & 2 & 3 & 6 & 5 & 2 & 888.60 & 0.009072 & NUI \\
\hline 3207.26 & 0.0215 & 0.0951 & 4 & 2 & 2 & 4 & 1 & 3 & $275 \cdot 52$ & 3.642913 & $2 N \cup 2 *$ \\
\hline 3207.32 & 0.1295 & 0.0840 & 5 & 4 & 2 & 6 & 3 & 3 & 661.56 & 0.303663 & 2NU2 \\
\hline 3207. & 0.0015 & 0.0509 & 10 & 2 & 9 & 10 & 5 & 6 & 1718.77 & 0.021485 & NUI \\
\hline 3207 . & 0.0173 & 0.1054 & 2 & 1 & 2 & 1 & 0 & 1 & 23.79 & 1.500000 & $2 \mathrm{NU} 2 * *$ \\
\hline 3208 . & 0.0183 & 0.0979 & 2 & 2 & 0 & 2 & 1 & 1 & 95.17 & 1.258755 & $2 \mathrm{NU} 2 *$ \\
\hline 3208 . & 0.0513 & 0.0671 & 10 & 4 & 7 & 11 & 5 & 6 & 1999.02 & 2.723522 & NUI \\
\hline 320 & 0.0149 & 0.0690 & 4 & 2 & 3 & 5 & 5 & 0 & 742.10 & 0.001619 & NU1 \\
\hline 320 & 0.0117 & 0.0603 & 10 & 5 & 5 & 11 & 6 & 6 & 2142.69 & 4.667626 & NUI \\
\hline 3209 & 40.9463 & 0.0994 & 3 & 0 & 3 & 2 & 1 & 2 & 79.48 & 1.741385 & $2 \mathrm{NU} 2$ \\
\hline 321 & 0.0029 & 0.0974 & 4 & 1 & 3 & 4 & 0 & 4 & 222.06 & 2.047924 & $2 \mathrm{NU} 2 * *$ \\
\hline 21 & 0.4528 & 0.0864 & 4 & 2 & 2 & 3 & 3 & 1 & 285.23 & 0.161593 & $2 \mathrm{NU} 2$ \\
\hline
\end{tabular}




\begin{tabular}{|c|c|c|c|c|c|c|c|c|c|c|c|}
\hline $\begin{array}{c}\text { FREQUENCY } \\
\mathrm{cm}^{-1}\end{array}$ & $\begin{array}{l}\text { LINE } \\
\text { STRENGTH } \\
\frac{\mathrm{cm}^{-1}}{\mathrm{gm} \mathrm{cm}^{-2}}\end{array}$ & $\begin{array}{l}\text { HALF } \\
\text { WIDTH } \\
\mathrm{cm}^{-1} \\
a+m .\end{array}$ & $J^{\prime}$ & $\mathrm{K}_{a}$ & $\mathrm{~K}_{\mathrm{c}}$ & $J^{\prime \prime}$ & $K_{0}$ & $K_{c}$ & $\begin{array}{c}E^{\prime \prime} \\
\mathrm{cm}^{-1}\end{array}$ & L & BAND \\
\hline 3211.19 & 0.0145 & 0.0684 & 10 & 4 & 6 & 11 & 6 & 5 & 2144.12 & 0.181046 & NU3 \\
\hline 3211.48 & 0.0134 & 0.0677 & 9 & 4 & 5 & 10 & 6 & 4 & 1875.53 & 0.124623 & NU3 \\
\hline 3212.70 & 0.0139 & 0.0949 & 3 & 2 & 1 & 3 & 1 & 2 & 173.36 & 2.538253 & $2 \mathrm{NU} 2 * *$ \\
\hline 3213.17 & 0.0056 & 0.0631 & 11 & 2 & 9 & 12 & 4 & 8 & 2205.65 & 0.209597 & NU3 \\
\hline 3213.83 & 0.0040 & 0.0951 & 4 & 2 & 2 & 4 & 1 & 3 & 275.52 & 3.642913 & $2 \mathrm{NU} 2 * *$ \\
\hline 3213.93 & 0.0229 & 0.0631 & 7 & 0 & 7 & 6 & 1 & 6 & 2042.77 & 5.983681 & $2 N \cup 2 * * *$ \\
\hline 3214.14 & 46.6563 & 0.1054 & 2 & 1 & 2 & 1 & 0 & 1 & 23.79 & 1.500000 & $2 \mathrm{NU} 2$ \\
\hline 3214.66 & 0.4485 & 0.0826 & 5 & 2 & 4 & 4 & 3 & 1 & 383.85 & 0.261722 & 2 NU 2 \\
\hline $3214 \cdot 68$ & 0.0402 & 0.0947 & 5 & 2 & 3 & 5 & 1 & 4 & 399.46 & 4.214107 & $2 \mathrm{NU} 2 *$ \\
\hline 3214.73 & 0.0433 & 0.1021 & 2 & 2 & 1 & 1 & 1 & 0 & 1640.51 & 1.500000 & $2 \mathrm{NU} 2 * * *$ \\
\hline 3215.23 & 0.0874 & 0.0482 & 9 & 1 & 9 & 10 & 3 & 8 & 1446.16 & 0.030178 & NU3 \\
\hline 3215.28 & 0.0034 & 0.0979 & 2 & 2 & 0 & 2 & 1 & 1 & 95.17 & 1.258755 & $2 \mathrm{NU} 2 * *$ \\
\hline 3215.41 & 0.0040 & 0.0815 & 5 & 2 & 4 & 5 & 1 & 5 & 1922.86 & 1.711792 & $2 \mathrm{~N} \cup 2 * * *$ \\
\hline 3215.54 & 0.0017 & 0.0831 & 6 & 2 & 5 & 5 & 3 & 2 & 508.81 & 0.312103 & $2 N \cup 2 *$ \\
\hline 3215.75 & 0.0040 & 0.0843 & 6 & 1 & 5 & 5 & 2 & 4 & 2024.17 & 2.671788 & $2 N \cup 2 * * *$ \\
\hline 3216.51 & 7.8852 & 0.0974 & 4 & 1 & 3 & 4 & 0 & 4 & 222.06 & 2.047924 & $2 \mathrm{NU} 2$ \\
\hline 3216.88 & 0.0410 & 0.1026 & 3 & 1 & 3 & 2 & 0 & 2 & 70.08 & 2.156792 & $2 \mathrm{NU} 2 *$ \\
\hline 3217.10 & 0.0077 & 0.0642 & 7 & 1 & 7 & 6 & 0 & 6 & 2041.76 & 5.990506 & $2 N \cup 2 * * *$ \\
\hline 3218.02 & 0.0078 & 0.0798 & 9 & 2 & 8 & 8 & 3 & 5 & 1050.15 & 0.126905 & $2 \mathrm{NU} 2$ \\
\hline 3218.67 & 0.0189 & 0.0456 & 6 & 5 & 2 & 7 & 7 & 1 & 1394.86 & 0.014987 & NU3 \\
\hline 3218.72 & 0.0567 & 0.0457 & 6 & 5 & 1 & 7 & 7 & 0 & 1394.86 & 0.014988 & NU3 \\
\hline 3218.87 & 0.0010 & 0.0539 & 9 & 1 & 9 & 9 & 4 & 6 & 1340.89 & 0.006488 & NU1 \\
\hline 3219.39 & 37.4362 & 0.0949 & 3 & 2 & 1 & 3 & 1 & 2 & 173.36 & 2.538253 & $2 N \cup 2$ \\
\hline 3219.68 & 0.0214 & 0.0776 & 5 & 1 & 5 & 6 & 4 & 2 & 757.78 & 0.007497 & NU1 \\
\hline 3219.98 & 0.3804 & 0.0727 & 5 & 4 & 1 & 6 & 3 & 4 & 648.97 & 0.278030 & 2 NU 2 \\
\hline 3220.22 & 0.0430 & 0.0601 & 8 & 4 & 5 & 9 & 6 & 4 & 1631.27 & 0.110400 & NU3+ \\
\hline 3220.40 & 0.0327 & 0.0961 & 4 & 0 & 4 & 3 & 1 & 3 & 142.28 & 2.831638 & $2 \mathrm{NU} 2 *$ \\
\hline 3220.41 & 10.7494 & 0.0951 & 4 & 2 & 2 & 4 & 1 & 3 & 275.52 & 3.642913 & $2 \mathrm{NU} 2$ \\
\hline 3220.80 & 0.0042 & 0.0927 & 7 & 3 & 4 & 7 & 2 & 5 & $2392 \cdot 58$ & 5.918411 & $2 N \cup 2{ }^{*} * * *$ \\
\hline 3221.07 & 0.0074 & 0.0947 & 5 & 2 & 3 & 5 & 1 & 4 & 399.46 & 4.214107 & $2 \mathrm{NU} 2 * *$ \\
\hline 3221.15 & 0.0123 & 0.0949 & 2 & 2 & 0 & 1 & 1 & 1 & 1634.97 & 1.244747 & $2 \mathrm{NU} 2 * * *$ \\
\hline 3222.02 & 9.1560 & 0.0979 & 2 & 2 & 0 & 2 & 1 & 1 & 95.17 & 1.258755 & $2 \mathrm{NU} 2$ \\
\hline 3223.26 & 0.0395 & 0.0952 & 2 & 2 & 1 & 2 & 1 & 2 & 79.48 & 0.833333 & $2 \mathrm{NU} 2 *$ \\
\hline $3223 \cdot 34$ & 0.0207 & 0.0679 & 7 & 2 & 5 & 8 & 1 & 8 & $744 \cdot 16$ & 0.024347 & 2 NU 2 \\
\hline 3223.37 & 0.0309 & 0.0511 & 9 & 0 & 9 & 10 & 2 & 8 & 1438.00 & 0.030629 & NU3 \\
\hline 3223.39 & 0.0076 & 0.1026 & 3 & 1 & 3 & 2 & 0 & 2 & 70.08 & 2.156792 & $2 \mathrm{NU} 2 * *$ \\
\hline 3224.48 & 0.0061 & 0.0703 & 4 & 2 & 2 & 5 & 5 & 1 & $742 \cdot 10$ & 0.001985 & NUI \\
\hline 3225.07 & 0.0028 & 0.0925 & 6 & 3 & 3 & 6 & 2 & 4 & $2211 \cdot 21$ & 4.829435 & $2 \mathrm{~N} \cup 2 * * *$ \\
\hline 3225.11 & 0.0086 & 0.0646 & 8 & 5 & 3 & 9 & 4 & 6 & 1340.89 & 0.597432 & $2 \mathrm{NU} 2$ \\
\hline 3225.72 & 0.3078 & 0.0711 & 7 & 1 & 6 & 8 & 4 & 5 & 1122.72 & 0.222677 & NU I \\
\hline 3226.13 & 0.3109 & 0.0650 & 7 & 0 & 7 & 8 & 3 & 6 & $1006 \cdot 12$ & 0.125531 & NUI \\
\hline $3226 \cdot 44$ & 0.0013 & 0.0947 & 4 & 1 & 3 & 3 & 2 & 2 & $206 \cdot 30$ & 0.835095 & $2 \mathrm{NU} 2 *$ \\
\hline 3226.70 & 0.0060 & 0.0961 & 4 & 0 & 4 & 3 & 1 & 3 & $142 \cdot 28$ & 2.831638 & $2 \mathrm{NU} 22^{*} *$ \\
\hline 3227.34 & 0.8624 & 0.0831 & 6 & 2 & 5 & 5 & 3 & 2 & 508.81 & 0.312103 & 2 NU 2 \\
\hline 3227.47 & 20.1179 & 0.0947 & 5 & 2 & 3 & 5 & 1 & 4 & 399.46 & 4.214107 & $2 N \cup 2$ \\
\hline 3227.77 & 0.0266 & 0.0914 & 5 & 1 & 4 & 5 & 0 & 5 & 325.35 & 1.912338 & $2 \mathrm{NU} 2 *$ \\
\hline 3228.89 & 0.2794 & 0.0454 & 8 & 6 & 3 & 9 & 7 & 2 & 1810.63 & 6.141896 & NUI \\
\hline 3228.94 & 0.0931 & 0.0454 & 8 & 6 & 2 & 9 & 7 & 3 & 1810.63 & 6.142105 & NU1 \\
\hline 3229.01 & 0.0035 & 0.0671 & 7 & 1 & 6 & 7 & 0 & 7 & 2180.65 & 1.838288 & $2 \mathrm{NU} 2 * * *$ \\
\hline 3229.33 & 0.1006 & 0.0819 & 8 & 2 & 7 & 7 & 3 & 4 & $842 \cdot 38$ & 0.192937 & 2 NU 2 \\
\hline 3229.36 & 0.0066 & 0.0920 & 6 & 2 & 4 & 6 & 1 & 5 & 542.91 & 4.225862 & $2 \mathrm{NU} 2 *$ \\
\hline 3229.91 & 20.4780 & 0.1026 & 3 & 1 & 3 & 2 & 0 & 2 & 70.08 & 2.156792 & 2 NU2 \\
\hline 3230.16 & 0.0987 & 0.0616 & 9 & 3 & 7 & 10 & 5 & 6 & 1718.77 & 0.128613 & NU3 \\
\hline 3230.41 & 0.1647 & 0.0659 & 8 & 4 & 4 & 9 & 6 & 3 & 1631.41 & 0.140500 & NU3+ \\
\hline
\end{tabular}




\begin{tabular}{|c|c|c|c|c|c|c|c|c|c|c|c|}
\hline $\begin{array}{c}\text { FREQUENCY } \\
\mathrm{cm}^{-1}\end{array}$ & $\begin{array}{l}\text { LINE } \\
\text { STRENGTH } \\
\frac{\mathrm{cm}^{-1}}{\mathrm{gm} \mathrm{cm}^{-2}}\end{array}$ & $\begin{array}{l}\text { HALF } \\
\text { WIDTH } \\
\frac{\mathrm{cm}^{-1}}{\text { atm. }}\end{array}$ & $J^{\prime}$ & $K_{0}$ & $K_{c}$ & $J^{\prime \prime}$ & $\mathrm{K}_{\mathrm{a}}$ & $K_{c}$ & $\begin{array}{c}E^{\prime \prime} \\
\mathrm{cm}^{-1}\end{array}$ & L & BAND \\
\hline $\begin{array}{l}3230.86 \\
3230.86\end{array}$ & $\begin{array}{l}0.1217 \\
0.3650\end{array}$ & $\begin{array}{l}0.0356 \\
0.0356\end{array}$ & $\begin{array}{l}7 \\
7\end{array}$ & $\begin{array}{l}7 \\
7\end{array}$ & $\begin{array}{l}1 \\
0\end{array}$ & $\begin{array}{l}8 \\
8\end{array}$ & $\begin{array}{l}8 \\
8\end{array}$ & $\begin{array}{l}0 \\
1\end{array}$ & $\begin{array}{l}1789 \cdot 10 \\
1789 \cdot 10\end{array}$ & $\begin{array}{l}7.402783 \\
7.402783\end{array}$ & $\begin{array}{l}\text { NU1 } \\
\text { NUI }\end{array}$ \\
\hline 3230.93 & 0.0409 & 0.0717 & 6 & 1 & 5 & 7 & 5 & 2 & 1059.85 & 0.002023 & $N \cup 3$ \\
\hline $3231 \cdot 34$ & 0.1191 & 0.0690 & 5 & 3 & 3 & 4 & 4 & 0 & $488 \cdot 13$ & 0.116422 & $2 \mathrm{NU} 2$ \\
\hline 3231.87 & 0.0045 & 0.0520 & 8 & 0 & 8 & 7 & 1 & 7 & $2181 \cdot 12$ & 6.998008 & $2 \mathrm{NU} 22^{*}{ }^{*} *$ \\
\hline 3231.89 & 0.1073 & 0.0976 & 4 & 1 & 4 & 3 & 0 & 3 & 136.77 & 3.004990 & $2 \mathrm{NU} 2 *$ \\
\hline 3232.06 & 0.0150 & 0.0939 & 3 & 2 & 2 & 3 & 1 & 3 & $142 \cdot 28$ & 1.297651 & $2 N \cup 2 *$ \\
\hline 3232.27 & 3.4961 & 0.0947 & 4 & 1 & 3 & 3 & 2 & 2 & 206.30 & 0.835095 & $2 \mathrm{NU} 2$ \\
\hline 3232.52 & 0.0132 & 0.0909 & 5 & 3 & 2 & 5 & 2 & 3 & 2053.98 & 3.442901 & $2 \mathrm{NU} 2 * * * *$ \\
\hline 3232.59 & 0.1165 & 0.0830 & 7 & 2 & 6 & 6 & 3 & 3 & 661.56 & 0.271048 & $2 \mathrm{~N} \cup 2$ \\
\hline 3232.76 & 0.0489 & 0.0569 & 9 & 5 & 5 & 10 & 6 & 4 & 1875.53 & 4.830967 & NU 1 \\
\hline 3233.00 & 16.3336 & 0.0961 & 4 & 0 & 4 & 3 & 1 & 3 & $142 \cdot 28$ & 2.831638 & $2 \mathrm{NU} 2$ \\
\hline 3233.39 & 0.0516 & 0.0729 & 9 & 3 & 7 & 10 & 4 & 6 & $1616 \cdot 51$ & 1.314980 & NU1 \\
\hline 3233.46 & 0.0135 & 0.0523 & 8 & 1 & 8 & 7 & 0 & 7 & 2180.65 & 7.000291 & $2 \mathrm{NU} 2 * * *$ \\
\hline 3233.50 & 0.0021 & 0.0859 & 7 & 2 & 5 & 6 & 3 & 4 & $2271 \cdot 70$ & 1.512999 & $2 \mathrm{NU} 2 * * *$ \\
\hline 3233.61 & 0.0068 & 0.0710 & 6 & 2 & 5 & 6 & 1 & 6 & 2042.77 & 1. .783035 & $2 \mathrm{~N} \cup 2 * * *$ \\
\hline 3233.75 & 0.0046 & 0.0886 & 5 & 2 & 3 & 4 & 3 & 2 & 382.52 & 0.436045 & $2 \mathrm{NU} 2^{*}$ \\
\hline 3233.77 & 0.1475 & 0.0583 & 9 & 5 & 4 & 10 & 6 & 5 & 1875.00 & 4.857731 & NU1 \\
\hline 3233.93 & 0.0049 & 0.0914 & 5 & 1 & 4 & 5 & 0 & 5 & 325.35 & 1.912338 & $2 \mathrm{~N} \cup 2 * *$ \\
\hline 3234.65 & 0.3668 & 0.0761 & 5 & 3 & 2 & 4 & 4 & 1 & 488.10 & 0.119405 & 2 NU2 2 \\
\hline 3235.54 & 0.0012 & 0.0920 & 6 & 2 & 4 & 6 & 1 & 5 & 542.91 & 4.225862 & $2 \mathrm{NU} 2 * *$ \\
\hline 3235.98 & 0.0014 & 0.0661 & 10 & 6 & 5 & 11 & 5 & 6 & 1999.02 & 0.799607 & $2 \mathrm{NU} 2$ \\
\hline 3236.40 & 0.1308 & 0.0560 & 9 & 2 & 8 & 10 & 4 & 7 & $1581 \cdot 34$ & 0.087251 & NU3 \\
\hline 3236.67 & 19.7590 & 0.0952 & 2 & 2 & 1 & 2 & 1 & 2 & 79.48 & 0.833333 & 2NU2 \\
\hline 3237.72 & 0.0139 & 0.0706 & 8 & 5 & 4 & 7 & 4 & 3 & $2572 \cdot 13$ & 9.52 & NU1*** \\
\hline 3237.94 & 0.3310 & 0.0787 & 4 & 4 & 1 & 5 & 3 & 2 & 508.81 & 0.119405 & 2NU2 \\
\hline 3238.42 & 0.0198 & 0.0976 & 4 & 1 & 4 & 3 & 0 & 3 & 136.77 & 3.004990 & $2 \mathrm{~N} \cup 2 * *$ \\
\hline 3238.73 & 0.0028 & 0.0939 & 3 & 2 & 2 & 3 & 1 & 3 & $142 \cdot 28$ & 1.297651 & 2 NU $2 * *$ \\
\hline 3240.09 & 13.2755 & 0.0914 & 5 & 1 & 4 & 5 & 0 & 5 & 325.35 & 1.912338 & 2NU2 \\
\hline 3240.36 & 0.0053 & 0.0884 & 4 & 3 & 1 & 4 & 2 & 2 & 1922.92 & 2.167225 & $2 \mathrm{NU} 2 * * * *$ \\
\hline 3241.39 & 0.0902 & 0.0880 & 5 & 0 & 5 & 4 & 1 & 4 & $224 \cdot 83$ & 3.911752 & 2 NU2* \\
\hline 3241.72 & 3.2965 & 0.0920 & 6 & 2 & 4 & 6 & 1 & 5 & $542 \cdot 91$ & $4 \cdot 225862$ & $2 \mathrm{NU} 2$ \\
\hline 3242.08 & 0.0016 & 0.0551 & 9 & 2 & 8 & 9 & 5 & 5 & 1474.99 & 0.019752 & NUI \\
\hline 3242.76 & 0.1104 & 0.0708 & 4 & 4 & 0 & 5 & 3 & 3 & 504.00 & 0.116422 & $2 N U 2$ \\
\hline 3242.86 & 0.0055 & 0.0721 & 11 & 3 & 8 & 12 & 5 & 7 & $2300 \cdot 80$ & 0.411017 & NU3 \\
\hline 3243.02 & 0.3081 & 0.0712 & 8 & 2 & 7 & 9 & 3 & 6 & 1282.92 & 0.493418 & NUI \\
\hline 3243.99 & 0.0362 & 0.0896 & 4 & 2 & 3 & 4 & 1 & 4 & 224.83 & 1.567300 & $2 N \cup 2 *$ \\
\hline 3244.41 & 2.2776 & 0.0886 & 5 & 2 & 3 & 4 & 3 & 2 & $382 \cdot 52$ & 0.436045 & $2 \mathrm{NU} 2$ \\
\hline $3244 \cdot 96$ & 53.6439 & 0.0976 & 4 & 1 & 4 & 3 & 0 & 3 & 136.77 & 3.004990 & $2 \mathrm{NU} 2$ \\
\hline 3245.40 & 7.5139 & 0.0939 & 3 & 2 & 2 & 3 & 1 & 3 & $142 \cdot 28$ & 1.297651 & $2 \mathrm{NU} 2$ \\
\hline 3245.90 & 0.0552 & 0.0644 & 7 & 5 & 2 & 8 & 4 & 5 & 1122.72 & 0.427306 & $2 \mathrm{NU} 2$ \\
\hline 3246.06 & 0.0136 & 0.0854 & 3 & 3 & 0 & 3 & 2 & 1 & $1819 \cdot 34$ & 1.092505 & $2 \mathrm{~N} \cup 2 * * *$ \\
\hline 3246.68 & 0.0748 & 0.0677 & 9 & 4 & 6 & 10 & 5 & 5 & $1724 \cdot 72$ & 3.272354 & NU1 \\
\hline 3247.31 & 0.1972 & 0.0596 & 7 & 4 & 4 & 8 & 6 & 3 & 1411.65 & 0.056441 & NU3 \\
\hline 3247.37 & 0.0310 & 0.0900 & 5 & 1 & 5 & 4 & 0 & 4 & 222.06 & 3.972803 & $2 \mathrm{NU} 2 *$ \\
\hline 3247.77 & 0.0167 & 0.0880 & 5 & 0 & 5 & 4 & 1 & 4 & $224 \cdot 83$ & 3.911752 & $2 \mathrm{NU} 2 * *$ \\
\hline 3247.83 & 0.0016 & 0.0596 & 6 & 3 & 4 & 6 & 6 & 1 & 1045.07 & 0.000772 & NU1 \\
\hline $3248 \cdot 36$ & 0.0087 & 0.0734 & 7 & 1 & 6 & 6 & 2 & 5 & $2161 \cdot 30$ & 3.799063 & $2 \mathrm{NU} 2 * * * *$ \\
\hline 3248.90 & 0.0070 & 0.0436 & 9 & 0 & 9 & 8 & 1 & 8 & $2337 \cdot 70$ & 8.007087 & $2 \mathrm{NU} 2 * * * *$ \\
\hline 3248.91 & 0.0299 & 0.0955 & 4 & 2 & 3 & 3 & 1 & 2 & 1772.41 & 1.970843 & $2 N \cup 2 * * * *$ \\
\hline 3249.15 & 0.0144 & 0.0673 & 11 & 4 & 7 & 12 & 5 & 8 & 2275.44 & 3.784189 & NU1 \\
\hline 3249.49 & 0.1344 & 0.0690 & 7 & 1 & 7 & 8 & 2 & 6 & 982.91 & 0.143980 & NU 1 \\
\hline 3249.72 & 0.0023 & 0.0437 & 9 & 1 & 9 & 8 & 0 & 8 & 2337.53 & 8.007851 & $2 \mathrm{NU} 2 * * * *$ \\
\hline 3250.61 & 0.0067 & 0.0896 & 4 & 2 & 3 & 4 & 1 & 4 & 224.83 & 1.567300 & $2 N \cup 2^{*} x^{*}$ \\
\hline
\end{tabular}




\begin{tabular}{|c|c|c|c|c|c|c|c|c|c|c|c|}
\hline $\begin{array}{c}\text { FREQUENCY } \\
\mathrm{cm}^{-1}\end{array}$ & $\begin{array}{l}\text { LINE } \\
\text { STRENGTH } \\
\frac{\mathrm{cm}^{-1}}{\mathrm{gm} \mathrm{cm}^{-2}}\end{array}$ & $\begin{array}{l}\text { HALF } \\
\text { WIDTH } \\
\mathrm{cm}^{-1} \\
a+m .\end{array}$ & $J^{\prime}$ & $\mathrm{K}_{\mathrm{a}}$ & $K_{c}$ & $J^{\prime \prime}$ & $k_{a}$ & $K_{c}$ & $\begin{array}{c}E^{\prime \prime} \\
\mathrm{cm}^{-1}\end{array}$ & L & BAND \\
\hline $3251 \cdot 30$ & 0.0084 & 0.0848 & 7 & 2 & 5 & 7 & 1 & 6 & $704 \cdot 22$ & 3.983004 & $2 \operatorname{NU} 2 *$ \\
\hline $251 \cdot 47$ & 0.0666 & 0.0628 & 7 & 4 & 3 & 8 & 6 & 2 & 1411.68 & 0.057059 & NU3 \\
\hline 3251.49 & 0.0044 & 0.0830 & 3 & 3 & 1 & 3 & 2 & 2 & 1813.81 & 1.035682 & $2 \mathrm{NU} 2 * * *$ \\
\hline 3252.43 & 0.0017 & 0.0422 & 7 & 6 & 1 & 8 & 7 & 2 & 1590.74 & 6.263444 & NUI* \\
\hline 3253.07 & 0.0022 & 0.0604 & 8 & 2 & 6 & 9 & 1 & 9 & 920.21 & 0.018354 & 2 NU2 2 \\
\hline 3253.25 & 0.0260 & 0.0918 & 5 & 1 & 4 & 4 & 2 & 3 & 300.35 & 1.638916 & $2 \operatorname{NU} 2 *$ \\
\hline 3254.15 & 45.0823 & 0.0880 & 5 & 0 & 5 & 4 & 1 & 4 & 224.83 & 3.911752 & 2 NU 2 \\
\hline 3254.60 & 0.4662 & 0.0713 & 6 & 3 & 4 & 5 & 4 & 1 & 610.35 & 0.278030 & $2 \mathrm{NU} 2$ \\
\hline 3254.62 & 0.0148 & 0.0829 & 4 & 3 & 2 & 4 & 2 & 3 & 1907.99 & 1.848533 & $2 \mathrm{~N} \cup 2 * * *$ \\
\hline 3254.63 & 0.0012 & 0.0601 & 7 & 2 & 6 & 7 & 1 & 7 & $2181 \cdot 12$ & 1.816721 & $2 \mathrm{~N} \cup 2 * * *$ \\
\hline 3256.01 & 0.2793 & 0.0422 & 7 & 6 & 2 & 8 & 7 & 1 & 1590.74 & 6.263414 & NU1 \\
\hline 3256.01 & 0.8380 & 0.0422 & 7 & 6 & 1 & 8 & 7 & 2 & 1590.74 & 6.263444 & NU 1 \\
\hline 3257.23 & 18.0800 & 0.0896 & 4 & 2 & 3 & 4 & 1 & 4 & 224.83 & 1.567300 & 2NU2 \\
\hline 3257.29 & 0.0016 & 0.0848 & 7 & 2 & 5 & 7 & 1 & 6 & $704 \cdot 22$ & 3.983004 & $2 \mathrm{NU} 2 * *$ \\
\hline 3258.02 & 0.4997 & 0.0557 & 8 & 5 & 4 & 9 & 6 & 3 & 1631.41 & 4.505 & NU I- \\
\hline $3258 \cdot 12$ & 0.1760 & 0.0566 & 8 & 5 & 3 & 9 & 6 & 4 & 1631.27 & $4 \cdot 76$ & NU1- \\
\hline 3258.72 & 0.0079 & 0.0815 & 5 & 2 & 4 & 5 & 1 & 5 & $326 \cdot 64$ & 1.711792 & $2 N \cup 2 *$ \\
\hline 3259.17 & 0.0048 & 0.0918 & 5 & 1 & 4 & 4 & 2 & 3 & $300 \cdot 35$ & 1.638916 & 2 NU $2 * *$ \\
\hline 3260.22 & 0.0866 & 0.1021 & 2 & 2 & 1 & 1 & 1 & 0 & $42 \cdot 37$ & 1.500000 & $2 \mathrm{NU} 2 *$ \\
\hline 3260.42 & 0.0037 & 0.0803 & 5 & 3 & 3 & 5 & 2 & 4 & $2024 \cdot 17$ & 2.492169 & $2 \mathrm{NU} 2 * * *$ \\
\hline 3260.44 & 15.5056 & 0.0900 & 5 & 1 & 5 & 4 & 0 & 4 & 222.06 & 3.972803 & $2 \mathrm{NU} 2$ \\
\hline 3260.50 & 0.0230 & 0.0760 & 6 & 0 & 6 & 5 & 1 & 5 & 326 & 4.958549 & $2 \mathrm{NU} 2 *$ \\
\hline $3261 \cdot 31$ & 0.0098 & 0.0724 & 5 & 1 & 4 & 6 & 5 & 1 & 888 & 0.000613 & NU3 \\
\hline 3262.02 & 0.0264 & 0.0909 & 5 & 3 & 2 & 5 & 2 & 3 & 446 & 3.442901 & $2 N \cup 2 *$ \\
\hline $3262 \cdot 50$ & 0.1820 & 0.0774 & 6 & 1 & 5 & 7 & 4 & 4 & 927.77 & 0.147419 & NUI \\
\hline 3262.78 & 0.0074 & 0.0927 & 5 & 2 & 4 & 4 & 1 & 3 & 1875.49 & 2.444582 & $2 N \cup 2 * * *$ \\
\hline $3263 \cdot 28$ & 4.1894 & 0.0848 & 7 & 2 & 5 & 7 & 1 & 6 & 704.22 & 3.983004 & $2 \mathrm{NU} 2$ \\
\hline 3263.37 & 0.0699 & 0.0779 & 6 & 1 & 6 & 5 & 0 & 5 & 35 & 4.979001 & 2 NU2* \\
\hline 3263.60 & 0.1704 & 0.0809 & 6 & 3 & 3 & 5 & 4 & 2 & 12 & 0.303663 & $2 \mathrm{NU} 2$ \\
\hline 3263.79 & 0.0016 & 0.0927 & 7 & 3 & 4 & 7 & 2 & 5 & 782.40 & 5.918411 & 2 NU2** \\
\hline 3263.99 & 0.0010 & 0.0925 & 6 & 3 & 3 & 6 & 2 & 4 & 602.77 & 4.829435 & 2 NU $2 * *$ \\
\hline 326 & 0.0233 & 0.0701 & 10 & 4 & 6 & & 5 & 7 & 83 & 3.652101 & NUI \\
\hline 3264.27 & 0.0870 & 0.0740 & 6 & 0 & 6 & 7 & 4 & 3 & .22 & 002241 & NU3 \\
\hline 3264.33 & 0.0100 & 0.0589 & 8 & 1 & 8 & 8 & 4 & 5 & 1122 & 0.007182 & NUI \\
\hline 3265.09 & 12.9917 & 0.0918 & 5 & 1 & 4 & 4 & 2 & 3 & $300 \cdot 35$ & 1.638916 & $2 \mathrm{NU} 2$ \\
\hline 3266.04 & 0.0937 & 0.0662 & 8 & 3 & 6 & 9 & 5 & 5 & 1474.99 & 0.109168 & NU3 \\
\hline 3266.11 & 0.0858 & 0.0704 & 6 & 5 & $\cdot 2$ & 7 & 4 & 3 & 931.22 & 0.253734 & 2 NU2 \\
\hline $3266 \cdot 35$ & 2.3549 & 0.0806 & 6 & 1 & 5 & 6 & 0 & 6 & 446.71 & 1.852055 & $2 \mathrm{NU} 2$ \\
\hline 3266.58 & 0.0246 & 0.0949 & 2 & 2 & 0 & 1 & 1 & 1 & 13 & 1.244747 & $2 \mathrm{~N} \cup 2 *$ \\
\hline 3266.96 & 0.0243 & 0.0760 & 6 & 0 & 6 & 5 & 1 & 5 & 326.64 & 4.958549 & $2 \mathrm{~N} \cup 2 * *$ \\
\hline 3267.00 & 0.0160 & 0.1021 & 2 & 2 & 1 & 1 & 1 & 0 & $42 \cdot 37$ & 1.500000 & $2 \mathrm{NU} 2 * *$ \\
\hline 326 & 0.0858 & 0.0795 & 4 & 1 & 4 & 5 & 4 & 1 & 35 & 0.004729 & NU 1 \\
\hline 326 & 0.0013 & 0.0620 & 9 & 6 & 4 & 10 & 5 & 5 & 1724 & 0.586260 & $2 \mathrm{NU} 2$ \\
\hline 326 & 0.0067 & 0.0751 & 6 & 3 & 4 & 6 & 2 & 5 & 2161 & 2.963539 & $2 \mathrm{NU} 2 * 2^{*} *$ \\
\hline 3268.62 & 0.0107 & 0.0884 & 4 & 3 & 1 & 4 & 2 & 2 & 315 & 2.167225 & 2 NU $2 *$ \\
\hline 3268.88 & 0.0037 & 0.0673 & 7 & 3 & 4 & 7 & 6 & 1 & 1216 & 0.004214 & NUI \\
\hline 3269.13 & 0.0049 & 0.0909 & 5 & 3 & 2 & 5 & 2 & 3 & 446 & 3.442901 & $2 \mathrm{NU} 2 * *$ \\
\hline 3269.67 & 0.0288 & 0.0626 & 6 & 5 & 1 & 7 & 4 & 4 & 927.77 & 0.250934 & $2 \mathrm{NU} 2$ \\
\hline 3269. & 0.0129 & 0.0779 & 6 & 1 & 6 & 5 & 0 & 5 & $325 \cdot 35$ & 4.979001 & $2 \mathrm{NU} 2 * *$ \\
\hline $3269 \cdot 94$ & 0.0017 & 0.0893 & 6 & 2 & 4 & 5 & 3 & 3 & 00 & 0.863547 & $2 \mathrm{NU} 2^{*}$ \\
\hline 3270.48 & 4.2197 & 0.0927 & 7 & 3 & 4 & 7 & 2 & 5 & $782 \cdot 40$ & 5.918411 & $2 N \cup 2$ \\
\hline 3270.78 & 0.0112 & 0.0591 & 8 & 2 & 7 & 8 & 5 & 4 & $1255 \cdot 16$ & 0.015469 & NUI \\
\hline 3270.95 & 2.8182 & 0.0925 & 6 & 3 & 3 & 6 & 2 & 4 & 602.77 & 4.829435 & $2 \mathrm{NU} 2$ \\
\hline 3271.40 & 0.0023 & 0.0497 & 10 & 0 & 10 & 10 & 4 & 7 & $1581 \cdot 34$ & 0.001525 & NU3 \\
\hline
\end{tabular}




\begin{tabular}{|c|c|c|c|c|c|c|c|c|c|c|c|}
\hline $\begin{array}{c}\text { FREQUENCY } \\
\mathrm{cm}^{-1}\end{array}$ & $\begin{array}{l}\text { LINE } \\
\text { STRENGTH } \\
\frac{\mathrm{cm}^{-1}}{\mathrm{gm} \mathrm{cm}^{-2}}\end{array}$ & $\begin{array}{l}\text { HALF } \\
\text { WIDTH } \\
\frac{\mathrm{cm}^{-1}}{a+m .}\end{array}$ & $J^{\prime}$ & $k_{0}$ & $\mathrm{~K}_{\mathrm{c}}$ & J" & $k_{a}$ & $K_{c}$ & $\mathrm{Em}^{\prime \prime}$ & L & BAND \\
\hline 3271.87 & 3.9743 & 0.0815 & 5 & 2 & 4 & 5 & 1 & 5 & $326 \cdot 64$ & 1.711792 & $2 \mathrm{NU} 2$ \\
\hline 3271.95 & 0.1032 & 0.0558 & 8 & 1 & 8 & 9 & 3 & 7 & $1216 \cdot 27$ & 0.033194 & NU3 \\
\hline 3272.03 & 0.0855 & 0.0704 & 10 & 2 & 8 & 11 & 4 & 7 & 1899.06 & 0.260837 & NU3 \\
\hline 3272.49 & 0.0775 & 0.0880 & 4 & 3 & 2 & 5 & 0 & 5 & 325.35 & 0.011053 & $2 \mathrm{NU} 2$ \\
\hline 3272.76 & 0.0206 & 0.0865 & 5 & 3 & 3 & 6 & 0 & 6 & 446.71 & 0.016149 & $2 \mathrm{NU} 2$ \\
\hline 3273.07 & 0.0020 & 0.0587 & 7 & 1 & 7 & 7 & 5 & 2 & 1059.85 & 0.000093 & NU3 \\
\hline 3273.36 & 0.0861 & 0.0590 & 6 & 4 & 3 & 5 & 5 & 0 & $742 \cdot 10$ & 0.098702 & $2 \mathrm{NU} 2$ \\
\hline 3273.42 & 11.5179 & 0.0760 & 6 & 0 & 6 & 5 & 1 & 5 & $326 \cdot 64$ & 4.958549 & 2 NU2 \\
\hline 3273.78 & 43.3084 & 0.1021 & 2 & 2 & 1 & 1 & 1 & 0 & $42 \cdot 37$ & 1.500000 & $2 \mathrm{NU} 2$ \\
\hline 3273.89 & 0.1059 & 0.0573 & 6 & 4 & 3 & 7 & 6 & 2 & $1216 \cdot 20$ & 0.033870 & NU3 \\
\hline 3274.25 & 0.0271 & 0.0854 & 3 & 3 & 0 & 3 & 2 & 1 & $212 \cdot 15$ & 1.092505 & $2 \mathrm{NU} 2 *$ \\
\hline $3274 \cdot 60$ & 0.0736 & 0.0752 & 10 & 3 & 7 & 11 & 5 & 6 & 1999.02 & 0.359370 & NU3 \\
\hline 3275.16 & 0.3186 & 0.0589 & 6 & 4 & 2 & 7 & 6 & 1 & $1216 \cdot 20$ & 0.033963 & NU3 \\
\hline 3275.48 & 0.0154 & 0.0873 & 6 & 2 & 5 & 5 & 1 & 4 & $2000 \cdot 90$ & 3.129447 & $2 \mathrm{~N} \cup 2 * * *$ \\
\hline 3275.76 & 0.0020 & 0.0884 & 4 & 3 & 1 & 4 & 2 & 2 & 315.79 & 2.167225 & $2 \mathrm{~N} \cup 2 * *$ \\
\hline 3275.80 & 0.1160 & 0.0740 & 7 & 3 & 5 & 6 & 4 & 2 & 757.78 & 0.431025 & $2 \mathrm{NU} 2$ \\
\hline 3275.87 & 0.0137 & 0.0710 & 6 & 2 & 5 & 6 & 1 & 6 & $447 \cdot 24$ & 1.783035 & $2 \mathrm{NU} 2 *$ \\
\hline 3276.25 & 13.1878 & 0.0909 & 5 & 3 & 2 & 5 & 2 & 3 & $446 \cdot 50$ & $3.44290^{\circ} 1$ & 2NU2 \\
\hline 3276.47 & 34.9525 & 0.0779 & 6 & 1 & 6 & 5 & 0 & 5 & $325 \cdot 35$ & 4.979001 & $2 \mathrm{NU} 2$ \\
\hline 3276.81 & 0.0072 & 0.0628 & 9 & 0 & 9 & 9 & 3 & 6 & $1282 \cdot 92$ & 0.011425 & NU1 \\
\hline 3276.99 & 0.0015 & 0.0769 & 8 & 3 & 6 & 9 & 4 & 5 & $1360 \cdot 28$ & 1.797448 & NU I* \\
\hline 3277.40 & 0.0016 & 0.0503 & 8 & 2 & 7 & 8 & 1 & 8 & 2337.70 & 1.833129 & $2 \mathrm{NU} 2 * * *$ \\
\hline 3277.56 & 0.5567 & 0.0898 & 8 & 3 & 5 & 8 & 2 & 6 & 982.91 & 6.368783 & $2 \mathrm{NU} 2$ \\
\hline 3277.58 & 0.2703 & 0.0711 & 9 & 4 & 5 & 10 & 5 & 6 & 1718.77 & 3.697402 & NU1 \\
\hline 3277.60 & 0.0016 & 0.0685 & 8 & 3 & 5 & 8 & 6 & 2 & 1411.68 & 0.014126 & NU 1 \\
\hline 3278.32 & 0.0459 & 0.0631 & 7 & 0 & 7 & 6 & 1 & 6 & $447 \cdot 24$ & 5.983681 & $2 \mathrm{NU} 2 *$ \\
\hline 3278.42 & 0.01 .63 & 0.0892 & 3 & 3 & 1 & 4 & 0 & 4 & 222.06 & 0.004153 & 2 NU2 2 \\
\hline 3278.47 & 0.0011 & 0.0682 & 7 & 3 & 5 & 7 & 2 & 6 & $2318 \cdot 53$ & 3.273499 & $2 \mathrm{~N} \cup 2 * * *$ \\
\hline 3278.53 & 0.0011 & 0.0759 & 8 & 2 & 6 & 8 & 1 & 7 & 882.93 & 3.773034 & $2 \mathrm{NU} 2 *$ \\
\hline 3278.58 & 0.2815 & 0.0735 & 6 & 0 & 6 & 7 & 3 & 5 & 816.72 & 0.130226 & NU1 \\
\hline 3278.69 & 0.0342 & 0.0845 & 6 & 3 & 4 & 7 & 0 & 7 & $586 \cdot 26$ & 0.017960 & 2 NU2 2 \\
\hline 3278.79 & 0.0692 & 0.0648 & 9 & 1 & 8 & 10 & 3 & 7 & $1538 \cdot 23$ & 0.110021 & NU3 \\
\hline 3278.87 & 0.0248 & 0.0984 & 3 & 2 & 2 & 2 & 1 & 1 & $95 \cdot 17$ & 1.666667 & $2 \mathrm{NU} 2 *$ \\
\hline 3279.07 & 0.8791 & 0.0676 & 8 & 4 & 5 & 9 & 5 & 4 & 1477.31 & 3.682999 & NUI \\
\hline 3279.22 & 0.0017 & 0.0613 & 8 & 1 & 7 & 7 & 2 & 6 & 2318.53 & 4.913532 & $2 \mathrm{NU} 2 * * *$ \\
\hline 3279.41 & 0.0015 & 0.0388 & 6 & 6 & 0 & 7 & 7 & 1 & 1394.86 & 6.401518 & NU $1 *$ \\
\hline 3279.41 & 0.0046 & 0.0388 & 6 & 6 & 1 & 7 & 7 & 0 & 1394.86 & 6.401516 & NU $1 *$ \\
\hline 3279.64 & 0.0154 & 0.0642 & 7 & 1 & 7 & 6 & 0 & 6 & 446.71 & 5.990506 & $2 \mathrm{NU} 2 *$ \\
\hline 3280.06 & $12 \cdot 3204$ & 0.0949 & 2 & 2 & 0 & 1 & 1 & 1 & 37.13 & 1.244747 & $2 \mathrm{NU} 2$ \\
\hline 3280.14 & 0.0034 & 0.0539 & 7 & 5 & 2 & 8 & 6 & 3 & 1411.65 & 5.144364 & NU 1* \\
\hline 3280.47 & 0.0070 & 0.0671 & 7 & 1 & 6 & 7 & 0 & 7 & 586.26 & 1.838288 & $2 N \cup 2 *$ \\
\hline 3280.63 & 0.8282 & 0.0893 & 6 & 2 & 4 & 5 & 3 & 3 & 504.00 & 0.863547 & 2 NU2 2 \\
\hline 3280.72 & 0.0011 & 0.0834 & 9 & 3 & 6 & 9 & 2 & 7 & 1201.95 & 6.247031 & $2 \mathrm{NU} 2 *$ \\
\hline $3281 \cdot 36$ & 0.0050 & 0.0854 & 3 & 3 & 0 & 3 & 2 & 1 & 212.15 & 1.092505 & $2 \mathrm{NU} 2 * *$ \\
\hline 3282.39 & 0.0025 & 0.0710 & 6 & 2 & 5 & 6 & 1 & 6 & $447 \cdot 24$ & 1.783035 & $2 \mathrm{NU} 2 * *$ \\
\hline 3282.91 & 5.3299 & 0.0884 & 4 & 3 & 1 & 4 & 2 & 2 & 315.79 & 2.167225 & $2 \mathrm{NU} 2$ \\
\hline 3283.01 & 0.7731 & 0.0388 & 6 & 6 & 0 & 7 & 7 & 1 & 1394.86 & 6.401518 & NU1 \\
\hline 3283.01 & 2.3193 & 0.0388 & 6 & 6 & 1 & 7 & 7 & 0 & 1394.86 & 6.401516 & NU1 \\
\hline 3283.52 & 0.0296 & 0.0829 & 4 & 3 & 2 & 4 & 2 & 3 & $300 \cdot 35$ & 1.848533 & $2 \mathrm{NU} 2 *$ \\
\hline 3283.74 & 0.7692 & 0.0769 & 8 & 3 & 6 & 9 & 4 & 5 & $1360 \cdot 28$ & 1.797448 & NUI \\
\hline $3284 \cdot 10$ & 0.5684 & 0.0537 & 7 & 5 & 3 & 8 & 6 & 2 & 1411.68 & 5.142711 & NU1 \\
\hline 3284.15 & 1.7058 & 0.0539 & 7 & 5 & 2 & 8 & 6 & 3 & 1411.65 & 5.144364 & NU1 \\
\hline 3284.69 & 0.0080 & 0.0843 & 6 & 1 & 5 & 5 & 2 & 4 & $416 \cdot 22$ & 2.671788 & $2 \mathrm{NU} 2 *$ \\
\hline 3284.75 & 0.0020 & 0.0543 & 9 & 2 & 7 & 10 & 1 & 10 & 1114.56 & 0.015027 & $2 \mathrm{NU} 2$ \\
\hline
\end{tabular}




\begin{tabular}{|c|c|c|c|c|c|c|c|c|c|c|c|}
\hline $\begin{array}{c}\text { FREQUENCY } \\
\mathrm{cm}^{-1}\end{array}$ & $\begin{array}{l}\text { LINE } \\
\text { STRENGTH } \\
\frac{\mathrm{cm}-1}{\mathrm{gm} \mathrm{cm}^{-2}}\end{array}$ & $\begin{array}{l}\text { HALF } \\
\text { WIDTH } \\
\frac{\mathrm{cm}^{-1}}{\text { atm. }}\end{array}$ & $J^{\prime}$ & $\mathrm{K}_{\mathrm{a}}$ & $\mathrm{K}_{\mathrm{c}}$ & $J^{\prime \prime}$ & $K_{a}$ & $K_{c}$ & $\begin{array}{c}E^{\prime \prime} \\
\mathrm{cm}^{-1}\end{array}$ & L & BAND \\
\hline 3284.83 & 0.0085 & 0.0631 & 7 & 0 & 7 & 6 & 1 & 6 & $447 \cdot 24$ & 5.983681 & $2 \mathrm{~N} \cup 2 * *$ \\
\hline 3285.01 & 0.1492 & 0.0623 & 8 & 2 & 7 & 9 & 4 & 6 & 1340.89 & 0.038564 & NU3 \\
\hline 3285.69 & 0.0046 & 0.0984 & 3 & 2 & 2 & 2 & 1 & 1 & 95.17 & 1.666667 & $2 N \cup 2 * *$ \\
\hline 3285.70 & 0.0092 & 0.0717 & 4 & 1 & 3 & 5 & 5 & 0 & $742 \cdot 10$ & 0.000091 & NU3 \\
\hline $3286 \cdot 20$ & 0.0028 & 0.0642 & 7 & 1 & 7 & 6 & 0 & 6 & 446.71 & 5.990506 & $2 \operatorname{NU} 2 * *$ \\
\hline $3286 \cdot 22$ & 0.3507 & 0.0602 & 8 & 0 & 8 & 9 & 2 & 7 & 1201.95 & 0.034667 & NU3 \\
\hline 3286.75 & 0.0013 & 0.0671 & 7 & 1 & 6 & 7 & 0 & 7 & 586.26 & $1: 838288$ & $2 \mathrm{NU} 2^{* *} *$ \\
\hline 3286.79 & 0.0044 & 0.0679 & 9 & 3 & 6 & 9 & 6 & 3 & 1631.41 & 0.039684 & NUI \\
\hline 3287.15 & 0.0016 & 0.0830 & 3 & 3 & 1 & 3 & 2 & 2 & $206 \cdot 30$ & 1.035682 & $2 N \cup 2 * *$ \\
\hline 3287.55 & 0.0013 & 0.0681 & 7 & 3 & 5 & 8 & 5 & 4 & $1255 \cdot 16$ & 0.084527 & NU3* \\
\hline 3288.47 & 13.5567 & 0.0854 & 3 & 3 & 0 & 3 & 2 & 1 & $212 \cdot 15$ & 1.092505 & 2NU 2 \\
\hline 3288.91 & 6.8306 & 0.0710 & 6 & 2 & 5 & 6 & 1 & 6 & $447 \cdot 24$ & 1.783035 & $2 \mathrm{NU} 2$ \\
\hline 3289.43 & 0.0075 & 0.0803 & 5 & 3 & 3 & 5 & 2 & 4 & $416 \cdot 22$ & 2.492169 & $2 N \cup 2 *$ \\
\hline 3289.47 & 0.0032 & 0.0781 & 7 & 2 & 6 & 6 & 1 & 5 & $2146 \cdot 28$ & 4.008107 & $2 \mathrm{~N} \cup 2 * * *$ \\
\hline 3289.49 & 0.0051 & 0.0818 & 7 & 3 & 5 & 8 & 0 & 8 & 744.09 & 0.017472 & $2 \mathrm{NU} 2$ \\
\hline $3289 \cdot 94$ & 0.0013 & 0.0799 & 5 & 1 & 4 & 6 & 4 & 3 & 756.75 & 0.071290 & NU I * \\
\hline 3290.35 & 0.5458 & 0.0759 & 8 & 2 & 6 & 8 & 1 & 7 & 882.93 & 3.773034 & $2 \mathrm{NU} 2$ \\
\hline 3290.50 & 0.0055 & 0.0829 & 4 & 3 & 2 & 4 & 2 & 3 & 300.35 & $1.8,48533$ & $2 \mathrm{NU} 2 * *$ \\
\hline 3290.76 & 0.0015 & 0.0843 & 6 & 1 & 5 & 5 & 2 & 4 & $416 \cdot 22$ & 2.671788 & $2 \mathrm{NU} 2 * *$ \\
\hline 3291.35 & 22.9399 & 0.0631 & 7 & 0 & 7 & 6 & 1 & 6 & $447 \cdot 24$ & 5.983681 & $2 \mathrm{NU} 2$ \\
\hline 3292.51 & 12.3883 & 0.0984 & 3 & 2 & 2 & 2 & 1 & 1 & 95.17 & 1.666667 & $2 N U 2$ \\
\hline 3292.65 & 0.5516 & 0.0834 & 9 & 3 & 6 & 9 & 2 & 7 & $1201 \cdot 95$ & 6.247031 & $2 \mathrm{NU} 2$ \\
\hline 3292.70 & 0.0268 & 0.0646 & 5 & 5 & 1 & 6 & 4 & 2 & 757.78 & 0.098971 & $2 N \cup 2$ \\
\hline 3292.76 & 7.6787 & 0.0642 & 7 & 1 & 7 & 6 & 0 & 6 & 446.71 & 5.990506 & 2NU2 \\
\hline 3292.80 & 0.6267 & 0.0799 & 5 & 1 & 4 & 6 & 4 & 3 & 756.75 & 0.071290 & NU1 \\
\hline 3293.04 & 3.5190 & 0.0671 & 7 & 1 & 6 & 7 & 0 & 7 & $586 \cdot 26$ & 1.838288 & $2 N \cup 2$ \\
\hline 3293.22 & 0.0063 & 0.0636 & 7 & 2 & 6 & 7 & 5 & 3 & 1059.65 & 0.009705 & NU1 \\
\hline 3293.40 & 0.1771 & 0.0750 & 8 & 3 & 6 & 7 & 4 & 3 & 931.22 & 0.519243 & 2 NU2 \\
\hline 3293.43 & 0.0010 & 0.0659 & 10 & 3 & 7 & 10 & 6 & 4 & 1875.53 & 0.095771 & NU1 \\
\hline 3293.66 & 0.0017 & 0.0679 & 5 & 3 & 3 & 6 & 5 & 2 & 2552.85 & 0.105000 & NU $3 * * *$ \\
\hline 3293.73 & 0.0806 & 0.0590 & 5 & 5 & 0 & 6 & 4 & 3 & 756.75 & 0.098702 & $2 \mathrm{NU} 2$ \\
\hline 3294.11 & 0.2648 & 0.0708 & 8 & 4 & 4 & 9 & 5 & 5 & 1474.99 & 3.26 & NUI- \\
\hline $3294 \cdot 18$ & 4.4189 & 0.0830 & 3 & 3 & 1 & 3 & 2 & 2 & $206 \cdot 30$ & 1.035682 & 2NU 2 \\
\hline 3294.63 & 0.0023 & 0.0601 & 7 & 2 & 6 & 7 & 1 & 7 & $586 \cdot 48$ & 1.816721 & $2 \mathrm{NU} 2 *$ \\
\hline 3294.89 & 0.0015 & 0.0629 & 11 & 3 & 8 & 11 & 6 & 5 & $2144 \cdot 12$ & 0.189337 & NU 1 \\
\hline 3294.96 & 0.0597 & 0.0955 & 4 & 2 & 3 & 3 & 1 & 2 & $173 \cdot 36$ & 1.970843 & $2 \mathrm{NU} 2 *$ \\
\hline $3295 \cdot 22$ & 0.0090 & 0.0520 & 8 & 0 & 8 & 7 & 1 & 7 & 586.48 & 6.998008 & $2 N \cup 2 *$ \\
\hline 3295.82 & 0.0015 & 0.0603 & 8 & 3 & 6 & 8 & 2 & 7 & $2495 \cdot 18$ & 3.456295 & $2 \operatorname{NU} 2 * * *$ \\
\hline 3295.84 & C.0269 & 0.0523 & 8 & 1 & 8 & 7 & 0 & 7 & $586 \cdot 26$ & 7.000291 & $2 N \cup 2 *$ \\
\hline 3296.09 & 0.0020 & 0.0561 & 10 & 2 & 8 & 10 & 6 & 5 & 1875.00 & 0.005881 & NU 3 \\
\hline 3296.13 & 0.4497 & 0.0848 & 7 & 3 & 4 & 6 & 4 & 3 & 756.75 & 0.550367 & $2 N \cup 2$ \\
\hline 3296.34 & 0.0014 & 0.0803 & 5 & 3 & 3 & 5 & 2 & 4 & $416 \cdot 22$ & 2.492169 & $2 N \cup 2 * *$ \\
\hline 3296.84 & 3.9939 & 0.0843 & 6 & 1 & 5 & 5 & 2 & 4 & $416 \cdot 22$ & 2.671788 & $2 \mathrm{NU} 2$ \\
\hline 3297.07 & 0.0506 & 0.0587 & 8 & 6 & 3 & 9 & 5 & 4 & 1477.31 & 2.26 & $2 \mathrm{NU} 2+$ \\
\hline 3297.49 & 14.7987 & 0.0829 & 4 & 3 & 2 & 4 & 2 & 3 & $300 \cdot 35$ & 1.848533 & 2NU2 \\
\hline $3297 \cdot 58$ & 0.0649 & 0.0766 & 9 & 3 & 6 & 10 & 5 & 5 & 1724.72 & 0.261892 & $\mathrm{NU}_{3}$ \\
\hline 3297.83 & 0.0354 & 0.0648 & 7 & 4 & 4 & 6 & 5 & 1 & 888.63 & 0.250934 & 2 NU2 \\
\hline 3298.11 & 0.6557 & 0.0681 & 7 & 3 & 5 & 8 & 5 & 4 & 1255.16 & 0.084527 & NU3 \\
\hline 3298.27 & 0.0027 & 0.0643 & 6 & 4 & 3 & 7 & 5 & 2 & $2724 \cdot 19$ & 4.131192 & $N \cup 1 * * *$ \\
\hline 3298.35 & 0.0012 & 0.0697 & 5 & 3 & 2 & 6 & 5 & 1 & 2552.87 & 0.221000 & NU $3 * * *$ \\
\hline 3298.37 & 0.0135 & 0.0751 & 6 & 3 & 4 & 6 & 2 & 5 & 552.92 & 2.963539 & $2 N \cup 2 *$ \\
\hline 3299.74 & 0.1073 & 0.0688 & 7 & 4 & 3 & 6 & 5 & 2 & 888.60 & 0.253734 & 2NU2 \\
\hline 3299.91 & 0.0449 & 0.0961 & 3 & 2 & 1 & 2 & 1 & 2 & 79.48 & 0.925282 & $2 N \cup 2 *$ \\
\hline $3300 \cdot 31$ & 0.3378 & 0.0535 & 5 & 4 & 2 & 6 & 6 & 1 & 1045.07 & 0.015207 & NU3 \\
\hline
\end{tabular}




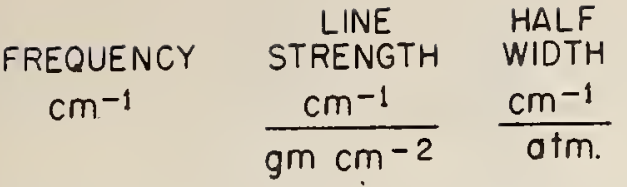

\begin{tabular}{|c|c|c|c|c|c|c|c|c|c|c|c|}
\hline $\begin{array}{l}3300.39 \\
3300.46\end{array}$ & $\begin{array}{l}0.0492 \\
0.1127\end{array}$ & $\begin{array}{l}0.0549 \\
0.0540\end{array}$ & $\begin{array}{l}8 \\
5\end{array}$ & $\begin{array}{l}6 \\
4\end{array}$ & $\begin{array}{l}2 \\
1\end{array}$ & $\begin{array}{l}9 \\
6\end{array}$ & $\begin{array}{l}5 \\
6\end{array}$ & $\begin{array}{l}5 \\
0\end{array}$ & $\begin{array}{l}1474.99 \\
1045.07\end{array}$ & $\begin{array}{l}6.42 \\
0.015215\end{array}$ & $\begin{array}{l}\text { NU2 }+ \\
\text { NU3 }\end{array}$ \\
\hline 3300.78 & 0.0073 & 0.0530 & 12 & 3 & 9 & 13 & 4 & 10 & 2426.22 & 5.75353 & NU1 \\
\hline 3301.79 & 0.0017 & 0.0520 & 8 & 0 & 8 & 7 & 1 & 7 & 586.48 & 6.998008 & $2 N \cup 2 * *$ \\
\hline 301.83 & 0.0111 & 0.0955 & 4 & 2 & 3 & 3 & 1 & 2 & 173.36 & 1.970843 & $2 N \cup 2 * *$ \\
\hline $302 \cdot 43$ & 0.0050 & 0.0523 & 8 & 1 & 8 & 7 & 0 & 7 & $586 \cdot 26$ & 7.000291 & $2 \mathrm{~N} \cup 2 * *$ \\
\hline 302.70 & 0.0074 & 0.0758 & 8 & 4 & 5 & 9 & & 8 & 1079.07 & 0.045574 & $2 \mathrm{NU} 2$ \\
\hline 302.72 & 0.4661 & 0.0784 & 7 & 2 & 6 & 8 & & 5 & $1050 \cdot 15$ & 0.688192 & NUI \\
\hline 303.25 & 3.7326 & 0.0803 & 5 & 3 & 3 & 5 & & 4 & 416.22 & 2.492169 & $2 \mathrm{NU} 2$ \\
\hline 303.53 & 0.0054 & 0.0757 & 7 & 4 & 4 & 8 & & 7 & $882 \cdot 93$ & 0.037317 & $2 \mathrm{NU} 2$ \\
\hline $4 \cdot 44$ & 0.0058 & 0.0770 & 8 & 3 & 6 & 9 & & 9 & $920 \cdot 18$ & 0.015979 & $2 \mathrm{NU} 2$ \\
\hline $304 \cdot 54$ & 0.0212 & 0.0760 & 9 & 3 & 7 & 8 & & 4 & 1131.76 & 0.505247 & $2 \mathrm{NU} 2$ \\
\hline 304.63 & 0.0089 & 0.0644 & 7 & 1 & 7 & 7 & & 4 & 927.77 & 0.007119 & NU 1 \\
\hline 5.20 & 0.0025 & 0.0751 & 6 & 3 & 4 & 6 & & 5 & $552 \cdot 92$ & 2.963539 & $2 \operatorname{NU} 2 * *$ \\
\hline $5 \cdot 36$ & 0.0054 & 0.0546 & 8 & 2 & 7 & 7 & & 6 & $2309 \cdot 74$ & 4.996649 & $2 \mathrm{NU} 2 * *$ \\
\hline 306.01 & 0.0011 & 0.0542 & 8 & 1 & 7 & 8 & & 8 & 744.09 & 1.839778 & $2 \mathrm{NU} 2 *$ \\
\hline 6.11 & 0.0028 & 0.0790 & 6 & 1 & 6 & 7 & & 5 & $782 \cdot 40$ & 0.182385 & NUI* \\
\hline 6.45 & 0.0094 & 0.0498 & 6 & 5 & 2 & 7 & & 1 & $1216 \cdot 20$ & 5.265222 & NUI* \\
\hline $306 \cdot 46$ & 0.0031 & 0.0499 & 6 & 5 & 1 & 7 & 0 & 2 & $1216 \cdot 20$ & 5.265494 & NU I* \\
\hline 306.59 & 0.0083 & 0.0961 & 3 & 2 & 1 & 2 & & 2 & 79.48 & 0.925282 & $2 \mathrm{~N} \cup 2 * *$ \\
\hline 7.65 & 0.0043 & 0.0859 & 7 & 2 & 5 & 6 & 3 & 4 & 648.97 & 1.512999 & $2 \operatorname{NU} 2 *$ \\
\hline 307.67 & 1.1631 & 0.0601 & 7 & 2 & 6 & 7 & 1 & 7 & 586.48 & 1.816721 & 2NU2 \\
\hline 307.72 & 0.0164 & 0.0770 & 10 & 3 & 8 & 9 & 4 & 5 & $1360 \cdot 28$ & 0.409296 & $2 \mathrm{NU} 2$ \\
\hline 8.03 & 0.9571 & 0.0665 & 7 & 4 & 4 & 8 & 5 & 3 & 1255.92 & 3.953657 & NU1 \\
\hline $308 \cdot 36$ & 4.4811 & 0.0520 & 8 & 0 & 8 & 7 & 1 & 7 & $586 \cdot 48$ & 6.998008 & $2 \mathrm{NU} 2$ \\
\hline $308 \cdot 40$ & 0.0189 & 0.0791 & 3 & 1 & 3 & 4 & 4 & 0 & $488 \cdot 13$ & 0.001674 & NUI \\
\hline $308 \cdot 70$ & 29.8740 & 0.0955 & 4 & 2 & 3 & 3 & 1 & 2 & $173 \cdot 36$ & 1.970843 & $2 \mathrm{NU} 2$ \\
\hline 309.02 & 13.4655 & 0.0523 & 8 & 1 & 8 & 7 & 0 & 7 & $586 \cdot 26$ & 7.000291 & $2 N \cup 2$ \\
\hline 309.16 & 0.0149 & 0.0927 & 5 & 2 & 4 & 4 & 1 & 3 & 275.52 & 2.444582 & $2 N \cup 2 *$ \\
\hline 309.82 & 0.0026 & 0.0507 & 9 & 1 & 8 & 8 & 2 & 7 & $2495 \cdot 18$ & 5.987889 & $2 \mathrm{NU} 2 *$ \\
\hline 310.50 & 4.6862 & 0.0498 & 6 & 5 & 2 & 7 & 6 & 1 & $1216 \cdot 20$ & 5.265222 & NU1 \\
\hline 310.51 & 1.5622 & 0.0499 & 6 & 5 & 1 & 7 & 6 & 2 & 1216.20 & 5.265494 & NU1 \\
\hline 3310.78 & 0.0202 & 0.0664 & 6 & 2 & 5 & 6 & 5 & 2 & 888.60 & 0.004423 & NUI \\
\hline 3311.00 & 0.0533 & 0.0747 & 10 & 3 & 7 & 10 & 2 & 8 & 1438.00 & 5.910419 & 2 NU2 \\
\hline 3311.24 & 0.0252 & 0.0756 & 6 & 4 & 3 & 7 & 1 & 6 & 704.22 & 0.023637 & 2 NU2 \\
\hline 3311.35 & 0.0140 & 0.0436 & 9 & 0 & 9 & 8 & 1 & 8 & 744.16 & 8.007087 & $2 N \cup 2 *$ \\
\hline 311.66 & 0.0047 & 0.0437 & 9 & 1 & 9 & 8 & 0 & 8 & 744.09 & 8.007851 & $2 N \cup 2 *$ \\
\hline 312.03 & 6.7403 & 0.0751 & 6 & 3 & 4 & 6 & 2 & 5 & 552.92 & 2.963539 & 2NU2 \\
\hline 312.99 & 0.0059 & 0.0686 & 7 & 4 & 3 & 8 & 5 & 4 & $1255 \cdot 16$ & 4.002077 & NU1* \\
\hline 313.27 & 22.4584 & 0.0961 & 3 & 2 & 1 & 2 & 1 & 2 & 79.48 & 0.925282 & 2 NU2 2 \\
\hline 3.43 & 1.4190 & 0.0790 & 6 & 1 & 6 & 7 & 2 & 5 & $782 \cdot 40$ & 0.182385 & NU1 \\
\hline 313.82 & 0.0174 & 0.0734 & 7 & 1 & 6 & 6 & 2 & 5 & 552.92 & 3.799063 & $2 \mathrm{NU} 2 *$ \\
\hline 4.03 & 0.0376 & 0.0616 & 11 & 3 & 8 & 12 & 4 & 9 & 2124.98 & 4.836759 & NUI \\
\hline 4.51 & 0.0032 & 0.0503 & 8 & 2 & 7 & 8 & 1 & 8 & 744.16 & 1.833129 & $2 \mathrm{NU} 2 *$ \\
\hline 314.51 & 0.0013 & 0.0578 & 10 & 1 & 10 & 10 & 3 & 7 & $1538 \cdot 23$ & 0.002006 & NU3 \\
\hline 3315.07 & 0.4359 & 0.0768 & 8 & 3 & 5 & 9 & 5 & $4^{\circ}$ & 1477.31 & 0.169518 & NU3 \\
\hline $3315 \cdot 12$ & 0.0011 & 0.0686 & 7 & 4 & 3 & 8 & 5 & 4 & $1255 \cdot 16$ & 4.002077 & NU $1 * *$ \\
\hline $3317 \cdot 26$ & 2.9302 & 0.0686 & 7 & 4 & 3 & 8 & 5 & 4 & $1255 \cdot 16$ & 4.002077 & NU1 \\
\hline 3317.69 & 0.1444 & 0.0806 & 4 & 1 & 3 & 5 & 4 & 2 & $610 \cdot 12$ & 0.023494 & NUI \\
\hline 3317.72 & 0.0046 & 0.0614 & 11 & 2 & 9 & 11 & 5 & 6 & 1999.02 & 0.234997 & NU I \\
\hline 3317.96 & 0.0026 & 0.0436 & 9 & 0 & 9 & 8 & 1 & 8 & $744 \cdot 16$ & 8.007087 & $2 \mathrm{NU} 2 * *$ \\
\hline $3318 \cdot 38$ & 0.0605 & 0.0783 & 5 & 0 & 5 & 6 & 4 & 2 & 757.78 & 0.001931 & NU3 \\
\hline $3318 \cdot 53$ & 2.1333 & 0.0859 & 7 & 2 & 5 & 6 & 3 & 4 & 648.97 & 1.512999 & $2 \mathrm{NU} 2$ \\
\hline 3318.77 & 0.5373 & 0.0542 & 8 & 1 & 7 & 8 & 0 & 8 & $7,44.09$ & 1.839778 & $2 \mathrm{NU} 2$ \\
\hline
\end{tabular}




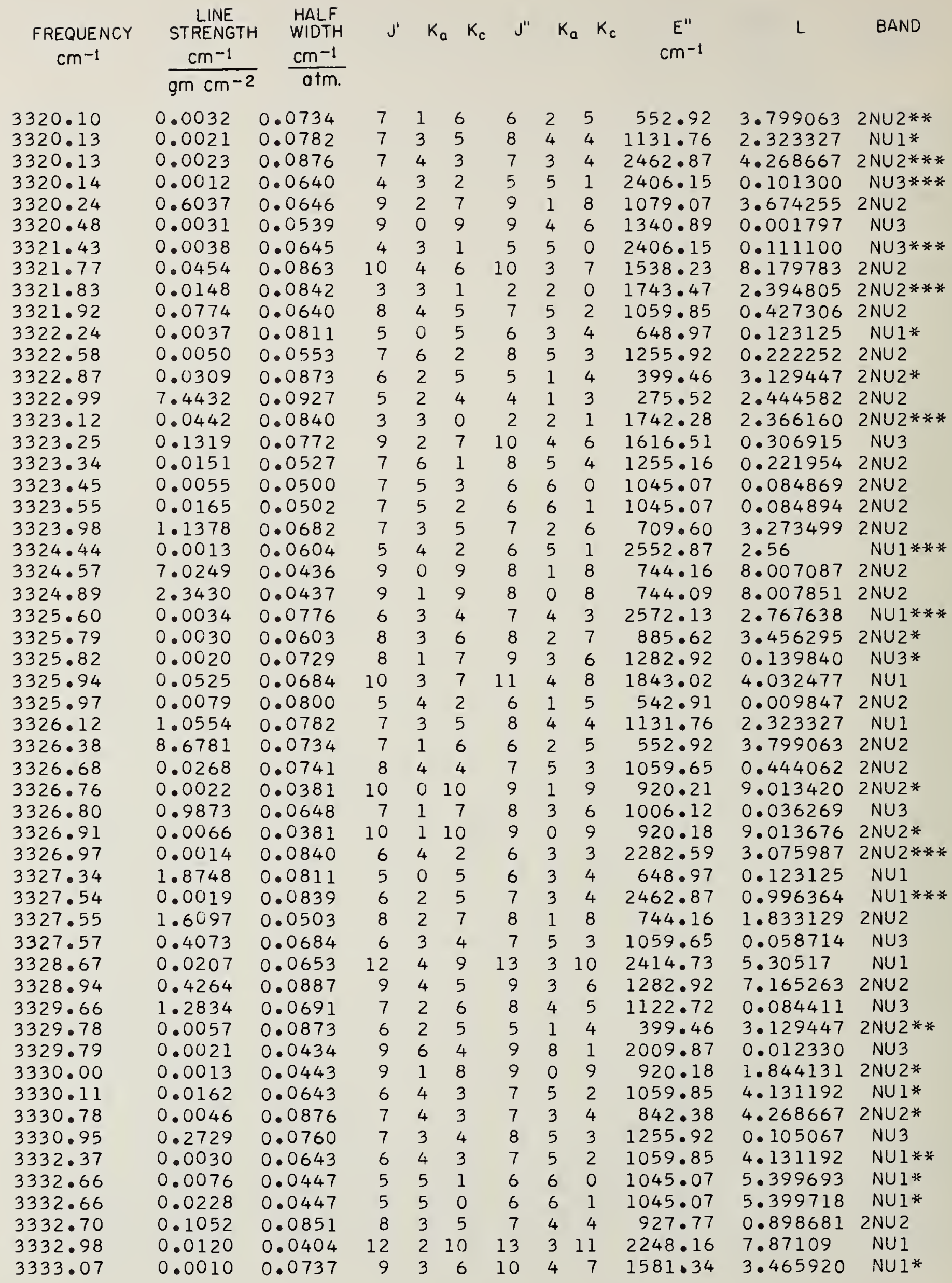




\begin{tabular}{|c|c|c|c|c|c|c|c|c|c|c|c|}
\hline $\begin{array}{c}\text { FREQUENCY } \\
\mathrm{cm}^{-1}\end{array}$ & $\begin{array}{l}\text { LINE } \\
\text { STRENGTH } \\
\frac{\mathrm{cm}^{-1}}{9 \mathrm{~cm} \mathrm{~cm}^{-2}}\end{array}$ & $\begin{array}{l}\text { HALF } \\
\text { WIDTH } \\
\frac{\mathrm{cm}^{-1}}{a+m .}\end{array}$ & $J^{\prime}$ & $K_{0}$ & $K_{c}$ & $J^{\prime \prime}$ & $\mathrm{K}_{0}$ & $K_{c}$ & $\mathrm{Em}^{-1}$ & $L$ & BAND \\
\hline 3333.26 & 0.0182 & 0.032 & 14 & 0 & 14 & 15 & 1 & 15 & $2358 \cdot 44$ & 14.00193 & NU1 \\
\hline 3333.26 & 0.0546 & 0.032 & 14 & 1 & 14 & 15 & 0 & 15 & $2358 \cdot 44$ & 14.00192 & NU1 \\
\hline 3333.31 & 0.0429 & 0.033 & 13 & 1 & 12 & 14 & 2 & 13 & 2327.90 & 11.01049 & NU1 \\
\hline 3333.37 & 0.0143 & 0.033 & 13 & 2 & 12 & 14 & 1 & 13 & 2327.88 & 11.00954 & NU1 \\
\hline 3333.44 & 0.0022 & 0.0417 & 8 & 6 & 2 & 8 & 8 & 1 & 1789.10 & 0.004043 & NU3 \\
\hline 3333.56 & 0.0012 & 0.0381 & 10 & 1 & 10 & 9 & 0 & 9 & 920.18 & 9.013676 & $2 \mathrm{NU} 2 * *$ \\
\hline 3333.75 & 0.0059 & 0.0787 & 5 & 4 & 1 & 5 & 3 & 2 & 2130.50 & 2.066632 & $2 \operatorname{NU} 2 * * * *$ \\
\hline 3334.64 & 8.0867 & 0.0643 & 6 & 4 & 3 & 7 & 5 & 2 & 1059.85 & 4.131192 & NU1 \\
\hline 3334.69 & 0.0014 & 0.0447 & 5 & 5 & 1 & 6 & 6 & 0 & 1045.07 & 5.399693 & NU $1 * *$ \\
\hline 3334.69 & 0.0042 & 0.0447 & 5 & 5 & 0 & 6 & 6 & 1 & 1045.07 & 5.399718 & NU $1 * *$ \\
\hline 3334.80 & 0.0014 & 0.0655 & 5 & 1 & 5 & 5 & 5 & 0 & 742.10 & 0.000013 & NU 3 \\
\hline 3336.02 & 0.0305 & 0.0470 & 12 & 3 & 10 & 13 & 2 & 11 & 2246.98 & 7.82232 & NU1 \\
\hline 3336.18 & 0.3080 & 0.0894 & 8 & 4 & 4 & 8 & 3 & 5 & 1050.15 & 4.85 & 2NU2- \\
\hline 3336.24 & 0.0020 & 0.0720 & 4 & 4 & 0 & 4 & 3 & 1 & 2005.92 & 1.088294 & $2 N \cup 2 * \ddot{x} *$ \\
\hline 3336.69 & 15.4453 & 0.0873 & 6 & 2 & 5 & 5 & 1 & 4 & 399.46 & 3.129447 & $2 \mathrm{~N} \cup 2$ \\
\hline 3336.73 & 3.7934 & 0.0447 & 5 & 5 & 1 & 6 & 6 & 0 & 1045.07 & 5.399693 & NUI \\
\hline 3336.73 & 11.3802 & 0.0447 & 5 & 5 & 0 & 6 & 6 & 1 & 1045.07 & 5.399718 & NUI \\
\hline 3336.86 & 0.5024 & 0.0737 & 9 & 3 & 6 & 10 & 4 & 7 & 1581.34 & 3.465920 & NUI \\
\hline 3337.10 & 0.0690 & 0.0683 & 8 & 0 & 8 & 8 & 3 & 5 & 1050.15 & 0.101 & NUIt \\
\hline 3337.34 & 0.0059 & 0.0689 & 4 & 4 & 1 & 4 & 3 & 2 & 2004.81 & 1.082946 & $2 \mathrm{~N} \cup 2 * * *$ \\
\hline 3337.46 & 0.0065 & 0.0781 & 7 & 2 & 6 & 6 & 1 & 5 & 542.91 & 4.008107 & $2 N \cup 2 *$ \\
\hline 3337.75 & 0.0020 & 0.0719 & 5 & 4 & 2 & 5 & 3 & 3 & 2126.41 & 2.024051 & $2 N \cup 2{ }^{*}{ }^{*} *$ \\
\hline 3337.85 & 0.0054 & 0.0656 & 6 & 4 & 2 & 7 & 5 & 3 & 1059.65 & 4.143594 & NUI* \\
\hline 3337.86 & 0.0041 & 0.0720 & 6 & 4 & 3 & 6 & 3 & 4 & 2271.70 & 2.878858 & $2 \mathrm{NU} 2 * * * *$ \\
\hline 3338.43 & 0.0026 & 0.0733 & 6 & 3 & 3 & 7 & 5 & 2 & 1059.85 & 0.063852 & NU $3 *$ \\
\hline 3339.00 & 1.5020 & 0.0603 & 8 & 3 & 6 & 8 & 2 & 7 & 885.62 & 3.456295 & 2 NU2 2 \\
\hline 3339.08 & 0.0523 & 0.0699 & 6 & 1 & 6 & 6 & 4 & 3 & 756.75 & 0.005871 & NUI \\
\hline 3339.30 & 0.1549 & 0.0784 & 3 & 1 & 2 & 4 & 4 & 1 & 488.10 & 0.004537 & NU1 \\
\hline 3339.36 & 0.0027 & 0.0840 & 6 & 4 & 2 & 6 & 3 & 3 & 661.56 & 3.075987 & $2 N \cup 2 *$ \\
\hline 3340.07 & 1.0982 & 0.0381 & 10 & 0 & 10 & 9 & 1 & 9 & 920.21 & 9.013420 & 2 NU2 \\
\hline 3340.22 & 3.2949 & 0.0381 & 10 & 1 & 10 & 9 & 0 & 9 & 920.18 & 9.013676 & $2 \mathrm{NU} 2$ \\
\hline 3340.30 & 0.0431 & 0.0655 & 11 & 3 & 8 & 11 & 2 & 9 & 1690.70 & 5.642326 & 2NU2 \\
\hline 3340.40 & 0.0034 & 0.0613 & 8 & 1 & 7 & 7 & 2 & 6 & 709.60 & 4.913532 & $2 N \cup 2 *$ \\
\hline 3340.51 & 0.0073 & 0.0970 & 4 & 2 & 2 & 3 & 1 & 3 & $142 \cdot 28$ & 0.610552 & $2 \operatorname{NU} 2 *$ \\
\hline 3341.36 & 0.9587 & 0.0729 & 8 & 1 & 7 & 9 & 3 & 6 & 1282.92 & 0.139840 & NU 3 \\
\hline 3342.03 & 0.0046 & 0.0558 & 9 & 1 & 8 & 9 & 5 & 5 & 1474.99 & 0.005298 & NU3 \\
\hline $3342 \cdot 28$ & 2.7098 & 0.0656 & 6 & 4 & 2 & 7 & 5 & 3 & 1059.65 & 4.143594 & NU1 \\
\hline 3342.38 & 0.0047 & 0.0962 & 5 & 2 & 3 & 4 & 1 & 4 & 1821.61 & 0.386397 & $2 \mathrm{NU} 2 * * *$ \\
\hline 3342.93 & 0.6747 & 0.0443 & 9 & 1 & 8 & 9 & 0 & 9 & 92 & 1.844131 & $2 \mathrm{~N} \cup 2$ \\
\hline 3343.27 & 0.0296 & 0.0868 & 4 & 3 & 2 & 3 & 2 & 1 & 1819.34 & 2.320352 & $2 N \cup 2 * * *$ \\
\hline $3344 \cdot 28$ & 0.0056 & 0.0649 & 10 & 2 & 8 & 10 & 5 & 5 & 1724.72 & 0.238204 & NU1 \\
\hline 3344.35 & 0.0012 & 0.0781 & 7 & 2 & 6 & 6 & 1 & 5 & 542.91 & 4.008107 & $2 \mathrm{NU} 2 * *$ \\
\hline 3344.42 & 0.0010 & 0.0775 & 8 & 3 & 5 & 9 & 4 & 6 & 1340.89 & 3.141455 & NU1* \\
\hline 3345.08 & 0.0136 & 0.0620 & 9 & 4 & 6 & 8 & 5 & 3 & 1255.92 & 0.597432 & 2 NU2 2 \\
\hline 3345.24 & 0.0119 & 0.0787 & 5 & 4 & 1 & 5 & 3 & 2 & 508.81 & 2.066632 & $2 \operatorname{Nu} 2 *$ \\
\hline 3345.45 & 0.0104 & 0.0679 & 5 & 3 & 3 & 6 & 5 & 2 & 888.60 & 0.105000 & NU3* \\
\hline 3345.96 & 2.3073 & 0.0876 & 7 & 4 & 3 & 7 & 3 & 4 & 842.38 & 4.268667 & 2 NU2 2 \\
\hline 3347.25 & 0.0155 & 0.0515 & 6 & 6 & 1 & 7 & 5 & 2 & 1059.85 & 0.084894 & $2 \mathrm{NU} 2$ \\
\hline 3347.29 & 0.0099 & 0.0834 & 4 & 4 & 1 & 5 & 1 & 4 & 399.46 & 0.001994 & $2 \mathrm{NU} 2$ \\
\hline 3347.70 & 0.0052 & 0.0497 & 6 & 6 & 0 & 7 & 5 & 3 & 1059.65 & 0.084869 & $2 \mathrm{NU} 2$ \\
\hline 3347.96 & 0.2250 & 0.0430 & 9 & 2 & 8 & 9 & 1 & 9 & 920.21 & 1.842036 & 2 NU2 \\
\hline 3348.15 & 0.0039 & 0.0720 & 4 & 4 & 0 & 4 & 3 & 1 & 383.85 & 1.088294 & $2 \mathrm{NU} 2 *$ \\
\hline 3348.20 & 1.3374 & 0.0733 & 6 & 3 & 3 & 7 & 5 & 2 & 1059.85 & 0.063852 & NU3 \\
\hline 3348.39 & 0.5063 & 0.0775 & 8 & 3 & 5 & 9 & 4 & 6 & 1340.89 & 3.141455 & NU 1 \\
\hline
\end{tabular}




\begin{tabular}{|c|c|c|c|c|c|c|c|c|c|c|c|}
\hline $\begin{array}{l}\text { FREQUENCY } \\
\mathrm{cm}^{-1}\end{array}$ & $\begin{array}{l}\text { LINE } \\
\text { STRENGTH } \\
\frac{\mathrm{cm}^{-1}}{g m \mathrm{~cm}^{-2}}\end{array}$ & $\begin{array}{l}\text { HALF } \\
\text { WIDTH } \\
\mathrm{cm}^{-1} \\
\text { atm. }\end{array}$ & $J^{\prime}$ & $k_{c}$ & $\mathrm{~K}_{\mathrm{c}}$ & J" & $k$ & $\mathrm{~K}_{\mathrm{c}}$ & $\begin{array}{c}E^{\prime \prime} \\
\mathrm{cm}^{-1}\end{array}$ & L & BAND \\
\hline 3348.56 & 0.0692 & 0.0534 & 10 & 2 & 8 & 10 & 1 & 9 & 1293.04 & 3.646815 & $2 N \cup 2$ \\
\hline 3348.90 & 0.0186 & 0.0516 & 8 & 5 & 4 & 7 & 6 & 1 & $1216 \cdot 20$ & 0.221954 & 2 NU2 \\
\hline 3349.47 & 0.0095 & 0.0861 & 4 & 3 & 1 & 3 & 2 & 2 & 1813.81 & 2.164905 & $2 \operatorname{N} \cup 2 * * *$ \\
\hline 3349.53 & 0.0073 & 0.0687 & 5 & 3 & 2 & 6 & 5 & 1 & 888.63 & 0.221000 & NU $3 *$ \\
\hline 3349.54 & 0.0117 & 0.0689 & 4 & 4 & 1 & 4 & 3 & 2 & $382 \cdot 52$ & 1.082946 & $2 N \cup 2 *$ \\
\hline 3349.79 & 0.0010 & 0.0656 & 8 & 4 & 5 & 8 & 3 & 6 & $2630 \cdot 21$ & 4.264036 & $2 N \cup 2 * * *$ \\
\hline 3349.80 & 0.0062 & 0.0542 & 8 & 5 & 3 & 7 & 6 & 2 & $1216 \cdot 20$ & 0.222252 & 2NU2 \\
\hline 3349.88 & 0.4189 & 0.0692 & 7 & 0 & 7 & 8 & 2 & 6 & 982.91 & 0.040852 & NU3 3 \\
\hline 3350.01 & 0.0116 & 0.0839 & 6 & 2 & 5 & 7 & 3 & 4 & $842 \cdot 38$ & 0.996364 & NU 1* \\
\hline 3350.09 & 0.0296 & 0.0842 & 3 & 3 & 1 & 2 & 2 & 0 & 136.17 & 2.394805 & $2 N \cup 2$ * \\
\hline 3350.22 & 0.0040 & 0.0719 & 5 & 4 & 2 & 5 & 3 & 3 & 504.00 & 2.024051 & $2 N \cup 2 *$ \\
\hline 3350.37 & 0.0113 & 0.0545 & 4 & 4 & 1 & 5 & 5 & 0 & $2406 \cdot 15$ & 3.52 & NUI $* \dot{*} * *$ \\
\hline 3350.40 & 0.0039 & 0.0546 & 4 & 4 & 0 & 5 & 5 & 1 & $2406 \cdot 15$ & 3.61 & NU $1 * * *$ \\
\hline 3350.58 & 0.0020 & 0.0679 & 5 & 3 & 3 & 6 & 5 & 2 & 888.60 & 0.105000 & NU $3 * *$ \\
\hline 3351.24 & 3.2323 & 0.0781 & 7 & 2 & 6 & 6 & 1 & 5 & 542.91 & 4.008107 & $2 \mathrm{NU} 2$ \\
\hline 3351.54 & 0.0884 & 0.0840 & 3 & 3 & 0 & 2 & 2 & 1 & 134.91 & 2.366160 & $2 N \cup 2 *$ \\
\hline 3351.80 & 0.0029 & 0.0812 & 8 & 2 & 6 & 9 & 4 & 5 & $1360 \cdot 28$ & 0.309042 & NU3* \\
\hline 3351.93 & 0.0082 & 0.0720 & 6 & 4 & 3 & 6 & 3 & 4 & 648.97 & 2.878858 & $2 \operatorname{N} \cup 2 *$ \\
\hline 3352.69 & 0.0022 & 0.0787 & 5 & 4 & 1 & 5 & 3 & 2 & 508.81 & 2.066632 & $2 \mathrm{NU} 2 * *$ \\
\hline 3353.26 & 1.7229 & 0.0613 & 8 & 1 & 7 & 7 & 2 & 6 & 709.60 & 4.913532 & $2 N \cup 2$ \\
\hline 3353.65 & 3.6532 & 0.0970 & 4 & 2 & 2 & 3 & 1 & 3 & $142 \cdot 28$ & 0.610552 & $2 N \cup 2$ \\
\hline 3353.70 & 0.0178 & 0.0703 & 5 & 2 & 3 & 5 & 5 & 0 & $742 \cdot 10$ & 0.001850 & NUI \\
\hline 3353.84 & 0.0108 & 0.0646 & 8 & 2 & 7 & 7 & 1 & 6 & 704.22 & 4.996649 & $2 \operatorname{N} \cup 2 *$ \\
\hline 3354.19 & 0.1379 & 0.0465 & 11 & 2 & 9 & 12 & 3 & 10 & 1962.55 & 7.003088 & NUI \\
\hline 3354.44 & 1.3721 & 0.0840 & 6 & 4 & 2 & 6 & 3 & 3 & 661.56 & 3.075987 & $2 \mathrm{NU} 2$ \\
\hline 3355.25 & 1.3923 & 0.0349 & 11 & 0 & 11 & 10 & 1 & 10 & 1114.56 & 10.018153 & $2 \mathrm{~N} \cup 2$ \\
\hline 3355.31 & 0.4641 & 0.0349 & 11 & 1 & 11 & 10 & 0 & 10 & 1114.55 & 10.018239 & 2 NU 2 \\
\hline 3355.36 & 0.0015 & 0.0696 & 7 & 4 & 4 & 7 & 3 & 5 & 816.72 & 3.637027 & $2 N \cup 2$ ̌ㅜ \\
\hline 3355.44 & 0.1535 & 0.032 & 13 & 0 & 13 & 14 & 1 & 14 & 2073.66 & 12.99957 & NUI \\
\hline 3355.72 & 5.1842 & 0.0679 & 5 & 3 & 3 & 6 & 5 & 2 & 888.60 & 0.105000 & $\mathrm{NU}_{3+}$ \\
\hline 3355.75 & 0.0511 & 0.0665 & 9 & 1 & 8 & 9 & 4 & 5 & $1360 \cdot 28$ & 0.116860 & NUI \\
\hline 3355.92 & 0.1955 & 0.0529 & 9 & 3 & 7 & 9 & 2 & 8 & 1080.38 & 3.554957 & 2 NU 2 \\
\hline 3356.21 & 0.0208 & 0.0776 & 6 & 3 & 4 & 7 & 4 & 3 & $931 \cdot 22$ & 2.767638 & NUI \\
\hline 3356.27 & 0.1577 & 0.0349 & 12 & 2 & 11 & 13 & 1 & 12 & 2042.33 & 9.99731 & NUI \\
\hline 3356.33 & 0.0045 & 0.0585 & 11 & 2 & 10 & 11 & 4 & 7 & 1899.06 & 0.013267 & NU3 \\
\hline 3356.56 & 0.4977 & 0.0793 & 8 & 2 & 6 & 7 & 3 & 5 & 816.72 & 2.422109 & $2 N U 2$ \\
\hline 3356.70 & 0.0461 & 0.0774 & 9 & 4 & 5 & 8 & 5 & 4 & $1255 \cdot 16$ & 0.669979 & $2 \mathrm{NU} 2$ \\
\hline 3357.00 & 5.8196 & 0.0839 & 6 & 2 & 5 & 7 & 3 & 4 & $842 \cdot 38$ & 0.996364 & NU 1 \\
\hline 3358.10 & 0.0087 & 0.0796 & 7 & 3 & 4 & 8 & 4 & 5 & 1122.72 & 3.022183 & NUI* \\
\hline 3358.62 & 0.0164 & 0.0840 & 3 & 3 & 0 & 2 & 2 & 1 & 134.91 & 2.366160 & $2 \mathrm{NU} 2 * *$ \\
\hline 3358.94 & 0.0038 & 0.0776 & 6 & 3 & 4 & 7 & 4 & 3 & $931 \cdot 22$ & 2.767638 & NU $1 * \ddot{*}$ \\
\hline 3359.55 & 3.6412 & 0.0697 & 5 & 3 & 2 & 6 & 5 & 1 & 888.63 & 0.221000 & $\mathrm{NU}_{3}+$ \\
\hline 3359.70 & 0.0172 & 0.0735 & 11 & 4 & 8 & 12 & 3 & 9 & 2105.90 & 4.355455 & NU1 \\
\hline 3360.14 & 5.9457 & 0.0787 & 5 & 4 & 1 & 5 & 3 & 2 & 508.81 & 2.066632 & $2 \mathrm{NU} 2$ \\
\hline 3360.39 & 0.0441 & 0.0688 & 9 & 2 & 7 & 9 & 5 & 4 & 1477.31 & 0.180415 & NU 1 \\
\hline 3360.66 & 0.0020 & 0.0646 & 8 & 2 & 7 & 7 & 1 & 6 & $704 \cdot 22$ & 4.996649 & $2 \operatorname{NU} 2 * *$ \\
\hline 3360.68 & 0.0550 & 0.0557 & 11 & 3 & 9 & 12 & 2 & 10 & 1960.22 & 6.964699 & NUI \\
\hline 3360.92 & 0.0165 & 0.0729 & 6 & 2 & 4 & 6 & 5 & 1 & 888.63 & $\cdot 0.010677$ & NUI \\
\hline 3361.68 & 10.3768 & 0.0776 & 6 & 3 & 4 & 7 & 4 & 3 & $931 \cdot 22$ & 2.767638 & NUI \\
\hline 3362.36 & 4.3544 & 0.0796 & 7 & 3 & 4 & 8 & 4 & 5 & 1122.72 & 3.022183 & NU 1 \\
\hline 3362.74 & 0.0106 & 0.0771 & 5 & 3 & 2 & 6 & 4 & 3 & 2398.39 & 3.53 & NU1*** \\
\hline 3362.91 & 1.9510 & 0.0720 & 4 & 4 & 0 & 4 & 3 & 1 & 383.85 & 1.088294 & 2 NU2 \\
\hline 3363.09 & 0.0164 & 0.0600 & 10 & 4 & 7 & 9 & 5 & 4 & 1477.31 & 0.720783 & 2NU2 \\
\hline 3363.10 & 0.0347 & 0.0608 & 5 & 4 & 1 & 6 & 5 & 2 & 888.60 & 3.63 & NUI* \\
\hline
\end{tabular}




\begin{tabular}{|c|c|c|c|c|c|c|c|c|c|c|c|}
\hline $\begin{array}{c}\text { FREQUENCY } \\
\mathrm{cm}^{-1}\end{array}$ & $\begin{array}{l}\text { LINE } \\
\text { STRENGTH } \\
\frac{\mathrm{cm}^{-1}}{\mathrm{gm} \mathrm{cm}^{-2}}\end{array}$ & $\begin{array}{l}\text { HALF } \\
\text { WIDTH } \\
\frac{\mathrm{cm}^{-1}}{\mathrm{~atm} .}\end{array}$ & $J^{\prime}$ & $K_{0}$ & $\mathrm{~K}_{\mathrm{c}}$ & J" & $K_{a}$ & $K_{c}$ & $\begin{array}{c}E^{\prime \prime} \\
\mathrm{cm}^{-1}\end{array}$ & $L$ & BAND \\
\hline 3363.15 & 0.0028 & 0.0695 & 8 & 5 & 4 & 9 & 2 & 7 & 1201.95 & 0.030877 & $2 N \cup 2$ \\
\hline 3363.80 & 0.0075 & 0.032 & 14 & 1 & 13 & 14 & 2 & 13 & 2327.90 & 1.90878 & NU? \\
\hline 3363.82 & 0.0025 & 0.032 & 14 & 0 & 14 & 14 & 1 & 13 & 2327.88 & 1.90883 & NUI \\
\hline 3363.85 & 0.3093 & 0.0907 & 3 & 3 & 0 & 3 & 0 & 3 & 136.77 & 0.016715 & 2 NU2 \\
\hline 3364.23 & 5.8655 & 0.0689 & 4 & 4 & 1 & 4 & 3 & 2 & 382.52 & 1.082946 & 2NU? \\
\hline 3364.27 & 0.2108 & 0.0803 & 4 & 0 & 4 & 5 & 4 & 1 & 610.35 & 0.001060 & NU3 \\
\hline 3364.31 & 14.8187 & 0.0842 & 3 & 3 & 1 & 2 & 2 & 0 & 136.17 & 2.394805 & 2NU2 \\
\hline 3364.77 & 1.4440 & 0.0812 & 8 & 2 & 6 & 9 & 4 & 5 & 1360.28 & 0.309042 & NU3 \\
\hline 3364.88 & 1.9911 & 0.0719 & 5 & 4 & 2 & 5 & 3 & 3 & 504.00 & 2.024051 & 2NU2 \\
\hline 3365.36 & 0.0064 & 0.0608 & 5 & 4 & 1 & 6 & 5 & 2 & 888.60 & 3.63 & NUI*** \\
\hline 3365.45 & 0.0306 & 0.0590 & 8 & 0 & 8 & 8 & 4 & 5 & 1122.72 & 0.001991 & NU3 \\
\hline 3365.71 & 44.2161 & 0.0840 & 3 & 3 & 0 & 2 & 2 & ]. & 134.91 & 2.366160 & 2NU? \\
\hline $3366 \cdot 12$ & 0.0020 & 0.0874 & 4 & 0 & 4 & 5 & 3 & 3 & 504.00 & 0.095779 & NU I* \\
\hline 3366.15 & 0.0859 & 0.0382 & 10 & 1 & 9 & 10 & 0 & 10 & 1114.55 & 1.848326 & 2NU2 \\
\hline $3366 \cdot 23$ & 0.0754 & 0.0734 & 7 & 2 & 5 & 7 & 5 & 2 & 1059.85 & 0.038149 & NUI \\
\hline 3366.49 & 4.1179 & 0.0720 & 6 & 4 & 3 & 6 & 3 & 4 & 648.97 & 2.878858 & $2 N \cup 2$ \\
\hline 3366.99 & 0.0242 & 0.0712 & 8 & 2 & 6 & 8 & 5 & 3 & 1255 & 0.098024 & NU1 \\
\hline 3367.22 & 0.0021 & 0.0840 & 6 & 2 & 4 & 7 & 4 & 3 & 2572 & 0.157817 & NU $3 * * * *$ \\
\hline $3367 \cdot 34$ & 0.0223 & 0.0743 & 5 & 1 & 5 & 5 & 4 & 2 & 610 & 0.003573 & NU1 \\
\hline 3367.49 & 5.4234 & 0.0646 & 8 & 2 & 7 & 7 & 1 & 6 & $704 \cdot 22$ & 4.996649 & $2 N \cup 2$ \\
\hline 3367.62 & 17.3426 & 0.0608 & 5 & 4 & 1 & 6 & 5 & 2 & .60 & 3.63 & NU1- \\
\hline 3368.72 & 0.2578 & 0.0378 & 10 & 2 & 9 & 10 & 1 & 10 & 1114.56 & 1.847651 & 2 NU2 \\
\hline 3368.80 & 2.3502 & 0.0744 & 6 & 2 & 5 & 7 & 4 & 4 & 927 & 0.173000 & NU3+ \\
\hline 3369.10 & 3.9693 & 0.0604 & 5 & 4 & 2 & 6 & 5 & 1 & .63 & 2.56 & NUI- \\
\hline 3369.12 & 0.0311 & 0.0608 & 8 & 1 & 7 & 8 & 5 & 4 & 1255 & 0.003922 & NU3 \\
\hline 3369.74 & 0.7503 & 0.0696 & 7 & 4 & 4 & 7 & 3 & 5 & 816.72 & 3.637027 & 2 NU2 \\
\hline 3369.91 & 0.1768 & 0.0332 & 12 & 0 & 12 & 11 & 1 & 11 & $1327 \cdot 14$ & 11.021861 & $2 \mathrm{NU} 2$ \\
\hline 3369.93 & 0.5304 & 0.0332 & 12 & 1 & 12 & 11 & 0 & 11 & $1327 \cdot 14$ & 11.021891 & $2 \mathrm{NU} 2$ \\
\hline 3370.98 & 1.0166 & 0.0874 & 4 & 0 & 4 & 5 & 3 & 3 & 504.00 & 0.095779 & NUI \\
\hline $3371 \cdot 36$ & 0.0593 & 0.0868 & 4 & 3 & 2 & 3 & 2 & 1 & 212.15 & 2.320352 & $2 N \cup 2 *$ \\
\hline 3371.69 & 0.0018 & 0.0539 & 9 & 2 & 8 & 8 & 1 & 7 & 882.93 & 6.019178 & $2 \mathrm{~N} \cup 2$ \\
\hline 3371.88 & 0.1900 & 0.0820 & 9 & 3 & 6 & 8 & 4 & 5 & 1122.72 & 1.416234 & 2NU2 \\
\hline 3372.81 & 0.0070 & 0.0640 & 4 & 3 & 2 & 5 & 5 & 1 & $742 \cdot 10$ & 0.101300 & NU3* \\
\hline 3373.15 & 0.0023 & 0.0864 & 5 & 2 & 4 & 6 & 3 & 3 & 2282 & 1.411171 & NU] $* * * *$ \\
\hline 3373.97 & 0.1651 & 0.0544 & 10 & 2 & 8 & 11 & 3 & 9 & 1695 & 5.954956 & NU 1 \\
\hline 3374.18 & 0.0230 & 0.0645 & 4 & 3 & 1 & 5 & 5 & 0 & $742 \cdot 10$ & 0.111100 & $N \cup 3 *$ \\
\hline $3374 \cdot 32$ & 0.0041 & 0.0529 & 9 & 5 & 5 & 8 & 6 & 2 & 1411.68 & 0.391127 & 2NU2 \\
\hline 3374.34 & 0.2056 & 0.0458 & 10 & 3 & 8 & 10 & 2 & 9 & 1293 & 3.605841 & $2 N \cup 2$ \\
\hline 3374.69 & 1.6486 & 0.0868 & 5 & 1 & 5 & 6 & 2 & 4 & 602 & 0.254241 & NU1 \\
\hline 3375.35 & 0.0124 & 0.0586 & 9 & 5 & 4 & 8 & 6 & 3 & 1411 & 0.393126 & $2 N \cup 2$ \\
\hline 3375.65 & 1.0261 & 0.0656 & 8 & 4 & 5 & 8 & 3 & 6 & 12 & 4.264036 & $2 N \cup 2$ \\
\hline 3375.68 & 0.0098 & 0.0896 & 6 & 3 & 4 & 5 & 2 & 3 & 2053 & 2.452523 & $2 N \cup 2 * * *$ \\
\hline 3376.57 & 0.0032 & 0.0515 & 11 & 5 & 7 & 11 & 7 & 4 & 2321.94 & 0.077402 & NU3 \\
\hline 3376.64 & 0.1926 & 0.0892 & 4 & 3 & 1 & 4 & 0 & 4 & 222.06 & 0.047652 & $2 N \cup 2$ \\
\hline 3377.09 & 0.0021 & 0.0459 & 8 & 7 & 2 & 9 & 6 & 3 & 1631 & 0.197898 & $2 N \cup 2$ \\
\hline 3377.49 & 2.6321 & 0.0507 & 9 & 1 & 8 & 8 & 2 & 7 & .885 .62 & 5.987889 & $2 \mathrm{NU} 2$ \\
\hline 3377.94 & 0.0013 & 0.0640 & 4 & 3 & 2 & 5 & 5 & 1 & $742 \cdot 10$ & 0.101300 & NU3** \\
\hline 3378.03 & 0.5830 & 0.0324 & 12 & 1 & 12 & 13 & 0 & 13 & $1806 \cdot 78$ & 11.99672 & NUI \\
\hline $3378 \cdot 31$ & 0.0190 & 0.0861 & 4 & 3 & 1 & 3 & 2 & 2 & $206 \cdot 30$ & 2.164905 & $2 N \cup 2 *$ \\
\hline 3378.41 & 0.1905 & 0.0325 & 12 & 0 & 12 & 13 & 1 & 13 & $1806 \cdot 78$ & 11.99676 & NU 1 \\
\hline 3378.47 & 0.0217 & 0.0628 & 9 & 1 & 9 & 9 & 3 & 6 & 1282.92 & 0.003124 & NU3 \\
\hline 3378.51 & 0.0153 & 0.0884 & 5 & 3 & 2 & 4 & 2 & 3 & 1907.99 & 1.841317 & $2 \mathrm{NU} 2 * * * *$ \\
\hline 3378.52 & 0.0110 & 0.0868 & 4 & 3 & 2 & 3 & 2 & 1 & 212.15 & 2.320352 & $2 N \cup 2 * * *$ \\
\hline $3378 \cdot 54$ & 0.0015 & 0.0794 & 6 & 0 & 6 & 7 & 2 & 5 & 2392.58 & 0.051494 & NU $3 * * * *$ \\
\hline
\end{tabular}




\begin{tabular}{|c|c|c|c|c|c|c|c|c|c|c|c|}
\hline $\begin{array}{c}\text { FREQUENCY } \\
\mathrm{cm}^{-1}\end{array}$ & $\begin{array}{l}\text { LINE } \\
\text { STRENGTH } \\
\frac{\mathrm{cm}^{-1}}{\mathrm{gm} \mathrm{cm}^{-2}}\end{array}$ & $\begin{array}{l}\text { HALF } \\
\text { WIDTH } \\
\frac{\mathrm{cm}^{-1}}{\text { atm. }}\end{array}$ & J' & $K_{c}$ & $K_{c}$ & ل" & $\mathrm{K}_{0}$ & $\mathrm{~K}_{\mathrm{c}}$ & $\mathrm{Em}^{-1}$ & $\mathrm{~L}$ & BAND \\
\hline 3378.79 & 0.5041 & 0.0361 & 11 & 1 & 10 & 12 & 2 & 11 & 1774.74 & 9.078065 & NUI \\
\hline 3379.10 & 0.1680 & 0.0379 & 11 & 2 & 10 & 12 & 1 & 11 & 1774.60 & 9.076485 & NU1 \\
\hline 3379.12 & 0.9184 & 0.0731 & 6 & 1 & 6 & 7 & 3 & 5 & 816.72 & 0.038676 & NU3 \\
\hline 3379.27 & 0.0043 & 0.0645 & 4 & 3 & 1 & 5 & 5 & 0 & $742 \cdot 10$ & 0.111100 & NU $3 * * *$ \\
\hline 3379.52 & 0.0026 & 0.0424 & 8 & 6 & 3 & 7 & 7 & 0 & 1394.86 & 0.074321 & $2 \mathrm{NU} 2$ \\
\hline 3380.41 & 2.7376 & 0.0790 & 6 & 3 & 3 & 7 & 4 & 4 & 927.77 & $2 \cdot 14$ & NU1- \\
\hline 3381.13 & 0.0659 & 0.0442 & 11 & 2 & 9 & 11 & 1 & 10 & 1524.87 & 3.646421 & 2NU2 \\
\hline $3381 \cdot 36$ & 0.0017 & 0.0698 & 9 & 2 & 7 & 8 & 3 & 6 & $1006 \cdot 12$ & 3.529968 & 2 NU $2 *$ \\
\hline 3381.67 & 0.0068 & 0.0734 & 4 & 3 & 1 & 5 & 4 & 2 & 2251.71 & 3.270203 & NUI $* * * *$ \\
\hline 3381.87 & 0.0022 & 0.0804 & 5 & 1 & 5 & 6 & 3 & 4 & 2271.70 & 0.038984 & NU $3 * * *$ \\
\hline 3382.87 & 0.0151 & 0.0516 & 9 & 5 & 5 & 9 & 7 & 2 & 1810.63 & 0.029706 & NU3 \\
\hline 3383.07 & 3.4956 & 0.0640 & 4 & 3 & 2 & 5 & 5 & $I$ & 742.10 & 0.101300 & NU3+ \\
\hline 3383.14 & 0.0204 & 0.0733 & 4 & 3 & 2 & 5 & 4 & 1 & 2251.86 & 3.250024 & NUI**** \\
\hline 3383.74 & 0.0605 & 0.0324 & 13 & 1 & 13 & 12 & 0 & 12 & 1557.91 & 11.99676 & 2 NU2 \\
\hline 3383.74 & 0.1814 & 0.0324 & 13 & 0 & 13 & 12 & 1 & 12 & 1557.91 & 11.99672 & 2 NU 2 \\
\hline 3383.87 & 0.0026 & 0.0804 & 7 & 1 & 6 & 8 & 3 & 5 & $1050 \cdot 15$ & 0.180748 & NU 3* \\
\hline 3384.37 & 11.5062 & 0.0645 & 4 & 3 & 1 & 5 & 5 & 0 & $742 \cdot 10$ & 0.111100 & NU3+ \\
\hline 3384.73 & 0.1328 & 0.0600 & 9 & 4 & 6 & 9 & 3 & 7 & $1216 \cdot 27$ & 4.734521 & $2 \mathrm{NU} 2$ \\
\hline 3385.01 & 0.0074 & 0.0785 & 10 & 4 & 6 & 9 & 5 & 5 & 1474.99 & 0.959644 & $2 \mathrm{NU} 2$ \\
\hline $3385 \cdot 24$ & 0.8958 & 0.0539 & 9 & 2 & 8 & 8 & 1 & 7 & 882.93 & 6.019178 & $2 \mathrm{NU} 2$ \\
\hline 3385.27 & 0.0071 & 0.0498 & 8 & 5 & 4 & 8 & 7 & 1 & 1590.74 & 0.014493 & NU3 \\
\hline 3385.35 & 0.0035 & 0.0861 & 4 & 3 & 1 & 3 & 2 & 2 & 206.30 & 2.164905 & $2 \mathrm{NU} 2 * *$ \\
\hline 3385.44 & 0.4891 & 0.0655 & 10 & 3 & 8 & 11 & 2 & 9 & 1690.70 & 5.859437 & NU1. \\
\hline 3385.69 & 29.6440 & 0.0868 & 4 & 3 & 2 & 3 & 2 & 1 & $212 \cdot 15$ & 2.320352 & $2 \mathrm{NU} 2$ \\
\hline 3385.98 & 0.0051 & 0.0538 & 9 & 5 & 4 & 9 & 7 & 3 & 1810.63 & 0.029861 & NU3 \\
\hline 3386.30 & 0.0215 & 0.0509 & 8 & 5 & 3 & 8 & 7 & 2 & 1590.74 & 0.014513 & NU3 \\
\hline 3386.62 & 0.0101 & 0.0325 & 13 & 1 & 13 & 13 & 2 & 12 & 2042.38 & 1.90640 & NUI \\
\hline 3386.67 & 0.0303 & 0.0327 & 13 & 0 & 13 & 13 & 1 & 12 & 2042.33 & 1.90653 & NUI \\
\hline 3387.26 & 0.0896 & 0.0348 & 11 & 1 & 10 & I I & 0 & 11 & $1327 \cdot 14$ & 1.851910 & $2 \mathrm{NU}_{2}$ \\
\hline $3387 \cdot 36$ & 0.0184 & 0.0769 & 5 & 3 & 3 & 6 & 4 & 2 & 757.78 & 3.066224 & NUI* \\
\hline 3387.72 & 0.0066 & 0.0563 & 10 & 5 & 5 & 10 & 7 & 4 & 2054.40 & 0.051622 & NU3 \\
\hline 3387.81 & 0.0189 & 0.0476 & 7 & 5 & 3 & 7 & 7 & 0 & 1394.86 & 0.004734 & $\mathrm{NU} 3$ \\
\hline 3388.05 & 0.0063 & 0.0479 & 7 & 5 & 2 & 7 & 7 & 1 & 1394.86 & 0.004736 & NU3 \\
\hline 3388.46 & 0.0299 & 0.0348 & 11 & 2 & 10 & 11 & 1 & 11 & $1327 \cdot 14$ & 1.851690 & $2 \mathrm{NU} 2$ \\
\hline 3389.17 & 0.0028 & 0.0795 & 7 & 2 & 5 & 8 & 3 & 6 & $2630 \cdot 21$ & 3.262769 & NU $1 * * * *$ \\
\hline 3389.18 & 0.0119 & 0.0892 & 5 & 3 & 3 & 4 & 2 & 2 & 315.79 & 2.324407 & $2 N \cup 2^{*}$ \\
\hline 3389.23 & 0.0093 & 0.0962 & 5 & 2 & 3 & 4 & 1 & 4 & 224.83 & 0.386397 & $2 \mathrm{NU} 2 *$ \\
\hline 3389.31 & 0.0153 & 0.0656 & 7 & 1 & 6 & 7 & 5 & 3 & 1059.65 & 0.002162 & NU3 \\
\hline 3389.46 & 0.0424 & 0.0762 & 4 & I & 4 & 4 & 4 & 1 & $488 \cdot 10$ & 0.001222 & NU1 \\
\hline 3389.87 & 0.1961 & 0.0741 & 7 & 0 & 7 & 7 & 3 & 4 & $842 \cdot 38$ & 0.033256 & NU 1 \\
\hline 3389.96 & 0.0034 & 0.0769 & 5 & 3 & 3 & 6 & 4 & 2 & 757.78 & 3.066224 & NUI** \\
\hline 3390.68 & 0.0024 & 0.0444 & 10 & 2 & 9 & 9 & 1 & 8 & 1079.07 & 7.043661 & $2 \mathrm{NU} 2 *$ \\
\hline 3391.54 & 1.4140 & 0.0637 & 9 & 2 & 7 & 10 & 3 & 8 & $1446 \cdot 16$ & 4.932263 & NUI \\
\hline 3392.09 & 0.0677 & 0.0709 & 8 & 1 & 7 & 8 & 4 & 4 & 1131.76 & 0.146118 & NU1 \\
\hline 3392.40 & 9.5122 & 0.0861 & 4 & 3 & 1 & 3 & 2 & 2 & $206 \cdot 30$ & 2.164905 & $2 \mathrm{NU} 2$ \\
\hline 3392.57 & 9.2083 & 0.0769 & 5 & 3 & 3 & 6 & 4 & 2 & 757.78 & 3.066224 & NUI \\
\hline 3392.70 & 11.7236 & 0.0546 & 4 & 4 & 0 & 5 & 5 & 1 & 742.10 & 3.61 & NUI- \\
\hline 3392.95 & 34.2967 & 0.0545 & 4 & 4 & 1 & 5 & 5 & 0 & 742.10 & 3.52 & NU1- \\
\hline 3393.19 & 0.8539 & 0.0698 & 9 & 2 & 7 & 8 & 3 & 6 & 1006.12 & 3.529968 & $2 \mathrm{NU} 2$ \\
\hline 3394.24 & 0.1346 & 0.0536 & 10 & 4 & 7 & 10 & 3 & 8 & $1446 \cdot 16$ & 5.051335 & $2 N \cup 2$ \\
\hline 3394.54 & 0.1520 & 0.0811 & 10 & 4 & 7 & 11 & 3 & 8 & $1813 \cdot 26$ & 3.178818 & NU1 \\
\hline 3394.87 & 0.0220 & 0.0406 & 11 & 3 & 9 & 11 & 2 & 10 & 1525.13 & 3.632655 & $2 \mathrm{NU} 2$ \\
\hline 3395.66 & C.0017 & 0.0962 & 5 & 2 & 3 & 4 & 1 & 4 & $224 \cdot 83$ & 0.386397 & $2 \mathrm{NU} 2 * *$ \\
\hline 3395.88 & 0.0052 & 0.0793 & 11 & 5 & 7 & 12 & 4 & 8 & 2205.65 & 1.975180 & NU1 \\
\hline
\end{tabular}




\begin{tabular}{|c|c|c|c|c|c|c|c|c|c|c|c|}
\hline $\begin{array}{c}\text { FREQUENCY } \\
\mathrm{cm}^{-1}\end{array}$ & $\begin{array}{l}\text { LINE } \\
\text { STRENGTH } \\
\frac{\mathrm{cm}^{-1}}{\mathrm{am} \mathrm{cm}^{-2}}\end{array}$ & $\begin{array}{l}\text { HALF } \\
\text { WIDTH } \\
\frac{\mathrm{cm}^{-1}}{\text { atm. }}\end{array}$ & $J^{\prime}$ & $\mathrm{K}_{\mathrm{c}}$ & $K_{c}$ & $J^{\prime \prime}$ & $K_{a}$ & $K_{c}$ & $\begin{array}{c}E^{\prime \prime} \\
\mathrm{cm}^{-1}\end{array}$ & L & BAND \\
\hline 3396.23 & $1 \cdot 2212$ & 0.0841 & 7 & 2 & 5 & 8 & 4 & 4 & $1131 \cdot 76$ & 0.247061 & NU 3 \\
\hline 3396.43 & 0.0022 & 0.0892 & 5 & 3 & 3 & 4 & 2 & 2 & 315.79 & 2.324407 & $2 N \cup 2 * *$ \\
\hline 3396.82 & 0.0138 & 0.0864 & 5 & 2 & 4 & 6 & 3 & 3 & 661.56 & 1.411171 & NU1* \\
\hline 3397.21 & 32.0157 & 0.0771 & 5 & 3 & 2 & 6 & 4 & 3 & 756.75 & 3.53 & NU1+ \\
\hline 3397.28 & 0.0193 & 0.032 & 14 & 0 & 14 & 13 & 1 & 13 & $1806 \cdot 78$ & 12.99955 & 2NU2 \\
\hline 3397.28 & 0.0578 & 0.032 & 14 & 1 & 14 & 13 & 0 & 13 & 1806.78 & 12.99957 & 2 NU2 \\
\hline 3397.40 & 0.4548 & 0.0880 & 5 & 3 & 2 & 5 & 0 & 5 & $325 \cdot 35$ & 0.062476 & $2 \mathrm{NU} 2$ \\
\hline 3397.59 & 0.0062 & 0.0532 & 10 & 5 & 6 & 9 & 6 & 3 & 1631.41 & 0.576235 & 2NU2 \\
\hline 3397.93 & 0.0088 & 0.0 .794 & 6 & 0 & 6 & 7 & 2 & 5 & 782.40 & 0.051494 & NU3* \\
\hline 3398.42 & 0.0024 & 0.0832 & 8 & 3 & 6 & 7 & 2 & 5 & 2392.58 & 3.262769 & $2 \operatorname{NU} 2 * * *$ \\
\hline 3398.81 & 1.3424 & 0.0804 & 7 & 1 & 6 & 8 & 3 & 5 & $1050 \cdot 15$ & 0.180748 & NU 3 \\
\hline 3399.68 & 0.0045 & 0.0348 & 9 & 8 & 1 & 9 & 9 & 0 & 2225.56 & 1.131403 & NU1 \\
\hline 3399.84 & 0.0026 & 0.0447 & 9 & 6 & 3 & 8 & 7 & 2 & 1590.74 & 0.197898 & $2 \mathrm{NU} 2$ \\
\hline 3400.05 & 0.0025 & 0.0864 & 5 & 2 & 4 & 6 & 3 & 3 & 661.56 & 1.411171 & NU $1 * *$ \\
\hline 3400.28 & 0.0015 & 0.0348 & 9 & 8 & 2 & 9 & 9 & 1 & 2225.56 & 1.131403 & NU 1 \\
\hline 3400.32 & 0.3918 & 0.0431 & 10 & 1 & 9 & 9 & 2 & 8 & 1080.38 & 7.032041 & $2 \mathrm{NU} 2$ \\
\hline 3400.65 & 0.5258 & 0.0401 & 10 & 1 & 9 & 11 & 2 & 10 & 1525.13 & 8.063213 & NU 1 \\
\hline 0.73 & 0.0021 & 0.0619 & 10 & 5 & 5 & 9 & 6 & 4 & $1631 \cdot 27$ & 0.586260 & 2NU2 \\
\hline 3400.83 & 0.0021 & 0.0835 & 6 & 2 & 4 & 7 & 3 & 5 & 2439.97 & 2.751216 & $N \cup 1 * * *$ \\
\hline 3401.02 & 0.6015 & 0.0331 & 11 & 1 & 11 & 12 & 0 & 12 & 1557.91 & 11.021861 & NU 1 \\
\hline 3401.02 & 1.8044 & 0.0335 & 11 & 0 & 11 & 12 & 1 & 12 & 1557.91 & 11.021891 & NUi \\
\hline 3401.19 & 0.0029 & 0.0795 & 4 & 2 & 2 & 5 & 4 & 1 & $2251 \cdot 86$ & 0.042579 & NU $3 * * *$ \\
\hline 3401.51 & 1.5770 & 0.0442 & 10 & 2 & 9 & 11 & 1 & 10 & 1524.87 & 8.058917 & NU 1 \\
\hline 3401.53 & 0.0024 & 0.0716 & 8 & 2 & 6 & 9 & 3 & 7 & 1216.27 & 4.004942 & NU 1* \\
\hline $3402 \cdot 10$ & 4.6556 & 0.0962 & 5 & 2 & 3 & 4 & 1 & 4 & $224 \cdot 83$ & 0.386397 & 2 NU2 \\
\hline $3402 \cdot 18$ & 0.0379 & 0.0694 & 6 & 1 & 5 & 6 & 5 & 2 & $888 \cdot 60$ & 0.000758 & NU3 \\
\hline 3402.72 & 0.0334 & 0.0795 & 3 & 0 & 3 & 4 & 4 & 0 & $488 \cdot 13$ & 0.000270 & NU3 \\
\hline 3403.29 & 6.8788 & 0.0864 & 5 & 2 & 4 & 6 & 3 & 3 & 661.56 & 1.411171 & NU 1 \\
\hline 3403.68 & 5.9268 & 0.0892 & 5 & 3 & 3 & 4 & 2 & 2 & 315.79 & 2.324407 & $2 N \cup 2$ \\
\hline 3403.76 & 0.0196 & 0.0896 & 6 & 3 & 4 & 5 & 2 & 3 & $446 \cdot 50$ & 2.452523 & 2NU2* \\
\hline $3404 \cdot 21$ & 0.0060 & 0.0890 & 3 & 0 & 3 & 4 & 3 & 2 & 382.52 & 0.051049 & NU I* \\
\hline 3404.21 & 1.1866 & 0.0444 & 10 & 2 & 9 & 9 & 1 & 8 & 1079.07 & 7.043661 & 2NU2 \\
\hline $3404 \cdot 27$ & 0.0017 & 0.0539 & 12 & 4 & 9 & 12 & 6 & 6 & 2437.56 & 0.124675 & NU3 \\
\hline 3405.02 & 0.0270 & 0.0646 & 7 & 0 & 7 & 7 & 4 & 4 & 927.77 & 0.001966 & NU3 \\
\hline 3405.51 & 0.0016 & 0.0794 & 6 & 0 & 6 & 7 & 2 & 5 & $782 \cdot 40$ & 0.051494 & NU3** \\
\hline 3406.64 & 1.2173 & 0.0716 & 8 & 2 & 6 & 9 & 3 & 7 & $1216 \cdot 27$ & 4.004942 & NU1 \\
\hline 3407.40 & 0.0063 & 0.0379 & 12 & 2 & 10 & 12 & 1 & 11 & 1774.60 & 3.652921 & 2NU2 \\
\hline 3407.84 & 0.0044 & 0.0694 & 5 & 1 & 4 & 5 & 5 & 1 & $742 \cdot 10$ & 0.000125 & NU3 \\
\hline 3407.97 & 0.0017 & 0.0348 & 10 & 1 & 10 & 11 & 0 & 11 & 2915.94 & 10.018153 & NU1 $* * * *$ \\
\hline 3408.11 & 0.0331 & 0.0752 & 10 & 3 & 7 & 9 & 4 & 6 & 1340.89 & 2.171641 & 2NU2 \\
\hline $3408 \cdot 17$ & 0.0128 & 0.0840 & 6 & 2 & 4 & 7 & 4 & 3 & 931.22 & 0.157817 & NU3* \\
\hline $3408 \cdot 54$ & 0.0306 & 0.0884 & 5 & 3 & 2 & 4 & 2 & 3 & $300 \cdot 35$ & 1.841317 & $2 N \cup 2 *$ \\
\hline $3408 \cdot 72$ & 1.4741 & 0.0774 & 5 & 2 & 4 & 6 & 4 & 3 & 756.75 & 0.015200 & NU3- \\
\hline 3408.83 & 3.0175 & 0.0890 & 3 & 0 & 3 & 4 & 3 & 2 & 382.52 & 0.051049 & NU1 \\
\hline 3409.09 & 0.0094 & 0.0331 & 12 & 1 & 11 & 12 & 0 & 12 & 1557.91 & 1.854934 & $2 \mathrm{NU} 2$ \\
\hline $3409 \cdot 19$ & 0.0395 & 0.0683 & 3 & 3 & 0 & 4 & 4 & 1 & .2129 .63 & 3.393148 & NU $1 * * * *$ \\
\hline $3409 \cdot 54$ & 0.0131 & 0.0680 & 3 & 3 & 1 & 4 & 4 & 0 & 2129.65 & 3.390390 & NU $1 * * * *$ \\
\hline 3409.67 & 0.0283 & 0.0331 & 12 & 2 & 11 & 12 & 1 & 12 & 1557.91 & 1.854861 & $2 \mathrm{NU} 2$ \\
\hline 3410.07 & 0.1040 & 0.0337 & 12 & 1 & 12 & 12 & 2 & 11 & 1774.74 & 1.854861 & NU 1 \\
\hline 3410.19 & 0.1288 & 0.0552 & 11 & 6 & 5 & 12 & 6 & 6 & 2437.56 & 9.054429 & NU3 \\
\hline 3410.44 & 0.0052 & 0.032 & 15 & 1 & 15 & 14 & 0 & 14 & 2073.66 & 14.00192 & $2 \mathrm{NU} 2$ \\
\hline 3410.44 & 0.0156 & 0.032 & 15 & 0 & 15 & 14 & 1 & 14 & 2073.66 & 14.00191 & 2 NIJ 2 \\
\hline 3410.59 & 0.0347 & 0.0337 & 12 & u & 12 & 12 & 1 & 11 & 1774.60 & 1.854934 & NU1 \\
\hline 3411.02 & 0.0096 & 0.0622 & 10 & 2 & 9 & 10 & 4 & 6 & 1616.51 & 0.021601 & NU3 \\
\hline
\end{tabular}




\begin{tabular}{|c|c|c|c|c|c|c|c|c|c|c|c|}
\hline $\begin{array}{c}\text { FREQUENCY } \\
\mathrm{cm}^{-1}\end{array}$ & $\begin{array}{l}\text { LINE } \\
\text { STRENGTH } \\
\frac{\mathrm{cm}^{-1}}{\mathrm{gm} \mathrm{cm}^{-2}}\end{array}$ & $\begin{array}{l}\text { HALF } \\
\text { WIDTH } \\
\frac{\mathrm{cm}^{-1}}{\text { atm. }}\end{array}$ & $J^{\prime}$ & $K_{0}$ & $\mathrm{~K}_{\mathrm{c}}$ & J" & $\mathrm{K}_{\mathrm{a}}$ & $K_{c}$ & $\begin{array}{c}E^{\prime \prime} \\
\mathrm{cm}^{-1}\end{array}$ & $L$ & BAND \\
\hline 3411.10 & 0.0036 & 0.0896 & 6 & 3 & 4 & 5 & 2 & 3 & 446.50 & 2.452523 & $2 \mathrm{NU} 2 * *$ \\
\hline 3411.13 & 0.0193 & 0.0871 & 4 & 2 & 3 & 5 & 3 & 2 & 2130.50 & 1.841317 & $N \cup 1 * * *$ \\
\hline 3411.83 & 0.0045 & 0.0869 & 6 & 1 & 5 & 7 & 3 & 4 & 2462.87 & 0.214427 & NU $3 * * * *$ \\
\hline 3411.85 & 0.3863 & 0.0516 & 11 & 6 & 6 & 12 & 6 & 7 & 2433.85 & 9.048040 & NU3 \\
\hline 3411.90 & 0.4713 & 0.0747 & 9 & 3 & 7 & 10 & 2 & 8 & 1438.00 & 4.706625 & NUI \\
\hline 3412.11 & 0.0022 & 0.0887 & 6 & 3 & 3 & 5 & 2 & 4 & 2024.17 & 1.411171 & $2 N \cup 2 * * * *$ \\
\hline 3413.09 & 0.0050 & 0.0351 & 13 & 2 & 12 & 13 & 3 & 1.1 & 2248.16 & 3.75319 & NU1 \\
\hline 3413.09 & 4.3976 & 0.0794 & 6 & 0 & 6 & 7 & 2 & 5 & 782.40 & 0.051494 & NU3 \\
\hline 3413.77 & 0.1431 & 0.0721 & 11 & 5 & 6 & 12 & 5 & 7 & 2300.80 & 10.047055 & NU3 \\
\hline 3413.84 & 0.0024 & 0.0840 & 6 & 2 & 4 & 7 & 4 & 3 & $931 \cdot 22$ & 0.157817 & NU $3 * *$ \\
\hline 3414.23 & 0.0150 & 0.0470 & 13 & 1 & 12 & 13 & 2 & 11 & 2246.98 & 3.76133 & NU 1 \\
\hline 3414.48 & 0.0128 & 0.0852 & 5 & 2 & 3 & 6 & 3 & 4 & 2271.70 & 2.452523 & NU1**** \\
\hline 3414.65 & 0.0020 & 0.0757 & 3 & 2 & 2 & 4 & 4 & 1 & 2129.63 & 0.015552 & NU3**** \\
\hline 3414.81 & 0.0130 & 0.0804 & 5 & 1 & 5 & 6 & 3 & 4 & 648.97 & 0.038984 & NU3* \\
\hline 3414.93 & 0.0026 & 0.0806 & 6 & 5 & 2 & 7 & 2 & 5 & 782.40 & 0.003511 & 2NU2 \\
\hline 3414.94 & 0.0171 & 0.0795 & 7 & 2 & 5 & 8 & 3 & 6 & $1006 \cdot 12$ & 3.262769 & NU $1 *$ \\
\hline 3415.38 & 0.1234 & 0.0733 & 4 & 3 & 2 & 5 & 4 & 1 & 610.35 & 3.250024 & NU $1 *$ \\
\hline 3415.46 & 0.4965 & 0.0566 & 12 & 3 & 9 & 13 & 3 & 10 & 2414.73 & 11.684530 & NU3 \\
\hline 3415.47 & 0.0057 & 0.0884 & 5 & 3 & 2 & 4 & 2 & 3 & $300 \cdot 35$ & 1.841317 & $2 N \cup 2 * *$ \\
\hline 3415.49 & 0.0249 & 0.0813 & 10 & 5 & 5 & 10 & 4 & 6 & 1616.51 & 6.465380 & 2 NU2 2 \\
\hline 3415.61 & 0.1625 & 0.0488 & 12 & 4 & 9 & 13 & 4 & 10 & 2426.22 & 11.473010 & NU 3 \\
\hline 3415.97 & 0.0034 & 0.0881 & 7 & 3 & 5 & 6 & 2 & 4 & 602.77 & 2.751216 & $2 \operatorname{NU} 2 * x$ \\
\hline 3416.00 & 0.0088 & 0.0463 & 9 & 1 & 8 & 10 & 2 & 9 & 1293.66 & 7.043661 & NU $1 *$ \\
\hline 3416.26 & 0.0190 & 0.0368 & 12 & 3 & 10 & 12 & 2 & 11 & 1774.74 & 3.648229 & 2 NU2 2 \\
\hline 3416.33 & 0.0042 & 0.0412 & 10 & 7 & 3 & 10 & 8 & 2 & 2254.34 & 3.150851 & NUI \\
\hline 3416.33 & 0.0126 & 0.0412 & 10 & 7 & 4 & 10 & 8 & 3 & 2254.34 & 3.150801 & NU1 \\
\hline 3416.71 & 0.0415 & 0.0734 & 4 & 3 & 1 & 5 & 4 & 2 & 610.12 & 3.270203 & $N \cup 1 *$ \\
\hline 3417.22 & 0.5182 & 0.0748 & 7 & 1 & 6 & 7 & 4 & 3 & 931.22 & 0.135960 & NU1 \\
\hline 3417.44 & 0.0029 & 0.0534 & 9 & 2 & 8 & 10 & 1 & 9 & 1293.04 & 7.032041 & NUI* \\
\hline 3417.45 & 0.0032 & 0.0795 & 7 & 2 & 5 & 8 & 3 & 6 & $1006 \cdot 12$ & 3.262769 & NU $1 * * *$ \\
\hline 3417.93 & 0.0228 & 0.0733 & 4 & 3 & 2 & 5 & 4 & 1 & 610.35 & 3.250024 & NUl** \\
\hline 3418.45 & 9.8020 & 0.0896 & 6 & 3 & 4 & 5 & 2 & 3 & 446.50 & 2.452523 & $2 N \cup 2$ \\
\hline 3419.18 & 0.0016 & 0.0463 & 9 & 1 & 8 & 10 & 2 & 9 & 1293.66 & 7.043661 & NU1*** \\
\hline 3419.22 & 0.0077 & 0.0734 & 4 & 3 & 1 & 5 & 4 & 2 & 610.12 & 3.270203 & NU $1 * * *$ \\
\hline 3419.36 & 0.0096 & 0.0561 & 11 & 4 & 8 & 11 & 6 & 5 & 2144.12 & 0.111521 & NU3 \\
\hline 3419.52 & 6.4141 & 0.0840 & 6 & 2 & 4 & 7 & 4 & 3 & $931 \cdot 22$ & 0.157817 & NU 3 \\
\hline 3419.96 & 8.5676 & 0.0795 & 7 & 2 & 5 & 8 & 3 & 6 & $1006 \cdot 12$ & 3.262769 & NU1 \\
\hline 3420.03 & 0.0116 & 0.0391 & 9 & 7 & 3 & 9 & 8 & 2 & 2009.87 & 2.165389 & NU 1 \\
\hline 3420.03 & 0.0348 & 0.0392 & 9 & 7 & 2 & 9 & 8 & 1 & 2009.87 & 2.165395 & NU1 \\
\hline 3420.49 & 61.6930 & 0.0733 & 4 & 3 & 2 & 5 & 4 & 1 & 610.35 & 3.250024 & NU1 \\
\hline 3420.74 & 0.4658 & 0.0378 & 11 & 1 & 10 & 10 & 2 & 9 & 1293.66 & 8.058917 & $2 N \cup 2$ \\
\hline 3421.37 & 0.0024 & 0.0804 & 5 & 1 & 5 & 6 & 3 & 4 & 648.97 & 0.038984 & NU $3 * *$ \\
\hline 3421.74 & 20.7259 & 0.0734 & 4 & 3 & 1 & 5 & 4 & 2 & 610.12 & 3.270203 & NU 1 \\
\hline 3422.25 & 0.0156 & 0.0595 & 11 & 3 & 9 & 11 & 5 & 6 & 1999.02 & 0.072485 & NU3 \\
\hline 3422.37 & 4.3866 & 0.0463 & 9 & 1 & 8 & 10 & 2 & 9 & 1293.66 & 7.043661 & NU1 \\
\hline 3422.40 & 15.2993 & 0.0884 & 5 & 3 & 2 & 4 & 2 & 3 & 300.35 & 1.841317 & $2 \mathrm{NU} 2$ \\
\hline 3422.56 & 0.1564 & 0.0385 & 11 & 2 & 10 & 10 & 1 & 9 & 1293.04 & 8.063213 & $2 N U 2$ \\
\hline 3423.07 & 0.4244 & 0.0564 & 11 & 5 & 7 & 12 & 5 & 8 & 2275.44 & 9.908577 & NU 3 \\
\hline 3423.23 & 1.7581 & 0.0354 & 10 & 0 & 10 & 11 & 1 & 11 & 1327.14 & 10.018239 & NU1 \\
\hline 3423.24 & 5.2744 & 0.0348 & 10 & 1 & 10 & 11 & 0 & 11 & 1327.14 & 10.018153 & NU1 \\
\hline 3423.94 & 0.0196 & 0.0369 & 8 & 7 & 1 & 8 & 8 & 0 & 1789.10 & 1.126216 & NU 1 \\
\hline 3423.94 & 0.0589 & 0.0369 & 8 & 7 & 2 & 8 & 8 & 1 & 1789.10 & 1.126215 & NU1 \\
\hline 3424.03 & 0.0334 & 0.0920 & 4 & 1 & 4 & 5 & 2 & 3 & $446 \cdot 50$ & 0.386397 & NU $1 *$ \\
\hline 3424.03 & 1.4699 & 0.0534 & 9 & 2 & 8 & 10 & 1 & 9 & 1293.04 & 7.032041 & NU 1 \\
\hline
\end{tabular}




$\begin{array}{cccccccccccc}\text { FREQUENCY } & \begin{array}{l}\text { LINE } \\ \mathrm{cm}^{-1}\end{array} & \begin{array}{c}\text { STRENGTH } \\ \text { WIDTH }\end{array} & \frac{\mathrm{cm} m^{-1}}{g m \mathrm{~cm}^{-2}} & \frac{\mathrm{cm}^{-1}}{a+m}\end{array}$

\begin{tabular}{|c|c|c|c|c|c|c|c|c|c|c|c|}
\hline $3424 \cdot 32$ & 0.0012 & 0.0646 & 6 & 5 & 1 & 6 & 4 & 2 & 757.78 & 2.089996 & $2 \mathrm{NU} 2 *$ \\
\hline $3424 \cdot 37$ & 0.0016 & 0.0460 & 10 & 6 & 5 & 9 & 7 & 2 & 1810.63 & 0.354752 & 2 NU 2 \\
\hline $3424 \cdot 88$ & 0.0039 & 0.0580 & 5 & 5 & 0 & 5 & 4 & 1 & 610.35 & 1.101100 & $2 N \cup 2 *$ \\
\hline $3425 \cdot 12$ & 0.0013 & 0.0566 & 5 & 5 & 1 & 5 & 4 & 2 & 610.12 & 1.100600 & $2 N \cup 2 *$ \\
\hline $3425 \cdot 32$ & 0.0036 & 0.0607 & 6 & 5 & 2 & 6 & 4 & 3 & 756.75 & 2.085226 & $2 N \cup 2 *$ \\
\hline $3425 \cdot 33$ & 0.1336 & 0.0590 & 10 & 2 & 8 & 9 & 3 & 7 & 1216.27 & 4.706625 & 2 NU 2 \\
\hline 3426.15 & 0.3077 & 0.0772 & 11 & 4 & 7 & 12 & 4 & 8 & 2205.65 & 10.766337 & NU 3 \\
\hline 3426.60 & 0.0014 & 0.0866 & 4 & 1 & 4 & 5 & 3 & 3 & 2126.41 & 0.035251 & NU $3 * * *$ \\
\hline 3426.72 & 0.2120 & 0.0794 & 9 & 5 & 4 & 9 & 4 & 5 & 1360.28 & 5.113359 & 2NU2 \\
\hline 3426.75 & 0.2825 & 0.0446 & 10 & 7 & 3 & 11 & 7 & 4 & 2321.94 & 6.588161 & NU3 \\
\hline 3426.80 & 0.0941 & 0.0425 & 10 & 7 & 4 & 11 & 7 & 5 & 2321.89 & 6.588139 & NU3 \\
\hline 3426.80 & 0.0020 & 0.0580 & 5 & 5 & 0 & 5 & 4 & 1 & 2251.86 & 1.101100 & $2 N \cup 2 * * *$ \\
\hline 3427.01 & 0.0692 & 0.0865 & 6 & 3 & 3 & 6 & 0 & 6 & 445.71 & 0.051901 & 2NU 2 \\
\hline 3427.01 & 0.0018 & 0.0607 & 6 & 5 & 2 & 6 & 4 & 3 & 2398.39 & 2.085226 & $2 N \cup 2 * * * *$ \\
\hline 3427.05 & 0.0056 & 0.0824 & 5 & 2 & 3 & 6 & 4 & 2 & 757.78 & 0.086601 & NU3* \\
\hline 3427.21 & 0.0011 & 0.0620 & 8 & 5 & 4 & 8 & 4 & 5 & $1122 \cdot 72$ & 3.901175 & $2 N \cup 2 *$ \\
\hline $3427 \cdot 34$ & 0.0049 & 0.0832 & 8 & 3 & 6 & 7 & 2 & 5 & 782.40 & 3.262769 & $2 N \cup 2 *$ \\
\hline $3427 \cdot 54$ & 0.0062 & 0.0920 & 4 & 1 & 4 & 5 & 2 & 3 & $446 \cdot 50$ & 0.386397 & NU $1 * *$ \\
\hline 3427.77 & 0.0125 & 0.0835 & 6 & 2 & 4 & 7 & 3 & 5 & 816.72 & 2.751216 & NU I* \\
\hline 3427.93 & 6.5170 & 0.0804 & 5 & 1 & 5 & 6 & 3 & 4 & 648.97 & 0.038984 & NU3 \\
\hline 3428.43 & 0.0047 & 0.0779 & 4 & 2 & 3 & 5 & 4 & 2 & 610.12 & 0.034972 & NU3* \\
\hline 3428.61 & 0.0036 & 0.0620 & 8 & 5 & 4 & 8 & 4 & 5 & 2764.71 & 6.79 & NU $1 * * * *$ \\
\hline 3429.14 & 0.0099 & 0.0578 & 10 & 4 & 7 & 10 & 6 & 4 & 1875.53 & 0.086992 & NU3 \\
\hline 3429.22 & 0.0061 & 0.0324 & 13 & 1 & 12 & 13 & 0 & 13 & $1806 \cdot 78$ & 1.90654 & 2NU2 \\
\hline 3429.47 & 0.0046 & 0.0388 & 9 & 0 & 9 & 10 & 1 & 10 & $2705 \cdot 18$ & 9.013676 & NU 1*** \\
\hline 3429.52 & 0.0029 & 0.0333 & 13 & 2 & 12 & 13 & 1 & 13 & $1806 \cdot 78$ & 1.90640 & $2 \mathrm{NU} 2$ \\
\hline 3429.60 & 0.0015 & 0.0382 & 9 & 1 & 9 & 10 & 0 & 10 & 2705.15 & 9.013420 & NU1**** \\
\hline 3430.30 & 0.0023 & 0.0835 & 6 & 2 & 4 & 7 & 3 & 5 & 816.72 & 2.751216 & NU 1 \\
\hline 3430.40 & 0.1349 & 0.0943 & 3 & 3 & 1 & 2 & 0 & 2 & 70.08 & 0.013376 & 2 NU2 \\
\hline 3430.81 & 1.6839 & 0.0881 & 7 & 3 & 5 & 6 & 2 & 4 & 602.77 & 2.751216 & $2 \mathrm{NU} 2$ \\
\hline 3431.06 & 16.6946 & 0.0920 & 4 & 1 & 4 & 5 & 2 & 3 & $446 \cdot 50$ & 0.386397 & NU 1 \\
\hline $3431 \cdot 44$ & 0.0084 & 0.0865 & 4 & 2 & 2 & 5 & 3 & 3 & 2126.41 & 2.324407 & NU I*** \\
\hline 3432.09 & 0.0012 & 0.0834 & 8 & 3 & 6 & 9 & 2 & 7 & 2818.40 & 3.529968 & NU $1 * * * *$ \\
\hline 3432.49 & 0.2575 & 0.0785 & 6 & 1 & 5 & 6 & 4 & 2 & 757.78 & 0.084732 & NU 1 \\
\hline 3432.83 & 6.2277 & 0.0835 & 6 & 2 & 4 & 7 & 3 & 5 & 816.72 & 2.751216 & NUI \\
\hline 3433.17 & 0.1342 & 0.0863 & 9 & 4 & 6 & 10 & 3 & 7 & $1538 \cdot 23$ & 2.171641 & NU 1 \\
\hline 3433.67 & 0.0056 & 0.0349 & 13 & 2 & 11 & 13 & 1 & 12 & 2042.33 & 3.76133 & $2 \mathrm{NU} 2$ \\
\hline 3433.74 & 0.2553 & 0.0793 & 6 & 0 & 6 & 6 & 3 & 3 & 661.56 & 0.051901 & NU 1 \\
\hline 3433.80 & 0.1219 & 0.0358 & 11 & 1 & 11 & 11 & 2 & 10 & 1525.13 & 1.851690 & NU 1 \\
\hline .00 & 0.0070 & 0.0834 & 8 & 3 & 6 & 9 & 2 & 7 & 1201.95 & 3.529968 & NU $1 *$ \\
\hline 3434.06 & 0.3658 & 0.0359 & 11 & 0 & 11 & 11 & 1 & 10 & 1524.87 & 1.851910 & NU 1 \\
\hline 3434.23 & 0.0274 & 0.0869 & 6 & 1 & 5 & 7 & 3 & 4 & $842 \cdot 38$ & 0.214427 & NU3* \\
\hline .24 & 0.1748 & 0.0756 & 8 & 5 & 3 & 8 & 4 & 4 & 1131.76 & 4.012859 & $2 \mathrm{NU} 2$ \\
\hline 3434.52 & 0.0307 & 0.0674 & 4 & 4 & 1 & 3 & 3 & 0 & 1907.63 & 3.393148 & $2 \mathrm{NU} 2 * * * *$ \\
\hline .69 & 0.0102 & 0.0665 & 4 & 4 & 0 & 3 & 3 & 1 & 1907.47 & 3.390390 & $2 N \cup 2 * \ddot{*} * *$ \\
\hline .65 & 0.0744 & 0.0590 & 9 & 4 & 6 & 9 & 6 & 3 & 1631.41 & 0.059724 & NU3 \\
\hline 3435.92 & 1.2000 & 0.0812 & 10 & 5 & 6 & 11 & 4 & 7 & 1899.06 & 37.2 & NU1+ \\
\hline 3436.05 & 0.0736 & 0.0381 & 12 & 2 & 11 & 12 & 3 & 10 & 1962.55 & 3.648229 & NU 1 \\
\hline $3436 \cdot 12$ & 0.0019 & 0.0343 & 13 & 3 & 11 & 13 & 2 & 12 & 2042.38 & 3.75319 & 2 NU2 \\
\hline $3436 \cdot 22$ & 0.0012 & 0.0787 & 5 & 1 & 4 & 5 & 4 & 1 & 610.35 & 0.031887 & NU 1 * \\
\hline 3436.29 & 0.5083 & 0.0411 & 12 & 2 & 10 & 13 & 2 & 11 & 2246.98 & 11.888730 & NU3 \\
\hline 3436 & 0.1892 & 0.0330 & 13 & 1 & 12 & 14 & 1 & 13 & 2327.88 & 13.271580 & NU 3 \\
\hline 3436 & 0.5674 & 0.0330 & 13 & 2 & 12 & 14 & 2 & 13 & 2327.90 & 13.271150 & NU3 \\
\hline 3436.48 & 0.1169 & 0.0871 & 4 & 2 & 3 & 5 & 3 & 2 & 508.81 & 1.841317 & NU $1 *$ \\
\hline
\end{tabular}




\begin{tabular}{|c|c|c|c|c|c|c|c|c|c|c|c|}
\hline $\begin{array}{c}\text { FREQUENCY } \\
\mathrm{cm}^{-1}\end{array}$ & $\begin{array}{l}\text { LINE } \\
\text { STRENGTH } \\
\frac{\mathrm{cm}^{-1}}{\mathrm{gm} \mathrm{cm}^{-2}}\end{array}$ & $\begin{array}{l}\text { HALF } \\
\text { WIDTH } \\
\mathrm{cm}^{-1} \\
\text { atm. }\end{array}$ & $J^{\prime}$ & K & K & J" & $\mathrm{K}_{\mathrm{c}}$ & k & $\begin{array}{c}\mathrm{E}^{\prime \prime} \\
\mathrm{cm}^{-1}\end{array}$ & L & BAND \\
\hline $\begin{array}{l}3437 \cdot 12 \\
3437.38\end{array}$ & $\begin{array}{l}0.0073 \\
0.0017\end{array}$ & $\begin{array}{l}0.0552 \\
0.0752\end{array}$ & $\begin{array}{r}8 \\
10\end{array}$ & $\begin{array}{l}1 \\
3\end{array}$ & $\begin{array}{l}7 \\
7\end{array}$ & $\begin{array}{r}9 \\
11\end{array}$ & $\begin{array}{l}2 \\
3\end{array}$ & $\begin{array}{l}8 \\
8\end{array}$ & $\begin{array}{l}1080.38 \\
3441.09\end{array}$ & $\begin{array}{l}6.019178 \\
9.899853\end{array}$ & $\begin{array}{l}\text { NU 1* } \\
\text { NU } 3 * * * *\end{array}$ \\
\hline 3437.40 & 1.0818 & 0.0704 & 7 & 5 & 2 & 7 & 4 & 3 & $931 \cdot 22$ & 3.039271 & 2NU2 \\
\hline 3437.42 & 0.6686 & 0.0536 & 10 & 6 & 4 & 11 & 6 & 5 & $2144 \cdot 12$ & 7.774282 & NU3 \\
\hline 3437.76 & 0.4621 & 0.0658 & 11 & 3 & 8 & 12 & 3 & 9 & 2105.90 & 10.744435 & NU 3 \\
\hline 3.02 & 0.0014 & 0.0430 & 9 & 7 & 3 & 10 & 7 & 4 & 2054.40 & 5.133658 & NU $3 *$ \\
\hline 3438.03 & 0.0013 & 0.0834 & 8 & 3 & 6 & 9 & 2 & 7 & 1201.95 & 3.529968 & NUl \\
\hline 8.07 & 0.0397 & 0.0681 & 8 & 1 & 8 & 8 & 3 & 5 & 1050.15 & 0.005295 & NU3 \\
\hline 3.08 & 0.2229 & 0.0515 & 10 & 6 & 5 & 11 & 6 & 6 & 2142.69 & 7.773013 & NU3 \\
\hline $3 \cdot 18$ & 2.8088 & 0.0824 & 5 & 2 & 3 & 6 & 4 & 2 & 757.78 & 0.086601 & NU3 \\
\hline 3438.28 & 0.0295 & 0.0391 & 12 & 1 & 1 & 12 & 2 & 10 & $1960 \cdot 22$ & 3.652921 & NUl \\
\hline 3438.50 & 0.9018 & 0.0548 & 11 & 4 & 8 & 12 & 4 & 9 & 2124.98 & 10.481399 & NU3 \\
\hline 3.58 & 0.0277 & 0.0388 & 9 & 0 & 9 & 10 & 1 & 10 & $1114 \cdot 56$ & 9.013676 & NU I * \\
\hline 3438.62 & 0.0092 & 0.0382 & 9 & 1 & 9 & 10 & 0 & 10 & $1114 \cdot 55$ & 9.013420 & NU l * \\
\hline $3438 \cdot 64$ & 0.2102 & 0.0320 & 14 & 1 & 4 & 15 & 1 & 15 & $2358 \cdot 44$ & $14 \cdot 743490$ & NU3 \\
\hline $3438 \cdot 64$ & 0.6306 & 0.0320 & 14 & 0 & 4 & 15 & 0 & 15 & 2358.44 & 14.743490 & NU3 \\
\hline 8.74 & 0.1561 & 0.0703 & 6 & 0 & 6 & 6 & 4 & 3 & $756 \cdot 75$ & 0.001595 & NU3 \\
\hline 3439.18 & 0.0074 & 0.0432 & 13 & 3 & 1 & 13 & 4 & 10 & $2426 \cdot 22$ & 5.48699 & NUl \\
\hline $3439 \cdot 20$ & 0.6086 & 0.0787 & 5 & 1 & 4 & 5 & 4 & 1 & 610.35 & 0.031887 & NU1 \\
\hline $3439 \cdot 50$ & 0.0216 & 0.0871 & 4 & 2 & 3 & 5 & 3 & 2 & 508.81 & 1.841317 & NU $1 * *$ \\
\hline 3439.51 & 0.0067 & 0.0465 & 11 & 6 & 6 & 11 & 7 & 5 & 2321.89 & 4.990193 & NUl \\
\hline 3439.66 & 0.5908 & 0.0646 & 6 & 5 & 1 & 6 & 4 & 2 & $757 \cdot 78$ & 2.089996 & $2 \mathrm{NU} 2$ \\
\hline 3439.68 & 0.0702 & 0.0777 & 4 & 1 & 3 & 4 & 4 & 0 & $488 \cdot 13$ & 0.005990 & NU1 \\
\hline 3439.81 & 0.0440 & 0.0592 & 8 & 4 & 5 & 8 & 6 & 2 & $1411 \cdot 68$ & 0.035676 & NU3 \\
\hline 3439.82 & 0.0520 & 0.0374 & 9 & 8 & 1 & 10 & 8 & 2 & $2254 \cdot 34$ & 3.624262 & NU3 \\
\hline 3439.82 & 0.1560 & 0.0373 & 9 & 8 & 2 & 10 & 8 & 3 & $2254 \cdot 34$ & 3.624262 & NU3 \\
\hline 3439.96 & 2.3745 & 0.0779 & 4 & 2 & 3 & 5 & 4 & 2 & 610.12 & 0.034972 & NU 3 \\
\hline 3440.08 & 0.0220 & 0.0646 & 8 & 2 & 7 & 9 & 1 & 8 & 1079.07 & 5.987889 & NU ${ }^{*} *$ \\
\hline 3440.13 & 1.9517 & 0.0580 & 5 & 5 & 0 & 5 & 4 & 1 & 610.35 & 1.101100 & 2NU 2 \\
\hline 3440.21 & 0.0202 & 0.0497 & 11 & 6 & 5 & 11 & 7 & 4 & 2321.94 & 4.999181 & NU1 \\
\hline 3440.36 & 0.6512 & 0.0566 & 5 & 5 & 1 & 5 & 4 & 2 & $610 \cdot 12$ & 1.100600 & 2 NU 2 \\
\hline $3440 \cdot 58$ & 1.7783 & 0.0607 & 6 & 5 & 2 & 6 & 4 & 3 & 756.75 & 2.085226 & $2 \mathrm{NU} 2$ \\
\hline 3440.65 & 0.4327 & 0.0895 & 2 & 0 & 2 & 3 & 3 & 1 & 285.23 & 0.013376 & NU1 \\
\hline 3440.75 & 0.3642 & 0.0624 & 7 & 5 & 3 & 7 & 4 & 4 & 927.77 & 3.012848 & 2NU2 \\
\hline $3440 \cdot 94$ & 0.0016 & 0.0875 & 5 & 0 & 5 & 6 & 2 & 4 & $2211 \cdot 21$ & 0.069891 & NU $3 * * *$ \\
\hline $3441 \cdot 31$ & 0.0051 & 0.0869 & 6 & 1 & 5 & 7 & 3 & 4 & $842 \cdot 38$ & 0.214427 & NU $3 * *$ \\
\hline 3441.62 & 0.0774 & 0.0852 & 5 & 2 & 3 & 6 & 3 & 4 & $648 \cdot 97$ & 2.452523 & NUI* \\
\hline 1.77 & 0.0080 & 0.0653 & 7 & 1 & 6 & 8 & 2 & 7 & $2495 \cdot 18$ & $4 \cdot 996649$ & NU $1 * * *$ \\
\hline 3441.87 & 0.0051 & 0.0388 & 9 & 0 & 9 & 10 & 1 & 10 & 1114.56 & 9.013676 & NU $1^{* *}$ \\
\hline 3441.87 & 0.0556 & 0.0350 & 12 & 1 & 1 & 11 & 2 & 10 & 1525.13 & 9.076485 & 2 NU 2 \\
\hline 3441.91 & 0.0017 & 0.0382 & 9 & 1 & 9 & 10 & 0 & 10 & 1114.55 & 9.013420 & NU $1 * * *$ \\
\hline 3441.97 & 0.0797 & 0.0680 & 3 & 3 & 1 & 4 & 4 & 0 & $488 \cdot 13$ & 3.390390 & NUl * \\
\hline 3442.07 & 3.5091 & 0.0834 & 8 & 3 & 6 & 9 & 2 & 7 & 1201.95 & 3.529968 & NU1 \\
\hline $3442 \cdot 15$ & 0.2393 & 0.0683 & 3 & 3 & 0 & 4 & 4 & 1 & $488 \cdot 10$ & 3.393148 & NU I* \\
\hline $3442 \cdot 22$ & 2.4484 & 0.0832 & 8 & 3 & 6 & 7 & 2 & 5 & 782.40 & 3.262769 & 2NU 2 \\
\hline $3442 \cdot 38$ & 0.5343 & 0.0620 & 8 & 5 & 4 & 8 & 4 & 5 & 1122.72 & 3.901175 & $2 \mathrm{NU} 2$ \\
\hline $3442 \cdot 52$ & 58.4557 & 0.0871 & 4 & 2 & 3 & 5 & 3 & 2 & 508.81 & 1.841317 & NU I \\
\hline $3442 \cdot 71$ & 0.1669 & 0.0350 & 12 & 2 & 1 & 11 & 1 & 10 & $1524 \cdot 87$ & 9.078065 & $2 \mathrm{NU} 2$ \\
\hline 3442.73 & 0.0164 & 0.0484 & 10 & 6 & 4 & 10 & 7 & 3 & 2054.40 & 4.061130 & NU1 \\
\hline 3442.76 & 0.1726 & 0.0578 & 7 & 4 & 4 & 7 & 6 & 1 & $1216 \cdot 20$ & 0.017491 & NU3 \\
\hline 3443.07 & 0.0143 & 0.0858 & 3 & 2 & 2 & 4 & 3 & 1 & 2005.92 & 2.164905 & NU $1 * * * *$ \\
\hline 3443.10 & 1.8952 & 0.0700 & 10 & 5 & 5 & 11 & 5 & 6 & 1999.02 & 8.798801 & NU 3 \\
\hline 3443.47 & 0.0041 & 0.0646 & 8 & 2 & 7 & 9 & 1 & 8 & 1079.07 & 5.987889 & NU I*** \\
\hline 3443.47 & 3.6582 & 0.0552 & 8 & 1 & 7 & 9 & 2 & 8 & 1080.38 & 6.019178 & NUl \\
\hline
\end{tabular}




\begin{tabular}{|c|c|c|c|c|c|c|c|c|c|c|c|}
\hline $\begin{array}{l}\text { FREQUENCY } \\
\qquad \mathrm{cm}^{-1}\end{array}$ & $\begin{array}{l}\text { LINE } \\
\text { STRENGTH } \\
\frac{\mathrm{cm}^{-1}}{\mathrm{gm} \mathrm{cm}^{-2}}\end{array}$ & 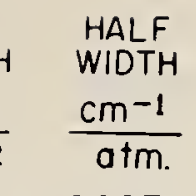 & $J^{\prime}$ & $\mathrm{K}_{0}$ & $\mathrm{~K}_{\mathrm{c}}$ & $J^{\prime \prime}$ & $\mathrm{K}_{0}$ & $K_{c}$ & $\begin{array}{c}E^{\prime \prime} \\
\mathrm{cm}^{-1}\end{array}$ & $L$ & BAND \\
\hline 3444.07 & 0.0044 & 0.0887 & 6 & 3 & 3 & 5 & 2 & 4 & $416 \cdot 22$ & 1.411171 & $2 \mathrm{NU} 2 *$ \\
\hline 3444.18 & 0.0010 & 0.0625 & 7 & 4 & 3 & 8 & 1 & 8 & $744 \cdot 16$ & 0.001069 & 2 NU2 \\
\hline $3444 \cdot 22$ & 0.0143 & 0.0852 & 5 & 2 & 3 & 6 & 3 & 4 & 648.97 & 2.452523 & NU1*** \\
\hline $3444 \cdot 52$ & 0.0147 & 0.0680 & 3 & 3 & 1 & 4 & 4 & 0 & $488 \cdot 13$ & 3.390390 & NU $1 * *$ \\
\hline 3444.64 & 0.0174 & 0.0795 & 4 & 2 & 2 & 5 & 4 & 1 & 610.35 & 0.042579 & NU $3 *$ \\
\hline $3444 \cdot 70$ & 0.0443 & 0.0683 & 3 & 3 & 0 & 4 & 4 & 1 & 488.10 & 3.393148 & NU $1 * *$ \\
\hline 3445.02 & 0.0441 & 0.0552 & 6 & 4 & 3 & 6 & 6 & 0 & 1045.07 & 0.005704 & NU3 \\
\hline 3445.11 & 0.0729 & 0.0600 & 9 & 5 & 5 & 9 & 4 & 6 & 1340.89 & 4.736397 & $2 N U 2$ \\
\hline 3445.17 & 13.8539 & 0.0388 & 9 & 0 & 9 & 10 & 1 & 10 & 1114.56 & 9.013676 & NU1 \\
\hline 3445.21 & 4.6179 & 0.0382 & 9 & 1 & 9 & 10 & 0 & 10 & 1114.55 & 9.013420 & NU 1 \\
\hline 3445.61 & 0.0504 & 0.0463 & 9 & 6 & 4 & 9 & 7 & 3 & 1810.63 & 3.116005 & NUI \\
\hline 3445.73 & 0.1513 & 0.0467 & 9 & 6 & 3 & 9 & 7 & 2 & 1810.63 & 3.116419 & NU1 \\
\hline 3445.79 & 0.0019 & 0.0915 & 4 & 3 & 2 & 3 & 0 & 3 & 136.77 & 051049 & 2 NU2* $*$ \\
\hline 3446.29 & 0.1327 & 0.0568 & 6 & 4 & 2 & 6 & 6 & 1 & 1045.07 & 721 & NU 3 \\
\hline $3446 \cdot 52$ & 0.0614 & 0.0674 & 4 & 4 & 1 & 3 & 3 & 0 & 285.43 & 148 & $2 \mathrm{NU} 2 *$ \\
\hline 3446.83 & 38.6857 & 0.0852 & 5 & 2 & 3 & 6 & 3 & 4 & 648.97 & $2 \cdot 4$ & NU 1 \\
\hline 3446.87 & 11.0009 & 0.0646 & 8 & 2 & 7 & 9 & 1 & 8 & 1079.07 & 89 & NUI \\
\hline 3446.95 & 0.0583 & 0.0610 & 7 & 4 & 3 & 7 & 6 & 2 & $1216 \cdot 20$ & 690 & NU3 \\
\hline 3447.08 & 39.8404 & 0.0680 & 3 & 3 & 1 & 4 & 4 & 0 & 488.13 & 90 & NUI \\
\hline 3447.25 & 119.6379 & 0.0683 & 3 & 3 & 0 & 4 & 4 & 1 & $488 \cdot 10$ & 148 & NU 1 \\
\hline $3447 \cdot 66$ & 0.0762 & 0.0563 & 10 & 5 & 6 & 10 & 4 & 7 & $1581 \cdot 34$ & 5.482102 & 2 NU2 \\
\hline 3448.40 & 13.7101 & 0.0869 & 6 & 1 & 5 & 7 & 3 & 4 & 842.38 & 214427 & NU3 \\
\hline $3448 \cdot 78$ & 0.3040 & 0.0440 & 8 & 6 & 3 & 8 & 7 & 2 & $1590 \cdot 74$ & 2.144879 & NU1 \\
\hline 3448.83 & 0.1013 & 0.0441 & 8 & 6 & 2 & 8 & 7 & 1 & 1590.74 & 2.144937 & NU 1 \\
\hline 3449.03 & 0.0015 & 0.0520 & 9 & 6 & 3 & 10 & 6 & 4 & 1875.53 & 6.440 & $3 *$ \\
\hline 3449.09 & 0.6291 & 0.0588 & 10 & 5 & 6 & 11 & 5 & 7 & 1985.83 & 8.747542 & NU3 \\
\hline 3449.36 & 0.0036 & 0.0889 & 5 & 1 & 4 & 6 & 3 & 3 & 2282.59 & 0.202769 & NU $3 * * *$ \\
\hline 3449.45 & 0.0013 & 0.0710 & 6 & 3 & 3 & 6 & 5 & 2 & 2552.85 & 0.077000 & NU $3 * * *$ \\
\hline 3449.47 & 0.0045 & 0.0508 & 9 & 6 & 4 & 10 & 6 & 5 & 1875.00 & 6.439836 & NU3* \\
\hline 3449.65 & 0.0024 & 0.0427 & 7 & 7 & 0 & 8 & 6 & 3 & 1411.65 & 0.074321 & $2 \mathrm{NU}_{2}$ \\
\hline 3449.66 & 0.0442 & 0.0674 & 11 & 3 & 8 & 10 & 4 & 7 & $1581 \cdot 34$ & 3.178818 & $2 \mathrm{NU} 2$ \\
\hline 3449.71 & 0.0462 & 0.0851 & 3 & 2 & 1 & 4 & 3 & 2 & 2004.81 & 2.320352 & NUI*** \\
\hline 3449.83 & 0.0021 & 0.0604 & 9 & 5 & 5 & 10 & 5 & 6 & 3383.36 & 7.532122 & NU $3 * * *$ \\
\hline 3449.87 & 0.0231 & 0.0459 & 13 & 2 & 11 & 1 & 3 & 10 & 2414.73 & 5.733 & NU 1 \\
\hline 3449.97 & 0.0023 & 0.0393 & 10 & 1 & 10 & 10 & 2 & 9 & 1293.66 & 51 & $1 *$ \\
\hline 3450.17 & 0.1377 & 0.0646 & 8 & 4 & 4 & 8 & 6 & 3 & 1411.65 & 0.037 & NU3 \\
\hline 3450.21 & 0.0032 & 0.0795 & 4 & 2 & 2 & 5 & 4 & 1 & 610.35 & 0.042 & NU $3 * *$ \\
\hline 3450.70 & 0.0031 & 0.0447 & 8 & 0 & 8 & 9 & 1 & 9 & 2512.43 & & NU $1 * * *$ \\
\hline $3451 \cdot 10$ & 0.0108 & 0.0443 & 8 & 1 & 8 & 9 & 0 & 9 & 2512.36 & 87 & NU $1 * * *$ \\
\hline $3451 \cdot 20$ & 0.0218 & 0.0620 & 10 & 3 & 8 & 10 & 5 & 5 & 1724.72 & 0.084234 & NC \\
\hline $3451 \cdot 24$ & 0.0022 & 0.032 & 14 & 2 & 13 & 14 & 1 & 14 & 2073.66 & 78 & $2 \mathrm{NC}$ \\
\hline 3451.89 & 0.1422 & 0.0409 & 7 & 6 & 2 & 7 & 7 & 1 & 1394.86 & 792 & NUI \\
\hline 3451.89 & 0.4266 & 0.0409 & 7 & 6 & 1 & 7 & 7 & 0 & 1394.86 & 1.119797 & NU1 \\
\hline 3452.08 & 0.0024 & 0.0879 & 7 & 3 & 4 & 6 & 2 & 5 & $2161 \cdot 30$ & 0.996364 & $2 \mathrm{NU} 2^{* * * *}$ \\
\hline 3453.15 & 0.2218 & 0.0431 & 9 & 7 & 2 & 10 & 7 & 3 & 2054.40 & 5.133660 & NU3 \\
\hline 3453.15 & 0.6654 & 0.0430 & 9 & 7 & 3 & 10 & 7 & 4 & 2054.40 & 5.133658 & NU3 \\
\hline 3453.39 & 0.3689 & 0.0754 & 9 & 3 & 7 & 8 & 2 & 6 & 982.91 & 4.004942 & $2 \mathrm{NU} 2$ \\
\hline 3453.81 & 0.0057 & 0.0948 & 3 & 1 & 3 & 4 & 2 & 2 & 1922.92 & 0.610552 & $N U 1 * * * *$ \\
\hline 3453.88 & 0.0269 & 0.0531 & 12 & 5 & 8 & 12 & 6 & 7 & 2433.85 & 6.631337 & NU1 \\
\hline 3453.92 & 0.0114 & 0.0674 & 4 & 4 & 1 & 3 & 3 & 0 & 285.43 & 3.393148 & $2 \mathrm{NU} 2 * *$ \\
\hline 3454.13 & 0.0038 & 0.0665 & 4 & 4 & 0 & 3 & 3 & 1 & 285.23 & 3.390390 & $2 \mathrm{NU} 2 * *$ \\
\hline 3454.51 & 0.0027 & 0.0759 & 7 & 2 & 6 & 8 & 1 & 7 & $2490 \cdot 37$ & 4.913532 & NU $1^{* * *}$ \\
\hline 3455.74 & 0.0280 & 0.0665 & 9 & 4 & 5 & 9 & 6 & 4 & 1631 . & 0.066893 & NU3 \\
\hline 3455.79 & 8.7020 & 0.0795 & 4 & 2 & 2 & 5 & 4 & 1 & 610.35 & 0.042579 & NU 3 \\
\hline
\end{tabular}




\begin{tabular}{|c|c|c|c|c|c|c|c|c|c|c|c|}
\hline $\begin{array}{l}\text { FREQUENCY } \\
\mathrm{cm}^{-1}\end{array}$ & $\begin{array}{l}\text { LINE } \\
\text { STRENGTH } \\
\frac{\mathrm{cm}^{-1}}{\mathrm{gm} \mathrm{cm}^{-2}}\end{array}$ & $\begin{array}{l}\text { HALF } \\
\text { WIDTH } \\
\mathrm{cm}^{-1} \\
\text { atm. }\end{array}$ & $J^{\prime}$ & $\mathrm{K}_{\mathrm{c}}$ & K & $J^{\prime \prime}$ & $K_{a}$ & $\mathrm{~K}_{\mathrm{c}}$ & $\begin{array}{c}E^{\prime \prime} \\
\mathrm{cm}^{-1}\end{array}$ & L & BAND \\
\hline 3456.25 & 2.0134 & 0.0810 & 10 & 4 & 6 & 11 & 4 & 7 & 1899.06 & 5.820000 & NU3- \\
\hline 3456.35 & 0.0039 & 0.0671 & 9 & 5 & 4 & 10 & 5 & 5 & 1724.72 & 7.545052 & NU $3 *$ \\
\hline 3456.57 & 0.0485 & 0.0653 & 7 & 1 & 6 & 8 & 2 & 7 & 885.62 & 4.996649 & NU $1 *$ \\
\hline 3456.72 & 1.1622 & 0.0393 & 10 & 1 & 10 & 10 & 2 & 9 & 1293.66 & 1.847651 & NU1 \\
\hline 3457.15 & 0.0118 & 0.0757 & 3 & 2 & 2 & 4 & 4 & 1 & $488 \cdot 10$ & 0.015552 & NU $3 *$ \\
\hline $3457 \cdot 18$ & 0.0507 & 0.0865 & 4 & 2 & 2 & 5 & 3 & 3 & 504.00 & 2.324407 & NU I* \\
\hline $3457 \cdot 21$ & 0.0030 & 0.0563 & 10 & 2 & 8 & 11 & 2 & 9 & $3314 \cdot 91$ & 9.932549 & NU $3 * * *$ \\
\hline $3457 \cdot 28$ & 0.0022 & 0.0634 & 6 & 2 & 5 & 5 & 5 & 0 & $742 \cdot 10$ & 0.000220 & NUI \\
\hline 3457.33 & 0.3901 & 0.0397 & 10 & 0 & 10 & 10 & 1 & 9 & 1293.04 & 1.848326 & NU 1 \\
\hline 3457.46 & 0.1608 & 0.0658 & 9 & 2 & 8 & 9 & 4 & 5 & $1360 \cdot 28$ & 0.033499 & NU3 \\
\hline $3457 \cdot 50$ & 2.2123 & 0.0887 & 6 & 3 & 3 & 5 & 2 & 4 & $416 \cdot 22$ & 1.411171 & $2 \mathrm{NU} 2$ \\
\hline 3457.99 & 0.6239 & 0.0928 & 6 & 2 & 4 & 5 & 1 & 5 & 326.64 & 0.254241 & $2 \mathrm{NU} 2$ \\
\hline 3458.02 & 0.0434 & 0.0467 & 12 & 3 & 10 & 12 & 4 & 9 & $2124 \cdot 98$ & 5.350112 & NUl \\
\hline 3458.15 & 0.0052 & 0.0764 & 6 & 1 & 5 & 7 & 2 & 6 & 2318.53 & 4.008107 & NU $1^{* * *}$ \\
\hline 3458.24 & 0.0061 & 0.0742 & 5 & 4 & 2 & 4 & 3 & 1 & 2005.92 & 3.270203 & $2 \mathrm{NU} 2^{* * *} *$ \\
\hline 3458.36 & 0.0010 & 0.0650 & 8 & 4 & 5 & 8 & 5 & 4 & 2919.63 & 3.901175 & NU1**** \\
\hline 3458.37 & 0.0099 & 0.0875 & 5 & 0 & 5 & 6 & 2 & 4 & 602.77 & 0.069891 & NU $3 *$ \\
\hline 3458.60 & 0.9428 & 0.0475 & 11 & 2 & 9 & 12 & 2 & 10 & 1960.22 & 10.893773 & NU3 \\
\hline 3458.67 & 0.1033 & 0.0425 & 11 & 2 & 10 & 11 & 3 & 9 & 1695.03 & 3.632655 & NU1 \\
\hline 3458.70 & 0.7092 & 0.0338 & 12 & 2 & 11 & 13 & 2 & 12 & $2042 \cdot 38$ & 12.275740 & NU3 \\
\hline 3458.70 & 2.1229 & 0.0338 & 12 & 1 & 11 & 13 & 1 & 12 & 2042.33 & 12.276740 & NU3 \\
\hline 3458.72 & 2.3526 & 0.0447 & 11 & 3 & 9 & 12 & 3 & 10 & $1962 \cdot 55$ & 10.873594 & NU3 \\
\hline 3458.84 & 0.0157 & 0.0549 & 11 & 5 & 7 & 11 & 6 & 6 & 2142.69 & 5.799382 & NU1 \\
\hline 3459.44 & 0.0182 & 0.0719 & 5 & 4 & 1 & 4 & 3 & 2 & $2004 \cdot 81$ & 3.250024 & $2 \mathrm{NU} 2 * * * *$ \\
\hline 3459.59 & 0.0124 & 0.0604 & 9 & 5 & 5 & 10 & 5 & 6 & $1718 \cdot 77$ & 7.532122 & NU3* \\
\hline 3459.69 & 0.0090 & 0.0653 & 7 & 1 & 6 & 8 & 2 & 7 & 885.62 & 4.996649 & NUI** \\
\hline 3459.84 & 0.1591 & 0.0494 & 11 & 2 & 9 & 10 & 3 & 8 & $1446 \cdot 16$ & 5.859437 & $2 \mathrm{NU} 2$ \\
\hline 3459.88 & 0.0094 & 0.0865 & 4 & 2 & 2 & 5 & 3 & 3 & 504.00 & 2.324407 & NUl ${ }^{*} *$ \\
\hline 3459.98 & 0.1597 & 0.0554 & 10 & 5 & 6 & 10 & 6 & 5 & 1875.00 & 4.913223 & NUI \\
\hline 3460.19 & 0.0654 & 0.0443 & 8 & 1 & 8 & 9 & 0 & 9 & $920 \cdot 18$ & 8.007087 & NUI \\
\hline $3460 \cdot 35$ & 0.0082 & 0.0866 & 4 & 1 & 4 & 5 & 3 & 3 & 504.00 & 0.035251 & NU $3 *$ \\
\hline 3460.36 & 5.1431 & 0.0752 & 10 & 3 & 7 & 11 & 3 & 8 & $1813 \cdot 26$ & 9.899853 & NU3 \\
\hline 3460.58 & 0.5918 & 0.0320 & 13 & 0 & 13 & 14 & 0 & 14 & 2073.66 & 13.743940 & NU3 \\
\hline 3460.58 & 1.7862 & 0.0320 & 13 & 1 & 13 & 14 & 1 & 14 & 2073.66 & 13.743930 & NU3 \\
\hline 3460.60 & 0.0014 & 0.0691 & 11 & 6 & 6 & 12 & 5 & 7 & $2300 \cdot 80$ & 1.049439 & NU1 \\
\hline $3461 \cdot 07$ & 0.9720 & 0.0915 & 4 & 3 & 2 & 3 & 0 & 3 & 136.77 & 0.051049 & $2 \mathrm{NU} 2$ \\
\hline 3461.26 & 0.0528 & 0.0332 & 13 & 1 & 12 & 12 & 2 & 11 & $1774 \cdot 74$ & 9.99731 & $2 N \cup 2$ \\
\hline $3461 \cdot 32$ & 30.7223 & 0.0674 & 4 & 4 & 1 & 3 & 3 & 0 & 285.43 & 3.393148 & 2 NU2 \\
\hline 3461.53 & 10.2433 & 0.0665 & 4 & 4 & 0 & 3 & 3 & 1 & $285 \cdot 23$ & 3.390390 & $2 \mathrm{NU} 2$ \\
\hline $3461 \cdot 65$ & $1 \cdot 3626$ & 0.0603 & 10 & 4 & 7 & 11 & 4 & 8 & 1843.02 & 9.438945 & NU3 \\
\hline 3461.70 & 0.0176 & 0.0331 & 13 & 2 & 12 & 12 & 1 & 11 & 1774.60 & 9.99954 & 2NU2 \\
\hline $3461 \cdot 94$ & 0.0040 & 0.0843 & 5 & 0 & 5 & 5 & 3 & 2 & $508 \cdot 81$ & 0.062476 & NUI* \\
\hline $3462 \cdot 58$ & 25.3423 & 0.0865 & 4 & 2 & 2 & 5 & 3 & 3 & 504.00 & 2.324407 & NU1 \\
\hline $3462 \cdot 82$ & 24.2543 & 0.0653 & 7 & 1 & 6 & 8 & 2 & 7 & 885.62 & 4.996649 & NUI \\
\hline 3462.83 & 0.3113 & 0.0445 & 11 & 1 & 10 & 11 & 2 & 9 & $1690 \cdot 70$ & 3.646421 & NU1 \\
\hline $3462 \cdot 90$ & 0.0161 & 0.0759 & 7 & 2 & 6 & 8 & 1 - & 7 & 882.93 & 4.913532 & NUI* \\
\hline $3462 \cdot 95$ & 0.0021 & 0.0500 & 8 & 6 & 2 & 9 & 6 & 3 & $3321 \cdot 00$ & 5.032058 & NU $3 * * *$ \\
\hline $3462 \cdot 98$ & 0.0045 & 0.0648 & 9 & 4 & 6 & 10 & 4 & 7 & $3224 \cdot 55$ & 8.354292 & NU $3 * * *$ \\
\hline 3463.19 & 0.0019 & 0.0887 & 8 & 4 & 5 & 9 & 3 & 6 & $1282 \cdot 92$ & 1.416234 & NUI* \\
\hline 3463.50 & 0.0041 & 0.0757 & 3 & 2 & 1 & 4 & 4 & 0 & $488 \cdot 13$ & 0.016542 & NU3* \\
\hline 3463.53 & 0.0121 & 0.0443 & 8 & 1 & 8 & 9 & 0 & 9 & 920.18 & 8.007087 & NU $1 *$ * \\
\hline $3464 \cdot 24$ & 0.0014 & 0.0616 & 7 & 2 & 6 & 6 & 5 & 1 & 888.63 & 0.000884 & NU 1 \\
\hline $3464 \cdot 25$ & 0.7443 & 0.0520 & 9 & 6 & 3 & 10 & 6 & 4 & 1875.53 & 6.440055 & NU3 \\
\hline $3464 \cdot 25$ & 0.0013 & 0.0407 & 8 & 7 & 2 & 9 & 7 & 3 & 1810.63 & 3.579253 & NU $3 *$ \\
\hline
\end{tabular}




\begin{tabular}{|c|c|c|c|c|c|c|c|c|c|c|c|}
\hline $\begin{array}{c}\text { FREQUENCY } \\
\mathrm{cm}^{-1}\end{array}$ & $\begin{array}{l}\text { LINE } \\
\text { STRENGTH } \\
\frac{\mathrm{cm}^{-1}}{\mathrm{gm} \mathrm{cm}^{-2}}\end{array}$ & $\begin{array}{l}\text { HALF } \\
\text { WIDTH } \\
\frac{\mathrm{cm}^{-1}}{a \dagger m .}\end{array}$ & $J^{\prime}$ & $K_{a}$ & $\mathrm{~K}_{\mathrm{c}}$ & J" & $\mathrm{K}_{\mathrm{a}}$ & $K_{c}$ & $\begin{array}{c}E^{\prime \prime} \\
\mathrm{cm}^{-1}\end{array}$ & $L$ & BAND \\
\hline 3464.25 & 0.0037 & 0.0408 & 8 & 7 & 1 & 9 & 7 & 2 & 1810.63 & 3.579254 & NU3* \\
\hline 3464.66 & 2.2332 & 0.0508 & 9 & 6 & 4 & 10 & 6 & 5 & 1875.00 & 6.439836 & NU3 \\
\hline 3465.88 & 0.1099 & 0.0354 & 8 & 8 & 1 & 9 & 8 & 2 & 2009.87 & 1.901685 & NU3 \\
\hline 3465.88 & 0.3298 & 0.0354 & 8 & 8 & 0 & 9 & 8 & 1 & 2009.87 & 1.901685 & NU3 \\
\hline 3465.88 & 0.0018 & 0.0875 & 5 & 0 & 5 & 6 & 2 & 4 & 602.77 & 0.069891 & NU $3 * *$ \\
\hline 3466.04 & 0.0630 & 0.0751 & 5 & 0 & 5 & 5 & 4 & 2 & 610.12 & 0.000921 & NU3 \\
\hline 3466.05 & 0.4584 & 0.0657 & 10 & 3 & 8 & 9 & 2 & 7 & 1201.95 & 4.932263 & $2 \mathrm{NU} 2$ \\
\hline 3466.09 & 0.0039 & 0.0585 & 8 & 2 & 7 & 7 & 5 & 2 & 1059.85 & 0.001936 & NU1 \\
\hline 3466.12 & 1.4345 & 0.0608 & 8 & 4 & 4 & 9 & 1 & 9 & 920.21 & 12.48 & NU2+ \\
\hline 3466.33 & 0.0048 & 0.0898 & 7 & 3 & 5 & 8 & 2 & 6 & 982.91 & 2.422109 & NUI $*$ \\
\hline 3466.62 & 0.0670 & 0.0845 & 7 & 3 & 4 & 7 & 0 & 7 & $586 \cdot 26$ & 0.033256 & $2 \mathrm{NU} 2$ \\
\hline 3466.87 & 32.7206 & 0.0443 & 8 & 1 & 8 & 9 & 0 & 9 & 920.18 & 8.007087 & NUI \\
\hline 3467.04 & 9.2767 & 0.0447 & 8 & 0 & 8 & 9 & 1 & 9 & 920.21 & 6.81 & NUI- \\
\hline 3467.50 & 1.9978 & 0.0843 & 5 & 0 & 5 & 5 & 3 & 2 & 508.81 & 0.062476 & NU1 \\
\hline 3467.96 & 0.0267 & 0.0534 & 12 & 4 & 9 & 12 & 5 & 8 & 2275.44 & 6.558344 & NUI \\
\hline 3468.28 & 0.0030 & 0.0530 & 8 & 1 & 8 & 8 & 2 & 7 & 2495.18 & 1.833129 & NUI**** \\
\hline 3468.60 & 5.8795 & 0.0757 & 3 & 2 & 2 & 4 & 4 & 1 & $488 \cdot 10$ & 0.015552 & NU3 \\
\hline 3469.14 & 0.0021 & 0.0680 & 7 & 4 & 3 & 7 & 5 & 2 & 2724.19 & 3.039271 & NUI $* * * *$ \\
\hline 3469.18 & 0.0211 & 0.0320 & 14 & 0 & 14 & 14 & 2 & 13 & 2327.90 & 0.488990 & NU3 \\
\hline 3469.20 & 0.0864 & 0.0858 & 3 & 2 & 2 & 4 & 3 & 1 & 383 & 2.164905 & NU $1 *$ \\
\hline 3469.20 & 0.0070 & 0.0320 & 14 & 1 & 14 & 14 & 1 & 13 & 2327.88 & 0.489010 & NU3 \\
\hline 3469.41 & 0.0121 & 0.0817 & 9 & 3 & 6 & 10 & 3 & 7 & 1538.23 & 9.029655 & NU3* \\
\hline 3469.49 & 0.1636 & 0.0834 & 5 & 4 & 1 & 5 & 1 & 4 & 399.46 & 0.031887 & $2 \mathrm{NU} 2$ \\
\hline 3469.61 & 0.0025 & 0.0620 & 6 & 4 & 3 & 6 & 5 & 2 & 2552.85 & $1 \cdot 56$ & NU $1 * * * *$ \\
\hline 3469.94 & 8.0734 & 0.0759 & 7 & 2 & 6 & 8 & 1 & 7 & 882.93 & 4.913532 & NU1 \\
\hline 3470.16 & 0.0122 & 0.0742 & 5 & 4 & 2 & 4 & 3 & 1 & 383.85 & 3.270203 & $2 N \cup 2 *$ \\
\hline 3470.50 & 3.0644 & 0.0824 & 9 & 4 & 5 & 10 & 4 & 6 & 1616.51 & 6.830000 & NU3- \\
\hline 3470.86 & 0.0270 & 0.0648 & 9 & 4 & 6 & 10 & 4 & 7 & $1581 \cdot 34$ & 8.354292 & NU $3 *$ \\
\hline $3471 \cdot 12$ & 0.0771 & 0.0841 & 2 & 2 & 1 & 3 & 3 & 0 & 1907.63 & 2.366160 & NU $1 * * * *$ \\
\hline 3471.21 & 0.0028 & 0.0578 & 5 & 4 & 1 & 5 & 5 & 0 & $2406 \cdot 15$ & 0.826 & NUl $* * * *$ \\
\hline $3471 \cdot 24$ & 0.0191 & 0.0840 & 4 & 4 & 0 & 4 & 1 & 3 & 275.52 & 0.005990 & $2 N \cup 2$ \\
\hline 3471.62 & 0.2181 & 0.0645 & 9 & 3 & 7 & 9 & 5 & 4 & 1477.31 & 0.080993 & NU3 \\
\hline 3471.64 & 0.0365 & 0.0719 & 5 & 4 & 1 & 4 & 3 & 2 & 382.52 & 3.250024 & $2 \mathrm{NU} 2^{*}$ \\
\hline 3471.87 & 0.0229 & 0.0534 & 7 & 0 & 7 & 8 & 1 & 8 & 2337.70 & 7.000291 & NU1 $* * * *$ \\
\hline 3471.89 & 1.9665 & 0.0671 & 9 & 5 & 4 & 10 & 5 & 5 & $1724 \cdot 72$ & 7.545052 & NU3 \\
\hline 3472.10 & 0.0160 & 0.0858 & 3 & 2 & 2 & 4 & 3 & 1 & 383.85 & 2.164905 & NU $1 \ddot{*} \ddot{*}$ \\
\hline 3472.32 & 0.0076 & 0.0542 & 7 & 1 & 7 & 8 & 0 & 8 & 2337.53 & 5.998008 & NU 1 㞔些 \\
\hline 3472.56 & 0.0023 & 0.0451 & 9 & 1 & 9 & 9 & 2 & 8 & $1080 \cdot 38$ & 1.842036 & NU $1 *$ \\
\hline 3472.83 & 0.0260 & 0.0830 & 2 & 2 & 0 & 3 & 3 & 1 & 1907.47 & 2.394805 & $N \cup 1 * * *$ \\
\hline 3472.85 & 0.0267 & 0.0842 & 5 & 1 & 4 & 6 & 2 & 5 & $2161 \cdot 30$ & 3.129447 & NUI \\
\hline 3473.09 & 0.0708 & 0.0800 & 6 & 4 & 2 & 6 & 1 & 5. & 542.91 & 0.084732 & $2 \mathrm{NU} 2$ \\
\hline $3473 \cdot 30$ & 4.1084 & 0.0866 & 4 & 1 & 4 & 5 & 3 & 3 & .00 & 0.035251 & NU3 \\
\hline 3473.39 & 4.9709 & 0.0875 & 5 & 0 & 5 & 6 & 2 & 4 & 602.77 & 0.069891 & NU3 \\
\hline 3473.46 & 0.9471 & 0.0887 & 8 & 4 & 5 & 9 & 3 & 6 & 1282.92 & 1.416234 & NU 1 \\
\hline 3473.69 & 0.0068 & 0.0459 & 9 & 0 & 9 & 9 & 1 & 8 & 1079.07 & 1.844131 & NUI* \\
\hline 3473.98 & 0.0343 & 0.0948 & 3 & 1 & 3 & 4 & 2 & 2 & 315.79 & 0.610552 & NUI* \\
\hline 3474.55 & 0.0314 & 0.0764 & 6 & 1 & 5 & 7 & 2 & 6 & 709.60 & 4.008107 & NUI* \\
\hline 3474.73 & 6.2214 & 0.0604 & 9 & 5 & 5 & 10 & 5 & 6 & 1718.77 & 7.532122 & NU3 \\
\hline $3474 \cdot 78$ & 2.0881 & 0.0757 & 3 & 2 & 1 & 4 & 4 & 0 & $488 \cdot 13$ & 0.016542 & NU3 \\
\hline 3474.90 & 0.0056 & 0.0640 & 8 & 5 & 3 & 9 & 5 & 4 & 3141.06 & 6.258032 & NU $3 * * *$ \\
\hline 3474.96 & 0.0215 & 0.0889 & 5 & 1 & 4 & 6 & 3 & $\underline{2}$ & $661 \cdot 56$ & 0.202769 & NU $3 *$ \\
\hline 3474.97 & 2.4177 & 0.0898 & 7 & 3 & 5 & 8 & 2 & 6 & 982.91 & 2.422109 & NU1 \\
\hline 3475.01 & 43.1955 & 0.0858 & 3 & 2 & 2 & 4 & 3 & 1 & 383.85 & 2.164905 & NUl \\
\hline 3475.63 & 0.0012 & 0.0739 & 7 & 1 & 7 & 7 & 3 & 4 & $842 \cdot 38$ & 0.009220 & NU3* \\
\hline
\end{tabular}




\begin{tabular}{|c|c|c|c|c|c|c|c|c|c|c|c|}
\hline $\begin{array}{c}\text { FREQUENCY } \\
\mathrm{cm}^{-1}\end{array}$ & $\begin{array}{l}\text { LINE } \\
\text { STRENGTH } \\
\frac{\mathrm{cm}^{-1}}{\mathrm{gm} \mathrm{cm}^{-2}}\end{array}$ & $\begin{array}{l}\text { HALF } \\
\text { WIDTH } \\
\frac{\mathrm{cm}^{-1}}{\text { atm. }}\end{array}$ & $J^{\prime}$ & $K_{a}$ & $K_{c}$ & Ј" & $k_{a}$ & $K_{c}$ & $\begin{array}{c}E^{\prime \prime} \\
\mathrm{cm}^{-1}\end{array}$ & L & BAND \\
\hline 3475.77 & 0.0018 & 0.0607 & 8 & 5 & 4 & 9 & 5 & 5 & 3139.50 & 6.255627 & NU $3 * * * *$ \\
\hline 3475.93 & 0.0043 & 0.0496 & 8 & 6 & 3 & 9 & 6 & 4 & 1631.27 & 5.032027 & NU $3 *$ \\
\hline 3475.94 & 0.0128 & 0.0500 & 8 & 6 & 2 & 9 & 6 & 3 & 1631.41 & 5.032058 & NU $3 *$ \\
\hline 3476.41 & 0.0541 & 0.0608 & 10 & 5 & 5 & 10 & 6 & 4 & 1875.53 & 4.974368 & NU1 \\
\hline 3476.44 & 0.0098 & 0.0644 & 12 & 5 & 7 & 12 & 6 & 6 & 2437.56 & 7.235377 & NUI \\
\hline 3476.67 & 0.2802 & 0.0851 & 3 & 2 & 1 & 4 & 3 & 2 & $382 \cdot 52$ & 2.320352 & NU ${ }^{*} *$ \\
\hline 3476.74 & 0.0022 & 0.0817 & 9 & 3 & 6 & 10 & 3 & 7 & $1538 \cdot 23$ & 9.029655 & NU3** \\
\hline 3477.02 & 0.1579 & 0.0558 & 9 & 5 & 5 & 9 & 6 & 4 & 1631.27 & 4.001691 & NUI \\
\hline 3477.17 & 0.0013 & 0.0459 & 9 & 0 & 9 & 9 & 1 & 8 & 1079.07 & 1.844131 & NU I*** \\
\hline 3477.27 & 0.0491 & 0.0629 & 11 & 5 & 6 & 11 & 6 & 5 & $2144 \cdot 12$ & 6.009423 & NU 1 \\
\hline $3477 \cdot 36$ & 0.0063 & 0.0948 & 3 & 1 & 3 & 4 & 2 & 2 & 315.79 & 0.610552 & NUI** \\
\hline 3477.36 & 0.4756 & 0.0583 & 9 & 5 & 4 & 9 & 6 & 3 & 1631.41 & 4.017231 & NUI \\
\hline 3477.43 & 0.0049 & 0.0477 & 6 & 5 & 2 & 6 & 6 & 1 & 1045.07 & 1.111607 & NUI $*$ \\
\hline 3477.44 & 0.0016 & 0.0478 & 6 & 5 & 1 & 6 & 5 & 0 & 1045.07 & 1.111654 & NU I* \\
\hline 3477.59 & 0.0022 & 0.0742 & 5 & 4 & 2 & 4 & 3 & 1 & 383.85 & 3.270203 & $2 \operatorname{NU} 2 * *$ \\
\hline 3477.61 & 0.0058 & 0.0764 & 6 & 1 & 5 & 7 & 2 & 6 & 709.60 & 4.008107 & NU $1 * *$ \\
\hline 3477.71 & 0.3601 & 0.0556 & 8 & 5 & 3 & 8 & 6 & 2 & $1411 \cdot 68$ & 3.077042 & NU 1 \\
\hline 3477.78 & 1.0793 & 0.0545 & 8 & 5 & 4 & 8 & 6 & 3 & 1411.65 & 3.073714 & NUI \\
\hline 3477.79 & 0.0157 & 0.0898 & 4 & 1 & 3 & 5 & 3 & 2 & 2130.50 & 0.137702 & $\operatorname{NU} 3 * * *$ \\
\hline 3477.88 & 0.0714 & 0.0528 & 11 & 3 & 9 & 11 & 4 & 8 & 1843.02 & 5.243402 & NU 1 \\
\hline 3478.29 & 0.0050 & 0.0648 & 9 & 4 & 6 & 10 & 4 & 7 & $1581 \cdot 34$ & 8.354292 & NU $3 \ddot{*} *$ \\
\hline 3478.67 & 0.0032 & 0.0666 & 9 & 2 & 7 & 10 & 2 & 8 & 3058.41 & 9.000870 & NU $3 * * *$ \\
\hline 3479.03 & 0.0067 & 0.0719 & 5 & 4 & 1 & 4 & 3 & 2 & $382 \cdot 52$ & 3.250024 & $2 N \cup 2 * *$ \\
\hline 3479.06 & 0.0095 & 0.0800 & 6 & 4 & 3 & 5 & 3 & 2 & 2130.50 & 3.153879 & $2 \mathrm{NU} 2 * * *$ \\
\hline 3479.37 & 0.6232 & 0.0407 & 8 & 7 & 2 & 9 & 7 & 3 & 1810.63 & 3.579253 & NU3 \\
\hline 3479.37 & 1.8697 & 0.0408 & 8 & 7 & 1 & 9 & 7 & 2 & 1810.63 & 3.579254 & $\mathrm{NU}_{3}$ \\
\hline $3479 \cdot 38$ & 1.1312 & 0.0451 & 9 & 1 & 9 & 9 & 2 & 8 & 1080.38 & 1.842036 & NU 1 \\
\hline 3479.45 & 0.0518 & 0.0851 & 3 & 2 & 1 & 4 & 3 & 2 & 382.52 & 2.320352 & NU $1 * * *$ \\
\hline 3479.58 & 0.6609 & 0.0518 & 7 & 5 & 3 & 7 & 6 & 2 & $1216 \cdot 20$ & 2.119434 & NU 1 \\
\hline 3479.60 & 1.9832 & 0.0521 & 7 & 5 & 2 & 7 & 6 & 1 & $1216 \cdot 20$ & 2.119964 & NUI \\
\hline 3479.77 & 0.0415 & 0.0579 & 11 & 4 & 8 & 11 & 5 & 7 & 1985.83 & 6.097371 & NUI \\
\hline 3480.22 & 1.0608 & 0.0486 & 10 & 2 & 9 & 10 & 3 & 8 & 1446.16 & 3.605841 & NU1 \\
\hline 3480.31 & 0.0400 & 0.0667 & 10 & 4 & 6 & 10 & 6 & 5 & 1875.00 & 0.114652 & NU3 \\
\hline 3480.39 & 9.0825 & 0.0563 & 10 & 2 & 8 & 11 & 2 & 9 & 1690.70 & 9.932549 & NU 3 \\
\hline 3480.65 & 2.2914 & 0.0361 & 11 & 1 & 10 & 12 & 1 & 11 & 1774.60 & 11.275820 & NU 3 \\
\hline 3480.65 & 6.8739 & 0.0360 & 11 & 2 & 10 & 12 & 2 & 11 & 1774.74 & 11.275004 & NU3 \\
\hline 3480.66 & 3.4212 & 0.0459 & 9 & 0 & 9 & 9 & 1 & 8 & 1079.07 & 1.844131 & NU 1 \\
\hline 3480.67 & 15.7126 & 0.0764 & 6 & 1 & 5 & 7 & 2 & 6 & 709.60 & 4.008107 & NUI \\
\hline 3480.74 & 17.1473 & 0.0948 & 3 & 1 & 3 & 4 & 2 & 2 & 315.79 & 0.610552 & NU1 \\
\hline 3480.89 & 3.0124 & 0.0515 & 10 & 3 & 8 & 11 & 3 & 9 & 1695.03 & 9.881611 & NU 3 \\
\hline 3481.06 & 0.0118 & 0.0815 & 8 & 4 & 4 & 9 & 4 & 5 & 2998.78 & 7.302001 & NU $3 * * *$ \\
\hline $3481 \cdot 37$ & 0.1388 & 0.0534 & 7 & 0 & 7 & 8 & 1 & 8 & 744.16 & 7.000291 & NU $1 *$ \\
\hline $3481 \cdot 37$ & 0.0073 & 0.0402 & 10 & 1 & 9 & 11 & 1 & 10 & 3135.82 & 10.283342 & NU $3 * * *$ \\
\hline 3481.55 & 0.0463 & 0.0542 & 7 & 1 & 7 & 8 & 0 & 8 & 744.09 & 6.998008 & NU1* \\
\hline 3481.63 & 2.4445 & 0.0477 & 6 & 5 & 2 & 6 & 6 & 1 & 1045.07 & 1.111607 & NU1 \\
\hline 3481.64 & 0.8149 & 0.0478 & 6 & 5 & 1 & 6 & 6 & 0 & 1045.07 & 1.111654 & NU 1 \\
\hline 3481.67 & 0.0040 & 0.0889 & 5 & 1 & 4 & 6 & 3 & 3 & $661 \cdot 56$ & 0.202769 & NU3** \\
\hline 3482.24 & 140.1098 & 0.0851 & 3 & 2 & 1 & 4 & 3 & 2 & 382.52 & 2.320352 & NU 1 \\
\hline 3482.41 & 2.2189 & 0.0325 & 12 & 1 & 12 & 13 & 1 & 13 & 1806.78 & 12.744430 & NU 3 \\
\hline 3482.41 & 6.6590 & 0.0325 & 12 & 0 & 12 & 13 & 0 & 13 & 1806.78 & 12.739050 & NU3 \\
\hline 3482.57 & 0.0148 & 0.033 & 14 & 2 & 13 & 13 & 1 & 12 & 2042.33 & 9.74091 & 2 NU2 2 \\
\hline 3482.73 & 0.0018 & 0.0675 & 7 & 3 & 5 & 7 & 5 & 2 & 1059.85 & 0.041795 & NU $3 *$ \\
\hline 3483.15 & 0.0031 & 0.0755 & 6 & 4 & 2 & 5 & 3 & 3 & 2126.41 & 3.066224 & $2 N \cup 2 * * *$ \\
\hline 3483.53 & 0.0023 & 0.0500 & 8 & 6 & 2 & 9 & 6 & 3 & 163.1 .41 & 5.032058 & NU $3 * *$ \\
\hline
\end{tabular}




\begin{tabular}{|c|c|c|c|c|c|c|c|c|c|c|c|}
\hline $\begin{array}{c}\text { FREQUENCY } \\
\mathrm{cm}^{-1}\end{array}$ & $\begin{array}{l}\text { LINE } \\
\text { STRENGTH } \\
\frac{\mathrm{cm}^{-1}}{\mathrm{gm} \mathrm{cm}^{-2}}\end{array}$ & $\begin{array}{l}\text { HALF } \\
\text { WIDTH } \\
\frac{\mathrm{cm}^{-1}}{a+m .}\end{array}$ & $J^{\prime}$ & $\mathrm{K}_{\mathrm{a}}$ & $K_{c}$ & J" & $\mathrm{K}_{\mathrm{a}}$ & $K_{c}$ & $\begin{array}{c}E^{\prime \prime} \\
\mathrm{cm}-1\end{array}$ & L & BAND \\
\hline 3484.07 & 6.0352 & 0.0817 & 9 & 3 & 6 & 10 & 3 & 7 & 1538.23 & 9.029655 & $N \cup 3$ \\
\hline $3484 \cdot 12$ & 0.1527 & 0.0756 & 7 & 4 & 3 & 7 & 1 & 6 & $704 \cdot 22$ & 0.135960 & $2 N \cup 2$ \\
\hline 3484.40 & 0.0340 & 0.0640 & 8 & 5 & 3 & 9 & 5 & 4 & 1477.31 & 6.258032 & NU $3 *$ \\
\hline 3484.73 & 0.0257 & 0.0534 & 7 & 0 & 7 & 8 & 1 & 8 & $744 \cdot 16$ & 7.000291 & NUl \\
\hline 3484.93 & 0.0086 & 0.0542 & 7 & 1 & 7 & 8 & 0 & 8 & 744.09 & 6.998008 & NU $1 * *$ \\
\hline 3485.03 & 6.0755 & 0.0742 & 5 & 4 & 2 & 4 & 3 & 1 & 383.85 & 3.270203 & 2NU2 \\
\hline 3485.11 & 0.1744 & 0.0669 & 8 & 3 & 6 & 8 & 5 & 3 & 1255.92 & 0.064192 & NU3 \\
\hline 3485.48 & 0.0123 & 0.0687 & 6 & 1 & 6 & 5 & 4 & 1 & 610.35 & 0.000636 & NU 1 \\
\hline 3485.72 & 13.4802 & 0.0648 & 9 & 4 & 6 & 10 & 4 & 7 & $1581 \cdot 34$ & 8.354292 & NU3 \\
\hline 3485.92 & 0.0710 & 0.0815 & 8 & 4 & 4 & 9 & 4 & 5 & 1360.28 & 7.302001 & NU3* \\
\hline 3486.06 & 0.0019 & 0.0870 & 4 & 0 & 4 & 4 & 3 & 1 & 383.85 & 0.047652 & NU I K \\
\hline 3486.43 & 18.2424 & 0.0719 & 5 & 4 & 1 & 4 & 3 & 2 & $382 \cdot 52$ & 3.250024 & 2 NU2 2 \\
\hline 3486.52 & 0.1040 & 0.0770 & 4 & 0 & 4 & 4 & 4 & 1 & $488 \cdot 10$ & 0.000274 & NU3 \\
\hline 3486.71 & 0.0037 & 0.0547 & 11 & 6 & 5 & 12 & 5 & 8 & $2275 \cdot 44$ & 0.908981 & NU1 \\
\hline 3486.74 & 0.0138 & 0.0901 & 4 & 1 & 3 & 5 & 2 & 4 & $2024 \cdot 17$ & 2.444582 & NU $1 * * * *$ \\
\hline 3486.97 & 0.0185 & 0.0424 & 12 & 2 & 10 & 11 & 3 & 9 & 1695.03 & 6.964699 & $2 \mathrm{NU} 2$ \\
\hline 3487.05 & 0.0048 & 0.0879 & 7 & 3 & 4 & 6 & 2 & 5 & 552.92 & 0.996364 & $2 N \cup 2 *$ \\
\hline 3487.68 & 0.0197 & 0.0666 & 9 & 2 & 7 & 10 & 2 & 8 & 1438.00 & 9.000870 & NU3* \\
\hline 3487.78 & 0.3733 & 0.0520 & 10 & 1 & 9 & 10 & 2 & 8 & 1438.00 & 3.646815 & NU1 \\
\hline 3487.82 & 0.0922 & 0.0848 & 6 & 2 & 5 & 7 & 1 & 6 & 704.22 & 3.799063 & NU $1 *$ \\
\hline 3488.09 & 69.4023 & 0.0534 & 7 & 0 & 7 & 8 & 1 & 8 & $744 \cdot 16$ & 7.000291 & NU1 \\
\hline $3488 \cdot 31$ & 23.1376 & 0.0542 & 7 & 1 & 7 & 8 & 0 & 8 & 744.09 & 6.998008 & NU 1 \\
\hline $3488 \cdot 38$ & 10.7964 & 0.0889 & 5 & 1 & 4 & 6 & 3 & 3 & 661.56 & 0.202769 & NU3 \\
\hline $3488 \cdot 74$ & 0.0044 & 0.0469 & 7 & 6 & 2 & 8 & 6 & 3 & $3101 \cdot 19$ & 3.522863 & NU $3 * * *$ \\
\hline 3489.03 & 0.4267 & 0.0613 & 10 & 4 & 7 & 10 & 5 & 6 & 1718.77 & 5.482102 & NU1 \\
\hline 3489.11 & 0.0056 & 0.0658 & 11 & 4 & 7 & 11 & 6 & 6 & 2142.69 & 0.195288 & NU 3 \\
\hline 3489.33 & 0.0026 & 0.0715 & 5 & 1 & 5 & 4 & 4 & 0 & $488 \cdot 13$ & 0.000220 & NU1 \\
\hline 3489.46 & 0.0012 & 0.0613 & 9 & 1 & 8 & 9 & 2 & 7. & $2818 \cdot 40$ & 3.674255 & NU $1 * * * *$ \\
\hline 3489.56 & 0.0191 & 0.0864 & 8 & 3 & 5 & 9 & 3 & 6 & 2904.69 & 8.066869 & NU $3 * * * *$ \\
\hline 3490.29 & 0.0020 & 0.0380 & 7 & 7 & 0 & 8 & 7 & 1 & $1590 \cdot 74$ & 1.887603 & NU $3 *$ \\
\hline 3490.29 & 0.0058 & 0.0380 & 7 & 7 & 1 & 8 & 7 & 2 & 1590.74 & 1.887603 & NU3* \\
\hline 3490.54 & 0.5967 & 0.0739 & 7 & 1 & 7 & 7 & 3 & 4 & $842 \cdot 38$ & 0.009220 & NU3 \\
\hline 3490.55 & 0.1619 & 0.0842 & 5 & 1 & 4 & 6 & 2 & 5 & 552.92 & 3.129447 & NU $1 *$ \\
\hline $3491 \cdot 10$ & 2.1246 & 0.0496 & 8 & 6 & 3 & 9 & 6 & 4 & 1631.27 & 5.032027 & NU3 \\
\hline $3491 \cdot 13$ & 0.9552 & 0.0870 & 4 & 0 & 4 & 4 & 3 & 1 & 383.85 & 0.047652 & NU1 \\
\hline 3491.13 & 6.3739 & 0.0500 & 8 & 6 & 2 & 9 & 6 & 3 & 1631.41 & 5.032058 & NU 3 \\
\hline $3491 \cdot 49$ & 0.0171 & 0.0848 & 6 & 2 & 5 & 7 & 1 & 6 & 704.22 & 3.799063 & NU $1 * *$ \\
\hline 3491.63 & 0.0189 & 0.0800 & 6 & 4 & 3 & 5 & 3 & 2 & 508.81 & 3.153879 & 2 NU2* \\
\hline $3491 \cdot 78$ & 0.1073 & 0.0814 & 9 & 5 & 5 & 10 & 4 & 6 & 1616.51 & 2.53 & NU1+ \\
\hline 3491.82 & 0.0079 & 0.0648 & 5 & 3 & 3 & 5 & 5 & 0 & $742 \cdot 10$ & 0.037100 & NU $3 *$ \\
\hline $3491 \cdot 86$ & 0.0283 & 0.0327 & 13 & 0 & 13 & 13 & 2 & 12 & 2042.38 & 0.486060 & NU3 \\
\hline 3491.91 & 0.0849 & 0.0327 & 13 & 1 & 13 & 13 & 1 & 12 & 2042.33 & 0.486110 & NU3 \\
\hline 3492.06 & 0.0063 & 0.0640 & 8 & 5 & 3 & 9 & 5 & 4 & 1477.31 & 6.258032 & NU3** \\
\hline $3492 \cdot 34$ & 0.0145 & 0.0647 & 6 & 0 & 6 & 7 & 1 & 7 & $2181 \cdot 12$ & 5.990506 & $N \cup 1 * * *$ \\
\hline $3493 \cdot 42$ & 0.9133 & 0.0675 & 7 & 3 & 5 & 7 & 5 & 2 & 1059.85 & 0.041795 & NU3 \\
\hline 3493.44 & 0.0021 & 0.0607 & 8 & 5 & 4 & 9 & 5 & 5 & 1474.99 & 6.255627 & NU $3 * *$ \\
\hline 3493.59 & 0.0299 & 0.0842 & 5 & 1 & 4 & 6 & 2 & 5 & 552.92 & 3.129447 & NU $1 * * *$ \\
\hline 3493.65 & 0.0434 & 0.0671 & 6 & 1 & 6 & 7 & 0 & 7 & 2180.65 & 5.983681 & NU $1 * * * *$ \\
\hline 3493.73 & 0.0131 & 0.0815 & 8 & 4 & 4 & 9 & 4 & 5 & 1360.28 & 7.302001 & NU $3 * *$ \\
\hline 3493.83 & 0.0016 & 0.0732 & 7 & 3 & 5 & 7 & 4 & 4 & 2569.52 & 3.637027 & NU $1 * * * *$ \\
\hline 3493.96 & 0.0022 & 0.0563 & 9 & 2 & 8 & 9 & 3 & 7 & 1216.27 & 3.554957 & NU $1 *$ \\
\hline 3494.11 & 0.0010 & 0.0420 & 12 & 1 & 12 & 11 & 2 & 9 & 1690.70 & 0.011432 & NU1 \\
\hline 3494.14 & 0.2294 & 0.0692 & 8 & 2 & 7 & 8 & 4 & 4 & 1131.76 & 0.045112 & NU3 3 \\
\hline $3494 \cdot 42$ & 0.1161 & 0.0864 & 8 & 3 & 5 & 9 & 3 & 6 & $1282 \cdot 92$ & 8.066869 & NU $3 *$ \\
\hline
\end{tabular}




\begin{tabular}{|c|c|c|c|c|c|c|c|c|c|c|c|}
\hline $\begin{array}{c}\text { FREQUENCY } \\
\mathrm{cm}^{-1}\end{array}$ & 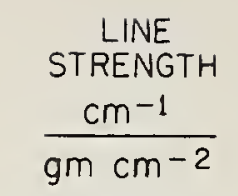 & $\begin{array}{l}\text { HALF } \\
\text { WIDTH } \\
\frac{\mathrm{cm}^{-1}}{a+m .}\end{array}$ & $J^{\prime}$ & $\mathrm{K}_{\mathrm{a}}$ & $\mathrm{K}_{\mathrm{c}}$ & $J^{\prime \prime}$ & $\mathrm{K}_{\mathrm{a}}$ & $\mathrm{K}_{\mathrm{c}}$ & $\begin{array}{c}E^{\prime \prime} \\
\mathrm{cm}^{-1}\end{array}$ & L & BAND \\
\hline 3494.57 & 0.0180 & 0.0530 & 8 & 1 & 8 & 8 & 2 & 7 & 885.62 & 1.833129 & NU I * \\
\hline 3494.80 & 0.7668 & 0.0590 & 10 & 3 & 8 & 10 & 4 & 7 & $1581 \cdot 34$ & 5.051335 & NUI \\
\hline 3495.16 & 46.0968 & 0.0848 & 6 & 2 & 5 & 7 & 1 & 6 & 704.22 & 3.799063 & NUI \\
\hline 3495.50 & 0.0104 & 0.0594 & 9 & 3 & 7 & 10 & 3 & 8 & $1446 \cdot 16$ & 8.878817 & NU3* $* *$ \\
\hline 3495.60 & 0.0260 & 0.0682 & 8 & 4 & 5 & 9 & 4 & 6 & 1340.89 & 7.216366 & NU3* \\
\hline 3495.93 & 0.0031 & $C .0666$ & 5 & 3 & 2 & 5 & 5 & 1 & 742.10 & 0.043300 & NU3* \\
\hline 3496.17 & 0.0437 & 0.0950 & 2 & 1 & 2 & 3 & 2 & 1 & 1819.34 & 0.925282 & NU $1 * \ddot{*} *$ \\
\hline 3496.41 & 0.0056 & 0.0616 & 11 & 5 & 6 & 12 & 4 & 9 & $2124 \cdot 98$ & 0.678814 & NUI \\
\hline 3496.41 & 0.4083 & 0.0639 & 9 & 4 & 6 & 9 & 5 & 5 & 1474.99 & 4.736397 & NU1 \\
\hline 3496.63 & 80.9344 & 0.0842 & 5 & 1 & 4 & 6 & 2 & 5 & 552.92 & 3.129447 & NU 1 \\
\hline 3497.02 & 0.0015 & 0.0648 & 5 & 3 & 3 & 5 & 5 & 0 & 742.10 & 0.037100 & NU $3 * *$ \\
\hline 3497.31 & 0.0063 & 0.0755 & 6 & 4 & 2 & 5 & 3 & 3 & 504.00 & 3.066224 & $2 \mathrm{NU} 2 *$ \\
\hline 3497.41 & 0.4010 & 0.0880 & 5 & 3 & 3 & 4 & 0 & 4 & .06 & 0.095779 & $2 \mathrm{NU} 2$ \\
\hline 3497.55 & 0.4671 & 0.0841 & 2 & 2 & 1 & 3 & 3 & 0 & 285.43 & 2.366160 & NU1* \\
\hline 3497.81 & 0.0017 & 0.0762 & 5 & 2 & 4 & 5 & 4 & 1 & 2251.86 & 0.024136 & NU $3 * * * *$ \\
\hline 3497.97 & 0.0164 & 0.0661 & 10 & 6 & 5 & 11 & 5 & 6 & .02 & 0.7996 & NU 1 \\
\hline 3498.00 & 0.0033 & 0.0530 & 8 & 1 & 8 & 8 & 2 & 7 & .62 & 1.833129 & NU $1 * *$ \\
\hline 3498.59 & 0.3673 & 0.0665 & 6 & 3 & 4 & 6 & 5 & 1 & .63 & 0.021403 & NU3 \\
\hline 3498.85 & 0.0011 & 0.0650 & 8 & 4 & 5 & 8 & 5 & 4 & 1255 & 3.9011 & NU $1 * * *$ \\
\hline 3499.06 & 0.1578 & 0.0830 & 2 & 2 & 0 & 3 & 3 & 1 & .23 & 2.394805 & NU $1 *$ \\
\hline 3499.14 & 0.0035 & 0.0800 & 6 & 4 & 3 & 5 & 3 & 2 & .81 & 3.153879 & $2 N \cup Z$ * \\
\hline 3499.34 & 0.0090 & 0.0739 & 6 & 3 & 4 & 6 & 4 & 3 & .39 & 2.878858 & NU $1 * * *$ \\
\hline 3499.41 & 0.0014 & 0.0834 & 7 & 4 & 4 & 6 & 3 & 3 & .59 & 3.050978 & $2 \mathrm{NU} 2 * * *$ \\
\hline 3499.65 & 0.0041 & 0.0645 & 7 & 4 & 4 & 7 & 5 & 3 & .65 & 3.012848 & NU $1 *$ \\
\hline 3499.73 & 16.9887 & 0.0640 & 8 & 5 & 3 & 9 & 5 & 4 & .31 & 6.258032 & NU3 \\
\hline 3499.96 & 2.3947 & 0.0879 & 7 & 3 & 4 & 6 & 2 & 5 & .92 & 0.996364 & 2NU2 \\
\hline 3500.23 & 0.0341 & 0.0886 & 3 & 1 & 3 & 4 & 3 & 2 & & 0.0262 & NU3* \\
\hline 3500.30 & 0.0561 & 0.0454 & 12 & 3 & 10 & 11 & 2 & 9 & .70 & 7.003088 & 2 NU 2 \\
\hline 3500.33 & 0.0290 & 0.0760 & 8 & 2 & 6 & 9 & 2 & 7 & .40 & 8.113471 & NU $3 * * *$ \\
\hline 3500.37 & 0.0047 & 0.0885 & 3 & 1 & 2 & 4 & 3 & 1 & .92 & 0.066374 & NU $3 * * *$ \\
\hline 3500.41 & 0.0864 & 0.0841 & 2 & 2 & 1 & 3 & 3 & 0 & & 2.366 & NU $1 \ddot{*} *$ \\
\hline 3500.65 & 0.0030 & 0.0482 & 11 & 7 & 4 & 12 & 6 & 7 & 85 & 0.724 & NU 1 \\
\hline 3500.80 & 1.1104 & 0.0563 & 9 & 2 & 8 & 9 & 3 & 7 & .27 & 3.5549 & NU 1 \\
\hline 3501.02 & 5.7541 & 0.0607 & 8 & 5 & 4 & 9 & 5 & 5 & .99 & 6.2556 & NU3 \\
\hline 3501.22 & 3.0147 & 0.0650 & 8 & 4 & 5 & 8 & 5 & 4 & .16 & 3.901 & NU 1 \\
\hline 3501 . & 0.0045 & 0.0608 & 7 & 5 & 2 & 8 & 5 & 3 & .16 & 4.90 & $3 * * *$ \\
\hline 3501.4 & 8.9974 & 0.0530 & 8 & 1 & 8 & 8 & 2 & 7 & 62 & 1.83 & NU 1 \\
\hline 3501 . & 35.5074 & 0.0815 & 8 & 4 & 4 & 9 & 4 & 5 & & 7 . & NU3 \\
\hline 3501 . & 0.0600 & 0.0930 & 3 & 1 & 2 & 4 & 2 & 3 & 99 & 1.97 & NU $1 * * * *$ \\
\hline 3501.76 & 9.8584 & 0.0566 & 9 & 2 & 7 & 10 & 2 & 8 & .00 & 9.00 & NU3 \\
\hline 3501 . & 0.0292 & 0.0830 & 2 & 2 & 0 & 3 & 3 & 1 & .23 & $2 \cdot 39$ & 1 前 \\
\hline & 0.0215 & 0.0864 & 8 & 3 & 5 & 9 & 3 & 6 & .92 & 69 & NU $3 * *$ \\
\hline 3502.04 & 0.0876 & 0.0647 & 6 & 0 & 6 & 7 & 1 & 7 & & & NU $1 *$ \\
\hline 3502 . & 0.0089 & 0.0469 & 7 & 6 & 1 & 8 & 6 & 2 & .68 & 3.522866 & NU3* \\
\hline 3502.22 & 3.9765 & C. 0648 & 5 & 3 & 3 & 5 & 5 & 0 & .10 & 0.037100 & NU3+ \\
\hline 3502 . & 0.0266 & 0.0469 & 7 & 6 & 2 & 8 & 6 & 3 & .65 & 3.522863 & NU3 \\
\hline 3502 . & 22.0795 & 0.0402 & 10 & 1 & 9 & 11 & 1 & 10 & .87 & 10.283342 & NU3 \\
\hline 3502 . & 7.3583 & 0.0397 & 10 & 2 & 9 & 11 & 2 & 10 & .13 & 10.2811 & NU3 \\
\hline 3502 . & 0.2630 & 0.0671 & 6 & 1 & 6 & 7 & 0 & 7 & .26 & 5.983681 & NU $1 *$ \\
\hline 3502 . & 28.0151 & 0.0594 & 9 & 3 & 7 & 10 & 3 & 8 & . 16 & 8.878817 & NU3 \\
\hline & 233.5445 & 0.0841 & 2 & 2 & 1 & 3 & 3 & 0 & & 2.366160 & NU1 \\
\hline & 0.4727 & 0.0757 & 8 & 4 & 4 & 8 & 1 & 7 & .93 & 3.07 & $2 \mathrm{NU} 2+$ \\
\hline 3503 . & 0.2785 & 0.0614 & 11 & 2 & 9 & 11 & 3 & 8 & & 5.6423 & NU 1 \\
\hline 3504.12 & 7.0357 & 0.0335 & 11 & 0 & 11 & 12 & 0 & 12 & 1557.91 & 11.742873 & NU3 \\
\hline
\end{tabular}




\begin{tabular}{|c|c|c|c|c|c|c|c|c|c|c|c|}
\hline $\begin{array}{c}\text { FREQUENCY } \\
\mathrm{cm}^{-1}\end{array}$ & $\begin{array}{l}\text { LINE } \\
\text { STRENGTH } \\
\frac{\mathrm{cm}^{-1}}{g m \mathrm{~cm}^{-2}}\end{array}$ & $\begin{array}{l}\text { HALF } \\
\text { WIDTH } \\
\frac{\mathrm{cm}^{-1}}{\mathrm{~atm} .}\end{array}$ & $J^{\prime}$ & $K_{0}$ & $K_{c}$ & $J^{\prime \prime}$ & $K_{0}$ & $K_{c}$ & $\begin{array}{c}\mathrm{E}^{\prime \prime} \\
\mathrm{cm}^{-1}\end{array}$ & L & BAND \\
\hline 3504.12 & 21.1072 & 0.0335 & 11 & 1 & 11 & 12 & 1 & 12 & 1557.91 & 11.742858 & NU3 \\
\hline $3504 \cdot 30$ & 2.0659 & 0.0645 & 7 & 4 & 4 & 7 & 5 & 3 & 1059.65 & 3.012848 & NU1 \\
\hline 3504.32 & 2.5886 & 0.0548 & 8 & 0 & 8 & 8 & 1 & 7 & 882.93 & 1.56 & NU1- \\
\hline $3504 \cdot 74$ & 78.9022 & 0.0830 & 2 & 2 & 0 & 3 & 3 & 1 & 285.23 & 2.394805 & NU1 \\
\hline 3505.37 & 0.0129 & 0.0699 & 4 & 3 & 2 & 4 & 4 & 1 & 2129.63 & 1.082946 & NU $1 * * * *$ \\
\hline 3505.42 & 0.9658 & 0.0380 & 7 & 7 & 0 & 8 & 7 & 1 & $1590 \cdot 74$ & 1.887603 & NU3 \\
\hline 3505.42 & 2.8974 & 0.0380 & 7 & 7 & 1 & 8 & 7 & 2 & 1590.74 & 1.887603 & NU3 \\
\hline 3505.43 & 0.0162 & 0.0647 & 6 & 0 & 6 & 7 & 1 & 7 & $586 \cdot 48$ & 5.990506 & NU I** \\
\hline $3505 \cdot 52$ & 0.0838 & 0.0901 & 4 & 1 & 3 & 5 & 2 & 4 & $416 \cdot 22$ & 2.444582 & NU $1 *$ \\
\hline 3505.89 & 7.5510 & 0.0620 & 6 & 4 & 3 & 5 & 5 & 2 & 888.60 & 1.56 & NUI- \\
\hline 3505.92 & 1.6510 & 0.0874 & 3 & 0 & 3 & 3 & 3 & 0 & 285.43 & 0.016715 & NU 1 \\
\hline 3506.08 & 1.5485 & 0.0666 & 5 & 3 & 2 & 5 & 5 & 1 & 742.10 & 0.043300 & NU3+ \\
\hline 3506.09 & 0.0953 & 0.0898 & 4 & 1 & 3 & 5 & 3 & 2 & 508.81 & 0.137702 & NU $3 *$ \\
\hline 3506.13 & 0.0486 & 0.0671 & 6 & 1 & 6 & 7 & 0 & 7 & $586 \cdot 26$ & 5.983681 & NU1*艹̈ \\
\hline 3506.65 & 9.4693 & 0.0800 & 6 & 4 & 3 & 5 & 3 & 2 & $508 \cdot 81$ & 3.153879 & $2 \mathrm{NU} 2$ \\
\hline 3507.75 & 0.0205 & 0.0354 & 10 & 0 & 10 & 11 & 0 & 11 & 2915.94 & 10.743597 & NU3*** \\
\hline $3508 \cdot 17$ & 0.0125 & 0.0680 & 7 & 4 & 3 & 7 & 5 & 2 & 1059.85 & 3.039271 & NU $1 *$ \\
\hline 3508.55 & 0.0155 & 0.0901 & 4 & 1 & 3 & 5 & 2 & 4 & 416.22 & 2.444582 & NUI*** \\
\hline 3508.81 & 0.1759 & 0.0760 & 8 & 2 & 6 & 9 & 2 & 7 & 1201.95 & 8.113471 & NU $3 *$ \\
\hline 3508.82 & 43.8189 & 0.0647 & 6 & 0 & 6 & 7 & 1 & 7 & 586.48 & 5.990506 & NUi \\
\hline 3509.01 & 0.7997 & 0.0651 & 9 & 3 & 7 & 9 & 4 & 6 & 1340.89 & 4.734521 & NU1 \\
\hline $\begin{array}{l}3509.04 \\
3509.46\end{array}$ & $\begin{array}{l}0.0179 \\
0.0080\end{array}$ & $\begin{array}{l}0.0889 \\
0.0920\end{array}$ & $\begin{array}{l}7 \\
5\end{array}$ & $\begin{array}{l}3 \\
2\end{array}$ & $\begin{array}{l}4 \\
4\end{array}$ & $\begin{array}{l}8 \\
6\end{array}$ & $\begin{array}{l}3 \\
1\end{array}$ & $\begin{array}{l}5 \\
5\end{array}$ & 2670.79 & $\begin{array}{l}6.985053 \\
2.671788\end{array}$ & NU $3 * * * *$ \\
\hline 3509 . & 58.0876 & 0.0864 & 8 & 3 & 5 & 9 & 3 & 6 & $\begin{array}{l}2146 \cdot 28 \\
1282.92\end{array}$ & 8.066869 & $\begin{array}{l}\text { NU } 1 * * * 2 \\
\text { NU } 3\end{array}$ \\
\hline 3509 . & 131.4829 & 0.0671 & 6 & 1 & 6 & 7 & 0 & 7 & 586.26 & 5.983681 & NU1 \\
\hline 3510.07 & 0.0473 & 0.0674 & 8 & 3 & 6 & 9 & 3 & 7 & 1216 & 7.0700 & NU $3 *$ \\
\hline 3510.50 & 12.4713 & 0.0927 & 6 & 3 & 4 & 7 & 2 & 5 & .40 & 1.512 & NUI \\
\hline 3510.60 & 12.9950 & 0.0682 & 8 & 4 & 5 & 9 & 4 & 6 & 1340.89 & 7.216366 & NU3 \\
\hline 3511.59 & 41.9220 & 0.0901 & 4 & 1 & 3 & 5 & 2 & 4 & $416 \cdot 22$ & 2.444582 & NU1 \\
\hline 3512.00 & 3.1481 & 0.0755 & 6 & 4 & 2 & 5 & 3 & 3 & 504.00 & 3.066224 & $2 \mathrm{NU} 2$ \\
\hline 3512.04 & 0.0727 & 0.0771 & 5 & 0 & 5 & 6 & 1 & 6 & 2042.77 & 4.979001 & $N \cup 1 * * * *$ \\
\hline $3512 \cdot 32$ & 0.0813 & 0.0594 & 7 & 5 & 3 & 8 & 5 & 4 & $1255 \cdot 16$ & 4.903948 & NU $3 *$ \\
\hline 3512.54 & 0.0176 & 0.0898 & 4 & 1 & 3 & 5 & 3 & 2 & 508.81 & 0.137702 & NU $3 * *$ \\
\hline 3512.57 & 6.2667 & 0.0680 & 7 & 4 & 3 & 7 & 5 & 2 & 1059.85 & 3.039271 & NU 1 \\
\hline 3513.00 & 0.0154 & 0.0743 & 6 & 1 & 6 & 6 & 2 & 5 & $2161 \cdot 30$ & 1.783035 & NU $1 * * * *$ \\
\hline 3513.03 & 17.0244 & 0.0886 & 3 & 1 & 3 & 4 & 3 & 2 & 382.52 & 0.026225 & NU 3 \\
\hline 3513.11 & 0.0320 & 0.0701 & 7 & 4 & 4 & 8 & 4 & 5 & 2764.71 & 6.014663 & NU $3 * * * *$ \\
\hline 3513.13 & 0.0187 & 0.0646 & 8 & 2 & 7 & 8 & 3 & 6 & $1006 \cdot 12$ & 3.456295 & NU 1 * \\
\hline 3513.18 & 0.8767 & 0.0715 & 8 & 4 & 4 & 8 & 5 & 3 & 1255.92 & $3 \cdot 41$ & NU1- \\
\hline 3513.27 & 0.0954 & 0.0587 & 9 & 6 & 3 & 9 & 5 & 4 & 1477.31 & 4.017231 & $2 N \cup 2$ \\
\hline 3513.30 & 3.3793 & 0.0637 & 6 & 4 & 2 & 6 & 5 & 1 & 888.63 & 2.089996 & NU 1 \\
\hline $3513 \cdot 38$ & 0.0914 & 0.0928 & 4 & 0 & 4 & 5 & 2 & 3 & $446 \cdot 50$ & 0.096503 & NU3* \\
\hline 3513.45 & 0.0174 & 0.0378 & 13 & 2 & 11 & 12 & 3 & 10 & 1962.55 & 7.82232 & 2NU2 \\
\hline 3513.80 & 0.6475 & 0.0894 & 7 & 4 & 4 & 8 & 3 & 5 & $1050 \cdot 15$ & 0.898681 & NU1 \\
\hline 3514 . & 3.7290 & 0.0613 & 9 & 1 & 8 & 9 & 2 & 7 & 1201.95 & 3.674255 & NU1 \\
\hline $3514 \cdot 12$ & 8.3355 & 0.0578 & 5 & 4 & 1 & 5 & 5 & 0 & 742.10 & 0.826 & NU1- \\
\hline 3514.19 & 0.0488 & 0.0920 & 5 & 2 & 4 & 6 & 1 & 5 & 542.91 & 2.671788 & NU1* \\
\hline 3514.45 & 0.3000 & 0.0337 & 12 & 0 & 12 & 12 & 2 & 11 & 1774.74 & 0.487254 & NU3 \\
\hline 3514.59 & 0.1000 & 0.0337 & 12 & 1 & 12 & 12 & 1 & 11 & 1774.60 & 0.487287 & NU3 \\
\hline 3514.87 & 0.0242 & 0.0806 & 5 & 1 & 5 & 6 & 0 & 6 & 2041.76 & 4.958549 & NU1**** \\
\hline 3515.53 & 0.0129 & 0.0705 & 8 & 3 & 6 & 8 & 4 & 5 & 1122.72 & 4.264036 & NUI* \\
\hline 3515.63 & 2.5912 & 0.0574 & 5 & 4 & 2 & 5 & 5 & 1 & 742.10 & 0.77 & NU1- \\
\hline 3515.89 & 0.0621 & 0.0787 & 7 & 4 & 3 & 8 & 4 & 4 & 1131.76 & 6.039574 & NU $3 *$ \\
\hline 3515.94 & 0.0144 & 0.0634 & 7 & 1 & 7 & 7 & 2 & 6 & 709.60 & 1.816721 & NUI* \\
\hline
\end{tabular}




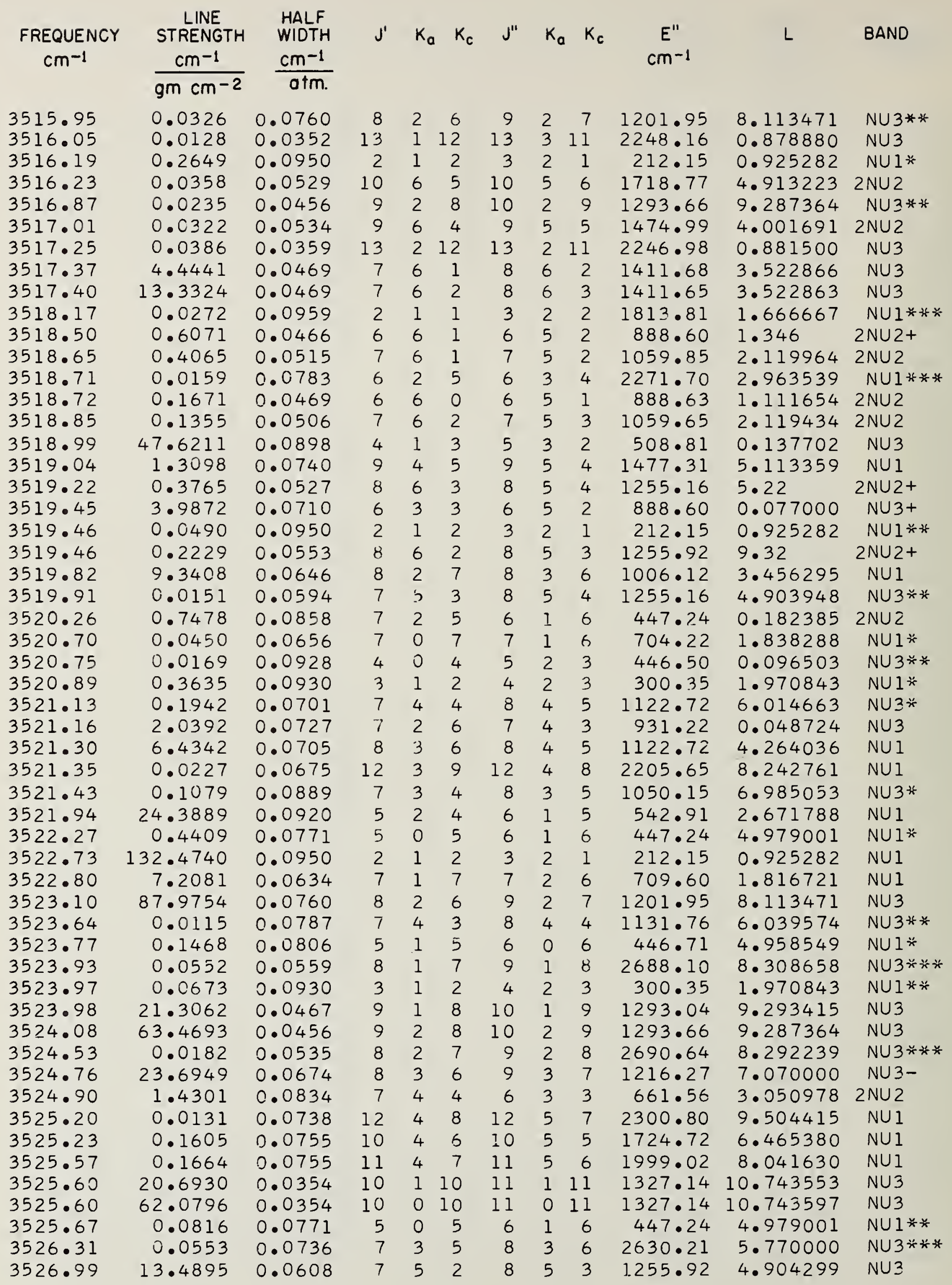




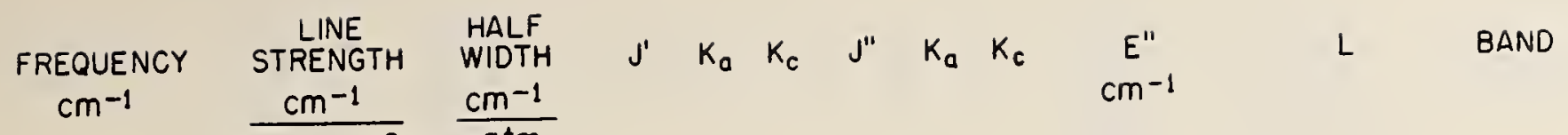

\begin{tabular}{|c|c|c|c|c|c|c|c|c|c|c|c|}
\hline 3527.05 & 181.7682 & 0.0930 & 3 & 1 & 2 & 4 & 2 & 3 & 300.35 & 1.970843 & NU 1 \\
\hline 3527.22 & 0.3897 & 0.0740 & 7 & 3 & 4 & 7 & 5 & 3 & 1059.65 & 0.052981 & NU3 \\
\hline 3527.43 & 0.1104 & 0.0641 & 9 & 5 & 4 & 10 & 4 & 7 & $1581 \cdot 34$ & 0.720783 & NU 1 \\
\hline 527.51 & 40.6879 & 0.0594 & 7 & 5 & 3 & 8 & 5 & 4 & $1255 \cdot 16$ & 4.903948 & NU3 \\
\hline 528.03 & 22.5150 & 0.0656 & 7 & 0 & 7 & 7 & 1 & 6 & $704 \cdot 22$ & 1.838288 & NU 1 \\
\hline 528.12 & 45.6895 & 0.0928 & 4 & 0 & 4 & 5 & 2 & 3 & $446 \cdot 50$ & 0.096503 & NU3 \\
\hline 528.49 & 0.0127 & 0.0430 & 6 & 6 & 1 & 7 & 6 & 2 & $1216 \cdot 20$ & 1.869458 & NU $3 *$ \\
\hline 528.49 & 0.0380 & 0.0430 & 6 & 6 & 0 & 7 & 6 & L & $1216 \cdot 20$ & 1.869458 & NU3* \\
\hline $528 \cdot 68$ & 0.0359 & 0.0 .701 & 7 & 4 & 4 & 8 & 4 & 5 & 1122.72 & 6.014663 & NU $3 \ddot{*} \ddot{x}$ \\
\hline 529.07 & 220.4414 & 0.0771 & 5 & 0 & 5 & 6 & 1 & 6 & $447 \cdot 24$ & 4.979001 & NU 1 \\
\hline 529.15 & 3.3984 & 0.0794 & 8 & 5 & 4 & 9 & 4 & 5 & $1360 \cdot 28$ & 7.39 & $\mathrm{~N} \cup 1+$ \\
\hline 529.67 & 0.0153 & 0.0722 & 7 & 2 & 6 & 7 & 3 & 5 & 816.72 & 3.273499 & NU I* \\
\hline 30.11 & 4.8586 & 0.0732 & 7 & 3 & 5 & 7 & 4 & 4 & 927.77 & 3.637027 & NU 1 \\
\hline 30.33 & 0.0288 & 0.0885 & 3 & 1 & 2 & 4 & 3 & 1 & 383.85 & 0.066374 & NU $3 *$ \\
\hline $30 \cdot 48$ & 0.1574 & 0.0838 & 7 & 2 & 5 & 8 & 2 & 6 & 982.91 & 7.251910 & NU $3 *$ \\
\hline 530.75 & 73.4053 & 0.0806 & 5 & 1 & 5 & 6 & 0 & 6 & 446.71 & 4.958549 & NU 1 \\
\hline 530.77 & 0.3757 & 0.0701 & 10 & 2 & 8 & 10 & 3 & 7 & 1538.23 & 5.910419 & NU 1 \\
\hline 0.81 & 0.3347 & 0.0559 & 8 & 1 & 7 & 9 & 1 & 8 & 1079.07 & 8.308658 & NU $3 *$ \\
\hline 0.98 & 0.1106 & 0.0535 & 8 & 2 & 7 & 9 & 2 & 8 & $1080 \cdot 38$ & 8.292239 & NU3* \\
\hline 31.39 & 31.0420 & 0.0787 & 7 & 4 & 3 & 8 & 4 & 4 & 1131.76 & 6.039574 & NU3 \\
\hline 32.29 & 0.3349 & 0.0736 & 7 & 3 & 5 & 8 & 3 & 6 & $1006 \cdot 12$ & 5.770000 & NU $3 *$ \\
\hline 2.33 & 0.1096 & 0.0388 & 9 & 0 & 9 & 10 & 0 & 10 & 1114.55 & 9.744485 & NU3* \\
\hline .34 & 0.3287 & 0.0388 & 9 & 1 & 9 & 10 & 1 & 10 & 1114.56 & 9.744353 & NU3* \\
\hline 2.79 & 0.0204 & 0.0758 & 9 & 4 & 5 & 9 & 1 & 8 & 1079.07 & 0.116860 & 2 NU 2 \\
\hline .48 & 0.0101 & 0.0739 & 6 & 3 & 4 & 6 & 4 & 3 & 756.75 & 2.878858 & NU I** \\
\hline $4 \cdot 28$ & 0.7996 & 0.0789 & 6 & 1 & 6 & 6 & 3 & 3 & 661.56 & 0.014823 & NU3 \\
\hline 4.99 & 0.0265 & 0.0737 & 5 & 3 & 3 & 5 & 4 & 2 & 610.12 & 2.024051 & NU 1 * \\
\hline .05 & 0.0934 & 0.0743 & 6 & 1 & 6 & 6 & 2 & 5 & 552.92 & 1.783035 & NU 1 * \\
\hline .15 & 7.6330 & 0.0722 & 7 & 2 & 6 & 7 & 3 & 5 & 816.72 & 3.273499 & NU I \\
\hline 6.15 & 27.1780 & 0.0739 & 6 & 3 & 4 & 6 & 4 & 3 & 756.75 & 2.878858 & NU1 \\
\hline .24 & 97.1271 & 0.0701 & 7 & 4. & 4 & 8 & 4 & 5 & 1122.72 & 6.014663 & NU3 \\
\hline .57 & 0.1058 & 0.0914 & 4 & 1 & 4 & 5 & 0 & 5 & $1920 \cdot 76$ & 3.911752 & NU $1 * * * *$ \\
\hline .72 & 53.9853 & 0.0889 & 7 & 3 & 4 & 8 & 3 & 5 & 1050.15 & 6.985053 & NU3 \\
\hline .90 & 0.3492 & 0.0358 & 11 & 0 & 11 & 11 & 2 & 10 & $1525 \cdot 13$ & 0.483142 & NU3 \\
\hline .16 & 1.0479 & 0.0359 & 11 & 1 & 11 & 11 & 1 & 10 & 1524.87 & 0.483240 & NU3 \\
\hline .21 & 0.0662 & 0.0748 & 6 & 4 & 2 & 7 & 4 & 3 & $2572 \cdot 13$ & 4.739627 & NU $3 * * * *$ \\
\hline 37.22 & 0.8921 & 0.0742 & 8 & 3 & 5 & 8 & 5 & 4 & $1255 \cdot 16$ & 0.107233 & NU3 \\
\hline $7 \cdot 34$ & 0.1055 & 0.0993 & 1 & 1 & 0 & 2 & 2 & 1 & $1742 \cdot 28$ & 1.500000 & $\mathrm{NU} 1 * * *$ \\
\hline .48 & 0.0171 & 0.0711 & 7 & 2 & 5 & 6 & 5 & 2 & 888.60 & 0.003511 & NU 1 \\
\hline .53 & 0.0785 & 0.0699 & 4 & 3 & 2 & 4 & 4 & 1 & 488.10 & 1.082946 & NU $1 *$ \\
\hline .59 & 0.0455 & 0.0555 & 9 & 6 & 3 & 10 & 5 & 6 & 1718.77 & 0.576235 & NU1 \\
\hline .78 & 0.0291 & 0.0838 & 7 & 2 & 5 & 8 & 2 & 6 & 982.91 & 7.251910 & NU 3* \\
\hline .89 & 0.1648 & 0.0959 & 2 & 1 & 1 & 3 & 2 & 2 & 206.30 & 1.666667 & NU $1 *$ \\
\hline 01 & 0.0619 & 0.0559 & 8 & 1 & 7 & 9 & 1 & 8 & 1079.07 & 8.308658 & NU $3 * *$ \\
\hline 25 & 0.0205 & 0.0535 & 8 & 2 & 7 & 9 & 2 & 8 & 1080.38 & 8.292239 & $N \cup 3 * *$ \\
\hline 37 & 0.0118 & 0.0925 & 5 & 3 & 3 & 5 & 2 & 4 & 602.77 & 0.863547 & NU 1 * \\
\hline 48 & 0.1933 & 0.0381 & 12 & 1 & 11 & 12 & 3 & 10 & 1962.55 & 0.873307 & NU3 \\
\hline 54 & 0.1534 & 0.0569 & 6 & 5 & 1 & 7 & 5 & 2 & 1059.85 & 3.450058 & NU $3 *$ \\
\hline .59 & 0.0263 & 0.0705 & 4 & 3 & 1 & 4 & 4 & 0 & $488 \cdot 13$ & 1.088294 & NU 1 * \\
\hline .61 & 0.0813 & 0.0759 & 5 & 3 & 2 & 5 & 4 & 1 & 610.35 & 2.066632 & $\mathrm{NU} 1 *$ \\
\hline .69 & 0.0164 & 0.0423 & 13 & 2 & 11 & 13 & 4 & 10 & $2426 \cdot 22$ & 1.112100 & NU3 \\
\hline & 0.0225 & 0.0700 & 6 & 4 & 3 & 7 & 4 & 4 & 2569.52 & 4.735129 & NU $3 * * * *$ \\
\hline .71 & 0.0511 & 0.0565 & 6 & 5 & 2 & 7 & 5 & 3 & 1059.65 & 3.450020 & NU 3* \\
\hline .79 & 1.3423 & 0.0755 & 6 & 2 & 5 & 6 & 4 & 2 & 757.78 & 0.040211 & NU3 \\
\hline
\end{tabular}




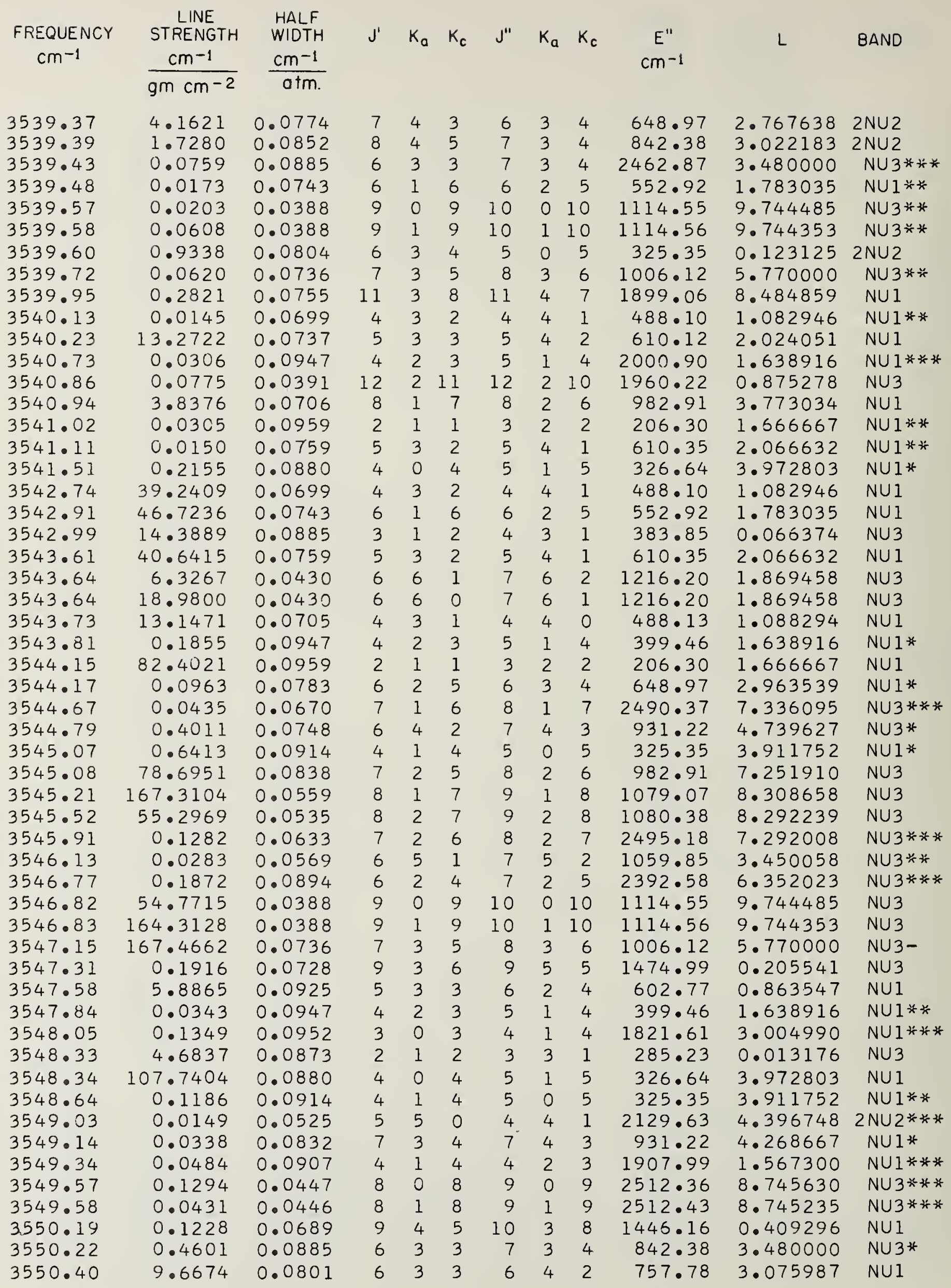




\begin{tabular}{|c|c|c|c|c|c|c|c|c|c|c|c|}
\hline $\begin{array}{c}\text { FREQUENCY } \\
\mathrm{cm}^{-1}\end{array}$ & $\begin{array}{l}\text { LINE } \\
\text { STRENGTH } \\
\frac{\mathrm{cm}^{-1}}{\mathrm{gm} \mathrm{cm}^{-2}}\end{array}$ & $\begin{array}{l}\text { HALF } \\
\text { WIDTH } \\
\frac{\mathrm{cm}^{-1}}{a+m .}\end{array}$ & $J^{\prime}$ & $\mathrm{K}_{\mathrm{a}}$ & $K_{c}$ & $J^{\prime \prime}$ & $k_{a}$ & $K_{c}$ & $\begin{array}{c}E^{\prime \prime} \\
\mathrm{cm}^{-1}\end{array}$ & L & BAND \\
\hline 3550.41 & 48.1511 & 0.0783 & 6 & 2 & 5 & 6 & 3 & 4 & 648.97 & 2.963539 & NU1 \\
\hline 550.68 & 0.0521 & 0.0451 & 13 & 3 & 11 & 13 & 3 & 10 & 2414.73 & 1.178730 & NU3 \\
\hline 3550.72 & 0.1754 & 0.0947 & 1 & 1 & 1 & 2 & 2 & 0 & $136 \cdot 17$ & 1.244747 & NUI* \\
\hline 3550.85 & 0.2124 & 0.0844 & 9 & 4 & 6 & 8 & 3 & 5 & 1050.15 & 3.141455 & 2NU2 \\
\hline 3550.87 & 0.2555 & 0.0852 & 8 & 3 & 5 & 7 & 2 & 6 & 709.60 & 0.688192 & 2NU2 \\
\hline 3551.24 & 0.0462 & 0.0885 & 2 & 1 & 1 & 3 & 3 & 0 & 285.43 & 0.021579 & NU 3* \\
\hline 3551.59 & 0.2640 & 0.0670 & 7 & 1 & 6 & 8 & 1 & 7 & 882.93 & 7.336095 & NU3* \\
\hline 3551.87 & 92.7404 & 0.0947 & 4 & 2 & 3 & 5 & 1 & 4 & 399.46 & 1.638916 & NUI \\
\hline 3552.11 & 85.8435 & 0.0876 & 6 & 4 & 3 & 7 & 3 & 4 & $842 \cdot 38$ & 13.89 & NUI+ \\
\hline 3552.11 & 0.7770 & 0.0633 & 7 & 2 & 6 & 8 & 2 & 7 & 885.62 & 7.292008 & NU $3 *$ \\
\hline 3552.21 & 320.6569 & 0.0914 & 4 & 1 & 4 & 5 & 0 & 5 & 325.35 & 3.911752 & NU1 \\
\hline 3552.39 & 17.0523 & 0.0777 & 6 & 0 & 6 & 6 & 1 & 5 & 542.91 & 1.852055 & NU1 \\
\hline 3552.45 & 0.3525 & 0.0806 & 10 & 3 & 7 & 10 & 4 & 6 & 1616.51 & 8.179783 & NUI \\
\hline 3552.83 & 2.4224 & 0.0720 & 8 & 4 & 4 & 9 & 3 & 7 & 1216.27 & 7.64 & NUI+ \\
\hline 3553.42 & 0.7839 & 0.0447 & 8 & 0 & 8 & 9 & 0 & 9 & $920 \cdot 18$ & 8.745630 & NU $3 *$ \\
\hline 3553.43 & 0.2613 & 0.0446 & 8 & 1 & 8 & 9 & 1 & 9 & 920.21 & 8.745235 & NU $3 *$ \\
\hline 3553.44 & 1.1343 & 0.0894 & 6 & 2 & 4 & 7 & 2 & 5 & $782 \cdot 40$ & 6.352023 & NU $3 *$ \\
\hline 3553.45 & 0.0320 & 0.0516 & 5 & 5 & 1 & 6 & 5 & 2 & 2552 & 1.845180 & NU $3 * * *$ \\
\hline 3553.73 & 76.6965 & 0.0569 & 6 & 5 & 1 & 7 & 5 & 2 & 1059.85 & 3.450058 & NU3 \\
\hline 3553.86 & 16.9197 & 0.0832 & 7 & 3 & 4 & 7 & 4 & 3 & $931 \cdot 22$ & 4.268667 & NU1 \\
\hline 3553.88 & 25.5662 & 0.0565 & 6 & 5 & 2 & 7 & 5 & 3 & 1059.65 & 3.450020 & NU3 \\
\hline $3554 \cdot 49$ & 0.0595 & 0.0839 & 5 & 1 & 5 & 5 & 2 & 4 & $416 \cdot 22$ & 1.711792 & NUI $*$ \\
\hline 3554.51 & 0.0134 & 0.0835 & 5 & 1 & 5 & 5 & 3 & 2 & 508.81 & 0.019115 & NU $3 *$ \\
\hline $3554 \cdot 74$ & 0.0251 & 0.0700 & 6 & 4 & 3 & 7 & 4 & 4 & .77 & 4.735129 & NU $3 * *$ \\
\hline 3554.75 & 0.0380 & 0.0576 & 12 & 3 & 9 & 12 & 5 & 8 & $2275 \cdot 44$ & 0.8553 & NU3 \\
\hline 3554.77 & 0.0559 & 0.0833 & 5 & 2 & 4 & 5 & 3 & 3 & 504.00 & 2.492169 & NUI* \\
\hline 3554.78 & 4.2757 & 0.0783 & 9 & 2 & 7 & 9 & 3 & 6 & 128 & 6.247031 & NU 1 \\
\hline 4.85 & 0.3121 & 0.0694 & 10 & 3 & 7 & 10 & 5 & 6 & .77 & 0.369 & NU3 \\
\hline 3554.90 & 0.0118 & 0.0466 & 9 & 7 & 2 & 10 & 6 & 5 & .00 & 0.354752 & NUI \\
\hline 3554.92 & 0.0086 & 0.0650 & 10 & 4 & 6 & 11 & 3 & 9 & 169 & 0.293096 & NU1 \\
\hline $5 \cdot 12$ & 5.0746 & 0.0762 & 5 & 2 & 4 & 5 & 4 & 1 & .35 & 0.024 & NU3 \\
\hline $5 \cdot 34$ & 0.0541 & 0.032 & 13 & 1 & 13 & 14 & 0 & 14 & .66 & 12.99 & NU1 \\
\hline 3555.52 & 0.2283 & 0.0771 & 6 & 3 & 4 & 7 & 3 & 5 & 816.72 & 4.550000 & NU3* \\
\hline 3555.68 & 0.0376 & 0.0884 & 5 & 2 & 3 & 5 & 3 & 2 & $\cdot 50$ & 3.442901 & NU $1 * * *$ \\
\hline 3556.51 & 0.0122 & 0.0886 & 7 & 2 & 5 & 7 & 3 & 4 & .87 & 5.918411 & NUl*** \\
\hline $3557 \cdot 14$ & 87.7136 & 0.0947 & 1 & 1 & 1 & 2 & 2 & 0 & .17 & 1.244747 & NUl \\
\hline 3557.27 & 0.6393 & 0.0993 & 1 & 1 & 0 & 2 & 2 & 1 & +.91 & 1.500000 & NU $1 *$ \\
\hline 3557.48 & 0.2204 & 0.0801 & 10 & 4 & 7 & 9 & 3 & 6 & 1282.92 & 3.465920 & 2NU2 \\
\hline 3557.52 & 2.7741 & 0.0844 & 8 & 3 & 5 & 8 & 4 & 4 & 1131.76 & 5.709388 & NU1 \\
\hline 3557.81 & 0.0103 & 0.0833 & 5 & 2 & 4 & 5 & 3 & 3 & 504.00 & 2.492169 & NUI** \\
\hline 3557.83 & 0.0445 & 0.0647 & 11 & 3 & 8 & 11 & 5 & 7 & 5.83 & 0.598994 & NU3 \\
\hline 3557.86 & 0.0110 & 0.0839 & 5 & 1 & 5 & 5 & 2 & 4 & $\cdot 22$ & 1.711792 & NUl** \\
\hline 3557.92 & 3.3222 & 0.0835 & 9 & 3 & 6 & 9 & 4 & 5 & 1360.28 & 7.165263 & NUI \\
\hline $3557 \cdot 94$ & 0.0851 & 0.0885 & 6 & 3 & 3 & 7 & 3 & 4 & $842 \cdot 38$ & 3.480000 & NU $3 * *$ \\
\hline 3558.12 & 0.0667 & 0.0802 & 7 & 1 & 6 & 7 & 2 & 5 & .40 & 3.983004 & NUI* \\
\hline 3558.29 & 0.0967 & 0.0477 & 12 & 2 & 10 & 12 & 4 & 9 & .98 & 1.085478 & NU3 \\
\hline 3558.81 & 0.0488 & 0.0670 & 7 & 1 & 6 & 8 & 1 & 7 & .93 & 7.336095 & NU $3 * *$ \\
\hline 3559.08 & 3.2993 & 0.0393 & 10 & 0 & 10 & 10 & 2 & 9 & 3.66 & 0.478039 & NU3 \\
\hline 3559.23 & 0.0431 & 0.0974 & 3 & 1 & 3 & 4 & 0 & 4 & $1817 \cdot 50$ & 2.831638 & NU $1 * * *$ \\
\hline 3559.43 & 0.1438 & 0.0633 & 7 & 2 & 6 & 8 & 2 & 7 & 885.62 & 7.292008 & NU $3 * * *$ \\
\hline 3559.59 & 0.0283 & 0.0876 & 6 & 4 & 3 & 7 & 3 & 4 & 2462.87 & 13.89 & NUI**** \\
\hline 3559.69 & 0.8176 & 0.0952 & 3 & 0 & 3 & 4 & 1 & 4 & 224.83 & 3.004990 & NU $1 *$ \\
\hline 3559.70 & 1.1078 & 0.0397 & 10 & 1 & 10 & 10 & 1 & 9 & 1293.04 & 0.478334 & NU3 \\
\hline 3560.14 & 200.5529 & 0.0748 & 6 & 4 & 2 & 7 & 4 & 3 & $931 \cdot 22$ & 4.739627 & NU3 \\
\hline
\end{tabular}




\begin{tabular}{|c|c|c|c|c|c|c|c|c|c|c|c|}
\hline $\begin{array}{c}\text { FREQUENCY } \\
\mathrm{cm}^{-1}\end{array}$ & $\begin{array}{l}\text { LINE } \\
\text { STRENGTH } \\
\frac{\mathrm{cm}^{-1}}{\mathrm{gm} \mathrm{cm}^{-2}}\end{array}$ & $\begin{array}{l}\text { HALF } \\
\text { WIDTH } \\
\mathrm{cm}^{-1} \\
\text { atm. }\end{array}$ & $J^{\prime}$ & $k_{0}$ & $k_{c}$ & $J^{\prime \prime}$ & $K_{a}$ & $K_{c}$ & $\mathrm{~cm}^{\prime \prime}$ & L & BAND \\
\hline $\begin{array}{l}3560.22 \\
3560.44 \\
3560.68\end{array}$ & $\begin{array}{l}0.2652 \\
0.1183 \\
0.0713\end{array}$ & $\begin{array}{l}0.0427 \\
0.0993 \\
0.0952\end{array}$ & $\begin{array}{r}11 \\
1 \\
3\end{array}$ & $\begin{array}{l}1 \\
1 \\
0\end{array}$ & $\begin{array}{r}10 \\
0 \\
3\end{array}$ & $\begin{array}{r}11 \\
2 \\
4\end{array}$ & $\begin{array}{l}3 \\
2 \\
2\end{array}$ & $\begin{array}{l}9 \\
1 \\
2\end{array}$ & $\begin{array}{r}1695.03 \\
134.91 \\
315.79\end{array}$ & $\begin{array}{l}0.850512 \\
1.500000 \\
0.116055\end{array}$ & $\begin{array}{l}\text { NU3 } \\
\text { NU } 1 * * \\
\text { NU 3* }\end{array}$ \\
\hline 3560.70 & 0.1450 & 0.0447 & 8 & 0 & 8 & 9 & 0 & 9 & 920.18 & 8.745630 & NU $3 * *$ \\
\hline 3560.72 & 0.0484 & 0.0446 & 8 & 1 & 8 & 9 & 1 & 9 & 920.21 & 8.745235 & NU $3 * *$ \\
\hline 3560.85 & 27.9425 & 0.0833 & 5 & 2 & 4 & 5 & 3 & 3 & 504.00 & 2.492169 & NU 1 \\
\hline 3560.89 & 0.2099 & 0.0894 & 6 & 2 & 4 & 7 & 2 & 5 & $782 \cdot 40$ & 6.352023 & NU $3 * *$ \\
\hline $3561 \cdot 24$ & 29.7705 & 0.0839 & 5 & 1 & 5 & 5 & 2 & 4 & $416 \cdot 22$ & 1.711792 & NU 1 \\
\hline 3561.45 & 0.0726 & 0.0753 & 5 & 1 & 4 & 4 & 4 & 1 & $488 \cdot 10$ & 0.001994 & NU1 \\
\hline $3561 \cdot 95$ & 1.1374 & 0.0750 & 4 & 2 & 3 & 4 & 4 & 0 & $488 \cdot 13$ & 0.008789 & NU3 \\
\hline 3562.21 & 0.1019 & 0.0587 & 8 & 6 & 3 & 9 & 5 & 4 & 1477.31 & 0.393126 & NU1 \\
\hline $3562 \cdot 32$ & 68.0243 & 0.0700 & 6 & 4 & 3 & 7 & 4 & 4 & 927.77 & 4.735129 & NU3 \\
\hline 3562 & 4.9576 & 0.0527 & 5 & 5 & 1 & 4 & 4 & 0 & $488 \cdot 13$ & 4.397010 & $2 \mathrm{NU} 2$ \\
\hline $3562 \cdot 38$ & 14.8739 & 0.0525 & 5 & 5 & 0 & 4 & 4 & 1 & $488 \cdot 10$ & 4.396748 & 2NU2 \\
\hline 3562.88 & 0.2288 & 0.0845 & 4 & 2 & 3 & 4 & 3 & 2 & $382 \cdot 52$ & 1.848533 & NU I* \\
\hline $3562 \cdot 92$ & 0.0214 & 0.0944 & 3 & 1 & 3 & 3 & 2 & 2 & 1813.81 & 1.297651 & $N \cup 1 * * *$ \\
\hline 3563.01 & 0.0423 & 0.0771 & 6 & 3 & 4 & 7 & 3 & 5 & $816 \cdot 72$ & 4.550000 & NU $3 * *$ \\
\hline 3563.10 & 0.1513 & 0.0952 & 3 & 0 & 3 & 4 & 1 & 4 & $224 \cdot 83$ & 3.004990 & NU $1 * *$ \\
\hline 3563.61 & 319.6552 & 0.0993 & 1 & 1 & 0 & 2 & 2 & 1 & $134 \cdot 91$ & 1.500000 & NU1 \\
\hline 3563.85 & 23.0900 & 0.0885 & 2 & 1 & 1 & 3 & 3 & 0 & 285.43 & 0.021579 & NU 3 \\
\hline 3563.94 & 0.0374 & 0.0699 & 5 & 4 & 1 & 6 & 4 & 2 & $2399 \cdot 17$ & 3.352166 & NU $3 * * *$ \\
\hline 3564.02 & 0.2162 & 0.0756 & 7 & 5 & 3 & 8 & 4 & 4 & $1131 \cdot 76$ & 0.444062 & NU1 \\
\hline $3564 \cdot 45$ & 0.0490 & 0.1006 & 2 & 0 & 2 & 3 & 1 & 3 & 1739.51 & 2.156792 & NU $1 * * * *$ \\
\hline 3564.63 & 0.1129 & 0.0679 & 5 & 4 & 2 & 6 & 4 & 3 & 2398.39 & 3.351648 & NU $3 * * *$ \\
\hline 3564.69 & 0.8018 & 0.0444 & 11 & 2 & 10 & 11 & 2 & 9 & 1690.70 & 0.856092 & NU3 \\
\hline $3564 \cdot 72$ & 46.4773 & 0.0792 & 6 & 6 & 1 & 7 & 3 & 4 & $842 \cdot 38$ & $81 \cdot 799998$ & $2 \mathrm{NU} 2+$ \\
\hline 3564.92 & 0.0647 & 0.0516 & 5 & 5 & 0 & 6 & 5 & 1 & 888.63 & 1.845182 & NU 3* \\
\hline $3564 \cdot 95$ & 0.1943 & 0.0516 & 5 & 5 & 1 & 6 & 5 & 2 & $888 \cdot 60$ & 1.845180 & NU 3* \\
\hline 3564.96 & 0.2798 & 0.0785 & 6 & 1 & 5 & 7 & 1 & 6 & $2309 \cdot 74$ & 6.391209 & $\mathrm{NU}_{3} * * *$ \\
\hline 3565.67 & 230.0716 & 0.0885 & 6 & 3 & 3 & 7 & 3 & 4 & $842 \cdot 38$ & 3.480000 & NU3- \\
\hline 3566.03 & 132.0104 & 0.0670 & 7 & 1 & 6 & 8 & 1 & 7 & 882.93 & 7.336095 & NU3 \\
\hline 3566.04 & 33.3505 & 0.0802 & 7 & 1 & 6 & 7 & 2 & 5 & $782 \cdot 40$ & 3.983004 & NUI \\
\hline 3566.30 & 27.1083 & 0.0741 & 7 & 4 & 3 & 8 & 3 & 6 & $1006 \cdot 12$ & 9.90 & NUI+ \\
\hline 3566.52 & 408.8145 & 0.0952 & 3 & 0 & 3 & 4 & 1 & 4 & 224.83 & 3.004990 & NUI \\
\hline 3566.76 & 388.5256 & 0.0633 & 7 & 2 & 6 & 8 & 2 & 7 & 885.62 & 7.292008 & NU3 \\
\hline $3567 \cdot 17$ & 0.2610 & 0.0974 & 3 & 1 & 3 & 4 & 0 & 4 & 222.06 & 2.831638 & NU $1^{*}$ \\
\hline 3567.23 & 0.0623 & 0.0734 & 6 & 2 & 5 & 7 & 2 & 6 & 2318.53 & 4.390000 & NU $3 * * *$ \\
\hline $3567 \cdot 45$ & 0.0192 & 0.0458 & 9 & 1 & 9 & 9 & 1 & 8 & 1079.07 & 0.472318 & NU $3 *$ \\
\hline 3567.99 & $391 \cdot 9608$ & 0.0447 & 8 & 0 & 8 & 9 & 0 & 9 & $920 \cdot 18$ & 8.745630 & NU3 \\
\hline 3568.01 & 130.6484 & 0.0446 & 8 & 1 & 8 & 9 & 1 & 9 & $920 \cdot 21$ & 8.745235 & NU3 \\
\hline 3568.09 & 6.7027 & 0.0835 & 5 & 1 & 5 & 5 & 3 & 2 & 508.81 & 0.019115 & NU3 \\
\hline $3568 \cdot 34$ & 567.1561 & 0.0894 & 6 & 2 & 4 & 7 & 2 & 5 & 782.40 & 6.352023 & NU3 \\
\hline 3568.81 & 114.3949 & 0.0845 & 4 & 2 & 3 & 4 & 3 & 2 & $382 \cdot 52$ & 1.848533 & NU 1 \\
\hline 3569.27 & 0.2179 & 0.0871 & 5 & 0 & 5 & 5 & 1 & 4 & 399.46 & 1.912338 & $N U l^{*}$ \\
\hline 3569.61 & 0.5077 & 0.0793 & 8 & 4 & 4 & 7 & 3 & 5 & 816.72 & 2.323327 & 2 NU2 2 \\
\hline 3570.06 & 0.0926 & 0.0535 & 7 & 0 & 7 & 8 & 0 & 8 & 2337.53 & 7.747248 & NU $3 * * *$ \\
\hline $3570 \cdot 14$ & 0.2779 & 0.0532 & 7 & 1 & 7 & 8 & 1 & 8 & 2337.70 & 7.746063 & NU $3 * * *$ \\
\hline 3570.50 & $114 \cdot 1599$ & 0.0771 & 6 & 3 & 4 & 7 & 3 & 5 & 816.72 & 4.550000 & NU3- \\
\hline 3570.55 & 0.2932 & 0.0907 & 4 & 1 & 4 & 4 & 2 & 3 & $300 \cdot 35$ & 1.5673 .00 & NU $1 *$ \\
\hline 3570.82 & 0.0483 & 0.0974 & 3 & 1 & 3 & 4 & 0 & 4 & 222.06 & 2.831638 & $N \cup 1 * *$ \\
\hline $3571 \cdot 18$ & 0.3481 & 0.0861 & 5 & 3 & 2 & 6 & 3 & 3 & 661.56 & 3.180000 & NU 3* \\
\hline 3571.60 & 0.0158 & 0.0598 & 6 & 5 & 2 & 5 & 4 & 1 & 610.35 & 4.266963 & $2 \mathrm{NU} 2 *$ \\
\hline 3572.04 & 1.6961 & 0.0785 & 6 & 1 & 5 & 7 & 1 & 6 & $704 \cdot 22$ & 6.391209 & NU 3* \\
\hline 3572.15 & 0.1301 & 0.0917 & 5 & 2 & 3 & 6 & 2 & 4 & 2211.21 & 5.358633 & $\mathrm{NU} 3 * * * *$ \\
\hline
\end{tabular}




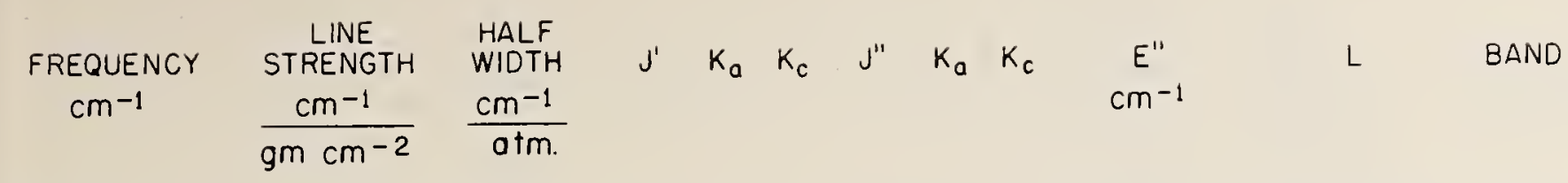

\begin{tabular}{|c|c|c|c|c|c|c|c|c|c|c|c|}
\hline 3572.23 & 0.3780 & 0.0734 & 6 & 2 & 5 & 7 & 2 & 6 & 709.60 & 4.390000 & NU $3 *$ \\
\hline 3572.49 & 0.2269 & 0.0699 & 5 & 4 & 1 & 6 & 4 & 2 & 757.78 & 3.352166 & NU $3 *$ \\
\hline 3572.50 & 0.0120 & 0.0516 & 5 & 5 & 0 & 6 & 5 & 1 & 888.63 & 1.845182 & NU $3 * *$ \\
\hline 3572.53 & 0.0359 & 0.0516 & 5 & 5 & 1 & 6 & 5 & 2 & 888.60 & 1.845180 & NU $3 * *$ \\
\hline 3572.76 & 4.6660 & 0.0840 & 8 & 2 & 6 & 8 & 3 & 5 & 1050.15 & 6.368783 & NU 1 \\
\hline 3573.06 & 0.0403 & 0.0871 & 5 & 0 & 5 & 5 & 1 & 4 & 399.46 & 1.912338 & NUI** \\
\hline 3573.08 & 0.6543 & 0.0644 & 7 & 5 & 2 & 8 & 4 & 5 & 1122.72 & 0.427306 & NU 1 \\
\hline 3573.23 & 0.0592 & 0.0955 & 2 & 1 & 2 & 2 & 2 & 1 & $1742 \cdot 28$ & 0.833333 & NU $1 * * *$ \\
\hline 3573.39 & 0.0743 & 0.0850 & 4 & 4 & 1 & 3 & 1 & 2 & 173.36 & 0.004537 & $2 \mathrm{NU} 2$ \\
\hline 3573.44 & 0.6844 & 0.0679 & 5 & 4 & 2 & 6 & 4 & 3 & 756.75 & 3.351648 & NU $3 *$ \\
\hline 3573.63 & 34.7936 & 0.0833 & 3 & 2 & 2 & 3 & 3 & 1 & $285 \cdot 23$ & 1.035682 & NU1 \\
\hline 3573.65 & 0.2203 & 0.0835 & 3 & 2 & 1 & 3 & 3 & 0 & 285.43 & 1.092505 & NU $1 *$ \\
\hline 3573.88 & 0.0542 & 0.0907 & 4 & 1 & 4 & 4 & 2 & 3 & $300 \cdot 35$ & 1.567300 & NU $1 * *$ \\
\hline 3574.07 & 0.5616 & 0.0535 & 7 & 0 & 7 & 8 & 0 & 8 & 744.09 & 7.747248 & NU $3 *$ \\
\hline 3574.11 & 1.6840 & 0.0532 & 7 & 1 & 7 & 8 & 1 & 8 & 744.16 & 7.746063 & NU $3 *$ \\
\hline 3574.47 & 130.4987 & 0.0974 & 3 & 1 & 3 & 4 & 0 & 4 & 222.06 & 2.831638 & NU 1 \\
\hline 3574.81 & 0.1950 & 0.0797 & 5 & 3 & 3 & 6 & 3 & 4 & 2271.70 & 3.370000 & NU $3 * * *$ \\
\hline 3575.06 & 35.6751 & 0.0952 & 3 & 0 & 3 & 4 & 2 & 2 & 315.79 & 0.116055 & NU 3 \\
\hline 3575.09 & 0.0591 & 0.0951 & 3 & 2 & 2 & 4 & 1 & 3 & 275.52 & 0.835095 & NU 1 * \\
\hline 3575.10 & 0.0394 & 0.0909 & 4 & 3 & 2 & 5 & 2 & 3 & 446.50 & 0.436045 & NU $1 *$ \\
\hline 3575.80 & 0.1464 & 0.0546 & 11 & 2 & 9 & 11 & 4 & 8 & 1843.02 & 0.981559 & NU3 \\
\hline 3576.49 & 0.0408 & 0.0835 & 3 & 2 & 1 & 3 & 3 & 0 & 285.43 & 1.092505 & NU $1 * * *$ \\
\hline 3576.73 & 0.2971 & 0.1006 & 2 & 0 & 2 & 3 & 1 & 3 & 142.28 & 2.156792 & NUI* \\
\hline 3576.85 & 108.9735 & 0.0871 & 5 & 0 & 5 & 5 & 1 & 4 & 399.46 & 1.912338 & NUI \\
\hline 3577.21 & 146.6057 & 0.0907 & 4 & 1 & 4 & 4 & 2 & 3 & 300.35 & 1.567300 & NU1 \\
\hline 3578.04 & 0.0240 & 0.0942 & 4 & 0 & 4 & 4 & 1 & 3 & 1875.49 & 2.047924 & NU $1 * * *$ \\
\hline 3578.04 & 4.2064 & 0.0762 & 4 & 2 & 2 & 4 & 4 & 1 & $488 \cdot 10$ & 0.010786 & NU3 \\
\hline 3578.06 & 0.7885 & 0.0917 & 5 & 2 & 3 & 6 & 2 & 4 & 602.77 & 5.358633 & NU3* \\
\hline 3578.73 & 0.0511 & 0.0509 & 12 & 3 & 10 & 12 & 3 & 9 & 2105.90 & 1.142616 & NU3 \\
\hline 3578.90 & 0.0644 & 0.0861 & 5 & 3 & 2 & 6 & 3 & 3 & 661.56 & 3.180000 & NU3* \\
\hline 3579.30 & 0.3138 & 0.0785 & 6 & 1 & 5 & 7 & 1 & 6 & $704 \cdot 22$ & 6.391209 & NU3** \\
\hline 3579.33 & 110.1731 & 0.0835 & 3 & 2 & 1 & 3 & 3 & 0 & 285.43 & 1.092505 & NU 1 \\
\hline 3579.60 & 0.0699 & 0.0734 & 6 & 2 & 5 & 7 & 2 & 6 & 709.60 & 4.390000 & NU $3 * *$ \\
\hline 3580.09 & 32.3977 & 0.0516 & 5 & 5 & 0 & 6 & 5 & 1 & 888.63 & 1.845182 & NU 3 \\
\hline 3580.12 & 97.1937 & 0.0516 & 5 & 5 & 1 & 6 & 5 & 2 & 888.60 & 1.845180 & NU3 \\
\hline 3580.26 & 1.1817 & 0.0797 & 5 & 3 & 3 & 6 & 3 & 4 & 648.97 & 3.370000 & NU $3 *$ \\
\hline 3580.43 & 0.0137 & 0.0886 & 7 & 2 & 5 & 7 & 3 & 4 & $842 \cdot 38$ & 5.918411 & NU $1 * *$ \\
\hline 3580.99 & 3.1751 & 0.0451 & 9 & 0 & 9 & 9 & 2 & 8 & 1080.38 & 0.471417 & NU3 \\
\hline 3581.03 & 0.1266 & 0.0679 & 5 & 4 & 2 & 6 & 4 & 3 & 756.75 & 3.351648 & NU $3 * *$ \\
\hline 3581.09 & 2.6402 & 0.0491 & 10 & 1 & 9 & 10 & 3 & 8 & 1446.16 & 0.818434 & NU3 \\
\hline 3581.29 & 0.1406 & 0.1037 & 1 & 0 & 1 & 2 & 1 & 2 & 1677.08 & 1.500000 & NU $1 * * * *$ \\
\hline 3581.32 & 0.2278 & 0.0884 & 5 & 2 & 3 & 5 & 3 & 2 & $508 \cdot 81$ & 3.442901 & NU I * \\
\hline $3581 \cdot 38$ & 0.1039 & 0.0535 & 7 & 0 & 7 & 8 & 0 & 8 & 744.09 & 7.747248 & NU $3 * *$ \\
\hline 3581.43 & 0.3115 & 0.0532 & 7 & 1 & 7 & 8 & 1 & 8 & $744 \cdot 16$ & 7.746063 & NU $3 * *$ \\
\hline $3581 \cdot 48$ & 0.0488 & 0.0971 & 2 & 0 & 2 & 3 & 2 & 1 & 1819.34 & 0.094498 & NU $3 * * *$ \\
\hline 3581.63 & 0.0241 & 0.0459 & 8 & 7 & 2 & 9 & 6 & 3 & 1631.41 & 0.197898 & NU1 \\
\hline 3582.01 & 0.0496 & 0.0893 & 6 & 2 & 4 & 6 & 3 & 3 & $661 \cdot 56$ & 4.829435 & NU $1 *$ \\
\hline 3582.32 & 9.6106 & 0.0458 & 9 & 1 & 9 & 9 & 1 & 8 & 1079.07 & 0.472318 & NU3 \\
\hline 3582.73 & 44.5822 & 0.0862 & 4 & 2 & 2 & 4 & 3 & 1 & 383.85 & 2.167225 & NU1 \\
\hline 3583.28 & 0.2945 & 0.0684 & 7 & 0 & 7 & 6 & 3 & 4 & 648.97 & 0.017960 & NU1 \\
\hline 3583.34 & 29.5317 & 0.0951 & 3 & 2 & 2 & 4 & 1 & 3 & 275.52 & 0.835095 & NUI \\
\hline 3583.60 & 148.5278 & 0.1006 & 2 & 0 & 2 & 3 & 1 & 3 & $142 \cdot 28$ & 2.156792 & NU1 \\
\hline 3583.70 & 36.9029 & 0.0886 & 7 & 2 & 5 & 7 & 3 & 4 & $842 \cdot 38$ & 5.918411 & NU 1 \\
\hline 3584.15 & 0.0421 & 0.0884 & 5 & 2 & 3 & 5 & 3 & 2 & 508.81 & 3.442901 & NU $1 * * *$ \\
\hline
\end{tabular}




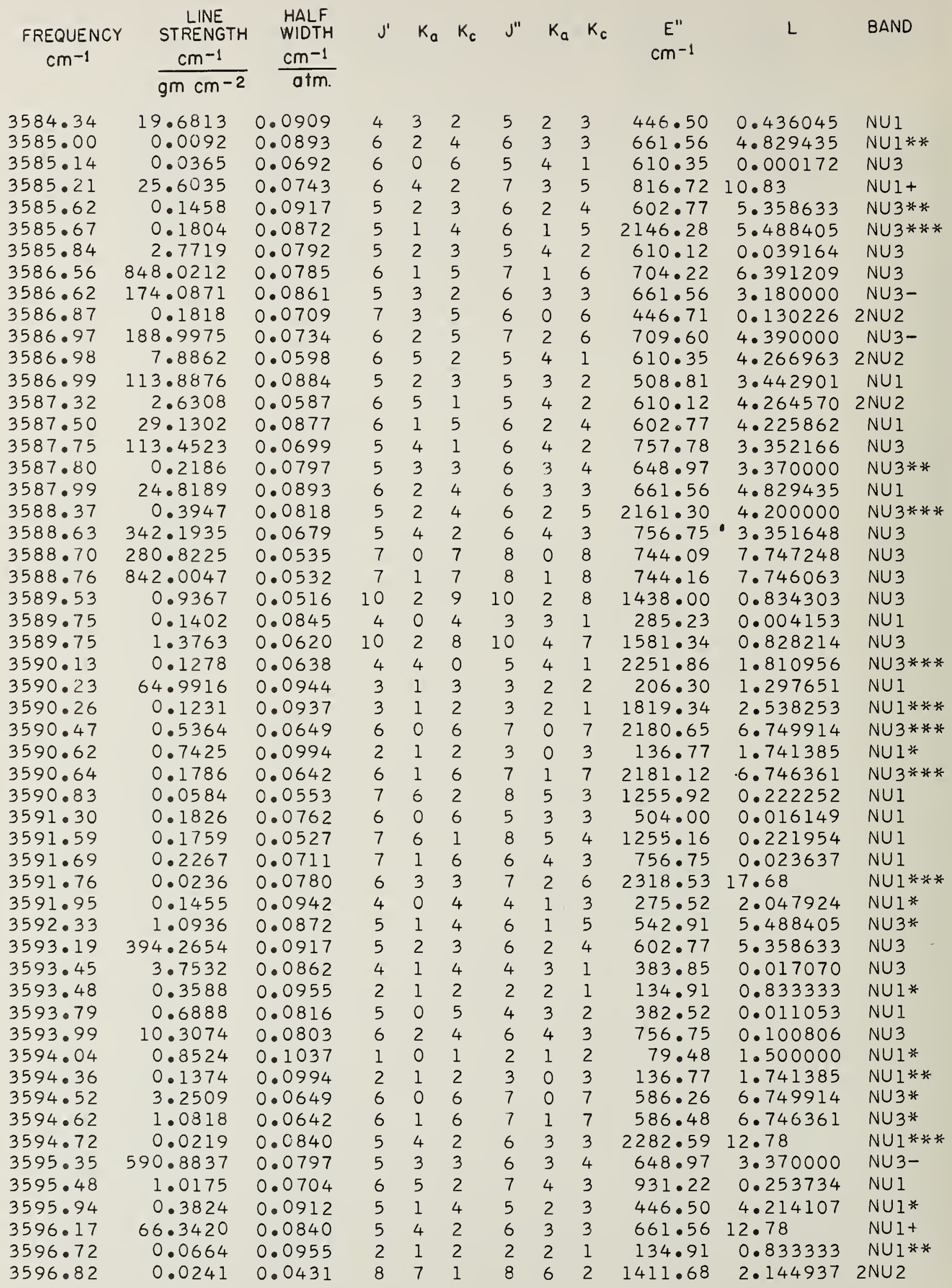




\begin{tabular}{|c|c|c|c|c|c|c|c|c|c|c|c|}
\hline $\begin{array}{c}\text { FREQUENCY } \\
\mathrm{cm}^{-1}\end{array}$ & $\begin{array}{l}\text { LINE } \\
\text { STRENGTH } \\
\frac{\mathrm{cm}^{-1}}{\mathrm{gm} \mathrm{cm}^{-2}}\end{array}$ & $\begin{array}{l}\text { HALF } \\
\text { WIDTH } \\
\frac{\mathrm{cm}^{-1}}{\mathrm{a+m} .}\end{array}$ & $J^{\prime}$ & $\mathrm{K}_{\mathrm{a}}$ & $K_{c}$ & J" & $\mathrm{K}_{\mathrm{a}}$ & $\mathrm{K}_{\mathrm{c}}$ & $\begin{array}{c}E^{\prime \prime} \\
\mathrm{cm}^{-1}\end{array}$ & L & BAND \\
\hline 3596.85 & 0.0723 & 0.0430 & 8 & 7 & 2 & 8 & 6 & 3 & 1411.65 & 2.144879 & 2NU2 \\
\hline 3597.08 & 0.3203 & 0.0826 & 4 & 3 & 1 & 5 & 3 & 2 & 2130.50 & 2.730000 & NU $3 * * *$ \\
\hline 3597.21 & 0.0183 & 0.0870 & 3 & 1 & 3 & 3 & 3 & 0 & 285.43 & 0.008443 & NU $3 *$ \\
\hline 3597.25 & 0.1288 & 0.0975 & 3 & 0 & 3 & 3 & 1 & 2 & 1772.41 & 2.185861 & NU 1 前䓋 \\
\hline 3597.51 & 0.1577 & 0.1037 & 1 & 0 & 1 & 2 & 1 & 2 & 79.48 & 1.500000 & NU $1 * *$ \\
\hline 3597.62 & 2.3918 & 0.0818 & 5 & 2 & 4 & 6 & 2 & 5 & 552.92 & 4.200000 & NU $3 *$ \\
\hline 3598.11 & 371.2599 & 0.0994 & 2 & 1 & 2 & 3 & 0 & 3 & 136.77 & 1.741385 & NU1 \\
\hline 3598.58 & 71.6598 & 0.0780 & 6 & 3 & 3 & 7 & 2 & 6 & 709.60 & 17.68 & NUl+ \\
\hline 3598.87 & 1.1526 & 0.0694 & 9 & 2 & 7 & 9 & 4 & 6 & 1340.89 & 0.624317 & NU 3 \\
\hline 3598.94 & 0.3418 & 0.0626 & 6 & 5 & 1 & 7 & 4 & 4 & 927.77 & 0.250934 & NUI \\
\hline 3598.97 & 0.2957 & 0.0971 & 2 & 0 & 2 & 3 & 2 & 1 & 212.15 & 0.094498 & NU 3 * \\
\hline 3599.07 & 0.6819 & 0.0920 & 4 & 2 & 2 & 5 & 2 & 3 & 2053.98 & 4.253846 & NU $3 * * *$ \\
\hline 3599.24 & 0.7743 & 0.0638 & 4 & 4 & 0 & 5 & 4 & 1 & 610.35 & 1.810956 & NU $3 *$ \\
\hline 3599.45 & 0.2584 & 0.0633 & 4 & 4 & 1 & 5 & 4 & 2 & $610 \cdot 12$ & 1.810924 & NU $3 *$ \\
\hline 3599.46 & 19.1232 & 0.0737 & 7 & 3 & 4 & 8 & 2 & 7 & 885.62 & 3.79 & NUI+ \\
\hline 3599.46 & 72.7472 & 0.0942 & 4 & 0 & 4 & 4 & 1 & 3 & 275.52 & 2.047924 & NU1 \\
\hline 3599.68 & 0.2023 & 0.0872 & 5 & 1 & 4 & 6 & 1 & 5 & 542.91 & 5.488405 & NU3** \\
\hline 3599.97 & 179.3981 & 0.0955 & 2 & 1 & 2 & 2 & 2 & 1 & 134.91 & 0.833333 & NU 1 \\
\hline $3600 \cdot 22$ & 3.1696 & 0.0791 & 7 & 2 & 5 & 7 & 4 & 4 & 927.77 & 0.218314 & NU3 \\
\hline 3600.75 & 2.6347 & 0.0570 & 9 & 1 & 8 & 9 & 3 & 7 & $1216 \cdot 27$ & 0.769552 & NU3 \\
\hline 3600.98 & $426 \cdot 1887$ & 0.1037 & 1 & 0 & 1 & 2 & 1 & 2 & 79.48 & 1.500000 & NUI \\
\hline 3601.04 & 265.5457 & 0.0814 & 5 & 3 & 2 & 6 & 2 & 5 & 552.92 & 9.87 & NUI+ \\
\hline 3601.87 & 0.6015 & 0.0649 & 6 & 0 & 6 & 7 & 0 & 7 & $586 \cdot 26$ & 6.749914 & NU $3 * *$ \\
\hline 3601.99 & 0.2001 & 0.0642 & 6 & 1 & 6 & 7 & 1 & 7 & 586.48 & 6.746361 & NU $3 * *$ \\
\hline $3602 \cdot 32$ & 1.9411 & 0.0826 & 4 & 3 & 1 & 5 & 3 & 2 & 508.81 & 2.730000 & NU $3 *$ \\
\hline 3602.33 & 6.6223 & 0.0747 & 8 & 2 & 6 & 8 & 4 & 5 & 1122.72 & 0.402567 & NU3 \\
\hline 3602.55 & 24.8739 & 0.0531 & 8 & 0 & 8 & 8 & 2 & 7 & 885.62 & 0.462205 & NU3 \\
\hline 3603.05 & 191.2005 & 0.0912 & 5 & 1 & 4 & 5 & 2 & 3 & $446 \cdot 50$ & 4.214107 & NUI \\
\hline 3603.86 & 0.0409 & 0.0663 & 8 & 1 & 7 & 8 & 3 & 6 & $1006 \cdot 12$ & 0.691047 & NU3* \\
\hline $3604 \cdot 36$ & 4.1325 & 0.0920 & 4 & 2 & 2 & 5 & 2 & 3 & $446 \cdot 50$ & 4.253846 & NU $3 *$ \\
\hline $3604 \cdot 48$ & 0.0108 & 0.0838 & 4 & 4 & 0 & 3 & 1 & 3 & $142 \cdot 28$ & 0.001674 & 2NU2 \\
\hline 3604.65 & 0.2061 & 0.0801 & 8 & 1 & 8 & 7 & 2 & 5 & $782 \cdot 40$ & 0.024347 & NUI \\
\hline 3605.08 & 0.4425 & 0.0818 & 5 & 2 & 4 & 6 & 2 & 5 & 552.92 & 4.200000 & NU $3 * *$ \\
\hline 3605.27 & 0.2123 & 0.0934 & 4 & 1 & 3 & 4 & 2 & 2 & 315.79 & 3.642913 & NU I* \\
\hline 3605.29 & 8.4594 & 0.0547 & 8 & 1 & 8 & 8 & 1 & 7 & 882.93 & 0.464983 & NU3 \\
\hline 3605.74 & 0.4620 & 0.0798 & 9 & 4 & 5 & 8 & 3 & 6 & 1006.12 & 1.797448 & 2NU2 \\
\hline 3606.01 & 0.7798 & 0.0803 & 4 & 3 & 2 & 5 & 3 & 3 & 504.00 & 3.208780 & NU3* \\
\hline 3606.02 & 0.0547 & 0.0971 & 2 & 0 & 2 & 3 & 2 & 1 & $212 \cdot 15$ & 0.094498 & NU3** \\
\hline $3606 \cdot 34$ & 0.0177 & 0.0949 & 2 & 2 & 1 & 3 & 1 & 2 & $1772 \cdot 4^{\prime} 1$ & 0.300506 & NU I*** \\
\hline 3607.03 & 546.7939 & 0.0872 & 5 & 1 & 4 & 6 & 1 & 5 & 542.91 & 5.488405 & NU3 \\
\hline 3607.25 & 4.5897 & 0.0727 & 5 & 4 & 1 & 6 & 3 & 4 & 648.97 & 0.278030 & NUI \\
\hline 3607.39 & 0.9355 & 0.0926 & 4 & 1 & 3 & 5 & 1 & 4 & $2000 \cdot 90$ & 4.601931 & NU $3 * * *$ \\
\hline 3607.86 & 0.1803 & 0.0957 & 2 & 1 & 1 & 2 & 2 & 0 & $136 \cdot 17$ & 1.258755 & NU $1 *$ \\
\hline 3608.01 & 0.6081 & 0.0596 & 11 & 3 & 9 & 11 & 3 & 8 & 1813.26 & 1.122765 & NU3 \\
\hline 3608.31 & 0.0271 & 0.0427 & 7 & 7 & 0 & 8 & 6 & 3 & 1411.65 & 0.074321 & NU1 \\
\hline 3608.46 & 0.0389 & 0.0636 & 7 & 0 & 7 & 7 & 2 & 6 & 709.60 & 0.448059 & NU3* \\
\hline 3608.64 & 0.0393 & 0.0934 & 4 & 1 & 3 & 4 & 2 & 2 & 315.79 & 3.642913 & NUI** \\
\hline 3608.73 & 0.7459 & 0.0937 & 3 & 1 & 2 & 3 & 2 & 1 & 212.15 & 2.538253 & NUI* \\
\hline $3609 \cdot 21$ & 0.0127 & 0.0829 & 4 & 3 & 1 & 5 & 2 & 4 & 2024.17 & 2.18 & NUI*** \\
\hline & 1625.4689 & 0.0649 & 6 & 0 & 6 & 7 & 0 & 7 & $586 \cdot 26$ & 6.749914 & NU3 \\
\hline $3609 \cdot 36$ & 540.9480 & 0.0642 & 6 & 1 & 6 & 7 & 1 & 7 & $586 \cdot 48$ & 6.746361 & NU3 \\
\hline 3609.99 & 0.3591 & 0.0826 & 4 & 3 & 1 & 5 & 3 & 2 & 508.81 & 2.730000 & NU $3 * *$ \\
\hline 3610.12 & 9.1514 & 0.0870 & 3 & 1 & 3 & 3 & 3 & 0 & 285.43 & 0.008443 & NU3 \\
\hline 3610.31 & 0.0604 & 0.1021 & 2 & 0 & 2 & 2 & 1 & 1 & 1693.65 & 2.074578 & NU $1 * * * *$ \\
\hline
\end{tabular}




\begin{tabular}{|c|c|c|c|c|c|c|c|c|c|c|c|}
\hline $\begin{array}{c}\text { FREQUENC } \\
\mathrm{cm}^{-1}\end{array}$ & $\begin{array}{l}\text { LINE } \\
\text { STRENGTH } \\
\frac{\mathrm{cm}^{-1}}{\mathrm{gm} \mathrm{cm}^{-2}}\end{array}$ & $\begin{array}{l}\text { HALF } \\
\text { WIDTH } \\
\frac{\mathrm{cm}^{-1}}{a+m .}\end{array}$ & $J^{\prime}$ & $\mathrm{K}_{0}$ & $\mathrm{~K}_{\mathrm{c}}$ & $J^{\prime \prime}$ & $k_{a}$ & $K_{c}$ & $\begin{array}{c}E^{\prime \prime} \\
\mathrm{cm}^{-1}\end{array}$ & L & BAND \\
\hline 3610.39 & 0.3080 & 0.0775 & 5 & 0 & 5 & 6 & 0 & 6 & 2041.76 & 5.755360 & NU $3 * * *$ \\
\hline 3610.66 & 0.7804 & 0.0975 & 3 & 0 & 3 & 3 & 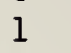 & 2 & 173.36 & 2.185861 & NUl* \\
\hline 0.74 & 1.2296 & 0.0660 & 7 & 5 & 3 & 6 & 4 & 2 & 757.78 & 4.143594 & $2 \mathrm{NU} 2$ \\
\hline 0.80 & 0.9199 & 0.0764 & 5 & 1 & 5 & 6 & 1 & $\overline{6}$ & 2042.77 & 5.744669 & NU $3 * * *$ \\
\hline 1.07 & 0.0333 & 0.0957 & 2 & 1 & 1 & 2 & 2 & 0 & 136.17 & 1.258755 & NUI** \\
\hline 1.18 & 0.0198 & 0.0949 & 2 & 2 & 1 & 3 & 1 & 2 & 173.36 & 0.300506 & NUl** \\
\hline 3611.41 & 0.0291 & 0.0994 & 1 & 0 & 1 & 2 & 2 & 0 & 1743.47 & 0.038246 & NU $3 * * *$ \\
\hline 1.87 & 3.6986 & 0.0628 & 7 & 5 & 2 & 6 & 4 & 3 & 756.75 & 4.131192 & $2 \mathrm{NU} 2$ \\
\hline 1.99 & 0.1380 & 0.0937 & 3 & 1 & 2 & 3 & 2 & 1 & $212 \cdot 15$ & 2.538253 & NU I** \\
\hline 12.00 & 0.7645 & 0.0920 & 4 & 2 & 2 & 5 & 2 & 3 & $446 \cdot 50$ & 4.253846 & NU $3 * *$ \\
\hline 3612.02 & $106 \cdot 1695$ & 0.0934 & 4 & 1 & 3 & 4 & 2 & 2 & 315.79 & 3.642913 & NU 1 \\
\hline 3612.55 & 1195.9086 & 0.0818 & 5 & 2 & 4 & 6 & 2 & 5 & 552.92 & 4.200000 & NU3- \\
\hline 3613.07 & 147.8754 & 0.0971 & 2 & 0 & 2 & 3 & 2 & 1 & 212.15 & 0.094498 & \\
\hline 3613.42 & 5.6696 & 0.0926 & 4 & 1 & 3 & 5 & 1 & 4 & 399.46 & 4.601931 & NU3* \\
\hline 3613.59 & 0.1442 & 0.0803 & 4 & 3 & 2 & 5 & 3 & 3 & 504.00 & 3.208 & NU $3 * *$ \\
\hline 3613.89 & 0.0177 & 0.0557 & 7 & 4 & 4 & 6 & 6 & 1 & 1045 & 0.000 & NU3 \\
\hline 3614.28 & .90 .1255 & 0.0957 & 2 & 1 & 1 & 2 & 2 & 0 & 136 & 1.258 & NL \\
\hline $3614 \cdot 44$ & 387.1051 & 0.0538 & 4 & 4 & 0 & 5 & 4 & 1 & 610 & 1.810 & NL \\
\hline 3614.64 & 129.2037 & 0.0633 & 4 & 4 & 1 & 5 & 4 & 2 & 610 & 810 & NL \\
\hline 3614.69 & 1.8667 & 0.0775 & 5 & 0 & 5 & 6 & 0 & 6 & 446 & 360 & $3 *$ \\
\hline 3614.84 & 5.5753 & 0.0764 & 5 & 1 & 5 & 6 & 1 & 6 & $447 \cdot 24$ & 5.744669 & $3 *$ \\
\hline 3615.25 & 372.9500 & 0.0937 & 3 & 1 & 2 & 3 & 2 & 1 & 212.15 & 2.538 & NL \\
\hline 3615.35 & 53.6079 & 0.0949 & 2 & 2 & 1 & 3 & 1 & 2 & $173 \cdot 36$ & 300506 & NU \\
\hline 3615.58 & 0.1841 & 0.0875 & 4 & 2 & 3 & 5 & 2 & 4 & 2024.17 & 950000 & $3 * *=$ \\
\hline 3615.60 & 0.1733 & 0.1044 & 1 & 1 & 1 & 2 & 0 & 2 & 70 & 0.755253 & \\
\hline 3615.64 & 38.4920 & 0.0829 & 4 & 3 & 1 & 5 & 2 & 4 & 416 & 2.18 & $1+$ \\
\hline 5.79 & 9.0578 & 0.0606 & 9 & 2 & 8 & 9 & 2 & 7 & 95 & 813942 & \\
\hline 6.00 & 0.3813 & 0.0846 & 6 & 4 & 3 & 5 & 1 & 4 & 46 & 0.071 & $2 N L$ \\
\hline 7.66 & .5790 & 0.0826 & 4 & 3 & 1 & 5 & 3 & 2 & & 2.73 & \\
\hline .7 .99 & 390.2244 & 0.0975 & 3 & 0 & 3 & 3 & 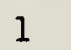 & 2 & 36 & $2 \cdot 18=$ & \\
\hline 8.16 & 20.4583 & 0.0663 & 8 & 1 & 7 & 8 & 3 & 6 & & 47 & NL \\
\hline 8.22 & 0.0601 & 0.0497 & 6 & 6 & 0 & 7 & 5 & 3 & & 69 & \\
\hline 8.36 & 0.0290 & 0.0743 & 7 & 1 & 6 & 7 & 3 & 5 & & 04 & \\
\hline 3.85 & 1.1157 & 0.0875 & 4 & 2 & 3 & 5 & & 4 & & 00 & $3 *$ \\
\hline 9.42 & 4.7191 & 0.0884 & 3 & 3 & 1 & 4 & 2 & 2 & & 93 & NL \\
\hline 9.64 & 2066.2326 & 0.0920 & 4 & 2 & 2 & 5 & 2 & 3 & & 46 & NL \\
\hline 9.95 & 141.6631 & 0.0996 & 0 & 0 & 0 & 1 & 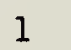 & 1 & & 1.00 & NL \\
\hline & 1.0489 & 0.0926 & 4 & 1 & 3 & 5 & 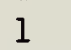 & 4 & & 4.60 & $* *$ \\
\hline & 389.8899 & 803 & 4 & 3 & 2 & 5 & 3 & 3 & & & NL \\
\hline & 3454 & 775 & 5 & 0 & 5 & 6 & 0 & 6 & & 60 & $3 * *$ \\
\hline 2.25 & 0314 & 0.0764 & 5 & 1 & 5 & 6 & & 6 & 24 & 69 & $3 * *$ \\
\hline & .4846 & 0.0636 & 7 & 0 & 7 & 7 & 2 & 6 & & 9 & NL \\
\hline 36 & 399 & 0.1044 & 1 & 1 & 1 & 2 & 0 & 2 & & 53 & \\
\hline 36 & 3663 & 0.1021 & 2 & 0 & 2 & 2 & 1 & 1 & & 2.074578 & $1 *$ \\
\hline 3624 & 2722 & 0.0646 & 5 & 5 & 1 & 6 & 4 & 2 & & 1.02 & $1+$ \\
\hline 3624 . & 1295 & 0.0784 & 3 & 3 & 0 & 4 & 3 & 1 & 2005 . & 1.758969 & NU $3 * *$ \\
\hline & 0560 & 0.0633 & 9 & 3 & 6 & 10 & 2 & 9 & 1293. & 0.084934 & NUI \\
\hline 36 & 3712 & 0.0590 & 5 & 5 & 0 & 6 & 4 & 3 & & 1.02 & $1+$ \\
\hline 36 & 3911 & 0.0774 & 3 & 3 & 1 & 4 & 3 & 2 & 200 & 1.758385 & NU $3 * * *$ \\
\hline 362 & 0.0150 & 0.0773 & 6 & 1 & 6 & 6 & 1 & 5 & $2146 \cdot 28$ & 0.450603 & NU $3 * *$ \\
\hline 3626 . & 153.1589 & 0.0787 & 4 & 4 & 1 & 5 & 3 & 2 & & 4.58 & NUl+ \\
\hline 3626 . & 0.2064 & 0.0875 & 4 & 2 & 3 & 5 & 2 & 4 & 416 & 2.950000 & NU $3 * *$ \\
\hline 362 & 180 & 0.0689 & 8 & 2 & 7 & 8 & 2 & 6 & 982.91 & 0.807542 & NU3* \\
\hline 3626 . & 0.3139 & 0.0914 & 3 & 2 & 1 & 4 & 2 & 2 & 1922.92 & 3.033776 & NU $3 * *$ \\
\hline
\end{tabular}




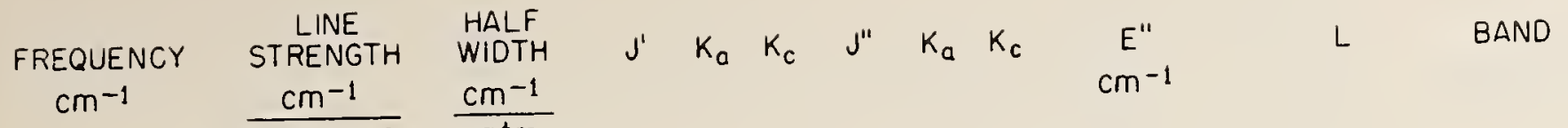

$\mathrm{gm} \mathrm{cm}-2$ atm

3627.12

$3628 \cdot 34$

3628.70

3629.45

3629.66

3629.91

3630.71

3630.80

3630.80

3631.00

3631.21

3631.79

3632.15

3632.24

3632.35

3632.49

3632.91

3633.85

3633.88

3634.42

3634.54

3635.00

3635.10

3635.22

3635.74

3636.18

3636.22

3636.29

3636.34

3636.47

3636.47

3637.45

3637.69

3638.09

3638.36

3638.84

3638.88

3639.45

3639.93

3639.94

3639.95

3641.24

3641.61

3641.81

3641.84

3642.57

3642.99

3643.20

3643.33

3643.55

3643.73

3643.77

$3644 \cdot 12$

3644.41
0.0678

2834.7985

61.3129

933.3359

2787.6639

1.4124

183.1462

1.3310

0.4647

1.0367

0.7850

1.9028

0.0343

14.4900

2.3704

0.1218

0.0541

557.8829

1.5055

8.5598

0.1918

12.4966

0.0262

0.0450

2.8170

0.0457

2.8166

1.0035

0.0326

0.0137

0.1789

0.0909

0.7973

518.3663

0.2655

0.1452

0.0226

0.3521

17.1625

26.7903

0.4386

6.0815

8.4447

70.8127

1.5835

121.6832

9.0052

0.5212

88.2494

0.1293

0.0397

0.5210

0.0610

0.0111
0.1021

0.0926

0.0654

0.0775

0.0764

0.0887

0.1021

0.0708

0.0957

0.1075

0.0784

0.0914

0.0914

0.0743

0.0774

0.0871

0.0857

0.0875

0.0706

0.0887

0.1075

0.0829

0.0813

0.0747

0.0872

0.0633

0.0957

0.0903

0.0994

0.0500

0.0394

0.0773

0.0671

0.1075

0.0614

0.0784

0.0871

0.0914

0.0914

0.0872

0.0774

0.0903

0.0860

0.0813

0.0887

0.0747

0.0689

0.0872

0.0994

0.0788

0.0786

0.0957

0.0812

0.0649

$\begin{array}{lll}2 & 0 & 2 \\ 4 & 1 & 3 \\ 7 & 1 & 7 \\ 5 & 0 & 5 \\ 5 & 1 & 5 \\ 4 & 0 & 4 \\ 2 & 0 & 2 \\ 4 & 4 & 0 \\ 3 & 1 & 2 \\ 1 & 0 & 1 \\ 3 & 3 & 0 \\ 3 & 2 & 1 \\ 3 & 2 & 1 \\ 7 & 1 & 6 \\ 3 & 3 & 1\end{array}$

$$
\begin{array}{lll}
2 & 1 & 1 \\
5 & 1 & 4
\end{array}
$$

95.17

2.074578

399.46

704.22

446.71

447.24

1920.76

95.17

504.00

1875.49

42.37

383.85

315.79

224.83

816.72

$382 \cdot 52$

382.52

504.00

$416 \cdot 22$

$931 \cdot 22$

325.35

42.37

300.35

648.97

552.92

326.64

2205.65

275.52

1907.99

136.17

$1216 \cdot 27$

$2248 \cdot 16$

542.91

1538.23

42.37

586.26

383.85

382.52

315.79

224.83

326.64

382.52

300.35

285.23

648.97

325.35

552.92

982.91

326.64

$136 \cdot 17$

542.91

$1216 \cdot 27$

275.52

224.83

552.92
4. 601931

0.456726

5.755360

5.744669

4.769122

2.074578

0.116422

3.667441

1.500000

1.758969

3.033776

0.123609

0.567704

1. 758385

0.090390

0.221254

2. 950000

4. 002077

4. 769122

1.500000

0.131472

0.398711

0.423826

4. 737042

1. 508618

3.667441

2. 984448

0.038246

0.003958

11.866070

0.450603

1.142576

1.500000

0.125531

1.758969

0.090390

3.033776

0.123609

0.963

1. 758385

2.984448

0.023148

0.398711

4. 769122

0.423826

0.807542

4. 737042

0.038246

0.147419 2NU2

1.314980 2NU2

3.667441 NU $3 * *$

0.004729 2NU2

0.0044232 NU2

NU 3

NU 3

NU3

NU $3 * * *$

NU 1

NU 1

NU $3 * * *$

NU $1 *$

$\mathrm{N} \cup 3 *$

NU $3 *$

NU $1 *$

NU $1 * * *$ 


\begin{tabular}{|c|c|c|c|c|c|c|c|c|c|c|c|}
\hline $\begin{array}{c}\text { FREQUENC } \\
\mathrm{cm}^{-1}\end{array}$ & $\begin{array}{l}\text { LINE } \\
\text { STRENGTH } \\
\frac{\mathrm{cm}^{-1}}{\mathrm{gm} \mathrm{cm}^{-2}}\end{array}$ & $\begin{array}{l}\text { HALF } \\
\text { WIDTH } \\
\frac{\mathrm{cm}^{-1}}{\text { atm. }}\end{array}$ & $J^{\prime}$ & $\mathrm{K}_{\mathrm{a}}$ & $k_{c}$ & $J^{\prime \prime}$ & $\mathrm{K}_{\mathrm{a}}$ & $K_{c}$ & $\begin{array}{c}E^{\prime \prime} \\
\mathrm{cm}^{-1}\end{array}$ & $\mathrm{~L}$ & BAND \\
\hline 3644.66 & 0.0299 & 0.0424 & 8 & 6 & 3 & 7 & 7 & 0 & 1394.86 & 0.074321 & NUI \\
\hline 3644.71 & .0100 & 0.0424 & 8 & 6 & 2 & 7 & 7 & 1 & 1394.86 & 0.074323 & NU 1 \\
\hline 3645.10 & 0.1019 & 0.0401 & 7 & 7 & 0 & 7 & 6 & 1 & $1216 \cdot 20$ & 1.119797 & $2 \mathrm{NU} 2$ \\
\hline $3645 \cdot 28$ & 60.8873 & 0.0871 & 4 & 1 & 3 & 4 & 3 & 2 & 382.52 & 0.090390 & NU3 \\
\hline 3645.41 & 0.1445 & 0.0846 & 5 & 0 & 5 & 5 & 2 & 4 & $416 \cdot 22$ & 0.379277 & NU $3 *$ \\
\hline 3645.62 & 0.0302 & 0.0628 & 8 & 4 & 4 & 7 & 6 & 1 & $1216 \cdot 20$ & 0.002886 & NU3 \\
\hline $3645 \cdot 94$ & 27.0677 & 0.0857 & 5 & 1 & 4 & 5 & 3 & 3 & 504.00 & 0.221254 & NU3 \\
\hline 3646.47 & 392.4581 & 0.0784 & 3 & 3 & 0 & 4 & 3 & 1 & 383.85 & 1.758 & NU3 \\
\hline 3647.12 & 951.3782 & 0.0914 & 3 & 2 & 1 & 4 & 2 & 2 & 315.79 & 3.033776 & NU3 \\
\hline 3647.55 & 1185.1957 & 0.0774 & 3 & 3 & 1 & 4 & 3 & 2 & 382.52 & 1.7583 & NU3 \\
\hline 3647.69 & 7.1283 & 0.0941 & 2 & 2 & 0 & 3 & 1 & 3 & $142 \cdot 28$ & 0.1016 & NUI \\
\hline 3648.56 & 51.0375 & 0.0812 & 5 & 2 & 3 & 6 & 1 & 6 & 447.24 & 1.115 & $\mathrm{NUl}+$ \\
\hline 3648.79 & 1.1250 & 0.0903 & 3 & 2 & 2 & 4 & 2 & 3 & $300 \cdot 35$ & 2.984448 & NU $3 * *$ \\
\hline $3649 \cdot 27$ & 4279.9025 & 0.0887 & 4 & 0 & 4 & 5 & 0 & 5 & 325 & 4.769122 & NU 3 \\
\hline 3650.66 & 1408.4906 & 0.0872 & 4 & 1 & 4 & 5 & 1 & 5 & 326 & 4.73 & NU3 \\
\hline 3651.32 & 1408.3050 & 0.0957 & 3 & 1 & 2 & 4 & 1 & 3 & 275 & 3.66 & NU 3 \\
\hline 3651.56 & 1.8287 & 0.0948 & 3 & 1 & 3 & 4 & 1 & 4 & 1821 & 3.713893 & $\mathrm{NU}_{3} * * *$ \\
\hline 3652.39 & 1.0352 & 0.0590 & 6 & 4 & 3 & 5 & 5 & 0 & 742 & 0.09 & NU1 \\
\hline 3652.67 & 0.0941 & 0.0863 & 5 & 1 & 5 & 5 & 1 & 4 & 2000 & 0.456689 & NU $3 * * *$ \\
\hline 3652.93 & 45.4636 & 0.0773 & 6 & 1 & 6 & 6 & 1 & 5 & 542 & 0.45 & NU3 \\
\hline 3653.64 & 0.0147 & 0.0960 & 3 & 1 & 3 & 2 & 2 & 0 & 136 & 0.10 & NU I* \\
\hline 3.66 & 0.1541 & 0.0782 & 7 & 2 & 6 & 7 & 2 & 5 & 782 & 0.83 & NU $3 *$ \\
\hline 3.87 & 3.8369 & 0.0960 & 3 & 0 & 3 & 4 & 0 & 4 & 222 & 3.80 & NU $3 *$ \\
\hline $4 \cdot 24$ & 0.1779 & 0.0750 & 9 & 5 & 5 & $\varepsilon$ & 4 & 4 & 1131 & 3.84 & 2 NU 2 \\
\hline 3654.32 & 0.8804 & 0.0907 & 2 & 2 & 0 & 3 & 2 & 1 & 1819 & 1.67 & NU $3 * \ddot{*} *$ \\
\hline 5.15 & 0.1180 & 0.0650 & 9 & 2 & 7 & 8 & 1 & 8 & 744.16 & 0.12 & $2 \mathrm{NU} 2$ \\
\hline 5.27 & 0.1328 & 0.0562 & 9 & 1 & 9 & 8 & 3 & 6 & 1006 & 0.00 & NU 3 \\
\hline 5.41 & 0.0259 & 0.0577 & 9 & 4 & 6 & 8 & 6 & 3 & 1411 & 0.00 & NU 3 \\
\hline 5.71 & 11.0833 & 0.0948 & 3 & 1 & 3 & 4 & 1 & 4 & 224 & 3.71 & NU $3 *$ \\
\hline 5.77 & 1.6872 & 0.0986 & 2 & 1 & 1 & 3 & 1 & 2 & 1772.41 & 2.64 & NU $3 * * *$ \\
\hline 6.35 & 3040.7787 & 0.0903 & 3 & 2 & 2 & 4 & 2 & 3 & 35 & 2.984448 & NU3 \\
\hline 3658.38 & 0.0468 & 0.0722 & 9 & 5 & 4 & 10 & 3 & 7 & $1538 \cdot 23$ & 0.06 & NU3 \\
\hline 8.39 & 0.0368 & 0.0948 & 4 & 1 & 4 & 3 & 2 & 1 & 15 & 0.12 & NU1* \\
\hline 8.53 & 0.3008 & 0.0915 & 2 & 2 & 1 & 3 & 2 & 2 & 1813.81 & 1.66 & NU3*长前 \\
\hline 8.54 & 0.0494 & 0.0977 & 2 & 0 & 2 & 2 & 2 & 1 & $1742 \cdot 28$ & 0.06 & NU3*关并 \\
\hline 3659.66 & 5.3353 & 0.0907 & 2 & 2 & 0 & 3 & 2 & 1 & 15 & 1.67 & NU $3 *$ \\
\hline 3659.83 & 0.3467 & 0.0629 & 6 & 4 & 2 & 5 & 5 & 1 & 742.10 & 0.098971 & NU1 \\
\hline 3659.86 & 0.0272 & 0.0825 & 4 & 2 & 3 & 3 & 3 & 0 & 285.43 & 0.131472 & NU1 ${ }^{2}$ \\
\hline 3659.87 & 1.0473 & 0.0763 & 8 & 4 & 4 & 9 & 2 & 7 & 1201.95 & 0.09 & $\mathrm{NU}_{3}$ \\
\hline 3659.90 & 0.6137 & 0.0916 & 4 & 0 & 4 & 4 & 2 & 3 & $300 \cdot 35$ & 0.29 & NU $3 *$ \\
\hline 3659.94 & 72.2409 & 0.0846 & 5 & 0 & 5 & 5 & 2 & 4 & 22 & & NU3 \\
\hline 3660.36 & 7.3739 & 0.0960 & 3 & 1 & 3 & 2 & 2 & 0 & $136 \cdot 17$ & 0.101693 & NUl \\
\hline 3660.71 & 10.2254 & 0.0986 & 2 & 1 & 1 & 3 & 1 & 2 & 173.36 & 2.645088 & NU3* \\
\hline 3661.07 & 0.1896 & 0.1111 & 1 & 1 & 0 & 1 & 0 & 1 & 1618.55 & 1.500000 & NU $1 * * * *$ \\
\hline $1 \cdot 33$ & 0.7099 & 0.0960 & 3 & 0 & 3 & 4 & 0 & 4 & & 3.80 & NU $3 \%$ * \\
\hline 1.67 & 2.6981 & 0.0929 & 5 & 1 & 5 & 4 & 2 & 2 & 315 & 0.091324 & NUI \\
\hline 1.82 & 0.0285 & 0.0782 & 7 & 2 & 6 & 7 & 2 & 5 & 782 & 0.838 & NU $3 * *$ \\
\hline 1.84 & 0.5702 & 0.0863 & 5 & 1 & 5 & 5 & 1 & 4 & 399 & 0.456689 & NU $3 *$ \\
\hline 3662.22 & 1.4535 & 0.0690 & 5 & 3 & 3 & 4 & 4 & 0 & 488.13 & 0.116422 & NU 1 \\
\hline 3663.07 & 0.2732 & 0.0747 & 6 & 2 & 4 & 7 & 1 & 7 & 586.48 & 0.035782 & NUI \\
\hline 3663.21 & 2.0504 & 0.0948 & 3 & 1 & 3 & 4 & 1 & 4 & 224.83 & 3.713893 & NU $3 * *$ \\
\hline 34.26 & 1.8233 & 0.0915 & 2 & 2 & 1 & 3 & 2 & 2 & 206.30 & 1.666667 & NU3 \\
\hline 3664.28 & 0.5369 & 0.0654 & 9 & 5 & 4 & 8 & 4 & 5 & 1122.72 & 3.682999 & $2 \mathrm{NU} 2$ \\
\hline 3664.42 & 0.6240 & 0.0688 & 11 & 4 & 8 & 11 & 4 & 7 & 1899.06 & 1.701473 & NU3 \\
\hline
\end{tabular}




\begin{tabular}{|c|c|c|c|c|c|c|c|c|c|c|c|}
\hline $\begin{array}{c}\text { FREQUENC } \\
\mathrm{cm}^{-1}\end{array}$ & $\begin{array}{l}\text { LINE } \\
\text { STRENGTH } \\
\frac{\mathrm{cm}^{-1}}{\mathrm{gm} \mathrm{cm}^{-2}}\end{array}$ & $\begin{array}{l}\text { HALF } \\
\text { WIDTH } \\
\frac{\mathrm{cm}^{-1}}{\text { ałm. }}\end{array}$ & $J^{\prime}$ & $\mathrm{K}_{\mathrm{a}}$ & $\mathrm{K}_{\mathrm{c}}$ & J" & $\mathrm{K}_{\mathrm{a}}$ & $\mathrm{K}_{\mathrm{c}}$ & $\begin{array}{c}E^{\prime \prime} \\
\mathrm{cm}^{-1}\end{array}$ & $L$ & BAND \\
\hline 3664.61 & 0.0723 & 0.0669 & 10 & 4 & 6 & 11 & 2 & 9 & 1690.70 & 0.075083 & NU3 \\
\hline 3664.67 & 0.2090 & 0.0652 & 7 & 3 & 5 & 6 & 5 & 2 & 888.60 & 0.003875 & NU3 \\
\hline 3665.00 & 4.5829 & 0.0431 & 6 & 6 & 1 & 5 & 5 & 0 & $742 \cdot 10$ & $4 \cdot 59$ & 2NU2- \\
\hline 3665.25 & 1.7589 & 0.0431 & 6 & 6 & 0 & 5 & 5 & 1 & 742.10 & 5.399693 & $2 \mathrm{NU} 2$ \\
\hline 3665.41 & 18.4141 & 0.0948 & 4 & 1 & 4 & 3 & 2 & 1 & 212.15 & 0.123609 & NU1 \\
\hline 3665.62 & 0.0298 & 0.0447 & 9 & 6 & 3 & 8 & 7 & 2 & 1590.74 & 0.197898 & NU 1 \\
\hline 3665.86 & 4.4771 & 0.0761 & 5 & 3 & 2 & 4 & 4 & 1 & $488 \cdot 10$ & 0.119405 & NU1 \\
\hline 3665.90 & 13.5789 & 0.0825 & 4 & 2 & 3 & 3 & 3 & 0 & 285.43 & 0.131472 & NU1 \\
\hline 3665.95 & 1.0990 & 0.0680 & 6 & 3 & 3 & 5 & 5 & 0 & $742 \cdot 10$ & 0.009800 & NU3+ \\
\hline 3666.01 & 9.2943 & 0.0740 & 9 & 3 & 7 & 9 & 3 & 6 & 1282.92 & 1.235624 & NU3 \\
\hline 3666.92 & 0.0954 & 0.0798 & 9 & 2 & 8 & 8 & 3 & 5 & 1050.15 & 0.126905 & NU1 \\
\hline 3667.01 & 0.0798 & 0.1044 & 2 & 1 & 1 & 2 & 0 & 2 & 1664.97 & 2.074578 & $N \cup 1 * * * *$ \\
\hline 3667.02 & 1.1491 & 0.1111 & 1 & 1 & 0 & 1 & 0 & 1 & 23.79 & 1.500000 & NU I* \\
\hline 3667.08 & 0.1136 & 0.0916 & 4 & 0 & 4 & 4 & 2 & 3 & 300.35 & 0.299684 & NU $3 * *$ \\
\hline 3667.31 & 0.9870 & 0.0907 & 2 & 2 & 0 & 3 & 2 & 1 & 212.15 & 1.676022 & NU $3 * \%$ \\
\hline 3667.32 & 0.0219 & 0.0951 & 3 & 1 & 2 & 2 & 2 & 1 & 1742.28 & 0.300506 & NU $1 * * * *$ \\
\hline 3668.31 & 1.8917 & 0.0986 & 2 & 1 & 1 & 3 & 1 & 2 & $173 \cdot 36$ & 2.645088 & NU $3 * *$ \\
\hline 3668.79 & 1918.4159 & 0.0960 & 3 & 0 & 3 & 4 & 0 & 4 & 222.06 & 3.805784 & NU 3 \\
\hline 3668.93 & 2.2135 & 0.1010 & 2 & 0 & 2 & 3 & 0 & 3 & 1731.89 & 2.880004 & NU $3 * * * *$ \\
\hline 3669.64 & 0.1055 & 0.0863 & 5 & 1 & 5 & 5 & 1 & 4 & 399.46 & 0.456689 & NU $3 * \ddot{*}$ \\
\hline 3669.98 & 77.0437 & 0.0782 & 7 & 2 & 6 & 7 & 2 & 5 & 782.40 & 0.838975 & NU3 \\
\hline 3670.37 & 0.1991 & 0.0948 & 3 & 0 & 3 & 3 & 2 & 2 & $206 \cdot 30$ & 0.181744 & NU3* \\
\hline 3670.72 & 5541.6353 & 0.0948 & 3 & 1 & 3 & 4 & 1 & 4 & 224.83 & 3.713893 & NU3 \\
\hline 3671.35 & 0.0181 & 0.0345 & 8 & 8 & 0 & 8 & 8 & 1 & $1789 \cdot 10$ & 15.079045 & NU3* \\
\hline 3671.50 & 0.1260 & 0.0620 & 8 & 1 & 8 & 7 & 3 & 5 & 816.72 & 0.004883 & NU3 \\
\hline 3671.85 & 0.3373 & 0.0915 & 2 & 2 & 1 & 3 & 2 & 2 & $206 \cdot 30$ & 1.666667 & NU3*** \\
\hline 3671.92 & 0.6619 & 0.0984 & 2 & 1 & 2 & 3 & 1 & 3 & 1739.51 & 2.653490 & NU $3 * * * *$ \\
\hline 3672.17 & 0.0104 & 0.0385 & 9 & 8 & 1 & 9 & 7 & 2 & 1810.63 & 2.165395 & $2 N U 2$ \\
\hline 3672.74 & 0.4835 & 0.1044 & 2 & 1 & 1 & 2 & 0 & 2 & 70.08 & 2.074578 & NU1* \\
\hline 3673.19 & 0.0732 & 0.0542 & 8 & 5 & 3 & 7 & 6 & 2 & $1216 \cdot 20$ & 0.222252 & NUI \\
\hline 3673.23 & 0.2192 & 0.0516 & 8 & 5 & 4 & 7 & 6 & 1 & $1216 \cdot 20$ & 0.221954 & NU 1 \\
\hline 3673.40 & 13.4152 & 0.1010 & 2 & 0 & 2 & 3 & 0 & 3 & 136.77 & 2.880004 & NU3* \\
\hline 3674.27 & 306.8374 & 0.0916 & 4 & 0 & 4 & 4 & 2 & 3 & 300.35 & 0.299684 & NU3 \\
\hline 3674.73 & 574.5293 & 0.1111 & 1 & 1 & 0 & 1 & 0 & 1 & 23.79 & 1.500000 & NU 1 \\
\hline 3674.97 & 2667.6552 & 0.0907 & 2 & 2 & 0 & 3 & 2 & 1 & .15 & 1.676022 & NU3 \\
\hline 3675.92 & 5112.6901 & 0.0986 & 2 & 1 & 1 & 3 & 1 & 2 & .36 & 2.645088 & NU3 \\
\hline 3676.55 & 0.0894 & 0.1044 & 2 & 1 & 1 & 2 & 0 & 2 & .08 & 2.074578 & NU1** \\
\hline 3677.37 & 0.6079 & 0.0729 & 5 & 2 & 4 & 4 & 4 & 1 & 488.10 & 0.001517 & Nu3 \\
\hline 3677.44 & 285.1285 & 0.0863 & 5 & 1 & 5 & 5 & 1 & 4 & .46 & 0.456689 & NU3 3 \\
\hline 3677.71 & 0.1577 & 0.0994 & 3 & 1 & 2 & 3 & 0 & 3 & 1731 & 2.185861 & NU $1 \ddot{*} \ddot{*} \ddot{x}$ \\
\hline $3678 \cdot 36$ & 0.0107 & 0.0749 & 9 & 4 & 6 & 10 & 1 & 9 & 1293.04 & 0.047764 & NUI \\
\hline 3678.46 & 0.0570 & 0.0605 & 9 & 0 & 9 & 8 & 2 & 6 & 982.91 & 0.005067 & NU3 \\
\hline 3679.44 & 911.6467 & 0.0915 & 2 & 2 & 1 & 3 & 2 & 2 & $206 \cdot 30$ & 1.666667 & NU3 \\
\hline 3680.24 & 0.7428 & 0.0784 & 7 & 4 & 3 & 8 & 2 & 6 & 982.91 & 0.065935 & NU3 \\
\hline 3680.37 & 241.7413 & 0.1044 & 2 & 1 & 1 & 2 & 0 & 2 & 70.08 & 2.074578 & NUI \\
\hline 3680.92 & 2.4818 & 0.1010 & 2 & 0 & 2 & 3 & 0 & 3 & 136.77 & 2.830004 & NU3** \\
\hline 3681.00 & 5.5316 & 0.082 .6 & 5 & 2 & 4 & 4 & 3 & 1 & 383.85 & 0.261722 & NU 1 \\
\hline 3681.02 & 0.0650 & 0.0757 & 7 & 4 & 4 & 8 & 1 & 7 & 882.93 & 0.037317 & NU 1 \\
\hline 3681.35 & 5.5923 & 0.0864 & 4 & 2 & 2 & 3 & 3 & 1 & 285.23 & 0.161593 & NU1 \\
\hline $3681 \cdot 38$ & 0.0652 & 0.0649 & 8 & 3 & 6 & 7 & 5 & 3 & 1059.65 & 0.008488 & NU 3 \\
\hline $3681 \cdot 52$ & 0.1849 & 0.0385 & 10 & 8 & 3 & 10 & 8 & 2 & $2254 \cdot 34$ & 12.044007 & NU 3 \\
\hline 3681 . & 0.5547 & 0.0385 & 10 & 8 & 2 & 10 & 8 & 3 & $2254 \cdot 34$ & 12.044007 & $\mathrm{NU}_{3}$ \\
\hline 3681 . & 0.1811 & 0.1032 & 2 & 0 & 2 & 1 & 1 & 1 & 37.13 & 0.755253 & NU1* \\
\hline 3681.74 & 0.4747 & 0.1037 & 1 & 1 & 0 & 2 & 1 & 1 & 1693.55 & 1.500000 & NU $3 * * * *$ \\
\hline
\end{tabular}




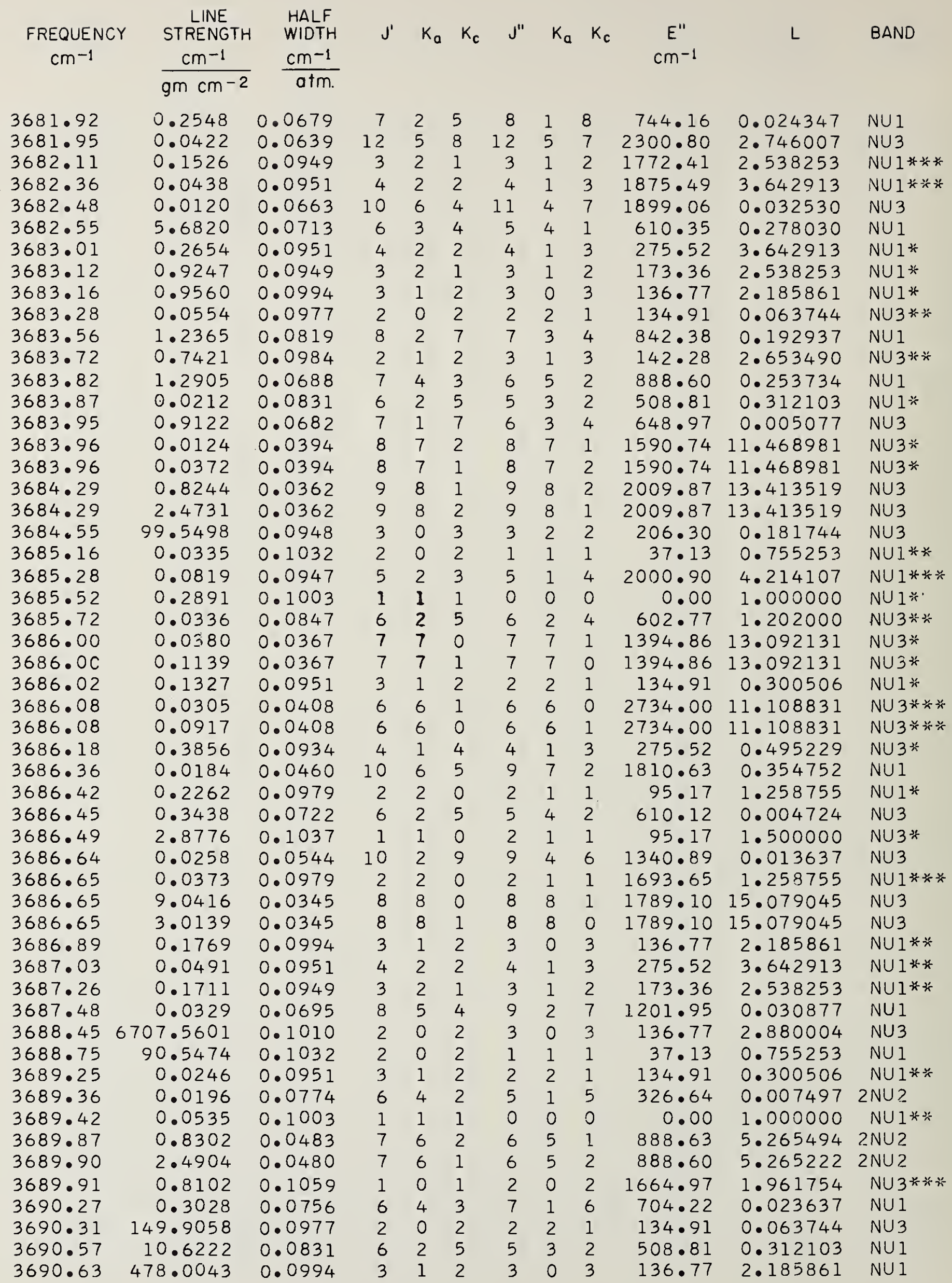




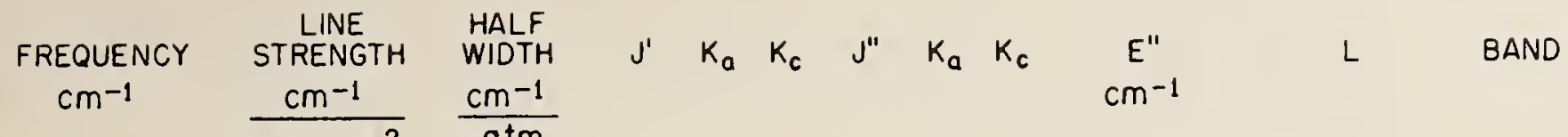

$\begin{array}{lll}3690.88 & 11.5899 & 0.0798\end{array}$

3691.06

3691.28

3691.40

3691.84

3692.07

3692.21

3692.49

3692.49

3692.58

3692.69

3692.93

3693.31

3693.77

3693.80

3694.12

$3694 \cdot 12$

3694.24

3694.29

3694.29

3694.38

3694.41

3694.52

3694.80

3695.02

3695.24

3695.39

3695.59

3695.63

3695.69

3696.34

3696.53

3696.61

3696.92

3696.92

3696.96

3697.01

3697.10

3697.12

3697.16

3697.44

3697.55

3697.55

3698.06

3698.13

3698.24

3698.33

3698.46

3698.54

3698.79

3698.83

3698.89

3699.26

3699.26
132.6981

2005.8259

462.3283

0.5808

0.5537

0.0378

0.0918

66.3550

0.1683

1. 5451

0.0196

144.5340

0.1230

90.9143

0.2496

0.5323

4. 9105

0.4194

1.2583

2. 3349

0.1029

0.2451

113.0859

0.2268

0.0170

0.0221

0.0664

0.9062

0.1889

248.1606

0.9272

0.0487

1.8803

5.6408

0.1915

9.3640

0.0143

0.1467

0.0499

0.0166

0.0676

0.2029

2.0800

0.1759

0.0886

0.3215

0.1957

0.0190

0.0136

0.0460

0.1379

6.1927

18.5782
0.0951

0.0984

0.0949

0.0757

0.0837

0.0531

0.0947

0.0 .951

0.0994

0.0989

0.0509

0.1003

0.0619

0.0847

0.0735

0.1037

0.1059

0.0439

0.0439

0.0809

0.0927

0.0827

0.0979

0.0594

0.0927

0.0487

0.0487

0.0691

0.0887

0.0947

0.0640

0.0529

0.0421

0.0421

0.1054

0.0989

0.0947

0.0586

0.0588

0.0588

0.0451

0.0451

0.0809

0.0642

0.0721

0.0909

0.0974

0.0927

0.0898

0.0546

0.0546

0.0394

0.0394

$\begin{array}{llllll}8 & 3 & 6 & 8 & 3 & 5 \\ 4 & 2 & 2 & 4 & 1 & 3 \\ 2 & 1 & 2 & 3 & 1 & 3 \\ 3 & 2 & 1 & 3 & 1 & 2 \\ 6 & 1 & 6 & 5 & 3 & 3 \\ 4 & 1 & 4 & 3 & 3 & 1 \\ 9 & 3 & 7 & 8 & 0 & 8 \\ 5 & 2 & 3 & 5 & 1 & 4 \\ 3 & 1 & 2 & 2 & 2 & 1 \\ 3 & 0 & 3 & 2 & 1 & 2 \\ 1 & 1 & 1 & 2 & 1 & 2 \\ 9 & 6 & 4 & 9 & 6 & 3 \\ 1 & 1 & 1 & 0 & 0 & 0 \\ 9 & 3 & 7 & 8 & 5 & 4\end{array}$

$\begin{array}{lll}8 & 5 & 4\end{array}$

624

936

$\begin{array}{lll}2 & 1 & 1\end{array}$

$\begin{array}{lll}2 & 0 & 2\end{array}$

$\begin{array}{lll}10 & 7 & 3\end{array}$

$\begin{array}{llllll}10 & 7 & 3 & 10 & 7 & 4\end{array}$

$\begin{array}{llllll}5 & 1 & 5 & 4 & 3 & 2 \\ 7 & 3 & 4 & 7 & 2 & 5\end{array}$

$\begin{array}{lll}7 & 3 & 5\end{array}$

$\begin{array}{lll}2 & 2 & 0\end{array}$

7

$\begin{array}{llllll}7 & 3 & 4 & 7 & 2 & 5 \\ 8 & 6 & 3 & 8 & 6 & 2\end{array}$

862

$\begin{array}{lll}7 & 2 & 6\end{array}$

$\begin{array}{lll}5 & 2 & 4\end{array}$

$\begin{array}{lll}5 & 2 & 3 \\ 8 & 4 & 5\end{array}$

955

$\begin{array}{lll}9 & 7 & 2\end{array}$

$\begin{array}{lll}9 & 7 & 3\end{array}$

$\begin{array}{lll}2 & 1 & 2\end{array}$

$\begin{array}{lll}1 & 1 & 1\end{array}$

$\begin{array}{lll}4 & 1 & 3\end{array}$

954

$\begin{array}{lll}7 & 5 & 3\end{array}$

$\begin{array}{lll}7 & 5 & 2\end{array}$

$\begin{array}{lll}7 & 6 & 1\end{array}$

$\begin{array}{lll}7 & 6 & 2\end{array}$

633

$\begin{array}{lll}8 & 2 & 7\end{array}$

$\begin{array}{lll}7 & 3 & 4\end{array}$

$\begin{array}{lll}5 & 3 & 2\end{array}$

$\begin{array}{lll}4 & 1 & 3 \\ 7 & 3 & 4\end{array}$

$\begin{array}{lll}7 & 3 & 4 \\ 8 & 3 & 5\end{array}$

$\begin{array}{lll}6 & 5 & 2\end{array}$

$\begin{array}{lll}6 & 5 & 1 \\ 8 & 7 & 2\end{array}$

$\begin{array}{llllll}8 & 7 & 2 & 8 & 7 & 1 \\ 8 & 7 & 1 & 8 & 7 & 2\end{array}$
1050.15

275.52

142.28

173.36

504.00

285.23

744.09

399.46

134.91

1677.08

1677.08

1631.41

0.00

1255.16

602.77

1282.92

95.17

70.08

2054.40

2054.40

382.52

782.40

842.38

95.17

1122.72

2392.58

1411.68

1411.65

756.75

2053.98

399.46

1059.85

1411.68

1810.63

1810.63

1618.55

79.48

1813.81

1411.65

2724.19

2724.05

1216.20

1216.20

610.12

927.77

888.63

446.50

222.06

782.40

982.91

2552.87

2552.85

1590.74

1590.74

1.436963

3. 642913

2.653490

2. 538253

0.004689

0.001497

0.116343

4. 214107

0.300506

1. 741385

1.500000

7.297946

1.000000

0.014160

1.202000

0.032937

1.500000

1.961754

9.075340

9.075340

0.003420

5. 918411

1. 781698

1.258755

0.013441

5. 918411

8.307617

8.307617

0.008618

1. 147395

4. 214107

0.427306

0.391127

10.162962

10.162962 


\begin{tabular}{|c|c|c|c|c|c|c|c|c|c|c|c|}
\hline $\begin{array}{l}\text { FREQUENCY } \\
\qquad \mathrm{cm}^{-1}\end{array}$ & 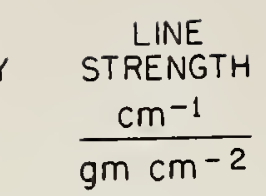 & $\begin{array}{l}\text { HALF } \\
\text { WIDTH } \\
\frac{\mathrm{cm}^{-1}}{a \dagger m .}\end{array}$ & $J^{\prime}$ & $k_{a}$ & $\mathrm{~K}_{\mathrm{c}}$ & $J^{\prime \prime}$ & $K_{a}$ & $K_{c}$ & $\begin{array}{c}E^{11} \\
\mathrm{~cm}^{-1}\end{array}$ & $L$ & BAND \\
\hline 3699.38 & 0.0812 & 0.0920 & 6 & 2 & 4 & 6 & 1 & 5 & 542.91 & 4.225862 & NUI* \\
\hline 3699.47 & & 0.0408 & 6 & 6 & 1 & 6 & 6 & 0 & 1045.07 & 11.108831 & NU $3 *$ \\
\hline 3699.47 & 0.5555 & 0.0408 & 6 & 6 & 0 & 6 & 6 & 1 & 1045.07 & 11.108831 & NU $3 *$ \\
\hline 3699.49 & 0.7748 & 0.0646 & 11 & 5 & 7 & 11 & 5 & 6 & 1999.02 & 3.347960 & NU3 \\
\hline 3700.10 & 1.4118 & 0.0740 & 7 & 3 & 5 & 6 & 4 & 2 & 757.78 & 0.431025 & NUI \\
\hline 3700.15 & 0.1145 & 0.0486 & 5 & 5 & 0 & 5 & 5 & 1 & 2406.15 & 9.130832 & NU $3 * * *$ \\
\hline 3700.15 & 0.3436 & 0.0486 & 5 & 5 & 1 & 5 & 5 & 0 & 2406.15 & 9.130832 & NU $3 * * *$ \\
\hline 3700.65 & 0.0274 & 0.0633 & 9 & 5 & 5 & 9 & 5 & 4 & 1477.31 & 4.762151 & NU3* \\
\hline 3700.76 & 0.3862 & 0.0971 & 3 & 1 & 3 & 3 & 1 & 2 & 1772.41 & 0.597949 & NU $3 * *+$ \\
\hline 3700.92 & 0.4878 & 0.0952 & 2 & 2 & 1 & 2 & 1 & 2 & 79.48 & 0.833333 & IUI* \\
\hline 3700.93 & 0.0190 & 0.0581 & 10 & 3 & 8 & 9 & 5 & 5 & 1474.99 & 0.019495 & VU3 \\
\hline 3701.30 & 18.9961 & 0.0367 & 7 & 7 & 0 & 7 & 7 & 1 & 1394.86 & 13.092131 & NU3 \\
\hline 3701.30 & 56.9884 & 0.0367 & 7 & 7 & 1 & 7 & 7 & 0 & 1394.86 & 13.09 & NU3 \\
\hline 3701.58 & 0.5239 & 0.0532 & 11 & 6 & 6 & 11 & 6 & 5 & $2144 \cdot 12$ & 5.65 & NU3 \\
\hline 3701.75 & 1438.7777 & 0.1037 & 1 & 1 & 0 & 2 & 1 & 1 & 95.17 & 1.50 & NU 3 \\
\hline 3701.78 & 192.7970 & 0.0934 & 4 & 1 & 4 & 4 & 1 & 3 & 275.52 & 0.495229 & NU3 \\
\hline 3702.68 & 51.4549 & 0.0927 & 7 & 3 & 4 & 7 & 2 & 5 & $782 \cdot 40$ & 5.918411 & NU1 \\
\hline 3702.89 & 0.0595 & 0.0909 & 5 & 3 & 2 & 5 & 2 & 3 & $446 \cdot 50$ & $3 \cdot 44$ & NU $1 * \ddot{*}$ \\
\hline 3703.01 & 0.0150 & 0.0920 & 6 & 2 & 4 & 6 & 1 & 5 & 542.91 & $4 \cdot 22$ & NUl $* *$ \\
\hline 3703.01 & 1.1452 & 0.0887 & 5 & 2 & 4 & 5 & 2 & 3 & $446 \cdot 50$ & 1.14 & NU 3* \\
\hline 3703.04 & 0.0041 & 0.0487 & 8 & 6 & 3 & 8 & 6 & 2 & 1411.68 & 8.30 & NU $3 * *$ \\
\hline 3703.23 & 1.1604 & 0.1054 & 2 & 1 & 2 & 1 & 0 & 1 & 23.79 & 1.50 & NUI* \\
\hline 3703.24 & 0.0123 & 0.0487 & 8 & 6 & 2 & 8 & 6 & 3 & 1411.65 & 8.30 & NU $3 * * *$ \\
\hline 3703.57 & 0.0727 & 0.0532 & 10 & 5 & 6 & 9 & 6 & 3 & 1631.41 & 0.57 & NU1 \\
\hline 3703.64 & 0.2508 & 0.0865 & 5 & 3 & 3 & 6 & 0 & & 446.71 & 0.01 & NUI \\
\hline 3703.77 & 0.0341 & 0.0751 & 8 & 4 & 5 & 8 & 4 & 4 & $1131 \cdot 76$ & 152 & NU3* \\
\hline 3703.83 & 8.3690 & 0.0801 & 6 & 3 & 3 & 7 & 1 & 6 & $704 \cdot 22$ & 0.06 & NU3 \\
\hline 3703.94 & 0.9218 & 0.0754 & 7 & 3 & 4 & 8 & 1 & 7 & 882.93 & 318 & NU 3 \\
\hline 3704.14 & 0.0102 & 0.0887 & 9 & 4 & 5 & 9 & 3 & 6 & $1282 \cdot 92$ & 7.16 & NU $1 *$ \\
\hline 3704.39 & 0.0132 & 0.0770 & 11 & 3 & 9 & 10 & 4 & 6 & 1616.51 & 0.25 & NU1 \\
\hline 3704.56 & 1.0200 & 0.0994 & 3 & 0 & 3 & 2 & 1 & 2 & 79.48 & 1.74 & NU I * \\
\hline 3704.60 & 1.7324 & 0.0989 & 1 & 1 & 1 & 2 & 1 & 2 & 79.48 & 1.50 & NU $3 * * *$ \\
\hline 3704.72 & 0.0323 & 0.0614 & 8 & 5 & 4 & 8 & 5 & 3 & 1255.92 & 5.56 & NU 3* \\
\hline 3705.06 & 0.1749 & 0.0532 & 11 & 6 & 5 & 11 & 6 & 6 & 2142.69 & 5.65 & NU3 \\
\hline 3705.07 & 0.0902 & 0.0952 & 2 & 2 & 1 & 2 & 1 & 2 & 79.48 & 0.83 & NU $1 * *$ \\
\hline 3705.20 & 0.2286 & 0.0536 & 12 & 6 & 6 & 12 & 6 & 7 & 2433.85 & 4.92 & NU 3 \\
\hline 3705.20 & 0.0126 & 0.0451 & 7 & 6 & 1 & 7 & 6 & 2 & $1216 \cdot 20$ & 9.53 & NU3** \\
\hline 3705.20 & 0.0376 & 0.0451 & 7 & 6 & 2 & 7 & 6 & 1 & $1216 \cdot 20$ & 9.53 & NU $3 * *$ \\
\hline 3705.24 & 0.7952 & 0.0520 & 10 & 6 & 5 & 10 & 6 & 4 & 1875.53 & 6.43 & NU3 \\
\hline 3705.27 & 5.5085 & 0.0845 & 5 & 3 & 2 & 6 & 1 & 5 & 542.91 & 0.053826 & NU3 \\
\hline 3705.41 & 17.1589 & 0.0925 & 6 & 3 & 3 & 6 & 2 & 4 & 602.77 & $2 \cdot 41$ & NUI+ \\
\hline 3705.49 & 0.9451 & 0.0880 & 4 & 3 & 2 & 5 & 0 & 5 & $325 \cdot 35$ & 0.011053 & NU1 \\
\hline 3705.75 & 97.8469 & 0.0974 & 4 & 1 & 3 & 4 & 0 & 4 & 222.06 & 24 & NUI \\
\hline 3705.77 & 0.6215 & 0.0680 & 8 & 0 & 8 & 7 & 2 & 5 & 782.40 & 0.00 & $\mathrm{NU}_{3}$ \\
\hline 3706.37 & 6.7804 & 0.0898 & 8 & 3 & 5 & 8 & 2 & 6 & 982.91 & 6.36 & NUI \\
\hline 3706.54 & 2.3864 & 0.0520 & 10 & 6 & 4 & 10 & 6 & 5 & 1875.00 & 6.43 & NU3 \\
\hline 3706.64 & 0.4168 & 0.0845 & 6 & 3 & 4 & 7 & 0 & 7 & 586.26 & 0.01 & NUI \\
\hline 3706.64 & 40.5980 & 0.0920 & 6 & 2 & 4 & 6 & 1 & 5 & 542.91 & 4.225862 & NU 1 \\
\hline 3706 & 0.0973 & 0.0614 & 8 & 5 & 3 & 8 & 5 & 4 & $1255 \cdot 16$ & 5.561462 & NU3* \\
\hline 3706.68 & 2.3406 & 0.0971 & 3 & 1 & 3 & 3 & 1 & 2 & 173.36 & 0.597949 & NU $3 *$ \\
\hline 3706.78 & 12.9838 & 0.0746 & 9 & 4 & 6 & 9 & 4 & 5 & 1360.28 & 2.522255 & NU3 \\
\hline 3706.88 & 0.1300 & 0.0884 & 4 & 3 & 1 & 4 & 2 & 2 & 315.79 & 2.167225 & NUI* \\
\hline 3707.12 & 0.0342 & 0.0408 & 6 & 6 & 1 & 6 & 6 & 0 & 1045.07 & 11.108831 & NU $3 * *$ \\
\hline 3707.12 & 0.1028 & 0.0408 & 6 & 6 & 0 & 6 & 6 & 1 & 1045.07 & $11 \cdot 108831$ & NU $3 * *$ \\
\hline
\end{tabular}




\begin{tabular}{|c|c|c|c|c|c|c|c|c|c|c|c|}
\hline $\begin{array}{l}\text { FREQUENCY } \\
\qquad \mathrm{cm}^{-1}\end{array}$ & $\begin{array}{l}\text { LINE } \\
\text { STRENGTH } \\
\frac{\mathrm{cm}^{-1}}{\mathrm{gm} \mathrm{cm}^{-2}}\end{array}$ & $\begin{array}{l}\text { HALF } \\
\text { WIDTH } \\
\frac{\mathrm{cm}^{-1}}{\mathrm{~atm} .}\end{array}$ & $J^{\prime}$ & $\mathrm{K}_{0}$ & $K_{c}$ & $J^{\prime \prime}$ & $\mathrm{K}_{0}$ & $k_{c}$ & $\begin{array}{c}E^{\prime \prime} \\
\mathrm{cm}^{-1}\end{array}$ & L & BAND \\
\hline 3707.15 & 0.0530 & 0.0909 & 5 & 3 & 2 & 5 & 2 & 3 & 2053.98 & 3.442901 & NU $1 \ddot{x} * * *$ \\
\hline 3707.16 & 0.2147 & 0.1054 & 2 & 1 & 2 & 1 & 0 & 1 & 23.79 & 1.500000 & NU 1 米关 \\
\hline 3707.46 & 160.7364 & 0.0909 & 5 & 3 & 2 & 5 & 2 & 3 & 446.50 & 3.442901 & NU1 \\
\hline 3707.50 & 0.3021 & 0.0588 & 7 & 5 & 3 & 7 & 5 & 2 & 1059.85 & 6.486001 & NU $3 *$ \\
\hline 3707.72 & 0.0562 & 0.0886 & 5 & 2 & 3 & 4 & 3 & 2 & 382.52 & 0.436045 & NU $1 *$ \\
\hline 3707.83 & 0.3283 & 0.0763 & 5 & 2 & 3 & 4 & 4 & 0 & 488.13 & 0.002437 & NU3 \\
\hline 3707.98 & 0.1007 & 0.0588 & 7 & 5 & 2 & 7 & 5 & 3 & 1059.65 & 6.486001 & NU3* \\
\hline 3708.21 & 0.1887 & 0.0994 & 3 & 0 & 3 & 2 & 1 & 2 & 79.48 & 1.741385 & NUI \\
\hline 3708.25 & 9.8188 & 0.0509 & 9 & 6 & 4 & 9 & 6 & 3 & 1631.41 & 7.297946 & NU3 \\
\hline $3708 \cdot 34$ & 0.1854 & 0.0939 & 3 & 2 & 2 & 3 & 1 & 3 & $142 \cdot 28$ & 1.297651 & NU $1 *$ \\
\hline 3708.35 & 0.0276 & 0.0793 & 12 & 5 & 7 & 12 & 4 & 8 & 2205.65 & 9.504415 & NUI \\
\hline 3708.51 & 3.2732 & 0.0509 & 9 & 6 & 3 & 9 & 6 & 4 & 1631.27 & 7.297946 & NU3 \\
\hline 3708.96 & 3.0116 & 0.0802 & 6 & 4 & 2 & 7 & 2 & 5 & 782.40 & 0.032449 & NU3 \\
\hline 3709.23 & 243.8891 & 0.0952 & 2 & 2 & 1 & 2 & 1 & 2 & 79.48 & 0.833333 & NU1 \\
\hline $3709 \cdot 42$ & 2455.2679 & 0.1059 & 1 & 0 & 1 & 2 & 0 & 2 & 70.08 & 1.961754 & NU3 \\
\hline 3709.68 & 0.8360 & 0.0546 & 6 & 5 & 1 & 6 & 5 & 2 & 888.60 & 7.626602 & NU $3 *$ \\
\hline 3710.20 & 1.1225 & 0.0642 & 10 & 5 & 6 & 10 & 5 & 5 & 1724.72 & 4.030316 & NU3 \\
\hline 3710.46 & 0.0214 & 0.0884 & 4 & 3 & 1 & 4 & 2 & 2 & 1922.92 & 2.167225 & NUI*** \\
\hline 3710.69 & 11.0561 & 0.0487 & 8 & 6 & 3 & 8 & 6 & 2 & 1411.68 & 8.307617 & NU3 \\
\hline 3710.89 & 33.1701 & 0.0487 & 8 & 6 & 2 & 8 & 6 & 3 & 1411.65 & 8.307617 & NU3 \\
\hline 3710.89 & 122.5898 & 0.0827 & 7 & 3 & 5 & 7 & 3 & 4 & $842 \cdot 38$ & 1.781698 & NU3 \\
\hline 3711.09 & 580.2141 & 0.1054 & 2 & 1 & 2 & 1 & 0 & 1 & 23.79 & 1.500000 & NUI \\
\hline $3711 \cdot 32$ & 0.6942 & 0.0486 & 5 & 5 & 0 & 5 & 5 & 1 & 742.10 & 9.130832 & NU $3 *$ \\
\hline 3711.32 & 2.0826 & 0.0486 & 5 & 5 & 1 & 5 & 5 & 0 & 742.10 & 9.130832 & NU3* \\
\hline 3711.40 & 0.1397 & 0.0667 & 5 & 4 & 1 & 5 & 4 & 2 & 2251.71 & 5.742440 & NU3*** \\
\hline 3711.72 & 0.5465 & 0.0863 & 10 & 4 & 6 & 10 & 3 & 7 & $1538 \cdot 23$ & 8.179783 & NU1 \\
\hline 3711.87 & 510.0087 & 0.0994 & 3 & 0 & 3 & 2 & 1 & 2 & 79.48 & 1.741385 & NU1 \\
\hline 3712.20 & 4682.0000 & 0.0989 & 1 & 1 & 1 & 2 & 1 & 2 & 79.48 & 1.500000 & NU3 \\
\hline 3712.66 & 1.5409 & 0.1054 & 0 & 0 & 0 & 1 & 0 & 1 & $1618 \cdot 55$ & 1.000000 & NU $3 * * *$ \\
\hline 3712.80 & 2.1502 & 0.0750 & 8 & 3 & 6 & 7 & 4 & 3 & 931.22 & 0.519243 & NUI \\
\hline 3712.85 & 33.8205 & 0.0451 & 7 & 6 & 1 & 7 & 6 & 2 & $1216 \cdot 20$ & 9.538170 & $\mathrm{NU}_{3}$ \\
\hline 3712.85 & 101.4617 & 0.0451 & 7 & 6 & 2 & 7 & 6 & 1 & $1216 \cdot 20$ & 9.538170 & $\mathrm{NU}_{3}$ \\
\hline 3713.15 & 0.1988 & 0.0892 & 3 & 3 & 1 & 4 & 0 & 4 & 222.06 & 0.004153 & NU1 \\
\hline $3713 \cdot 28$ & 28.0766 & 0.0886 & 5 & 2 & 3 & 4 & 3 & 2 & 382.52 & 0.436045 & NUI \\
\hline 3713.31 & 0.7199 & 0.0694 & 8 & 3 & 5 & 9 & 1 & 8 & 1079.07 & 0.034132 & NU3 \\
\hline 3713.39 & 0.0542 & 0.0914 & 5 & 1 & 4 & 5 & 0 & 5 & 1920.76 & 1.912338 & NU $1 * * * *$ \\
\hline 3713.43 & 5.1230 & 0.0887 & 9 & 4 & 5 & 9 & 3 & 6 & 1282.92 & 7.165263 & NUI \\
\hline 3713.79 & 0.0615 & 0.0818 & 7 & 3 & 5 & 8 & 0 & 8 & 744.09 & 0.017472 & NUI \\
\hline 3714.02 & 0.0670 & 0.0961 & 4 & 0 & 4 & 3 & 1 & 3 & 1739.51 & 2.831638 & NU1 ${ }^{*} * *$ \\
\hline 3714.08 & 0.3306 & 0.0854 & 3 & 3 & 0 & 3 & 2 & 1 & $212 \cdot 15$ & 1.092505 & NUI \\
\hline 3714.26 & 0.0180 & 0.0614 & 8 & 5 & 3 & 8 & 5 & 4 & 1255.16 & 5.561462 & NU $3 *$ * \\
\hline $3714 \cdot 43$ & 0.4330 & 0.0971 & 3 & 1 & 3 & 3 & 1 & 2 & 173.36 & 0.597949 & NU3** \\
\hline 3714.53 & 1.0415 & 0.0528 & 8 & 6 & 3 & 7 & 5 & 2 & 1059.85 & 5.144364 & 2 NU2 \\
\hline 3714.77 & 92.5882 & 0.0408 & 6 & 6 & 1 & 6 & 6 & 0 & 1045.07 & 11.108831 & NU3 \\
\hline 3714.77 & 277.7646 & 0.0408 & 6 & 6 & 0 & 6 & 6 & 1 & 1045.07 & 11.108831 & NU3 \\
\hline 3715.13 & 0.0177 & 0.0751 & 8 & 4 & 4 & 8 & 4 & 5 & 2764.71 & 3.145152 & NU $3 *$ 幽* \\
\hline 3715.16 & 0.0559 & 0.0588 & 7 & 5 & 3 & 7 & 5 & 2 & 1059.85 & 6.486001 & NU3灾并 \\
\hline 3715.48 & 0.1624 & 0.0620 & 9 & 4 & 6 & 8 & 5 & 3 & 1255.92 & 0.597432 & NU 1 \\
\hline 3715.62 & 0.0187 & 0.0588 & 7 & 5 & 2 & 7 & 5 & 3 & 1059.65 & 6.486001 & NU $3 * *$ \\
\hline 3715.73 & 0.3472 & 0.0502 & 8 & 6 & 2 & 7 & 5 & 3 & 1059.65 & 5.142711 & $2 \mathrm{NU} 2$ \\
\hline 3715.86 & 0.1982 & 0.0770 & 10 & 3 & 8 & 9 & 4 & 5 & $1360 \cdot 28$ & 0.409296 & NU 1 \\
\hline 3716.01 & 0.2714 & 0.0829 & 5 & 3 & 3 & 5 & 3 & 2 & 2130.50 & 2.240000 & NU $3 * * 3$ \\
\hline 3716.07 & 64.9813 & 0.0884 & 4 & 3 & 1 & 4 & 2 & 2 & 315.79 & 2.167225 & NU1 \\
\hline 3716.19 & 13.7273 & 0.0533 & 9 & 5 & 5 & 9 & 5 & 4 & 1477.31 & 4.762151 & NU3 \\
\hline
\end{tabular}




\begin{tabular}{|c|c|c|c|c|c|c|c|c|c|c|c|}
\hline $\begin{array}{c}\text { FREQUENCY } \\
\mathrm{cm}^{-1}\end{array}$ & $\begin{array}{l}\text { LINE } \\
\text { STRENGTH } \\
\frac{\mathrm{cm}^{-1}}{\mathrm{gm} \mathrm{cm}^{-2}}\end{array}$ & $\begin{array}{l}\text { HALF } \\
\text { WIDTH } \\
\frac{\mathrm{cm}^{-1}}{a \dagger m .}\end{array}$ & $J^{\prime}$ & $\mathrm{K}_{\mathrm{a}}$ & $K_{c}$ & Ј" & $K_{a}$ & $K_{c}$ & $\begin{array}{c}E^{\prime \prime} \\
\mathrm{cm}^{-1}\end{array}$ & $L$ & BAND \\
\hline 3716.25 & 6.7049 & 0.0834 & 9 & 3 & 6 & 9 & 2 & 7 & 1201.95 & 6.247031 & NUI \\
\hline 3716.58 & 92.6790 & 0.0939 & 3 & 2 & 2 & 3 & 1 & 3 & 142.28 & 1.297651 & NU1 \\
\hline 3716.83 & 0.1610 & 0.0907 & 4 & 2 & 3 & 4 & 2 & 2 & 1922.92 & 1.519518 & NU $3 * \ddot{*}$ 前 \\
\hline 3716.87 & 9.3386 & 0.1054 & 0 & 0 & 0 & 1 & 0 & 1 & 23.79 & 1.000000 & NU $3 *$ \\
\hline 3716.93 & 0.3314 & 0.0711 & 6 & 4 & 3 & 6 & 4 & 2 & 757.78 & 4.707057 & NU $3 *$ \\
\hline 3716.98 & 0.3287 & 0.0914 & 5 & 1 & 4 & 5 & 0 & 5 & 325.35 & 1.912338 & NU $1 *$ \\
\hline 3717.02 & 0.0120 & 0.0583 & 11 & 1 & 10 & 10 & 3 & 7 & $1538 \cdot 23$ & 0.016855 & NU 3 \\
\hline 3717.25 & 0.0515 & 0.0546 & 6 & 5 & 2 & 6 & 5 & 1 & 888.63 & 7.626602 & NU $3 * *$ \\
\hline 3717.33 & 0.1546 & 0.0546 & 6 & 5 & 1 & 6 & 5 & 2 & 888.60 & 7.626602 & NU $3 * *$ \\
\hline 3717.71 & 0.0339 & 0.0829 & 6 & 3 & 4 & 6 & 3 & 3 & $661 \cdot 56$ & 1.610000 & NU $3 * *$ \\
\hline 3717.78 & 0.2663 & 0.0999 & 2 & 1 & 2 & 2 & 1 & 1 & 1693.65 & 0.833333 & NU $3 * * *$ \\
\hline 3718.07 & 0.0440 & 0.0607 & 9 & 4 & 6 & 8 & 1 & 7 & 882.93 & 0.269351 & 2 NU 2 \\
\hline 3718.14 & 0.2566 & 0.0760 & 9 & 3 & 7 & 8 & 4 & 4 & 1131.76 & 0.505247 & NU 1 \\
\hline 3718.23 & 0.0160 & 0.0947 & 4 & 1 & 3 & 3 & 2 & 2 & $206 \cdot 30$ & 0.835095 & NU $1 * *$ \\
\hline 3718.36 & 0.4456 & 0.0896 & 4 & 2 & 3 & 4. & 1 & 4 & $224 \cdot 83$ & 1.567300 & NU $1 *$ \\
\hline $3718 \cdot 42$ & 0.0190 & 0.0848 & 7 & 2 & 5 & 7 & 1 & 6 & $704 \cdot 22$ & 3.983004 & NUI** \\
\hline 3718.52 & 0.5089 & 0.1026 & 3 & 1 & 3 & 2 & 0 & 2 & 70.08 & 2.156792 & NU $1 *$ \\
\hline 3718.60 & 0.9759 & 0.0907 & 4 & 2 & 3 & 4 & 2 & 2 & 315.79 & 1.519518 & NU3* \\
\hline 3718.64 & 0.0612 & 0.0854 & 3 & 3 & 0 & 3 & 2 & 1 & $212 \cdot 15$ & 1.092505 & NUI** \\
\hline 3718.95 & 4.3541 & 0.0894 & 8 & 4 & 4 & 8 & 3 & 5 & 1050.15 & 5.709388 & NUI \\
\hline 3718.97 & 572.6216 & 0.0887 & 5 & 2 & 4 & 5 & 2 & 3 & $446 \cdot 50$ & 1.147395 & NU3 \\
\hline 3719.39 & 1.0003 & 0.0711 & 6 & 4 & 2 & 6 & 4 & 3 & $756 \cdot 75$ & 4.707057 & NU $3 *$ \\
\hline 3719.48 & 0.0546 & 0.0854 & 3 & 3 & 0 & 3 & 2 & 1 & 1819.34 & 1.092505 & NU I **** \\
\hline 3719.72 & 2.5369 & 0.0667 & 5 & 4 & 2 & 5 & 4 & 1 & 610.35 & 5.742440 & NU $3 *$ \\
\hline 3719.73 & 17.0275 & 0.0751 & 8 & 4 & 5 & 8 & 4 & 4 & 1131.76 & 3.145152 & NU3 \\
\hline 3719.95 & 0.1077 & 0.0830 & 3 & 3 & 1 & 3 & 2 & 2 & $206 \cdot 30$ & 1.035682 & NU I* \\
\hline 3719.95 & 0.0635 & 0.0737 & 7 & 4 & 4 & 7 & 4 & 3 & $931 \cdot 22$ & 3.870291 & NU $3 * *$ \\
\hline 3719.96 & 0.0554 & 0.0876 & 7 & 4 & 3 & 7 & 3 & 4 & $842 \cdot 38$ & 4.268667 & NUI* \\
\hline 3719.96 & 1.6446 & 0.0829 & 5 & 3 & 3 & 5 & 3 & 2 & 508.81 & 2.240000 & NU $3 *$ \\
\hline 3720.02 & 0.0735 & 0.0896 & 4 & 2 & 3 & 4 & 1 & 4 & 1821.61 & 1.567300 & $\mathrm{NU} 1 * * * *$ \\
\hline 3720.09 & 16.1346 & 0.0614 & 8 & 5 & 4 & 8 & 5 & 3 & 1255.92 & 5.561462 & NU3 \\
\hline 3720.10 & 0.0360 & 0.0735 & 12 & 4 & 8 & 12 & 3 & 9 & 2105.90 & 8.242761 & NUI \\
\hline 3720.12 & 0.8468 & 0.0667 & 5 & 4 & 1 & 5 & 4 & 2 & 610.12 & 5.742440 & NU3* \\
\hline 3720.35 & 0.1166 & 0.0737 & 7 & 4 & 3 & 7 & 4 & 4 & 927.77 & 3.870291 & NU $3 *$ \\
\hline 3720.37 & 0.2501 & 0.0800 & 4 & 3 & 2 & 4 & 3 & 1 & 2005.92 & 3.310000 & NU $3 * * * *$ \\
\hline 3720.59 & 0.0608 & 0.0914 & 5 & 1 & 4 & 5 & 0 & 5 & 325.35 & 1.912338 & NU 1** \\
\hline 3720.67 & 0.0248 & 0.0619 & 10 & 5 & 5 & 9 & 6 & 4 & 1631.27 & 0.586260 & NU1 \\
\hline 3721.33 & 1.9441 & 0.0605 & 4 & 4 & 1 & 4 & 4 & 0 & $488 \cdot 13$ & 7.160948 & NU3* \\
\hline 3721.39 & 5.8332 & 0.0605 & 4 & 4 & 0 & 4 & 4 & 1 & $488 \cdot 10$ & 7.160948 & NU3* \\
\hline 3721.51 & 43.3543 & 0.0947 & 4 & 1 & 3 & 3 & 2 & 2 & $206 \cdot 30$ & 0.835095 & NU1 \\
\hline $3721 \cdot 62$ & 0.3606 & 0.0829 & 4 & 3 & 2 & 4 & 2 & 3 & 300.35 & 1.848533 & NU $1 *$ \\
\hline 3721.62 & 4.6564 & 0.0633 & 9 & 5 & 4 & 9 & 5 & 5 & 1474.99 & 4.762151 & NU3 \\
\hline 3721.86 & 51.4633 & 0.0848 & 7 & 2 & 5 & 7 & 1 & 6 & $704 \cdot 22$ & 3.983004 & NUI \\
\hline 3721.88 & 48.6859 & 0.0614 & 8 & 5 & 3 & 8 & 5 & 4 & $1255 \cdot 16$ & 5.561462 & NU3 \\
\hline 3722.19 & 1170.3233 & 0.0971 & 3 & 1 & 3 & 3 & 1 & 2 & $173 \cdot 36$ & 0.597949 & NU 3 \\
\hline 3722.35 & 1.9306 & 0.0758 & 3 & 3 & 1 & 3 & 3 & 0 & 1907.63 & 5.203793 & NU $3 * * * *$ \\
\hline 3722.69 & 0.6443 & 0.0758 & 3 & 3 & 0 & 3 & 3 & 1 & 1907.47 & 5.203793 & NU3* \\
\hline 3722.77 & 0.7557 & 0.0800 & 4 & 3 & 1 & 4 & 3 & 2 & 2004.81 & 3.310000 & $N \cup 3 * * *$ \\
\hline 3722.82 & 151.0475 & 0.0588 & 7 & 5 & 3 & 7 & 5 & 2 & 1059.85 & 6.486001 & NU3 \\
\hline 3722.98 & 1.6144 & 0.0999 & 2 & 1 & 2 & 2 & 1 & 1 & 95.17 & 0.833333 & NU3* \\
\hline 3723.20 & 165.3169 & 0.0854 & 3 & 3 & 0 & 3 & 2 & 1 & $212 \cdot 15$ & 1.092505 & NU1 \\
\hline 3723.26 & 50.3551 & 0.0588 & 7 & 5 & 2 & 7 & 5 & 3 & 1059.65 & 6.486001 & NU3 \\
\hline 3723.35 & 3.5672 & 0.0642 & 10 & 5 & 5 & 10 & 5 & 6 & 1718.77 & 4.030316 & NU3 \\
\hline 3723.74 & 0.0110 & 0.0848 & 7 & 3 & 4 & 6 & 4 & 3 & 756.75 & 0.550367 & NU 1 * \\
\hline
\end{tabular}




\begin{tabular}{|c|c|c|c|c|c|c|c|c|c|c|c|}
\hline $\begin{array}{c}\text { FREQUENC } \\
\mathrm{cm}^{-1}\end{array}$ & $\begin{array}{l}\text { LINE } \\
\text { STRENGTH } \\
\frac{\mathrm{cm}^{-1}}{\mathrm{gm} \mathrm{cm}^{-2}}\end{array}$ & $\begin{array}{l}\text { HALF } \\
\text { WIDTH } \\
\frac{\mathrm{cm}^{-1}}{\mathrm{~atm} .}\end{array}$ & $J^{\prime}$ & $K_{a}$ & $\mathrm{~K}_{\mathrm{c}}$ & J" & $K_{a}$ & $K_{c}$ & $\begin{array}{c}\mathrm{E}^{\prime \prime} \\
\mathrm{cm}^{-1}\end{array}$ & L & BAND \\
\hline 3724.20 & $164 \cdot 3495$ & 0.0914 & 5 & 1 & 4 & 5 & 0 & 5 & 325.35 & 1.912338 & NU1 \\
\hline 3724.50 & 1.7277 & 0.1054 & 0 & 0 & 0 & 1 & 0 & 1 & 23.79 & 1.000000 & NU3** \\
\hline 3724.62 & 0.0613 & 0.0711 & 6 & 4 & 3 & 6 & 4 & 2 & 757.78 & 4.707057 & NU3** \\
\hline 3724.90 & 139.3240 & 0.0546 & 6 & 5 & 2 & 6 & 5 & 1 & 888.63 & 7.626602 & NU3 \\
\hline $3724 \cdot 94$ & 1.1515 & 0.0910 & 3 & 2 & 2 & 3 & 2 & 1 & $1819 \cdot 34$ & 2.151589 & NU $3 * * * *$ \\
\hline $3724 \cdot 98$ & 417.9811 & 0.0546 & 6 & 5 & 1 & 6 & 5 & 2 & 888.60 & 7.626602 & NU3 \\
\hline 3725.20 & 0.4062 & 0.0961 & 4 & 0 & 4 & 3 & 1 & 3 & $142 \cdot 28$ & 2.831638 & NU $1 *$ \\
\hline 3725.40 & 0.0431 & 0.0879 & 4 & 2 & 2 & 5 & 0 & 5 & 325.35 & 0.023440 & NU $3 *$ \\
\hline 3725.41 & 0.0909 & 0.0803 & 5 & 3 & 3 & 5 & 2 & 4 & 416.22 & 2.492169 & NUI* \\
\hline 3725.44 & 0.2198 & 0.0976 & 4 & 1 & 4 & 3 & 0 & 3 & 1731.89 & 3.004990 & NUl**** \\
\hline 3725.66 & 91.5553 & 0.0829 & 6 & 3 & 4 & 6 & 3 & 3 & $661 \cdot 56$ & 1.610000 & NU3- \\
\hline 3725.95 & 1.5155 & 0.0800 & 4 & 3 & 2 & 4 & 3 & 1 & 383.85 & 3.310000 & NU $3 *$ \\
\hline 3726.05 & 0.0667 & 0.0829 & 4 & 3 & 2 & 4 & 2 & 3 & 300.35 & 1.848533 & NU $1 * * *$ \\
\hline 3726.45 & 254.4680 & 0.1026 & 3 & 1 & 3 & 2 & 0 & 2 & 70.08 & 2.156792 & NUI \\
\hline 3726.50 & 222.7880 & 0.0896 & 4 & 2 & 3 & 4 & 1 & 4 & 224.83 & 1.567300 & NU 1 \\
\hline 3726.62 & 347.1002 & 0.0486 & 5 & 5 & 0 & 5 & 5 & 1 & $742 \cdot 10$ & 9.130832 & NU3 \\
\hline 3726.62 & 1041.3007 & 0.0486 & 5 & 5 & 1 & 5 & 5 & 0 & $742 \cdot 10$ & 9.130832 & NU 3 \\
\hline 3727.00 & 0.1850 & 0.0711 & 6 & 4 & 2 & 6 & 4 & 3 & 756.75 & 4.707057 & NU $3 * *$ \\
\hline 3727.01 & 17.8522 & 0.0854 & 4 & 3 & 1 & 5 & 1 & 4 & 399.46 & 0.028214 & NU 3 \\
\hline 3727.37 & 0.4693 & 0.0667 & 5 & 4 & 2 & 5 & 4 & 1 & 610.35 & 5.742440 & $N \cup 3 * *$ \\
\hline 3727.73 & 0.3042 & 0.0829 & 5 & 3 & 3 & 5 & 3 & 2 & $508 \cdot 81$ & 2.240000 & NU $3 * *$ \\
\hline 3727.74 & 171.4775 & 0.0737 & 7 & 4 & 4 & 7 & 4 & 3 & $931 \cdot 22$ & 3.870291 & NU3 \\
\hline 3728.33 & 5.4781 & 0.0848 & 7 & 3 & 4 & 6 & 4 & 3 & 756.75 & 0.550367 & NUI \\
\hline 3728.72 & 4.5800 & 0.0800 & 4 & 3 & 1 & 4 & 3 & 2 & $382 \cdot 52$ & 3.310000 & NU $3 *$ \\
\hline 3728.74 & 0.2604 & 0.0646 & 11 & 5 & 6 & 11 & 5 & 7 & 1985.83 & 3.347960 & NU3 \\
\hline 3728.87 & 0.8541 & 0.0912 & 2 & 2 & 1 & 2 & 2 & 0 & 1743.47 & 3.269589 & NU $3 * * *$ \\
\hline 3728.91 & 53.8748 & 0.0830 & 3 & 3 & 1 & 3 & 2 & 2 & $206 \cdot 30$ & 1.035682 & NUI \\
\hline 3728.95 & 0.0752 & 0.0961 & 4 & 0 & 4 & 3 & 1 & 3 & $142 \cdot 28$ & 2.831638 & NU1** \\
\hline 3728.98 & 0.3597 & 0.0605 & 4 & 4 & 1 & 4 & 4 & 0 & 488.13 & 7.160948 & NU3** \\
\hline 3729.04 & 1.0791 & 0.0605 & 4 & 4 & 0 & 4 & 4 & 1 & $488 \cdot 10$ & 7.160948 & NU3** \\
\hline 3729.08 & 6.9788 & 0.0910 & 3 & 2 & 2 & 3 & 2 & 1 & 212.15 & 2.151589 & NU3* \\
\hline 3729.13 & 0.5629 & 0.0829 & 5 & 3 & 2 & 5 & 3 & 3 & 504.00 & 2.240000 & NU3* \\
\hline 3729.26 & 0.0564 & 0.0637 & 9 & 3 & 6 & 10 & 1 & 9 & 1293.04 & 0.023266 & NU3 \\
\hline 3729.26 & 1.8787 & 0.1027 & 1 & 1 & 1 & 1 & 1 & 0 & 1640.51 & 1.500000 & $N \cup 3 * * *$ \\
\hline 3729.33 & 11.7012 & 0.0758 & 3 & 3 & 1 & 3 & 3 & 0 & 285.43 & 5.203793 & NU3 $*$ \\
\hline 3729.47 & 0.0162 & 0.0855 & 4 & 1 & 3 & 3 & 3 & 0 & 285.43 & 0.007215 & NU3* \\
\hline 3729.77 & 0.0168 & 0.0803 & 5 & 3 & 3 & 5 & 2 & 4 & $416 \cdot 22$ & 2.492169 & NU $1 * *$ \\
\hline 3729.80 & 3.9047 & 0.0758 & 3 & 3 & 0 & 3 & 3 & 1 & 285.23 & 5.203793 & NU3* \\
\hline 3730.02 & 0.7482 & 0.0749 & 7 & 0 & 7 & 6 & 2 & 4 & 602.77 & 0.009797 & NU3 \\
\hline 3730.04 & 27.7032 & 0.0876 & 7 & 4 & 3 & 7 & 3 & 4 & $842 \cdot 38$ & 4.268667 & NUI \\
\hline 3730.16 & 0.0978 & 0.0815 & 5 & 2 & 4 & 5 & 1 & 5 & 326.64 & 1.711792 & NU $I^{*}$ \\
\hline 3730.49 & 0.1957 & 0.0600 & 10 & 4 & 7 & 9 & 5 & 4 & 1477.31 & 0.720783 & NUI \\
\hline 3730.49 & 180.3214 & 0.0829 & 4 & 3 & 2 & 4 & 2 & 3 & $300 \cdot 35$ & 1.848533 & NU 1 \\
\hline 3730.68 & 0.2987 & 0.0999 & 2 & 1 & 2 & 2 & 1 & 1 & 95.17 & 0.833333 & NU3** \\
\hline 3730.96 & 0.6472 & 0.0747 & 10 & 3 & 7 & 10 & 2 & 8 & 1438.00 & 5.910419 & NUI \\
\hline 3731.33 & 0.0149 & 0.0922 & 4 & 0 & 4 & 3 & 2 & 1 & 1819.34 & 0.027572 & NU $3 * * *$ \\
\hline 3731.38 & 2.5800 & 0.0912 & 2 & 2 & 0 & 2 & 2 & i & $1742 \cdot 28$ & 3.269589 & $N \cup 3 * * *$ \\
\hline 3731.42 & 0.1640 & 0.0751 & 6 & 3 & 4 & 6 & 2 & 5 & $552 \cdot 92$ & 2.963539 & NU $I^{*}$ \\
\hline 3732.13 & 4669.2922 & 0.1054 & 0 & 0 & 0 & 1 & 0 & 1 & 23.79 & 1.000000 & NU3 \\
\hline 3732.31 & 165.7268 & 0.0711 & 6 & 4 & 3 & 6 & 4 & 2 & 757.78 & 4.707057 & NU3 \\
\hline 3732.70 & 203.1144 & 0.0961 & 4 & 0 & 4 & 3 & 1 & 3 & $142 \cdot 28$ & 2.831638 & NUI \\
\hline 3732.82 & 1.3321 & 0.0976 & 4 & 1 & 4 & 3 & 0 & 3 & 136.77 & 3.004990 & NU I * \\
\hline 3732.88 & 0.0161 & 0.0815 & 5 & 2 & 4 & 5 & 1 & 5 & 1922.86 & 1.711792 & NU $1 * * *$ \\
\hline 3733.20 & 0.1847 & 0.0880 & 5 & 0 & 5 & 4 & 1 & 4 & 1821.61 & 3.911752 & NU $I^{* * * *}$ \\
\hline
\end{tabular}




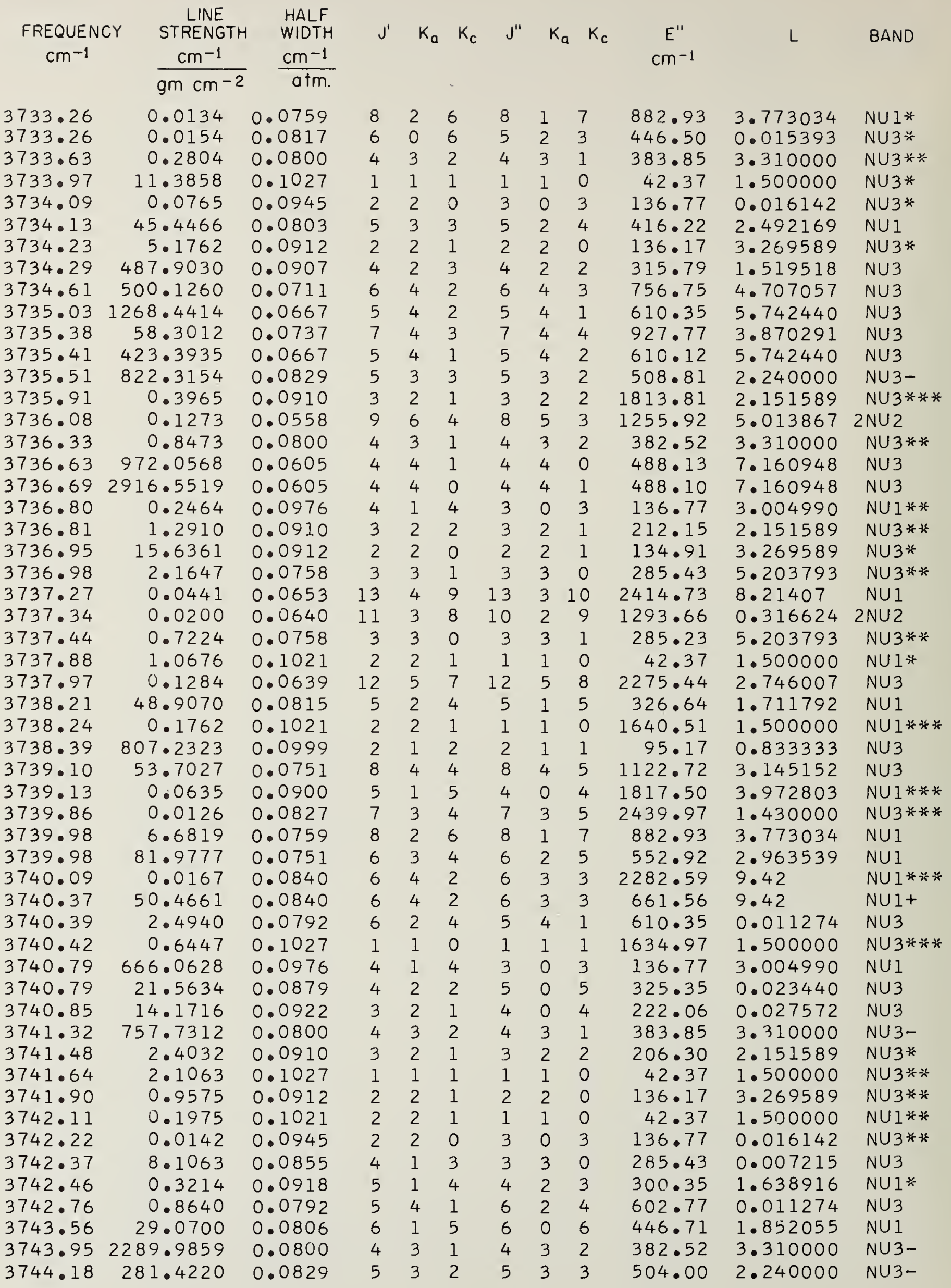




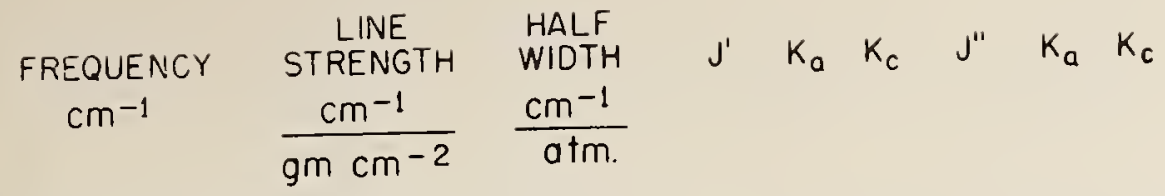

$\mathrm{E}^{\prime \prime}$

BAND

3744.553489 .4356 3744.645850 .5869 3745.091952 .3806 $3745.52 \quad 139.8683$

3745.55

3746.12

$3746 \cdot 34$

3746.86

3747.23

3747.41

3747.43

3747.74

3747.82

3748.17

3748.28

3748.31

3748.49

3748.65

3748.75

3748.99

3749.20

3749.31

3749.57

3750.26

3750.29

3750.35

3750.60

3750.63

3750.88

3750.95

3751.10

3751.48

$3752 \cdot 14$

3752.21

3752.53

3752.84

3753.54

3753.64

3753.73

3754.18

3754.61

3754.68

3754.71

3754.80

3755.13

3755.15

3755.40

3755.92

3756.61

3757.63

3757.69

3758.13

3758.32

3758.33
10.1844

4.8467

533.7904

0.1023

16.9013

$309 \cdot 9884$

0.3847

0.0899

0.0437

0.0311

13.8194

0.5214

2.4978

0.0562

0.0387

7.7443

160.6755

5692.9022

2588.0843

0.0364

38.2258

0.0471

0.0458

3. 1891

138.9137

0.8717

559.7738

83.9324

7818.0682

420.3516

151.8258

0.1430

1.6436

105.4296

0.0346

5. 2830

12.7948

0.0866

2.8489

1.5863

0.3050

192.3580

0.0758

1201.5765

2. 0594

0.1234

16.9430

0.0213

0.5899
12.2782
0.0910

0.0758

0.0758

0.0720

0.0893

0.0746

0.1021

0.0787

0.0696

0.0787

0.0900

0.0922

0.0457

0.0710

0.0682

0.0655

0.0794

0.0949

0.0583

0.0817

0.0918

0.1027

0.0912

0.0656

0.0603

0.0945

0.0760

0.0720

0.0907

0.0720

0.0999

0.0880

0.0710

0.0912

0.0689

0.0949

0.0779

0.0563

0.0719

0.0704

0.0999

0.0945

0.0671

0.0855

0.0600

0.0984

0.0900

0.0827

0.0910

0.0756

0.0586

0.0645

0.0567

0.0907 $\begin{array}{rrrrrr}3 & 2 & 2 & 3 & 2 & 1 \\ 3 & 3 & 1 & 3 & 3 & 0 \\ 3 & 3 & 0 & 3 & 3 & 1 \\ 6 & 4 & 3 & 6 & 3 & 4 \\ 6 & 2 & 4 & 5 & 3 & 3 \\ 9 & 4 & 5 & 9 & 4 & 6 \\ 2 & 2 & 1 & 1 & 1 & 0 \\ 5 & 4 & 1 & 5 & 3 & 2 \\ 7 & 4 & 4 & 7 & 3 & 5 \\ 5 & 4 & 1 & 5 & 3 & 2 \\ 5 & 1 & 5 & 4 & 0 & 4 \\ 4 & 0 & 4 & 3 & 2 & 1 \\ 10 & 3 & 8 & 9 & 0 & 9 \\ 6 & 2 & 5 & 6 & 1 & 6 \\ 7 & 3 & 5 & 7 & 2 & 6 \\ 11 & 3 & 8 & 11 & 2 & 9 \\ 9 & 5 & 4 & 9 & 4 & 5 \\ 2 & 2 & 0 & 1 & 1 & 1 \\ 10 & 3 & 7 & 11 & 1 & 10\end{array}$

606

$\begin{array}{lll}5 & 2\end{array}$

423

110

220

836

827

303

515

431

423

$\begin{array}{lll}4 & 3\end{array}$

212

414

$$
\begin{array}{lll}
6 & 1 & 6
\end{array}
$$$$
\begin{array}{lll}
2 & 2
\end{array}
$$$$
\begin{array}{lll}
4 & 3 & 2
\end{array}
$$$$
111
$$

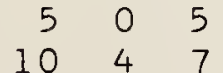

212.15

285.43

285.23

648.97

504.00

1340.89

42.37

2130.50

816.72

508.81

222.06

212.15

920.18

447.24

709.60

1690.70

1360.28

37.13

1524.87

446.50

$300 \cdot 35$

$42 \cdot 37$

136.17

1006.12

885.62

136.77

1922.86

2005.92

300.35

383.85

1677.08

224.83

447.24

134.91

382.52

37.13

1920.76

1581.34

504.00

931.22

79.48

136.17

586.26

275.52

1216.27

95.17

222.06

816.72

206.30

1131.76

1477.31

648.97

1581.34

300.35

2. 151589

5. 203793

5.203793

8.16

0.863547

2. 522255

1.500000

8.97

6.84

8.97

3. 972803

0.027572

0.106449

1. 783035

3.273499

5.642326

5.113359

1. 244747

0.016855

0.015393

1.638916

1.500000

3.269589

4.264036

3.456295

0.016142

4.958549

6.45

1.519518

6.45

0.833333

3.911752

1.783035

3.269589

6.46

1.244747

4.979001

10.08

8.92

4.12

0.833333

0.016142

1.838288

0.007215

4.734521

1.666667

3.972803

1.430000

2.151589

4.012859

4.857731

10.80

0.012276 


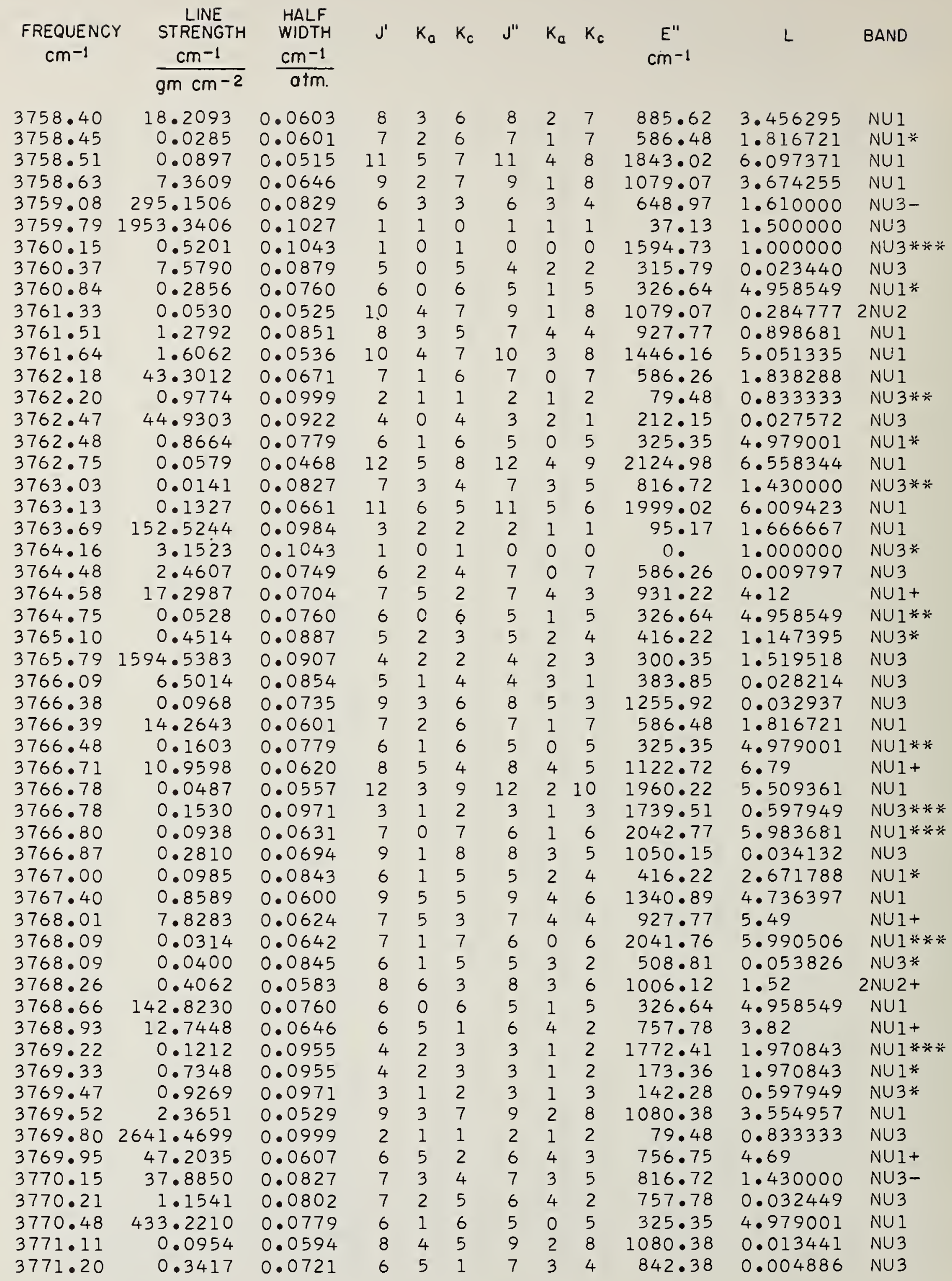




\begin{tabular}{|c|c|c|c|c|c|c|c|c|c|c|c|}
\hline $\begin{array}{c}\text { FREQUENCY } \\
\mathrm{cm}^{-1}\end{array}$ & $\begin{array}{l}\text { LINE } \\
\text { STRENGTH } \\
\frac{\mathrm{cm}^{-1}}{g m \mathrm{~cm}^{-2}}\end{array}$ & $\begin{array}{l}\text { HALF } \\
\text { WIDTH } \\
\frac{\mathrm{cm}^{-1}}{a+m .}\end{array}$ & $J^{\prime}$ & $\mathrm{K}_{\mathrm{a}}$ & $K_{c}$ & $J^{\prime \prime}$ & $\mathrm{K}_{\mathrm{a}}$ & $K_{c}$ & $\begin{array}{c}E^{\prime \prime} \\
\mathrm{cm}^{-1}\end{array}$ & L & BAND \\
\hline 3771.45 & 78.2781 & 0.0580 & 5 & 5 & 0 & 5 & 4 & 1 & 610.35 & 3.74 & NUI+ \\
\hline 3771.68 & $26 \cdot 1273$ & 0.0566 & 5 & 5 & 1 & 5 & 4 & 2 & 610.12 & 3.74 & NU I + \\
\hline 3771.83 & 0.5831 & 0.1043 & 1 & 0 & 1 & 0 & 0 & 0 & 0 & 1.000000 & NU $3 * * *$ \\
\hline 3772.10 & 0.0132 & 0.0542 & 8 & 1 & 7 & 8 & 0 & 8 & 744.09 & 1.839778 & $\mathrm{NU} 1$ * \\
\hline 3772.41 & 0.1322 & 0.0620 & 10 & 6 & 4 & 10 & 5 & 5 & 1724.72 & 4.974368 & NU 1 \\
\hline 3772.42 & 0.0835 & 0.0887 & 5 & 2 & 3 & 5 & 2 & 4 & 416.22 & 1.147395 & NU $3 * *$ \\
\hline 3772.46 & 0.0890 & 0.0798 & 8 & 3 & 5 & 8 & 3 & 6 & $1006 \cdot 12$ & 1.436963 & NU3* \\
\hline $3773 \cdot 28$ & 0.0156 & 0.0607 & 6 & 5 & 2 & 6 & 4 & 3 & 2398.39 & 4.69 & NU $1 * * * *$ \\
\hline 3773.34 & 0.6647 & 0.0642 & 7 & 4 & 4 & 8 & 2 & 7 & 885.62 & 0.011793 & NU 3 \\
\hline 3773.40 & 0.0998 & 0.0544 & 9 & 4 & 6 & 10 & 2 & 9 & 1293.66 & 0.013637 & NU 3 \\
\hline 3773.65 & 0.1359 & 0.0955 & 4 & 2 & 3 & 3 & 1 & 2 & 173.36 & 1.970843 & NU $1 * \ddot{x}$ \\
\hline 3773.84 & 0.0394 & 0.0503 & 8 & 2 & 7 & 8 & 1 & 8 & 744.16 & 1.833129 & NU I* \\
\hline 3773.92 & 0.0294 & 0.0486 & 12 & 6 & 7 & 12 & 5 & 8 & 2275.44 & 6.631337 & NUI \\
\hline 3773.97 & 2.8476 & 0.0726 & 10 & 4 & 6 & 10 & 4 & 7 & 1581.36 & 1.530000 & NU3- \\
\hline 3774.05 & 49.2429 & 0.0843 & 6 & 1 & 5 & 5 & 2 & 4 & $416 \cdot 22$ & 2.671788 & NUI \\
\hline 3774.69 & 0.0258 & 0.0580 & 5 & 5 & 0 & 5 & 4 & 1 & $2251 \cdot 86$ & 3.74 & NU $1 * * * *$ \\
\hline 3774.96 & 0.0893 & 0.0785 & 10 & 4 & 6 & 9 & 5 & 5 & 1474.99 & 0.959644 & NUI \\
\hline 3775.57 & 0.0428 & 0.0515 & 11 & 6 & 6 & 11 & 5 & 7 & 1985.83 & 5.799382 & NU 1 \\
\hline 3775.65 & 0.0352 & 0.0734 & 7 & 1 & 6 & 6 & 2 & 5 & $2161 \cdot 30$ & 3.799063 & NUl* $* * *$ \\
\hline 3775.96 & 0.8407 & 0.0534 & 10 & 2 & 8 & 10 & 1 & 9 & 1293.04 & 3.646815 & NUI \\
\hline 3776.46 & 0.6509 & 0.0989 & 2 & 1 & 2 & 1 & 1 & 1 & 1634.97 & 1.500000 & NU $3 * * * *$ \\
\hline 3776.55 & 0.2623 & 0.0649 & 7 & 5 & 3 & 8 & 3 & 6 & 1006.12 & 0.008488 & NU3 \\
\hline 3777.01 & 0.1714 & 0.0971 & 3 & 1 & 2 & 3 & 1 & 3 & 142.28 & 0.597949 & NU $3 * *$ \\
\hline 3777.02 & 0.5527 & 0.0961 & 3 & 2 & 1 & 2 & 1 & 2 & $79 \cdot 48$ & 0.925282 & NU.1* \\
\hline 3777.08 & 0.5683 & 0.0631 & 7 & 0 & 7 & 6 & 1 & 6 & $447 \cdot 24$ & 5.983681 & NUI* \\
\hline 3777.11 & 26.1526 & 0.0859 & 7 & 2 & 5 & 6 & 3 & 4 & 648.97 & 1.512999 & NU 1 \\
\hline 3777.44 & 0.0912 & 0.0961 & 3 & 2 & 1 & 2 & 1 & 2 & 1677.08 & 0.925282 & NU $1 * * * *$ \\
\hline 3777.97 & 367.3966 & 0.0955 & 4 & 2 & 3 & 3 & 1 & 2 & 173.36 & 1.970843 & NUI \\
\hline 3778.22 & 0.4141 & 0.0529 & 10 & 6 & 5 & 10 & 5 & 6 & 1718.77 & 4.913223 & NUI \\
\hline 3778.29 & 1.2356 & 0.0763 & 4 & 4 & 0 & 5 & 2 & 3 & 446.50 & 0.002437 & NU3 \\
\hline 3778.67 & 0.0116 & 0.0466 & 6 & 6 & 1 & 6 & 5 & 2 & 888.60 & 1.111607 & NUl* \\
\hline 3779.05 & 1.1050 & 0.0587 & 9 & 6 & 3 & 9 & 5 & 4 & 1477.31 & 4.017231 & NUI \\
\hline 3779.50 & $1576 \cdot 1857$ & 0.1043 & 1 & 0 & 1 & 0 & 0 & 0 & 0 & 1.000000 & NU3 \\
\hline 3779.74 & 225.6980 & 0.0887 & 5 & 2 & 3 & 5 & 2 & 4 & 416.22 & 1.147395 & NU3 \\
\hline 3779.76 & 6.5911 & 0.0542 & 8 & 1 & 7 & 8 & 0 & 8 & 744.09 & 1.839778 & NU 1 \\
\hline 3780.25 & 0.0302 & 0.0927 & 5 & 2 & 4 & 4 & 1 & 3 & 1875.49 & 2.444582 & NU I $* * * *$ \\
\hline 30.49 & 0.3911 & 0.0691 & 6 & 4 & 3 & 7 & 2 & 6 & 709.60 & 0.008618 & NU3 \\
\hline 80.60 & 0.1828 & 0.0927 & 5 & 2 & 4 & 4 & 1 & 3 & 275.52 & 2.444582 & $1 *$ \\
\hline 80.85 & 0.1187 & 0.0427 & 12 & 4 & 9 & 12 & 3 & 10 & 1962.55 & 5.350112 & NUI \\
\hline 3781.02 & $3 \cdot 9448$ & 0.0989 & 2 & 1 & 2 & 1 & 1 & 1 & 37.13 & 1.500000 & NU3* \\
\hline 3781.04 & 0.1051 & 0.0631 & 7 & 0 & 7 & 6 & $i$ & 6 & $447 \cdot 24$ & 5.983681 & NU $1 * *$ \\
\hline 3781.15 & 0.1023 & 0.0961 & 3 & 2 & 1 & 2 & 1 & 2 & 79.48 & 0.925282 & NUI** \\
\hline 3781.25 & 0.3731 & 0.0534 & 9 & 6 & 4 & 9 & 5 & 5 & 1474.99 & 4.001691 & NUI \\
\hline 3781.48 & 0.0128 & 0.0577 & 8 & 6 & 3 & 9 & 4 & 6 & 1340.89 & 0.006585 & NU3 \\
\hline 3781.68 & 0.0352 & 0.0642 & 7 & 1 & 7 & 6 & 0 & 6 & 446.71 & 5.990506 & NU $1 * *$ \\
\hline 3781.78 & 19.7042 & 0.0503 & 8 & 2 & 7 & 8 & 1 & 8 & $744 \cdot 16$ & 1.833129 & NUI \\
\hline 3781.90 & 3.4583 & 0.0754 & 8 & 1 & 7 & 7 & 3 & 4 & $842 \cdot 38$ & 0.049318 & NU3 \\
\hline 3781.97 & 20.0055 & 0.0845 & 6 & 1 & 5 & 5 & 3 & 2 & 508.81 & 0.053826 & NU3 \\
\hline $3782 \cdot 27$ & 2.7335 & 0.1059 & 2 & 0 & 2 & 1 & 0 & 1 & $1618 \cdot 55$ & 1.961754 & NU $3 * * *$ \\
\hline 3782.48 & 2.4819 & 0.0458 & 10 & 3 & 8 & 10 & 2 & 9 & 1293.66 & 3.605841 & NU1 \\
\hline 3782.70 & 0.0613 & 0.0810 & 8 & 5 & 4 & 7 & 2 & 5 & 782.40 & 0.074366 & $2 \mathrm{NU} 2$ \\
\hline 3782.81 & 0.0550 & 0.0523 & 8 & 1 & 8 & 7 & 0 & 7 & 2180.65 & 7.000291 & $\mathrm{NU} \mathbf{l}^{*} * *$ \\
\hline 3783.38 & 0.5625 & 0.0847 & 6 & 2 & 4 & 6 & 2 & 5 & 552.92 & 0.939536 & NU3* \\
\hline 3783.65 & 0.8520 & 0.0553 & 8 & 6 & 2 & 8 & 5 & 3 & 1255.92 & 3.077042 & NU1 \\
\hline
\end{tabular}




\begin{tabular}{|c|c|c|c|c|c|c|c|c|c|c|c|}
\hline $\begin{array}{c}\text { FREQUENCY } \\
\mathrm{cm}^{-1}\end{array}$ & $\begin{array}{l}\text { LINE } \\
\text { STRENGTH } \\
\frac{\mathrm{cm}^{-1}}{\mathrm{gm} \mathrm{cm}^{-2}}\end{array}$ & $\begin{array}{l}\text { HALF } \\
\text { WIDTH } \\
\mathrm{cm}^{-1} \\
\text { atm. }\end{array}$ & $J^{\prime}$ & $\mathrm{K}_{\mathrm{a}}$ & $K_{c}$ & $J^{\prime \prime}$ & $K_{a}$ & $K_{c}$ & $\begin{array}{c}E^{\prime \prime} \\
\mathrm{cm}^{-1}\end{array}$ & $\mathrm{~L}$ & BAND \\
\hline 83.90 & 0.2562 & 0.0680 & 7 & 2 & 5 & 8 & 0 & 8 & 744.09 & 0.006703 & NU3 \\
\hline & 0.0254 & 0.0470 & 13 & 3 & 10 & 13 & 2 & 11 & 2246.98 & & NUI \\
\hline $34 \cdot 36$ & 2.5674 & 0.0527 & 8 & 6 & 3 & 8 & 5 & 4 & $1255 \cdot 16$ & 3.073714 & NU 1 \\
\hline 3784.56 & 463.4236 & 0.0971 & 3 & 1 & 2 & 3 & 1 & 3 & $142 \cdot 28$ & 0.597949 & NU3 \\
\hline 35.01 & 284.1359 & 0.0631 & 7 & 0 & 7 & 6 & 1 & 6 & $447 \cdot 24$ & 5.983681 & NUI \\
\hline 5.28 & 276.3516 & 0.0961 & 3 & 2 & 1 & 2 & 1 & 2 & 79.48 & 0.925282 & NUI \\
\hline 5.69 & 95.0855 & 0.0642 & 7 & 1 & 7 & 6 & 0 & 6 & 446.71 & 5.990506 & NUI \\
\hline 6.01 & 16.5667 & 0.1059 & 2 & 0 & 2 & 1 & 0 & 1 & 23.79 & 1.961754 & NU3* \\
\hline 6.26 & 44.5172 & 0.0798 & 8 & 3 & 5 & 8 & 3 & 6 & 100 & 1.436963 & NU3 \\
\hline $6 \cdot 68$ & 0.2526 & 0.0934 & 4 & 1 & 3 & 4 & 1 & 4 & 182 & 0.495229 & NU $3 * * *$ \\
\hline 36.90 & 4.7126 & 0.0515 & 7 & 6 & 1 & 7 & 5 & 2 & 105 & 2.119964 & NU1 \\
\hline 3787.10 & 1.5705 & 0.0506 & 7 & 6 & 2 & 7 & 5 & 3 & 1055 & 2.119434 & NUI \\
\hline 7.40 & 3.5309 & 0.0801 & 7 & 1 & 6 & 6 & 3 & 3 & 56 & 0.061 & NU3 \\
\hline 3787.61 & 0.0263 & 0.0459 & 12 & 7 & 6 & 12 & 6 & 7 & 243 & 5.91 & NU1 \\
\hline 3787.67 & 1.9075 & 0.1037 & 2 & 1 & 1 & 1 & 1 & 0 & $164 C$ & 1.500000 & NU $3 \ddot{*} * *$ \\
\hline 3787.97 & 1.5311 & 0.0934 & 4 & 1 & 3 & 4 & 1 & 4 & .83 & 0.49 & NU3* \\
\hline 3788.02 & 0.0165 & 0.0443 & 9 & 1 & 8 & 9 & 0 & 9 & .18 & 1.844131 & $\mathrm{NU}{ }_{1 *}^{*}$ \\
\hline 3788.06 & 0.2133 & 0.0734 & 7 & 1 & 6 & 6 & 2 & 5 & .92 & 3.799063 & NUI* \\
\hline 3788.72 & 0.7298 & 0.0989 & 2 & 1 & 2 & 1 & 1 & 1 & 37.13 & 1.500000 & NU $3 * *$ \\
\hline 3788.78 & 0.2689 & 0.0688 & 11 & 4 & 7 & 11 & 4 & 8 & 1843 & 1.701473 & NU3 \\
\hline $3789 \cdot 24$ & 1.9386 & 0.0469 & 6 & 6 & 0 & 6 & 5 & 1 & 888.63 & 1.111654 & NUI \\
\hline 3789.27 & 5.8155 & 0.0466 & 6 & 6 & 1 & 6 & 5 & 2 & $888 \cdot 60$ & 1.111607 & NUI \\
\hline 3789.33 & 91.4180 & 0.0927 & 5 & 2 & 4 & 4 & 1 & 3 & . 52 & 2.444 & NU1 \\
\hline 3789.92 & 0.3605 & 0.0842 & 3 & 3 & 1 & 2 & 2 & 0 & $\cdot 17$ & 2.394805 & NU1* \\
\hline 90.38 & 0.0445 & 0.0488 & 11 & 7 & 4 & 11 & 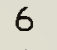 & 5 & 2144 & 4.999 & NU1 \\
\hline 3790.60 & 0.1040 & 0.0847 & 6 & 2 & 4 & 6 & 2 & 5 & 55 & 0.939 & NU3** \\
\hline 3791.20 & 0.3789 & 0.0873 & 6 & 2 & 5 & 5 & 1 & 4 & 39 & 3.129447 & NUI* \\
\hline 3791.37 & 1.0755 & 0.0840 & 3 & 3 & 0 & 2 & 2 & 1 & 134.91 & 2.366160 & NU $1 *$ \\
\hline 3791.44 & 0.0154 & 0.0442 & 11 & 2 & 9 & 10 & 1 & 10 & 111 & .09 & $2 N \cup 2$ \\
\hline 3791.57 & 11.5607 & 0.1037 & 2 & 1 & 1 & 1 & 1 & 0 & 37 & - 500000 & $\mathrm{NU} 3 *$ \\
\hline 3791.7 & 0.0395 & 0.0734 & 7 & 1 & 6 & $t$ & 2 & 5 & • 92 & 3.799063 & NU $1 * \ddot{*}$ \\
\hline 91.79 & 0.0148 & 0.0473 & 11 & 7 & 5 & 11 & 6 & 6 & 214 & 4.990193 & NU1 \\
\hline 3791.87 & 0.7954 & 0.0442 & 11 & 2 & 9 & 11 & 1 & 10 & .87 & 3.646421 & NU1 \\
\hline $3792 \cdot 30$ & 0.1976 & 0.0410 & 8 & 7 & 1 & 7 & 6 & 2 & .20 & .263414 & $2 \mathrm{NU} 2$ \\
\hline $3792 \cdot 30$ & 0.5929 & 0.0410 & 8 & 7 & 2 & 7 & $\epsilon$ & 1 & $\cdot 20$ & 6.263444 & $2 N \cup 2$ \\
\hline 3792.46 & 1.4121 & 0.0722 & 5 & 4 & 2 & 6 & 2 & 5 & .92 & 0.004724 & NU3 \\
\hline 3792.71 & 0.3332 & 0.0523 & 8 & 1 & 8 & 7 & 0 & 7 & $\cdot 26$ & 7.000291 & NU $1 *$ \\
\hline 3792.73 & 0.0943 & 0.0520 & 8 & 0 & 8 & 7 & 1 & 7 & .48 & .95 & NUI* \\
\hline 3793.7 & 3.0648 & 0.1059 & 2 & 0 & 2 & 1 & 0 & 1 & 23.79 & 1.961754 & NU3** \\
\hline 3793.83 & 2.9731 & 0.0784 & 8 & 2 & 6 & 7 & 4 & 3 & .22 & 0.065935 & NU3 \\
\hline 379 & 0.0667 & 0.0842 & 3 & 3 & 1 & 2 & 2 & 0 & - 17 & 2.394805 & NU $1 * *$ \\
\hline 3795. & 0.0483 & 0.0475 & 10 & 7 & 3 & 10 & 6 & 4 & .53 & 4.061130 & NU1 \\
\hline 3795.4 & 0.2833 & 0.0934 & 4 & 1 & 3 & 4 & 1 & 4 & +.83 & 0.495229 & NU3*** \\
\hline $3795 \cdot 48$ & 2.3034 & 0.0820 & 9 & 3 & 6 & 8 & 4 & 5 & 1122 & 1.416234 & NUI \\
\hline 3795. & 106.6507 & 0.0734 & 7 & 1 & 6 & 6 & 2 & 5 & .92 & 3.799063 & NUI \\
\hline $3795 \cdot 6$ & 0.1447 & 0.0463 & 10 & 7 & 4 & 10 & 6 & 5 & 187 & 4.058996 & NUI \\
\hline 3795.7 & 0.2644 & 0.0406 & 11 & 3 & 9 & 11 & 2 & 10 & 152 & 3.632655 & NU1 \\
\hline 3795.85 & 8.2511 & 0.0443 & 9 & 1 & 8 & 9 & 0 & 9 & 92 & 1.844131 & NU 1 \\
\hline 3795.90 & 0.1990 & 0.0840 & 3 & 3 & 0 & 2 & 2 & 1 & 134 & 2.366160 & NU $1 * * *$ \\
\hline 3796.09 & 2.8097 & 0.0984 & 3 & 1 & 3 & 2 & 1 & 2 & 1677 & 2.653490 & $\mathrm{NU} 3 * * *$ \\
\hline 3796.43 & 1972.3763 & 0.0989 & 2 & 1 & 2 & 1 & 1 & 1 & .13 & 1.500000 & NU3 \\
\hline 3796.86 & 2.7480 & 0.0430 & 9 & 2 & 8 & 9 & 1 & 9 & 92 & 1.842036 & NUI \\
\hline 3796. & 0.0287 & 0.0436 & 9 & 0 & 9 & 8 & 1 & 8 & 2337 & 8.007087 & NU $1 * * * *$ \\
\hline 3797.08 & 2.3801 & 0.0757 & 5 & 3 & 3 & 6 & 1 & 6 & $447 \cdot 24$ & 0.004689 & NU3 \\
\hline
\end{tabular}




$\begin{array}{ccc}\text { FREQUENCY } & \text { LINE } & \text { HALF } \\ \mathrm{cm}^{-1} & \frac{\mathrm{cm} \mathrm{m}^{-1}}{\mathrm{gm} \mathrm{cm}-2} & \frac{\mathrm{cm}-1}{\mathrm{otm} .}\end{array}$

$\begin{array}{rrrrrr}3797.29 & 0.0159 & 0.0628 & 7 & 6 & 1 \\ 3797.82 & 281.2435 & 0.0847 & 6 & 2 & 4 \\ 3798.49 & 0.4030 & 0.0459 & 9 & 7 & 2 \\ 3798.53 & 1.0581 & 0.0809 & 4 & 3 & 2 \\ 3798.58 & 3.7621 & 0.0907 & 3 & 3 & 0 \\ 3798.60 & 0.0131 & 0.0781 & 7 & 2 & 6 \\ 3798.63 & 0.1343 & 0.0454 & 9 & 7 & 3 \\ 3799.04 & 180.2308 & 0.0842 & 3 & 3 & 1 \\ 3799.24 & 2.1387 & 0.1037 & 2 & 1 & 1 \\ 3799.85 & 7.9446 & 0.0760 & 8 & 4 & 4 \\ 3799.92 & 189.4512 & 0.0873 & 6 & 2 & 5 \\ 3800.44 & 537.7475 & 0.0840 & 3 & 3 & 0 \\ 3800.58 & 17.0284 & 0.0984 & 3 & 1 & 3 \\ 3800.74 & 0.4287 & 0.0682 & 6 & 3 & 4 \\ 3800.77 & 47.1439 & 0.0520 & 8 & 0 & 8 \\ 3800.79 & 166.5854 & 0.0523 & 8 & 1 & 8 \\ 3801.43 & 883.3461 & 0.1059 & 2 & 0 & 2 \\ 3802.00 & 1.3395 & 0.0915 & 3 & 2 & 2 \\ 3802.31 & 0.0369 & 0.0669 & 11 & 2 & 9 \\ 3802.97 & 765.5725 & 0.0934 & 4 & 1 & 3 \\ 3803.51 & 0.8768 & 0.0948 & 3 & 2 & 2 \\ 3803.76 & 0.3817 & 0.0401 & 7 & 7 & 1 \\ 3803.76 & 1.1452 & 0.0401 & 7 & 7 & 0 \\ 3804.11 & 0.0770 & 0.0782 & 7 & 2 & 5 \\ 3804.87 & 0.2422 & 0.0605 & 8 & 2 & 6 \\ 3805.22 & 7.4254 & 0.1010 & 3 & 0 & 3 \\ 3805.24 & 2.3152 & 0.0837 & 3 & 3 & 1 \\ 3805.62 & 0.0146 & 0.0781 & 7 & 2 & 6 \\ 3805.82 & 0.1163 & 0.0766 & 11 & 4 & 7 \\ 3806.03 & 4.4716 & 0.0740 & 9 & 3 & 6 \\ 3806.19 & 6.0791 & 0.0793 & 8 & 2 & 6 \\ 3806.25 & 0.4469 & 0.0907 & 3 & 2 & 1 \\ 3806.33 & 0.0483 & 0.0573 & 7 & 6 & 2 \\ 3806.37 & 8.1183 & 0.0915 & 3 & 2 & 2 \\ 3806.91 & 5780.3612 & 0.1037 & 2 & 1 & 1 \\ 3807.45 & 0.1737 & 0.0436 & 9 & 0 & 9 \\ 3807.54 & 0.0579 & 0.0437 & 9 & 1 & 9 \\ 3808.00 & 0.5153 & 0.0763 & 9 & 2 & 7 \\ 3808.04 & 0.0184 & 0.0422 & 11 & 8 & 3 \\ 3808.26 & 0.2284 & 0.0368 & 12 & 3 & 10 \\ 3808.30 & 0.2844 & 0.0863 & 5 & 1 & 4 \\ 3808.32 & 3.1503 & 0.0984 & 3 & 1 & 3 \\ 3808.54 & 0.3006 & 0.0729 & 4 & 4 & 1 \\ 3808.58 & 0.1438 & 0.0633 & 12 & 4 & 8 \\ 3808.98 & 0.0304 & 0.0687 & 5 & 4 & 1 \\ 3809.09 & 0.0469 & 0.0863 & 5 & 1 & 4 \\ 3809.11 & 0.5633 & 0.0620 & 7 & 3 & 5 \\ 3809.46 & 0.7202 & 0.0868 & 4 & 3 & 2 \\ 3809.80 & 2.3407 & 0.0892 & 4 & 3 & 1 \\ 3809.96 & 39.5794 & 0.0781 & 7 & 2 & 6 \\ 3810.81 & 0.4963 & 0.0718 & 10 & 2 & 8 \\ 3811.20 & 0.0141 & 0.0410 & 10 & 8 & 2 \\ 3811.20 & 0.0423 & 0.0408 & 10 & 8 & 3 \\ 3811.23 & 1.0476 & 0.0382 & 10 & 1 & 9\end{array}$

\begin{tabular}{|c|c|c|}
\hline 8 & 4 & 4 \\
\hline 6 & 2 & 5 \\
\hline 9 & 6 & 3 \\
\hline 5 & 1 & 5 \\
\hline 3 & 0 & 3 \\
\hline 6 & 1 & 5 \\
\hline 9 & 6 & 4 \\
\hline 2 & 2 & 0 \\
\hline 1 & 1 & 0 \\
\hline 7 & 1 & 7 \\
\hline 5 & 1 & 4 \\
\hline 2 & 2 & 1 \\
\hline 2 & 1 & 2 \\
\hline 7 & 1 & 7 \\
\hline 7 & 1 & 7 \\
\hline 7 & 0 & 7 \\
\hline 1 & 0 & 1 \\
\hline 2 & 2 & 1 \\
\hline 10 & 4 & 6 \\
\hline 4 & 1 & 4 \\
\hline 3 & 0 & 3 \\
\hline 7 & 6 & 2 \\
\hline 7 & 5 & 1 \\
\hline 7 & 2 & 6 \\
\hline 9 & 0 & 9 \\
\hline 2 & 0 & 2 \\
\hline 4 & 1 & 4 \\
\hline 6 & 1 & 5 \\
\hline 10 & 5 & 6 \\
\hline 9 & 3 & 7 \\
\hline 7 & 3 & 5 \\
\hline 2 & 2 & 0 \\
\hline 8 & 4 & 5 \\
\hline 2 & 2 & 1 \\
\hline 1 & 1 & 0 \\
\hline 8 & 1 & 8 \\
\hline 8 & 0 & 8 \\
\hline 8 & 4 & 4 \\
\hline 11 & 7 & 4 \\
\hline 12 & 2 & 11 \\
\hline 5 & 1 & 5 \\
\hline 2 & 1 & 2 \\
\hline 5 & 2 & 4 \\
\hline 12 & 4 & 9 \\
\hline 6 & 1 & \\
\hline 5 & 1 & \\
\hline 8 & 1 & 8 \\
\hline 3 & 2 & \\
\hline 4 & 0 & \\
\hline 6 & 1 & \\
\hline 9 & 4 & \\
\hline 10 & $\begin{array}{l}7 \\
7\end{array}$ & \\
\hline & 7 & \\
\hline
\end{tabular}

1131.76 552.92

1631.41

326.64

136.77

2146.28

1631.27

136.17

42.37

586.4

399.46

134.91

79.48

586.48

586.48

586.26

23.79

1742.28

1616.51

224.83

136.77

$1216 \cdot 20$

$1216 \cdot 20$

709.60

920.18

70.08

224.83

542.91

1718.77

1216.27

816.72

1743.47

1122.72

134.91

42.37

744.16

744.09

1131.76

2321.94

1774.74

326.64

79.48

416.22

2124.98

447.24

1922.86

744.16

212.15

222.06

542.91

$1360 \cdot 28$

2054.40

2054.40

1114.55
0.002886

0.939536

3. 116419

0.003420

0.016715

4.008107

3. 116005

2.394805

1.500000

10.90

3. 129447

2. 366160

2. 653490

0.005077

5.95

7.000291

1.961754

1. 666667

0.075083

0.495229

0.181744

1. 119792

1. 119797

0.838975

0.005067

2. 880004

0.001497

4.008107

1. 370216

1. 235624

2. 422109

1.676022

0.002778

1.666667

1. 500000

8.007087

8.007851

0.092972

4. 105208

3. 648229

0.456689

2.653490

0.001517

1. 508618

0.000636

0.456689

0.004883

2. 320352

0.047652

4.0008107

0.093773

3.150851

3. 150801

1.848326
NU3

Nu 3

NUI

NU3

NUI

NU1****

NU 1

NU1

NU $3 * *$

$2 \mathrm{NU} 2+$

NUI

NU1

NU3*

NU3

NU1-

NU1

NU3

NU $3 * * *$

NU 3

NU 3

NU $3 *$

NU1

NU1

NU3*

NU 3

NU3*

NU3

NU1**

NU 1

NU3

NU1

NU3 $3 * *$

NU 3

NU3*

NU 3

NUI*

NUI*

NU3

NU 1

NU 1

NU $3 *$

NU $3 * *$

NU 3

NU3

NU1

NU3 ${ }^{*} *$ *

NU 3

NU1*

NU1

NU1

NU3

NU 1

NU1

NU1 


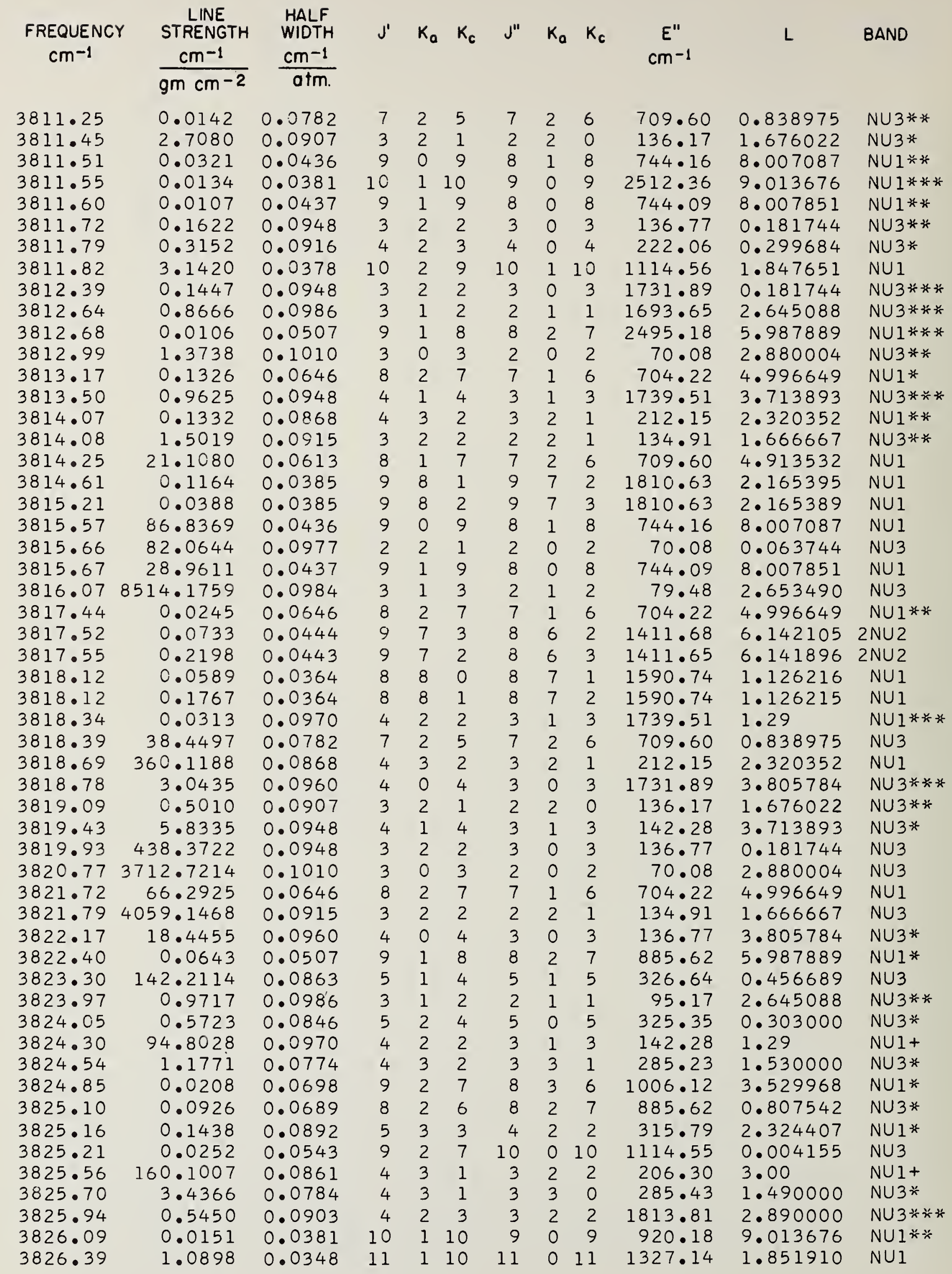




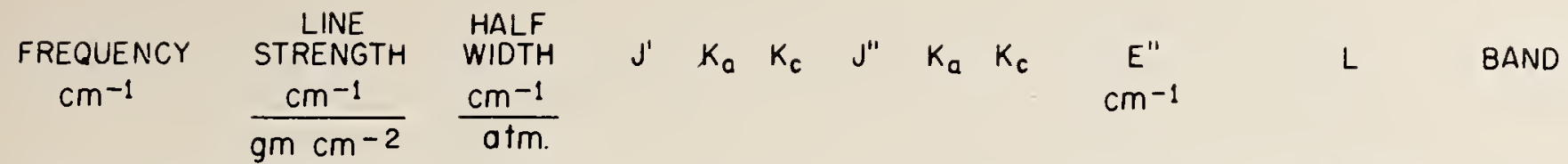

3827.22

3827.29

3827.46

3828.02

3828.07

3828.29

3828.42

3828.61

3828.91

3829.78

3829.86

3830.01

3830.16

3830.20

3830.41

3831.58

3831.67

3831.96

3832.24

3832.26

3832.33

3833.37

3833.39

3833.46

3833.71

3833.96

3834.14

3834.56

3835.02

3835.72

3835.88

3835.98

3836.03

3836.07

3836.39

3836.81

3836.90

3837.85

3838.35

3838.72

3838.80

3838.88

3838.94

3839.43

3839.94

3840.12

3840.59

3840.69

3841.04

3841.60

3841.68

3841.70

3841.80
1353.9804

1.0792

0.2311

3.9394

157.6210

0.4002

3.3028

0.4631

67.3233

0.0945

0.0247

0.0266

3.4125

13.5642

40.6942

32.1513

10.3849

2626.1743

2.4478

0.2177

0.0172

0.1486

0.6358

0.2513

0.0838

1.6711

0.1032

10.9281

71.9165

2916.7265

0.0134

2.4530

0.0857

0.6110

0.0427

14.8350

0.2376

0.0169

9222.7358

5.0508

10.1279

0.0939

0.0289

14.8661

46.3159

588.5700

$286 \cdot 1386$

0.1143

0.3429

1718.3218

0.0440

1.2546

0.6429

0.0270
0.0907

0.0948

0.0857

0.0671

0.0916

0.0752

0.0903

0.0773

0.0880

0.0846

0.0574

0.0892

0.0960

0.0381

0.0381

0.0507

0.0698

0.0986

0.0872

0.0774

0.0689

0.0743

0.0784

0.0633

0.0638

0.0914

0.0747

0.0539

0.0892

0.0948

0.0813

0.0957

0.0773

0.0903

0.0857

0.0872

0.0896

0.034

0.0960

0.0887

0.0914

0.0860

0.0444

0.0957

0.0689

0.0774

0.0846

0.0331

0.0331

0.0784

0.0896

0.0875

0.0803

0.0663 $\begin{array}{lll}3 & 2 & 1\end{array}$

$\begin{array}{lll}4 & 1 & 4\end{array}$

$\begin{array}{lll}5 & 3 & 3\end{array}$

$\begin{array}{lll}10 & 3 & 7\end{array}$

423

1037

423

615

$\begin{array}{lll}5 & 3 & 2\end{array}$

524

1349

533

404

10010

10110

$\begin{array}{lll}9 & 1 & 8 \\ 9 & 2 & 7\end{array}$

312

$\begin{array}{lll}5 & 1 & 5\end{array}$

$\begin{array}{lll}4 & 3 & 2\end{array}$

826

$\begin{array}{lll}7 & 3 & 5\end{array}$

$\begin{array}{lll}4 & 3 & 1\end{array}$

$\begin{array}{lll}5 & 4 & 2\end{array}$

$\begin{array}{lll}5 & 4 & 1\end{array}$

$\begin{array}{lll}4 & 2\end{array}$

625

$\begin{array}{lll}9 & 2 & 8\end{array}$

533

$\begin{array}{lll}4 & 1 & 4\end{array}$

634

$\begin{array}{lll}4 & 1 & 3 \\ 6 & 1 & 5\end{array}$

$\begin{array}{lll}6 & 1 & 5 \\ 4 & 2 & 3\end{array}$

$\begin{array}{lll}5 & 3 & 3\end{array}$

$\begin{array}{lll}5 & 1 & 5\end{array}$

$\begin{array}{lll}6 & 3 & 4\end{array}$

14

312

$\begin{array}{lll}4 & 0 & 4 \\ 5 & 0 & 5\end{array}$

$\begin{array}{lll}4 & 2\end{array}$

$\begin{array}{lll}3 & 3 & 1\end{array}$

1029

$\begin{array}{lll}4 & 1 & 3\end{array}$

826

$\begin{array}{lll}4 & 3 & 2\end{array}$

$\begin{array}{lll}5 & 2 & 4\end{array}$

$\begin{array}{lll}12 & 1 & 11\end{array}$

$\begin{array}{lll}12 & 211\end{array}$

$\begin{array}{lll}4 & 3 & 1\end{array}$

634

$\begin{array}{lll}5 & 2 & 4\end{array}$

$\begin{array}{lll}5 & 3 & 3 \\ 8 & 3 & 6\end{array}$ $\begin{array}{rrr}2 & 2 & 0 \\ 3 & 1 & 3 \\ 5 & 1 & 4 \\ 10 & 3 & 8 \\ 4 & 0 & 4 \\ 9 & 4 & 6 \\ 3 & 2 & 2 \\ 6 & 1 & 6 \\ 5 & 0 & 5 \\ 5 & 0 & 5 \\ 13 & 4 & 10 \\ 4 & 2 & 2 \\ 3 & 0 & 3\end{array}$

$\begin{array}{lll}4 & 2 & 2 \\ 3 & 0 & 3\end{array}$

$\begin{array}{lll}9 & 1 & 9\end{array}$

$\begin{array}{lll}9 & 0 & 9\end{array}$

$\begin{array}{lll}8 & 2\end{array}$

836

$\begin{array}{lll}2 & 1 & 1\end{array}$

$\begin{array}{lll}4 & 1 & 4\end{array}$

$\begin{array}{lll}3 & 3 & 1\end{array}$

$\begin{array}{lll}8 & 2 & 7\end{array}$

$\begin{array}{lll}7 & 1 & 6\end{array}$

330

$\begin{array}{lll}4 & 4 & 1\end{array}$

$\begin{array}{lll}4 & 4 & 0\end{array}$

$\begin{array}{lll}3 & 2 & 1\end{array}$

606

$\begin{array}{lll}8 & 1 & 7\end{array}$

$\begin{array}{lll}4 & 2 & 2\end{array}$

$\begin{array}{lll}3 & 1 & 3\end{array}$

615

3

6116

322

514

$\begin{array}{lll}4 & 1 & 4\end{array}$

523

14213

$\begin{array}{lll}3 & 0 & 3\end{array}$

$\begin{array}{lll}4 & 0 & 4\end{array}$

$\begin{array}{lll}3 & 2 & 1\end{array}$

$\begin{array}{lll}3 & 1 & 2\end{array}$

$\begin{array}{lll}9 & 1 & 8\end{array}$

$\begin{array}{lll}3 & 1 & 2\end{array}$

$\begin{array}{lll}8 & 2 & 7\end{array}$

$\begin{array}{lll}3 & 3 & 1\end{array}$

$\begin{array}{lll}5 & 0 & 5\end{array}$

$\begin{array}{lll}12 & 0 & 12\end{array}$

$\begin{array}{lll}12 & 1 & 12\end{array}$

$\begin{array}{lll}3 & 3 & 0\end{array}$

$\begin{array}{lll}5 & 2 & 3\end{array}$

423

$\begin{array}{lll}4 & 3 & 2\end{array}$

136.17

142.28

399.46

1446.16

222.06

1340.89

206.30

447.24

325.35

1920.76

2426.22

315.79

136.77

920.21

920.18

885.62

$1006 \cdot 12$

95.17

1821.61

285.23

885.62

704.22

285.43

2129.63

2129.65

1819.34

446.71

882.93

315.79

142.28 


\begin{tabular}{|c|c|c|c|c|c|c|c|c|c|c|c|}
\hline $\begin{array}{c}\text { FREQUENC } \\
\mathrm{cm}^{-1}\end{array}$ & $\begin{array}{l}\text { LINE } \\
\text { STRENGTH } \\
\frac{\mathrm{cm}^{-1}}{a m \mathrm{~cm}^{-2}}\end{array}$ & $\begin{array}{l}\text { HALF } \\
\text { WIDTH } \\
\frac{\mathrm{cm}^{-1}}{\text { atm. }}\end{array}$ & $J^{\prime}$ & $\mathrm{K}_{\mathrm{a}}$ & $K_{c}$ & $J^{\prime \prime}$ & $\mathrm{K}_{\mathrm{a}}$ & $K_{c}$ & $\begin{array}{c}E^{\prime \prime} \\
\mathrm{cm}^{-1}\end{array}$ & $\mathrm{~L}$ & BAND \\
\hline 3841.87 & 1.5227 & 0.0633 & 5 & 4 & 2 & 4 & 4 & 1 & $488 \cdot 10$ & 1.810924 & NU3* \\
\hline & 0.0191 & 0.0747 & 6 & 2 & 5 & 6 & 0 & 6 & 446.71 & 0.299000 & NU3** \\
\hline 2.00 & 0.5076 & 0.0638 & 5 & 4 & 1 & 4 & 4 & 0 & $488 \cdot 13$ & 1.810956 & NU $3 *$ \\
\hline$+3 \cdot 54$ & 231.5447 & 0.0773 & 6 & 1 & 5 & 6 & 1 & 6 & 447.24 & 0.450603 & NU3 \\
\hline 3843.78 & 1651.4006 & 0.0903 & 4 & 2 & 3 & 3 & 2 & 2 & $206 \cdot 30$ & 2.890000 & NU3- \\
\hline 3844.23 & 2.7445 & 0.0872 & 5 & 1 & 5 & 4 & 1 & 4 & 224.83 & 4.737042 & NU3** \\
\hline 3844.31 & 36.1015 & 0.0813 & 6 & 3 & 4 & 6 & 1 & 5 & 542.91 & 0.339000 & NU3- \\
\hline 3844.37 & 17.1819 & 0.0349 & 11 & 0 & 11 & 10 & 1 & 10 & 1114.56 & 10.018153 & NU1 \\
\hline 3844.38 & 5.7274 & 0.0349 & 11 & 1 & 11 & 10 & 0 & 10 & 1114.55 & 10.018239 & NU \\
\hline 3844.86 & 115.5386 & 0.0857 & 5 & 3 & 3 & 5 & 1 & 4 & 399.46 & 0.177000 & NU3- \\
\hline 845.30 & 0.1888 & 0.0826 & 5 & 3 & 2 & 4 & 3 & 1 & 2005.92 & 2.420000 & $3 * * *$ \\
\hline 845.37 & 0.0408 & 0.0881 & 7 & 3 & 5 & 6 & 2 & 4 & 602.77 & 2.751216 & NU $1 *$ \\
\hline 3845.40 & 4.7726 & 0.0431 & 10 & 1 & 9 & 9 & 2 & 8 & 1080.38 & 7.032041 & NU \\
\hline 845.55 & 0.0241 & 0.0 .516 & 6 & 5 & 2 & 5 & 5 & 1 & $2406 \cdot 15$ & 1.845180 & NU $3 * * *$ \\
\hline 3845.59 & 0.0721 & 0.0516 & 6 & 5 & 1 & 5 & 5 & 0 & $2406 \cdot 15$ & 1.845 & NU $3 \ddot{*} \ddot{*}$ \\
\hline 845.61 & 0.0381 & 0.0857 & 5 & 3 & 3 & 5 & 1 & 4 & $2000 \cdot 90$ & 0.177000 & NU $3 \ddot{*} * *$ \\
\hline $846 \cdot 22$ & 0.9344 & 0.0887 & 5 & 0 & 5 & 4 & 0 & 4 & 222.06 & 4.769122 & NU $3 * \%$ \\
\hline $3846 \cdot 35$ & 1.8736 & 0.0914 & 4 & 2 & 2 & 3 & 2 & 1 & $212 \cdot 15$ & 3.033776 & NU $3 * *$ \\
\hline $846 \cdot 36$ & 3.8963 & 0.0803 & 5 & 3 & 3 & 4 & 3 & 2 & 382.52 & 2.730000 & NU $3 *$ \\
\hline $3846 \cdot 40$ & 118.7903 & 0.0896 & 6 & 3 & 4 & 5 & 2 & 3 & 446 & 2.452523 & \\
\hline 846.69 & 2.7503 & 0.0957 & 4 & 1 & 3 & 3 & 1 & 2 & $173 \cdot 36$ & 3.657441 & NU $3 * *$ \\
\hline 3846.70 & 0.3282 & 0.0994 & 2 & 2 & 0 & 1 & 0 & 1 & .79 & 0.038246 & $3 *$ \\
\hline 3846.78 & 0.0245 & 0.0743 & 7 & 3 & 5 & 7 & 1 & 6 & 2309.74 & 0.522000 & NU $3 * * * *$ \\
\hline $3847 \cdot 14$ & 0.0783 & 0.0654 & 7 & 1 & 6 & 7 & 1 & 7 & 586.48 & 0.456 & $3 *$ \\
\hline 3847.31 & 14.4440 & 0.0444 & 10 & 2 & 9 & 9 & 1 & 8 & 1079.07 & 7.043 & 1 \\
\hline 3847.75 & 0.0174 & 0.0860 & 3 & 3 & 1 & 3 & 1 & 2 & 36 & 0.02 & $13 \ddot{*}$ \\
\hline 3848.63 & 0.3784 & 0.0596 & 11 & 3 & 8 & 11 & 3 & 9 & 1695 & 1.122765 & 3 \\
\hline 3848.89 & 0.2144 & 0.0674 & 4 & 4 & 1 & 3 & 3 & 0 & 1907.63 & 5.89 & $1 * * * *$ \\
\hline $3848 \cdot 90$ & 0.5974 & 0.0764 & 6 & 1 & 6 & 5 & 1 & 5 & 1922.86 & 5.744 & $3 \div \div \div$ \\
\hline 3849.05 & 74.3308 & 0.0743 & 7 & 3 & 5 & 7 & 1 & 6 & $704 \cdot 22$ & 0.522 & 13- \\
\hline 3849.07 & 1.1444 & 0.0826 & 5 & 3 & 2 & 4 & 3 & 1 & 383.85 & 2.420000 & $3 *$ \\
\hline 3849.08 & 0.0656 & 0.0665 & 4 & 4 & 0 & 3 & 3 & 1 & 47 & 5.49 & $1 * * * *$ \\
\hline 3849.53 & 7.6040 & 0.0875 & 5 & 2 & 4 & 4 & 2 & 3 & 300.35 & 3.530 & $3 *$ \\
\hline 3849.57 & 198.6771 & 0.0665 & 4 & 4 & 0 & 3 & 3 & 1 & $285 \cdot 23$ & 5.49 & NU I+ \\
\hline 3849.62 & 649.6736 & 0.0674 & 4 & 4 & 1 & 3 & 3 & c & 43 & 5.89 & \\
\hline 3849.65 & 31.1338 & 0.0871 & 4 & 3 & 2 & 4 & 1 & 3 & .52 & 0.076 & \\
\hline 3849.86 & 51.6049 & 0.0747 & 6 & 2 & 5 & 6 & 0 & 6 & 71 & 0.299 & \\
\hline 3850.25 & 0.2312 & 0.0636 & 7 & 2 & 6 & 7 & 0 & 7 & 26 & 0.44 & \\
\hline 3850.36 & 1.3076 & 0.0775 & 6 & 0 & 6 & 5 & 0 & 5 & 76 & 5.75 & 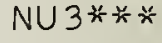 \\
\hline 3852.07 & 7417.5214 & 0.0872 & 5 & 1 & 5 & 4 & 1 & 4 & 83 & $4.73^{-1}$ & \\
\hline 3852.73 & 1.6180 & 0.0590 & 10 & 2 & 8 & 9 & 3 & 7 & 27 & 4.706625 & \\
\hline 3853.14 & 0.3522 & 0.0884 & 5 & 3 & 2 & 4 & 2 & 3 & 1907.99 & 10.59 & $1 * * \ddot{*}$ \\
\hline 3853.42 & 3.6207 & 0.0764 & 6 & 1 & 6 & 5 & 1 & 5 & 64 & 5.7744669 & \\
\hline 3.61 & 1067.1312 & 0.0884 & 5 & 3 & 2 & 4 & 2 & 3 & 35 & 10.59 & \\
\hline 353. & 5063.9614 & 0.0914 & 4 & 2 & 2 & 3 & 2 & 1 & 15 & 3.03 & \\
\hline 10 & 2525.4405 & 0.0887 & 5 & 0 & 5 & 4 & 0 & 4 & 06 & $4 \cdot 76$ & \\
\hline 30 & 10.9552 & 0.0775 & 6 & 0 & 6 & 5 & 0 & 5 & & 5.75 & \\
\hline $3854 \cdot 44$ & 7433.0627 & 0.0957 & 4 & 1 & 3 & 3 & 1 & 2 & $173 \cdot 36$ & 3.667441 & \\
\hline 3855 . & 0.0607 & 0.0994 & 2 & 2 & 0 & 1 & 0 & 1 & .79 & 0.038 & $\operatorname{NU} 3 \ddot{x} \ddot{x}$ \\
\hline 13 & 20.3797 & 0.0881 & 7 & 3 & 5 & 6 & 2 & 4 & 77 & 2.75 & \\
\hline 1 & 0.0541 & 0.0994 & 2 & 2 & 0 & 1 & 0 & 1 & 1618.55 & 0.038 & NU $3 * * *$ \\
\hline+7 & 0.0345 & 0.0333 & 13 & 2 & 12 & 13 & 1 & 13 & 1806.78 & 1.9064 & NU1 \\
\hline 93 & 0.0161 & 0.0430 & 7 & 6 & 2 & 6 & 6 & 1 & 2734.00 & 1.869458 & NU $3 * * *$ \\
\hline 3856.00 & 0.1457 & 0.0516 & 6 & 5 & 2 & 5 & 5 & 1 & 742.10 & 1.845180 & NU $3 *$ \\
\hline
\end{tabular}




\begin{tabular}{|c|c|c|c|c|c|c|c|c|c|c|c|}
\hline $\begin{array}{l}\text { FREQUENCY } \\
\qquad \mathrm{cm}^{-1}\end{array}$ & $\begin{array}{l}\text { LINE } \\
\text { STRENGTH } \\
\frac{\mathrm{cm}^{-1}}{\mathrm{gm} \mathrm{cm}^{-2}}\end{array}$ & $\begin{array}{l}\text { HALF } \\
\text { WIDTH } \\
\frac{\mathrm{cm}^{-1}}{\mathrm{~atm} .}\end{array}$ & $J^{\prime}$ & $K_{a}$ & $K_{c}$ & $J^{\prime \prime}$ & $\mathrm{K}_{\mathrm{a}}$ & $K_{c}$ & $\begin{array}{c}E^{\prime \prime} \\
\mathrm{cm}^{-1}\end{array}$ & L & BAND \\
\hline 3856.05 & 0.4372 & 0.0516 & 6 & 5 & 1 & 5 & 5 & 0 & 742.10 & 1.845182 & NU 3 * \\
\hline 3856.46 & 0.6187 & 0.0926 & 5 & 1 & 4 & 4 & 1 & 3 & 1875.49 & 4.601931 & NU $3 *$ 头并 \\
\hline 3856.52 & 0.0847 & 0.0679 & 6 & 4 & 3 & 5 & 4 & 2 & 2251.71 & 3.351648 & NU $3 * * *$ \\
\hline 3856.70 & 0.2117 & 0.0826 & 5 & 3 & 2 & 4 & 3 & 1 & 383.85 & 2.420000 & NU3*** \\
\hline 3856.71 & 46.9431 & 0.0860 & 3 & 3 & 1 & 3 & 1 & 2 & 173.36 & 0.023148 & NU3 \\
\hline 3856.76 & 268.5014 & 0.0834 & 5 & 4 & 1 & 5 & 1 & 4 & 399.46 & $4 \cdot 37$ & NUI+ \\
\hline $3857 \cdot 28$ & 761.3661 & 0.0633 & 5 & 4 & 2 & 4 & 4 & 1 & $488 \cdot 10$ & 1.810924 & NU3 \\
\hline $3857 \cdot 32$ & 1.4067 & 0.0875 & 5 & 2 & 4 & 4 & 2 & 3 & $300 \cdot 35$ & 3.530000 & NU3** \\
\hline 3857.40 & 253.7719 & 0.0638 & 5 & 4 & 1 & 4 & 4 & 0 & $488 \cdot 13$ & 1.810956 & NU 3 \\
\hline 3857.48 & 0.2539 & 0.0699 & 6 & 4 & 2 & 5 & 4 & 1 & 2251.86 & 3.352166 & NU $3 * \ddot{*}$ \\
\hline 3857.67 & 0.5327 & 0.0674 & 11 & 3 & 8 & 10 & 4 & 7 & $1581 \cdot 34$ & 3.178818 & NU 1 \\
\hline 3857.67 & 6.5393 & 0.0332 & 12 & 1 & 12 & 11 & 0 & 11 & $1327 \cdot 14$ & 11.021891 & NU 1 \\
\hline 3858.05 & 2.1800 & 0.0332 & 12 & 0 & 12 & 11 & 1 & 11 & $1327 \cdot 14$ & 11.021 & NU 1 \\
\hline 3858.10 & 13.4536 & 0.0663 & 8 & 3 & 6 & 8 & 1 & 7 & 882.93 & 0.691047 & NU3 \\
\hline $3858 \cdot 18$ & 0.0427 & 0.0636 & 7 & 2 & 6 & 7 & 0 & 7 & $586 \cdot 26$ & 0.448059 & NU $3 \%$ 丷ㅜㅇ \\
\hline 3858.74 & 3.7502 & 0.0926 & 5 & 1 & 4 & 4 & 1 & 3 & 275.52 & 4.601931 & NU $3 *$ \\
\hline 3859.02 & 6.3714 & 0.0800 & 6 & 4 & 2 & 6 & 1 & 5 & .91 & 0.637 & NU I+ \\
\hline 3859.28 & 4.9618 & 0.0840 & 4 & 4 & 0 & 4 & 1 & 3 & .52 & 0.1303 & NUI+ \\
\hline 3859.38 & 5.9083 & 0.0606 & 9 & 2 & 7 & 9 & 2 & 8 & 1080 & 0.813 & NU 3 \\
\hline 3859.87 & 5.6605 & 0.0378 & 11 & 1 & 10 & 10 & 2 & 9 & 1293 & 8.058917 & NUI \\
\hline 3860.44 & 0.0381 & 0.0636 & 7 & 2 & 6 & 7 & 0 & 7 & 218 & 0.448 & NU $3 \ddot{*} \ddot{*} *$ \\
\hline 3860.44 & 0.4683 & 0.0920 & 5 & 2 & 3 & 4 & 2 & 2 & 1922 & 4.253846 & NU $3 * 2 * 2$ \\
\hline 3860.66 & 1.9004 & 0.0385 & 11 & 2 & 10 & 10 & 1 & 9 & .04 & 8.063 & NUI \\
\hline $3861 \cdot 31$ & 0.6698 & 0.0764 & 6 & 1 & 6 & 5 & 1 & 5 & .64 & 5.744669 & NU $3 * *$ \\
\hline 3861.47 & 19.4285 & 0.0865 & 6 & 3 & 3 & 6 & 0 & 6 & .71 & 1.20 & NUI+ \\
\hline 3861.62 & 29.5843 & 0.0832 & 8 & 3 & 6 & 7 & 2 & 5 & .40 & 3.262 & NUI \\
\hline 3861.80 & 1948.1717 & 0.0803 & 5 & 3 & 3 & 4 & 3 & 2 & .52 & 2.730 & NU3- \\
\hline 3862.22 & 2.0267 & 0.0775 & 6 & 0 & 6 & 5 & 0 & 5 & .35 & 5.75 & NU 3 长茂 \\
\hline $3862 \cdot 48$ & 39.1902 & 0.0654 & 7 & 1 & 6 & 7 & 1 & 7 & .48 & 0.456 & NU3 \\
\hline 3863.33 & 164.1211 & 0.0994 & 2 & 2 & 0 & 1 & 0 & 1 & .79 & 0.038246 & NU3 \\
\hline 3863.71 & 0.0270 & 0.0516 & 6 & 5 & 2 & 5 & 5 & 1 & .10 & 1.845180 & $\mathrm{NU}_{3} * *$ \\
\hline 3863.76 & 0.0809 & 0.0516 & 6 & 5 & 1 & 5 & 5 & 0 & .10 & 1.845182 & NU3** \\
\hline 3863.80 & 0.1548 & 0.0797 & 6 & 3 & 4 & 5 & 3 & 3 & 2126.41 & 3.600 & NU $3 *$ 长关关 \\
\hline 3864.33 & 572.2053 & 0.0826 & 5 & 3 & 2 & 4 & 3 & 1 & 383.85 & 2.420 & NU3- \\
\hline $3864 \cdot 68$ & 1.5832 & 0.0818 & 6 & 2 & 5 & 5 & 2 & 4 & 416.22 & 3.920 & NU $3 *$ \\
\hline 3864.74 & 0.0110 & 0.0657 & 10 & 3 & 8 & 9 & 2 & 7 & 1201.95 & 4.932263 & NU 1 * \\
\hline 3864.85 & 2.8384 & 0.0920 & 5 & 2 & 3 & 4 & 2 & 2 & 315.79 & 4.253846 & NU3* \\
\hline 3865.11 & 7.4267 & 0.0694 & 9 & 4 & 6 & 9 & 2 & 7 & 1201.95 & 0.624 & $\mathrm{NU}_{3}$ \\
\hline 3865.12 & 3801.9877 & 0.0875 & 5 & 2 & 4 & 4 & 2 & 3 & .35 & 3.530000 & NU3- \\
\hline 3865.67 & 1.5388 & 0.0699 & 6 & 4 & 2 & 5 & 4 & 1 & .35 & 3.352 & NU $3 *$ \\
\hline 3865.83 & 0.3867 & 0.0649 & 7 & 0 & 7 & 6 & 0 & 6 & 2041.76 & 6.749 & NU $3 * * *$ \\
\hline 3865.84 & 0.2932 & 0.0351 & 8 & 8 & 1 & 7 & 7 & 0 & 1394.86 & 7.402783 & 2NU2 \\
\hline 3865.84 & 0.0977 & 0.0351 & 8 & 8 & 0 & 7 & 7 & 1 & 135 & 7.402 & $2 \mathrm{NU} 2$ \\
\hline 3866.12 & 115.5784 & 0.0636 & 7 & 2 & 6 & 7 & 0 & 7 & 26 & 59 & NU3 \\
\hline 3866.58 & 0.6938 & 0.0926 & 5 & 1 & 4 & 4 & 1 & 3 & .52 & 4.60 & NU $3 * *$ \\
\hline 3866.67 & 1.0017 & 0.0620 & 10 & 4 & 7 & 10 & 2 & 8. & .00 & 0.828 & NU3 \\
\hline 3866.99 & $4 \cdot 4486$ & 0.0754 & 9 & 3 & 7 & 8 & 2 & 6 & .91 & 4.004 & NUI \\
\hline 3867.64 & 0.2765 & 0.0509 & 12 & 3 & 9 & 12 & 3 & 10 & 1962.55 & 1.142616 & NU3 \\
\hline 3867.71 & 0.9382 & 0.0797 & 6 & 3 & 4 & 5 & 3 & 3 & 504.00 & 3.600000 & NU3* \\
\hline 3867.84 & 0.0260 & 0.032 & 14 & 2 & 13 & 14 & 1 & 14 & 2073.66 & 1.90878 & NU1 \\
\hline 3867.94 & 0.0160 & 0.0803 & 6 & 4 & 3 & 6 & 2 & 4 & 602.77 & 0.100806 & NU $3 *$ \\
\hline 3868.20 & 7.4799 & 0.0756 & 7 & 4 & 3 & 7 & 1 & 6 & $704 \cdot 22$ & 0.557 & NUI+ \\
\hline & 0.0652 & 0.0565 & 7 & 5 & 3 & 6 & 5 & 2 & 2552.85 & 3.450020 & NU $3 \ddot{*} * *$ \\
\hline 3868.53 & 0.0326 & 0.0430 & 7 & 6 & 1 & 6 & 6 & 0 & 1045.07 & 1.869458 & NU $3 *$ \\
\hline
\end{tabular}




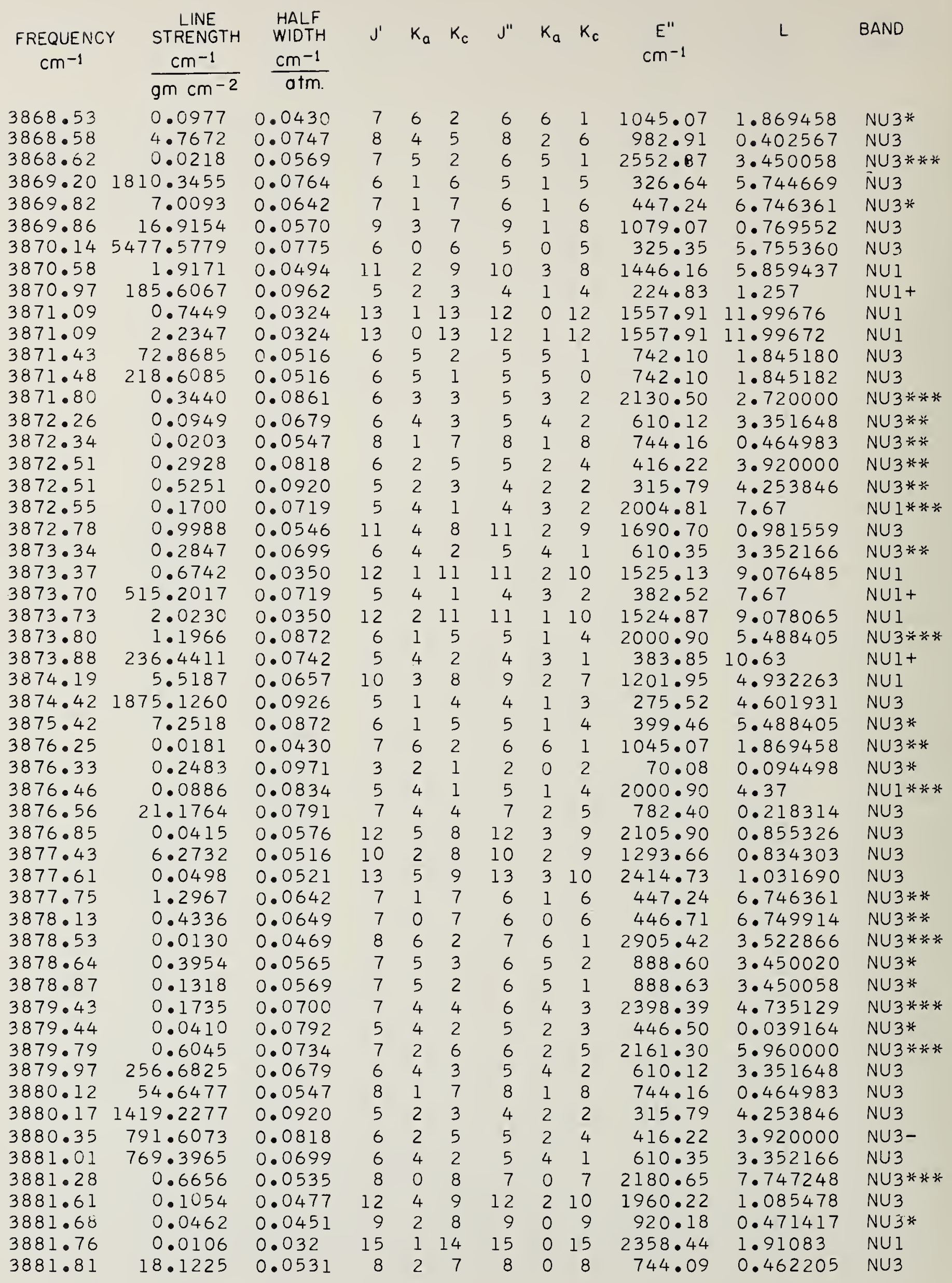




\begin{tabular}{|c|c|c|c|c|c|c|c|c|c|c|}
\hline $\begin{array}{l}\text { FREQUENCY } \\
\mathrm{cm}^{-1}\end{array}$ & $\begin{array}{l}\text { LINE } \\
\text { STRENGTH } \\
\mathrm{cm}^{-1}\end{array}$ & $\begin{array}{l}\text { HALF } \\
\text { WIDTH } \\
\mathrm{cm}^{-1}\end{array}$ & $J^{\prime}$ & $k_{a}$ & $\mathrm{~K}_{\mathrm{c}}$ & $J^{\prime \prime}$ & $K_{a}$ & $K_{c}$ & $\begin{array}{c}E^{\prime \prime} \\
\mathrm{cm}^{-1}\end{array}$ & L \\
\hline
\end{tabular}

\begin{tabular}{|c|c|c|c|c|c|c|c|c|c|c|c|}
\hline 3882.00 & 0.0576 & 0.0748 & 7 & 4 & 3 & 6 & 4 & 2 & 2399.17 & 4.739627 & NU $3 \ddot{2}$ 前并 \\
\hline 3882.88 & 2.0675 & 0.0491 & 10 & 3 & 8 & 10 & 1 & 9 & 1293.04 & 0.818434 & NU3 \\
\hline 3882.90 & 0.6787 & 0.0547 & 11 & 3 & 9 & 10 & 2 & 8 & 1438.00 & 5.954956 & NU1 \\
\hline 3883.22 & 469.0807 & 0.0797 & 6 & 3 & 4 & 5 & 3 & 3 & 504.00 & 3.600000 & NU3- \\
\hline 3883.37 & 1.3416 & 0.0872 & 6 & 1 & 5 & 5 & 1 & 4 & 399.46 & $5.488 /+05$ & NU $3 * *$ \\
\hline 3883.42 & 0.1167 & 0.0527 & 5 & 5 & 1 & 4 & 4 & 0 & $488 \cdot 13$ & 4.397010 & NU $1 *$ \\
\hline 3883.45 & 0.3502 & 0.0525 & 5 & 5 & 0 & 4 & 4 & 1 & $488 \cdot 10$ & 4.396748 & NU $1 *$ \\
\hline 3883.60 & 3.6637 & 0.0734 & 7 & 2 & 6 & 6 & 2 & 5 & 552.92 & 5.960000 & NU $3 *$ \\
\hline 3883.98 & 0.0662 & 0.0605 & 12 & 3 & 9 & 11 & 4 & 8 & 1843.02 & 4.355455 & NUI \\
\hline 3883.98 & 16.2910 & 0.0430 & 7 & 6 & 1 & 6 & 6 & 0 & 1045.07 & 1.869458 & NU 3 \\
\hline 3883.98 & 48.8730 & 0.0430 & 7 & 6 & 2 & 6 & 6 & 1 & 1045.07 & 1.869458 & NU3 \\
\hline 3884.08 & 2.0845 & 0.0861 & 6 & 3 & 3 & 5 & 3 & 2 & 508.81 & 2.720000 & $\mathrm{NU} 3 *$ \\
\hline 3884.20 & 0.0541 & 0.0862 & 4 & 3 & 1 & 4 & 1 & 4 & 224.83 & 0.017070 & NU3* \\
\hline 3884.58 & 0.0460 & 0.0971 & 3 & 2 & 1 & 2 & 0 & 2 & 70.08 & 0.094498 & NU $3 * *$ \\
\hline 3884.75 & 0.0410 & 0.0971 & 3 & 2 & 1 & 2 & 0 & 2 & 1664.97 & 0.094498 & NU $3 * * *$ \\
\hline 3884.82 & 0.3211 & 0.0771 & 7 & 3 & 5 & 6 & 3 & 4 & $2271 \cdot 70$ & 5.110000 & NU $3 \ddot{*} * *$ \\
\hline 3884.92 & 0.2374 & 0.032 & 14 & 0 & 14 & 13 & 1 & 13 & $1806 \cdot 78$ & 12.99955 & NU 1 \\
\hline 3884.92 & 0.7116 & 0.032 & 14 & 1 & 14 & 13 & 0 & 13 & $1806 \cdot 78$ & 12.99957 & NUI \\
\hline 3885.37 & 0.9264 & 0.0917 & 6 & 2 & 4 & 5 & 2 & 3 & 2053.98 & 5.358633 & NU $3 * \ddot{*} *$ \\
\hline 3885.68 & 543.9519 & 0.0800 & 6 & 4 & 3 & 5 & 3 & 2 & 508.81 & $15 \cdot 18$ & NU I+ \\
\hline 3885.68 & 3504.6611 & 0.0642 & 7 & 1 & 7 & 6 & 1 & 6 & $447 \cdot 24$ & 6.746361 & NU3 \\
\hline $3885 \cdot 94$ & 4.0338 & 0.0535 & 8 & 0 & 8 & 7 & 0 & 7 & 586.26 & 7.747248 & NU $3 *$ \\
\hline 3886.08 & 1172.0190 & 0.0649 & 7 & 0 & 7 & 6 & 0 & 6 & 446.71 & 6.749914 & NU3 \\
\hline 3886.47 & 0.6388 & 0.0332 & 13 & 1 & 12 & 12 & 2 & 11 & 1774.74 & 9.99731 & NU1 \\
\hline 3886.65 & 0.2130 & 0.0331 & 13 & 2 & 12 & 12 & 1 & 11 & $1774 \cdot 60$ & 9.99954 & NU1 \\
\hline 3886.77 & 1.0514 & 0.0700 & 7 & 4 & 4 & 6 & 4 & 3 & 756.75 & 4.735129 & NU3* \\
\hline 3887.32 & 8.0240 & 0.0803 & 6 & 4 & 3 & 6 & 2 & 4 & 602.77 & 0.100806 & NU3 \\
\hline 3888.04 & 6.7224 & 0.0870 & 3 & 3 & 0 & 3 & 1 & 3 & $142 \cdot 28$ & 0.008443 & NU3 \\
\hline $3888 \cdot 54$ & 0.0216 & 0.0527 & 5 & 5 & 1 & 4 & 4 & 0 & $488 \cdot 13$ & 4.397010 & NU $1 * *$ \\
\hline 3888.57 & 0.0648 & 0.0525 & 5 & 5 & 0 & 4 & 4 & 1 & 488.10 & 4.396748 & NUl $* \ddot{*}$ \\
\hline 3888.68 & 1.9460 & 0.0771 & 7 & 3 & 5 & 6 & 3 & 4 & 648.97 & 5.110000 & NU3* \\
\hline 3888.76 & 0.2275 & 0.0785 & 7 & 1 & 6 & 6 & 1 & 5 & $2146 \cdot 28$ & 6.391209 & NU $3 * * * *$ \\
\hline 3888.77 & 5.6151 & 0.0917 & 6 & 2 & 4 & 5 & 2 & 3 & $446 \cdot 50$ & 5.358633 & NU $3 *$ \\
\hline 3889.97 & 1.3791 & 0.0785 & 7 & 1 & 6 & 6 & 1 & 5 & 542.91 & 6.391209 & NU $3 *$ \\
\hline 3890.12 & 0.3492 & 0.0748 & 7 & 4 & 3 & 6 & 4 & 2 & 757.78 & 4.739627 & NU $3 *$ \\
\hline 3891.32 & 3625.9114 & 0.0872 & 6 & 1 & 5 & 5 & 1 & 4 & 399.46 & 5.488405 & NU3 \\
\hline 3891.66 & 0.3857 & 0.0861 & 6 & 3 & 3 & 5 & 3 & 2 & 508.81 & 2.720000 & NU $3 * *$ \\
\hline 3891.96 & 256.0173 & 0.0887 & 6 & 3 & 3 & 5 & 2 & 4 & $416 \cdot 22$ & 13.47 & NU I+ \\
\hline 3892 & 0.6720 & 0.0454 & 12 & 3 & 10 & 11 & 2 & 9 & 1690.70 & 7.003088 & NUI \\
\hline 3892.83 & 124.1187 & 0.0971 & 3 & 2 & 1 & 2 & 0 & 2 & 70.08 & 0.094498 & NU3 \\
\hline 3893.67 & 58.3632 & 0.0527 & 5 & 5 & 1 & 4 & 4 & 0 & $488 \cdot 13$ & 4.397010 & NU1 \\
\hline 3893 & 0.6812 & 0.0444 & 11 & 2 & 9 & 11 & 2 & 10 & 1525.13 & 0.856092 & NU3 \\
\hline 3893.70 & 175.1005 & 0.0525 & 5 & 5 & 0 & 4 & 4 & 1 & 488.10 & 4.396748 & NU1 \\
\hline 3893 & 0.2484 & 0.0532 & 8 & 1 & 8 & 7 & 1 & 7 & 586.48 & 7.746063 & NU $3 \ddot{*} \ddot{x}$ \\
\hline 3893 & 0.7463 & 0.0535 & 8 & 0 & 8 & 7 & 0 & 7 & 586.26 & 7.747248 & NU $3 * *$ \\
\hline 3894.07 & 11.7787 & 0.0915 & 4 & 3 & 2 & 3 & 0 & 3 & 136.77 & 0.051049 & NUI \\
\hline 3894.07 & 197.6637 & 0.0565 & 7 & 5 & 3 & 6 & 5 & 2 & 888.60 & 3.450020 & NU3 \\
\hline 3894.28 & 65.8922 & 0.0569 & 7 & 5 & 2 & 6 & 5 & 1 & 888.63 & 3.450058 & NU3 \\
\hline 3894.49 & 0.1945 & 0.0700 & 7 & 4 & 4 & 6 & 4 & 3 & 756.75 & 4.735129 & NU $3 * *$ \\
\hline 3895.14 & 2.8823 & 0.0380 & 8 & 7 & 2 & 7 & 7 & 1 & 1394.86 & 1.887603 & NU 3 \\
\hline 3895.14 & 8.6468 & 0.0380 & 8 & 7 & 1 & 7 & 7 & 0 & 1394.86 & 1.887603 & NU3 \\
\hline 3896 & 0.0460 & 0.0755 & 6 & 4 & 2 & 5 & 3 & 3 & 2126.41 & 11.35 & NU I $* * * *$ \\
\hline & 0.3424 & 0.0446 & 9 & 1 & 9 & 8 & 1 & 8 & 2337.70 & 8.745235 & NU $3 * * *$ \\
\hline 3896 & 2.0316 & 0.0427 & 11 & 3 & 9 & 11 & 1 & 10 & 1524.87 & 0.850512 & NU3 \\
\hline
\end{tabular}




\begin{tabular}{|c|c|c|c|c|c|c|c|c|c|c|c|}
\hline $\begin{array}{c}\text { FREQUENCY } \\
\mathrm{cm}^{-1}\end{array}$ & $\begin{array}{l}\text { LINE } \\
\text { STRENGTH } \\
\frac{\mathrm{cm}^{-1}}{\mathrm{gm} \mathrm{cm}^{-2}}\end{array}$ & $\begin{array}{l}\text { HALF } \\
\text { WIDTH } \\
\frac{\mathrm{cm}^{-1}}{\text { otm. }}\end{array}$ & $J^{\prime}$ & $k_{a}$ & $\mathrm{~K}_{\mathrm{c}}$ & J" & $K_{a}$ & $K_{c}$ & $\begin{array}{c}\mathrm{E}^{\prime \prime} \\
\mathrm{cm}^{-1}\end{array}$ & $L$ & BAND \\
\hline 3896.46 & 0.1142 & 0.0447 & 9 & 0 & 9 & 8 & 0 & 8 & 2337.53 & 8.745630 & NU $3 * * * *$ \\
\hline 3896.49 & 0.3600 & 0.0771 & 7 & 3 & 5 & 6 & 3 & 4 & 648.97 & 5.110000 & NU3*** \\
\hline 3896.50 & 1.0388 & 0.0917 & 6 & 2 & 4 & 5 & 2 & 3 & $446 \cdot 50$ & 5.358633 & NU $3 * *$ \\
\hline 3896.54 & 0.1131 & 0.0633 & 8 & 2 & 7 & 7 & 2 & 6 & 2318.53 & 7.292008 & NU $3 * * *$ \\
\hline 3896.69 & 0.2760 & 0.0594 & 10 & 5 & 6 & 10 & 3 & 7 & $1538 \cdot 23$ & 0.369280 & NU3 \\
\hline 3896.81 & 7.7064 & 0.0458 & 9 & 1 & 8 & 9 & 1 & 9 & 920.21 & 0.472318 & NU 3 \\
\hline 3896.81 & 0.0177 & 0.0397 & 10 & 1 & 9 & 10 & 1 & 10 & 1114.56 & 0.478334 & NU $3 *$ \\
\hline 3896.90 & 0.0193 & 0.0527 & 5 & 5 & 1 & 4 & 4 & 0 & 2129.65 & 4.397010 & NU1*** \\
\hline 3896.92 & 0.0578 & 0.0525 & 5 & 5 & 0 & 4 & 4 & 1 & 2129.63 & 4.396748 & NU $1 * * * *$ \\
\hline 3897.24 & 0.0778 & 0.032 & 15 & 1 & 15 & 14 & 0 & 14 & 2073.66 & 14.00192 & NU 1 \\
\hline 3897.24 & 0.1921 & 0.032 & 15 & 0 & 15 & 14 & 1 & 14 & 2073.66 & 14.00191 & NU1 \\
\hline 3897.24 & 0.1054 & 0.0885 & 7 & 3 & 4 & 6 & 3 & 3 & 2282.59 & 5.330000 & NU $3 * * * *$ \\
\hline 3897.56 & 23.0795 & 0.0451 & 9 & 2 & 8 & 9 & 0 & 9 & $920 \cdot 18$ & 0.471417 & NU3 \\
\hline 3897.93 & 139.3042 & 0.0755 & 6 & 4 & 2 & 5 & 3 & 3 & 504.00 & 11.35 & NUI+ \\
\hline 3898.01 & 0.2552 & 0.0785 & 7 & 1 & 6 & 6 & 1 & 5 & 542.91 & 6.391209 & NU $3 * *$ \\
\hline 3898.29 & 214.0639 & 0.0725 & 6 & 6 & 1 & 5 & 3 & 2 & 508.81 & 64.02 & $2 \mathrm{~N} \cup 2+$ \\
\hline 3898.82 & 5.8341 & 0.0845 & 7 & 3 & 4 & 7 & 0 & 7 & $586 \cdot 26$ & 0.239 & NUI+ \\
\hline 3898.82 & 0.0301 & 0.0692 & 5 & 4 & 1 & 6 & 0 & 6 & 446.71 & 0.000172 & NU3 \\
\hline 3898.88 & 20.4906 & 0.0792 & 5 & 4 & 2 & 5 & 2 & 3 & $446 \cdot 50$ & 0.039164 & NU 3 \\
\hline 3899.24 & 1042.2919 & 0.0861 & 6 & 3 & 3 & 5 & 3 & 2 & 508.81 & 2.720000 & NU3- \\
\hline 3899.46 & 1831.8316 & 0.0734 & 7 & 2 & 6 & 6 & 2 & 5 & 552.92 & 5.960000 & NU3- \\
\hline 3900.35 & 0.6856 & 0.0633 & 8 & 2 & 7 & 7 & 2 & 6 & 709.60 & 7.292008 & NU $3 *$ \\
\hline 3900.60 & 0.0070 & 0.0496 & 9 & 6 & 4 & 8 & 6 & 3 & 3101.19 & 5.032027 & NU $3 * * *$ \\
\hline 3900.95 & 0.0801 & 0.0594 & 8 & 5 & 4 & 7 & 5 & 3 & 1059.65 & 4.903948 & NU $3 *$ \\
\hline 3901.21 & 2.0752 & 0.0446 & 9 & 1 & 9 & 8 & 1 & 8 & $744 \cdot 16$ & 8.745235 & NU3* \\
\hline 3901.25 & 0.6921 & 0.0447 & 9 & 0 & 9 & 8 & 0 & 8 & 744.09 & 8.745630 & NU3* \\
\hline $3901 \cdot 33$ & 0.0123 & 0.0407 & 9 & 7 & 3 & 8 & 7 & 2 & 1590.74 & 3.579253 & NU $3 *$ \\
\hline 3901.64 & 27.0726 & 0.0862 & 4 & 3 & 1 & 4 & 1 & 4 & 224.83 & 0.017070 & NU3 \\
\hline 3901.74 & 671.4213 & 0.0532 & 8 & 1 & 8 & 7 & 1 & 7 & 586.48 & 7.746063 & NU3 \\
\hline 3901.91 & 2016.9288 & 0.0535 & 8 & 0 & 8 & 7 & 0 & 7 & $586 \cdot 26$ & 7.747248 & NU3 \\
\hline 3502.21 & 525.6870 & 0.0700 & 7 & 4 & 4 & 6 & 4 & 3 & 756.75 & 4.735129 & NU3 \\
\hline 3902.39 & 41.0263 & 0.0834 & 7 & 4 & 4 & 6 & 3 & 3 & 661.56 & $7 \cdot 34$ & NUI+ \\
\hline 3903.35 & 4.7486 & 0.0662 & 6 & 6 & 0 & 5 & 3 & 3 & 504.00 & 4.25 & $2 N \cup 2+$ \\
\hline 3903.46 & 0.0858 & 0.0852 & 8 & 4 & 5 & 7 & 3 & 4 & 842.38 & $6 \cdot 30$ & NÚl* \\
\hline 3903.90 & 2.1279 & 0.0670 & 8 & 1 & 7 & 7 & 1 & 6 & $704 \cdot 22$ & 7.336095 & NU $3 *$ \\
\hline 3904.24 & 2807.5183 & 0.0917 & 6 & 2 & 4 & 5 & 2 & 3 & 446.50 & 5.358633 & NU3 \\
\hline 3904.30 & 972.9716 & 0.0771 & 7 & 3 & 5 & 6 & 3 & 4 & 648.97 & 5.110000 & NU3- \\
\hline 3905.06 & 0.4026 & 0.0354 & 9 & 8 & 1 & 8 & 8 & 0 & $1789 \cdot 10$ & 1.901685 & NU3 \\
\hline 3905.06 & 1.2079 & 0.0354 & 9 & 8 & 2 & 8 & 8 & 1 & 1789.10 & 1.901685 & NU3 \\
\hline 3905.10 & 0.0713 & 0.0656 & 6 & 4 & 2 & 7 & 0 & 7 & $586 \cdot 26$ & 0.000274 & NU3 \\
\hline 3905.37 & 174.6112 & 0.0748 & 7 & 4 & 3 & 6 & 4 & 2 & 757.78 & 4.739627 & NU3 \\
\hline 3906.05 & 689.5227 & 0.0785 & 7 & 1 & 6 & 6 & 1 & 5 & 542.91 & 6.391209 & NU3 \\
\hline 3906.17 & 13.1418 & 0.0469 & 8 & 6 & 3 & 7 & 6 & 2 & $1216 \cdot 20$ & 3.522863 & NU3 \\
\hline 3906.32 & 0.0619 & 0.0587 & 6 & 5 & 1 & 5 & 4 & 2 & 610.12 & 4.264570 & NUI* \\
\hline 3906.34 & 39.4272 & 0.0469 & 8 & 6 & 2 & 7 & 6 & 1 & $1216 \cdot 20$ & 3.522866 & NU3 \\
\hline 3906.48 & 0.1100 & 0.0757 & 8 & 5 & 3 & 8 & 2 & 6 & 982.91 & 0.098024 & NUI \\
\hline 3906.82 & 0.2036 & 0.0695 & 9 & 5 & 4 & 9 & 2 & 7 & 1201.95 & 0.180415 & NUI \\
\hline 3907.40 & 0.1684 & 0.0894 & 7 & 2 & 5 & 6 & 2 & 4 & $2211 \cdot 21$ & 6.352023 & NU $3 * * * *$ \\
\hline 3907.71 & 0.0839 & 0.0787 & 8 & 4 & 4 & 7 & 4 & 3 & $2572 \cdot 13$ & 5.440000 & NU $3 * * *$ \\
\hline 3908.23 & 0.1903 & 0.0701 & 8 & 4 & 5 & 7 & 4 & 4 & 927.77 & 6.014663 & NU $3 \ddot{*}$ \\
\hline 3908.32 & 0.1268 & 0.0633 & 8 & 2 & 7 & 7 & 2 & 6 & 709.60 & 7.292008 & NU3** \\
\hline 3908.53 & 0.5993 & 0.0391 & 12 & 2 & 10 & 12 & 2 & 11 & 1774.74 & 0.875278 & NU3 \\
\hline 3908.57 & 0.2984 & 0.0736 & 8 & 3 & 6 & 7 & 3 & 5 & 816.72 & 5.410000 & NU3* \\
\hline 3908.65 & 0.0149 & 0.0594 & 8 & 5 & 4 & 7 & 5 & 3 & 1059.65 & 4.903948 & NU $3 * *$ \\
\hline
\end{tabular}




\begin{tabular}{|c|c|c|c|c|c|c|c|c|c|c|c|}
\hline $\begin{array}{c}\text { FREQUENCY } \\
\mathrm{cm}^{-1}\end{array}$ & $\begin{array}{l}\text { LINE } \\
\text { STRENGTH } \\
\frac{\mathrm{cm}^{-1}}{\mathrm{gm} \mathrm{cm}^{-2}}\end{array}$ & $\begin{array}{l}\text { HALF } \\
\text { WIDTH } \\
\frac{\mathrm{cm}^{-1}}{\text { atm. }}\end{array}$ & $J^{\prime}$ & $K_{0}$ & $K_{c}$ & $J^{\prime \prime}$ & $k_{a}$ & $K_{c}$ & $\begin{array}{c}E^{\prime \prime} \\
\mathrm{cm}^{-1}\end{array}$ & L & BAND \\
\hline 3908.73 & 0.0159 & 0.0852 & 8 & 4 & 5 & 7 & 3 & 4 & $842 \cdot 38$ & $6 \cdot 30$ & NU I * \\
\hline 3908.97 & 3.6252 & 0.0762 & 4 & 4 & 1 & 4 & 2 & 2 & 315.79 & 0.010786 & NU3 3 \\
\hline 3909.22 & 0.3840 & 0.0446 & 9 & 1 & 9 & 8 & 1 & 8 & 744.16 & 8.745235 & NU $3 * * *$ \\
\hline 3905 & 0.1280 & 0.0447 & 9 & 0 & 9 & 8 & 0 & 8 & 744.09 & 8.745630 & NU $3 * *$ \\
\hline 3905 & 0.0286 & 0.0637 & 6 & 5 & 1 & 7 & 1 & 6 & 704.22 & 0.000197 & NU3 \\
\hline 390 & 0.0445 & 0.0608 & 8 & 5 & 3 & 7 & 5 & 2 & 1059.85 & 4.904299 & $N \cup 3 * *$ \\
\hline 3909 & 1.0211 & 0.0894 & 7 & 2 & 5 & 6 & 2 & 4 & 602.77 & 6.352023 & NU3* \\
\hline 390 & 0.0229 & 0.032 & 16 & 0 & 16 & 15 & 1 & 15 & $2358 \cdot 44$ & 15.003 & NU1 \\
\hline 3910 & 0.1994 & 0.0381 & 12 & 3 & 10 & 12 & 1 & 11 & 1774.60 & 3307 & NU3 \\
\hline 3910.16 & 0.6387 & 0.0885 & 7 & 3 & 4 & 6 & 3 & 3 & $661 \cdot 56$ & 5.330000 & NU3* \\
\hline 3915 & 0.1823 & 0.0795 & 4 & 4 & 1 & 4 & 1 & 4 & 224.83 & 1222 & NUI \\
\hline $39^{\circ}$ & 1.6492 & 0.0728 & 9 & 5 & 5 & 9 & 3 & 6 & 1282.92 & 0.2 & NU3 \\
\hline 391 & 0.0135 & 0.0834 & 7 & 4 & 4 & 6 & 3 & 3 & 2.59 & 7.34 & NU $1 * * * *$ \\
\hline 391 & 0.0687 & 0.032 & 16 & 1 & 16 & 15 & 0 & 15 & $8 \cdot 44$ & 15.00394 & NU1 \\
\hline 391$]$ & 0.0343 & 0.0598 & 6 & 5 & 2 & 5 & 4 & 1 & 0.35 & $4 \cdot 2$ & NU $1 * * *$ \\
\hline 3911.27 & 0.0530 & 0.0388 & 10 & 1 & 10 & 9 & 1 & 9 & 2512.43 & 9.744353 & NU $3 * * * *$ \\
\hline 3911.33 & 0.1589 & 0.0388 & 10 & 0 & 10 & 9 & 0 & 9 & $2512 \cdot 36$ & 9.744485 & NU $3 * * *$ \\
\hline 3911.45 & 0.0114 & 0.0587 & 6 & 5 & 1 & 5 & 4 & 2 & 610.12 & 4.264570 & NUI $* *$ \\
\hline 3911.98 & 0.3936 & 0.0670 & 8 & 1 & 7 & 7 & 1 & 6 & 704.22 & 7.336 & NU $3 * * *$ \\
\hline 3912.30 & 0.0670 & 0.032 & 15 & 1 & 14 & 14 & 2 & 13 & 2327.90 & 12.018 & NU1 \\
\hline 3912.32 & 0.0183 & 0.032 & 15 & 2 & 14 & 14 & 1 & 13 & 2327.88 & 12.0 & NU1 \\
\hline $3912 \cdot 34$ & 0.1607 & 0.0535 & 9 & 2 & 8 & 8 & 2 & 7 & 2495.18 & 8.292239 & NU $3 * * * *$ \\
\hline 391 & 0.0425 & 0.0496 & 9 & 6 & 4 & 8 & 6 & 3 & 1411.65 & 5.032027 & NU3* \\
\hline 391 & 8.8978 & 0.0397 & 10 & 1 & 9 & 10 & 1 & 1 & +.56 & 0.478334 & NU3 \\
\hline 391 & 0.0142 & 0.0500 & 9 & 6 & 3 & 8 & 6 & 2 & 1.68 & $5 \cdot 0$ & NU3 $\ddot{*}$ \\
\hline $39:$ & 2.9643 & 0.0393 & 10 & 2 & 9 & 10 & 0 & 10 & +.55 & 0.478039 & NU3 \\
\hline 391 & 0.5759 & 0.0952 & 4 & 2 & 2 & 3 & 0 & & .77 & 6055 & NU $3 *$ \\
\hline 3913 & 0.3505 & 0.0806 & 7 & 5 & 2 & 7 & 2 & 5 & 782.40 & 8149 & NUI \\
\hline 56 & 0.0191 & 0.0607 & 9 & 5 & 5 & 8 & 5 & 4 & 63 & 5627 & NU $3 * * *$ \\
\hline 391 & 0.0312 & 0.0340 & 10 & 9 & 2 & 9 & 9 & 1 & .56 & 1.9 & NU3 \\
\hline 3913 & 0.0937 & 0.0340 & 10 & 9 & 1 & 9 & $\varsigma$ & 0 & .56 & 1.912938 & NU3 \\
\hline 3914 & 42.9027 & 0.0852 & 8 & 4 & 5 & 7 & 3 & 4 & $842 \cdot 38$ & $6 \cdot 30$ & NUI+ \\
\hline $391<$ & 0.2876 & 0.0774 & 7 & 4 & 3 & 6 & 3 & 4 & 648.97 & 8.01 & NUI * \\
\hline 391 & 0.0142 & 0.0852 & 8 & 4 & 5 & 7 & 3 & 4 & $2462 \cdot 87$ & $6 \cdot 30$ & NU $1 * * * * *$ \\
\hline 3915 & 0.0152 & 0.0928 & 6 & 2 & 4 & 5 & 1 & 5 & 326.64 & 0.254241 & NUI* \\
\hline .50 & 0.5083 & 0.0787 & 8 & 4 & 4 & 7 & 4 & 3 & 1.22 & 5.440000 & NU3* \\
\hline 3915.65 & 0.0544 & 0.0559 & 9 & 1 & 8 & 8 & 1 & 7 & 0.37 & 8.308658 & NU3***x \\
\hline 3915.97 & 0.0353 & 0.0701 & 8 & 4 & 5 & 7 & 4 & 4 & 7.77 & 14663 & NU3*** \\
\hline 3916.06 & 0.9741 & 0.0535 & 9 & 2 & 8 & 8 & 2 & 7 & 5.62 & 8.292239 & NU3* \\
\hline $3916 \cdot 30$ & 342.7574 & 0.0633 & 8 & 2 & 7 & 7 & 2 & 6 & 9.60 & 7.292008 & NU3 \\
\hline 3916.35 & 92.7388 & 0.0598 & 6 & 5 & 2 & 5 & 4 & 1 & 0.35 & 4.266963 & NUI \\
\hline $3916 \cdot 36$ & 40.0471 & 0.0594 & 8 & 5 & 4 & 7 & 5 & 3 & 105 & 4.903948 & NU3 \\
\hline $3916 \cdot 44$ & 0.0552 & 0.0736 & 8 & 3 & 6 & 7 & 3 & 5 & 816.72 & 5.410000 & NU $3 * *$ \\
\hline 3916.45 & 0.9627 & 0.0388 & 10 & 0 & 10 & 9 & 0 & 9 & 920.18 & 9.744485 & NU3* \\
\hline 3916 & 30.9367 & 0.0587 & 6 & 5 & 1 & 5 & 4 & 2 & 0.12 & 4.264570 & NUI \\
\hline 391 & 2.0463 & 0.0408 & 9 & 7 & 2 & 8 & 7 & 1 & $1590 \cdot 74$ & 3.579254 & NU3 \\
\hline 391 & 6.1389 & 0.0407 & 9 & 7 & 3 & 8 & 7 & 2 & 0.74 & 3.579253 & NU3 \\
\hline .19 & 120.1755 & 0.0608 & 8 & 5 & 3 & 7 & 5 & 2 & 9.85 & 4.904299 & NU3 \\
\hline 391 & 1037.6231 & 0.0446 & 9 & 1 & 9 & 8 & 1 & 8 & $744 \cdot 16$ & 8.745235 & NU3 \\
\hline$\cdot 28$ & 346.0373 & 0.0447 & 9 & 0 & 9 & 8 & 0 & 8 & 744.09 & 8.745630 & NU3 \\
\hline 3917.73 & 0.1182 & 0.0885 & 7 & 3 & 4 & 6 & 3 & 3 & $661 \cdot 56$ & 5.330000 & NU $3 * *$ \\
\hline 3917.82 & 0.0408 & 0.037 & 14 & 3 & 12 & 13 & 2 & 11 & 2246.98 & 8.90961 & NU1 \\
\hline 3917.89 & 0.3299 & 0.0559 & 9 & 1 & 8 & 8 & 1 & 7 & 882.93 & 8.308658 & NU $3 *$ \\
\hline 3918.78 & 0.0532 & 0.0774 & 7 & 4 & 3 & 6 & 3 & 4 & 648.97 & 8.01 & NUI*** \\
\hline
\end{tabular}




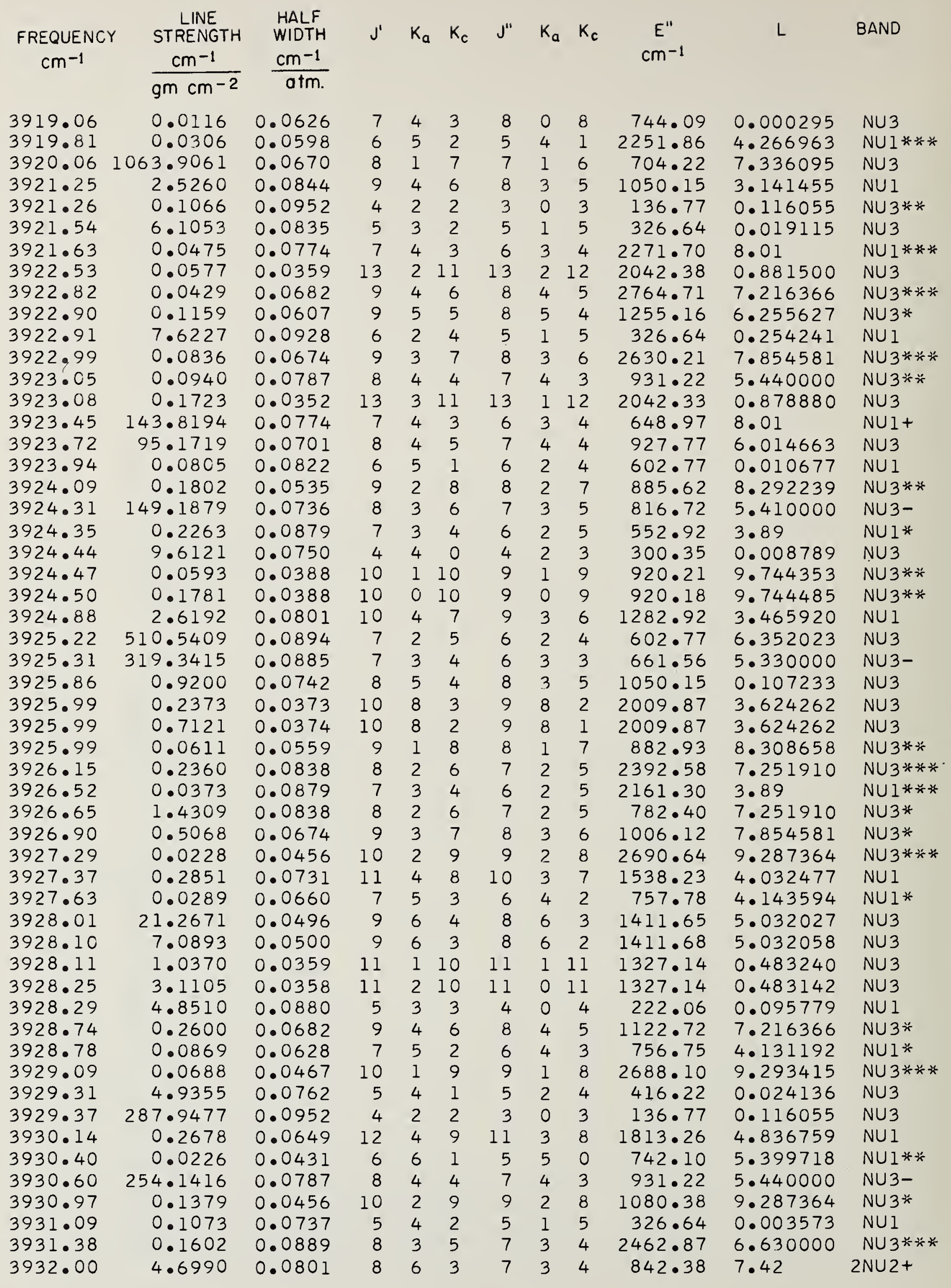




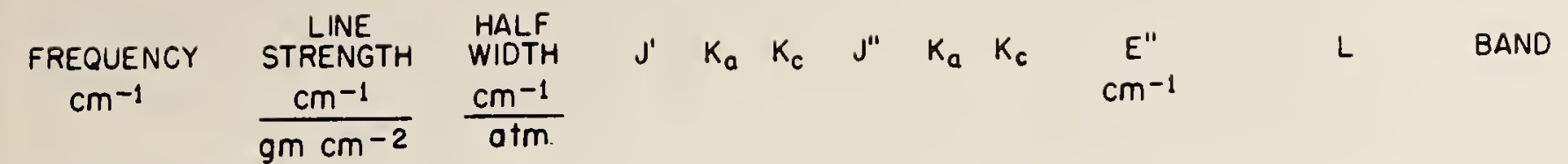

3932.12 3932.16 3932.53 3932.56 3934.09 3934.65 3934.69 3934.75 3934.85 3935.15 3935.17 3935.30 3935.77 3935.77 3936.54 3938.00 3938.06 3938.06 3938.34 3938.44 3939.05 3940.05 3940.07 3940.27 3940.29 3940.54 3940.69 3941.27 3941.64 3941.64 3941.93 3942.34 3942.65 3942.81 3943.12 3943.17 3943.57 3943.58 3944.14 3944.34 3944.41 3944.47 3945.19 3946.50 3946.50 3946.64 3947.15 3947.25 3947.47 3947.48 3947.76 3947.97 3948.18 3948.69

\begin{tabular}{|c|c|c|c|c|c|c|}
\hline 487.0765 & 0.0535 & 9 & 2 & 8 & 8 & 2 \\
\hline $\begin{array}{l}113.1350 \\
160.4471\end{array}$ & $\begin{array}{l}0.0879 \\
0.0388\end{array}$ & $\begin{array}{r}7 \\
10\end{array}$ & 1 & $\begin{array}{r}4 \\
10\end{array}$ & $\begin{array}{l}6 \\
9\end{array}$ & 2 \\
\hline 481.3514 & 0.0388 & 10 & 0 & 10 & & \\
\hline 64.9429 & 0.0559 & 9 & & 8 & & \\
\hline 0.2647 & 0.0838 & 8 & & 6 & & \\
\hline 0.9711 & 0.0889 & 8 & & 5 & & \\
\hline 0.0184 & 0.0520 & 10 & & 4 & & \\
\hline 0.0938 & 0.0674 & 9 & & 7 & & \\
\hline 22.0035 & 0.0885 & 3 & 3 & 0 & & \\
\hline 0.2344 & 0.0885 & 4 & 3 & 1 & & \\
\hline 0.0917 & 0.0815 & 5 & 5 & 0 & & \\
\hline 61.0285 & 0.0431 & 6 & 6 & 1 & 5 & \\
\hline 0.0438 & 0.0340 & 14 & 2 & 12 & 14 & ? \\
\hline 0.0480 & 0.0682 & 9 & 4 & 6 & 8 & \\
\hline 14.4444 & 0.0660 & 7 & 5 & 3 & 6 & \\
\hline 1.0117 & 0.0430 & 10 & 7 & 4 & 9 & \\
\hline 3.0352 & 0.0431 & 10 & 7 & 3 & 9 & \\
\hline 57.9477 & 0.0607 & 9 & 5 & 5 & 8 & 5 \\
\hline 12.4824 & 0.0755 & 6 & 4 & 2 & 6 & \\
\hline 43.4443 & 0.0628 & 7 & 5 & 2 & 6 & \\
\hline 0.0771 & 0.0467 & 10 & 1 & 9 & 9 & \\
\hline 0.0201 & 0.0431 & 6 & 6 & 1 & 5 & 5 \\
\hline 0.0638 & 0.0815 & 9 & 4 & 5 & 8 & 4 \\
\hline 3.8707 & 0.0740 & 7 & 5 & 3 & 7 & 3 \\
\hline 0.1965 & 0.0760 & 9 & 2 & 7 & 8 & \\
\hline 19.2321 & 0.0640 & 9 & 5 & 4 & 8 & \\
\hline 0.0325 & 0.0760 & 9 & 2 & 7 & 8 & 2 \\
\hline 0.0118 & 0.0608 & 8 & 4 & 4 & 9 & 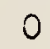 \\
\hline 0.0162 & 0.0873 & 3 & 3 & 1 & 2 & 1 \\
\hline 0.0143 & 0.0628 & 7 & 5 & 2 & 6 & 4 \\
\hline 0.1797 & 0.0889 & 8 & 3 & 5 & 7 & 3 \\
\hline 715.4263 & 0.0838 & 8 & 2 & 6 & 7 & 2 \\
\hline 253.3967 & 0.0674 & 9 & 3 & 7 & 8 & 3 \\
\hline 0.9857 & 0.0337 & 12 & 1 & 11 & 12 & 1 \\
\hline 0.3286 & 0.0337 & 12 & 2 & 11 & 12 & 0 \\
\hline 0.0783 & 0.0793 & 8 & 4 & 4 & 7 & 3 \\
\hline 0.0668 & 0.0594 & 10 & 3 & 8 & 9 & 3 \\
\hline 0.0434 & 0.0885 & 4 & 3 & 1 & 3 & 1 \\
\hline 129.9811 & 0.0682 & 9 & 4 & 6 & 8 & \\
\hline 0.0157 & 0.0789 & 6 & 3 & 3 & 6 & \\
\hline 0.0157 & 0.0604 & 10 & 5 & 6 & 9 & 5 \\
\hline 0.0722 & 0.0818 & 8 & 3 & 5 & 8 & 0 \\
\hline 0.0858 & 0.0392 & 11 & 8 & 3 & 10 & 8 \\
\hline 0.2576 & 0.0391 & 11 & 8 & 4 & 10 & 8 \\
\hline 0.0772 & 0.0658 & 11 & 6 & 6 & 11 & 4 \\
\hline 68.9486 & 0.0456 & 10 & 2 & 9 & 9 & 2 \\
\hline 0.2907 & 0.0681 & 6 & 4 & 3 & 6 & 1 \\
\hline 201.6251 & 0.0354 & 11 & 1 & 11 & 10 & 1 \\
\hline 67.2088 & 0.0354 & 11 & 0 & 11 & 10 & 0 \\
\hline 0.0118 & 0.0815 & 9 & 4 & 5 & 8 & 4 \\
\hline 0.0145 & 0.0793 & 8 & 4 & 4 & 7 & 3 \\
\hline 208.4119 & 0.0467 & 10 & 1 & 9 & 9 & 1 \\
\hline 0.0364 & 0.0760 & 9 & 2 & 7 & 8 & 2 \\
\hline
\end{tabular}

$\begin{array}{lll}487.0765 & 0.0535\end{array}$ 113.1350 160.4471 481.3514 0.2647

0.9711

0.0184

0.0938

2.0035

0.2344

0.0917

1.0285

0.0438

1.0117

3.0352

57.9477

0.0771

0.0201

0.0638

3.8707

0.1965

0.0325

0.0118

0.0162

0.0143

0.1797

715.4263

3.3967

0.3286

0.0783

0.0668

0.0434

0.0157

0.0157

0.0722

0.0858

.2576

8.9486

0.2907

67.2088

0.0118

08.4119

0.0364
0.0388

0.0559

0.0838

0.0520

0.0674

.0885

0.0815

0.0431

0.0340

0.0682

0.0660

0.0430

0.0431

0.0755

0.0628

0.0431

0.0815

0.0760

0.0640

0.0760

0.0873

0.0628

0.0838

0.0674

0.0337

0.0337

0.0793

0.0594
0.0885

0.0682

0.0789

0.0818

0.0392

0.0658

0.0456

0.0760
0.0354
885.62

552.92

920.21

920.18

882.93

782.40

842.38

1631.41

1006.12

95.17

$173 \cdot 36$

446.50

742.10

2327.90

1122.72

757.78

1810.63

1810.63

$1255 \cdot 16$

552.92

756.75

1079.07

2406.15

1131.76

842.38

982.91

1255.92

2595.81

$920 \cdot 18$

79.48

2398.39

842.38

782.40

1006.12

1557.91

1557.91

816.72

1216.27

173.36

1122.72

447.24

1474.99

744.09

2254.34

2254.34

1899.06

1080.38

447.24

1114.56

1114.55

1131.76

816.72

1079.07

982.91
8.292239

3.89

9.744353

9.744485

8.308658

7.251910

6.630000

6.440055

7.854581

0.021579

0.056400

0.001850

5.399718

0.895030

7.216366

4. 143594

5.133658

5.133660

6.255627

0.040211

4. 131192

9.293415

5.399718

5.540000

0.052981

8.113471

6.258032

8. 113471

0.000238

0.013176

4.131192

6.630000

7.251910

7.854581

0.487287

0.487254

5.02

8.878817

0.056400

7.216366

0.014823

7.532122

0.019318

5. 216541

5.216540

0.195288

9. 287364

0.005871

10.743553

10.743597

5.540000

5.02

9.293415

8. 113471

NU3

NU1+

NU3

NU3

NU 3

NU $3 * *$

NU $3 *$

NU $3 *$

NU $3 * *$

NU 3

NU $3 *$

NU 1

NU 1

NU 3

NU $3 * *$

NU1

NU3

NU3

NU 3

NU 3

NU 1

NU $3 * *$

NUI****

NU $3 *$

NU3

NU $3 *$

NU 3

NU $3 *$ 前前

NU 3

NU $3 * *$

NU1****

NU $3 * *$

NU 3

NU3

NU3

NU3

NU 1 *

NU $3 *$

NU $3 \ddot{*} *$

NU 3

NU3* 


\begin{tabular}{|c|c|c|c|c|c|c|c|c|c|c|c|}
\hline $\begin{array}{c}\text { FREQUENCY } \\
\mathrm{cm}^{-1}\end{array}$ & 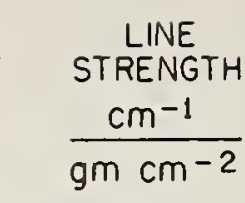 & $\begin{array}{l}\text { HALF } \\
\text { WIDTH } \\
\frac{\mathrm{cm}^{-1}}{\text { atm. }}\end{array}$ & $J^{\prime}$ & $\mathrm{K}_{\mathrm{a}}$ & $K_{c}$ & $J^{\prime \prime}$ & $K_{a}$ & $K_{c}$ & $\begin{array}{c}E^{\prime \prime} \\
\mathrm{cm}^{-1}\end{array}$ & L & BAND \\
\hline 3948.89 & 0.0105 & 0.0662 & 11 & 6 & 5 & 11 & 3 & 8 & 1813.26 & 0.189337 & NU1 \\
\hline 3949.39 & 0.0379 & 0.0850 & 4 & 4 & 1 & 3 & 1 & 2 & 173.36 & $0 \cdot 097$ & NU $1 *$ \\
\hline 3949.50 & 3.0760 & 0.0508 & 10 & 6 & 5 & 9 & 6 & 4 & $1631 \cdot 27$ & 6.439836 & NU3 \\
\hline 3950.00 & 485.5714 & 0.0889 & 8 & 3 & 5 & 7 & 3 & 4 & $842 \cdot 38$ & 6.630000 & NU3- \\
\hline 3950.13 & 9.2298 & 0.0520 & 10 & 6 & 4 & 9 & 6 & 3 & $1631 \cdot 41$ & 6.440055 & Nu3 \\
\hline 3950.59 & 43.7681 & 0.0873 & 3 & 3 & 1 & 2 & 1 & 2 & $79 \cdot 48$ & 0.013176 & NU3 \\
\hline 3951.39 & 0.0041 & 0.0669 & 8 & 5 & 3 & 8 & 3 & 6 & $1006 \cdot 12$ & 0.064192 & NU $3 *$ \\
\hline 3951.43 & 0.0119 & 0.0643 & 8 & 5 & 3 & 7 & 4 & 4 & 927.77 & 3.953657 & NUl* \\
\hline 3951.61 & 0.0123 & 0.0594 & 10 & 3 & 8 & 9 & 3 & 7 & $1216 \cdot 27$ & 8.878817 & NU $3 * *$ * \\
\hline 3951.62 & 0.0014 & 0.0612 & 10 & 5 & 5 & 9 & 2 & 8 & 1080.38 & 0.021627 & $2 \mathrm{NU} 2$ \\
\hline 3951.97 & 1.4100 & 0.0710 & 6 & 5 & 2 & 6 & 3 & 3 & 661.56 & 0.023375 & NU3 \\
\hline 3952.08 & 0.0051 & 0.0781 & 10 & 6 & 5 & 9 & 3 & 6 & 1282.92 & 0.071662 & 2 NU2 2 \\
\hline 3952.38 & 39.1454 & 0.0793 & 8 & 4 & 4 & 7 & 3 & 5 & 816.72 & 15.02 & NUI+ \\
\hline 3952.62 & 0.2199 & 0.0666 & 10 & 2 & 8 & 9 & 2 & 7 & 1201.95 & 9.000870 & NU $3 *$ \\
\hline 3952.77 & 0.0106 & 0.0480 & 7 & 6 & 1 & 6 & 5 & 2 & 888.60 & 5.265222 & NU 1** \\
\hline 3953.11 & 117.2356 & 0.0885 & 4 & 3 & 1 & 3 & 1 & 2 & $173 \cdot 36$ & 0.056400 & NU3- \\
\hline 3953.55 & 2.3121 & 0.0727 & 7 & 4 & 3 & 7 & 2 & 6 & 709.60 & 0.048724 & NU3 \\
\hline 3953.72 & 0.0363 & 0.0666 & 10 & 2 & 8 & 9 & 2 & 7 & 2818.40 & 9.000870 & NU $3 * * * *$ \\
\hline 3953.74 & 0.0232 & 0.0864 & 9 & 3 & 6 & 8 & 3 & 5 & 2670.79 & 8.066869 & NU $3 * * \ddot{*}$ \\
\hline 3954.55 & 0.0015 & 0.0665 & 6 & 5 & 1 & 6 & 3 & 4 & 648.97 & 0.021 & NU $3 * *$ \\
\hline 3955.17 & 0.0387 & 0.0885 & 4 & 3 & 1 & 3 & 1 & 2 & 1772.41 & 0.056400 & NU $3 * * * *$ \\
\hline 3955.25 & 31.8921 & 0.0815 & 9 & 4 & 5 & 8 & 4 & 4 & 1131.76 & 5.540000 & NU3- \\
\hline 3955.54 & 0.0070 & 0.0850 & 4 & 4 & 1 & 3 & 1 & 2 & $173 \cdot 36$ & 0.097 & $1 * *$ \\
\hline 3955.91 & 0.0063 & 0.0648 & 10 & 4 & 7 & 9 & 4 & 6 & 1340.89 & 8.354292 & NU $3 * \ddot{x}$ \\
\hline 3956.14 & 0.0028 & 0.0588 & 11 & 5 & 7 & 10 & 5 & 6 & 3383.36 & 8.747 & U $3 * * *$ \\
\hline 3956.52 & 0.0022 & 0.0643 & 8 & 5 & 3 & 7 & 4 & 4 & 927 & 3.953 & NU1 $\ddot{*} *$ \\
\hline 3956.59 & 0.1401 & 0.0864 & 9 & 3 & 6 & 8 & 3 & 5 & $1050 \cdot 15$ & 8.066 & NU $3 *$ \\
\hline 3956.85 & 98.2710 & 0.0760 & 9 & 2 & 7 & 8 & 2 & 6 & 982.91 & 8.113 & \\
\hline 7.28 & 0.0086 & 0.0671 & 10 & 5 & 5 & 9 & 5 & 4 & 31 & 7.545 & $3 * *$ \\
\hline 7.43 & 0.0962 & 0.0327 & 13 & 1 & 12 & 13 & 1 & 13 & 78 & 0.486 & \\
\hline 7.45 & 0.2887 & 0.0327 & 13 & 2 & 12 & 13 & 0 & 13 & $1806 \cdot 78$ & 0.486 & \\
\hline 3957.85 & 0.0225 & 0.0804 & 6 & 3 & 4 & 5 & 0 & 5 & 325.35 & 0.123125 & $1 *$ \\
\hline 3958.04 & 0.1054 & 0.0928 & 5 & 2 & 3 & 4 & 0 & 4 & 222.06 & 0.096503 & $3 *$ \\
\hline $3958 \cdot 12$ & 9.5916 & 0.0483 & 7 & 6 & 2 & 6 & 5 & 1 & 888.63 & 5.265 & NL \\
\hline $3958 \cdot 15$ & .7734 & 0.0480 & 7 & 6 & 1 & 6 & 5 & 2 & 888.60 & 5.265222 & \\
\hline 8.21 & .0275 & 0.0706 & 8 & 5 & 4 & 7 & 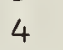 & 3 & $931 \cdot 22$ & & $1+$ \\
\hline $8 \cdot 30$ & .0471 & 0.0337 & 9 & 9 & 0 & 8 & 8 & 1 & $1789 \cdot 10$ & 8.403719 & \\
\hline 8.66 & 6727 & 0.0630 & 8 & 6 & 2 & 7 & 3 & 5 & & 15.00 & $2+$ \\
\hline 8.90 & 0068 & 0.0725 & 10 & 6 & 4 & 10 & 3 & 7 & & 0.095771 & \\
\hline 8.95 & 9790 & 425 & 11 & 7 & 5 & 10 & 7 & 4 & 40 & $6.58 \varepsilon$ & \\
\hline 9.05 & 3264 & 0.0446 & 11 & 7 & 4 & 10 & 7 & 3 & 2054.40 & 6.588161 & N \\
\hline & .0054 & 0.0692 & 8 & 4 & 4 & 8 & 2 & 7 & & 0.045 & J $3 *$ \\
\hline 9.27 & 0.1106 & 0725 & 13 & 5 & 8 & 12 & 5 & 7 & 0.80 & 11.22 & \\
\hline 9.65 & 33.4287 & 594 & 10 & 3 & 8 & 9 & 3 & 7 & 1216.27 & 8.878817 & \\
\hline 9.91 & 2.8344 & 0.0666 & 5 & 5 & 1 & 5 & 3 & 2 & 3.81 & 283 & NU \\
\hline 9.93 & 7.8365 & 0.0604 & 10 & 5 & 6 & 9 & 5 & 5 & 1474.99 & 7.532 & \\
\hline 0.81 & 7.8490 & 0.0789 & 6 & 3 & 3 & 6 & 1 & 6 & $447 \cdot 24$ & 0.014 & NU3 \\
\hline 0.88 & 0.0407 & 0.0666 & 10 & 2 & 8 & 9 & 2 & 7 & 1201.95 & 9.000870 & NU $3 \ddot{x} \ddot{x}$ \\
\hline 396 & .9272 & 0.0643 & 8 & 5 & 3 & 7 & 4 & 4 & 927.77 & 3.953657 & NU 1 \\
\hline 396 & 9596 & 0.0850 & 4 & 4 & 1 & 3 & 1 & 2 & 173.36 & 0.097 & NUI+ \\
\hline 396 & 9862 & 0.0397 & 11 & 2 & 10 & 10 & 2 & 9 & 1293.66 & 10.281117 & \\
\hline 396 & 4178 & 0.0335 & 12 & 1 & 12 & $1]$ & 1 & 11 & 1327.14 & 11.742858 & NU 3 \\
\hline & 76.2535 & 0.0335 & 12 & 0 & 12 & 11 & 0 & 11 & 1327.14 & 11.742873 & NU3 \\
\hline 3962.21 & 26.5075 & 0.0402 & 11 & 1 & 10 & 10 & 1 & 9 & 1293.04 & 10.283342 & $\mathrm{NU}_{3}$ \\
\hline
\end{tabular}




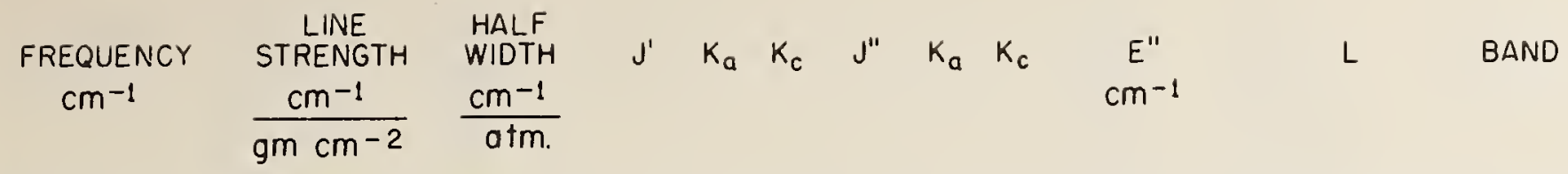

\begin{tabular}{|c|c|c|c|c|c|c|c|c|c|c|c|}
\hline 3962.70 & 0.0042 & 0.0804 & 6 & 3 & 4 & 5 & 0 & 5 & 325.35 & 0.123125 & NU $1 * *$ \\
\hline 963.35 & 0.0467 & 0.0775 & 10 & 5 & 6 & 9 & 4 & 5 & $1360 \cdot 28$ & $2 \cdot 80$ & NU $1 *$ \\
\hline 3.49 & 0.0032 & 0.0483 & 7 & 6 & 2 & 6 & 5 & 1 & $2552 \cdot 87$ & 5.265494 & NU $1 * * *$ \\
\hline 3.51 & 0.0095 & 0.0480 & 7 & 6 & 1 & 6 & 5 & 2 & 2552.85 & 5.265222 & $\mathrm{NU} 1 * \ddot{*} *$ \\
\hline 3.69 & 0.0321 & 0.0378 & 7 & 7 & 0 & 6 & 6 & 1 & 1045.07 & 6.401516 & NU I * \\
\hline 3.78 & 16.9862 & 0.0648 & 10 & 4 & 7 & 9 & 4 & 6 & 1340.89 & 8.354292 & NU3 3 \\
\hline 4.08 & 0.0020 & 0.0643 & 8 & 5 & 3 & 7 & 4 & 4 & $2569 \cdot 52$ & 3.953657 & $N \cup 1 * * *$ \\
\hline 4.26 & 0.0588 & 0.0667 & 10 & 6 & 5 & 10 & 4 & 6 & 1616.51 & 0.114652 & NU3 \\
\hline 3964.37 & 0.0259 & 0.0864 & 9 & 3 & 6 & 8 & 3 & 5 & 1050.15 & 8.066869 & $\mathrm{NU} 3 * *$ \\
\hline 3964.61 & 4.1382 & 0.0665 & 6 & 5 & 1 & 6 & 3 & 4 & 648.97 & 0.021403 & NU3 \\
\hline 3964.72 & 0.9442 & 0.0648 & 5 & 5 & 0 & 5 & 3 & 3 & 504.00 & 0.007097 & NU3 \\
\hline 3964.81 & 23.2044 & 0.0671 & 10 & 5 & 5 & 9 & 5 & 4 & 1477.31 & 7.545052 & NU3 \\
\hline 3965.09 & 0.0427 & 0.0886 & 4 & 3 & 2 & 3 & 1 & 3 & $142 \cdot 28$ & 0.026225 & NU $3 *$ \\
\hline 3965.58 & 0.0222 & 0.0698 & 5 & 5 & 1 & 5 & 2 & 4 & $416 \cdot 22$ & 0.001149 & NU1 \\
\hline 965.66 & 0.0189 & 0.0750 & 9 & 5 & 5 & 8 & 4 & 4 & 1131.76 & 7.37 & NU I* \\
\hline 3965.86 & 0.0174 & 0.0928 & 5 & 2 & 3 & 4 & 0 & 4 & $1817 \cdot 50$ & 0.096503 & NU $3 * * *$ \\
\hline 3965.97 & 0.0195 & 0.0928 & 5 & 2 & 3 & 4 & 0 & 4 & 222.06 & 0.096503 & NU $3 * *$ \\
\hline 3966.19 & 1.1648 & 0.0675 & 7 & 5 & 2 & 7 & 3 & 5 & 816.72 & 0.041795 & NU3 \\
\hline & 11.2730 & 0.0804 & 6 & 3 & 4 & 5 & 0 & 5 & 325.35 & 0.123125 & NU 1 \\
\hline 3968.88 & 0.0240 & 0.0528 & 8 & 6 & 3 & 7 & 5 & 2 & 1059.85 & 5.144364 & NU $1 *$ \\
\hline 3969.14 & 109.9538 & 0.0666 & 10 & 2 & 8 & 9 & 2 & 7 & 1201.95 & 9.000870 & NU3 \\
\hline 3970.00 & 0.0365 & 0.0859 & 5 & 4 & 2 & 4 & 1 & 3 & 275.52 & 0.465 & NU $1 *$ \\
\hline 3970.70 & 3.0892 & 0.0515 & 11 & 6 & 6 & 10 & 6 & 5 & 1875.00 & 7.773013 & NU3 \\
\hline 3970.92 & 2.0857 & 0.0669 & 8 & 5 & 3 & 8 & 3 & 6 & $1006 \cdot 12$ & 0.064192 & NU3 \\
\hline 3971.09 & 0.0035 & 0.0750 & 9 & 5 & 5 & 8 & 4 & 4 & 1131.76 & $7 \cdot 37$ & $N \cup 1 * *$ \\
\hline 3971.36 & 0.0182 & 0.0858 & 7 & 2 & 5 & 6 & 1 & 6 & $447 \cdot 24$ & 0.182385 & NU $1 *$ \\
\hline 3971.49 & 0.0242 & 0.0320 & 14 & 2 & 13 & 14 & 0 & 14 & 2073.66 & 0.488990 & NU3 \\
\hline 1.49 & 0.0729 & 0.0320 & 14 & 1 & 13 & 14 & 1 & 14 & 2073.66 & 0.489010 & NU3 \\
\hline 3972.15 & 70.0220 & 0.0864 & 9 & 3 & 6 & 8 & 3 & 5 & $1050 \cdot 15$ & 8.066869 & NU3 \\
\hline 3972.22 & 1.0302 & 0.0536 & 11 & 6 & 5 & 10 & 6 & 4 & 1875.53 & 7.774282 & NU3 \\
\hline 3972.66 & 37.8942 & 0.0898 & 5 & 3 & 2 & 4 & 1 & 3 & 275.52 & 0.089700 & NU3 \\
\hline $3973 \cdot 44$ & 0.0304 & 0.0779 & 9 & 6 & 3 & 9 & 3 & 6 & $1282 \cdot 92$ & 0.039684 & NU1 \\
\hline 3973.78 & 0.1300 & 0.0649 & 6 & 5 & 2 & 6 & 2 & 5 & 552.92 & 0.004423 & NU1 \\
\hline 3973.90 & 52.6906 & 0.0928 & 5 & 2 & 3 & 4 & 0 & 4 & 222.06 & 0.096503 & NU3 \\
\hline 3974.70 & 23.3262 & 0.0775 & 10 & 5 & 6 & 9 & 4 & 5 & $1360 \cdot 28$ & $42 \cdot 8$ & NU1t \\
\hline 3974.76 & 0.1479 & 0.0817 & 10 & 3 & 7 & 9 & 3 & 6 & 1282.92 & 9.029655 & NU3* \\
\hline 3974.89 & 5.3573 & 0.0378 & 7 & 7 & 1 & 6 & 6 & 0 & 1045.07 & 6.401518 & NU1 \\
\hline 3974.89 & 16.0718 & 0.0378 & 7 & 7 & 0 & 6 & 6 & 1 & 1045.07 & 6.401516 & NU1 \\
\hline 3975.11 & 35.3837 & 0.0515 & 11 & 3 & 9 & 10 & 3 & 8 & $1446 \cdot 16$ & 9.881611 & $\mathrm{NU}_{3}$ \\
\hline 3975.73 & 0.0126 & 0.0898 & 5 & 3 & 2 & 4 & 1 & 3 & 1875.49 & 0.089700 & NU $3 * * *$ \\
\hline 3975.95 & 9.1607 & 0.0360 & 12 & 2 & 11 & 11 & 2 & 10 & $1525 \cdot 13$ & 11.275004 & NU 3 \\
\hline 3976.08 & 0.0239 & 0.0654 & 9 & 5 & 4 & 8 & 4 & 5 & 1122.72 & 6.99 & NU $1 *$ \\
\hline 3976.16 & 27.4855 & 0.0361 & 12 & 1 & 11 & 11 & 1 & 10 & 1524.87 & 11.275820 & NU3 \\
\hline 3976.20 & 2.6796 & 0.0692 & 8 & 4 & 4 & 8 & 2 & 7 & 885.62 & 0.045112 & NU3 3 \\
\hline 3976.33 & 8.4533 & 0.0325 & 13 & 0 & 13 & 12 & 0 & 12 & 1557.91 & 12.744460 & NU3 3 \\
\hline $3976 \cdot 33$ & 25.3539 & 0.0325 & 13 & 1 & 13 & 12 & 1 & 12. & 1557.91 & 12.744430 & NU3 \\
\hline 3976.53 & 9.4335 & 0.0750 & 9 & 5 & 5 & 8 & 4 & 4 & $1131 \cdot 76$ & 17.37 & $\mathrm{NU} 1+$ \\
\hline 3976.97 & 0.0037 & 0.0804 & 6 & 3 & 4 & 5 & 0 & 5 & 1920.76 & 0.123125 & $N \cup 1 * * * *$ \\
\hline 3977.47 & 0.0590 & 0.0630 & 7 & 4 & 4 & 7 & 1 & 7 & $586 \cdot 48$ & 0.007119 & NU 1 \\
\hline 3978.56 & 0.0031 & 0.0750 & 9 & 5 & 5 & 8 & 4 & 4 & $2771 \cdot 71$ & $17 \cdot 37$ & NU1*** \\
\hline 3978.84 & 9.1041 & 0.0858 & 7 & 2 & 5 & 6 & 1 & 6 & $447 \cdot 24$ & 0.182385 & NU 1 \\
\hline 3975 & 0.3697 & 0.0665 & 9 & 6 & 4 & 9 & 4 & 5 & $1360 \cdot 28$ & 0.066893 & NU3 3 \\
\hline 3979.66 & 0.1322 & 0.0429 & 12 & 7 & 6 & 11 & 7 & 5 & 2321.89 & 7.967474 & NU3 \\
\hline 3979.67 & 12.0178 & 0.0528 & 8 & 6 & 3 & 7 & 5 & 2 & 1059.85 & 5.144364 & NUI \\
\hline
\end{tabular}




\begin{tabular}{|c|c|c|c|c|c|c|c|c|c|c|c|}
\hline $\begin{array}{c}\text { FREQUENCY } \\
\mathrm{cm}^{-1}\end{array}$ & $\begin{array}{l}\text { LINE } \\
\text { STRENGTH } \\
\frac{\mathrm{cm}^{-1}}{\mathrm{gm} \mathrm{cm}^{-2}}\end{array}$ & $\begin{array}{l}\text { HALF } \\
\text { WIDTH } \\
\frac{\mathrm{cm}^{-1}}{a+m .}\end{array}$ & $J^{\prime}$ & $K_{a}$ & $\mathrm{~K}_{\mathrm{c}}$ & Ј" & $k_{a}$ & $K_{c}$ & $E^{\mathrm{cm}^{-1}}$ & $L$ & BAND \\
\hline 3979.68 & 3.0846 & 0.0852 & 8 & 3 & 5 & 7 & 2 & 6 & 709.60 & 0.688192 & NU1 \\
\hline 3979.74 & 8.2754 & 0.0588 & 11 & 5 & 7 & 10 & 5 & 6 & 1718.77 & 8.747542 & NU3 \\
\hline 3979.91 & 0.3967 & 0.0453 & 12 & 7 & 5 & 11 & 7 & 4 & 2321.94 & 7.967606 & NU3 \\
\hline 3979.92 & 4.0049 & 0.0502 & 8 & 6 & 2 & 7 & 5 & 3 & 1059.65 & 5.142711 & NU1 \\
\hline 3980.34 & 0.3065 & 0.0645 & 9 & 5 & 4 & 9 & 3 & 7 & $1216 \cdot 27$ & 0.080993 & NU3 \\
\hline 3980.43 & 0.0112 & 0.0838 & 4 & 4 & 0 & 3 & 1 & 3 & $142 \cdot 28$ & 0.0731 & NU1* \\
\hline 3980.82 & 12.3671 & 0.0563 & 11 & 2 & 9 & 10 & 2 & 8 & 1438.00 & 9.932549 & NU3 \\
\hline 3981.06 & 0.0044 & 0.0654 & 9 & 5 & 4 & 8 & 4 & 5 & 1122.72 & 6.99 & NU I** \\
\hline $3981 \cdot 36$ & 0.0018 & 0.0378 & 7 & 7 & 1 & 6 & 6 & 0 & 2734.00 & 6.401518 & NU1**** \\
\hline $3981 \cdot 36$ & 0.0053 & 0.0378 & 7 & 7 & 0 & 6 & 6 & 1 & 2734.00 & 6.401516 & NU1**** \\
\hline $3982 \cdot 14$ & 17.3995 & 0.0603 & 11 & 4 & 8 & 10 & 4 & 7 & $1581 \cdot 34$ & 9.438945 & NU3 \\
\hline 3982.21 & $18 \cdot 2742$ & 0.0859 & 5 & 4 & 2 & 4 & 1 & 3 & 275.52 & 0.465 & NU1 + \\
\hline 3982.73 & 0.0273 & 0.0817 & 10 & 3 & 7 & 9 & 3 & 6 & 1282.92 & 9.029655 & NU $3 * *$ \\
\hline 3982.89 & 21.3901 & 0.0886 & 4 & 3 & 2 & 3 & 1 & 3 & $142 \cdot 28$ & 0.026225 & NU3 \\
\hline 3983.09 & 0.0682 & 0.0846 & 6 & 4 & 3 & 5 & 1 & 4 & 399.46 & 0.536 & NU $1 *$ \\
\hline 3983.35 & 0.0018 & 0.0798 & 9 & 4 & 5 & 8 & 3 & 6 & 2630.21 & 1.797448 & NU $1 * * * *$ \\
\hline 3983.47 & 0.0709 & 0.0679 & 13 & 6 & 8 & 12 & 5 & 7 & $2300 \cdot 80$ & 4.44317 & NU1 \\
\hline 3984.11 & 0.0063 & 0.0850 & 4 & 4 & 1 & 3 & 1 & 2 & 1772.41 & 0.097 & NU $1 * * * *$ \\
\hline 3984.46 & 0.0055 & 0.0590 & 9 & 6 & 3 & 8 & 3 & 6 & $1006 \cdot 12$ & 0.019449 & 2 NU2 \\
\hline 3984.58 & 0.0195 & 0.0451 & 13 & 3 & 10 & 13 & 3 & 11 & $2248 \cdot 16$ & 1.178730 & NU3 \\
\hline 3984.64 & 0.0260 & 0.0785 & 6 & 6 & 1 & 5 & 1 & 4 & 399.46 & 2.857 & $2 N \cup 2 *$ \\
\hline 3984.70 & 0.0016 & 0.0739 & 7 & 3 & 4 & 7 & 1 & 7 & 586.48 & 0.009220 & NU3* \\
\hline 3985.02 & 1.2442 & 0.0778 & 11 & 5 & 7 & 10 & 4 & 7 & 1616.51 & 25.73 & NU1 + \\
\hline $3985 \cdot 16$ & 0.0040 & 0.0528 & 8 & 6 & 3 & 7 & 5 & 2 & 2724.19 & 5.144364 & NUI $* * * *$ \\
\hline 3985.33 & 0.0013 & 0.0502 & 8 & 6 & 2 & 7 & 5 & 3 & 2724.05 & 5.142711 & NU $1 * * *$ \\
\hline 3985.37 & 0.0082 & 0.0320 & 15 & 1 & 14 & 15 & 1 & 15 & $2358 \cdot 44$ & 0.491510 & NU3 \\
\hline 3985.37 & 0.0244 & 0.0320 & 15 & 2 & 14 & 15 & 0 & 15 & $2358 \cdot 44$ & 0.491500 & NU3 \\
\hline 3985.62 & 0.0045 & 0.0410 & 8 & 7 & 1 & 7 & 6 & 2 & $1216 \cdot 20$ & 6.263414 & NU ${ }^{*}$ \\
\hline 3985.62 & 0.0135 & 0.0410 & 8 & 7 & 2 & 7 & 6 & 1 & $1216 \cdot 20$ & 6.263444 & NUI* \\
\hline 3986.05 & 11.9396 & 0.0654 & 9 & 5 & 4 & 8 & 4 & 5 & 1122.72 & 6.99 & NUI+ \\
\hline 3986.18 & 0.0436 & 0.0600 & 7 & 5 & 3 & 7 & 2 & 6 & $709 \cdot 60$ & 0.009705 & NU1 \\
\hline 3986.20 & 0.0020 & 0.0798 & 9 & 4 & 5 & 8 & 3 & 6 & $1006 \cdot 12$ & 1.797448 & NUI $\ddot{*} *$ \\
\hline 3986.47 & 0.0021 & 0.0838 & 4 & 4 & 0 & 3 & 1 & 3 & $142 \cdot 28$ & 0.0731 & NU I** \\
\hline 3986.78 & 0.0070 & 0.0886 & 4 & 3 & 2 & 3 & 1 & 3 & 1739.51 & 0.026225 & NU $3 \ddot{*} \ddot{x} *$ \\
\hline 3986.84 & 0.0039 & 0.0654 & 9 & 5 & 4 & 8 & 4 & 5 & 2764.71 & 6.99 & NUI**** \\
\hline 3988.67 & 0.1418 & 0.0754 & 12 & 5 & 8 & 11 & 4 & 7 & 1899.06 & 3.784189 & NU1 \\
\hline 3989.06 & 0.0126 & 0.0846 & 6 & 4 & 3 & 5 & 1 & 4 & 399.46 & 0.536 & NUI \\
\hline 3989.42 & 0.0116 & 0.0803 & 8 & 6 & 2 & 8 & 3 & 5 & 1050.15 & 0.014126 & NU1 \\
\hline 3989.49 & 24.5023 & 0.0346 & 13 & 2 & 12 & 12 & 2 & 11 & $1774 \cdot 74$ & 12.275740 & Nu3 \\
\hline 3989.60 & 3.7992 & 0.0447 & 12 & 3 & 10 & 11 & 3 & 9 & 1695.03 & 10.873594 & NU3 \\
\hline 3989.61 & 8.1702 & 0.0338 & 13 & 1 & 12 & 12 & 1 & 11 & $1774 \cdot 60$ & 12.276740 & NU3 \\
\hline 3989.85 & 2.6353 & 0.0700 & 11 & 5 & 6 & 10 & 5 & 5 & $1724 \cdot 72$ & 8.798801 & NU3 \\
\hline 3990.23 & 5.5069 & 0.0798 & 9 & 4 & 5 & 8 & 3 & 6 & $1006 \cdot 12$ & 1.797448 & NU1 \\
\hline 3990.30 & 2.7420 & 0.0320 & 14 & 1 & 14 & 13 & 1 & 13 & $1806 \cdot 78$ & 13.743930 & NU3 \\
\hline 3990.30 & 8.2322 & 0.0320 & 14 & 0 & 14 & 13 & 0 & 13 & $1806 \cdot 78$ & 13.743940 & NU3 \\
\hline 3990.43 & 0.0088 & 0.0536 & 9 & 6 & 3 & 8 & 5 & 4 & 1255.16 & 5.006530 & NU1* \\
\hline 3990.61 & 0.2154 & 0.0646 & 8 & 6 & 3 & 8 & 4 & 4 & 1131.76 & 0.037084 & NU3 \\
\hline 3990.70 & 73.9364 & 0.0817 & 10 & 3 & 7 & 9 & 3 & 6 & 1282.92 & 9.029655 & NU3 \\
\hline $3991 \cdot 12$ & 0.3012 & 0.0516 & 12 & 6 & 7 & 11 & 6 & 6 & 2142.69 & 9.048040 & NU3 \\
\hline $3991 \cdot 23$ & 0.0025 & 0.0410 & 8 & 7 & 2 & 7 & 6 & 1 & $1216 \cdot 20$ & 6.263444 & NU $1 * * *$ \\
\hline 3991.41 & 0.2760 & 0.0889 & 6 & 3 & 3 & 5 & 1 & 4 & 399.46 & 0.202769 & NU3* \\
\hline 3992.30 & 0.0077 & 0.0775 & 10 & 5 & 6 & 9 & 4 & 5 & $2998 \cdot 78$ & $42 \cdot 80$ & NUI $* * * *$ \\
\hline 3992.52 & 5.6070 & 0.0838 & 4 & 4 & 0 & 3 & 1 & 3 & $142 \cdot 28$ & 0.0731 & NU1+ \\
\hline 3992.57 & 11.4273 & 0.0475 & 12 & 2 & 10 & 11 & 2 & 9 & 1690.70 & 10.893773 & NU3 \\
\hline
\end{tabular}



$\begin{array}{ccc}\begin{array}{c}\text { FREQUENCY } \\ \mathrm{cm}^{-1}\end{array} & \begin{array}{c}\text { LINE } \\ \text { STRENGTH } \\ \mathrm{cm}^{-1}\end{array} & \begin{array}{c}\text { HALF } \\ \text { WIDTH }\end{array} \\ & \frac{\mathrm{cm}^{-1}}{\mathrm{gm} \mathrm{cm}-2} & \end{array}$
$J^{\prime} \quad K_{a} \quad K_{c} \quad J^{\prime \prime} \quad K_{a} \quad K_{c}$
$\mathrm{cm}^{\prime \prime}$
L
BAND

3993.32

3994.93

3995.03

3995.03

3995.96

3996.14

3996.35

3996.84

3996.84

3996.92

3997.83

3998.02

3998.81

3998.89

3999.31

3999.72

3999.82

4000.00

4000.20

4000.32

4000.39

4000.48

4000.75

4001.20

4001.28

4001.40

4001.62

4001.69

4001.82

4001.84

4002.06

4002.47

4002.47

4002.70

4002.71

4002.77

4002.82

4002.86

4002.86

4002.86

4002.98

4003.09

4003.81

4004.37

4004.69

4004.73

4005.43

4006.63

4006.97

4007.00

4007.37

4007.64

4008.59

4009.56
0.0575

0.9051

34.1141

23.8564

0.3032

0.0048

0.0138

2.2435

6.7304

0.8258

0.8406

0.0538

1.7479

0.1225

0.0185

0.0112

0.6517

0.0510

0.1610

1.4678

0.8194

0.0018

0.0021

4.4214

0.2822

0.0455

0.0013

0.0044

0.0060

0.0824

0.2160

0.0022

0.0066

0.1506

0.0022

0.8874

2.6561

0.7343

2.2163

2.8563

0.0106

0.6496

0.0868

0.0294

1.1902

0.0187

7.6069

0.2524

0.0017

0.0050

0.0015

12.9864

137.9963

0.0031
0.0757

0.0552

0.0846

0.0824

0.0620

0.0785

0.0700

0.0410

0.0410

0.0564

0.0610

0.0770

0.0548

0.0590

0.0757

0.0726

0.0592

0.0889

D. 0578

0.0558

0.0739

0.0751

0.0587

0.0536

0.0578

0.0889

0.0661

0.0709

0.0859

0.0866

0.0568

0.0351

0.0351

0.0770

0.0658

0.0330

0.0330

0.0320

0.0320

0.0394

0.0757

0.0552

0.0553

0.0792

0.0411

0.0561

0.0752

0.0658

0.0444

0.0443

0.0536

0.0785

0.0889

0.0788

$\begin{array}{rrrrrr}4 & 4 & 0 & 3 & 2 & 1 \\ 12 & 6 & 6 & 11 & 6 & 5 \\ 6 & 4 & 3 & 5 & 1 & 4 \\ 10 & 4 & 6 & 9 & 4 & 5 \\ 10 & 5 & 5 & 10 & 3 & 8 \\ 6 & 6 & 1 & 5 & 1 & 4 \\ 13 & 5 & 9 & 12 & 4 & 8 \\ 8 & 7 & 1 & 7 & 6 & 2 \\ 8 & 7 & 2 & 7 & 6 & 1 \\ 12 & 5 & 8 & 11 & 5 & 7 \\ 7 & 6 & 2 & 7 & 4 & 3 \\ 9 & 3 & 6 & 9 & 0 & 9\end{array}$

1249

11

$\begin{array}{lll}9 & 4 & 6\end{array}$

$\begin{array}{lll}3 & 2 & 2\end{array}$

$\begin{array}{lll}11 & 1 & 10\end{array}$

$$
\begin{array}{lll}
8 & 4 & 5 \\
5 & 1 & 4
\end{array}
$$$$
\begin{array}{lll}
5 & 1 & 4
\end{array}
$$

10

8

$$
7
$$$$
5
$$

12

8

$$
7
$$$$
5
$$$$
9
$$$$
1055
$$$$
735
$$$$
\begin{array}{lll}
5 & 4 & 2
\end{array}
$$$$
533
$$$$
\begin{array}{lll}
6 & 6 & 1
\end{array}
$$$$
\begin{array}{lll}
8 & 8 & 0
\end{array}
$$$$
\begin{array}{lll}
8 & 8 & 1
\end{array}
$$$$
\begin{array}{lll}
4 & 4 & 1
\end{array}
$$

$\begin{array}{lll}12 & 3 & 9\end{array}$

14213

$\begin{array}{lll}14 & 1 & 13\end{array}$

$\begin{array}{lll}15 & 0 & 15\end{array}$

$\begin{array}{lll}15 & 1 & 15\end{array}$

13311

$$
\begin{array}{rrrrrr}
4 & 4 & 0 & 3 & 2 & 1
\end{array}
$$$$
\begin{array}{llllll}
6 & 6 & 0 & 6 & 4 & 3
\end{array}
$$$$
\begin{array}{llllll}
8 & 5 & 4 & 8 & 2 & 7
\end{array}
$$$$
\begin{array}{llllll}
7 & 6 & 1 & 7 & 3 & 4
\end{array}
$$

$\begin{array}{lll}13 & 2 & 11\end{array}$

$\begin{array}{lll}11 & 6 & 5\end{array}$

$\begin{array}{lll}12 & 2 & 10\end{array}$

$\begin{array}{lll}11 & 4 & 8\end{array}$

$\begin{array}{lll}10 & 3 & 7\end{array}$

$\begin{array}{lll}9 & 2 & 8\end{array}$

$\begin{array}{lll}8 & 6 & 2\end{array}$

$\begin{array}{lll}8 & 6 & 3\end{array}$

$\begin{array}{lll}8 & 5 & 4\end{array}$

$\begin{array}{lll}5 & 1 & 4\end{array}$

$\begin{array}{lll}5 & 1 & 4\end{array}$
$212 \cdot 15$

2144.12

399.46

1360.28

1446.16

399.46

2205.65

1216.20

1216.20

1985.83

931.22

920.18

1843.02

1340.89

$206 \cdot 30$

1524.87

1122.72

399.46

1581.34

1255.92

586.48

325.35

2300.80

1255.16

927.77

2000.90

1340.89

446.71

1875.49

224.83

757.78

1394.86

1394.86

222.06

3441.09

2042.38

2042.33

2073.66

2073.66

1962.55

212.15

756.75

885.62

842.38

1960.22

1843.02

1538.23

1080.38

1411.68

1411.65

2919.63

399.46

399.46

542.91

0.016542

9. 054429

0.536

4. 300000

0.084234

2. 857

4.43448

6.263414

6.263444

9. 908577

0.017690

0.011425

10.481399

0.059724 


\begin{tabular}{|c|c|c|c|c|c|c|c|c|c|c|c|}
\hline $\begin{array}{l}\text { FREQUENCY } \\
\qquad \mathrm{cm}^{-1}\end{array}$ & $\begin{array}{l}\text { LINE } \\
\text { STRENGTH } \\
\frac{\mathrm{cm}^{-1}}{\mathrm{gm} \mathrm{cm}^{-2}}\end{array}$ & $\begin{array}{l}\text { HALF } \\
\text { WIDTH } \\
\frac{\mathrm{cm}^{-1}}{\text { atm. }}\end{array}$ & $J^{\prime}$ & $\mathrm{K}_{\mathrm{a}}$ & $\mathrm{K}_{\mathrm{c}}$ & J" & $\mathrm{K}_{\mathrm{a}}$ & $\mathrm{K}_{c}$ & $\begin{array}{c}E^{\prime \prime} \\
\mathrm{cm}^{-1}\end{array}$ & $L$ & BAND \\
\hline 4009.81 & 0.1384 & 0.0875 & 6 & 2 & 4 & 5 & 0 & 5 & 325.35 & 0.069891 & NU $3 *$ \\
\hline 4010.29 & 0.0295 & 0.0795 & 5 & 4 & 1 & 4 & 2 & 2 & 315.79 & 0.042579 & NU $3 *$ \\
\hline 4010.66 & 0.0152 & 0.0866 & 5 & 3 & 3 & 4 & 1 & 4 & 224.83 & 0.025200 & NU $3 * \ddot{*}$ \\
\hline 4010.78 & 0.5121 & 0.0503 & 13 & 6 & 8 & 12 & 6 & 7 & 2433.85 & 10.260880 & NU3 \\
\hline 4011.05 & 0.6318 & 0.0661 & 10 & 5 & 5 & 9 & 4 & 6 & 1340.89 & 3.272354 & NUI \\
\hline 4011.17 & 2.1902 & 0.0709 & 7 & 3 & 5 & 6 & 0 & 6 & 446.71 & 0.130226 & NU 1 \\
\hline 4012.22 & 0.0819 & 0.0588 & 8 & 4 & 5 & 8 & 1 & 8 & $744 \cdot 16$ & 0.007182 & NUI \\
\hline 4012.64 & 28.7486 & 0.0757 & 4 & 4 & 0 & 3 & 2 & 1 & 212.15 & 0.016542 & NU 3 \\
\hline 4014.00 & 1.0931 & 0.0351 & 8 & 8 & 0 & 7 & $\overline{7}$ & 1 & 1394.86 & 7.402783 & NU1 \\
\hline 4014.00 & 3.2793 & 0.0351 & 8 & 8 & 1 & 7 & 7 & 0 & 1394.86 & 7.402783 & NU1 \\
\hline 4014.00 & 1.1512 & 0.0488 & 13 & 4 & 10 & 12 & 4 & 9 & 2124.98 & 11.473010 & NU3 \\
\hline 4014.07 & 0.0126 & 0.0539 & 12 & 6 & 6 & 12 & 4 & 9 & $2124 \cdot 98$ & 0.124675 & $\mathrm{~N}_{3}$ \\
\hline 4014.33 & 0.0205 & 0.0562 & 11 & 7 & 5 & 11 & 5 & 6 & 1999.02 & 0.081625 & NU3 \\
\hline 4014.39 & 2.5230 & 0.0721 & 12 & 5 & 7 & 11 & 5 & 6 & 1999.02 & 10.047055 & NU3 \\
\hline 4015.29 & 5.0402 & 0.0810 & 11 & 4 & 7 & 10 & 4 & 6 & 1616.51 & 9.709460 & $\mathrm{~N} \cup 3$ \\
\hline 4015.73 & 0.2142 & 0.0360 & 14 & 3 & 12 & 13 & 3 & 11 & $2248 \cdot 16$ & 12.856270 & NU3 \\
\hline 4015.91 & 0.7129 & 0.0330 & 15 & 2 & 14 & 14 & 2 & 13 & 2327.90 & 14.267060 & NU 3 \\
\hline 4015.93 & 0.2377 & 0.0320 & 15 & 1 & 14 & 14 & 1 & 13 & 2327.88 & 14.267240 & NU3 \\
\hline 4016.31 & 0.0048 & 0.0755 & 6 & 6 & 0 & 6 & 3 & 3 & 661.56 & 0.000843 & NUI \\
\hline 4016.66 & 0.2622 & 0.0320 & 16 & 1 & 16 & 15 & 1 & 15 & $2358 \cdot 44$ & 15.743100 & $\mathrm{NU}_{3}$ \\
\hline 4016.66 & 0.7867 & 0.0320 & 16 & 0 & 16 & 15 & 0 & 15 & 2358.44 & 15.743100 & NU3 \\
\hline 4016.69 & 0.6430 & 0.0370 & 14 & 2 & 12 & 13 & 2 & 11 & 2246.98 & 12.866510 & NU3 \\
\hline 4016.90 & 0.5487 & 0.0531 & 13 & 5 & 9 & 12 & 5 & 8 & 2275.44 & 10.979590 & NU3 \\
\hline 4016.93 & 6.4797 & 0.0658 & 12 & 3 & 9 & 11 & 3 & 8 & 1813.26 & 10.744435 & NU3 \\
\hline 4017.60 & 0.0256 & 0.0875 & 6 & 2 & 4 & 5 & 0 & 5 & 325.35 & 0.069891 & NU3* $3 *$ \\
\hline 4018.02 & 0.0011 & 0.0351 & 8 & 8 & 1 & 7 & 7 & 0 & 3109.98 & 7.402783 & NU $1 * * * *$ \\
\hline 4018.04 & 0.1735 & 0.0573 & 13 & 6 & 7 & 12 & 6 & 6 & 2437.56 & 10.408370 & NU 3 \\
\hline 4018.22 & 0.8306 & 0.0444 & 9 & 7 & 3 & 8 & 6 & 2 & 1411.68 & 6.142105 & NU1 \\
\hline 4018.25 & 2.4918 & 0.0443 & 9 & 7 & 2 & 8 & 6 & 3 & 1411.65 & 6.141896 & NUI \\
\hline 4018.46 & 9.2903 & 0.0757 & 4 & 4 & 1 & 3 & 2 & 2 & $206 \cdot 30$ & 0.015552 & NU3 \\
\hline 4018.59 & 0.0228 & 0.0875 & 6 & 2 & 4 & 5 & 0 & 5 & 1920.76 & 0.069891 & NU3* $* *$ \\
\hline 4018.83 & 0.0010 & 0.0543 & 10 & 6 & 4 & 9 & 3 & 7 & 1216.27 & 0.029596 & $2 \mathrm{NU} 2$ \\
\hline 4019.49 & 41.1748 & 0.0866 & 5 & 3 & 3 & 4 & 1 & 4 & 224.83 & 0.025200 & NU3- \\
\hline 4019.54 & 0.0255 & 0.0595 & 11 & 5 & 6 & 11 & 3 & 9 & 1695.03 & 0.072485 & NU3 \\
\hline 4019.68 & 1.4213 & 0.0586 & 10 & 6 & 5 & 9 & 5 & 4 & 1477.31 & 4.857731 & NU 1 \\
\hline 4020.01 & 0.0054 & 0.0795 & 5 & 4 & 1 & 4 & 2 & 2 & 315.79 & 0.042579 & NU $3 * *$ \\
\hline 4020.03 & 0.9109 & 0.0751 & 5 & 4 & 2 & 5 & 0 & 5 & $325 \cdot 35$ & 0.000921 & NU3 \\
\hline 4021.04 & 1.5364 & 0.0788 & 7 & 4 & 4 & 6 & 1 & 5 & 542.91 & 0.147419 & NUI \\
\hline 4021.48 & 0.0014 & 0.0594 & 5 & 5 & 1 & 6 & 1 & 6 & $447 \cdot 24$ & 0.000003 & NU3 \\
\hline 4021.56 & 0.0113 & 0.0846 & 6 & 4 & 3 & 5 & 1 & 4 & $2000 \cdot 90$ & 0.536 & NU $1 * * *$ \\
\hline 4022.14 & 0.4791 & 0.0540 & 10 & 6 & 4 & 9 & 5 & 5 & 1474.99 & 4.830967 & NU1 \\
\hline 4022.65 & 0.0094 & 0.0757 & 4 & 4 & 0 & 3 & 2 & 1 & $1819 \cdot 34$ & 0.016542 & NU $3 * * *$ \\
\hline 4022.94 & 0.0024 & 0.0371 & 9 & 8 & 1 & 8 & 7 & 2 & 1590.74 & 7.261215 & NU1* \\
\hline 4023.97 & 0.0156 & 0.0563 & 10 & 7 & 4 & 10 & 5 & 5 & 1724.72 & 0.051622 & NU3 \\
\hline 4024.90 & 0.0136 & 0.0866 & 5 & 3 & 3 & 4 & 1 & 4 & 1821.61 & 0.025200 & NU $3 * * * *$ \\
\hline 4025.39 & 69.1862 & 0.0875 & 6 & 2 & 4 & 5 & 0 & 5 & 325.35 & 0.069891 & NU3 \\
\hline 4025.39 & 0.0940 & 0.0824 & 6 & 4 & 2 & 5 & 2 & 3 & 446.50 & 0.086601 & NU3* \\
\hline 4025.96 & 0.0788 & 0.0779 & 5 & 4 & 2 & 4 & 2 & 3 & $300 \cdot 35$ & 0.034972 & NU $3 *$ \\
\hline 4026.41 & 0.0054 & 0.0502 & 12 & 7 & 5 & 12 & 5 & 8 & 2275.44 & 0.108157 & NU3 \\
\hline 4026.84 & 0.7200 & 0.0566 & 13 & 3 & 10 & 12 & 3 & 9 & 2105.90 & 11.684530 & NU3 \\
\hline 4027.53 & 0.0479 & 0.0869 & 7 & 3 & 4 & 6 & 1 & 5 & 542.91 & 0.214427 & NU $3 *$ \\
\hline 4027.62 & 0.0065 & 0.0515 & 11 & 7 & 4 & 11 & 5 & 7 & 1985.83 & 0.077402 & NU3 \\
\hline 4027.91 & 0.0140 & 0.0522 & 9 & 5 & 5 & 9 & 2 & 8 & $1080 \cdot 38$ & 0.019752 & NUI \\
\hline 4027.95 & 0.0017 & 0.0465 & 10 & 7 & 4 & 9 & 6 & 3 & 1631.41 & 6.019433 & NU ${ }^{*} *$ \\
\hline
\end{tabular}




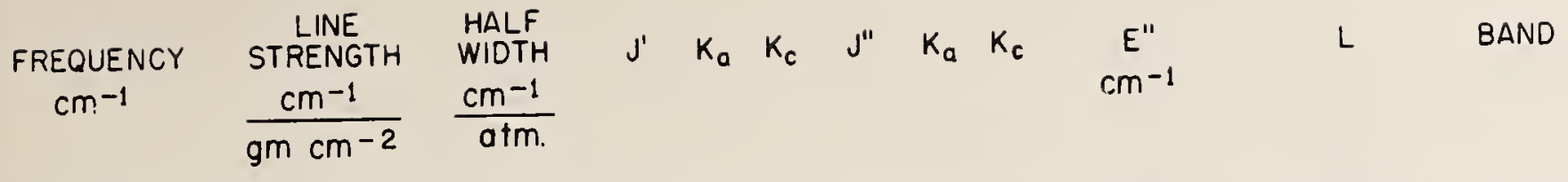

\begin{tabular}{|c|c|c|c|c|c|c|c|c|c|c|c|}
\hline $\begin{array}{l}4028.08 \\
4028 \cdot 16\end{array}$ & $\begin{array}{l}0.2084 \\
0.0031\end{array}$ & $\begin{array}{l}0.0430 \\
0.0757\end{array}$ & $\begin{array}{r}14 \\
4\end{array}$ & $\begin{array}{l}4 \\
4\end{array}$ & $\begin{array}{r}11 \\
1\end{array}$ & $\begin{array}{r}13 \\
3\end{array}$ & $\begin{array}{l}4 \\
2\end{array}$ & $\begin{array}{r}10 \\
2\end{array}$ & $\begin{array}{l}2426.22 \\
1813.81\end{array}$ & $\begin{array}{r}12.476350 \\
0.015552\end{array}$ & $\begin{array}{l}\text { NU } 3 \\
\text { NU } 3 * * *\end{array}$ \\
\hline 4028.81 & 0.0024 & 0.0764 & 8 & 2 & 6 & 7 & 1 & 7 & 586.48 & 0.143980 & NU $1 *$ \\
\hline 4028.90 & 0.0142 & 0.0645 & 6 & 6 & 1 & 6 & 3 & 4 & 648.97 & 0.000772 & NUI \\
\hline 4029.14 & 0.0010 & 0.0797 & 9 & 3 & 6 & 8 & 2 & 7 & 885.62 & 0.493418 & NU $1 * *$ \\
\hline 4029.38 & 0.0015 & 0.0523 & 13 & 6 & 7 & 13 & 4 & 10 & 2426.22 & 0.089990 & NU3 \\
\hline 4029.74 & 14.7533 & 0.0795 & 5 & 4 & 1 & 4 & 2 & 2 & 315.79 & 0.042579 & $\mathrm{NU}_{3}$ \\
\hline 4029.92 & 0.0486 & 0.0519 & 10 & 7 & 3 & 10 & 5 & 6 & 1718.77 & 0.050729 & NU3 \\
\hline 4030.03 & 0.0089 & 0.0619 & 7 & 6 & 2 & 7 & 3 & 5 & 816.72 & 0.003337 & NU1 \\
\hline 4030.24 & 0.0933 & 0.0538 & 9 & 7 & 3 & 9 & 5 & 4 & 1477.31 & 0.029861 & NU3 \\
\hline 4031.39 & 14.8720 & 0.0812 & 5 & 4 & 1 & 4 & 1 & 4 & 224.83 & 0.0967 & NUIt \\
\hline 4032.56 & 0.0314 & 0.0516 & 9 & 7 & 2 & 9 & 5 & 5 & 1474.99 & 0.029706 & NU3 \\
\hline 4032.58 & 2.7892 & 0.0797 & 9 & 3 & 6 & 8 & 2 & 7 & 885.62 & 0.493418 & NUI \\
\hline 4033.17 & 0.0013 & 0.0681 & 8 & 3 & 5 & 8 & 1 & 8 & $744 \cdot 16$ & 0.005295 & NU $3 *$ \\
\hline 4033.40 & 0.0267 & 0.0583 & 8 & 6 & 3 & 8 & 3 & 6 & $1006 \cdot 12$ & 0.008606 & NU1 \\
\hline 4033.55 & 0.0079 & 0.0869 & 7 & 3 & 4 & 6 & 1 & 5 & $2146 \cdot 28$ & 0.214427 & NU $3 * * * *$ \\
\hline 4033.68 & 0.4733 & 0.0786 & 10 & 4 & 6 & 9 & 3 & 7 & 1216.27 & 1.314980 & NUI \\
\hline 4034.08 & 0.0456 & 0.0509 & 8 & 7 & 2 & 8 & 5 & 3 & 1255.92 & 0.014513 & NU3 \\
\hline 4034.50 & 1.2038 & 0.0371 & 9 & 8 & 1 & 8 & 7 & 2 & 1590.74 & 7.261215 & NUI \\
\hline 4034.50 & $4 \cdot 3475$ & 0.0772 & 12 & 4 & 8 & 11 & 4 & 7 & 1899.06 & 10.766337 & NU3 \\
\hline 4034.84 & 0.1375 & 0.0498 & 8 & 7 & 1 & 8 & 5 & 4 & $1255 \cdot 16$ & 0.014493 & NU3 \\
\hline 4035.10 & 0.4013 & 0.0371 & 9 & 8 & 2 & 8 & 7 & 1 & 1590.74 & 7.261219 & NU1 \\
\hline 4035.12 & 0.0174 & 0.0824 & 6 & 4 & 2 & 5 & 2 & 3 & $446 \cdot 50$ & 0.086601 & NU3** \\
\hline 4035.44 & 0.0015 & 0.0759 & 11 & 7 & 4 & 11 & 4 & 7 & 1899.06 & 0.039893 & NU1 \\
\hline 4035.49 & 0.0146 & 0.0779 & 5 & 4 & 2 & 4 & 2 & 3 & 300.35 & 0.034972 & NU $3 * *$ \\
\hline 4035.74 & 0.0089 & 0.0869 & 7 & 3 & 4 & 6 & 1 & 5 & 542.91 & 0.214427 & NU3** \\
\hline 4036.31 & 0.1196 & 0.0479 & 7 & 7 & 1 & 7 & 5 & 2 & 1059.85 & 0.004736 & NU3 \\
\hline 4036.43 & 1.2115 & 0.0764 & 8 & 2 & 6 & 7 & 1 & 7 & 586.48 & 0.143980 & NUI \\
\hline 4036.51 & 0.0399 & 0.0476 & 7 & 7 & 0 & 7 & 5 & 3 & 1059.65 & 0.004734 & NU3 \\
\hline 4036.68 & 0.1327 & 0.0618 & 11 & 6 & 6 & 10 & 5 & 5 & 1724.72 & 4.667626 & NUI \\
\hline 4036.96 & 0.0010 & 0.0337 & 9 & 9 & 0 & 8 & 8 & 1 & $1789 \cdot 10$ & 8.403719 & NUI* \\
\hline 4038.51 & 0.0020 & 0.0533 & 7 & 5 & 3 & 8 & 1 & 8 & $744 \cdot 16$ & 0.000016 & NU3 \\
\hline 4039.26 & 0.8278 & 0.0465 & 10 & 7 & 4 & 9 & 6 & 3 & 1631.41 & 6.019433 & NUI \\
\hline 4039.40 & 0.2759 & 0.0460 & 10 & 7 & 3 & 9 & 6 & 4 & 1631.27 & 6.018378 & NUI \\
\hline 4039.97 & 0.0060 & 0.0540 & 9 & 6 & 4 & 9 & 3 & 7 & 1216.27 & 0.016664 & NUI \\
\hline 4040.05 & 0.4780 & 0.0676 & 11 & 5 & 6 & 10 & 4 & 7 & 1581.34 & 2.723522 & NU1 \\
\hline 4040.19 & 0.0048 & 0.0795 & 5 & 4 & 1 & 4 & 2 & 2 & 1922.92 & 0.042579 & NU $3 * * *$ \\
\hline 4041.13 & 0.0263 & 0.0840 & 7 & 4 & 3 & 6 & 2 & 4 & 602.77 & 0.157817 & NU $3 *$ \\
\hline 4041.27 & 0.0132 & 0.0692 & 8 & 4 & 5 & 7 & 1 & 6 & 704.22 & 0.47 & NU $1 *$ \\
\hline $4041 \cdot 32$ & 0.0158 & 0.0496 & 10 & 5 & 6 & 10 & 2 & 9 & 1293.66 & 0.021485 & NU1 \\
\hline 4043.10 & 0.0199 & 0.0804 & 6 & 3 & 4 & 5 & 1 & 5 & 326.64 & 0.030200 & NU $3 *$ \\
\hline 4043.38 & 0.4133 & 0.0523 & 11 & 6 & 5 & 10 & 5 & 6 & 1718.77 & 4.582064 & NU1 \\
\hline $4043 \cdot 38$ & 0.2882 & 0.0703 & 6 & 4 & 3 & 6 & 0 & 6 & 446.71 & 0.001595 & NU3 \\
\hline 4043.96 & 23.9504 & 0.0869 & 7 & 3 & 4 & 6 & 1 & 5 & 542.91 & 0.214427 & NU3 \\
\hline 4044.86 & 47.0066 & 0.0824 & 6 & 4 & 2 & 5 & 2 & 3 & $446 \cdot 50$ & 0.086601 & NU3 \\
\hline 4045.03 & 39.4195 & 0.0779 & 5 & 4 & 2 & 4 & 2 & 3 & $300 \cdot 35$ & 0.034972 & Nu3 \\
\hline 4046.71 & 0.0024 & 0.0692 & 8 & 4 & 5 & 7 & 1 & 6 & $704 \cdot 22$ & 0.47 & NU $1 * * *$ \\
\hline 4048.22 & 0.6493 & 0.0681 & 8 & 3 & 5 & 8 & 1 & 8 & $744 \cdot 16$ & 0.005295 & NU3 \\
\hline 4048.43 & 0.0064 & 0.0614 & 8 & 3 & 6 & 7 & 0 & 7 & $586 \cdot 26$ & 0.125531 & NU $1 *$ \\
\hline 4048.81 & 0.1731 & 0.0337 & 9 & 9 & 1 & 8 & 8 & 0 & 1789.10 & 8.403719 & NU1 \\
\hline 4048.81 & 0.5193 & 0.0337 & 9 & 9 & 0 & 8 & 8 & 1 & 1789.10 & 8.403719 & NU1 \\
\hline 4050.34 & 0.1060 & 0.0655 & 12 & 6 & 7 & 11 & 5 & 6 & 1999.02 & 4.458582 & NUI \\
\hline 4050.35 & 0.3876 & 0.0719 & 13 & 4 & 9 & 12 & 4 & 8 & 2205.65 & 11.537610 & NU3 \\
\hline 4050.75 & 0.0048 & 0.0840 & 7 & 4 & 3 & 6 & 2 & 4 & 602.77 & 0.157817 & NU $3 * *$ \\
\hline
\end{tabular}




\begin{tabular}{|c|c|c|c|c|c|c|c|c|c|c|c|}
\hline $\begin{array}{c}\text { FREQUENCY } \\
\mathrm{cm}^{-1}\end{array}$ & $\begin{array}{l}\text { LINE } \\
\text { STRENGTH } \\
\frac{\mathrm{cm}^{-1}}{9 m \mathrm{~cm}^{-2}}\end{array}$ & $\begin{array}{l}\text { HALF } \\
\text { WIDTH } \\
\mathrm{cm}^{-1} \\
\text { atm. }\end{array}$ & $J^{\prime}$ & $\mathrm{K}_{\mathrm{a}}$ & $K_{c}$ & J" & $\mathrm{K}_{\mathrm{a}}$ & $K_{c}$ & $\begin{array}{c}E^{\prime \prime} \\
\mathrm{cm}^{-1}\end{array}$ & $L$ & BAND \\
\hline 4050.85 & 0.0090 & 0.0496 & 10 & 6 & 5 & 10 & 3 & 8 & $1446 \cdot 16$ & 0.026178 & NUI \\
\hline 4050.86 & 0.0135 & 0.0572 & 12 & 5 & 7 & 12 & 3 & 10 & $1962 \cdot 55$ & 0.053237 & NU3 \\
\hline 4051.19 & 0.0103 & 0.0544 & 9 & 4 & 6 & 9 & 1 & 9 & 920.21 & 0.006488 & NU1 \\
\hline $4051 \cdot 64$ & 0.0012 & 0.0482 & 12 & 8 & 5 & 12 & 6 & 6 & 2437.56 & 0.066727 & NU3 \\
\hline 4051.71 & 0.0014 & 0.0798 & 5 & 5 & 1 & 4 & 2 & 2 & 315.79 & 0.0215 & NU $1 *$ \\
\hline 4051.84 & 0.0037 & 0.0804 & 6 & 3 & 4 & 5 & 1 & 5 & 326.64 & 0.030200 & NU $3 * *$ \\
\hline $4052 \cdot 16$ & 6.6138 & 0.0692 & 8 & 4 & 5 & 7 & 1 & 6 & $704 \cdot 22$ & 0.47 & NU1+ \\
\hline 4053.09 & 0.0012 & 0.0614 & 8 & 3 & 6 & 7 & 0 & 7 & 586.26 & 0.125531 & NU $1 * * *$ \\
\hline 4054.41 & 0.0044 & 0.0707 & 10 & 3 & 7 & 10 & 0 & 10 & 1114.55 & 0.007326 & NU 1 \\
\hline 4054.97 & 0.1359 & 0.0396 & 10 & 8 & 2 & 9 & 7 & 3 & 1810.63 & 7.138077 & NU1 \\
\hline 4054.97 & 0.4078 & 0.0396 & 10 & 8 & 3 & 9 & 7 & 2 & 1810.63 & 7.138103 & NU 1 \\
\hline $4054 \cdot 98$ & 0.0236 & 0.0774 & 6 & 4 & 3 & 5 & 2 & 4 & $416 \cdot 22$ & 0.0555 & NU 3 * \\
\hline 4055.03 & 0.0130 & 0.0779 & 5 & 4 & 2 & 4 & 2 & 3 & 1907.99 & 0.034972 & NU $3 * * *$ \\
\hline 4055.35 & 0.0033 & 0.0459 & 12 & 8 & 4 & 12 & 6 & 7 & 2433.85 & 0.066188 & NU3 \\
\hline 4055.36 & 0.0156 & 0.0824 & 6 & 4 & 2 & 5 & 2 & 3 & 2053.98 & 0.086601 & NU $3 * * * *$ \\
\hline 4055.75 & 0.0049 & 0.0812 & 5 & 4 & 1 & 4 & 1 & 4 & 1821.61 & 0.096 & NUI $* * *$ \\
\hline 4056.45 & 0.0040 & 0.0719 & 5 & 5 & 0 & 5 & 0 & 5 & 325.35 & 0.000043 & NU 1 \\
\hline 6.72 & 0.0044 & 0.0471 & 11 & 8 & 4 & 11 & 6 & 5 & $2144 \cdot 12$ & 0.04 & NU 3 \\
\hline 7.76 & 3.1892 & 0.0614 & 8 & 3 & 6 & 7 & 0 & 7 & $586 \cdot 26$ & 0.12 & NUI \\
\hline 8.15 & 0.0015 & 0.0459 & 11 & 8 & 3 & 11 & 6 & 6 & 2142.69 & 0.04 & NU3 \\
\hline 8.95 & 0.0747 & 0.0475 & 11 & 7 & 5 & 10 & 6 & 4 & 1875.53 & 5.881509 & NU1 \\
\hline 4059.50 & 0.2241 & 0.0468 & 11 & 7 & 4 & 10 & 6 & 5 & 1875.00 & 5.877228 & NUI \\
\hline 0.38 & 13.1213 & 0.0840 & 7 & 4 & 3 & 6 & 2 & 4 & 602.77 & 0.157817 & NU3 \\
\hline 4060.58 & 9.9877 & 0.0804 & 6 & 3 & 4 & 5 & 1 & 5 & $326 \cdot 64$ & 0.030200 & NU3- \\
\hline 4060.77 & 0.0505 & 0.0841 & 8 & 4 & 4 & 7 & 2 & 5 & 782.40 & 0.247061 & NU3* \\
\hline 4060.86 & 0.0103 & 0.0448 & 10 & 8 & 2 & 10 & 6 & 5 & 1875.00 & 0.025193 & NU3 \\
\hline 4061.65 & 0.1702 & 0.0622 & 10 & 4 & 6 & 10 & 2 & 9 & 1293.66 & 0.021601 & NU3 \\
\hline 4062.75 & 0.0182 & 0.0437 & 9 & 8 & 2 & 9 & 6 & 3 & 1631.41 & 0.012 & NU3 \\
\hline 2.89 & 0.0061 & 0.0434 & 9 & 8 & 1 & 9 & 6 & 4 & $1631 \cdot 27$ & 0.012330 & NU3 \\
\hline 3.73 & 0.0049 & 0.0774 & 6 & 4 & 2 & 5 & 1 & 5 & 326.64 & 0.07 & NU $1 *$ \\
\hline 4064.07 & 0.0059 & 0.0417 & 8 & 8 & 1 & 8 & 6 & 2 & 1411.68 & 0.004043 & NU3 \\
\hline 4064.10 & 0.0176 & 0.0416 & 8 & 8 & 0 & 8 & 6 & 3 & 1411.65 & 0.00 & NU3 \\
\hline 4064.42 & 0.0044 & 0.0774 & 6 & 4 & 3 & 5 & 2 & 4 & $416 \cdot 22$ & 0.05 & NU3** \\
\hline 4064.46 & 0.0078 & 0.0645 & 5 & 5 & 0 & 4 & 3 & 1 & .85 & 0.015576 & NU3* \\
\hline 4065.42 & 0.0335 & 0.0530 & 12 & 6 & 6 & 11 & 5 & 7 & 1985.83 & 4.215931 & NU 1 \\
\hline 4065.84 & 0.0188 & 0.0794 & 7 & 2 & 5 & 6 & 0 & 6 & $446 \cdot 71$ & 0.05 & NU3 $3 *$ \\
\hline 4065.86 & 0.0234 & 0.0640 & 5 & 5 & 1 & 4 & 3 & 2 & . 52 & 0.01 & NU3* \\
\hline 4066.01 & 0.7051 & 0.0798 & 5 & 5 & 1 & 4 & 2 & 2 & .79 & 0.02 & NU1+ \\
\hline 4066.37 & 0.0012 & 0.0462 & 11 & 6 & 6 & 11 & 3 & 9 & 1695.03 & 0.03 & NU 1 \\
\hline 4067.35 & 0.0033 & 0.0804 & 6 & 3 & 4 & 5 & 1 & 5 & 1922.86 & 0.03 & NU $3 * * * *$ \\
\hline 4067.75 & 0.0014 & 0.0795 & 4 & 4 & 0 & 3 & 0 & 3 & 136.77 & 0.000270 & NU $3 *$ \\
\hline 4068.25 & 0.0022 & 0.0692 & 8 & 4 & 5 & 7 & 1 & 6 & 2309.74 & 0.47 & NU $1 * * * *$ \\
\hline 4069.26 & 0.0865 & 0.0694 & 5 & 5 & 1 & 5 & 1 & 4 & 399.46 & 0.000 & NU3 \\
\hline 4069.62 & 0.0036 & 0.0739 & 9 & 7 & 2 & 9 & 4 & 5 & $1360 \cdot 28$ & 0.00 & NU 1 \\
\hline 4069.84 & 0.0011 & 0.0614 & 8 & 3 & 6 & 7 & 0 & 7 & 2180.65 & 0.125531 & NU $1 * * *$ \\
\hline 4069.96 & 0.0044 & 0.0840 & 7 & 4 & 3 & 6 & 2 & 4 & $2211 \cdot 21$ & 0.157817 & NU $3 * * *$ \\
\hline 4070.09 & 0.0093 & 0.0841 & 8 & 4 & 4 & 7 & 2 & 5 & 782.40 & 0.247061 & NU3** \\
\hline 4070.16 & 0.2192 & 0.0627 & 8 & 6 & 3 & 7 & 1 & 6 & 704.22 & 0.17 & $2 \mathrm{NU} 2+$ \\
\hline 4070.61 & 0.0526 & 0.0350 & 10 & 9 & 1 & 9 & 8 & 2 & 2009.87 & 8.259078 & NU 1 \\
\hline 4070.61 & 0.1579 & 0.0350 & 10 & 9 & 2 & 9 & 8 & 1 & 2009.87 & 8.259079 & NU1 \\
\hline 4070.62 & 0.0852 & 0.0694 & 6 & 5 & 2 & 6 & 1 & 5 & 542.91 & 0.000758 & NU3 \\
\hline 4070.98 & 0.0339 & 0.0690 & 12 & 5 & 7 & 11 & 4 & 8 & 1843.02 & 2.124861 & NU 1 \\
\hline 4072.50 & 0.0465 & 0.0804 & 8 & 3 & 5 & 7 & 1 & 6 & 704.22 & 0.154000 & NU $3 *$ \\
\hline 4072.70 & 0.5343 & 0.0646 & 7 & 4 & 4 & 7 & 0 & 7 & $5 \cdot 86.26$ & 0.001966 & NU3 \\
\hline
\end{tabular}




\begin{tabular}{|c|c|c|c|c|c|c|c|c|c|c|c|}
\hline $\begin{array}{c}\text { FREQUENCY } \\
\mathrm{cm}^{-1}\end{array}$ & $\begin{array}{l}\text { LINE } \\
\text { STRENGTH } \\
\frac{\mathrm{cm}^{-1}}{9 m \mathrm{~cm}^{-2}}\end{array}$ & $\begin{array}{l}\text { HALF } \\
\text { WIDTH } \\
\mathrm{cm}-1^{\text {atm. }}\end{array}$ & $J^{\prime}$ & $K_{a}$ & $K_{c}$ & J" & $k_{a}$ & $K_{c}$ & $\begin{array}{c}E^{\prime \prime} \\
\mathrm{cm}^{-1}\end{array}$ & $L$ & BAND \\
\hline 4073.87 & 11.7820 & 0.0774 & 6 & 4 & 3 & 5 & 2 & 4 & $416 \cdot 22$ & 0.055572 & NU3 \\
\hline 4074.66 & 0.0015 & 0.0645 & 5 & 5 & 0 & 4 & 3 & 1 & 383.85 & 0.015576 & NU $3 * *$ \\
\hline 075.29 & 2.4512 & 0.0774 & 6 & 4 & 2 & 5 & 1 & 5 & 326.64 & 0.0787 & NU1+ \\
\hline 4075.58 & 0.0336 & 0.0413 & 11 & 8 & 4 & 10 & 7 & 3 & 2054.40 & 7.018690 & NU1 \\
\hline 4075.58 & 0.1007 & 0.0412 & 11 & 8 & 3 & 10 & 7 & 4 & 2054.40 & 7.018546 & NU1 \\
\hline 4076.03 & 0.0044 & 0.0640 & 5 & 5 & 1 & 4 & 3 & 2 & 382.52 & 0.015487 & NU $3 * *$ \\
\hline 4076.40 & 0.0016 & 0.0470 & 11 & 5 & 7 & 11 & 2 & 10 & 1525.13 & 0.020871 & NU1 \\
\hline 4076.85 & 0.0031 & 0.0794 & 7 & 2 & 5 & 6 & 0 & 6 & 2041.76 & 0.051494 & NU $3 * * * *$ \\
\hline 4077.28 & 0.0015 & 0.0659 & 8 & 7 & 2 & 7 & 4 & 3 & 931.22 & 0.003551 & $2 \mathrm{NU} 2$ \\
\hline 4077.34 & 0.0547 & 0.0481 & 12 & 7 & 6 & 11 & 6 & 5 & 2144.12 & 5.716270 & NU1 \\
\hline 4078.14 & 0.0010 & 0.0607 & 9 & 4 & 6 & 8 & 1 & 7 & 882.93 & 0.269351 & NU $1 *$ \\
\hline 4078.43 & 0.3292 & 0.0754 & 11 & 4 & 7 & 10 & 3 & 8 & 1446.16 & 0.954846 & NUI \\
\hline 4078.45 & 0.3262 & 0.0656 & 7 & 5 & 3 & 7 & 1 & 6 & 704.22 & 0.002162 & NU3 \\
\hline 4079.11 & 0.0182 & 0.0470 & 12 & 7 & 5 & 11 & 6 & 6 & 2142.69 & 5.701369 & NUI \\
\hline 4079.42 & 25.2190 & 0.0841 & 8 & 4 & 4 & 7 & 2 & 5 & 782.40 & 0.247061 & NU3 \\
\hline 4080.00 & 0.0028 & 0.0626 & 6 & 5 & 1 & 6 & 0 & 6 & 446.71 & 0.000164 & NU1 \\
\hline $4080 \cdot 20$ & 0.4661 & 0.0822 & 6 & 5 & 2 & 5 & 2 & 3 & $446 \cdot 50$ & 0.009072 & NU 1 \\
\hline 4080.33 & 0.0086 & 0.0804 & 8 & 3 & 5 & 7 & 1 & 6 & $704 \cdot 22$ & 0.154000 & NU3** \\
\hline $4081 \cdot 28$ & 9.3902 & 0.0794 & 7 & 2 & 5 & 6 & 0 & 6 & 446.71 & 0.051494 & NU3 \\
\hline 4081.45 & 0.1728 & 0.0743 & 5 & 5 & 0 & 4 & 2 & 3 & 300.35 & 0.001619 & NU1 \\
\hline 4083.61 & 0.0012 & 0.0405 & 11 & 9 & 3 & 11 & 7 & 4 & 2321.94 & 0.021839 & NU3 \\
\hline 4084.06 & 0.0039 & 0.0774 & 6 & 4 & 3 & 5 & 2 & 4 & 2024.17 & 0.055572 & NU $3 * * *$ \\
\hline 4084.19 & 0.0290 & 0.0697 & 6 & 5 & 1 & 5 & 3 & 2 & 508.81 & 0.036022 & NU3* \\
\hline 4084.51 & 0.0077 & 0.0804 & 8 & 3 & 5 & 7 & 1 & 6 & 2309.74 & 0.154000 & NU $3 * * *$ \\
\hline 4084.87 & 3.8931 & 0.0645 & 5 & 5 & 0 & 4 & 3 & 1 & 383.85 & 0.015576 & NU3 \\
\hline 4085.56 & 0.0028 & 0.0650 & 9 & 2 & 7 & 8 & 1 & 8 & $744 \cdot 16$ & 0.122288 & NU $1 *$ \\
\hline 4085.57 & 0.0022 & 0.0378 & 9 & 9 & 1 & 9 & 7 & 2 & 1810.63 & 0.003527 & NU3 \\
\hline 4086.20 & 11.6938 & 0.0640 & 5 & 5 & 1 & 4 & 3 & 2 & 382.52 & 0.015487 & NU3 \\
\hline 4086.47 & 0.0062 & 0.0812 & 9 & 4 & 5 & 8 & 2 & 6 & 982.91 & 0.240000 & NU3* \\
\hline 4086.81 & 0.0010 & 0.0442 & 12 & 6 & 7 & 12 & 3 & 10 & 1962.55 & 0.040149 & NU 1 \\
\hline 4087.26 & 0.0083 & 0.0841 & 8 & 4 & 4 & 7 & 2 & 5 & $2392 \cdot 58$ & 0.247061 & NU $3 * * * *$ \\
\hline 4087.38 & 0.0473 & 0.0744 & 7 & 4 & 4 & 6 & 2 & 5 & 552.92 & 0.073173 & NU3* \\
\hline 4088.02 & 0.6971 & 0.0795 & 4 & 4 & 0 & 3 & 0 & 3 & 136.77 & 0.000270 & NU3 \\
\hline 4088.16 & 23.2913 & C. 0804 & 8 & 3 & 5 & 7 & 1 & 6 & 704.22 & 0.154000 & NU3- \\
\hline 4088.47 & 0.5215 & 0.0607 & 9 & 4 & 6 & 8 & 1 & 7 & 882.93 & 0.269351 & NU1 \\
\hline 4088.58 & 0.2745 & 0.0722 & 10 & 3 & 7 & 9 & 2 & 8 & $1080 \cdot 38$ & 0.380414 & NU1 \\
\hline 4088.71 & 0.0384 & 0.0731 & 7 & 3 & 5 & 6 & 1 & 6 & $447 \cdot 24$ & 0.034900 & NU $3 *$ \\
\hline 4088.74 & 0.0023 & 0.0655 & 7 & 7 & 0 & 7 & 4 & 3 & 931.22 & 0.000515 & NU 1 \\
\hline 4089.01 & 0.0012 & 0.0553 & 9 & 7 & 3 & 9 & 4 & 6 & 1340.89 & 0.006153 & NU 1 \\
\hline 4089.20 & 0.0097 & 0.0679 & 6 & 5 & 2 & 5 & 3 & 3 & 504.00 & 0.035127 & $\mathrm{NU}_{3} *$ \\
\hline 4089.33 & 0.0023 & 0.0525 & 10 & 7 & 4 & 10 & 4 & 7 & $1581 \cdot 34$ & 0.013111 & NU 1 \\
\hline 4089.97 & 0.0130 & 0.0369 & 11 & 9 & 3 & 10 & 8 & 2 & $2254 \cdot 34$ & 8.133537 & NU 1 \\
\hline 4089.97 & 0.0391 & 0.0369 & 11 & 9 & 2 & 10 & 8 & 3 & $2254 \cdot 34$ & 8.133535 & NU1 \\
\hline 4090.32 & 0.0039 & 0.0569 & 8 & 7 & 2 & 8 & 4 & 5 & $1122 \cdot 72$ & 0.002252 & NU I \\
\hline 4092.95 & 0.0014 & 0.0810 & 8 & 5 & 4 & 7 & 2 & 5 & $782 \cdot 40$ & 0.074366 & NU $1 *$ \\
\hline 4093.01 & 0.2257 & 0.0836 & 7 & 5 & 3 & 6 & 2 & 4 & 602.77 & 0.028700 & NU1 \\
\hline 4093.08 & 0.0810 & 0.0608 & 8 & 5 & 4 & 8 & 1 & 7 & 882.93 & 0.003922 & NU3 \\
\hline 4093.24 & 0.0101 & 0.0494 & 10 & 4 & 7 & 10 & 1 & 10 & 1114.56 & 0.005517 & NU1 \\
\hline 4093.54 & 1.4228 & 0.0650 & 9 & 2 & 7 & 8 & 1 & 8 & $744 \cdot 16$ & 0.122288 & NU1 \\
\hline 4094.48 & 0.0054 & 0.0697 & 6 & 5 & 1 & 5 & 3 & 2 & 508.81 & 0.036022 & NU $3 * * *$ \\
\hline 4096.71 & 0.0088 & 0.0744 & 7 & 4 & 4 & 6 & 2 & 5 & 552.92 & 0.073173 & NU $3 * *$ \\
\hline 4097.37 & 0.0071 & 0.0731 & 7 & 3 & 5 & 6 & 1 & 6 & $447 \cdot 24$ & 0.034900 & NU3** \\
\hline 4099.36 & 0.0018 & C. 0679 & 6 & 5 & 2 & 5 & 3 & 3 & 504.00 & 0.035127 & NU $3 * *$ \\
\hline 4100.38 & 0.0013 & 0.0645 & 5 & 5 & 0 & 4 & 3 & 1 & 2005.92 & 0.015576 & NU $3 * * *$ \\
\hline
\end{tabular}




\begin{tabular}{|c|c|c|c|c|c|c|c|c|c|c|c|}
\hline $\begin{array}{c}\text { FREQUENCY } \\
\mathrm{cm}^{-1}\end{array}$ & $\begin{array}{c}\text { LINE } \\
\text { STRENGTH } \\
\frac{\mathrm{cm}^{-1}}{\mathrm{gm} \mathrm{cm}^{-2}}\end{array}$ & $\begin{array}{l}\text { HALF } \\
\text { WIDTH } \\
\mathrm{cm}^{-1} \\
\text { ołm. }\end{array}$ & J' & $\mathrm{K}_{\mathrm{a}}$ & $K_{c}$ & $J^{\prime \prime}$ & $\mathrm{K}_{\mathrm{a}}$ & $K_{c}$ & $\begin{array}{c}E^{\prime \prime} \\
\mathrm{cm}^{-1}\end{array}$ & L & BAND \\
\hline 4100.52 & U.0081 & 0.0733 & 7 & 5 & 2 & 6 & 3 & 3 & 661.56 & 0.063852 & NU $3 *$ \\
\hline 4101.49 & 0.0038 & 0.0640 & 5 & 5 & 1 & 4 & 3 & 2 & 2004.81 & 0.015487 & NU3弗长头 \\
\hline 4102.09 & 0.0537 & 0.0628 & 9 & 3 & 6 & 9 & 1 & 9 & 920.21 & 0.003124 & NU3 \\
\hline 4103.48 & 0.0012 & 0.0803 & 5 & 4 & 1 & 4 & 0 & 4 & 222.06 & 0.001060 & NU $3 *$ \\
\hline $4104 \cdot 10$ & 3.1030 & 0.0812 & 9 & 4 & 5 & 8 & 2 & 6 & 982.91 & 0.247000 & NU3- \\
\hline 4104.77 & 14.5311 & 0.0697 & 6 & 5 & 1 & 5 & 3 & 2 & 508.81 & 0.036022 & NU3 \\
\hline 4105.81 & 0.4528 & 0.0531 & 9 & 3 & 7 & 8 & 0 & 8 & 744.09 & 0.116343 & NUI \\
\hline 4106.03 & 19.1581 & 0.0731 & 7 & 3 & 5 & 6 & 1 & 6 & $447 \cdot 24$ & 0.034900 & NU3- \\
\hline 4106.04 & 23.6813 & 0.0744 & 7 & 4 & 4 & 6 & 2 & 5 & 552.92 & 0.073173 & NU3 \\
\hline 4106.67 & 0.0112 & 0.0585 & 11 & 4 & 7 & 11 & 2 & 10 & 1525.13 & 0.013267 & NU3 \\
\hline 4107.03 & 0.7171 & 0.0810 & 8 & 5 & 4 & 7 & 2 & 5 & $782 \cdot 40$ & 0.074366 & NU1 \\
\hline 4107.40 & 0.0826 & 0.0590 & 8 & 4 & 5 & 8 & 0 & 8 & 744.09 & 0.001991 & NU3 \\
\hline 4109.53 & 4.8438 & 0.0679 & 6 & 5 & 2 & 5 & 3 & 3 & 504.00 & 0.035127 & NU3 \\
\hline 4109.54 & 0.0083 & 0.0572 & 7 & 5 & 2 & 7 & 0 & 7 & 586.26 & 0.000324 & NU1 \\
\hline 4110.49 & 0.1130 & 0.0709 & 6 & 5 & 1 & 5 & 2 & 4 & $416 \cdot 22$ & 0.005627 & NUI \\
\hline 4110.93 & 0.0015 & 0.0733 & 7 & 5 & 2 & 6 & 3 & 3 & 661.56 & 0.063852 & NU $3 x^{2} *$ \\
\hline 4111.86 & 0.0017 & 0.0754 & 9 & 5 & 5 & 8 & 2 & 6 & 982.91 & 0.7281 & NUI* \\
\hline 4111.87 & 0.0033 & 0.0625 & 11 & 3 & 8 & 11 & 0 & 11 & 1327.14 & 0.005168 & NU1 \\
\hline 4112.99 & 0.0013 & 0.0437 & 12 & 5 & 8 & 12 & 2 & 11 & 1774.74 & 0.018820 & NUI \\
\hline $4113 \cdot 45$ & 0.0236 & 0.0684 & 7 & 5 & 3 & 6 & 3 & 4 & 648.97 & 0.058714 & NU3* \\
\hline 4113.62 & 0.0161 & 0.0760 & 8 & 5 & 3 & 7 & 3 & 4 & $842 \cdot 38$ & 0.105067 & NU $3 *$ \\
\hline 4113.75 & 0.0063 & 0.0731 & 7 & 3 & 5 & 6 & 1 & 6 & 2042.77 & 0.034900 & NU $3 * * * *$ \\
\hline $4114 \cdot 15$ & 0.0045 & 0.0756 & 7 & 4 & 3 & 6 & 1 & 6 & $447 \cdot 24$ & 0.0432 & NU1* \\
\hline 4114.43 & 0.1238 & 0.0558 & 9 & 5 & 5 & 9 & 1 & 8 & 1079.07 & 0.005298 & NU3 \\
\hline 4115.52 & 0.0186 & 0.0684 & 13 & 5 & 8 & 12 & 4 & 9 & 2124.98 & 1.92132 & Nul \\
\hline $4116 \cdot 52$ & 0.0078 & 0.0744 & 7 & 4 & 4 & 6 & 2 & 5 & $2161 \cdot 30$ & 0.073173 & NU $3 * * * * x$ \\
\hline 4118.86 & 0.0013 & 0.0525 & 10 & 4 & 7 & 9 & 1 & 8 & 1079.07 & 0.284777 & NU1* \\
\hline 4120.36 & 0.0033 & 0.0670 & 10 & 5 & 6 & 9 & 2 & 7 & 1201.95 & $1 \cdot 386$ & NU $1 *$ \\
\hline $4121 \cdot 24$ & 0.0048 & 0.0697 & 6 & 5 & 1 & 5 & 3 & 2 & $2130 \cdot 50$ & 0.036022 & NU $3 * * * *$ \\
\hline 4121.35 & 4.0167 & 0.0733 & 7 & 5 & 2 & 6 & 3 & 3 & 661.56 & 0.063852 & NU3 \\
\hline 4123.24 & 0.0226 & 0.0692 & 8 & 2 & 6 & 7 & 0 & 7 & 586.26 & 0.040852 & NU3* \\
\hline 4123.47 & 0.6004 & 0.0803 & 5 & 4 & 1 & 4 & 0 & 4 & 222.06 & 0.001060 & NU3 \\
\hline 4123.57 & 0.0044 & 0.0684 & 7 & 5 & 3 & 6 & 3 & 4 & 648.97 & 0.058714 & NU $3 * *$ \\
\hline 4123.60 & 0.0084 & 0.0691 & 8 & 4 & 5 & 7 & 2 & 6 & 709.60 & 0.084411 & NU $3 *$ \\
\hline $4124 \cdot 14$ & 0.0030 & 0.0760 & 8 & 5 & 3 & 7 & 3 & 4 & $842 \cdot 38$ & 0.105067 & NU $3 * *$ \\
\hline 4124.31 & 0.0059 & 0.0729 & 9 & 3 & 6 & 8 & 1 & 7 & 882.93 & 0.139840 & NU $3 *$ \\
\hline $4125 \cdot 18$ & 2.2357 & 0.0756 & 7 & 4 & 3 & 6 & 1 & 6 & $447 \cdot 24$ & 0.0432 & NUIt \\
\hline $4125 \cdot 38$ & 0.8625 & 0.0754 & 9 & 5 & 5 & 8 & 2 & 6 & 982.91 & 0.7281 & NUIt \\
\hline 4125.46 & 0.0031 & 0.0768 & 9 & 5 & 4 & 8 & 3 & 5 & 1050.15 & 0.169518 & NU3* \\
\hline 4128.11 & 0.0075 & 0.0540 & 6 & 6 & 0 & 5 & 4 & 1 & 610.35 & 0.015215 & NU3* \\
\hline 4128.35 & 0.0025 & 0.0535 & 6 & 6 & 1 & 5 & 4 & 2 & 610.12 & 0.015207 & NU3* \\
\hline 4128.73 & 0.6267 & 0.0525 & 10 & 4 & 7 & 9 & 1 & 8 & 1079.07 & 0.284777 & NUI \\
\hline 4130.97 & 0.0245 & 0.0696 & 12 & 4 & 8 & 11 & 3 & 9 & 1695.03 & 0.720922 & NUI \\
\hline 4131.01 & 0.0041 & 0.0692 & 8 & 2 & 6 & 7 & 0 & 7 & 586.26 & 0.040852 & NU3长* \\
\hline $4132 \cdot 74$ & 0.0015 & .0 .0691 & 8 & 4 & 5 & 7 & 2 & 6 & $709 \cdot 60$ & 0.084411 & $\mathrm{NU} 3 * *$ \\
\hline 4133.03 & 1.6544 & 0.0670 & 10 & 5 & 6 & 9 & 2 & 7 & 1201.95 & $1 \cdot 386$ & NUl+ \\
\hline 4133.70 & 11.8363 & 0.0684 & 7 & 5 & 3 & 6 & 3 & 4 & 648.97 & 0.058714 & NU3 \\
\hline 4134.66 & 8.0547 & 0.0760 & 8 & 5 & 3 & 7 & 3 & 4 & $842 \cdot 38$ & 0.105067 & NU3 \\
\hline 4137.35 & 0.0067 & 0.0648 & 8 & 3 & 6 & 7 & 1 & 7 & $586 \cdot 48$ & 0.036269 & NU3* \\
\hline 4137.71 & 0.0384 & 0.0613 & 9 & 6 & 4 & 9 & 2 & 7 & 1201.95 & 0.003008 & NU3 \\
\hline 4138.08 & 0.0037 & 0.0692 & 8 & 2 & 6 & 7 & 0 & 7 & 2180.65 & 0.040852 & NU $3 * * * * *$ \\
\hline 4138.46 & 0.0010 & 0.0448 & 11 & 4 & 8 & 11 & 1 & 11 & 1327.14 & 0.004565 & NU1 \\
\hline 4138.79 & 11.2811 & 0.0692 & 8 & 2 & 6 & 7 & 0 & 7 & $586 \cdot 26$ & 0.040852 & NU3 \\
\hline 4138.90 & 0.0014 & 0.0733 & 7 & 5 & 2 & 6 & 3 & 3 & 2282.59 & 0.063852 & NU $3 \ddot{*} * * *$ \\
\hline
\end{tabular}




\begin{tabular}{|c|c|c|c|c|c|c|c|c|c|c|c|}
\hline $\begin{array}{c}\text { FREQUENCY } \\
\mathrm{cm}^{-1}\end{array}$ & $\begin{array}{l}\text { LINE } \\
\text { STRENGTH } \\
\frac{\mathrm{cm}^{-1}}{\mathrm{gm}^{\prime} \mathrm{cm}^{-2}}\end{array}$ & $\begin{array}{l}\text { HALF } \\
\text { WIDTH } \\
\frac{\mathrm{cm}^{-1}}{a+m .}\end{array}$ & $J^{\prime}$ & $\mathrm{K}_{\mathrm{a}}$ & $\mathrm{K}_{\mathrm{c}}$ & $J^{\prime \prime}$ & $\mathrm{K}_{\mathrm{a}}$ & $K_{c}$ & $\begin{array}{c}E^{\prime \prime} \\
\mathrm{cm}^{-1}\end{array}$ & $L$ & BAND \\
\hline $\begin{array}{l}4139.25 \\
4139.37 \\
4139.46 \\
4141.88 \\
4141.89\end{array}$ & $\begin{array}{l}0.0050 \\
2.9209 \\
0.0137 \\
0.0159 \\
4.1963\end{array}$ & $\begin{array}{l}0.0681 \\
0.0729 \\
0.0642 \\
0.0513 \\
0.0691\end{array}$ & $\begin{array}{r}8 \\
9 \\
8 \\
10 \\
8\end{array}$ & $\begin{array}{l}5 \\
3 \\
6 \\
5 \\
4\end{array}$ & $\begin{array}{l}4 \\
6 \\
3 \\
6 \\
5\end{array}$ & $\begin{array}{r}7 \\
8 \\
8 \\
10 \\
7\end{array}$ & $\begin{array}{l}3 \\
1 \\
2 \\
1 \\
2\end{array}$ & $\begin{array}{l}5 \\
7 \\
6 \\
9 \\
6\end{array}$ & $\begin{array}{r}816.72 \\
882.93 \\
982.91 \\
1293.04 \\
709.60\end{array}$ & $\begin{array}{l}0.084527 \\
0.139840 \\
0.001082 \\
0.005888 \\
0.084411\end{array}$ & $\begin{array}{l}\text { NU3* } \\
\text { NU3 } \\
\text { NU3 } \\
\text { NU3 } \\
\text { NU3 }\end{array}$ \\
\hline 4142.08 & 0.0045 & 0.0655 & 5 & 5 & 0 & 5 & 1 & 5 & 326.64 & 0.000013 & NU3 \\
\hline 4142.53 & 0.0024 & 0.0617 & 7 & 6 & 1 & 7 & 1 & 6 & 704.22 & 0.000170 & NU1 \\
\hline 4142.77 & 0.0076 & 0.0561 & 10 & 6 & 5 & 10 & 2 & 8 & 1438.00 & 0.005881 & NU3 \\
\hline 4142.88 & 0.3617 & 0.0666 & 7 & 5 & 2 & 6 & 2 & 5 & 552.92 & 0.011802 & NU1 \\
\hline 4145.30 & 0.0017 & 0.0552 & 8 & 5 & 3 & 8 & 0 & 8 & 744.09 & 0.000443 & NU1 \\
\hline $4145 \cdot 35$ & 0.2388 & 0.0640 & 11 & 3 & 8 & 10 & 2 & 9 & 1293.66 & 0.316624 & NU1 \\
\hline 4145.95 & 0.0013 & 0.0648 & 8 & 3 & 6 & 7 & 1 & 7 & 586.48 & 0.036269 & NU3** \\
\hline 4146.43 & 0.0039 & 0.0783 & 6 & 4 & 2 & 5 & 0 & 5 & 325.35 & 0.001931 & NU3* \\
\hline 4146.46 & 1.5361 & 0.0768 & 9 & 5 & 4 & 8 & 3 & 5 & 1050.15 & 0.169518 & NU3 \\
\hline 4146.65 & 0.0263 & 0.0664 & 7 & 6 & 2 & 7 & 2 & 5 & 782.40 & 0.000253 & NU3 \\
\hline 4146.88 & 0.0937 & 0.0539 & 9 & 4 & 6 & 9 & 0 & 9 & 920.18 & 0.001797 & NU3 \\
\hline 4148.79 & 0.1765 & 0.0536 & 10 & 2 & 8 & 9 & 1 & 9 & 920.21 & 0.108 & NUI \\
\hline 4149.49 & 3.7339 & 0.0540 & 6 & 6 & 0 & 5 & 4 & 1 & 610.35 & 0.015215 & NU3 \\
\hline 4149.65 & 0.0039 & 0.0684 & 7 & 5 & 3 & 6 & 3 & 4 & 2271.70 & 0.058 & NU $3 * * *$ \\
\hline 4149.72 & 1.2455 & 0.0535 & 6 & 6 & 1 & 5 & 4 & 2 & 610.12 & 0.01 & NU3 \\
\hline 4149.78 & 0.0026 & 0.0589 & 7 & 6 & 1 & 6 & 4 & 2 & 757.78 & 0.03 & NU $3 *$ \\
\hline 4150.89 & 0.0081 & 0.0573 & 7 & 6 & 2 & 6 & 4 & 3 & 756.75 & 0.033 & NU $3 *$ \\
\hline 4152.61 & 0.0014 & 0.0691 & 8 & 4 & 5 & 7 & 2 & 6 & 2318.53 & 0.084 & NU $3 * * * *$ \\
\hline 4153.09 & 0.0026 & 0.0760 & 8 & 5 & 3 & 7 & 3 & 4 & 2462.87 & 0.105067 & NU $3 * * *$ \\
\hline 4153 & $2 \cdot 3648$ & 0.0772 & 10 & 4 & 6 & 9 & 2 & 7 & .95 & 0.18 & Nu3- \\
\hline 4154.55 & 3.3475 & 0.0648 & 8 & 3 & 6 & 7 & 1 & 7 & 586.48 & 0.03 & Nu3 \\
\hline 4155.00 & 0.0096 & 0.0517 & 11 & 6 & 6 & 11 & 2 & 9 & .70 & 0.0 & NU3 \\
\hline 4155.96 & 0.5215 & 0.0457 & 10 & 3 & 8 & 9 & 0 & 9 & 920.18 & 0.106449 & NUI \\
\hline 4156.64 & 0.0010 & 0.0550 & 8 & 6 & 2 & 8 & 1 & 7 & 882.93 & 0.000504 & NU'1 \\
\hline 4157.07 & 0.0028 & 0.0660 & 6 & 6 & 1 & 6 & 2 & 4 & 602.77 & 0.00 & NU3 \\
\hline 4158.82 & 0.0061 & 0.0547 & 12 & 4 & 8 & 12 & 2 & 11 & $1774 \cdot 74$ & 0.00 & NU3 \\
\hline 4159.06 & 0.0396 & 0.0578 & 10 & 3 & 7 & 10 & 1 & 10 & 1114.56 & 0.00 & NU3 \\
\hline 4159.20 & 2.2350 & 0.0766 & 10 & 5 & 5 & 9 & 3 & 6 & 1282.92 & 0.261892 & NU3 \\
\hline 4159.29 & 2.4705 & 0.0681 & 8 & 5 & 4 & 7 & 3 & 5 & 816.72 & 0.084527 & NU3 \\
\hline 4159.74 & 0.0027 & 0.0802 & 5 & 5 & 1 & 4 & 0 & 4 & 222.06 & 0.000050 & NU 1 \\
\hline 4161.59 & 0.0015 & 0.0573 & 7 & 6 & 2 & 6 & 4 & 3 & 756.75 & 0.033870 & NU3 $* *$ \\
\hline 4163.50 & 0.0111 & 0.0623 & 9 & 4 & 6 & 8 & 2 & 7 & 885.62 & 0.088564 & NU $3 *$ \\
\hline 4163.53 & 0.1178 & 0.0573 & 11 & 5 & 7 & 10 & 2 & 8 & 1438.00 & 0.963 & NU1+ \\
\hline 4166.01 & 1.9787 & 0.0783 & 6 & 4 & 2 & 5 & 0 & 5 & 325.35 & 0.001931 & NU3 \\
\hline $4166 \cdot 34$ & 0.0268 & 0.0625 & 6 & 5 & 1 & 6 & 1 & 6 & 447.24 & 0.000048 & NU3 \\
\hline 4167.64 & 0.0075 & 0.0662 & 9 & 5 & 5 & 8 & 3 & 6 & $1006 \cdot 12$ & 0.109168 & NU $3 *$ \\
\hline 4168.22 & 0.0013 & 0.0540 & 6 & 6 & 0 & 5 & 4 & 1 & 2251.86 & 0.015215 & NU $3 * * * *$ \\
\hline 4169.06 & 0.0494 & 0.0725 & 6 & 6 & 1 & 5 & 3 & 2 & 508.81 & 0.001286 & NU1 \\
\hline 4169.63 & 0.0056 & 0.0628 & 8 & 6 & 2 & 7 & 4 & 3 & 931.22 & 0.05 & NU $3 *$ \\
\hline 4171.27 & 1.3364 & 0.0589 & 7 & 6 & 1 & 6 & 4 & 2 & 757.78 & 0.033963 & NU3 \\
\hline $4172 \cdot 30$ & 4.0204 & 0.0573 & 7 & 6 & 2 & 6 & 4 & 3. & 756.75 & 0.033870 & NU3 \\
\hline 4172.56 & 0.0713 & 0.0462 & 11 & 4 & 8 & 10 & 1 & 9 & 1293.04 & 0.280036 & NU1 \\
\hline 4173.18 & 0.0020 & 0.0596 & 8 & 6 & 3 & 7 & 4 & 4 & 927.77 & 0.056441 & NU $3 *$ \\
\hline 4173.59 & 0.0012 & 0.0474 & 12 & 6 & 7 & 12 & 2 & 10 & 1960.22 & 0.01 & NU3 \\
\hline 4173.64 & 0.0147 & 0.0478 & 11 & 5 & 7 & 11 & 1 & 10 & 1524.87 & 0.005757 & NU3 \\
\hline 4173.87 & 0.0165 & 0.0662 & 6 & 6 & 0 & 5 & 3 & 3 & 504.00 & 0.001253 & NUI \\
\hline $4176 \cdot 34$ & 0.2879 & 0.0752 & 11 & 5 & 6 & 10 & 3 & 7 & $1538 \cdot 23$ & 0.359370 & NU 3 \\
\hline 4177.29 & 0.0022 & 0.0485 & 9 & 6 & 3 & 9 & 1 & 8 & 1079.07 & 0.000971 & NU 1 \\
\hline 4177.51 & 0.0014 & 0.0662 & 9 & 5 & 5 & 8 & 3 & 6 & $1006 \cdot 12$ & 0.109168 & NU $3 *-3$ \\
\hline
\end{tabular}




\begin{tabular}{|c|c|c|c|c|c|c|c|c|c|c|c|}
\hline $\begin{array}{c}\text { FREQUENCY } \\
\mathrm{cm}^{-1}\end{array}$ & $\begin{array}{l}\text { LINE } \\
\text { STRENGTH } \\
\frac{\mathrm{cm}^{-1}}{9 m \mathrm{~cm}^{-2}}\end{array}$ & $\begin{array}{l}\text { HALF } \\
\text { WIDTH } \\
\frac{\mathrm{cm}^{-1}}{\text { atm. }}\end{array}$ & $J^{\prime}$ & $K_{0}$ & $K_{c}$ & J" & $K_{a}$ & $K_{c}$ & $\begin{array}{c}E^{\prime \prime} \\
\mathrm{cm}^{-1}\end{array}$ & L & BAND \\
\hline 4179.75 & 0.0053 & 0.0648 & 10 & 3 & 7 & 9 & 1 & 8 & 1079.07 & 0.110021 & NU $3 \ddot{*}$ \\
\hline 4179.78 & 0.0030 & 0.0602 & 9 & 2 & 7 & 8 & 0 & 8 & 744.09 & 0.034667 & NU $3 *$ \\
\hline 4179.79 & 0.0861 & 0.0622 & 8 & 5 & 3 & 7 & 2 & 6 & 709.60 & 0.018297 & NU 1 \\
\hline $4181 \cdot 44$ & 5.5320 & 0.0623 & 9 & 4 & 6 & 8 & 2 & 7 & 885.62 & 0.088564 & NU3 \\
\hline 4182.62 & 0.0598 & 0.0760 & 8 & 4 & 4 & 7 & 1 & 7 & $586 \cdot 48$ & 0.006860 & NU1 \\
\hline 4185.19 & 0.0298 & 0.0771 & 7 & 6 & 2 & 6 & 3 & 3 & 661.56 & 0.004975 & NU 1 \\
\hline 4185.26 & 0.0018 & 0.0456 & 7 & 7 & 1 & 6 & 5 & 2 & $888 \cdot 60$ & 0.014987 & NU 3 * \\
\hline $4187 \cdot 38$ & 3.7403 & 0.0662 & 9 & 5 & 5 & 8 & 3 & 6 & $1006 \cdot 12$ & 0.109168 & NU3 3 \\
\hline 4187.61 & 0.0084 & 0.0558 & 9 & 3 & 7 & 8 & 1 & 8 & $744 \cdot 16$ & 0.033194 & NU3* \\
\hline 4188.59 & 0.0023 & 0.0562 & 9 & 5 & 4 & 9 & 0 & 9 & $920 \cdot 18$ & 0.000466 & NU1 \\
\hline 4189.45 & 0.0182 & 0.0630 & 13 & 4 & 9 & 12 & 3 & 10 & 1962.55 & 0.73 & NU 1 \\
\hline 4190.12 & 0.0101 & $0^{\circ} .0497$ & 10 & 4 & 7 & 10 & 0 & 10 & 1114.55 & 0.001525 & NU3 \\
\hline 4191.32 & 2.8424 & 0.0628 & 8 & 6 & 2 & 7 & 4 & 3 & $931 \cdot 22$ & 0.05 & NU3 \\
\hline 4192.35 & 0.0018 & 0.0623 & 9 & 4 & 6 & 8 & 2 & 7 & $2495 \cdot 18$ & 0.088554 & NU $3 * * *$ \\
\hline 4193.20 & 0.0403 & 0.0717 & 5 & 5 & 0 & 4 & 1 & 3 & 275.52 & 0.00 & NU3 \\
\hline 4193.80 & 0.2571 & 0.0704 & 11 & 4 & 7 & 10 & 2 & 8 & 1438.00 & 0.19 & NU3- \\
\hline 4194.55 & 2.6213 & 0.0648 & 10 & 3 & 7 & 9 & 1 & 8 & .07 & 0.110 & NU3 \\
\hline 4194.60 & 0.9548 & 0.0596 & 8 & 6 & 3 & 7 & 4 & 4 & 927.77 & 0.056441 & NU3 \\
\hline .58 & 0.0032 & 0.0601 & 9 & 6 & 4 & 8 & 4 & 5 & 1122.72 & 0.082913 & NU $3 *$ \\
\hline 4195.67 & 1.4691 & 0.0602 & 9 & 2 & 7 & 8 & 0 & 8 & 744.09 & 0.03 & NU3 \\
\hline 4196.19 & 0.0016 & 0.0558 & 9 & 3 & 7 & 8 & 1 & 8 & $744 \cdot 16$ & 0.03 & NU $3 * *$ \\
\hline 4196.43 & 0.0086 & 0.0587 & 7 & 5 & 2 & 7 & 1 & 7 & 586.48 & 0.00 & NU3 \\
\hline 4197.03 & 0.0442 & 0.0488 & 12 & 5 & 8 & 11 & 2 & 9 & .70 & 0.42 & NU1 \\
\hline 4197.14 & 0.0980 & 0.0801 & 8 & 6 & 3 & 7 & 3 & 4 & .38 & 0.013 & NUI \\
\hline 7.78 & 0.0875 & 0.0653 & 7 & 6 & 1 & 6 & 3 & 4 & .97 & 0.00 & NUI \\
\hline 8.86 & 0.0015 & 0.0677 & 10 & 6 & 4 & 9 & 4 & 5 & $\cdot 28$ & 0.12 & NU $3 *$ \\
\hline .09 & 0.0021 & 0.0563 & 11 & 7 & 5 & 11 & 3 & 8 & .26 & 0.00 & NU3 \\
\hline 4200 & 0.2592 & 0.0721 & 12 & 5 & 7 & 11 & 3 & 8 & .26 & 0.41 & NU3 \\
\hline 4201.35 & 0.0205 & 0.0728 & 6 & 5 & 2 & 5 & 0 & 5 & .35 & 0.00 & NU 1 \\
\hline 87 & 0.0225 & 0.0548 & 12 & 3 & 9 & 11 & 2 & 10 & .13 & 0.27 & NU1 \\
\hline 420 & 0.1835 & 0.0442 & 11 & 2 & 9 & 10 & 1 & 10 & 1114.56 & 0.05 & NU1 \\
\hline 4202 & 0.0013 & 0.0662 & 9 & 5 & 5 & 8 & 3 & 6 & .21 & 0.10 & NU $3 * * * *$ \\
\hline 4204.77 & 4.2275 & 0.0558 & 9 & 3 & 7 & 8 & 1 & 8 & .16 & 0.033194 & NU3 \\
\hline 420 & 0.0276 & 0.0808 & 9 & 6 & 4 & 8 & 3 & 5 & • 15 & 0.0 & NU1 \\
\hline 420 & 0.0609 & 0.0404 & 11 & 3 & 9 & 10 & 0 & 10 & .55 & 0.0 & NU1 \\
\hline 67 & 0.0014 & 0.0560 & 10 & 4 & 7 & 9 & 2 & 8 & .38 & 0.08 & NU $3 *$ \\
\hline 420 & 0.0043 & 0.0595 & 6 & 6 & 0 & 6 & 2 & 5 & 552.92 & 0.000013 & NU3 \\
\hline .53 & 0.3093 & 0.0457 & 7 & 7 & 0 & 6 & 5 & 1 & 3.63 & 0.01 & NU3 \\
\hline 7.56 & 0.9278 & 0.0456 & 7 & 7 & 1 & 6 & 5 & 2 & .60 & 0.01 & NU3 \\
\hline .79 & 0.0017 & 0.0493 & 8 & 7 & 1 & 7 & 5 & 2 & .85 & 0.03 & NU3* \\
\hline 4208.02 & 0.5267 & 0.0659 & 9 & 6 & 3 & 8 & 4 & 4 & 1131.76 & 0.08 & NU3 \\
\hline 4208.15 & 0.0013 & 0.0448 & 12 & 5 & 8 & 12 & 1 & 11 & 1774.60 & 0.00 & NU3 \\
\hline 4214.07 & 0.0581 & 0.0781 & 10 & 6 & 5 & 9 & 3 & 6 & 1282.92 & 0.07 & NU1 \\
\hline 4214.12 & 0.4385 & 0.0724 & 6 & 5 & 1 & 5 & 1 & 4 & 399.46 & 0.000613 & NU3 \\
\hline 4215.50 & 0.0014 & 0.0558 & 9 & 3 & 7 & 8 & 1 & 8 & 2337.70 & & NU $3 * * * *$ \\
\hline 4216.44 & 0.4221 & 0.0740 & 7 & 4 & 3 & 6 & 0 & 6 & 446.71 & 0.002241 & NU3 \\
\hline 4216.52 & 0.0032 & 0.0528 & 11 & 3 & 8 & 11 & 1 & 11 & $1327 \cdot 14$ & 0.001419 & NU3 \\
\hline 4216.94 & 1.5967 & 0.0601 & 9 & 6 & 4 & 8 & 4 & 5 & 1122.72 & 0.082913 & NU3 \\
\hline 4218.53 & 0.0645 & 0.0415 & 12 & 4 & 9 & 11 & 1 & 10 & 1524.87 & 0.265959 & NU 1 \\
\hline 4218.65 & 0.5159 & 0.0616 & 10 & 5 & 6 & 9 & 3 & 7 & $1216 \cdot 27$ & 0.128613 & NU3 \\
\hline 4219.45 & 0.0031 & 0.0568 & 7 & 6 & 1 & 7 & 2 & 6 & 709.60 & 0.000062 & NU3 \\
\hline $4221 \cdot 26$ & 0.7306 & 0.0677 & 10 & 6 & 4 & 9 & 4 & 5 & 1360.28 & 0.124623 & NU3 \\
\hline 4222.85 & 0.0296 & 0.0630 & 8 & 6 & 2 & 7 & 3 & 5 & 816.72 & 0.010619 & NUI \\
\hline 4223.15 & 0.1316 & 0.0603 & 9 & 5 & 4 & 8 & 2 & 7 & 885.62 & 0.022231 & NU 1 \\
\hline
\end{tabular}




\begin{tabular}{|c|c|c|c|c|c|c|c|c|c|c|c|}
\hline $\begin{array}{c}\text { FREQUENCY } \\
\mathrm{cm}^{-1}\end{array}$ & $\begin{array}{l}\text { LINE } \\
\text { STRENGTH } \\
\frac{\mathrm{cm}^{-1}}{\mathrm{gm} \mathrm{cm}^{-2}}\end{array}$ & $\begin{array}{l}\text { HALF } \\
\text { WIDTH } \\
\frac{\mathrm{cm}^{-1}}{\text { atm. }}\end{array}$ & $J^{\prime}$ & $\mathrm{K}_{\mathrm{a}}$ & $K_{c}$ & $J^{\prime \prime}$ & $K_{a}$ & $K_{c}$ & $\begin{array}{c}E^{\prime \prime} \\
\mathrm{cm}^{-1}\end{array}$ & $L$ & BAND \\
\hline 4223.17 & 0.0112 & 0.0725 & 11 & 6 & 6 & 10 & 3 & 7 & 1538.23 & 0.147349 & NU1 \\
\hline 4224.24 & 0.0017 & 0.0460 & 13 & 3 & 10 & 13 & 0 & 13 & 1806.78 & 0.02938 & NUI \\
\hline $4224 \cdot 29$ & 0.6933 & 0.0560 & 10 & 4 & 7 & 9 & 2 & 8 & 1080.38 & 0.087251 & NU3 \\
\hline 4224.63 & 0.0030 & 0.0633 & 9 & 7 & 3 & 9 & 3 & 6 & $1282 \cdot 92$ & 0.000341 & NU3 \\
\hline 4230.15 & 0.8683 & 0.0493 & 8 & 7 & 1 & 7 & 5 & 2 & 1059.85 & 0.032814 & NU3 \\
\hline 4230.35 & 0.2894 & 0.0489 & 8 & 7 & 2 & 7 & 5 & 3 & 1059.65 & 0.032804 & NU3 \\
\hline $4231 \cdot 24$ & 0.0991 & 0.0684 & 11 & 6 & 5 & 10 & 4 & 6 & 1616.51 & 0.181046 & NU3 \\
\hline 4232.88 & 0.0160 & 0.0553 & 8 & 5 & 3 & 8 & 1 & 8 & $744 \cdot 16$ & 0.000125 & NU3 \\
\hline 4233.37 & 0.0098 & 0.0662 & 13 & 5 & 8 & 12 & 3 & 9 & 2105.90 & 0.310600 & NU3 \\
\hline 4233.56 & 0.0043 & 0.0565 & 13 & 7 & 8 & 12 & 5 & 7 & 2300.80 & 0.244900 & NU3 \\
\hline 4234.53 & 0.0032 & 0.0511 & 10 & 2 & 8 & 9 & 0 & 9 & $920 \cdot 18$ & 0.030629 & NU $3 *$ \\
\hline 4236.10 & 0.0158 & 0.0657 & 12 & 6 & 7 & 11 & 3 & 8 & $1813 \cdot 26$ & 0.264753 & NU 1 \\
\hline $4236 \cdot 34$ & 0.0088 & 0.0460 & 11 & 4 & 8 & 11 & 0 & 11 & $1327 \cdot 14$ & 0.001260 & NU3 \\
\hline $4236 \cdot 92$ & 0.0101 & 0.0533 & 8 & 6 & 2 & 8 & 2 & 7 & 885.62 & 0.000159 & NU3 \\
\hline 4237.39 & 0.0002 & 0.0573 & 10 & 5 & 5 & 10 & 0 & 10 & 1114.55 & 0.000396 & NU1 \\
\hline 4239.88 & 0.2443 & 0.0589 & 10 & 6 & 5 & 9 & 4 & 6 & 1340.89 & 0.112325 & NU3 \\
\hline 4239.99 & 0.1124 & 0.0678 & 12 & 6 & 6 & 11 & 4 & 7 & 1899.06 & 0.265013 & NU3 \\
\hline 4240.00 & 0.2369 & 0.0717 & 7 & 5 & 2 & 6 & 1 & 5 & 542.91 & 0.00 .2023 & NU3 \\
\hline $4241 \cdot 78$ & 0.0040 & 0.0423 & 13 & 5 & 9 & 12 & 2 & 10 & 1960.22 & 0.40461 & NU1 \\
\hline $4242 \cdot 86$ & 0.2336 & 0.0631 & 12 & 4 & 8 & 11 & 2 & 9 & $1690 \cdot 70$ & 0.209597 & $\mathrm{NU}_{3}$ \\
\hline 4243.89 & 0.0314 & 0.0703 & 5 & 5 & 1 & 4 & 1 & 4 & 224.83 & 0.000018 & NU3 \\
\hline 4249.07 & 0.0079 & 0.0642 & 7 & 5 & 3 & 6 & 0 & 6 & 446.71 & 0.000443 & NU1 \\
\hline 4249.95 & 0.0139 & 0.0668 & 13 & 6 & 7 & 12 & 4 & 8 & 2205.65 & 0.394450 & NU3 \\
\hline $4250 \cdot 24$ & 0.0635 & 0.0590 & 9 & 6 & 3 & 8 & 3 & 6 & $1006 \cdot 12$ & 0.019449 & NU1 \\
\hline 4250.62 & 0.2534 & 0.0571 & 11 & 3 & 8 & 10 & 1 & 9 & 1293.04 & 0.091645 & NU 3 \\
\hline 4250.91 & 1.6355 & 0.0511 & 10 & 2 & 8 & 9 & 0 & 9 & $920 \cdot 18$ & 0.030629 & NU3 \\
\hline $4251 \cdot 43$ & 0.0013 & 0.0676 & 8 & 5 & 3 & 7 & 1 & 6 & 704.22 & 0.004077 & NU $3 *$ \\
\hline 4251.63 & 0.1789 & 0.0523 & 9 & 7 & 2 & 8 & 5 & 3 & 1255.92 & 0.053971 & NU 3 \\
\hline 4252.19 & 0.0550 & 0.0763 & 9 & 4 & 5 & 8 & 1 & 8 & $744 \cdot 16$ & 0.004551 & NU1 \\
\hline 4252.35 & 0.5368 & 0.0566 & 11 & 5 & 7 & 10 & 3 & 8 & $1446 \cdot 16$ & 0.140157 & NU 3 \\
\hline 4252.39 & 0.5391 & 0.0512 & 9 & 7 & 3 & 8 & 5 & 4 & $1255 \cdot 16$ & 0.053898 & NU3 \\
\hline 4254.00 & 0.0195 & 0.0382 & 12 & 2 & 10 & 11 & 1 & 11 & 1327.14 & 0.089480 & NU1 \\
\hline 4255.71 & 0.5378 & 0.0482 & 10 & 3 & 8 & 9 & 1 & 9 & 920.21 & 0.030178 & NU3 \\
\hline 4255.86 & 0.0585 & 0.0367 & 12 & 3 & 10 & 11 & 0 & 11 & $1327 \cdot 14$ & 0.089303 & NU1 \\
\hline $4256 \cdot 26$ & 0.0192 & 0.0464 & 13 & 3 & 10 & 12 & 2 & 11 & 1774.74 & 0.27474 & NU1 \\
\hline 4257.57 & 0.0075 & 0.0553 & 13 & 6 & 8 & 12 & 3 & 9 & 2105.90 & 0.37068 & NU1 \\
\hline 4259.40 & 0.0023 & 0.0502 & 9 & 6 & 3 & 9 & 2 & 8 & $1080 \cdot 38$ & 0.000288 & NU 3 \\
\hline 4259.55 & 0.0604 & 0.0398 & 8 & 8 & 1 & 7 & 6 & 2 & $1216 \cdot 20$ & 0.014826 & NU3 \\
\hline 4259.55 & 0.1810 & 0.0398 & 8 & 8 & 0 & 7 & 6 & 1 & $1216 \cdot 20$ & 0.014826 & NU3 \\
\hline $4262 \cdot 18$ & 0.0037 & 0.0610 & 7 & 7 & 1 & 6 & 4 & 2 & 757.78 & 0.000981 & NU1 \\
\hline 4263.21 & 0.0111 & 0.0570 & 7 & 7 & 0 & 6 & 4 & 3 & 756.75 & 0.000979 & NU1 \\
\hline 4264.36 & 0.2809 & 0.0574 & 11 & 6 & 6 & 10 & 4 & 7 & 1581.34 & 0.142262 & NU3 \\
\hline 4265.40 & 0.0059 & 0.0381 & 13 & 4 & 10 & 12 & 1 & 11 & 1774.60 & 0.25444 & NU1 \\
\hline 4269.82 & 0.6860 & 0.0499 & 11 & 4 & 8 & 10 & 2 & 9 & 1293.66 & 0.082847 & NU3 \\
\hline $4271 \cdot 38$ & 0.2618 & 0.0548 & 10 & 7 & 3 & 9 & 5 & 4 & 1477.31 & 0.079027 & NU3 \\
\hline 4271.56 & 0.0465 & 0.0612 & 10 & 5 & 6 & 9 & 2 & 8 & $1080 \cdot 38$ & 0.0617 & NUI+ \\
\hline 4272.28 & 0.0024 & 0.0465 & 12 & 3 & 9 & 12 & 1 & 12 & 1557.91 & 0.001084 & NU3 \\
\hline 4272.82 & 0.6444 & 0.0676 & 8 & 5 & 3 & 7 & 1 & 6 & $704 \cdot 22$ & 0.004077 & NU3 \\
\hline 4273.70 & 0.0882 & 0.0524 & 10 & 7 & 4 & 9 & 5 & 5 & 1474.99 & 0.078635 & NU3 \\
\hline 4275.56 & 0.5363 & 0.0696 & 8 & 4 & 4 & 7 & 0 & 7 & $586 \cdot 26$ & 0.001880 & NU3 \\
\hline 4276.40 & 0.0023 & 0.0533 & 9 & 5 & 4 & 9 & 1 & 9 & 920.21 & 0.000130 & NU3 \\
\hline 4278.41 & 0.0015 & 0.0785 & 6 & 6 & 1 & 5 & 1 & 4 & 399.46 & 0.000022 & NU1 \\
\hline 4280.86 & 0.0113 & 0.0543 & 10 & 6 & 4 & 9 & 3 & 7 & 1216.27 & 0.029596 & NU1 \\
\hline $4281 \cdot 82$ & 0.0170 & 0.0659 & 8 & 7 & 2 & 7 & 4 & 3 & $931 \cdot 22$ & 0.003551 & NU1 \\
\hline
\end{tabular}




\begin{tabular}{|c|c|c|c|c|c|c|c|c|c|c|c|}
\hline $\begin{array}{l}\text { FREQUENCY } \\
\mathrm{cm}^{-1}\end{array}$ & $\begin{array}{l}\text { LINE } \\
\text { STRENGTH } \\
\frac{\mathrm{cm}^{-1}}{\mathrm{gm} \mathrm{cm}^{-2}}\end{array}$ & $\begin{array}{l}\text { HALF } \\
\text { WIDTH } \\
\mathrm{cm}^{-1} \\
\text { atm. }\end{array}$ & $J^{\prime}$ & $K_{a}$ & $K_{c}$ & $J^{\prime \prime}$ & $k_{0}$ & $k_{c}$ & $\begin{array}{c}\mathrm{E}^{\prime \prime} \\
\mathrm{cm}^{-1}\end{array}$ & $\mathrm{~L}$ & BAND \\
\hline 4282.48 & 0.0492 & 0.0425 & 9 & 8 & 1 & 8 & 6 & 2 & 1411.68 & 0.032025 & NU3 \\
\hline & & 0.0424 & 9 & 8 & 2 & 8 & 6 & 3 & & & NU3 \\
\hline 4283.88 & 0.0015 & 0.0554 & 8 & 7 & 1 & 8 & 3 & 6 & $1006 \cdot 12$ & 0.000044 & $\mathrm{~N} \cup 3$ \\
\hline 4285.27 & 0.0057 & 0.0579 & 8 & 7 & 1 & 7 & 4 & 4 & 927.77 & 0.003511 & NUI \\
\hline 4286.89 & 0.0230 & 0.0685 & 6 & 5 & 2 & 5 & 1 & 5 & 326.64 & 0.000066 & NU3 \\
\hline 4287.72 & 0.0539 & 0.0513 & 12 & 5 & 8 & 11 & 3 & 9 & 1695.03 & 0.143644 & NU3 \\
\hline 4287 & 0.0033 & 0.0474 & 10 & 6 & 4 & 10 & 2 & 9 & .66 & 0.000402 & NU3 \\
\hline 4288.73 & 0.0350 & 0.0569 & 11 & 7 & 4 & 10 & 5 & 5 & .72 & 0.10 & NU3 \\
\hline 4290. & 0.0303 & 0.0546 & 12 & 6 & 7 & 11 & 4 & 8 & .02 & 0.16 & NU3 \\
\hline 4294.58 & 0.1094 & 0.0504 & 11 & 7 & 5 & 10 & 5 & 6 & .77 & 0.10 & NU3 \\
\hline 4295.78 & 0.0199 & 0.0563 & 13 & 4 & 9 & 12 & 2 & 10 & $\cdot 22$ & 0.18 & NU3 \\
\hline 4298.14 & 0.0051 & 0.0708 & 9 & 7 & 3 & 8 & 4 & 4 & .76 & 0.00 & NUI \\
\hline 4302.53 & 0.0012 & 0.0497 & 10 & 7 & 3 & 10 & 3 & 8 & .16 & 10 & NU3 \\
\hline 4302.83 & 0.0393 & 0.0584 & 12 & 7 & 5 & 11 & 5 & 6 & .02 & 0.14 & $N \cup 3$ \\
\hline 4303.17 & 0.0172 & 0.0567 & 8 & 5 & 4 & 7 & 0 & 7 & $\cdot 26$ & 0.00 & NU1 \\
\hline 4303.84 & 0.0018 & 0.0728 & 7 & 6 & 2 & 6 & 1 & 5 & 542.91 & 0.00 & NUI \\
\hline 4304.27 & 0.1885 & $0.044 \mathrm{C}$ & 11 & 2 & 9 & 10 & 0 & 10 & .55 & 0.02 & NU3 \\
\hline $4304 \cdot 45$ & 0.0811 & 0.0448 & 10 & 8 & 2 & 9 & 6 & 3 & .41 & 0.05 & NU3 \\
\hline $4304 \cdot 59$ & 0.0271 & 0.0445 & 10 & 8 & 3 & 9 & 6 & 4 & $\cdot 27$ & 0.05 & $\mathrm{NU} 3$ \\
\hline 4305.32 & 0.2121 & 0.0489 & 12 & 3 & 9 & 11 & 1 & 10 & .87 & 0.08 & NU3 \\
\hline 4305.46 & 0.0278 & 0.0364 & 9 & 9 & 1 & 8 & 7 & 2 & 1590.74 & 0.01 & NU3 \\
\hline 4305.51 & 0.0092 & 0.0364 & 9 & 9 & 0 & 8 & 7 & 1 & 74 & 0.01 & NU3 \\
\hline 4306.69 & 0.0179 & 0.0348 & 13 & 2 & 11 & 12 & 1 & 12 & 91 & 0.0 & NUI \\
\hline 4306.71 & 0.5629 & 0.0421 & 11 & 3 & 9 & 10 & 1 & 10 & .56 & 89 & NU3 \\
\hline 4307.18 & 0.0153 & 0.0578 & 9 & 7 & 2 & 8 & 4 & 5 & .72 & 0.00 & NUI \\
\hline 4307.49 & 0.0059 & 0.0342 & 13 & 3 & 11 & 12 & 0 & 12 & 91 & 0.08 & NU1 \\
\hline 4310.39 & 0.0101 & 0.0742 & 10 & 7 & 4 & 9 & 4 & 5 & $\cdot 28$ & 0.01 & NU1 \\
\hline $\begin{array}{l}4313 \\
4373\end{array}$ & 0.0157 & 0.0665 & 6 & $\begin{array}{l}6 \\
5\end{array}$ & 0 & $\begin{array}{l}5 \\
8\end{array}$ & 2 & $\begin{array}{l}3 \\
7\end{array}$ & $\begin{array}{r}50 \\
.02\end{array}$ & 0.00 & NU3 \\
\hline 4313 & 0.1226 & 0.0634 & & 5 & 4 & 8 & 1 & 7 & 93 & 0.00 & NU3 \\
\hline 4315.72 & 0.0126 & 0.0513 & 12 & 7 & 6 & 11 & 5 & 7 & .83 & 0.13 & NU3 \\
\hline 4315.99 & 0.0139 & 0.0508 & 11 & 6 & 5 & 10 & 3 & 8 & $\cdot 16$ & 0.03 & NU1 \\
\hline 43 & 0.0682 & 0.0446 & 12 & 4 & 9 & 11 & 2 & 10 & .13 & 0.07 & NU3 \\
\hline 43 & 0.0018 & 0.0753 & 11 & 7 & 5 & 10 & 4 & 6 & . 51 & 0.03 & NUI \\
\hline 9.65 & 0.0196 & 0.0505 & 13 & 6 & 8 & 12 & 4 & 9 & - 98 & 0.18 & NU3 \\
\hline 4322.40 & 0.0028 & 0.0737 & 12 & 7 & 6 & 11 & 4 & 7 & .06 & 0.06 & NU1 \\
\hline 4325.31 & 0.0108 & 0.0464 & 11 & 8 & 3 & 10 & 5 & 4 & $\cdot 53$ & 4997 & NU3 \\
\hline 4325.84 & 0.0325 & 0.0454 & 11 & 8 & 4 & 10 & 6 & 5 & 1875.00 & 0.074947 & NU3 \\
\hline 432 & 0.0167 & 0.0681 & 7 & 6 & 1 & 6 & 2 & 4 & 77 & 89 & NU3 \\
\hline 432 & 022 & 0.0526 & 10 & 5 & 5 & 10 & 1 & 10 & .56 & 09 & NU3 \\
\hline 432 & 135 & 0.0638 & 11 & 5 & 6 & 10 & 2 & 9 & .66 & 174 & NU1 \\
\hline 43 & 068 & 0.0383 & 10 & 9 & 2 & 9 & 7 & 3 & 63 & +44 & NU3 \\
\hline 4328.73 & 0.0204 & 0.0384 & 10 & 9 & 1 & 9 & 7 & 2 & 181 & 0.02 & NU3 \\
\hline 43 & 0.0045 & 0.0755 & 10 & 4 & 6 & 9 & 1 & 9 & .21 & 27 & NU1 \\
\hline 4329.78 & 0.0033 & 0.0556 & 10 & 7 & 3 & $9^{\prime}$ & 4 & 6 & 89 & 23 & $N \cup 1$ \\
\hline 4329.79 & 0.0362 & 0.0464 & 13 & 5 & 9 & 12 & 3 & 10 & .55 & 0.13 & NU3 \\
\hline $4335 \cdot 30$ & 0.0082 & 0.0627 & 8 & 6 & 3 & 7 & 1 & 6 & 704.22 & 0.000543 & NU1 \\
\hline 4335.43 & 0.0755 & 0.0644 & 7 & 5 & 3 & 6 & 1 & 6 & $\cdot 24$ & 0.00 & NU3 \\
\hline 4340.14 & 0.0874 & 0.0682 & 8 & 6 & 2 & 7 & 2 & 5 & $782 \cdot 40$ & 0.00 & NU3 \\
\hline 4342.92 & 0.0546 & 0.0654 & 9 & 4 & 5 & 8 & 0 & 8 & 744.09 & 0.00 & NU3 \\
\hline $4343 \cdot 62$ & 0.0038 & 0.0631 & 6 & 6 & 1 & 5 & 2 & 4 & $416 \cdot 22$ & 0.000017 & NU3 \\
\hline 4345.08 & 0.0111 & 0.0474 & 12 & 8 & 4 & 11 & 6 & 5 & $2144 \cdot 12$ & 0.101574 & NU3 \\
\hline 4345.85 & 0.0032 & 0.0436 & 10 & 1 & 0 & 9 & 8 & 1 & 2009.87 & 0.014608 & $\mathrm{~N} \cup 3$ \\
\hline $4346 \cdot 51$ & 0.0037 & 0.0462 & 12 & 8 & 5 & 11 & 6 & 6 & 2142.69 & 0.101336 & NU3 \\
\hline 4349.01 & 0.0020 & 0.0490 & 8 & 8 & 1 & 7 & 5 & 2 & 1059.85 & 0.000796 & $\mathrm{NUI}$ \\
\hline
\end{tabular}




\begin{tabular}{|c|c|c|c|c|c|c|c|c|c|c|c|}
\hline $\begin{array}{c}\text { FREQUENCY } \\
\mathrm{cm}^{-1}\end{array}$ & 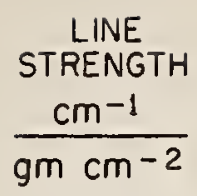 & $\begin{array}{l}\text { HALF } \\
\text { WIDTH } \\
\frac{\mathrm{cm}^{-1}}{\text { atm. }}\end{array}$ & $J^{\prime}$ & $K_{0}$ & $K_{c}$ & J" & $K_{a}$ & $\mathrm{~K}_{\mathrm{c}}$ & $\begin{array}{c}E^{\prime \prime} \\
\mathrm{cm}^{-1}\end{array}$ & $L$ & BAND \\
\hline 4349.39 & 0.0086 & 0.0510 & 14 & 4 & 10 & 13 & 2 & 11 & 2246.98 & 0.160530 & NU3 \\
\hline 4350.78 & 0.0033 & 0.0450 & 14 & 6 & 9 & 13 & 4 & 10 & 2426.22 & 0.186890 & NU3 \\
\hline $4351 \cdot 15$ & 0.0083 & 0.0398 & 11 & 9 & 3 & 10 & 7 & 4 & 2054.40 & 0.050502 & NU3 \\
\hline 4351.15 & 0.0028 & 0.0399 & 11 & 9 & 2 & 10 & 7 & 3 & 2054.40 & 0.050503 & $\mathrm{NU}_{3}$ \\
\hline 4353.16 & 0.0051 & 0.0522 & 11 & 7 & 4 & 10 & 4 & 7 & $1581 \cdot 34$ & 0.026914 & NU 1 \\
\hline 4356.13 & 0.1801 & 0.0389 & 12 & 2 & 10 & 11 & 0 & 11 & 1327.14 & 0.025215 & NU 3 \\
\hline 4356.22 & 0.0015 & 0.0496 & 12 & 6 & 6 & 11 & 3 & 9 & 1695.03 & 0.041150 & NU 1 \\
\hline 4356.87 & 0.0326 & 0.0655 & 9 & 6 & 3 & 8 & 2 & 6 & 982.91 & 0.002446 & NU3 \\
\hline 4357.49 & 0.0599 & 0.0379 & 12 & 3 & 10 & 11 & 1 & 11 & 1327.14 & 0.025171 & NU3 \\
\hline 4357.52 & 0.0016 & 0.034 & 14 & 2 & 12 & 13 & 1 & 13 & 1806.78 & 0.08004 & NUI \\
\hline 4358.02 & 0.0049 & 0.034 & 14 & 3 & 12 & 13 & 0 & 13 & 1806.78 & 0.07 & NUI \\
\hline 4358.14 & 0.0540 & 0.0438 & 13 & 3 & 10 & 12 & 1 & 11 & 1774.60 & 0.074330 & NU3 \\
\hline 4362.97 & 0.0028 & 0.0481 & 13 & 8 & 5 & 12 & 6 & 6 & 2437.56 & 0.150080 & NU3 \\
\hline 4363.05 & 0.1431 & 0.0597 & 10 & 5 & 5 & 9 & 1 & 8 & 1079.07 & 0.0057 & NU3 \\
\hline 4364.20 & 0.0030 & 0.0524 & 9 & 5 & 5 & 8 & 0 & 8 & 744.09 & 0.000727 & $\mathrm{NU} 1$ \\
\hline 4364.24 & 0.1541 & 0.0402 & 13 & 4 & 10 & 12 & 2 & 11 & 1774.74 & 0.0706 & NU3 \\
\hline 4366.67 & 0.0021 & 0.0457 & 13 & 8 & 6 & 1 & 6 & 7 & 2433.85 & 0.037 & NU3 \\
\hline 4369.22 & 0.0017 & 0.0549 & 11 & 10 & 2 & 10 & 8 & 3 & 2254.34 & 0.030 & NU3 \\
\hline 4370.08 & 0.0027 & 0.0495 & 9 & 8 & 1 & 8 & 5 & 4 & 1255.16 & 0.00 & NU \\
\hline 4372.96 & 0.0039 & 0.0410 & 12 & 9 & 3 & 11 & 7 & 4 & 2321.94 & 0.07 & NU \\
\hline 4373.01 & 0.0013 & 0.0390 & 12 & 9 & 4 & 11 & 7 & 5 & 2321.89 & 0.07 & $\mathrm{~N} \cup 3$ \\
\hline 4373.31 & 0.0024 & 0.0549 & 9 & 6 & 4 & 8 & 1 & 7 & 882.93 & 0.00 & NU \\
\hline 4374.44 & 0.0023 & 0.0440 & 14 & 5 & 10 & 13 & 3 & 11 & 2248.16 & 0.12 & NU \\
\hline 4376.13 & 0.0264 & 0.0617 & 7 & 6 & 2 & 5 & 2 & 5 & 2.92 & 0.00 & NU \\
\hline 4379.59 & 0.0721 & 0.0625 & 10 & 6 & 4 & 9 & 2 & 7 & 120 & 0.00 & NU \\
\hline 4388.29 & 0.0021 & 0.0559 & 10 & 8 & 3 & 9 & 5 & 4 & 14 & 0.0 & NU \\
\hline 4388.87 & 0.0010 & 0.0662 & 12 & 5 & 7 & 11 & 2 & 10 & 152 & 0.0 & NU \\
\hline 4389 & 0.0177 & 0.0592 & 8 & 5 & 4 & 7 & 1 & 7 & 58 & 0.00 & NU \\
\hline 4407 & 0.0170 & 0.0357 & 13 & 2 & 11 & 12 & 0 & 12 & 155 & 0.02 & NU \\
\hline 4407.50 & 0.0510 & 0.0350 & 13 & 3 & 11 & 12 & 1 & 12 & 155 & 0.023120 & NU \\
\hline 4408.80 & 0.0135 & 0.0410 & 14 & 3 & 11 & 1 & 1 & 12 & 204 & 0.06 & NU \\
\hline 4409.75 & 0.0118 & 0.0578 & 11 & 6 & 5 & 1 & 2 & 8 & 3.00 & 0.008540 & NU \\
\hline 4410.03 & 0.0028 & 0.0728 & 11 & 4 & 7 & 10 & 1 & 10 & +.56 & 0.001448 & NU \\
\hline 4411.21 & 0.0012 & 0.0504 & 11 & 8 & 3 & 1 & $\begin{array}{l}1 \\
5\end{array}$ & 6 & 1718.77 & 0.012538 & NU \\
\hline 4411.92 & 0.0048 & 0.0370 & 14 & 4 & 11 & 13 & 2 & 12 & $2042 \cdot 38$ & 0.065890 & NU \\
\hline 4412.77 & 0.0105 & 0.0586 & 8 & 6 & 3 & 7 & 2 & 6 & 709.60 & 0.000198 & NU? \\
\hline 4414.80 & 0.0037 & 0.0497 & 10 & 5 & 6 & 9 & 0 & 9 & 920.18 & 0.000705 & NU \\
\hline 4417.92 & 0.0042 & 0.0481 & 10 & 6 & 5 & 9 & 1 & 8 & 1079.07 & 0.001773 & NU \\
\hline $4421 \cdot 53$ & 0.0134 & 0.0571 & 11 & 5 & 6 & 10 & 1 & 9 & 1293.04 & 0.004657 & NU \\
\hline 4435.13 & 0.0399 & 0.0617 & 10 & 4 & 6 & 9 & 0 & 9 & 920.18 & 0.000715 & NU \\
\hline
\end{tabular}





\title{
Part II. Atlas of Computed Transmission Spectra for 2.7 $\mu \mathrm{H}_{2} \mathrm{O}$ Band
}

\author{
David M. Gates, Robert F. Calfee, and David W. Hansen
}

\begin{abstract}
Theoretical transmission spectra of the $2.7 \mu$ atmospheric water vapor band at infinite spectral resolution have been determined for specific values of pressure, temperature, and water vapor concentration. A triangular slit function has been applied to the theoretical spectra to show the effect of slit width upon the resolution of an actual instrument. Both the theoretical and "degraded" spectra are displayed in graphic form.
\end{abstract}

\section{Introduction}

The transparency of the Earth's atmosphere to infrared radiation of various frequencies is of great importance to astronomers, meteorologists, atmospheric physicists, communications engineers, ecologists, and others. The infrared spectral region covers a broad wavelength span from $7000 \AA$ or $0.7 \mu$ to $1000 \mu$ or $1 \mathrm{~mm}$, and a frequency span from $14,300 \mathrm{~cm}^{-1}\left(4.28 \times 10^{14} \mathrm{c} / \mathrm{s}\right)$ to $10 \mathrm{~cm}^{-1}(3.0 \times$ $\left.10^{11} \mathrm{c} / \mathrm{s}\right)$. The infrared region spans 10.5 octaves compared with the visible which spans less than 1 octave. If the atmosphere were transparent this would present a magnificent opportunity for communication beyond the very crowded radiofrequency region and beyond the very narrow optical region of the electromagnetic spectrum. Both fortunately and unfortunately, the Earth's atmosphere possesses many strong and broad absorption bands throughout the infrared spectrum due to water vapor, carbon dioxide, ozone, and weaker absorption bands arising from methane, nitrous oxide, heavy water, and other very minor constituents. Sunlight is absorbed by these gases in the atmosphere and the atmosphere reradiates this heat toward space and toward the ground. The climate of the Earth is greatly modified by this absorbed radiation. Without this warmth the Earth's surface would become intensely cold at night, and without the shielding affect of the atmosphere from the infrared solar radiation the daylight side would become very much hotter.

The location of many of the absorption bands at infrared frequencies are seen in figure 1 , which represents a direct recording of solar radiation transmitted by the atmosphere. Although the absorption bands are numerous, there are "windows" between some of these bands where radiation passes relatively unattenuated through the atmosphere. The best window of the entire infrared spectrum occurs at 11.08 microns, but other windows are good, particularly when the path length is not too great. A detailed analysis of the spectrum shown in figure 1 giving attenuation coefficients as a function of the frequency has been given by Gates [1960] and by Gates and Harrop [1963j.

The absorption spectrum is actually very much more complex than it would appear to be in figure 1. Each absorption band when fully resolved is seen to be comprised of many hundreds of individual absorption lines. A typical segment of the highly resolved absorption spectrum is shown in figure 2 as taken from the Migeotte Atlas [1956]. A thorough understanding of the infrared transmission by the atmosphere involves a detailed analysis of the high resolution spectrum. This requires that one deal with each and every line in the spectrum and with the simultaneous collective effect of the wings of many lines on the transmission of the atmosphere at any specific frequency. The precise nature of the observed spectrum will depend upon the instrument used for the observation, the spectrum being degraded by the influence of the slit or instrument factor. Before a careful analysis can be made of the spectral transmission as observed, it is first necessary to establish in detail the theoretical spectral transmission, which would be recorded with an instrument of infinite resolving power. This requires that one calculate the detailed absorption spectrum for each of the major absorbing molecules present in the Earth's atmosphere. Since water vapor is one of the most important constituents, these calculations have been performed for this molecule first.

Calculations have been made of the line positions, line strengths, and line half-widths of the water vapor spectrum from the quantum mechanical analysis of the asymmetric rotator. These results have been presented in tabular form by Gates, Calfee, Hansen, and Benedict [1963] for the frequency span 2857 to $4444 \mathrm{~cm}^{-1}$. 


\section{Transmission}

The monochromatic transmission at a frequency $\nu$, of $\mathrm{m} \mathrm{g} \mathrm{cm}{ }^{-2}$ of absorbing gas at a constant pressure and temperature is

$$
T_{\nu}(m)=\exp \left(-k_{\nu} m\right)
$$

where $k_{0}$ is the monochromatic absorption coefficient.

Each absorption line is affected by collisions which shorten the natural lifetime of excited vibrational states. The following Lorentz formula is taken to reasonably represent the spectral line shape which gives the monochromatic absorption coefficient in the following form:

$$
k_{\nu}=\frac{S}{\pi} \frac{\alpha}{\left[\left(\nu-\nu_{0}\right)^{2}+\alpha^{2}\right]}
$$

where $\nu_{0}$ is the frequency of the line center, $S$ is the line strength which depends upon temperature according to

$$
S=S_{0}\left(\frac{T_{0}}{T}\right)^{3 / 2} \exp \left[\frac{-E^{\prime \prime}}{k}\left(\frac{T_{0}-T}{T T_{0}}\right)\right]
$$

$\alpha$ is the half-width of the line which depends upon pressure and temperature in the following manner:

$$
\alpha=\alpha_{0}\left(\frac{P}{P_{0}}\right)\left(\frac{T_{0}}{T}\right)^{n}
$$

where $P / P_{0}$ is the total pressure of the gas in atmospheres, $T$ the absolute temperature, and $n$ depends upon the nature of the broadening gas and the frequency of the transition. For $\mathrm{N}_{2-}$ $\mathrm{H}_{2} \mathrm{O}$ collisions, Benedict and Kaplan [1959] show $n=0.62$ to be a good representative value. Here $S_{0}$ and $\alpha_{0}$ refer to the strength and half-width, respectively, calculated at a reference temperature $T_{0}$.

The line strength, $S$, is composed of vibrational and rotational contributions, $S_{r}$ and $S_{v}$, determined by

$$
S=\frac{S_{r} S_{v}}{Q_{r}} \frac{\nu}{\nu_{0}}
$$

The central frequency of the band is $\nu_{0}$. The rotational factor $S_{\tau}$ for a particular rotational transition is

$$
S_{r}=L g \exp \left(-E^{\prime \prime} / k T\right)
$$

where $L$ is the rotational transition strength defined to be the sum of the squares of the magnitude of the direction cosine matrix elements evaluated for the asymmetric rotator. A full discussion of the evaluation of $L$ for a rigid asymmetric rotator has been given by Cross, Hainer, and King [1944]. Schwendeman and Laurie [1958] have published a very useful table giving values of $L$ as a function of the asymmetry parameter, $\kappa$, for all transitions up to $J=12$. Wacker and Pratto [1963] have extended the computations of $L$ to higher $J$ values. For the ground state of $\mathrm{H}_{2} \mathrm{O}, \kappa=-0.4377$ was used for the present computations.

The statistical weighting factor $g$ depends upon the symmetry of the ground energy level $E^{\prime \prime}$ [Townes and Schawlow, 1955].

The rotational partition function $Q_{r}$ is given by

$$
Q_{r}=\sum_{J}(2 J+1) g \exp \left(-E^{\prime \prime} \mid k T\right) .
$$

The vibrational transition probability, $S_{v}$, is common to all lines in a band.

However, the transmission at any frequency in the spectrum depends not only on the shape, halfwidth, and strength of the nearest line, but is also influenced by the wings of many dozens of lines on either side. Therefore, to obtain the actual absorption coefficient at any frequency, it is necessary to sum the influence of many lines as follows:

$$
k(\nu)=\sum_{\nu_{0}} k_{\nu}=\sum_{\nu_{0}} \frac{S}{\pi} \frac{\alpha}{\left[\left(\nu-\nu_{0}\right)^{2}+\alpha^{2}\right]} .
$$

The transmission for a given pressure, temperature, and water vapor concentration is now given as a function of the frequency $\nu$,

$$
T(\nu)=\exp [-k(\nu) m] \text {. }
$$

The total absorption across an entire band is invariant to the spectral slit width of the instrument [Nielson, Thornton, and Dale, 1944]. For this reason the total band absorption is often given by experimentalists rather than the specific absorption within a narrow spectral interval. The total band absorption is given by

$$
A=\int_{\nu_{1}}^{\nu_{2}} A(\nu) d \nu=\int_{\nu_{1}}^{\nu_{2}}[1-T(\nu)] d \nu
$$

where $\nu_{1}$ and $\nu_{2}$ are the frequency limits for the band.

\section{Computational Procedure}

It was desired to compute the transmission as a function of the frequency across a definite frequency interval for a given pressure, temperature, and water vapor concentration. To avoid any end effects, the computation was extended to include the strong lines out to $150 \mathrm{~cm}^{-1}$ on either side of the interval selected. Starting with the first point of the interval, the transmission was computed by calculating directly the contributions of all lines by means of the Lorentz line shape formula. This was a straight sum of the $k_{\nu}$ 's at this position. The frequency value was then 
increased by a fixed increment, $0.01 \mathrm{~cm}^{-1}$, and the transmission again calculated. When the point came within $0.05 \mathrm{~cm}^{-1}$ of a line center, the increment was decreased to $0.0005 \mathrm{~cm}^{-1}$ in order to gain the full influence of the line on the calculations. Furthermore, at the position of each line the influence of all other lines was summed up to give an added effective strength to the single line absorption coefficient. By using a coarse increment when far from line centers and a fine increment when close, a marked saving of computer time was realized. After calculating the transmissions throughout the total interval, the total absorption was calculated using Simpson's rule, taking into account the two sizes of step intervals.
The computed theoretical water vapor spectra for the frequency interval 3500 to $4000 \mathrm{~cm}^{-1}$ are shown in figure 3 for a pressure of 0.1 atmosphere and figure 4 for a pressure of 1.0 atmosphere at a temperature of $287.7^{\circ} \mathrm{K}$. The water vapor concentration was changed in certain sections of the spectrum in order to display the line structure to better advantage. The values of water vapor concentration are indicated on each plate in figures 3 and 4 .

The necessary data for computation are the pressure in atmospheres, the water vapor concentration in precipitable centimeters, and the absolute temperature along with the line positions, line strengths, half-widths, and energy values of the initial state.

\section{Degraded Spectra}

A spectrometer scanning an absorption spectrum will not record it with the sharp detail of the natural line shape but will degrade the spectrum because of finite slit widths and limited dispersion of the grating or prism. Since all observed spectra are of this degraded nature to various degrees, it is desirable to modify the theoretical spectrum described earlier with a slit function or instrument factor. Several authors have discussed slit factors, including Dennison [1928], Nielsen, Thornton, and Dale [1944], Kostkowski and Bass [1956], and Rautian [1958].

The slit function, $\rho(\nu)$, is defined according to the degraded transmission $\bar{T}_{v_{i}}$ recorded by the spectrometer at a frequency, $\nu_{i}$, as follows:

$$
\bar{T}_{\nu_{l}}=\frac{\int_{\nu_{i}-a}^{\nu_{i}+a} \rho(\nu) T_{\nu} d \nu}{\int_{\nu_{i}-a}^{\nu_{i}+a} \rho(\nu) d \nu}
$$

where $T_{\nu}$ is the theoretical transmission at infinite resolving power at any frequency $\nu, \nu_{i}$ is the frequency at the center of the spectral interval being momentarily scanned by the exit slit, and $a$ is the spectral slit width (defined as one-half of the total frequency span passed by the spectrometer slits).

Various approximations to the slit function can be derived such as a Gaussian distribution, a trapezoidal type of distribution, and a triangular function. The simplicity of the triangular function and the fact that it represents reasonably well the slit distortions across the central maximum make it attractive for computational purposes. The triangular slit function was used in the following form:

$$
\left.\rho^{\prime} \nu\right)=a-\left|\nu-\nu_{i}\right| \text {. }
$$

To compute the degraded spectrum from the theoretical spectrum, the triangle slit function was superimposed upon the theoretical spectrum.
Then the average transmission was calculated across the frequency span passed by the slit. The slit function was shifted across the spectrum by increments of approximately 0.2 to $0.4 \mathrm{~cm}^{-1}$, depending upon the slit width used, and at each position the average transmission across the slit was computed, using (8). The averaging process within the spectral interval was calculated in steps of $0.05 \mathrm{~cm}^{-1}$.

The theoretical water vapor spectra of figures 3 and 4 covering the frequency range from 3500 to $4000 \mathrm{~cm}^{-1}$ are shown in degraded form in figures 5 to 7 . The degraded spectra were calculated for spectral slit widths, $0.5 \mathrm{~cm}^{-1}$ and 1.0 $\mathrm{cm}^{-1}$, or a full slit span of $1 \mathrm{~cm}^{-1}$ and $2 \mathrm{~cm}^{-1}$. Again pressures of 0.1 and 1.0 atmosphere were used at a temperature of $287.7^{\circ} \mathrm{K}$.

Within the figures on a single plate the effect of the difference in water concentration can be seen. By a comparison of figures 5 and 6 , the pressure effect on the spectrum can be observed since the same spectral slit width was used for each. Figures 6 and 7 show the effect of a change in slit width at constant pressure. These figures are presented as examples of the type of theoretical analysis possible. ${ }^{2}$ Given any pressure, temperature, and water vapor concentration, the theoretical spectrum can be computed. With additional information about spectral slit conditions, this theoretical spectrum can be degraded to any degree.

Figure 8 shows a small portion of the spectrum degraded by a triangular half slit width of 0.05 $\mathrm{cm}^{-1}$. As narrower slit widths are used (below about $0.1 \mathrm{~cm}^{-1}$ ) the triangular function becomes more and more inadequate as a slit function. This figure is included to show the type of spectral structure to be expected from a high resolution spectrometer. For a more correct presentation of the narrow slit spectrum, a more refined slit function, e.g., a Gaussian function, could be used.

\footnotetext{
2 Spectral data for any specific region in the $2.7 \mu \mathrm{H}_{2} \mathrm{O}$ band and any conditions of pressure, temperature, and slit conditions can be furnished in either graphic form or as numerical tables.
} 
The authors particularly thank W. S. Benedict of Johns Hopkins University and the National Bureau of Standards, Washington, D.C., for his invaluable assistance in providing the necessary information for determining the line positions, strengths, and half-widths required for the above analysis. The authors acknowledge the assistance of Charles C. Runner in preparing the figures.

\section{References}

Benedict, W. S., and L. D. Kaplan (1959), Calculations of line widths in $\mathrm{H}_{2} \mathrm{O}-\mathrm{N}_{2}$ collisions, J. Chem. Phys. 30, 388-399.

Cross, Paul C., R. M. Hainer, and Gilbert W. King (1944), The asymmetric rotor, J. Chem. Phys. 12, 210-243.

Dennison, D. M. (1928), The shape and intensities of infrared absorption lines, Phys. Rev. 31, 503-519.

Gates, D. M. (1960), Near infrared atmospheric transmission of solar radiation, J. Opt. Soc. Amer. 50, 1299-1304.

Gates, D. M., Robert F. Calfee, David W. Hansen, and W. S. Benedict (1963), Line positions, strengths, and half-widths for water vapor bands $\nu_{1}, 2 \nu_{2}$, and $\nu_{3}$ in the interval 2857 to $4444 \mathrm{~cm}^{-1}$, NBS Monograph No. 71, part I.

Gates, D. M., and Walter J. Harrop (1963), Infrared transmission of the atmosphere to solar radiation, Appl. Optics, 2, No. 9, 887-898.
Kostkowski, H. J., and A. M. Bass (1956), Silt function effects in the direct measurement of absorption line halfwidths and intensities, J. Opt. Soc. Amer. 46, 1060-1064.

Migeotte, M., L. Neven, and J. Swensson (1956), The solar spectrum from 2.8 to 23.7 microns, Part I, Photometric Atlas, University of Liège.

Nielsen, J. Rud, V. Thornton, and E. Brock Dale (1944), The absorption laws for gases in the infrared, Rev. Mod. Phys. 16, 307-324.

Schwendeman, R. H., and V. W. Laurie (1958), Tables of line strengths, Pergamon Press, Inc., New York, 39 pp.

Townes, C. H., and A. L. Schawlow (1955), Microwave spectroscopy, McGraw-Hill Book Company, Inc., New York, 102.

Wacker, Paul F., and Marlene R. Pratto (1963), Microwave spectral tables, Vol. II, Line strengths of asymmetric rotors, NBS Monograph No. 70. 


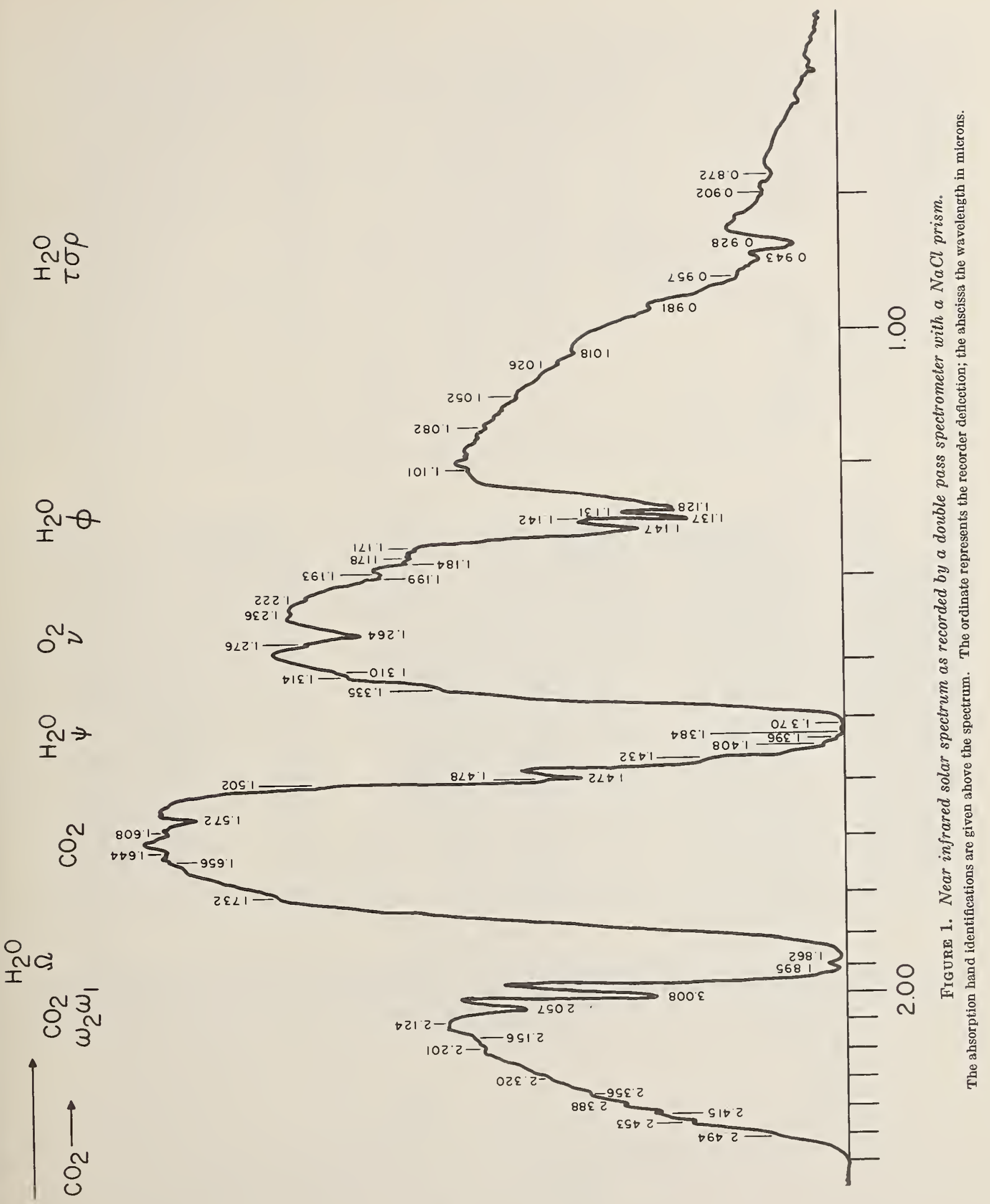



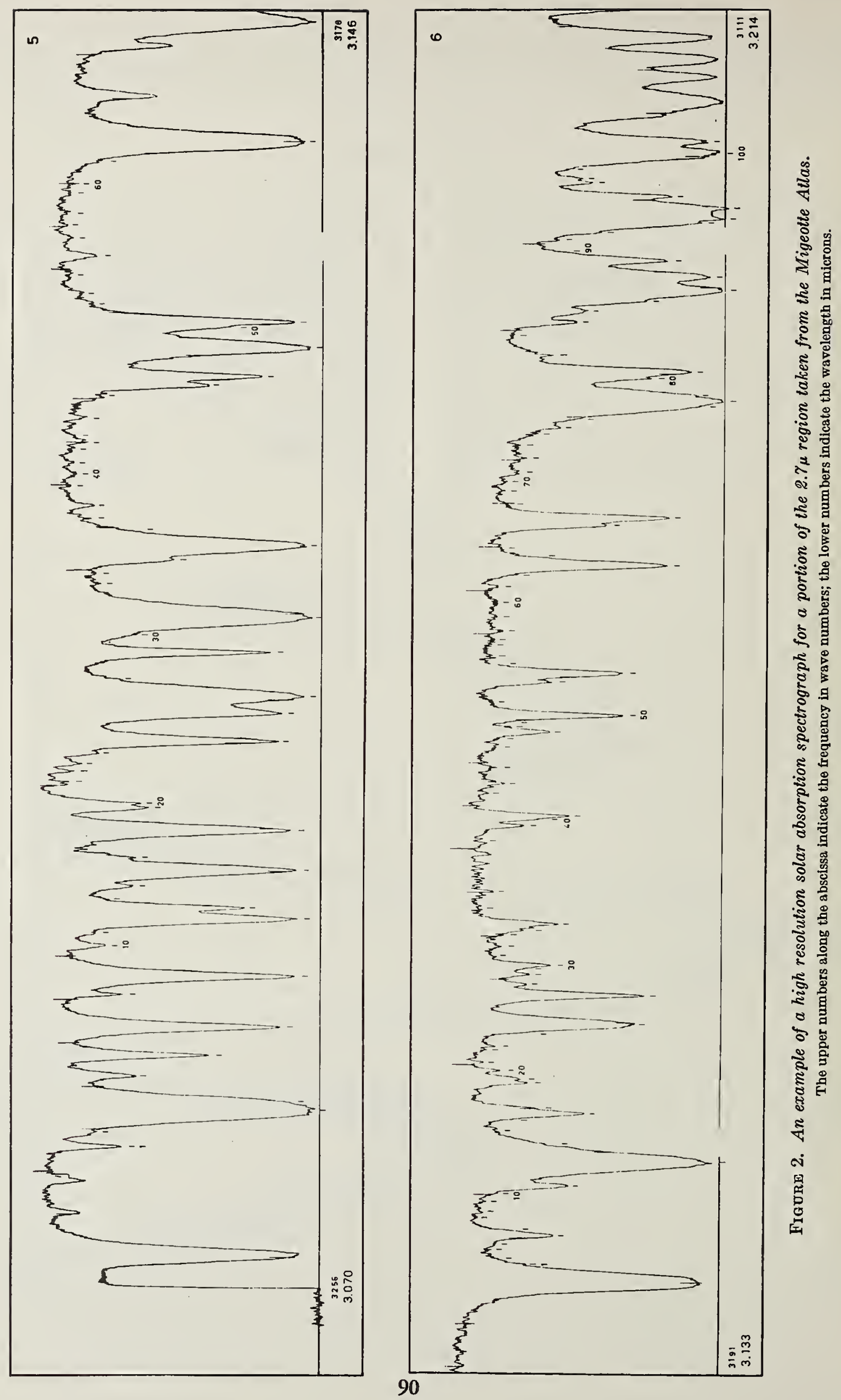

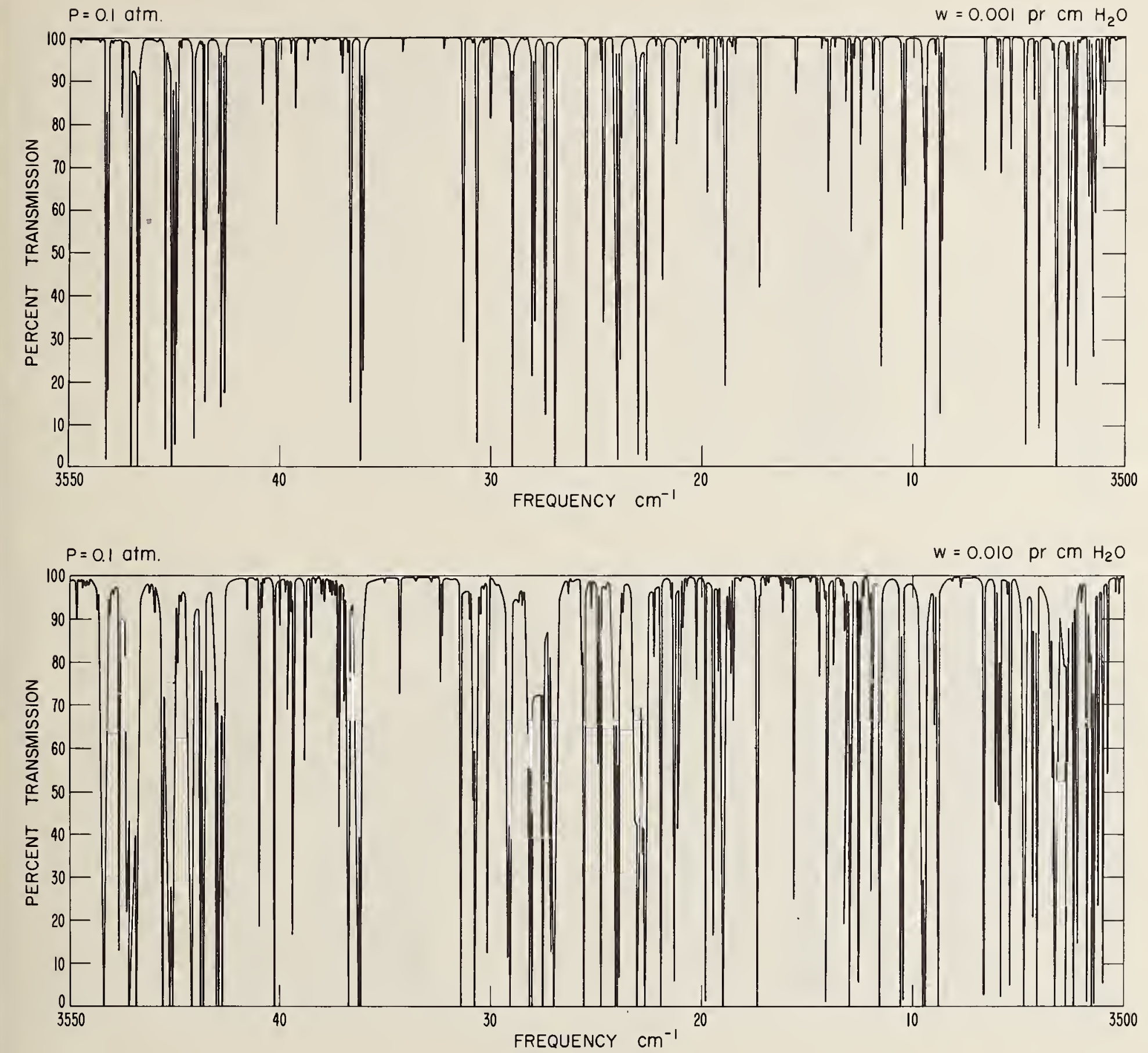

FIGURE 3. Computed high resolution theoretical spectra for the frequency range 3500 to $4000 \mathrm{~cm}-1$ at a pressure of 0.1 atmosphere and at concentrations of 0.001 and $0.01 \mathrm{~cm}$ of precipitable water vapor for a temperature of $287.7^{\circ} \mathrm{K}$.

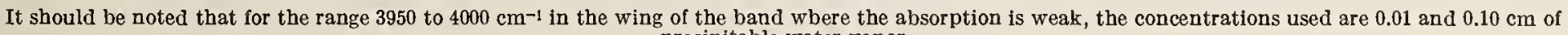
precipitable water vapor. 

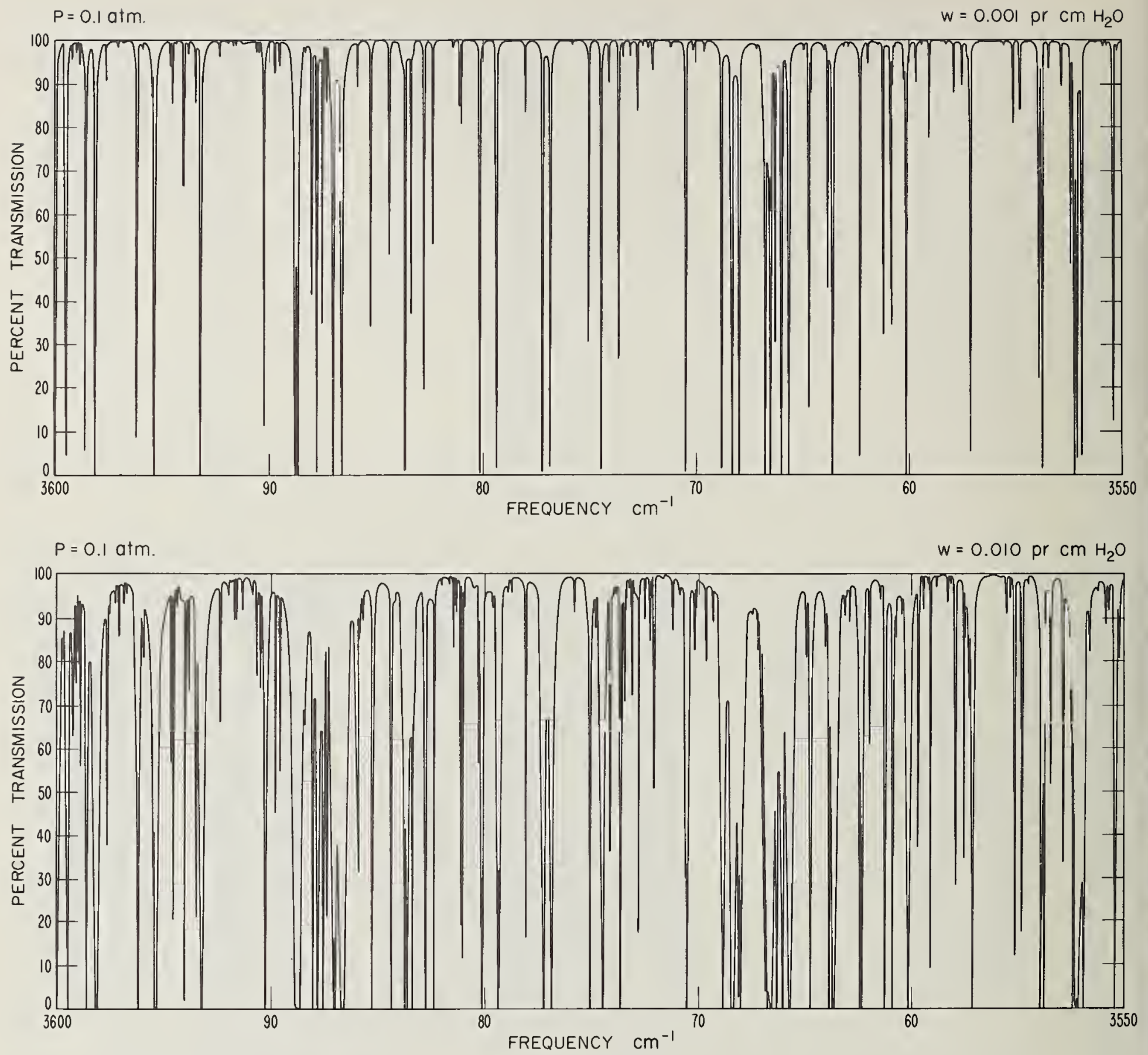

Figure 3.-Continued 

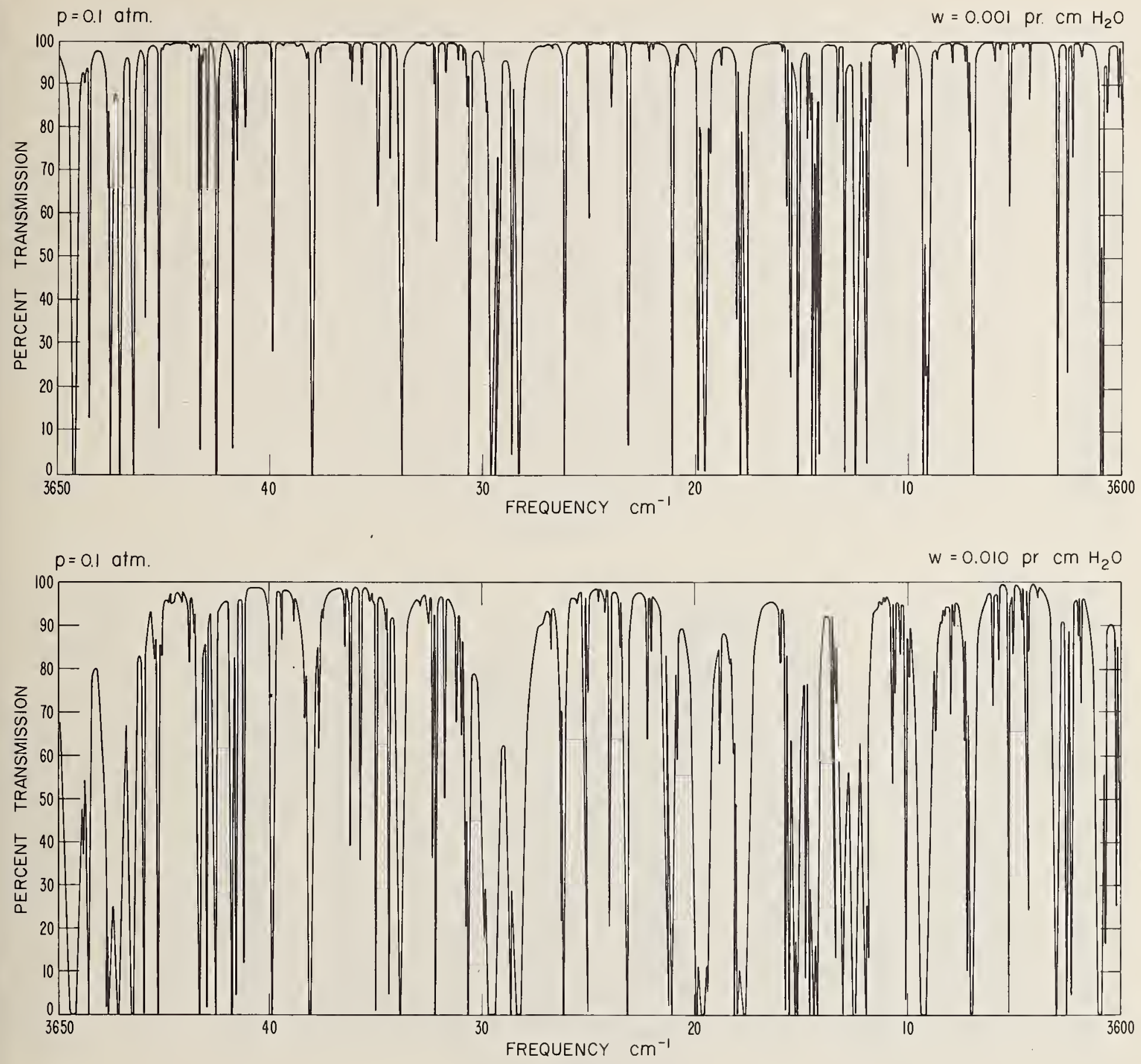

Figure 3.-Continued 

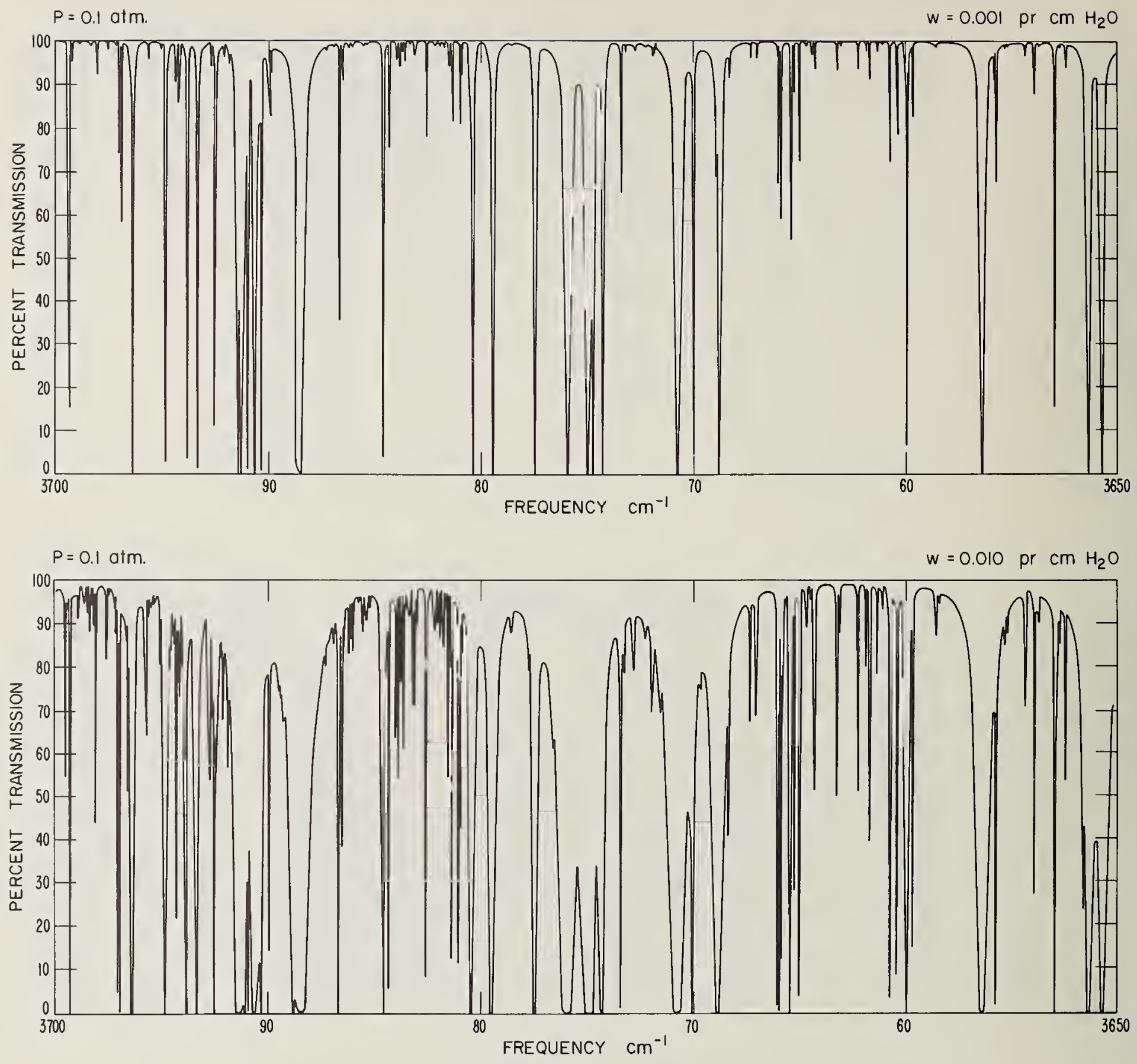

Figure 3.-Continued 

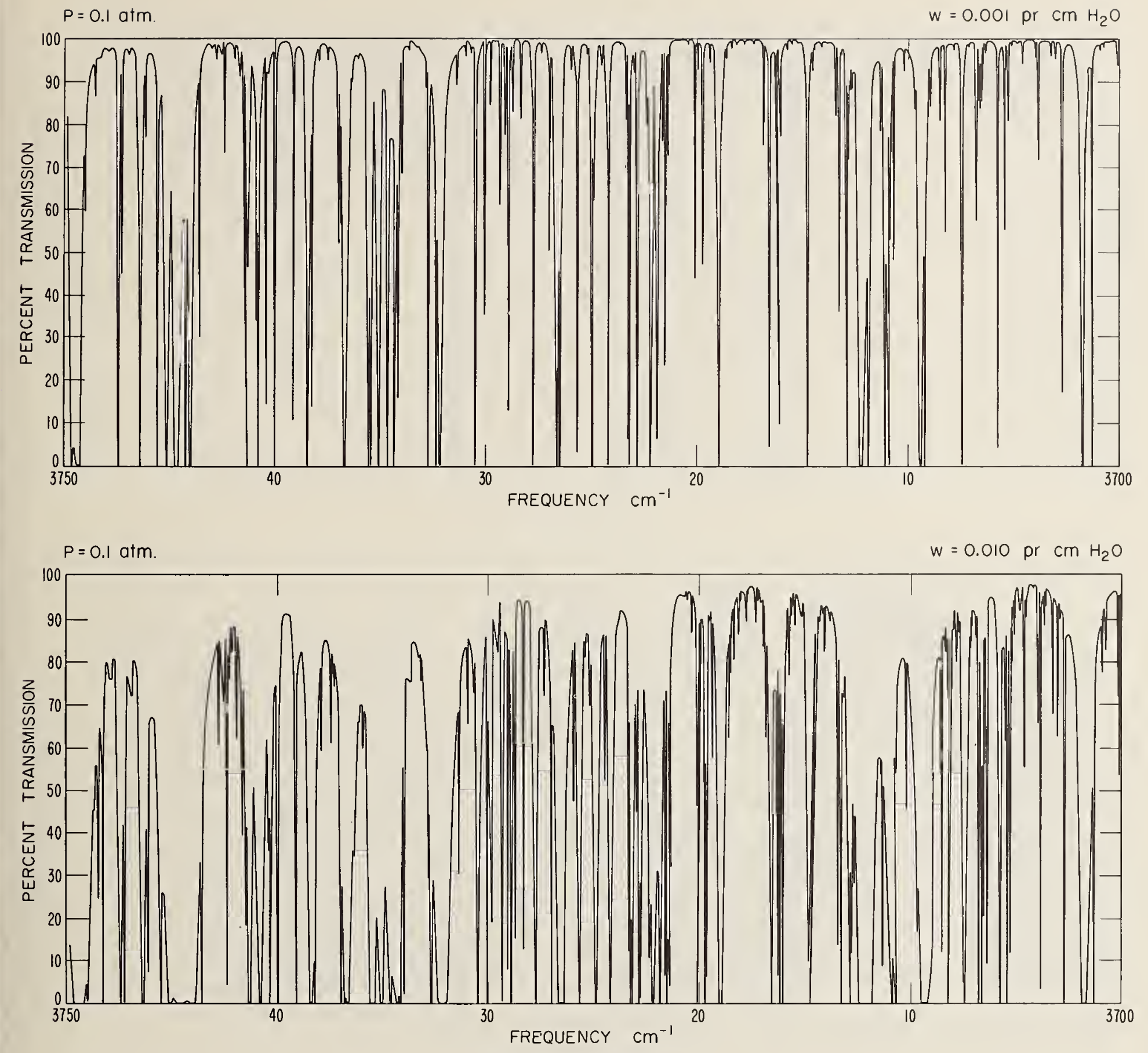

Figure 3.-Continued 

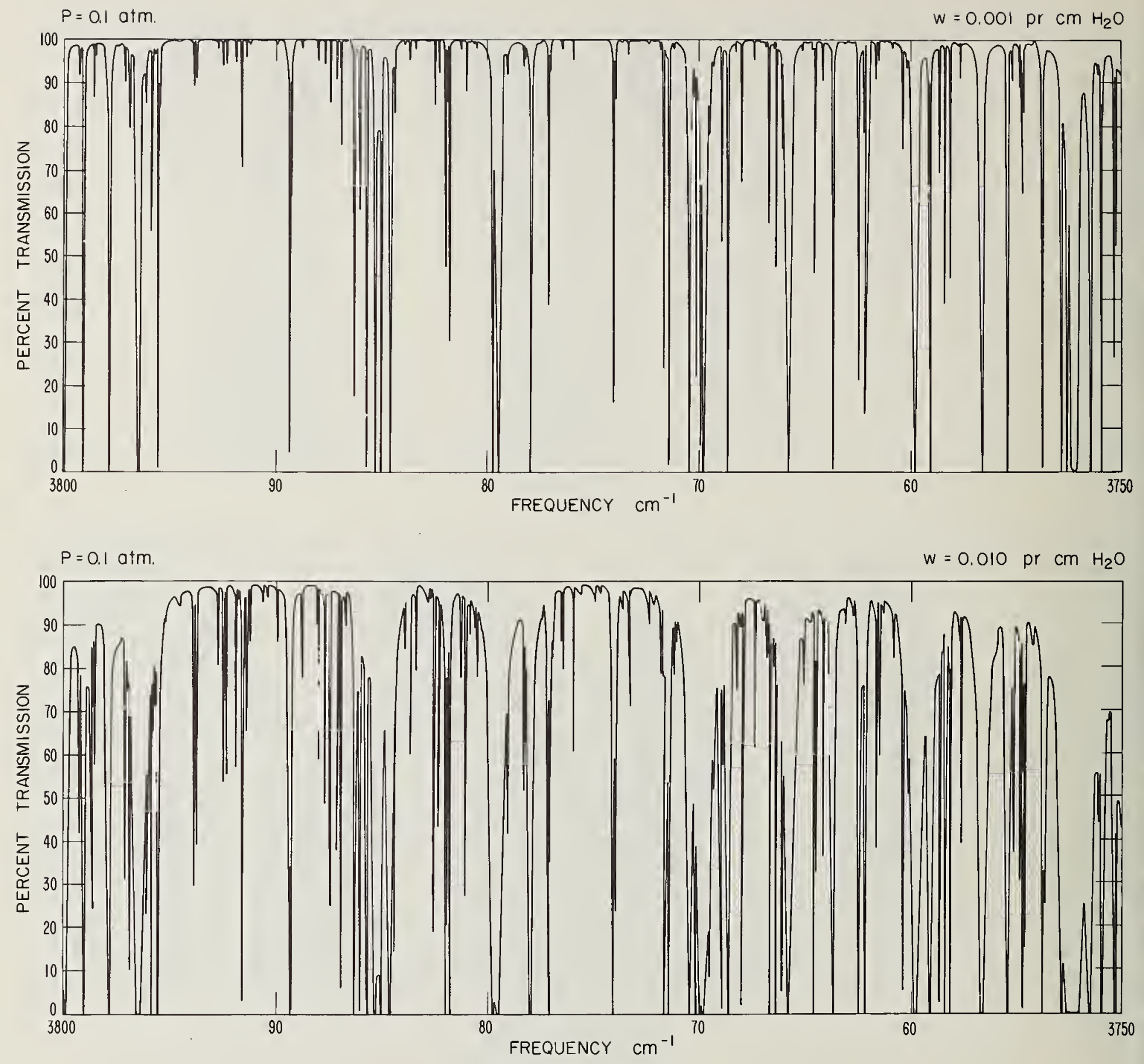

Figure 3.-Continued 

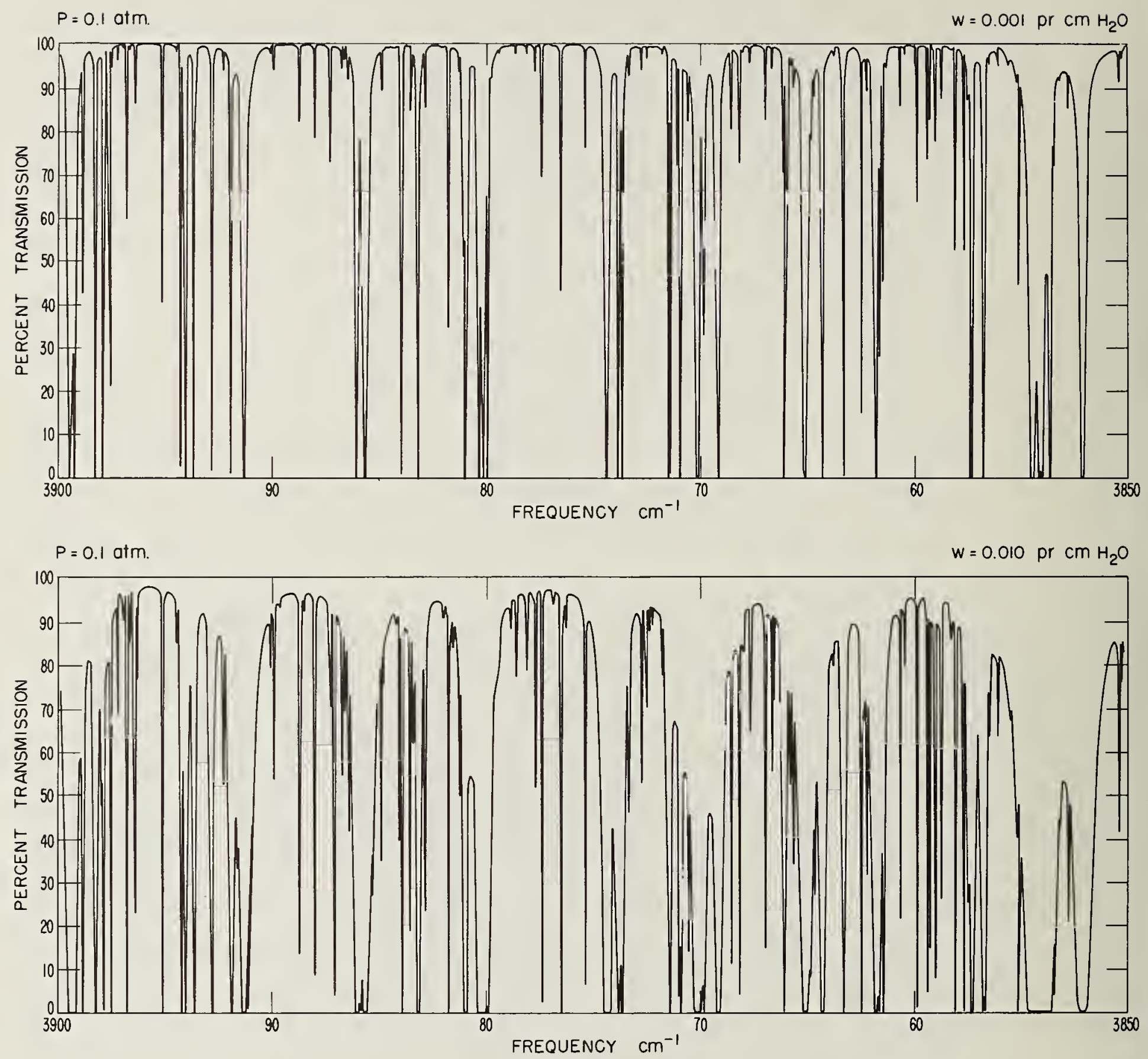

Frgure 3.-Continued 

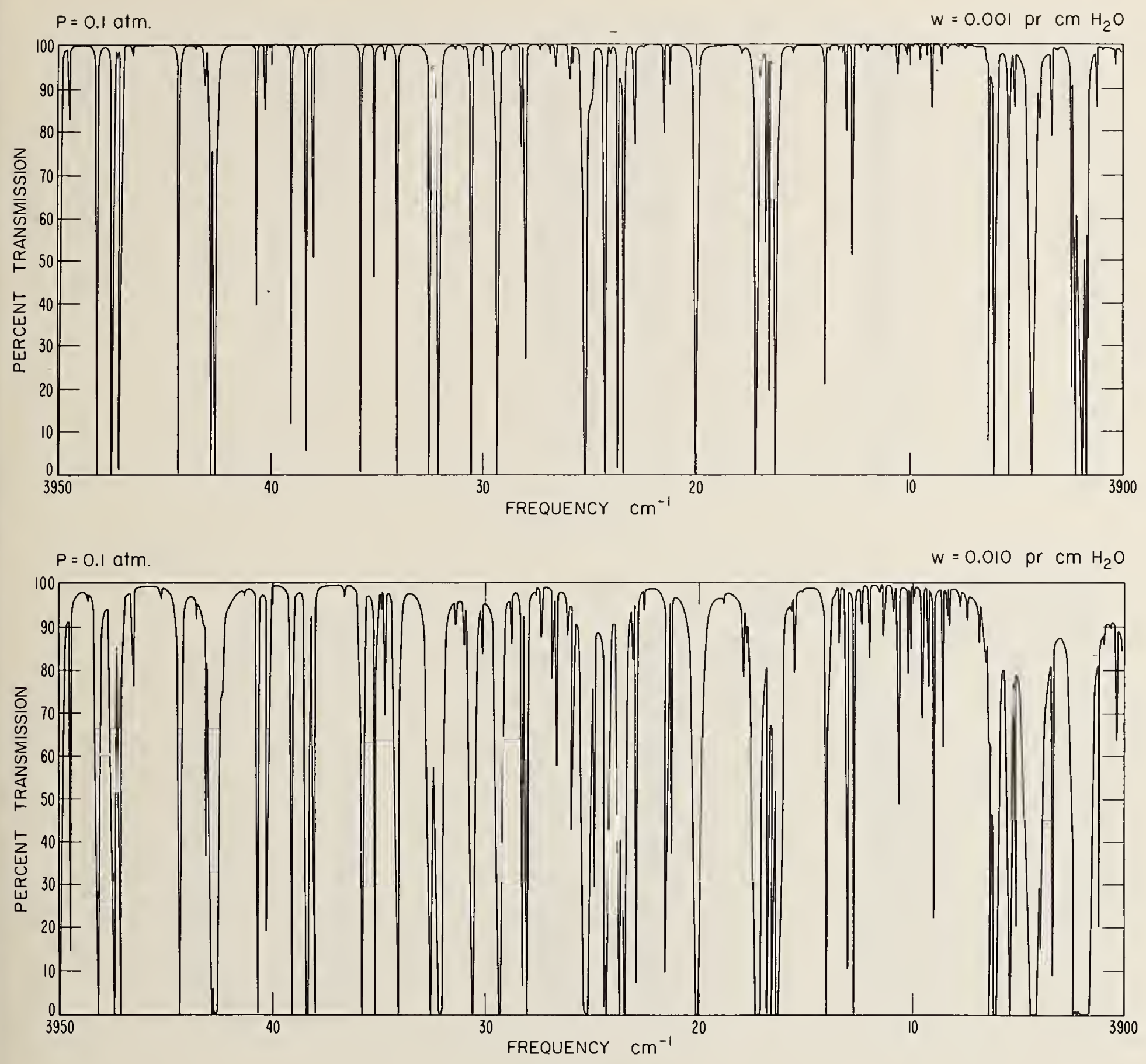

Figdre 3.-Continued 

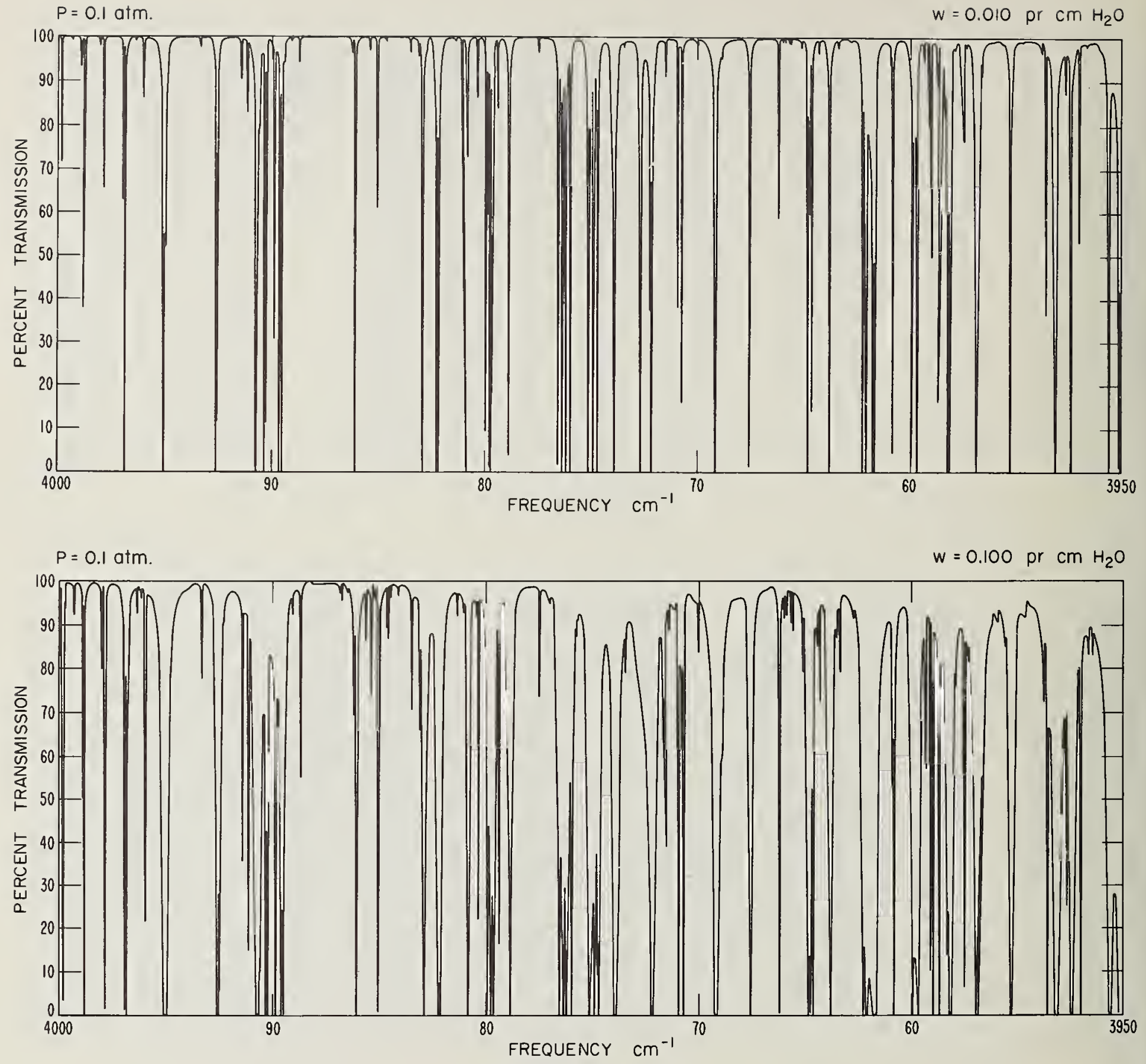

Figdre 3.-Continued 

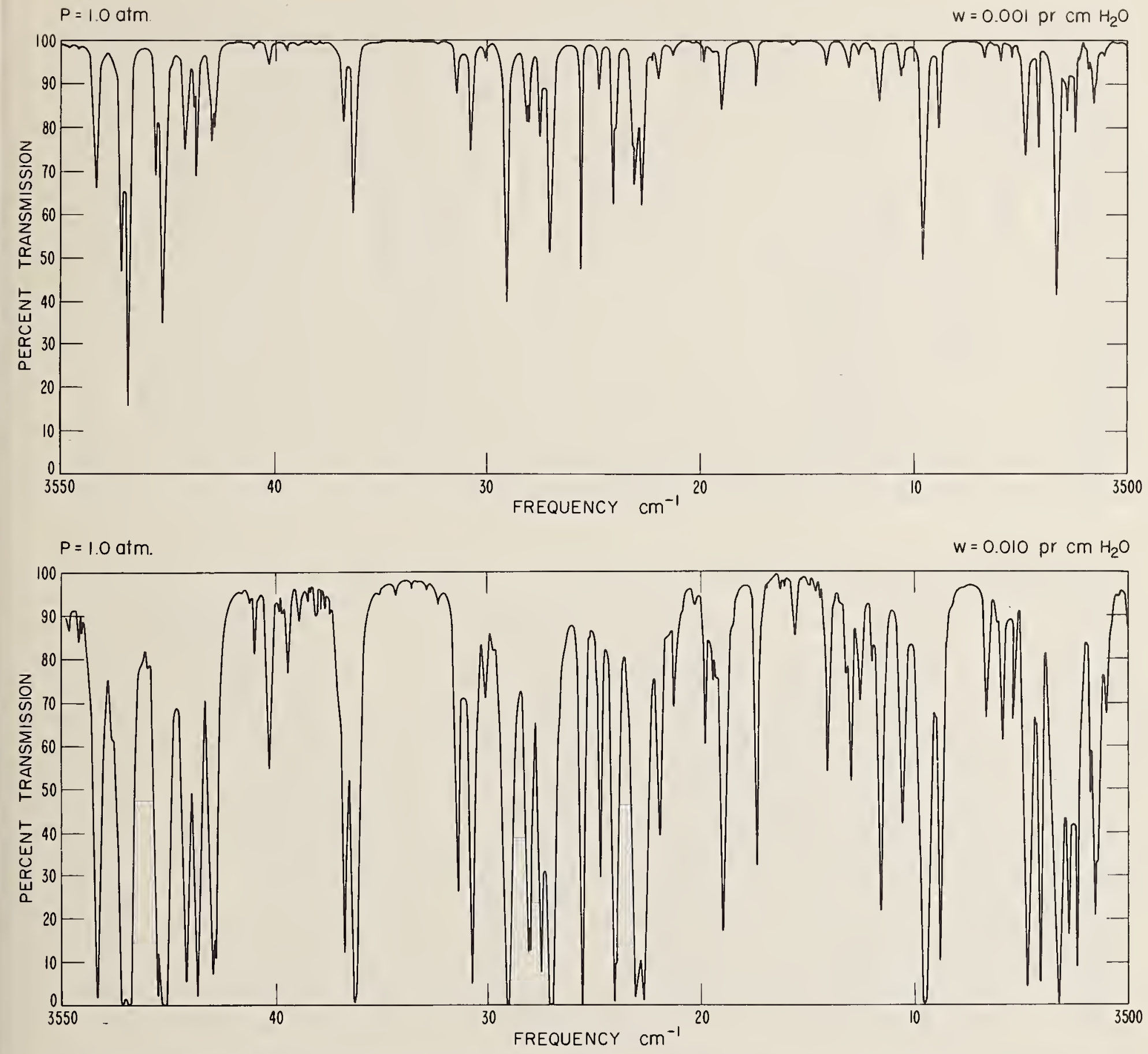

FIGURE 4. Computed high resolution theoretical spectra for the frequency range 3500 to $4000 \mathrm{~cm}-1$ at a pressure of 1.0 atmosphere and at concentrations of 0.001 and $0.01 \mathrm{~cm}$ of precipitable water vapor for a temperature of $287.7^{\circ} \mathrm{K}$.

It should be noted that for the range 3950 to $4000 \mathrm{~cm}^{-1}$ in the wing of the band where the absorption is weak, the concentrations used are 0.01 and $0.10 \mathrm{~cm}$ of precipitable water vapor. 

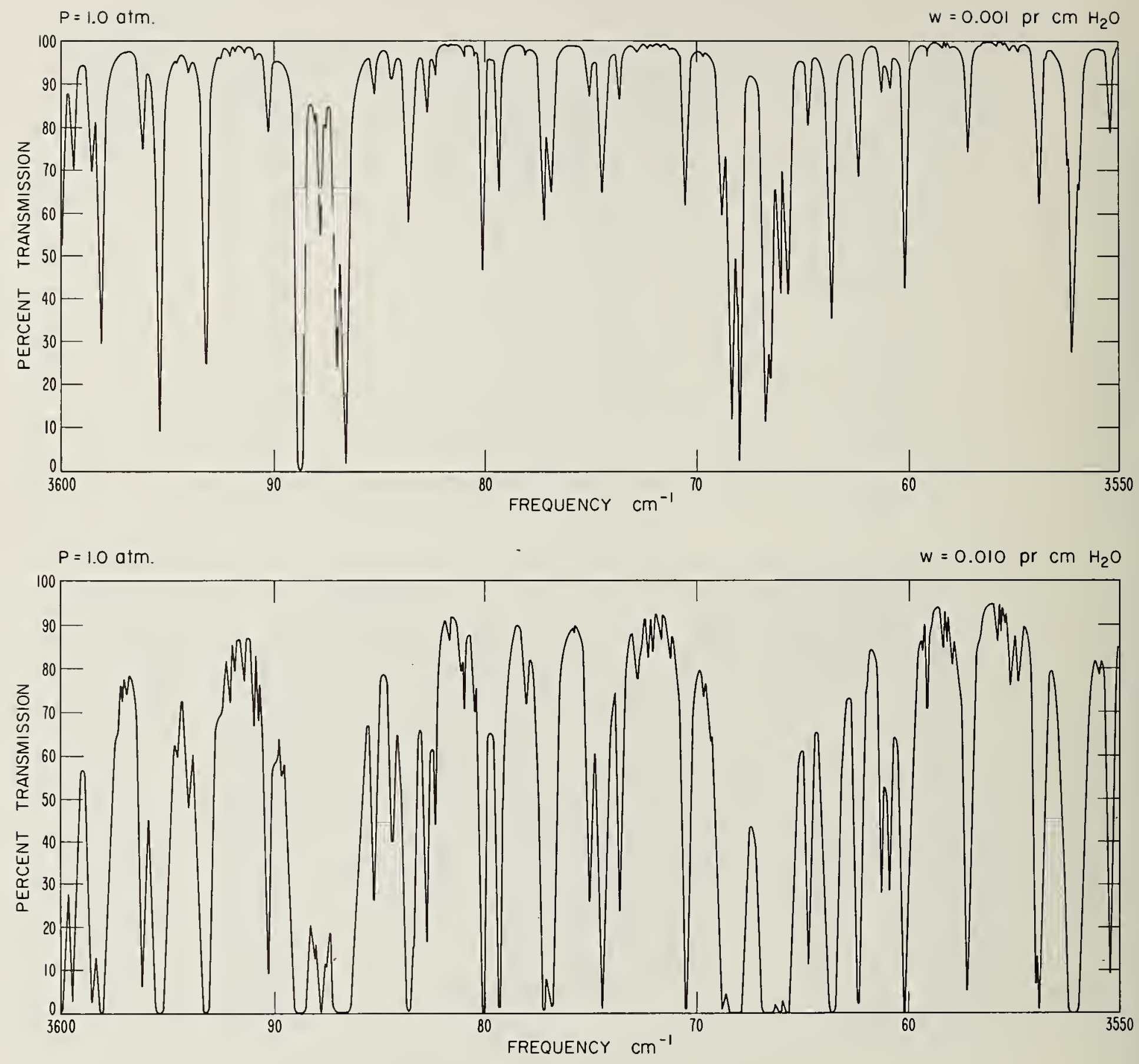

Figure 4.-Continued 

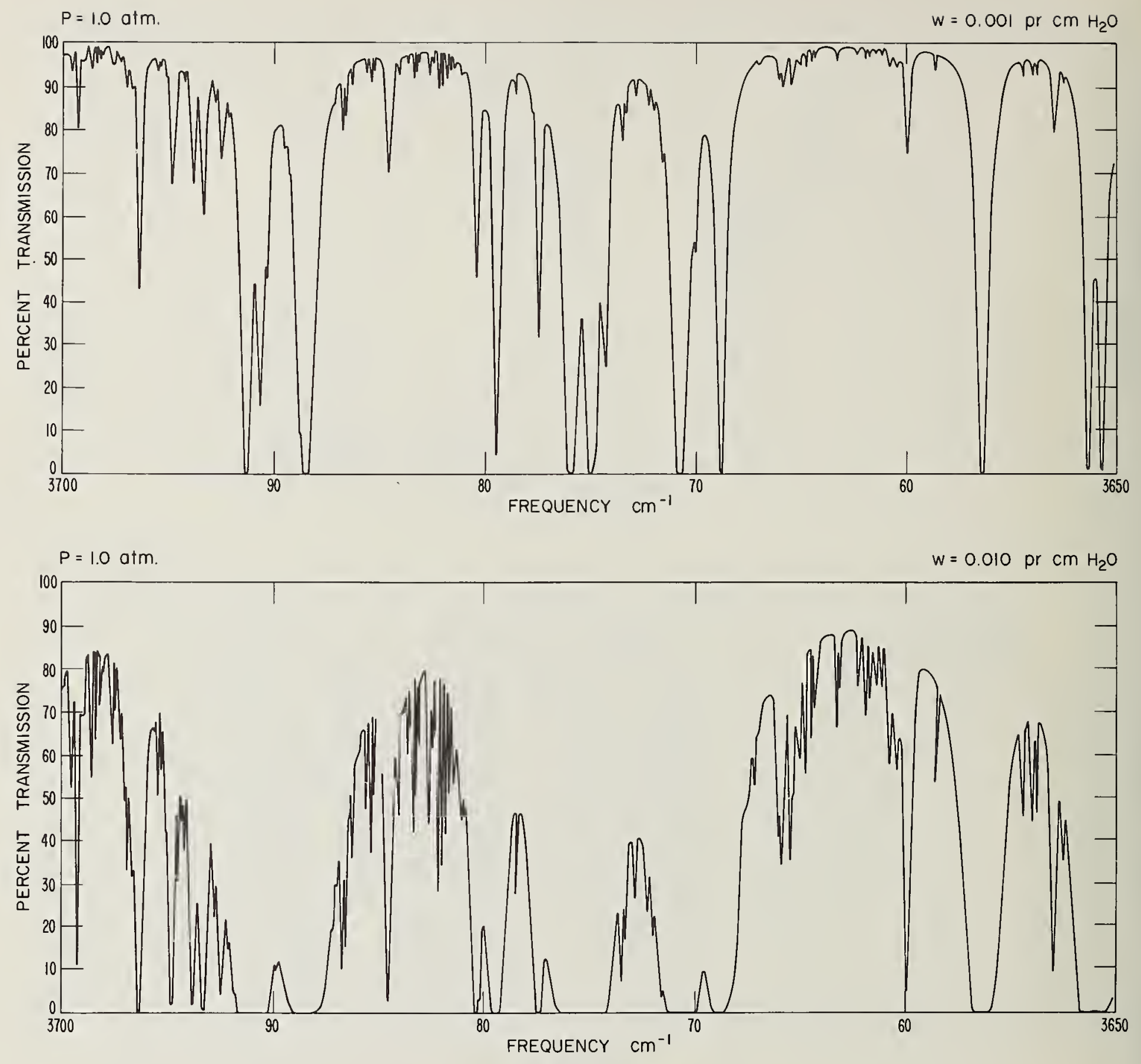

Figure 4.-Continued 

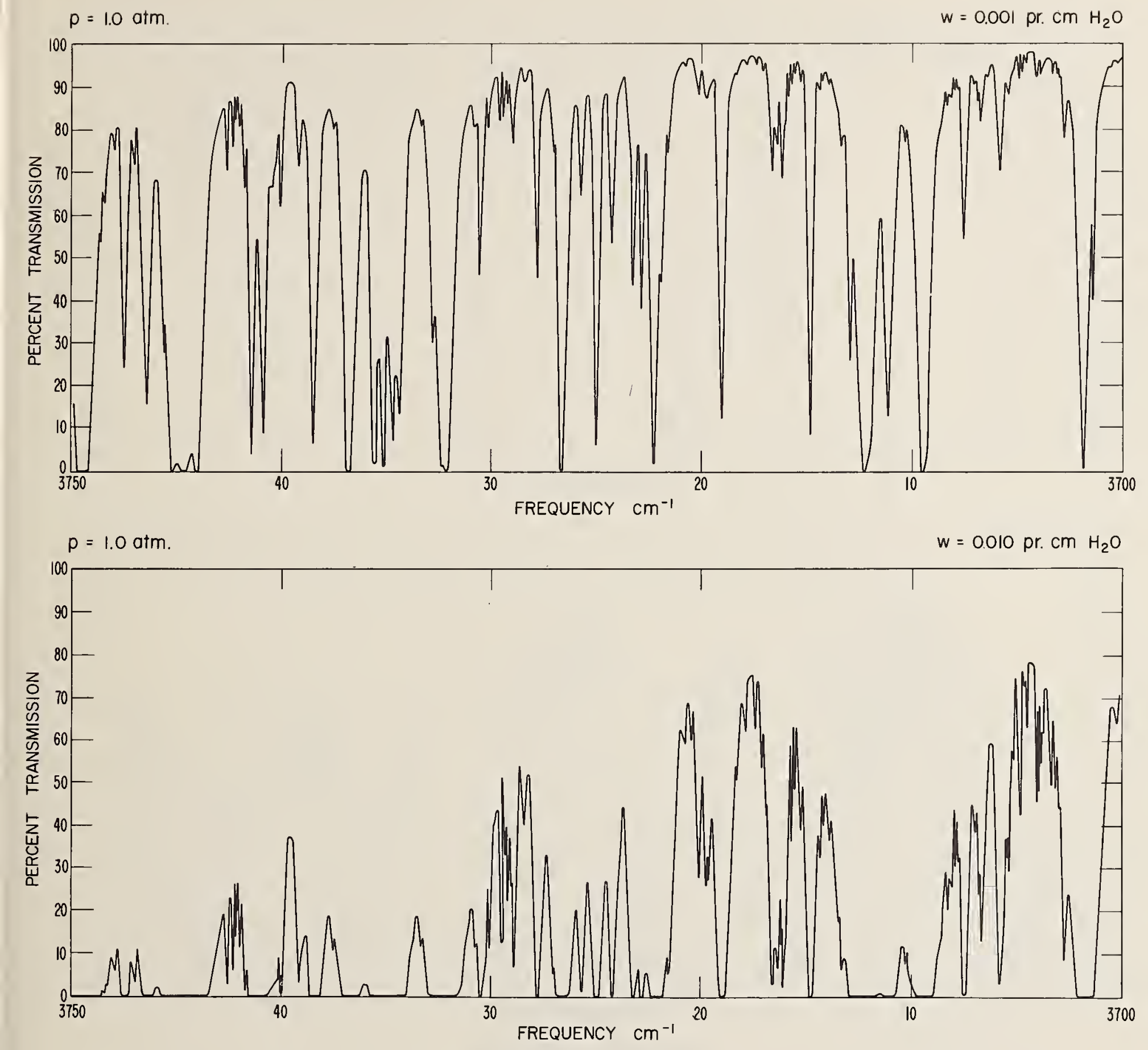

Figure 4.-Continued . 

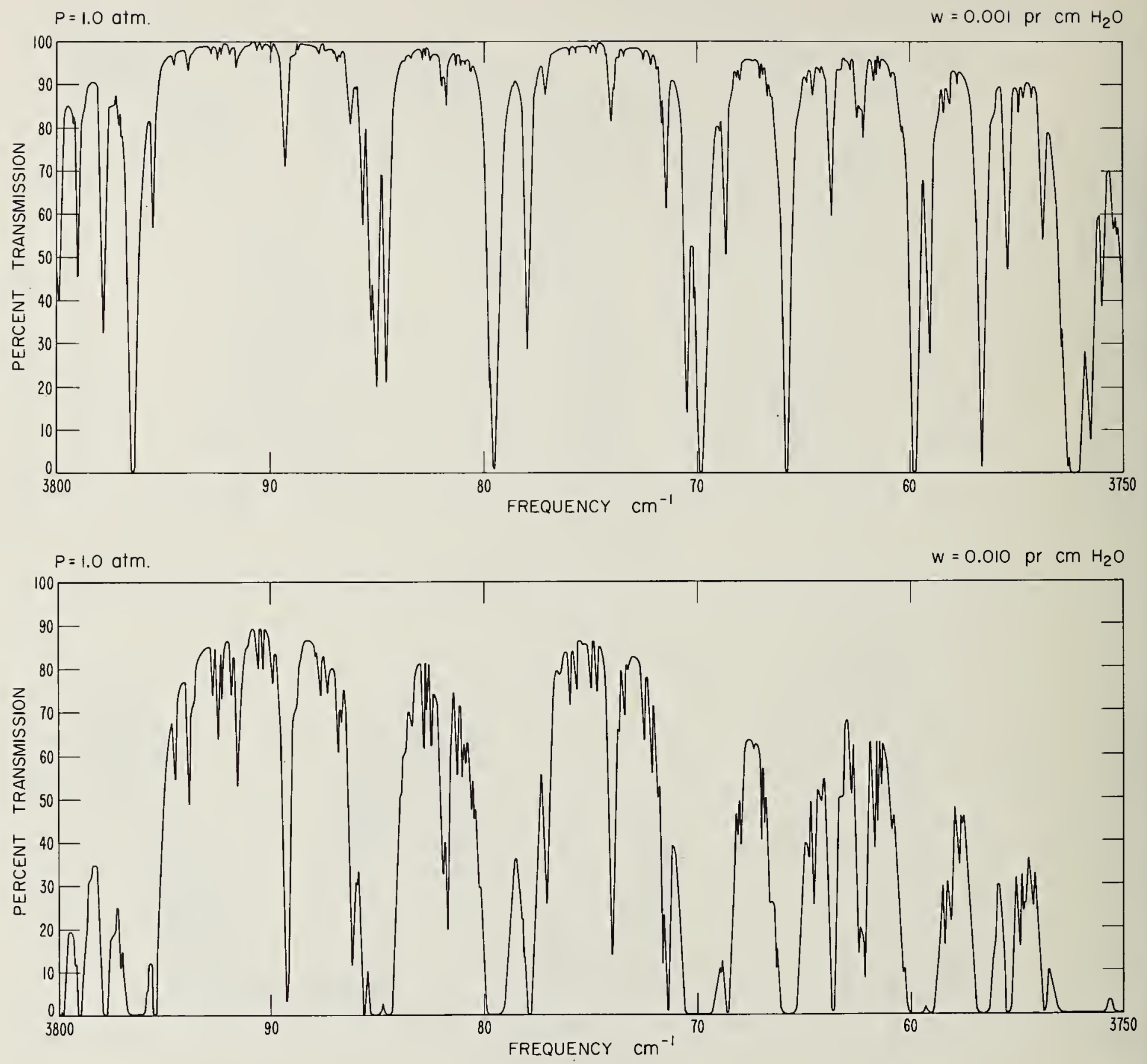

Figdre 4.-Continued 

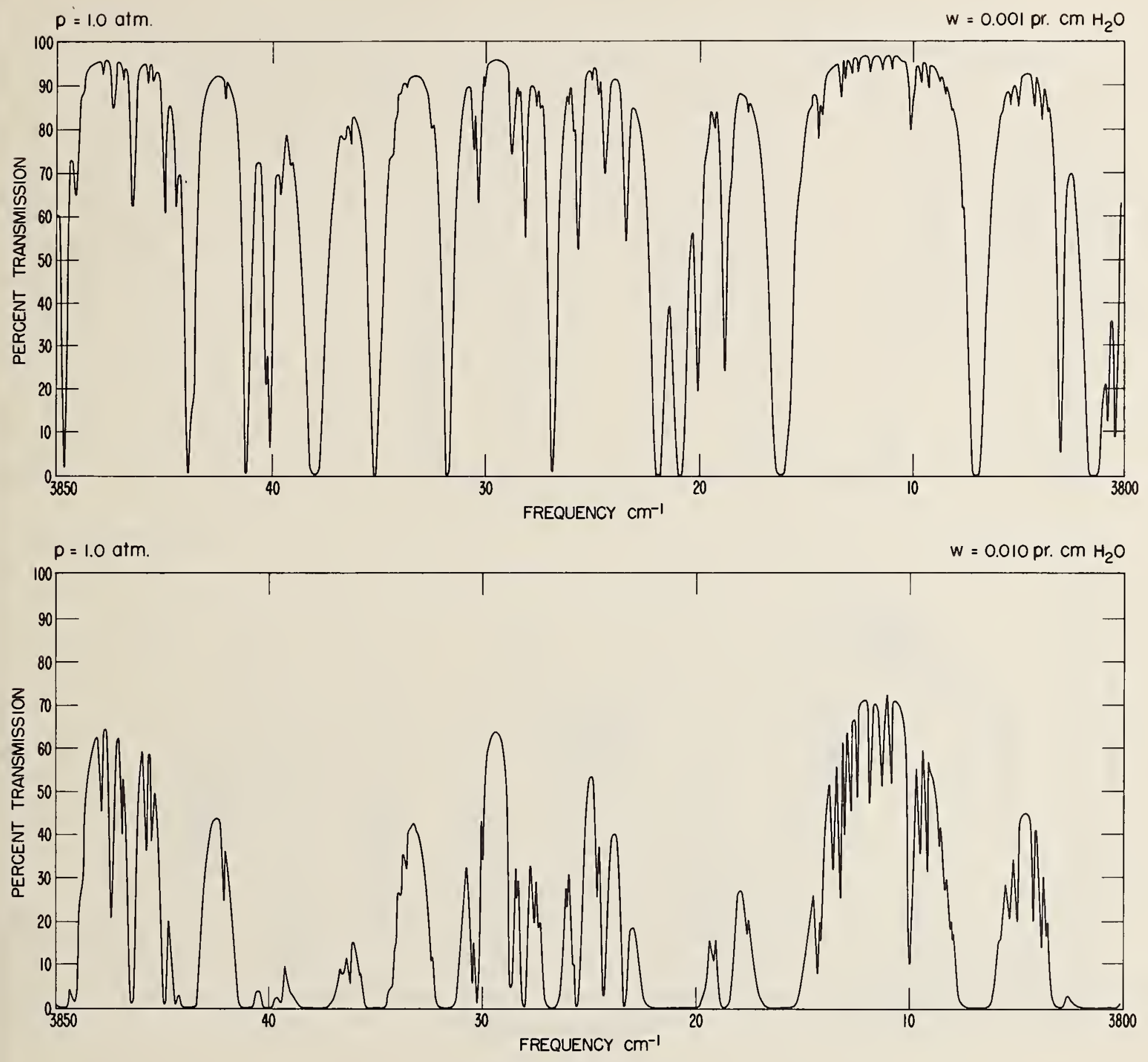

Figdre 4.-Continued 

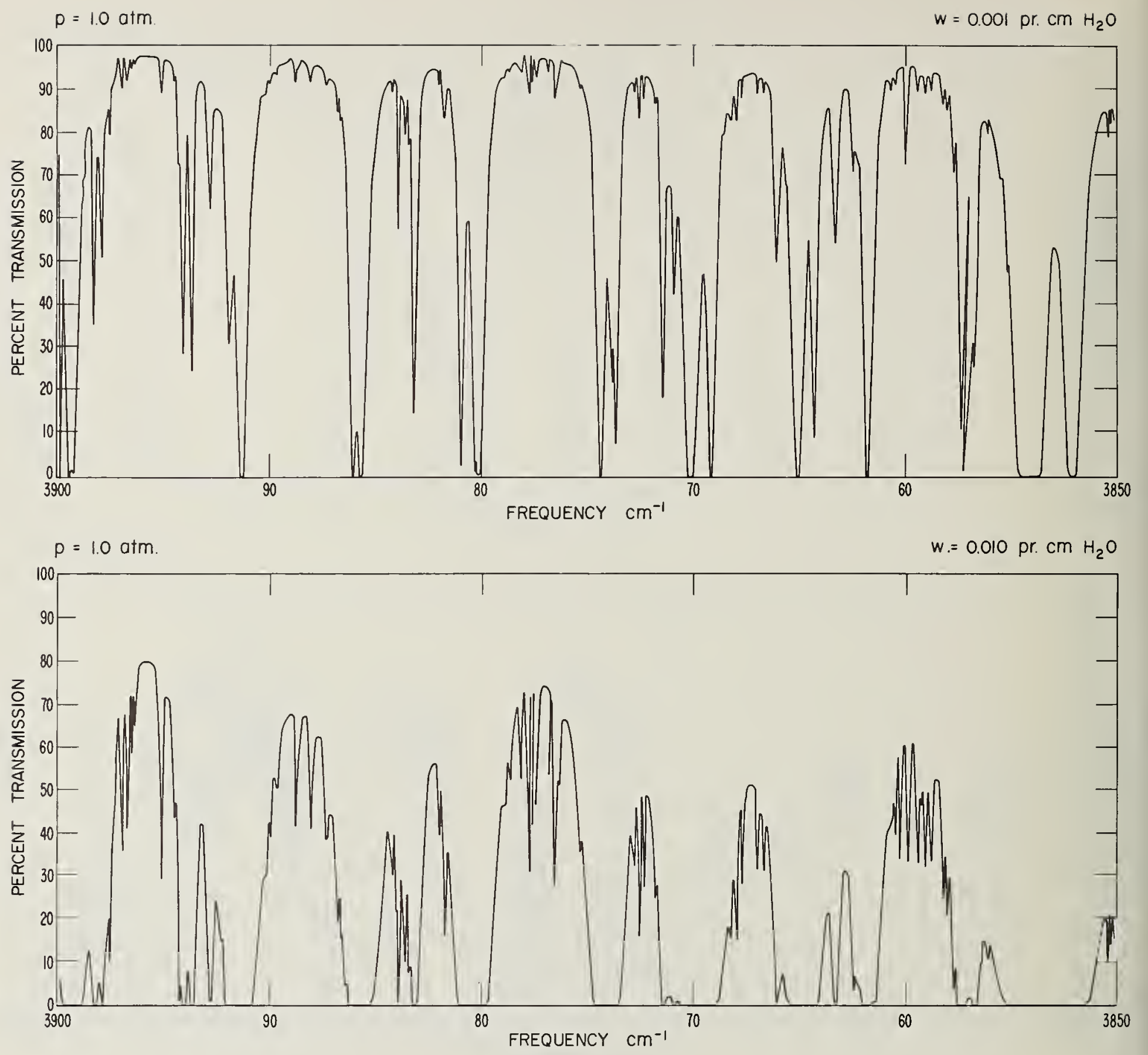

Figure 4.-Continued

108 

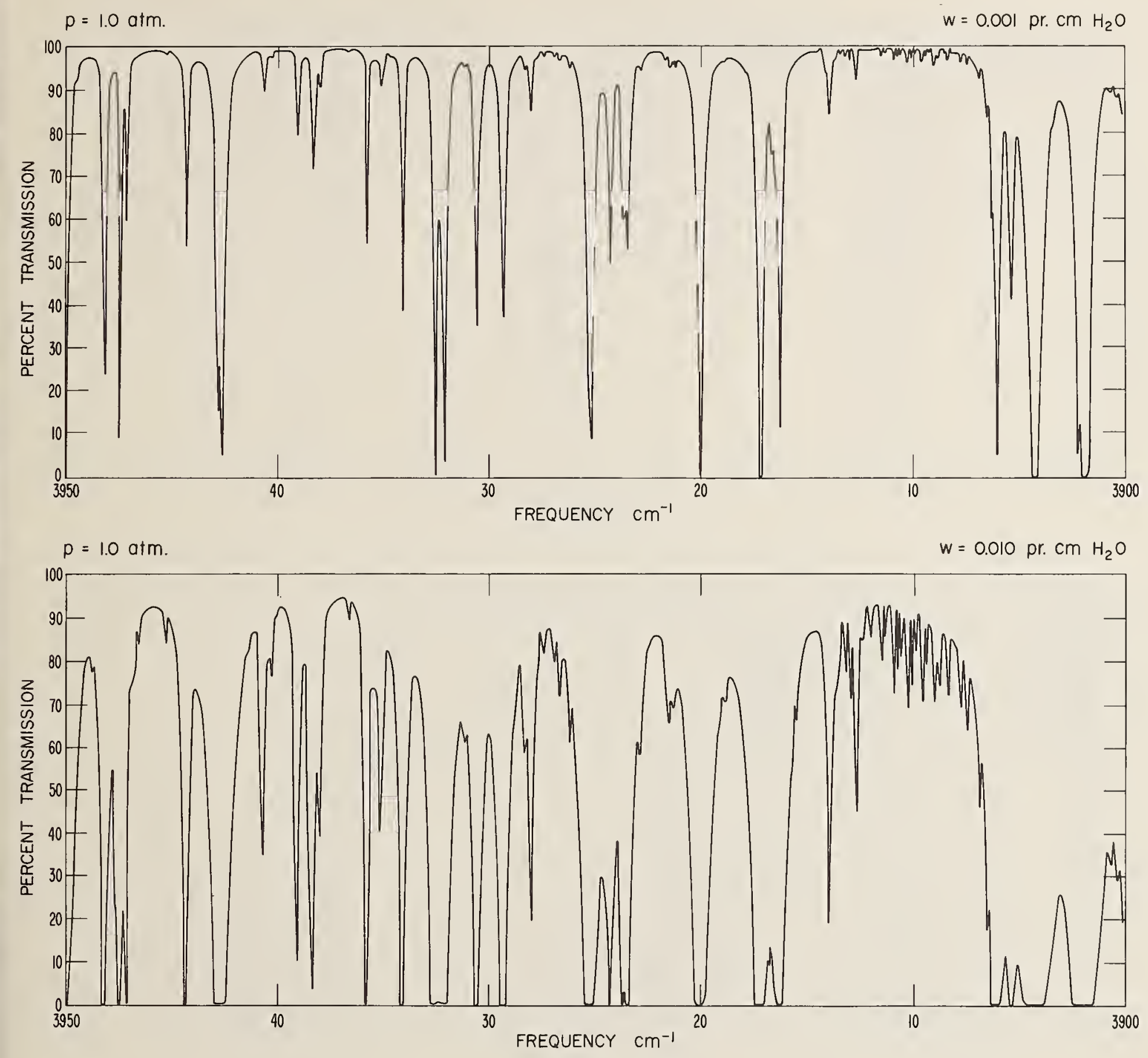

Frgure 4.-Continued 

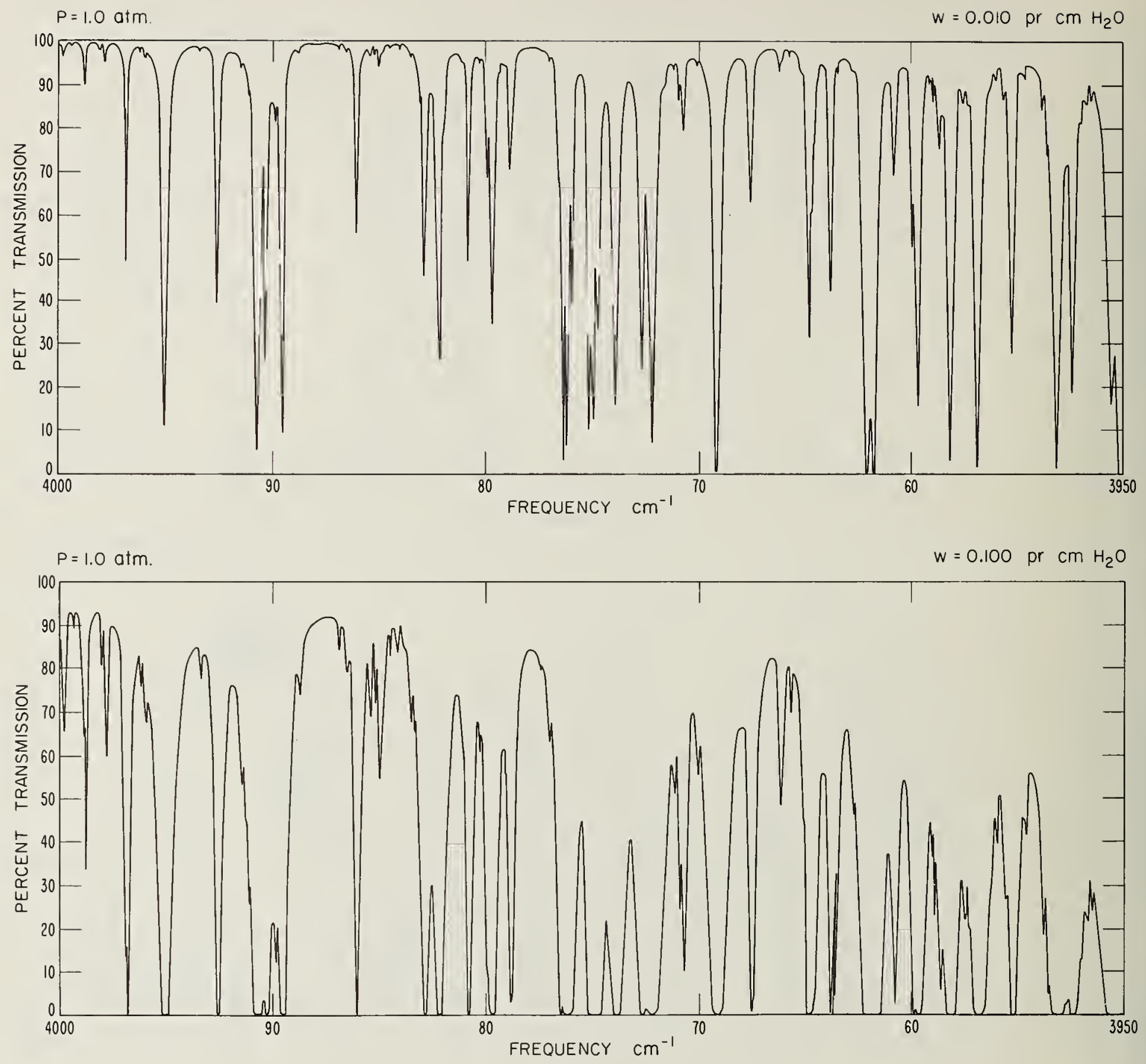

Figdre 4.-Continued 

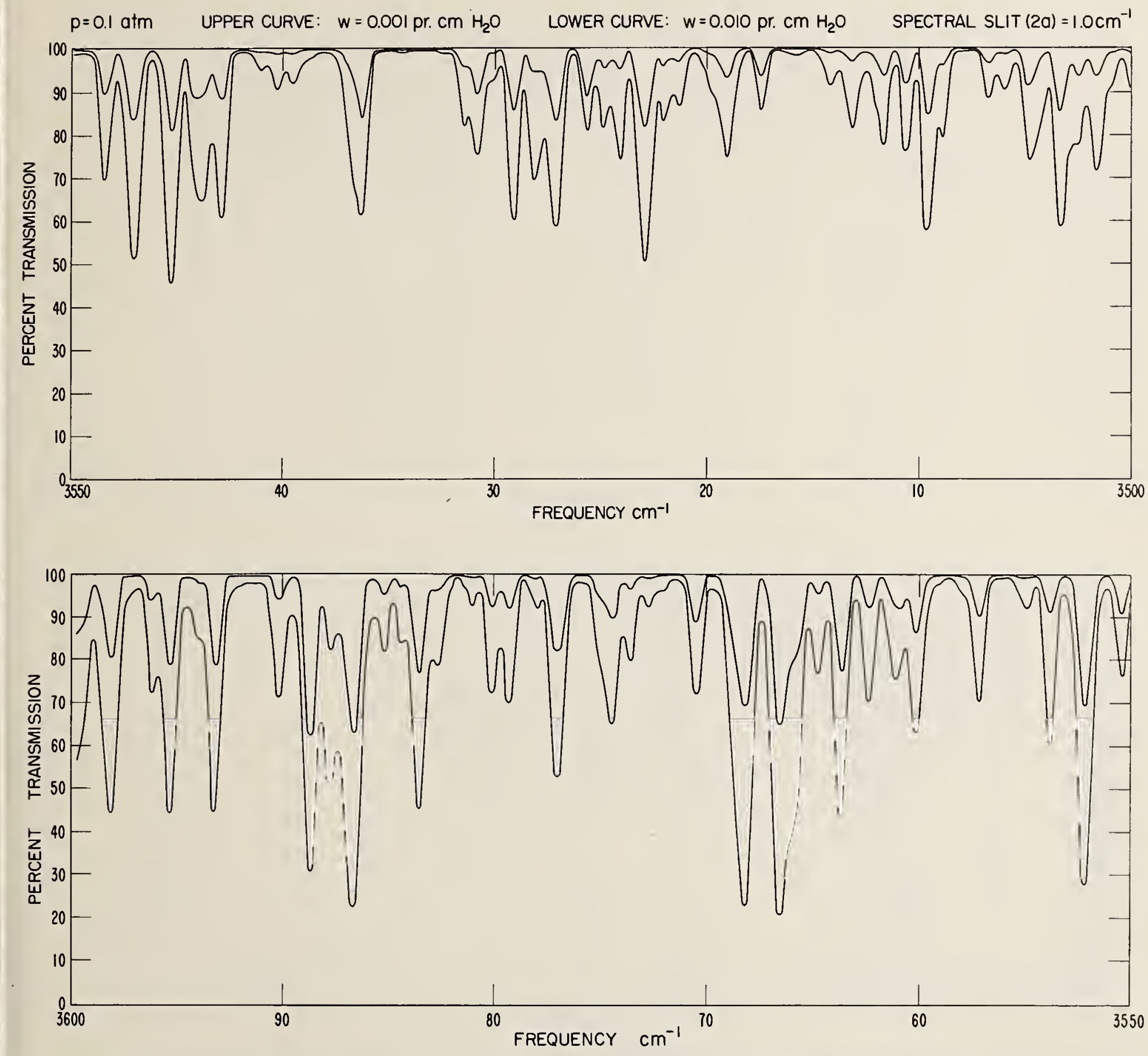

FIGURE 5. Computed degraded water vapor transmission spectra for the frequency range 3500 to $4000 \mathrm{~cm}-1$ at a pressure of 0.1 atmosphere, at concentrations of 0.001 and $0.01 \mathrm{~cm}$ of precipitable water. vapor, for a temperature of $287.7{ }^{\circ} \mathrm{K}$, and at a full spectral slit (triangular) span of $1.0 \mathrm{~cm}-1$. 

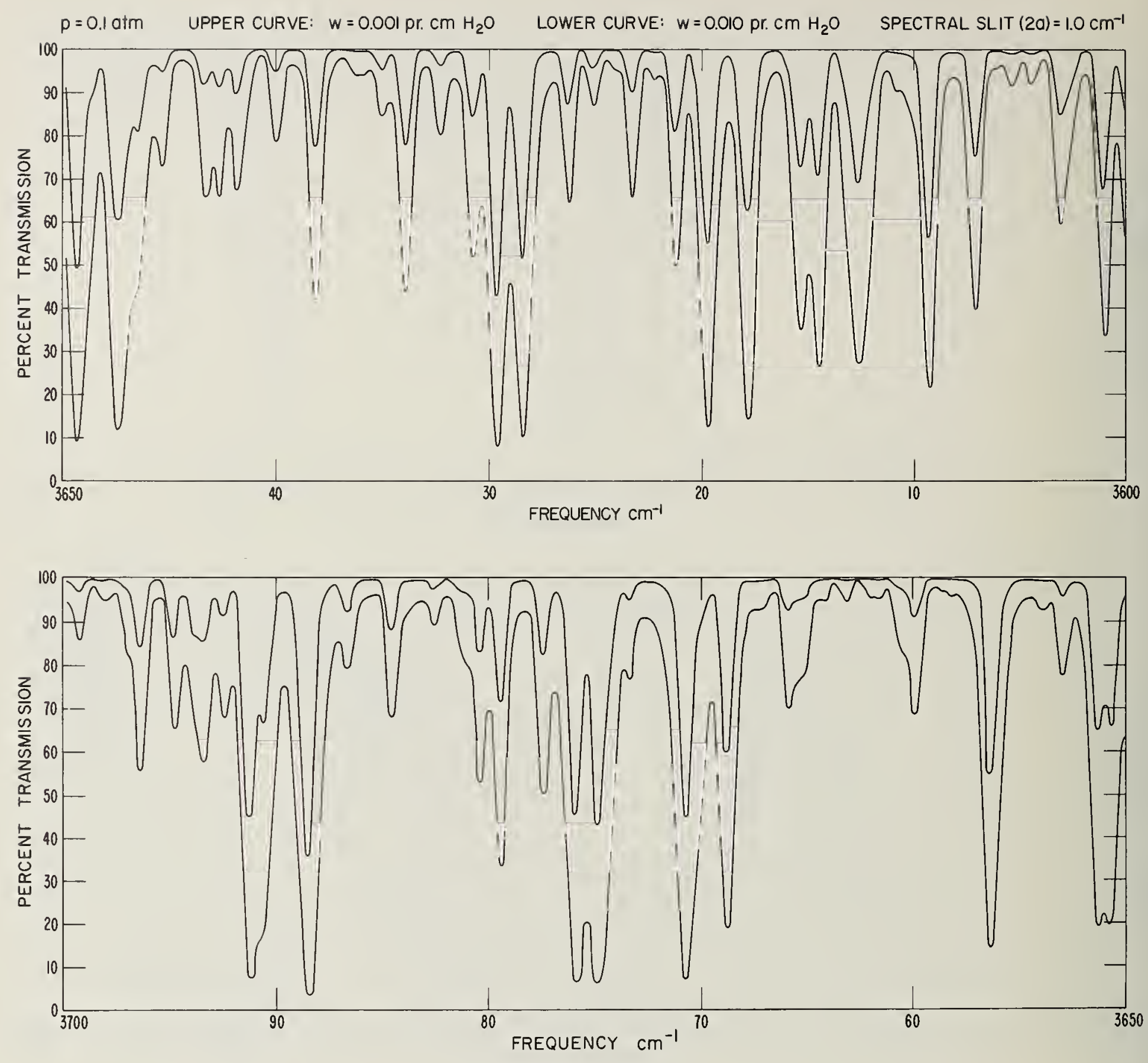

Figdre 5.-Continued 

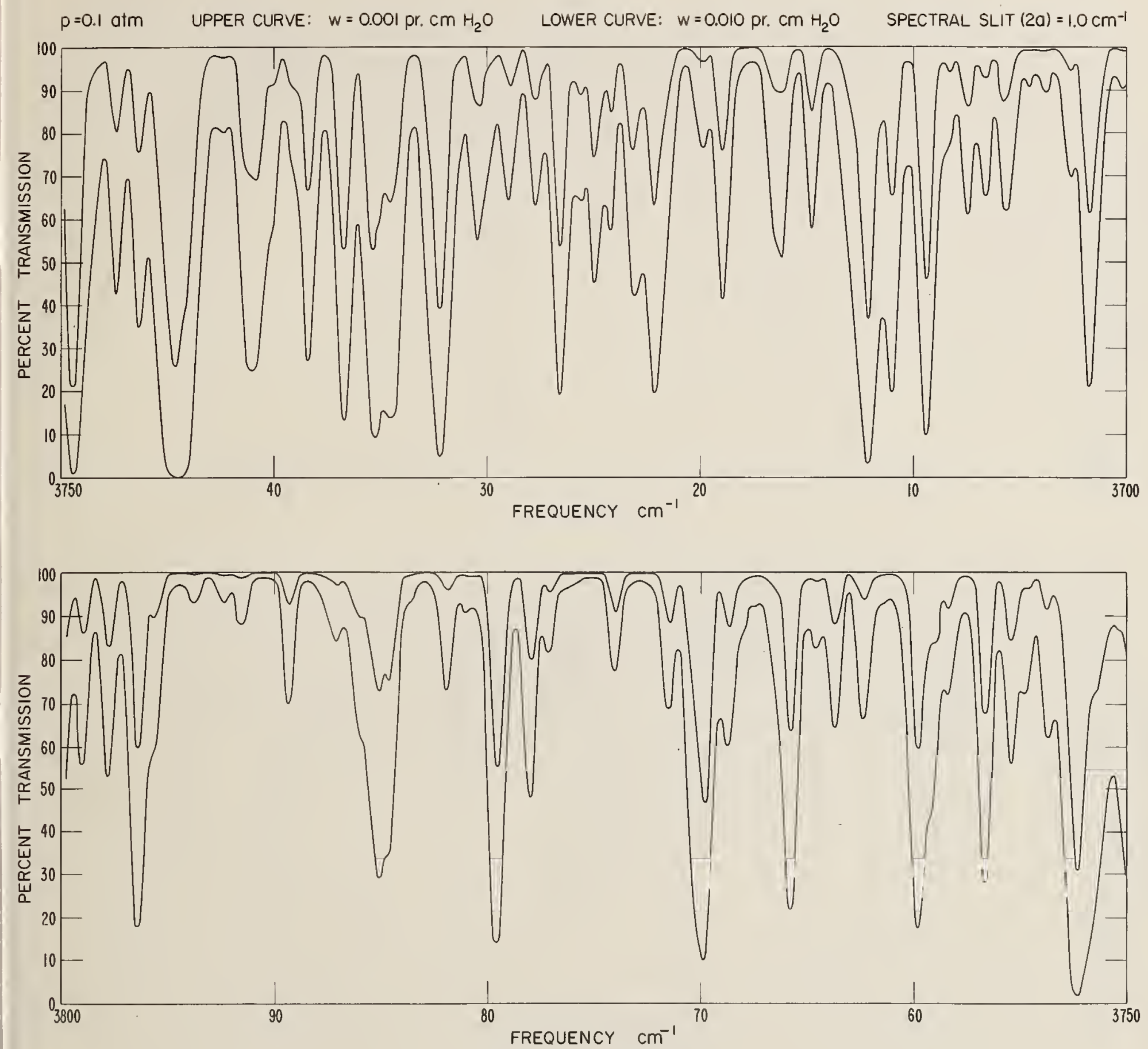

Figure 5.-Continued 

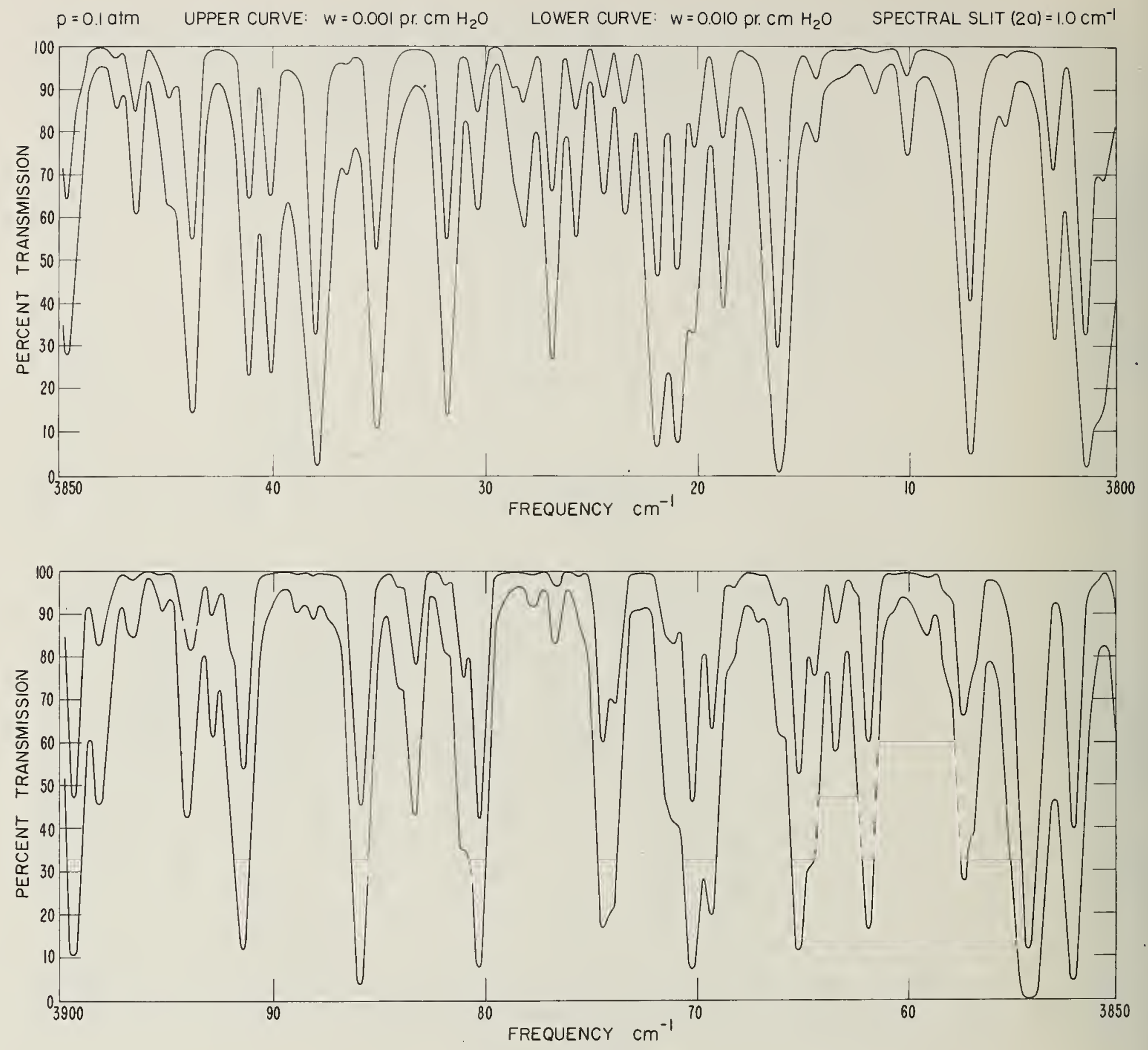

Figure 5.-Continued 

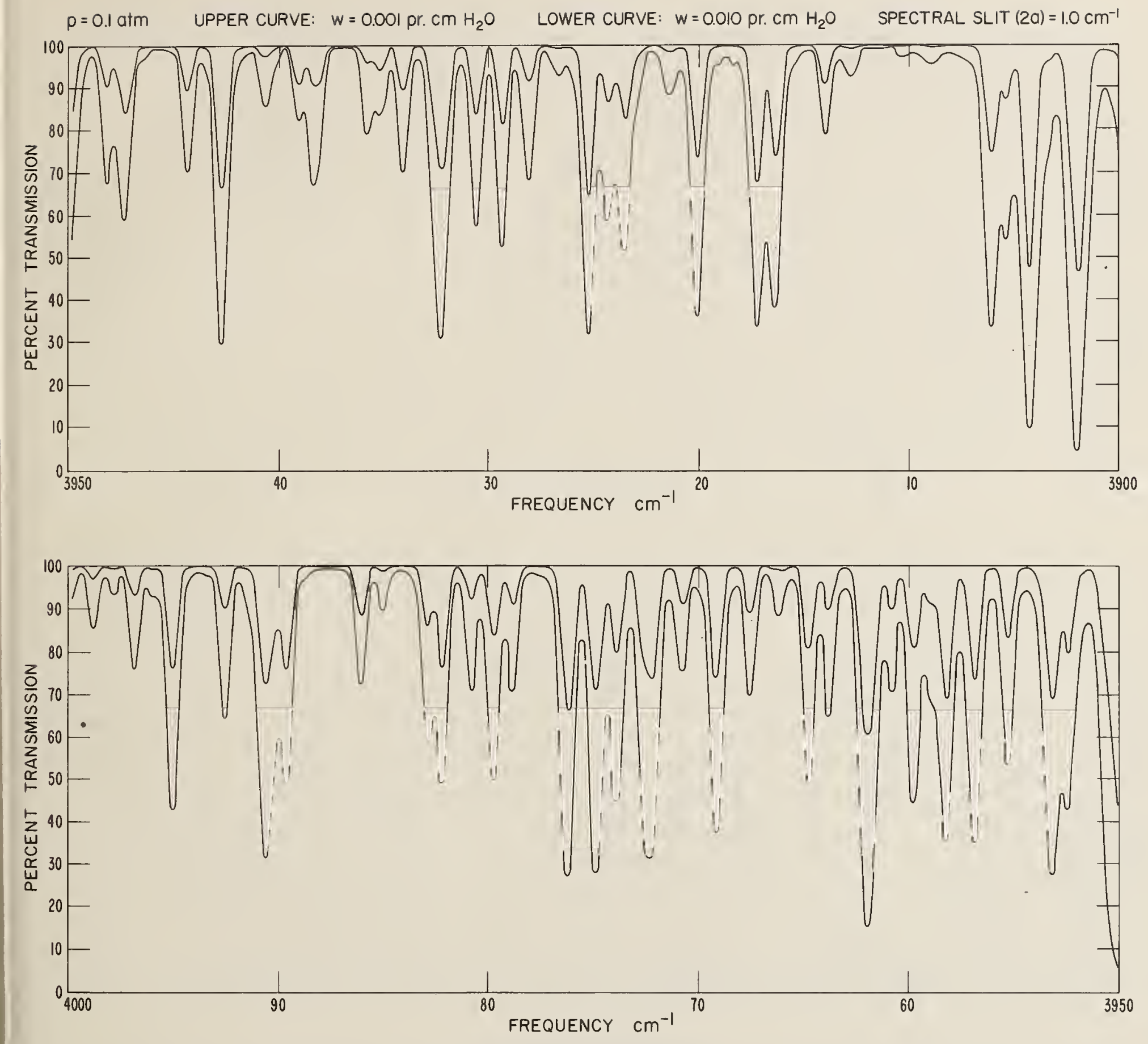

Figure 5.-Continued. 
$p=1.0 \mathrm{~atm} \quad$ UPPER CURVE: $w=0.001 \mathrm{pr} . \mathrm{cm} \mathrm{H}_{2} \mathrm{O}$ LOWER CURVE: $W=0.010 \mathrm{pr} . \mathrm{cm} \mathrm{H}_{2} \mathrm{O} \quad$ SPECTRAL SLIT $(2 \mathrm{O})=1.0 \mathrm{~cm}^{-1}$
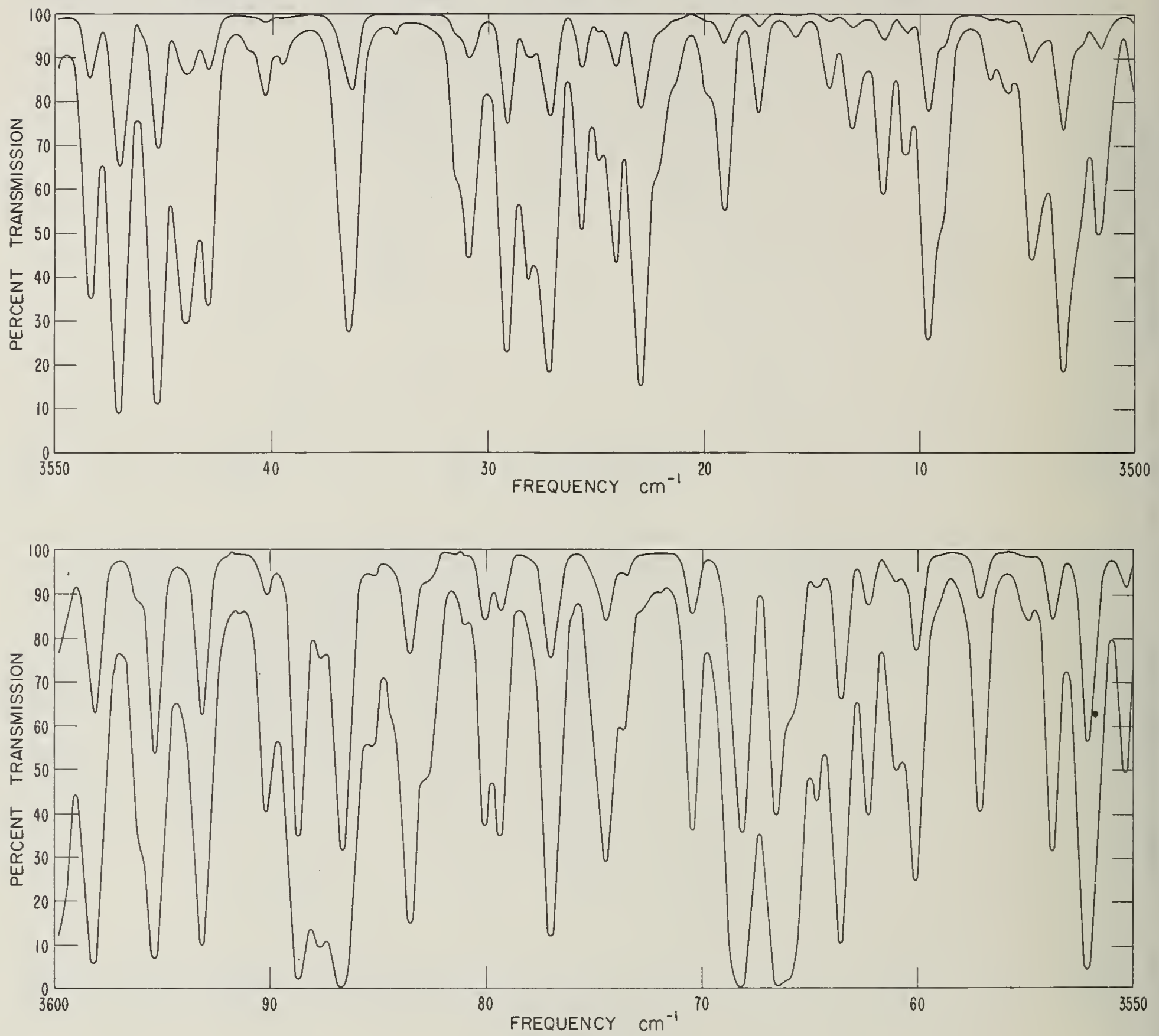

FIGURE 6. Computed degraded water vapor transmission spectra for the frequency range 8500 to $4000 \mathrm{~cm}-{ }^{1}$ at a pressure of 1.0 atmosphere, at concentrations of 0.001 and $0.01 \mathrm{~cm}$ of precipitable water vapor, for a temperature of $28 \% .7^{\circ} \mathrm{K}$, and at a full spectral slit (triangular) span of $1.0 \mathrm{~cm}-1$. 

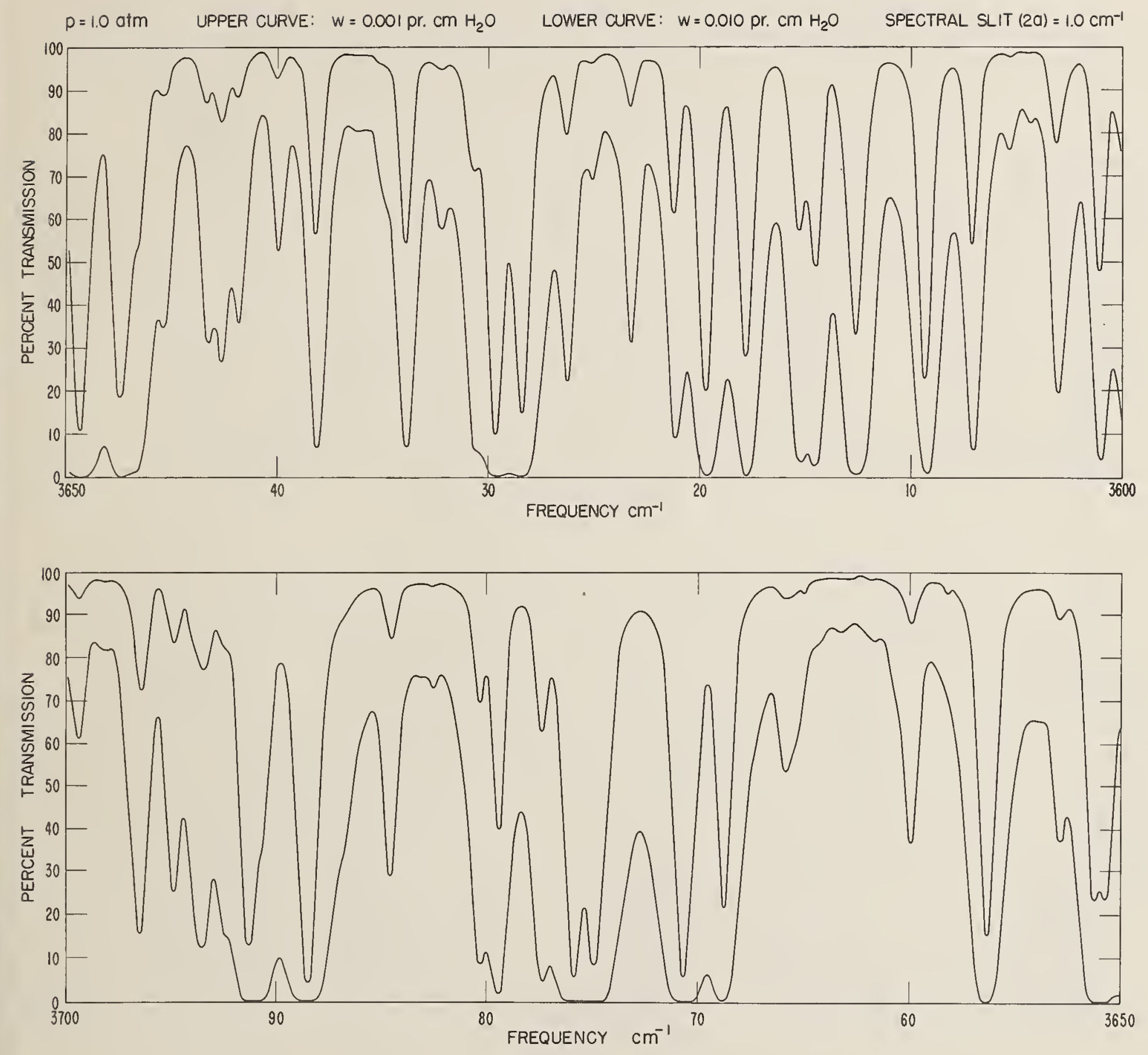

Figure 6.-Continued 

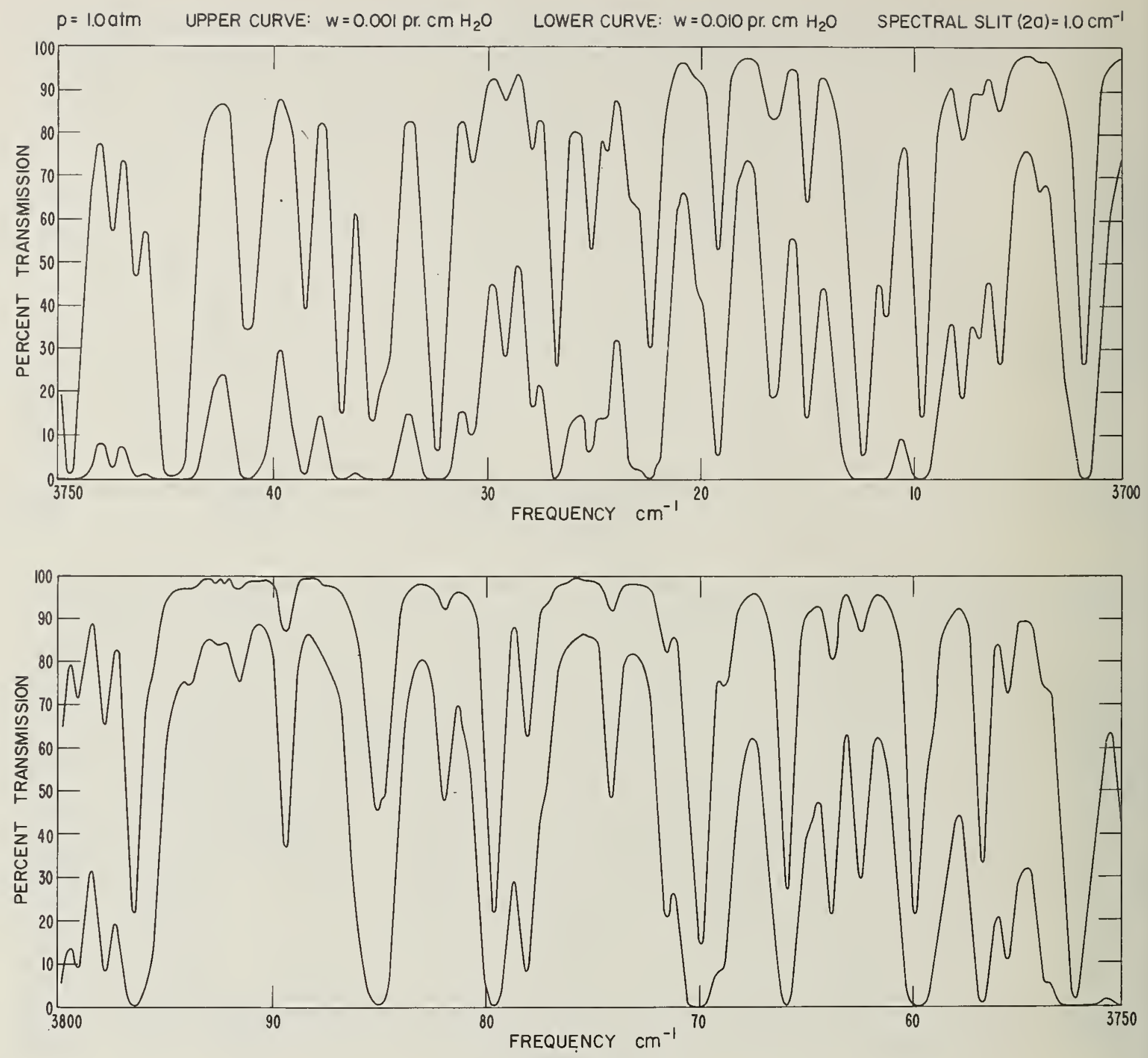

FIgURE 6.-Continued 

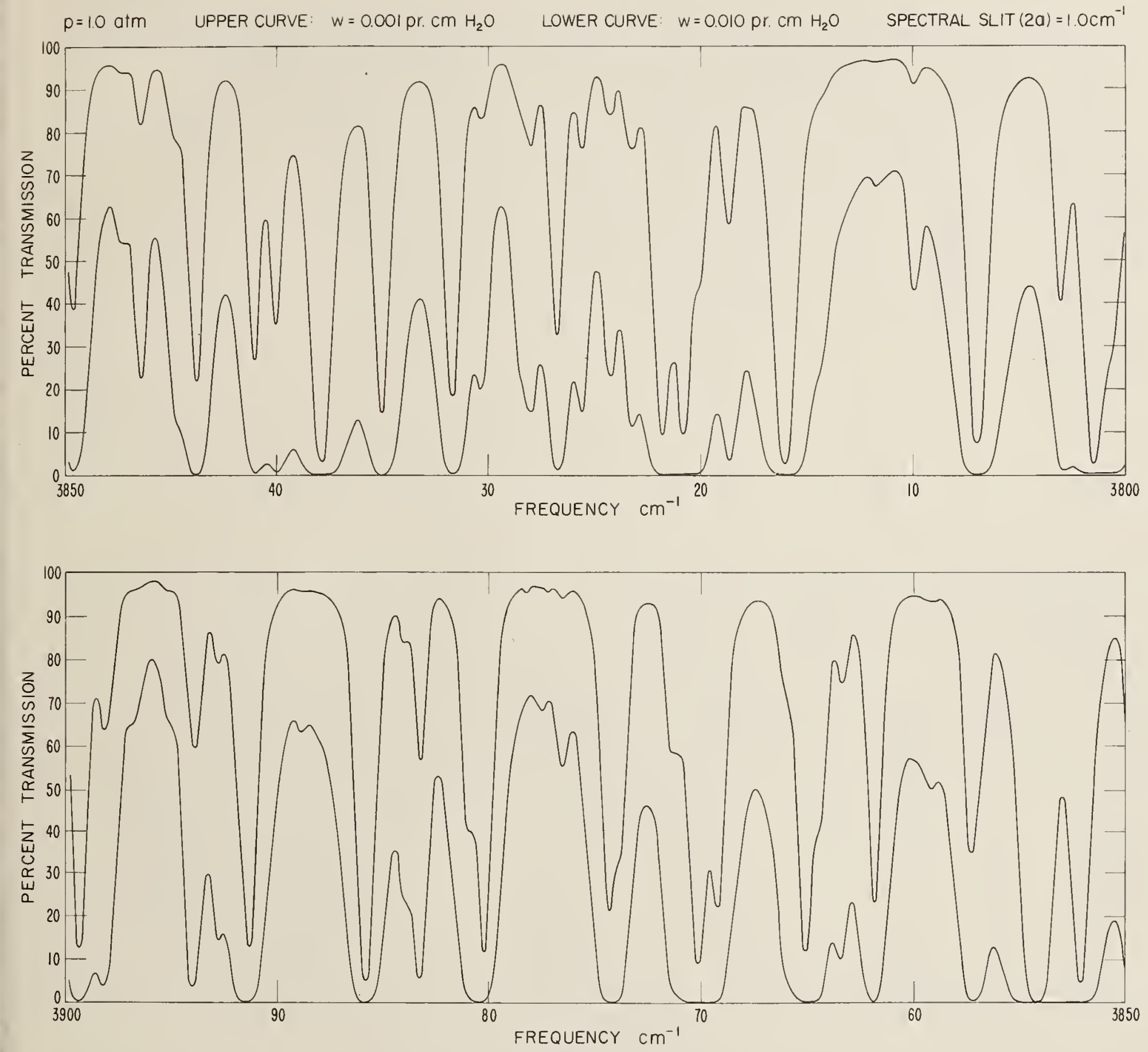

Figure 6.-Continued 

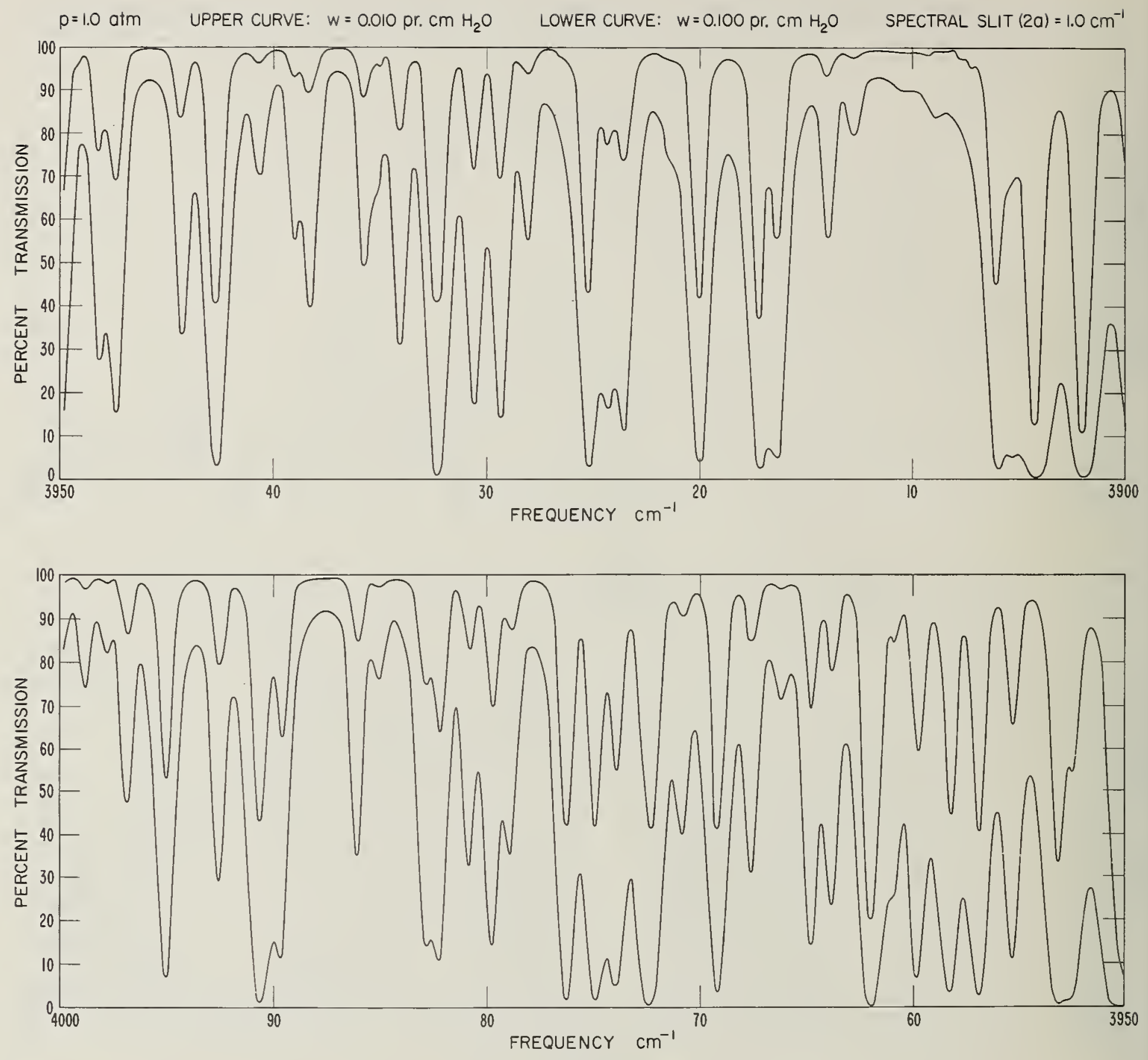

FIGURE 6.-Continued 

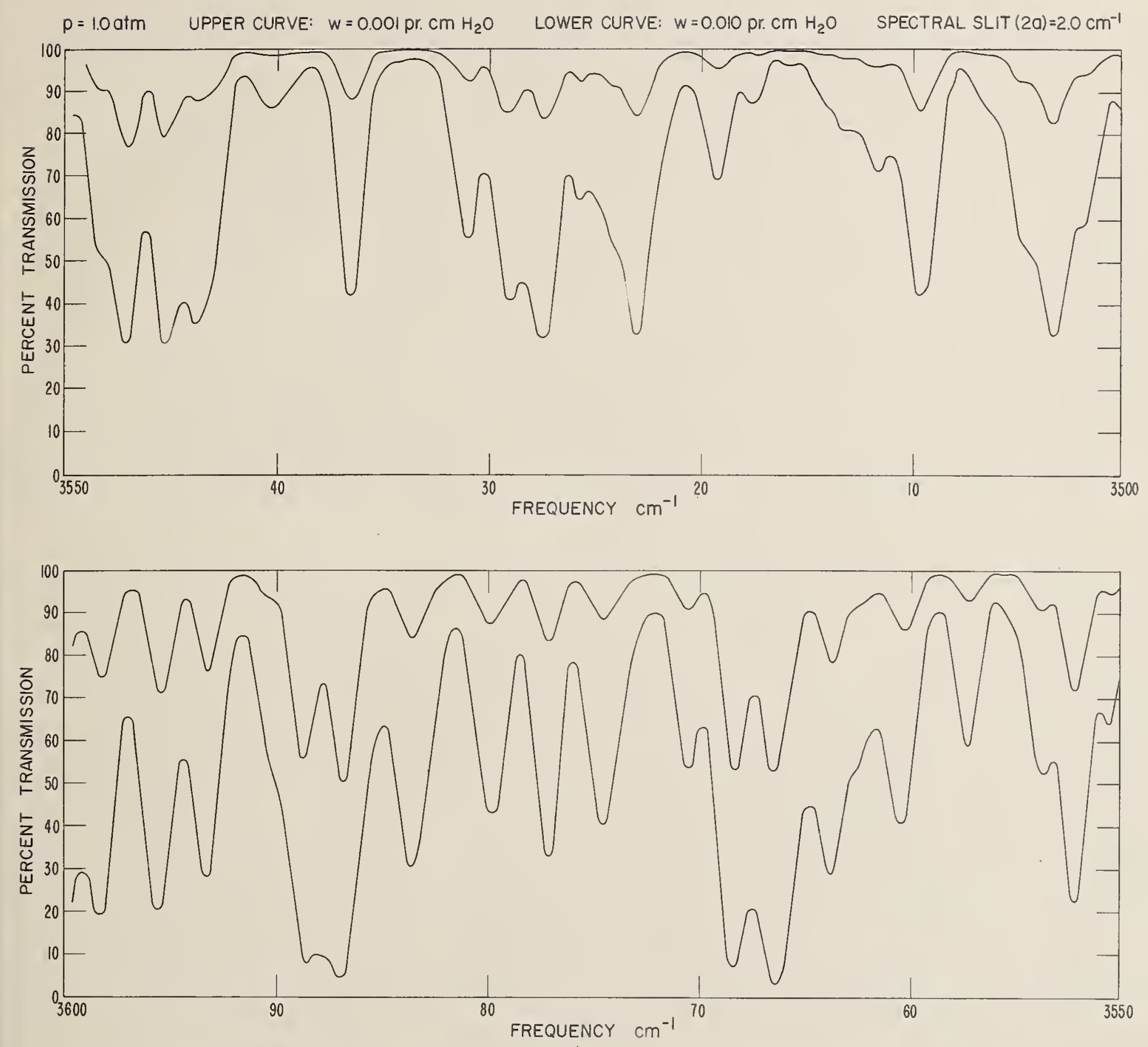

FIGURE 7. Computed degraded water vapor transmission spectra for the frequency range 3500 to $4000 \mathrm{~cm}-1$ at a pressure of 1.0 atmosphere, at concentraticns of 0.001 and $0.01 \mathrm{~cm}$ of precipitable water vapor, for a temperature of $28 \% .7{ }^{\circ} \mathrm{K}$, and at a full spectral slit (triangular) span of $2.0 \mathrm{~cm}-1$. 

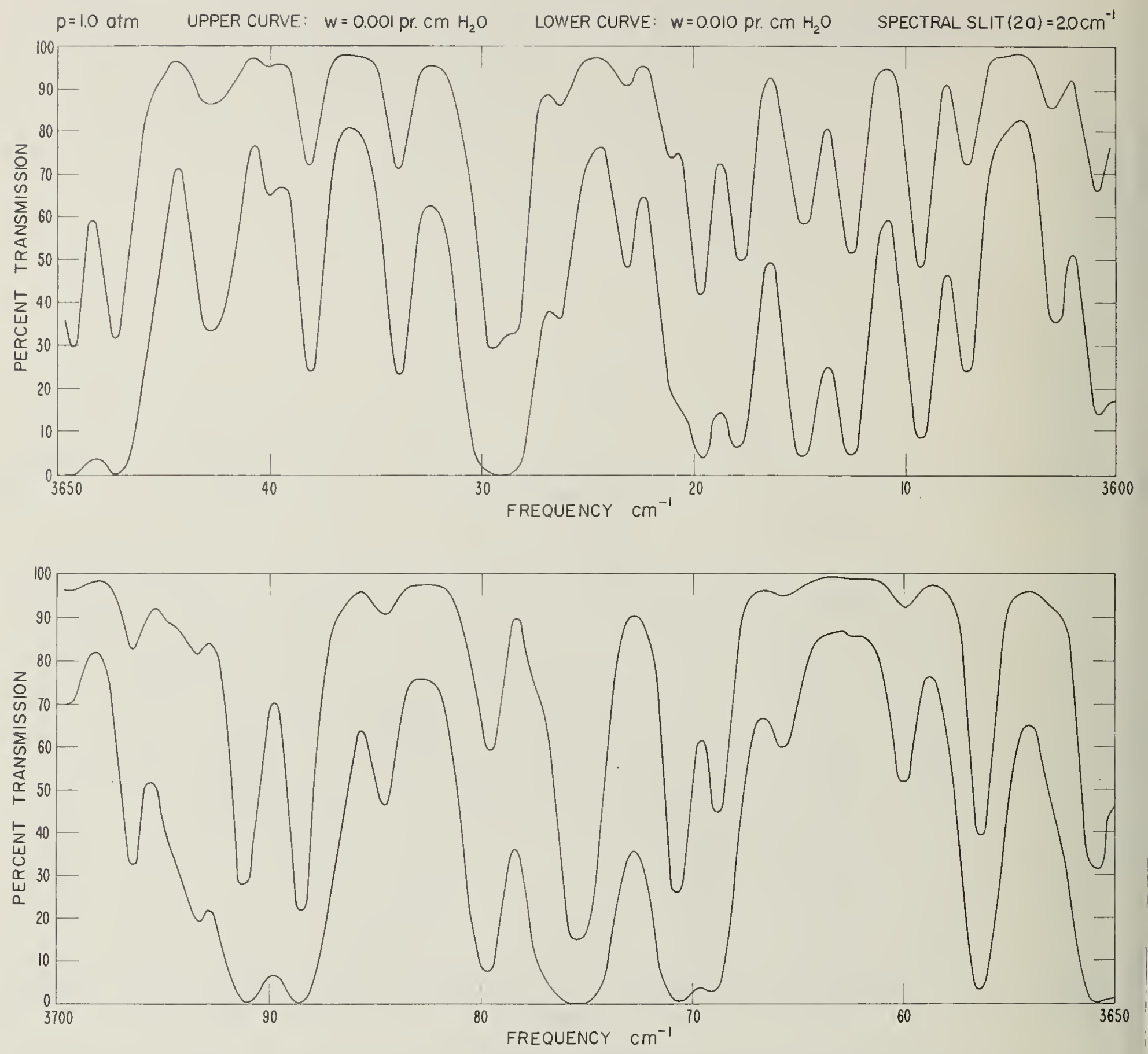

Figure 7.-Continued 

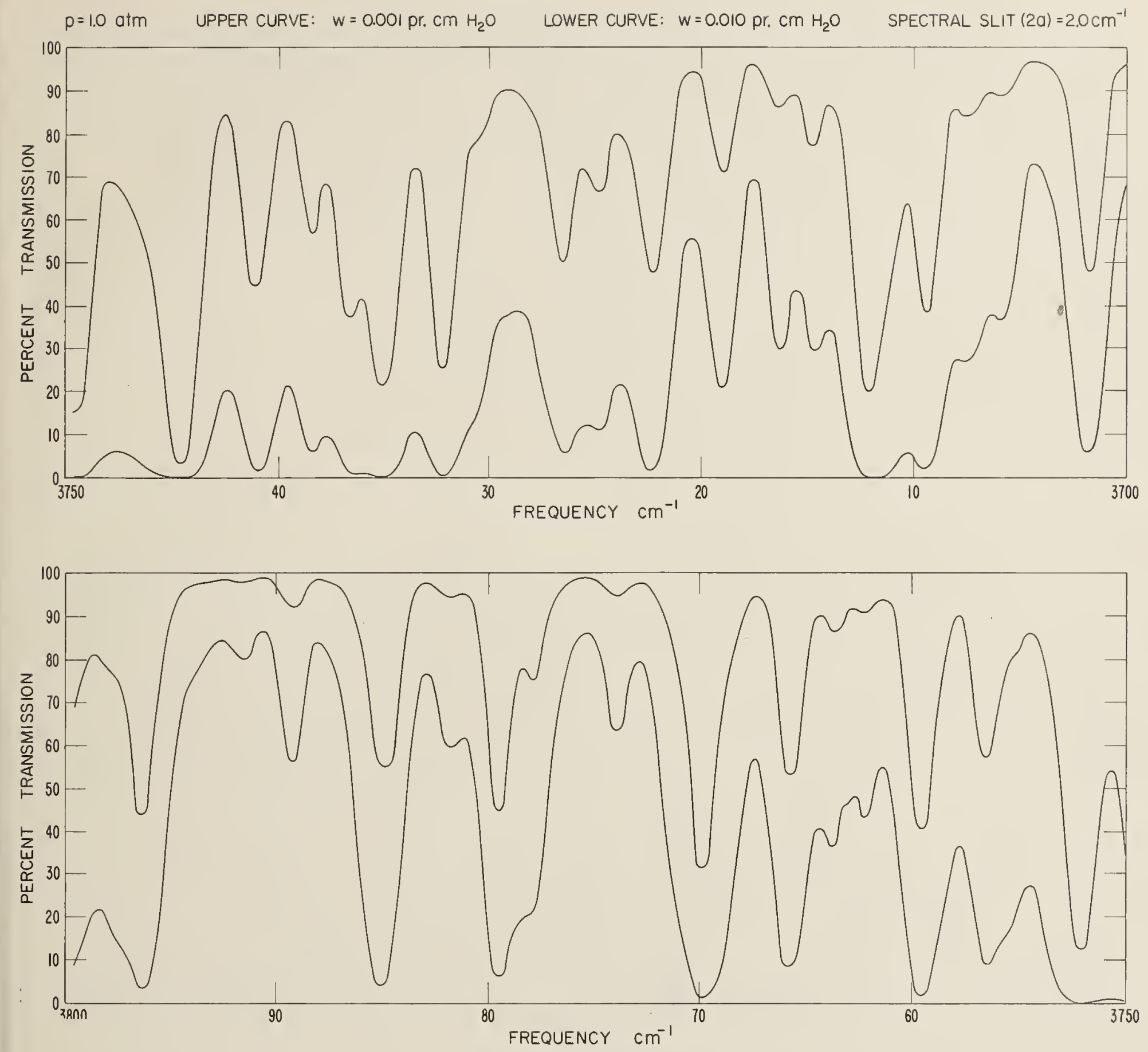

Figdre 7.-Continued 

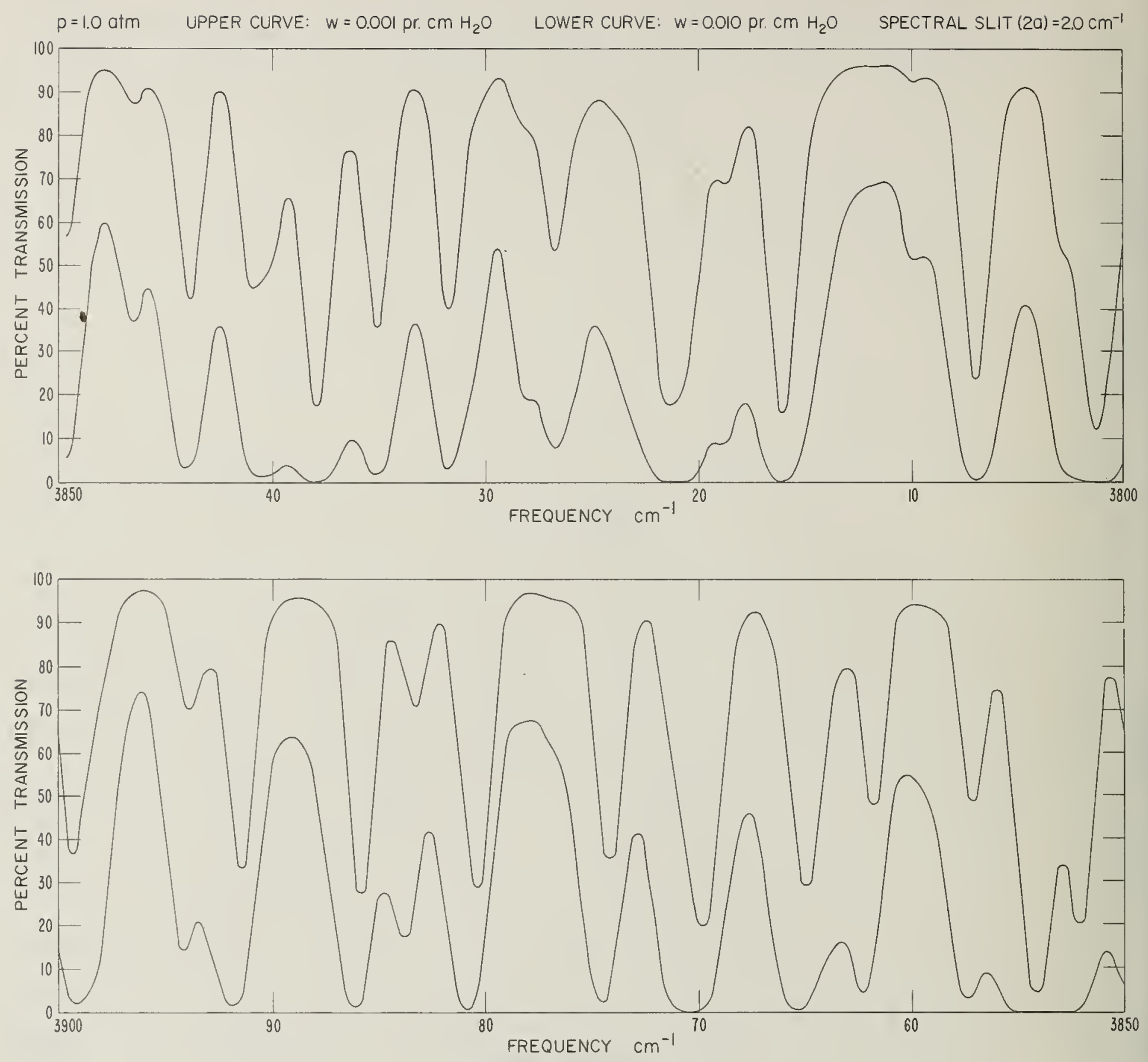

Figure 7.-Continued 

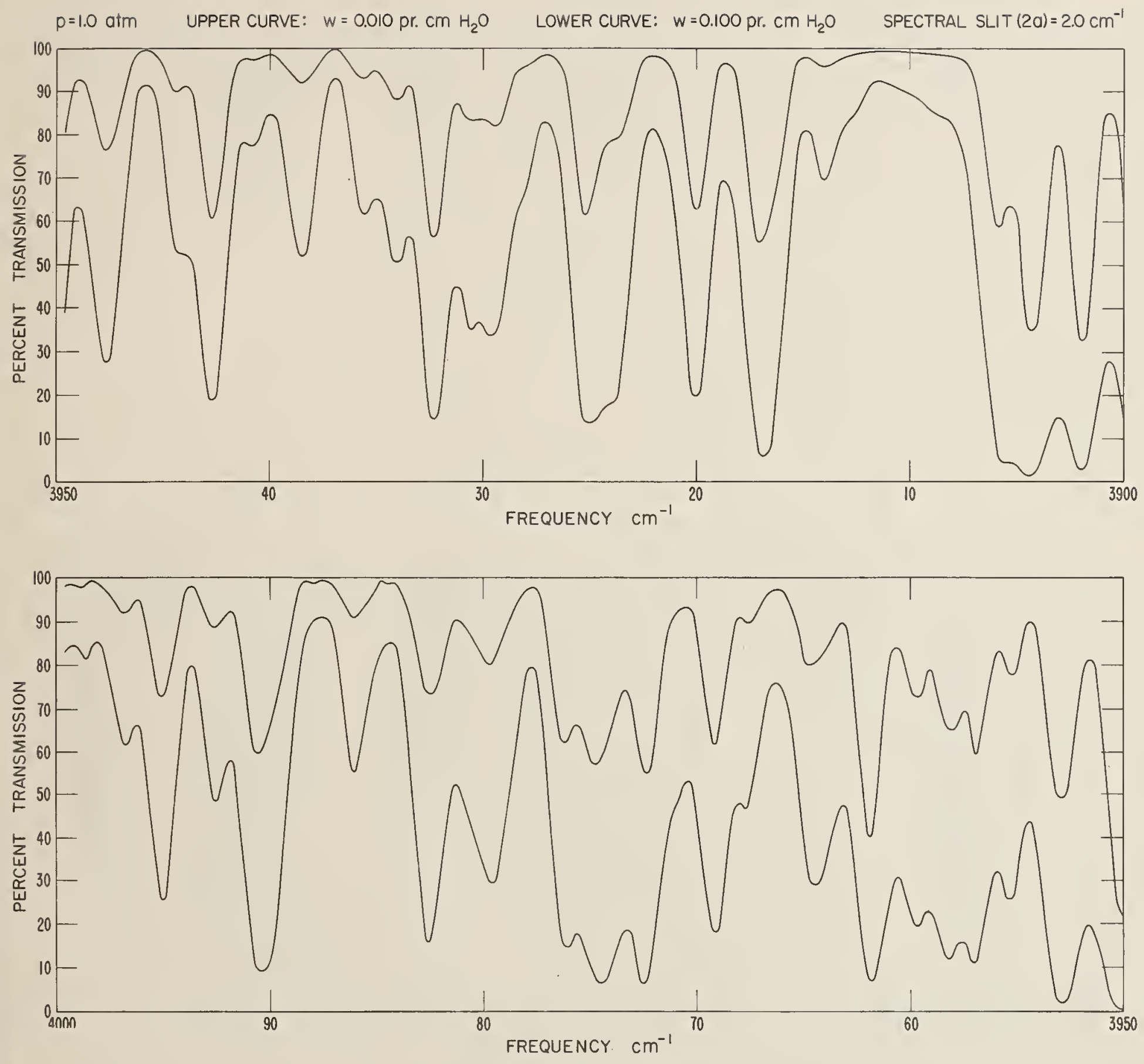

Figure 7.-Continued 

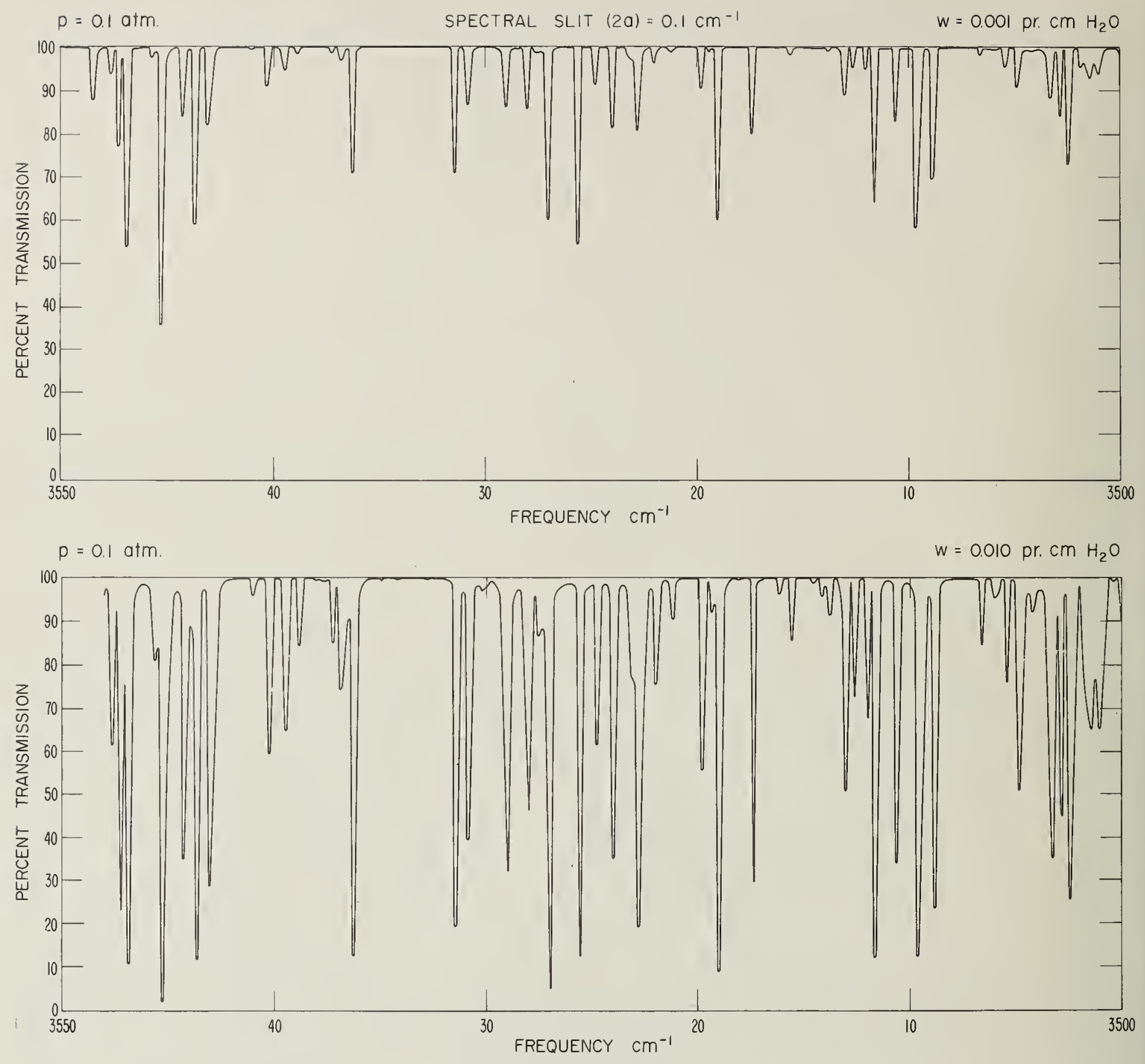

FIGURE 8. Computed degraded water vapor transmission spectra for the frequency range 3500 to $4000 \mathrm{~cm}-1$ at a pressure of 0.1 atmosphere, at concentrations of 0.001 and $0.01 \mathrm{~cm}$ of precipitable water vapor, for a temperature of $287.7^{\circ} \mathrm{K}$, and at a full spectral slit (triangular) span of $0.1 \mathrm{~cm}^{-1}$. 


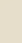





